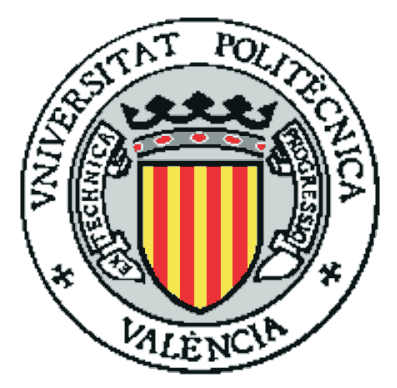

\title{
SIMULACIÓN ASISTIDA POR AGENTES PARA SISTEMAS DE FABRICACIÓN INTELIGENTES
}

Autor: Nancy Ruiz Vega

Directores: Dr. Vicente J. Botti Navarro

Dra. Adriana S. Giret Boggino

\author{
PARA LA OBTENCIÓN DEL GRADO DE \\ DOCTOR EN INFORMÁTICA \\ POR LA \\ UNIVERSIDAD POLITÉCNICA DE VALENCIA \\ Valencia, España \\ ENERO 2009
}



Fecha: Enero 2009

Autor: Nancy Ruiz Vega

Directores: $\quad$ Dr. Vicente J. Botti Navarro

Dra. Adriana S. Giret Boggino

Título: $\quad$ Simulación asistida por Agentes para Sistemas de Fabricación Inteligentes

Departamento: Sistemas Informáticos y Computación

Universidad: $\quad$ Universidad Politécnica de Valencia

Grado: Doctor $\quad$ Mes: Enero Año: 2009 

A mi familia. 



\section{Índice general}

Índice de Tablas

$\begin{array}{ll}\text { Índice de Figuras } & \text { IX }\end{array}$

Resumen $\quad$ XV

$\begin{array}{lll}\text { Abstract } & \text { XVII }\end{array}$

Resum

$\begin{array}{ll}\text { Agradecimientos } & \text { XXI }\end{array}$

1. Introducción 1

1.1. Motivación .................. 4

1.2. Objetivo ..................... 6

1.3. Estructura del Trabajo . . . . . . . . . . . . . . . . 8

I Marco Teórico. Estado del Arte. 11

2. Simulación $\quad 17$

2.1. Modelos y los Modelos de Simulación . . . . . . . . . . . . 18

2.2. El Modelado de un Sistema y el Modelado de una Organización 19

2.3. La Teoría del Modelado y la Simulación . . . . . . . . . . . . . 21

2.4. Etapas de un Estudio de Simulación . . . . . . . . . . . . . 22

2.4.1. Formulación del Problema, Objetivos y Plan de proyecto 25

2.4.2. Conceptualización del Modelo . . . . . . . . . . . . 26

2.4.3. Desarrollo de un Modelo de Simulación . . . . . . . . . 28

2.4.4. Recolección de Datos. El Modelado de las Entradas para la Simulación . . . . . . . . . . . . . . . 28 
2.4.5. Traducción del Modelo en formato Informatizado. La Simulación de Modelos. . . . . . . . . . . . . . . . 29

2.4.6. Verificación de Código del Simulador. . . . . . . . . . . 40

2.4.7. Validación del Modelo. . . . . . . . . . . . . . 40

2.4.8. Diseño de Experimentos. . . . . . . . . . . . . . . . 41

2.4.9. Análisis de los Resultados de una Simulación. . . . . . . 41

2.4.10. Documentación y Reportes. . . . . . . . . . . . . 43

2.4.11. Implementación. . . . . . . . . . . . . . . 44

2.5. Ventajas e Inconvenientes del Uso de la Simulación . . . . . . . 44

2.6. Áreas de aplicación . . . . . . . . . . . . . . . . . . . . . . 45

2.7. Conclusiones ................... . . 46

3. Los Sistemas Multiagente y la Simulación 47

3.1. Agentes y Sistemas Multiagente . . . . . . . . . . . . . . 47

3.2. Comunicación entre agentes . . . . . . . . . . . . . . 49

3.2.1. Métodos de Comunicación. . . . . . . . . . . . . . . 49

3.2.2. Detección de Situaciones de Cambios de Estado. . . . . 50

3.2.3. Negociación entre agentes. . . . . . . . . . . . . 51

3.3. El Proceso de Aprendizaje en los Sistemas Multiagente. . . . . . 55

3.4. Áreas de Aplicación de los Sistemas Multiagente . . . . . . . . 58

3.5. La Simulación y los Sistemas Multiagente . . . . . . . . . . . 61

3.5.1. El Proceso de Diseño de Simulación basada en Agentes. 63

3.5.2. Tratamiento del Entorno en la Simulación Multiagente. . 68

3.5.3. Áreas de aplicación de la Simulación guiada por Agentes. 70

3.6. Entornos de Simulación de Sistemas Multiagente . . . . . . . . 78

3.7. Conclusiones . . . . . . . . . . . . . . . . 79

4. Simulación de Sistemas de Fabricación y los Sistemas Multiagente 81

4.1. Simulación de Sistemas de Fabricación . . . . . . . . . . . . . 82

4.1.1. Sistemas de Colas y su Simulación. . . . . . . . . . . . 86

4.2. Estándares de Simulación y Fabricación . . . . . . . . . . . . . 90

4.3. Requisitos Actuales de Fabricación y la Simulación con Agentes 101

4.3.1. Posibilidades de mejora mediante MAS con respecto a los Requisitos de Fabricación . . . . . . . . . . . 102

4.4. Enfoque Holónico para Fabricación . . . . . . . . . . . . 106

4.4.1. Fabricación Virtual y los Sistemas Holónicos de Fabricación . . . . . . . . . . . . . . . . . 107

4.4.2. Sistemas Holónicos de Fabricación y los Sistemas Multiagente . . . . . . . . . . . . . 110 
4.4.3. Arquitectura de Fabricación basada en Agentes . . . . . 111

4.4.4. Arquitectura de Referencia PROSA . . . . . . . . . . 113

4.5. Características de la Simulación de Fabricación . . . . . . . . . 116

4.6. Modelo de Simulación de Fabricación . . . . . . . . . . . . . . 117

4.7. Herramientas de Simulación para Sistemas de Fabricación . . . 118

4.7.1. Clasificación de Herramientas de Simulación . . . . . . 119

4.7.2. Características de las Herramientas de Simulación . . . 124

4.7.3. Elementos clave de las Herramientas de Simulación basada en Agentes para la Fabricación . . . . . . . . . . . 125

4.7.4. Entornos de Simulación de Fabricación basadas en Agentes ...................... 126

4.8. Conclusiones . . . . . . . . . . . . . . . 126

II Marco de la Propuesta. 129

5. Modelo de la Arquitectura del Entorno de Simulación 135

5.1. Identificación y Definición de Agentes . . . . . . . . . . . . . 137

5.1.1. Características contempladas en la propuesta. . . . . . . 138

5.1.2. Identificación de Roles . . . . . . . . . . . . . . . . . . . . . . . . . . . . . . .

5.2. Funciones de Roles . . . . . . . . . . . . . . . . . . . . 143

5.3. Integración de Roles en la Simulación . . . . . . . . . . . 145

5.4. Secuencia de Ejecución . . . . . . . . . . . . . . . . . . . . . . 146

5.5. Definición de Escenarios . . . . . . . . . . . . . . . 147

5.6. Las Fases de Simulación y el Agente de Sincronización . . . . . 155

5.7. Fase I. Configuración preliminar del Modelo . . . . . . . . . . . 157

5.8. Fase I. Creación del Modelo . . . . . . . . . . . . . . . . 160

5.8.1. Detección y Control de Cambios de Estado . . . . . . . 161

5.8.2. Reglas de modelado aplicadas durante la Creación y Verificación del Modelo. . . . . . . . . . . . . . 166

5.9. Conclusiones . . . . . . . . . . . . . . . . . . 169

6. Metamodelo para la Simulación de Planta 171

6.1. Identificación de Elementos para el Metamodelo . . . . . . . . . 173

6.2. Definición de Taxonomía de los Holones del Metamodelo . . . . 181

6.3. Vinculación entre el Entorno Real y los Agentes del Metamodelo de Simulación . . . . . . . . . . . . . . . . . 208

6.4. Notación de los Elementos del Metamodelo . . . . . . . . . . . 210 
6.5. Interacciones entre el Metamodelo de la Planta de Fabricación y el Simulador . . . . . . . . . . . . . . . 210

6.6. Aprendizaje por refuerzo para los Agentes del Modelo . . . . . 211

6.7. Conclusiones . . . . . . . . . . . . . . . . . . 214

7. Arquitectura del Entorno para la Simulación de Modelos 215

7.1. Estrategias y Protocolos de Comunicación Entorno SimulaciónSistema Fabricación . . . . . . . . . . . . . . . . . 215

7.1.1. Apertura del Modelo a Simular . . . . . . . . . . . . . 218

7.1.2. Configuración preliminar en la Simulación del Modelo . 218

7.1.3. Control y Detección de Cambios de Estado . . . . . . . 219

7.1.4. Efectos de animación de Iconos . . . . . . . . . . . 223

7.1.5. Control de Avance de Tiempo . . . . . . . . . . . 226

7.1.6. Creación de Trazas . . . . . . . . . . . . . . . . 228

7.2. Estrategias y Protocolos de Comunicación durante la Simulación del Modelo . . . . . . . . . . . . . . . . . . . 229

7.2.1. Simulando el Lanzamiento de una Orden de Producción 230

7.2.2. Procesos de Negociación entre Orden de Producción y Recursos . . . . . . . . . . . . . . . . . 242

7.2.3. Tratamiento de Trabajos Aceptados por los Recursos . . 249

7.2.4. Reserva y Entrega de Materiales . . . . . . . . . . . . 249

7.2.5. Simulación de Supervisión de operaciones en el Sistema de Fabricación . . . . . . . . . . . . . . 250

7.3. Visualización de Resultados de la Simulación . . . . . . . . . 251

7.4. Exportación de Resultados de la Simulación de un Modelo . . . 253

7.5. Conclusiones . . . . . . . . . . . . . . . 254

\section{SimIShopF: Un prototipo de Entorno de Simulación soportado por} Agentes $\quad 257$

8.1. Funcionalidad del Entorno de Simulación . . . . . . . . . . 257

8.1.1. Alcance del Sistema . . . . . . . . . . . . . 260

8.1.2. Los Holones . . . . . . . . . . . . . . . . . . 269

8.1.3. Relaciones con el Entorno . . . . . . . . . . . . 291

8.1.4. La Arquitectura del Sistema . . . . . . . . . . . . 292

8.2. Implementación del Simulador . . . . . . . . . . . . . . . . . 297

8.2.1. Fase I. Creación de Modelo . . . . . . . . . . . . . . . 297

8.2.2. Fase II- Simulación de Modelo . . . . . . . . . . . . . . 305

8.3. Ontología . . . . . . . . . . . . . . . . 317

8.4. Conclusiones . . . . . . . . . . . . . . . . 319 
9.1. Evaluación del código del prototipo del Entorno de Simulación . 321

9.2. Bases para la Evaluación de Funcionalidad del entorno de Simulación ... . . . . . . . . . . . . . . . . . 323

9.2.1. Criterios de Evaluación con respecto al paradigma de Sistemas Multiagente y los requisitos de la Nueva Era de la Fabricación . . . . . . . . . . . . . . . . . . 324

9.2.2. Criterios de Evaluación del Entorno de Simulación en la Fase de Modelado . . . . . . . . . . . . . . 326

9.2.3. Criterios de Evaluación del Entorno en la fase de Simulación del Modelo . . . . . . . . . . . . . . . 328

9.3. Bases para la Evaluación de Resultados obtenidos durante la Simulación . . . . . . . . . . . . . . 330

9.3.1. Criterios para la Evaluación de Modelos . . . . . . . . . 330

9.3.2. Criterios para la Evaluación de Configuraciones de Planta previa y posterior a la Creación de Ordenes de Producción. . . . . . . . . . . . . . 333

9.3.3. Aplicación de técnicas multicriterio . . . . . . . . . . 340

9.3.4. Criterios para la Evaluación de los Resultados relacionados con Datos de Producción . . . . . . . . . . 341

9.4. Conclusiones . . . . . . . . . . . . . . . . . . 347

10. Caso de Estudio 349

10.1. Definición del Caso de Estudio . . . . . . . . . . . . . . . . 349

10.2. Simulación de la Planta Silos. . . . . . . . . . . . . . 350

10.2.1. Formulación del Problema. . . . . . . . . . . . . 350

10.2.2. Formulación del Modelo. . . . . . . . . . . . . . . 350

10.2.3. Representación y Programación del Modelo. . . . . . . 354

10.2.4. Diseño de Experimentos. . . . . . . . . . . . . . . 358

10.2.5. Simulando el Modelo. . . . . . . . . . . . . . . . 359

10.2.6. Lanzando a Simular una Orden de Producción . . . . . . 362

10.2.7. Modificando el Modelo . . . . . . . . . . . . . . 366

10.2.8. Deteniendo la Simulación . . . . . . . . . . . 367

10.2.9. Análisis de Resultados de la Simulación . . . . . . . . 368

10.2.10.Análisis de Configuraciones de Planta . . . . . . . . . . 371

10.2.11.Análisis de Tiempos Muertos por Configuraciones de Planta . . . . . . . . . . . . . 378

10.2.12.Aprendizaje de Agentes por refuerzo . . . . . . . . 382

10.3. Conclusiones . . . . . . . . . . . . . . . . . . 384 
11.1. Resultados de la Evaluación Global de la Implementación del

Prototipo . . . . . . . . . . . . . . . . 387

11.2. Resultados de la Evaluación de la Funcionalidad del Prototipo . 389

11.3. Conclusiones . . . . . . . . . . . . . . . 396

12. Conclusiones y Trabajo Futuro 401

12.1. Aportaciones . . . . . . . . . . . . . . . . 401

12.2. Líneas Futuras de Investigación . . . . . . . . . . . . . . 405

12.3. Publicaciones . . . . . . . . . . . . . . . 406

$\begin{array}{ll}\text { Bibliografía } & 411\end{array}$ 


\section{Índice de tablas}

2.1. Tipos de Mecanismos de Control de Tiempo . . . . . . . . . . 39

4.1. Elementos de un Sistema de Fabricación. . . . . . . . . . . . 83

4.2. Acciones potenciales de la unidad a su llegada. . . . . . . . . . . . 90

4.3. Programas de Simulación de Eventos Discretos . . . . . . . . 120

4.4. Programas de Simulación Geométrica . . . . . . . . . . . 123

4.5. Programas de Simulación Mixta . . . . . . . . . . . . . 123

5.1. Características de los Roles asociados al la funcionalidad del Entorno de Simulación . . . . . . . . . . . . . . . . . . . . 142

5.2. Fases de Simulación y los Roles que actúan en cada una . . . . . 145

5.3. Principales Acciones del AModelador y el ASimulador . . . . . 155

6.1. Relaciones de Tiempo entre tareas. . . . . . . . . . . . . . . 189

7.1. Tipos de Animación acorde a los Subestados de los Agentes Transformadores y el Agente de Orden de Producción. . . . . . 224

7.2. Tipos de Animación Asociado al Tipo de Elemento. . . . . . . . 225

7.3. Velocidad y Tiempos de Ejecución / Simulación. . . . . . . . . 227

7.4. Información estadística de negociaciones para una Orden. . . . . 253

8.1. Condiciones de Operación . . . . . . . . . . . . . . . . 261

8.2. Abreviaturas de las Entidades del Simulador . . . . . . . . . . 262

8.3. Iteración 1 - Objetivos del Sistema . . . . . . . . . . . 263

8.4. Esquemas Básicos acorde al tipo de Escenario . . . . . . . . . . 302

10.1. Centros de Trabajo y Elementos Asociados. . . . . . . . . . . . 352

10.2. Operarios y su grado de destreza en los Centros de Trabajo asociados. . . . . . . . . . . . . . 353

10.3. Tareas asociadas a Procesos. . . . . . . . . . . . 353

10.4. Ruta de Fabricación y Lista de Materiales de Silo. . . . . . . . . 354 
10.5. Evaluación de Modelo relacionado con el tipo de instancias . . . 358

10.6. Configuraciones utilizadas en los experimentos. . . . . . . . . 359

10.7. Carga de Trabajo de la Cuadrilla ID 43 por Tareas. . . . . . . . . 370

10.8. Carga de Trabajo de Centros de Trabajo para el Modelo PlantaSycsa1. . . . . . . . . . . . . 370

10.9. Comparativa de Carga de Trabajo en Centros de Trabajo para el Producto Semiremolque en dos Modelos de la Planta Silos. . . . 371

10.10Tipo de Instancias Seleccionada por operación . . . . . . . 372

10.11 Porcentajes de Reducción/Aumento de Tiempo de Procesamiento por Tipo de Instancias . . . . . . . . . . . . . . 372

10.12.Valoración de Tipo de Instancias de Configuraciones . . . . . . 373

10.13.Tiempo de Procesamiento Esperado de Configuraciones y porcentaje de Reducción respecto al Tiempo Estándar . . . . . . . . 374

10.14Coste de Configuraciones . . . . . . . . . . . . . . . 375

10.15Evaluación Global de Configuraciones . . . . . . . . . . . 376

10.16.Tipo de Proceso por Operación y Agentes que pueden atender las solicitudes. . . . . . . . . . . . . . . . . . 384

11.1. Resultados de Evaluación de Características relacionadas con la Creación del Modelo . . . . . . . . . . . . . . . . . . . . 392

11.2. Resultados de Evaluación de Características relacionadas con el Entorno de Ejecución de Simulación del Modelo . . . . . . . . 393

11.3. Resultados de Evaluación de Características relacionadas con la Animación y Distribución de Objetos del Modelo . . . . . . . . 394

11.4. Resultados de Evaluación de Características relacionadas con la Experimentación y Análisis de Modelos . . . . . . . . . . . . 395

11.5. Grado de Fortaleza en las Fases I y II. . . . . . . . . . . . . 396

11.6. Evaluación del Paradigma de Agentes y los requisitos de la nueva era de la Fabricación. . . . . . . . . . . . . . . . . . 397 


\section{Índice de figuras}

1.1. Identificación de Áreas Clave y sus Relaciones . . . . . . . . . 13

2.1. Entidades del Marco para el Modelado y la Simulación. . . . . . 21

2.2. Etapas de un Estudio de Simulación. . . . . . . . . . . . . 23

2.3. Actividades globales del Estudio de Simulación. . . . . . . . . . 24

2.4. Procedimiento para la Formulación del Modelo. . . . . . . . . . 26

2.5. Flujo del Desarrollo de un Modelo de Simulación . . . . . . . . 28

2.6. Modelado de las entradas de la Simulación . . . . . . . . . . . . 29

2.7. Aproximación Orientada a Eventos. . . . . . . . . . . . . . 32

2.8. Aproximación Orientada a Procesos. . . . . . . . . . . . . . 33

2.9. Aproximación Orientada a Actividades. . . . . . . . . . . . . 34

2.10. Aproximación en Tres Fases. . . . . . . . . . . . . . . 35

2.11. Control de Tiempo basado en Lista de Eventos . . . . . . . . . . 38

3.1. Simulación Dirigida por Agentes . . . . . . . . . . . . . 62

3.2. Proceso de Diseño Completo. . . . . . . . . . . . . . . . 66

3.3. Áreas que intervienen en la simulación social . . . . . . . . 71

4.1. Ejemplos de Sistemas de Colas. . . . . . . . . . . . . . . 88

4.2. Diagrama de Flujo de servicio finalizado . . . . . . . . . . . . 89

4.3. Diagrama de Flujo de cuando una unidad entra al sistema. . . . 90

4.4. Holonificación de un Sistema de Fabricación mediante la teoría de Fabricación Virtual. . . . . . . . . . . . . . . . . . 108

4.5. Diagrama de Estudio de una Simulación. . . . . . . . . . . . . 132

4.6. Elementos de la Propuesta. . . . . . . . . . . . . . . . . 133

5.1. Actividades a las que dará soporte la Arquitectura del Entorno de Simulación. . . . . . . . . . . . . . . . 136

5.2. Esquema Global de la Secuencia de Simulación y sus Agentes o roles . . . . . . . . . . . . . . . . . 146 
5.3. Esquema Global de Escenarios y sus Agentes (roles) . . . . . . 148

5.4. Subescenarios, Roles e Interacciones entre Agentes (roles) del Escenario de Edición del Modelo . . . . . . . . . . . . . . . . . 149

5.5. Agentes (roles) en los Escenarios Edición de Escenarios e Importación de Datos $(\mathrm{F} 1 \mathrm{c})$. . . . . . . . . . . . . . 150

5.6. Agentes (roles) e interacciones en los Subescenarios de la Configuración de Simulación (F2a) . . . . . . . . . . . . 151

5.7. Agentes (roles) e interacciones en los escenarios de Animación (Simulación) del Modelo (F2b) y el Análisis de los Resultados de Simulación $(\mathrm{F} 2 \mathrm{c})$. . . . . . . . . . . . . . . . 152

5.8. Agentes (roles) e interacciones en los escenarios Edición de Interfaces (F2d), Generación de Reportes y Gráficas (F2e) y Re-

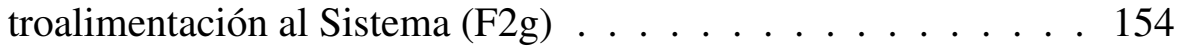

5.9. Diagrama de Cooperación entre Usuario y Agente de Sincronización . . . . . . . . . . . . . . . 156

5.10. Diagrama de Cooperación entre el Gestor de Importación / Exportación, Gestor de Iconos, el Sincronizador y el Usuario durante la Importación de Datos. . . . . . . . . . . . . 158

5.11. Diagrama de Cooperación entre el AGestorIconos y el Usuario durante la Creación de instancias para un Modelo. . . . . . . . . 160

5.12. Diagrama de Estados del Sistema durante la Fase I. . . . . . . . 161

5.13. FASE I. Diagrama de Cooperación entre el AModelador(sus roles), el AGestorIconos y el Usuario durante la Creación de Modelos . . . . . . . . . . . . . . . . . 162

5.14. Diagrama de Cooperación entre los Agentes durante la Creación del Modelo . . . . . . . . . . . . . . . . . . 167

6.1. Diagrama de Estudio de una Simulación. . . . . . . . . . . . . . 172

6.2. Áreas consideradas en la Definición del Metamodelo. . . . . . . 174

6.3. Principales Relaciones entre los Holones . . . . . . . . . . . . . 182

6.4. Entidades Básicas del Modelo . . . . . . . . . . . . . . . 183

6.5. Metamodelo del Holón Producto . . . . . . . . . . . . . . . . 184

6.6. Metamodelo del Holón ARecurso . . . . . . . . . . . . . . . . 192

6.7. Metamodelo del Holón AOrden . . . . . . . . . . . . . 200

6.8. Metamodelo del Holón Staff. . . . . . . . . . . . . . . . . . 207

6.9. Elementos de la Notación Gráfica del Modelo a Simular. . . . . 210

7.1. Estados del Sistema durante la Fase II . . . . . . . . . . . . 220 
7.2. Diagrama de Cooperación entre el Agente de Simulación (sus roles) y su Entorno durante la Simulación del Modelo . . . . . . 222

7.3. Estados generales de los Agentes . . . . . . . . . . . . . 223

7.4. Subestados de un Transformador . . . . . . . . . . . . . 223

7.5. Subestados de una Orden de Producción . . . . . . . . . . . . . 224

7.6. Sincronización de Relojes. . . . . . . . . . . . . . . . . . . . 228

7.7. Protocolo de Negociación entre Orden de Producción y Recursos. 242

7.8. Protocolo FIPA Contract Net . . . . . . . . . . . . . . . . 244

7.9. Diagrama de Cooperación entre el Agente de Reportes/gráficas y su Entorno durante la Visualización de Reportes y Gráficas . . 252

7.10. Diagrama de Cooperación entre el Agente de Importación/Exportación y su Entorno durante la Exportación de Resultados de la Simulación de un Modelo . . . . . . . . . . . . . . . . . . . 254

8.1. Procesos del Sistema -Parte 1- . . . . . . . . . . . . . 258

8.2. Procesos del Sistema -Parte 2- . . . . . . . . . . . . 260

8.3. Iteración 1 - Diagrama de Casos de Uso . . . . . . . . . . . 262

8.4. Iteración 1 - Diagrama de Organización . . . . . . . . . . . . 264

8.5. Iteración 1 - Diagrama de Interacción . . . . . . . . . . . 266

8.6. Iteración 1 - Tareas Abstractas en el Modelo de Organización . . 267

8.7. Diagrama de Tareas y Objetivos . . . . . . . . . . . . . 268

8.8. Iteración 1 - Agentes Abstractos y Modelo de Organización . . . 269

8.9. Modelo de Holon de Gestión de Importación/Exportación HGIE 270

8.10. Diagrama de Tareas y Objetivos así como de las Tareas y Creencias del Holón de Gestión de Importación/Exportación . . . . 270

8.11. Iteración 1 - Modelo de Holon de Análisis HANA . . . . . . . . 271

8.12. Iteración 1 - Diagrama de Tareas y Objetivos así como de las Tareas y Creencias del Holón de Análisis . . . . . . . . . . 271

8.13. Iteración 1 - Modelo de Holon de Iconos HICO . . . . . . . . . 272

8.14. Iteración 1 - Diagrama de Tareas y Objetivos así como de las Tareas y Creencias del Holón de Iconos . . . . . . . . . . . 272

8.15. Iteración 1 - Modelo de Agente del Holón de Interface . . . . . 273

8.16. Iteración 1 - Modelo de Holón Modelador HMOD . . . . . . . . 274

8.17. Iteración 1 - Diagrama de Tareas y Objetivos así como de las Tareas y Creencias del Holón Modelador . . . . . . . . . . . . 274

8.18. Iteración 1 - Modelo de Holón de Velocidad HVEL . . . . . . . 275

8.19. Iteración 1 - Diagrama de Tareas y Objetivos así como de las Tareas y Creencias del Holón de Velocidad . . . . . . . . . . . 275 8.20. Iteración 1 - Modelo de Holón de Animación HANI . . . . . . . 276 
8.21. Iteración 1 - Diagrama de Tareas y Objetivos así como de las Tareas y Creencias del Holón de Animación . . . . . . . . . . . 276

8.22. Iteración 1 - Modelo de Holón Gestor de Eventos HEVE . . . . 277

8.23. Iteración 1 - Diagrama de Tareas y Objetivos así como de las Tareas y Creencias del Holón de Eventos . . . . . . . . . . . . 277

8.24. Iteración 1 - Modelo de Holón de Resultados HRES . . . . . . . 278

8.25. Iteración 1 - Diagrama de Tareas y Objetivos así como de las Tareas y Creencias del Holón de Resultados . . . . . . . . . . . 278

8.26. Iteración 1 - Modelo de Holón de Sincronización HSIN . . . . . 279

8.27. Iteración 1 - Diagrama de Tareas y Objetivos así como de las Tareas y Creencias del Holón de Sincronización . . . . . . . . 279

8.28. Iteración 1 - Modelo de Holón de Simulación HSIM . . . . . . . 280

8.29. Iteración 1 - Diagrama de Tareas y Objetivos así como de las Tareas y Creencias del Holón de Simulación . . . . . . . . . . . 280

8.30. Iteración 1 - Modelo de Agente del Holón de Orden de Importación 281

8.31. Iteración 1 - Modelo de Agente del Holón de Orden de Modelo . 282

8.32. Iteración 1 - Modelo de Agente del Holón de Orden de Configuración . . . . . . . . . . . . . . . . 282

8.33. Iteración 1 - Modelo de Agente del Holón de Orden de Evento . 283

8.34. Iteración 1 - Modelo de Agente del Holón de Orden de Reporte/Gráfica . . . . . . . . . . . . . . . 283

8.35. Iteración 1 - Modelo de Agente del Holón de Orden de Exportación 284

8.36. Iteración 1 - Modelo de Agente del Holón de Modelo . . . . . . 285

8.37. Iteración 1 - Diagrama de la Interacción Gestionar la Importación de Datos Reales . . . . . . . . . . . . . . . . . . 286

8.38. Iteración 1 - Diagrama de la Interacción Creación de Modelo . 287

8.39. Iteración 1 - Diagrama de la Interacción Configuración de Simulación de Modelo . . . . . . . . . . . . . . . . . . . . . 288

8.40. Iteración 1 - Diagrama de la Interacción Simulación del Modelo 290

8.41. Iteración 1 - Diagrama de la Interacción Análisis de Resultados . 291

8.42. Iteración 1 - Modelo de Entorno del Holon GIE . . . . . . . . . 292

8.43. Plantilla JADE- Producto . . . . . . . . . . . . . . . . 293

8.44. Plantilla JADE- Transformador . . . . . . . . . . . . . . . . . . 294

8.45. Plantilla JADE- Modelador . . . . . . . . . . . . . . . . . 295

8.46. Plantilla JADE- Holón de Simulación . . . . . . . . . . . . . . 296

8.47. Agentes activos en la Fase I y relación entre el entorno de agentes modelado y la Planta física . . . . . . . . . . . . . . . 298

8.48. Definición de un Producto . . . . . . . . . . . . . . . . . . . . 299

8.49. Reutilización de Modelos . . . . . . . . . . . . . . . . . 300 
8.50. Notación Gráfica de Elementos . . . . . . . . . . . . . . . . 301

8.51. Seleccionando esquemas de escenarios . . . . . . . . . . . 301

8.52. Escenarios en un Modelo . . . . . . . . . . . . . . . . . . 303

8.53. Asignación de Recursos en el Escenario de Corte . . . . . . . . 304

8.54. Definiendo el Comportamiento de un Recurso . . . . . . . . . . 305

8.55. Interfaz de Apertura de Modelo y su representación gráfica . . . 306

8.56. Interfaz de Definición de Condición de Parada y Velocidad . . . 307

8.57. Interfaz de Lanzamiento de Orden de Producción y su representación gráfica . . . . . . . . . . . . . . . . . . . 308

8.58. Interfaz para la Visualización de Datos de un Recurso . . . . . . 309

8.59. Interfaz para la Definición del Tipo de Animación de un Operario 310

8.60. Interfaz para la Visualización del Progreso de una Orden de Pro-

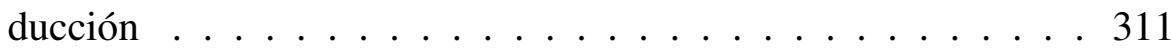

8.61. Interfaz de Visualización de Datos de un Semiproducto . . . . . 311

8.62. Interfaz de Visualización de finalización y calificación de Orden de Producción . . . . . . . . . . . . . . . . . 312

8.63. Interfaz de Visualización de Datos de Producto Final . . . . . . 313

8.64. Agentes de Escenarios de Corte Negociando op 10 con la Orden de Producción . . . . . . . . . . . . . . . . . . . 314

8.65. Agentes de Corte procesando op 10, Agentes de Soldadura procesando op 20 y Agentes de Moldeo negociando op 30 con la Orden de Producción . . . . . . . . . . . . . . . . . . 314

8.66. Interfaz para la solicitud de Reportes/Gráficas . . . . . . . . 315

8.67. Interfaz de Control de Ejecución de Simulación y comportamiento de Agentes al detenerla . . . . . . . . . . . . 316

8.68. ConfigurarElementoMod . . . . . . . . . . . . . 317

8.69. ConfigurarElementoMod . . . . . . . . . . . . 318

9.1. Diagrama de Estudio de Simulación. . . . . . . . . . . . . 326

10.1. Distribución de Planta de Fabricación. . . . . . . . . . . . . 351

10.2. Opciones para añadir Elementos al Modelo. . . . . . . . . . . . . . 355

10.3. Definiendo Relaciones Operario-Máquina. . . . . . . . . . . . . 355

10.4. Asignando Instancia a Icono de Modelo. . . . . . . . . . . . 356

10.5. Creando Instancia en Base de Datos. . . . . . . . . . . . 356

10.6. Modelo de la Planta. . . . . . . . . . . . . . . . . 357

10.7. Abriendo el Modelo. . . . . . . . . . . . . . . . . . . 360

10.8. Agentes activos relacionados con el Modelo. . . . . . . . . . . . 361

10.9. Definiendo Condición de Parada y Velocidad de Simulación. . . 361 
10.10Sincronización del Reloj del Agente Simulador y los Relojes de los Agentes de la Planta de Fabricación. . . . . . . . . . . . . 362

10.11 Lanzando una Orden de Producción. . . . . . . . . . . . . . . . 363

10.12Negociación entre Agente de Orden de Producción y Agentes de

Operarios. . . . . . . . . . . . . . . . 364

10.13Animación Gráfica de Negociación entre Agentes. . . . . . . . . 365

10.14Datos del Agente y datos de su configuración de animación . . . 366

10.15Elementos agregados en el Centro de Trabajo S26-Soldadura. . . 367

10.16Deteniendo la Simulación. . . . . . . . . . . . . . . . 368

10.17Proceso de Negociación y Ruta de Producción de Orden 225. . . 369

10.18Comparativa de Configuraciones acorde al Tipo de Instancias . . 373

10.19.Comparativa de Configuraciones acorde al Tiempo de Procesa-

miento . . . . . . . . . . . . . . . . 374

10.20.Comparativa de Configuraciones acorde al Coste . . . . . . . 376

10.21.Comparativa de Calificación Global de Configuraciones . . . . . 377

10.22 Comparativa de Uso de Recursos con Diferentes Configuraciones para 5 Ordenes . . . . . . . . . . . . . 378

10.23Distribución de Carga de Trabajo mediante Asignación directa para cinco órdenes . . . . . . . . . . . . . . . . . . 379

10.24Distribución de Carga de Trabajo mediante Negociación para cinco órdenes . . . . . . . . . . . . . . . . . . 380

10.25Distribución de Carga de Trabajo mediante Asignación y Negociación para cinco órdenes . . . . . . . . . . . . . . 381 


\section{Resumen}

En el área de la fabricación, la simulación es una herramienta esencial para la validación de métodos y arquitecturas antes de aplicarlos en un Entorno de Fabricación. Las herramientas de simulación actuales llevan a cabo la simulación de entornos de fabricación basándose en modelos estáticos que hacen uso de la programación de procesos de fabricación secuenciales y centralizados tradicionales, donde los mecanismos de planificación y control ofrecen una flexibilidad insuficiente para responder a los estilos de fabricación cambiantes y a los entornos de fabricación altamente mezclados y de bajo volumen. En consecuencia, las herramientas de simulación convencionales limitan la escalabilidad y reconfigurabilidad para el modelado de Sistemas de Fabricación que permitan adaptarlos ante las necesidades cambiantes del Cliente. Resulta difícil encontrar una herramienta de simulación que pueda ejecutar "inteligentemente" la simulación de tareas cada vez más complejas. La dificultad radica en integrar en la herramienta el conocimiento necesario del sistema original y que a la vez actúe como un asistente que proporcione consejos y guíe al usuario durante la simulación. Por ello, surge la necesidad de nuevas herramientas de simulación para fábricas que contemplen características tales como: a) flexibilidad y adaptabilidad, para modelar comportamientos complejos propios de un Sistema de Fabricación, b) escalabilidad para la integración transparente de funcionalidades adicionales, c) proactividad y reactividad para la adaptación automática ante los cambios del entorno y d) características de aprendizaje (inteligencia) basado en la experiencia adquirida durante la simulación.

Por su parte, las técnicas de Inteligencia Artificial han sido utilizadas en la Fabricación Inteligente por más de dos décadas. Las técnicas del área de Inteligencia Artificial permiten la definición de unidades de fabricación distribuidas, autónomas, inteligentes, flexibles, tolerantes a fallos y reutilizables, las cuales operan como un conjunto de entidades que cooperan entre sí. Además, los recientes desarrollos en el área de los Sistemas Multiagente han traído consigo 
nuevas e interesantes posibilidades. Algunos investigadores han aplicado la tecnología de agentes en la integración de la fabricación empresarial, la colaboración, la planificación de procesos de fabricación, la programación para el control de planta, el manejo de materiales y la gestión de inventarios, así como la implementación de nuevos tipos de sistemas de fabricación tales como los Sistemas de Fabricación Holónicos. Teniendo en cuenta estas aplicaciones exitosas de los Sistemas Multiagente en la Fabricación Inteligente, estamos convencidos de que esta tecnología puede mejorar también el desempeño de la Simulación de Sistemas de Fabricación Inteligente.

En esta tesis proponemos la definición de una Arquitectura para un Entorno de Simulación de Sistemas de Fabricación asistido por agentes. Esta arquitectura integra la funcionalidad de una herramienta de simulación tradicional, permite además la simulación de comportamientos complejos asociados a los Sistemas de Fabricación Inteligentes y proporciona soluciones y mejoras que se adaptan a las necesidades de la nueva era de fabricación. Por tanto, la arquitectura se centra en la mejora de actividades del proceso global de la simulación que incluyen: a) asistencia durante la representación y programación de modelos de sistemas de fabricación, b) aportar flexibilidad para plantear las hipótesis y el diseño de experimentos, c) la simulación del modelo, d) aporta métricas para la evaluación de modelos, configuraciones de planta y datos de producción que sirven de ayuda para la toma de decisiones, y e) la validación de los resultados contra la hipótesis. Asimismo, se propone un Metamodelo soportado por agentes que da soporte a la Arquitectura durante la Representación y Programación de Modelo de Sistemas de Fabricación Inteligentes. El metamodelo ofrece flexibilidad para la definición de modelos con un amplio rango de variantes que permiten diseñar experimentos que consideran los requisitos de la nueva fabricación. Mediante el diseño individual de los elementos del sistema de fabricación es posible la definición de comportamientos complejos para Sistemas de Fabricación reales. El metamodelo ofrece la posibilidad de integrar patrones para la definición de las interacciones entre las órdenes de producción y los recursos de fábrica durante la asignación de tareas. De este modo, la Arquitectura utiliza el metamodelo para facilitar el diseño de Modelos Simulación de Sistemas de Fabricación soportados por agentes y la animación de estos modelos. Finalmente, con el fin de validar la arquitectura y el metamodelo se ha implementado un prototipo que ofrece interfaces que dan soporte a la creación y simulación de modelos. El prototipo se ha utilizado para la simulación de un caso de estudio: un sistema de fabricación de silos. 


\section{Abstract}

In the area manufacturing area, the simulation is a essential tool for the validation of methods and architectures before apply them in a manufacturing environment. Current Simulation Tools conduct simulation of manufacturing environments based on static models that make use of the traditional sequential and centralized programming for manufacturing processes, where the mechanisms of planning and control offer insufficient flexibility to respond to the manufacturing styles that change continually and manufacturing environments highly mixed and low volume. Consequently, conventional simulation tools limit the scalability and reconfigurability for manufacturing systems modeling that allow to adapt models to the changing needs of the client. It is difficult to find a simulation tool that can "intelligently" execute simulation tasks more and more complex. The difficulty lies in the inclusion of the knowledge managed by the original system in the tool and that the tool acts as a wizard that provides advices and guides the User during the simulation. Therefore, the need for new simulation tools for factories emerges in order to cover features such as: a) flexibility and adaptability, to model complex behaviors of a manufacturing system, b) scalability for the inclusion of additional functionality, c) proactive and reactivity to the automatical adaptation faced with the environment changes, and d) learning features (intelligence) based on the experience gained during the simulation.

The artificial intelligence techniques have been used for the intelligent manufacturing for more than two decades. The techniques of the artificial intelligence area allow the definition of manufacturing entities that are distributed, autonomous, intelligent, flexible, fault tolerant and reusable, which operate as a set of entities that work together. In addition, the recent developments in the Multiagent Systems area have brought with them new and interesting possibilities. Some researchers have applied agent technology in the integration of the business manufacturing, collaboration, planning of manufacturing processes, the scheduling for shop floor control, material handling and inventory management, 
as well as the implementation of new types of Manufacturing Systems such as Holonic Manufacturing Systems. Taking into account these successful applications of the Multi-agent Systems in Intelligent Manufacturing, we are convinced that this technology can also improve the performance of the Intelligent Manufacturing Systems Simulation.

In this thesis we propose the definition of an architecture for a Simulation Environment for Manufacturing Systems supported by agents. This architecture integrates the functionality of a traditional simulation tool, it also enables the simulation of complex behaviors linked to Intelligent Manufacturing Systems and provides solutions and improvements that are adapted to the requirements of the new era of manufacturing. Therefore, the architecture focuses on improving tasks of the global simulation process that include: a) assistance during the representation and programming models of Manufacturing Systems, b) provide flexibility to set out the scenarios (hypothesis) and the experiment designs, c) the model simulation, d) provides metric set for the evaluation of models, shop floor configuration and production data in order to help the User during decisionmaking process, and e) the validation of the results against the hypothesis.

Also, it is proposed a Metamodel supported by agents that supports to the Architecture during the design and the programming of Models of Intelligent Manufacturing Systems. The Metamodel offers flexibility to the definition of models with a wide range of variants that allows the User to design experiments that take into account the requirements of the new manufacturing era. Through the individual design of the system entities it is possible to define complex behaviors of real Manufacturing Systems. The Metamodel offers the possibility to include patterns to define the interactions among the Production Orders and Factory Resources during the allocation of tasks. In this way, the architecture uses the Metamodel to facilitate the design of Simulation Models of Manufacturing Systems supported by agents and the graphical animation of these models. Finally, to validate the architecture and the Metamodel has been implemented a prototype that provides easy to use interfaces that provide support to the creation and simulation models. The prototype has been used for the simulation of a study case: a manufacturing system for silos (a product container). 


\section{Resum}

En l'àrea de la fabricació, la simulació és una eina fonamental per a la validació de mètodes i arquitectures abans d'aplicar-los dins d'un Entorn de Fabricació. Les eines de simulació actuals duen a terme la simulació d'entorns de fabricació basant-se en models estàtics que fan ús de la programació de processos de fabricació seqüencials i centralitzats tradicionals, on els mecanismes de planificació i control ofereixen una flexibilitat insuficient per a donar suport als estils de fabricació canviants i als entorns de fabricació altament barrejats i de baix volum. Com a conseqüència, les eines de simulació convencionals limiten l' escalabilitat i reconfigurabilitat per al modelat de Sistemes de Fabricació que permeten la seua adaptació front a les necessitats canviants del Client. Resulta difícil trobar una eina de simulació que puga executar "intel-ligentment"la simulació de tasques cada vegada més complexes. La dificultat radica en integrar en l'eina el coneixement necessari del sistema original i que alhora actue com a un assistent que proporcione consells y guie el procés de simulació. Per això, sorgeix la necessitat de noves eines de simulació per a fàbriques que contemplen noves característiques tals com: a) flexibilitat i adaptabilitat, per modelar comportaments complexos propis d'un Sistema de Fabricació, b) escalabilitat per a la integració transparent de funcionalitats addicionals, c) proactivitat i reactivitat per a l'adaptació automàtica davant els canvis de l'entorn i d) característiques d'aprenentatge (intel-ligència) basat en l'experiència adquirida al llarg de la simulació.

D'altra banda, les tècniques de la Intel-ligència Artificial han sigut emprades en la Fabricació Intel-ligent durant més de dues dècades. Les tècniques de l'àrea de la Intel-ligència Artificial permeten la definició d'unitats de fabricació distribuïdes, autònomes, intel-ligents, flexibles, tolerants a fallades i reutilitzables, les quals operen com a un conjunt d'entitats que cooperen entre sí. A més, els recents desenvolupaments dins de l'àrea dels Sistemes Multiagent han dut amb ells noves i interessants possibilitats. Alguns investigadors han aplicat la tecnologia 
d'agents a la integració de la fabricació empresarial, la col·laboració, la planificació de processos de fabricació, la programació per al control de planta, el maneig de materials i la gestió d inventaris, així com la implementació de nous tipus de sistemes de fabricació tals com els Sistemes de Fabricació Holònics. Tenint en compte aquestes aplicacions reeixides dels Sistemes Multiagent en la Fabricació Intel-ligent, estem convençuts de que amb aquesta tecnologia es pot millorar també l'acompliment de la Simulació dels Sistemes de Fabricació Intel-ligent.

En aquesta tesi proposem la definició d'una Arquitectura per a un entorn de Simulació de Sistemes de Fabricació assistit per agents. Aquesta arquitectura integra la funcionalitat d'una eina de simulació tradicional, a més permet la simulació de comportaments complexos associats als Sistemes de Fabricació Intel-ligents i que proporcionen solucions i millores que s'adapten a les necessitats de la nova era de fabricació. Tanmateix, l'arquitectura es centra en la millora d' activitats del procés global de la simulació que inclouen: a) assistència durant la representació i programació de models de sistemes de fabricació, b) aportar flexibilitat per plantejar les hipòtesis i el disseny d'experiments, c) la simulació del model, d) aporta mètriques per a l'avaluació de models, configuracions de planta i dades de producció que serveixen d'ajuda per a la presa de decisions, i e) la validació dels resultats contra la hipòtesi. Així mateix, es proposa un Metamodel suportat per agents que dona suport a l'Arquitectura durant la Representació i Programació del Model de Sistemes de Fabricació Intel-ligents. El metamodel ofereix flexibilitat per a la definició de models amb un ampli rang de variants que permeten el disseny d'experiments que consideren els requeriments de la nova fabricació. Mitjançant el disseny individual dels elements del sistema de fabricació és possible la definició de comportaments complexos per a Sistemes de Fabricació reals. El metamodel ofereix la possibilitat d' integrar patrons per a la definició de les interaccions entre les ordres de producció i els recursos de fàbrica al llarg de l'assignació de tasques. D'aqueta manera, l'Arquitectura empra el metamodel per a facilitar el disseny de Models de Simulació de Sistemes de Fabricació suportats per agents i l'animació d'aquests models. Per acabar, amb l'objectiu de validar l'arquitectura i el metamodel s'ha implementat un prototip que ofereix interfícies que donen suport a la creació i simulació de models. El prototip ha sigut emprat per a la simulació d'un cas d'estudi: un sistema de fabricació de sitges. 


\section{Agradecimientos}

A mis directores de tesis, D. Vicente Botti Navarro y Da. Adriana S. Giret Boggino, gracias a quienes debo toda mi labor como investigadora. A D. Vicente Botti Navarro que me permitió dar mis primeros pasos en el mundo de los Sistemas Multiagente. A Da. Adriana S. Giret Boggino, que ha revisado con paciencia y dedicación cada una de mis aportaciones e ideas y que ha sabido orientarme en todo momento. Quiero agradecerles el interés y confianza que han mostrado siempre por mi persona, así como su constante apoyo y orientación en mi trabajo.

A mi familia, en especial a mi padre y mis hermanos que han compartido conmigo esta gratificante experiencia, que me han animado y apoyado en presencia y en la distancia durante este largo proceso de aprendizaje. Y en especial a mi esposo y mi hijo, por su apoyo, por escucharme y compaginar nuestra la vida en familia y este proyecto. A mi madre, por su paciencia y comprensión que estuvo y siempre estará a mi lado. Y finalmente, a mis compañeros del grupo de investigación GTI-IA, con quienes trabajé, aprendí y compartí experiencias durante el proceso de elaboración de este trabajo. En especial, quiero agradecer a Sole, Luis y Marti con quienes desde mi llegada me apoyaron y ayudaron a integrarme además de compartir buenos momentos. 



\section{Capítulo 1}

\section{Introducción}

El reciente enfoque de sistemas conocidos como Sistemas de Producción Flexible (FMS) (Colombo, Schoop \& Neubert 2006), considera la presencia de un grupo de unidades de fabricación distribuidas, autónomas, inteligentes, tolerantes a fallos y reutilizables, las cuales operan como un conjunto de entidades que cooperan entre sí. En dichos sistemas cada entidad es capaz de interactuar dinámicamente entre ellas para el logro de objetivos tanto particulares como globales. Los objetivos relacionados con la fabricación van desde el nivel de control de máquinas físicas en la planta, hasta niveles superiores de gestión de la producción. De tal manera que a esta nueva generación de sistemas de fabricación se les referencia como Sistemas de Fabricación Inteligente (IMS) (Hayashi 1993).

En el área de la fabricación, la simulación es una herramienta esencial para la validación de métodos y arquitecturas antes de aplicarlos en la Planta de Producción. Las herramientas de simulación actuales (por ejemplo, ARENA (Hammann \& Markovitch 1995), Labview (Paris \& Reeves-Hardcastle 1996), AnyLogic (Wartha, Peev, Borshchev \& Filippov 2002) y MATLAB (The Mathworks 2006)) ofrecen técnicas útiles para la simulación de entornos de fabricación mediante el uso de modelos estáticos. Dichas herramientas se aplican al modelado de eventos discretos y/o continuos así como en aplicaciones geométricas. Su principal característica es el proceso de simulación basado en modelos estáticos. Algunas herramientas ofrecen una función de optimización para mejorar el modelo antes de la simulación, mientras que otras permiten al Usuario la modificación de dicho modelo durante la simulación. Sin embargo, debido a que el modelado de los requisitos de fabricación (especificación) cambia de acuerdo con las necesidades del Cliente, las herramientas de simulación deben permitir su adaptación ante tales necesidades. 
La programación de procesos de fabricación secuenciales y centralizados tradicionales, y los mecanismos de planificación y control ofrecen una flexibilidad insuficiente para responder a los estilos de fabricación cambiantes y a los entornos de fabricación altamente mezclados y de bajo volumen. Los enfoques tradicionales limitan la ampliación y reconfigurabilidad de los Sistemas de Fabricación. Por tanto, la Simulación de Sistemas de Fabricación debe dirigirse hacia la integración de los requisitos de la nueva era de la fabricación (Shen $\&$ Norrie 1999). Tales requisitos incluyen la integración de la empresa, organización distribuida, entornos heterogéneos, inter-operabilidad, cooperación, integración de humanos con el software y el hardware, agilidad, escalabilidad y tolerancia a fallos.

De modo que, ante tal escenario, surge la necesidad de nuevas herramientas de simulación para fábricas que contemplen dichas características. Tales características incluyen: a) flexibilidad y adaptabilidad, para modelar comportamientos complejos propios de un Sistema de Fabricación, b) escalabilidad para la integración transparente de funcionalidades adicionales, c) proactividad y reactividad para la adaptación automática ante los cambios del entorno y d) características de aprendizaje (inteligencia) basado en la experiencia adquirida durante la simulación.

La tecnología de agentes, por su parte, proporciona una forma natural para tratar tales problemas, así como para diseñar e implementar eficientes sistemas de fabricación inteligentes distribuidos. La tecnología de agentes ha sido considerada como un importante enfoque para el desarrollo de sistemas de fabricación distribuidos (Jennings, Corera \& Laresgoti 1995, Jennings \& Wooldridge $1998 b$ ). Ésta es particularmente reconocida como un prometedor paradigma para los sistemas de fabricación de la siguiente generación (Shen \& Norrie 1999, Shen, Norrie \& Barthes 2001), es decir, Sistemas de Fabricación con un mayor grado de adaptabilidad a las necesidades cambiantes del entorno. Las técnicas de Inteligencia Artificial han sido utilizadas en la Fabricación Inteligente por más de dos décadas. Sin embargo, los recientes desarrollos en el área de los Sistemas Multiagente han traído consigo nuevas e interesantes posibilidades (Wooldridge \& Jennings 1995, Panurak 1996, Jennings \& Wooldridge 1998b, Shen et al. 2001).

Los Sistemas Multiagente (SMA) se han aplicado exitosamente en diversas áreas tales como desarrollo de aplicaciones distribuidas (redes de sensores 
distribuidos (Mason \& Jonhson 1989), programación de fábrica (Neiman, Hildum, Lesser \& Sandholm 1994, Sycara, Roth, Sadeh \& Fox 1991), diagnóstico de red (Sugawara \& Murakami 1992, Oates, Nagendra \& Lesser 1997), ingeniería concurrente (Lander \& Lesser 1997)), integración de comportamientos complejos (extracción de reglas de bases de datos en sistemas complejos (Ryoke \& Nakamori 2005)), proactividad en cada uno de los elementos que integran el Sistema Multiagente así como la flexibilidad en la estructura de la organización. Específicamente es posible identificar las oportunidades brindadas con respecto a la simulación (Yilmaz \& Tuncer 2007, ADS 2008, Drogoul, Vanbergue $\&$ Meurisse 2003), área que también ha sido muy fructífera en cuanto a la simulación de entornos reales en la proyección de escenarios y resultados palpables. Entre las áreas donde ha sido aplicado el paradigma de agentes para la simulación podemos mencionar: la simulación social (Gans, Jarke, Alkemeyer \& Schmitz 2005, IDS-scheer 2003, Castelfranchi, Falcone \& Marzo 2006, Sansores \& Pavón 2006), el diseño de plantas de fabricación (Gabar, Shinohara, Shimada \& Suzuki 2003), la simulación de mercados (Chaturvedi, Mehta, Dolk \& Ayer 2005, Raberto, Cincotti, Focardi \& Marchesi 2001), la simulación espacial (Deadman 1999, Ligtenberg, Wachowicz, Bregt, Beulens \& Kettenis 2004), la simulación de transporte (Bazzan, Klugl \& Ossowski 2005, Hallé \& Chaibdraa 2005) y la simulación de Sistemas de Fabricación (Calderón \& García 2005) (Marík, Vrba, Maturana \& Hall 2005). Específicamente para el modelado de Sistemas de Fabricación entre algunos de los ejemplos más relevantes podemos mencionar HOLOS Architecture (Rabelo \& Camarinha-Matos 1994), Methamorph II (Shen, Xue \& Norrie 1998), MASCADA (Valckenaers, P., Baumgaertel, McFarlane \& Courtois 1999), PABADIS (Sauter \& Massotte 2001) y Factory Broker (Colombo et al. 2006). Tomando en consideración tales oportunidades de mejora con respecto a las herramientas de simulación actuales, se plantea la posibilidad de la definición de una Arquitectura para un Entorno de Simulación y un Metamodelo que permita definir un Sistema de Fabricación, ambos soportados por agentes para simular Sistemas de Fabricación. En consecuencia se pretenden integrar ventajas adicionales con respecto a los requisitos de la nueva era de la fabricación.

Las herramientas de simulación utilizan modelos para representar el sistema real a simular. Mientras que por un lado un modelo de simulación es una representación de un mundo real que incorpora el control del tiempo y sus cambios, por otro lado, mediante la simulación soportada por agentes, es posible emular un sistema del mundo real utilizando un modelo multiagente en el que los elementos del sistema real son representados por agentes situados en un entorno 
simulado. Los Sistemas Multiagente ofrecen la posibilidad de representar un entorno dinámico, el cual cambia continuamente como consecuencia de las acciones de sus elementos internos y externos. Tales características resultan clave en la resolución de la problemática que surge al intentar simular comportamientos complejos presentes en una planta de fabricación. Los agentes actúan como entidades inteligentes capaces de tomar decisiones y actuar de forma proactiva, reaccionando ante el estado actual del entorno y aprendiendo de tales decisiones. El conocimiento adquirido va formando parte de su base de conocimiento, el cual es utilizado posteriormente en las sucesivas tomas de decisiones. De esta forma el sistema multiagente aporta a la vez la flexibilidad necesaria para adaptarse a los cambios del entorno propios de un Sistema de Fabricación.

La Fabricación Holónica (HMS 2004) es un paradigma desarrollado en el marco del programa Intelligent Manufacturing Systems (IMS - Sistemas de Fabricación Inteligente). Trasladando los conceptos holónicos al mundo de la fabricación, se considera al sistema de fabricación como un compuesto de módulos autónomos con control distribuido. Algunos investigadores han equiparado el término de holón con el de agente (Giret \& Botti 2004a) debido a las características que comparten (autonomía, control distribuido, proactividad, habilidad social, racionalidad, cooperación, aprendizaje, etc.).

Por otra parte, la Fabricación Holónica (Holonic Manufacturing - HM) es una organización altamente distribuida, donde la inteligencia se distribuye entre las entidades individuales. Se la puede comparar con los sistemas distribuidos, sin embargo, el elemento nuevo en la Fabricación Holónica es el hecho que las entidades individuales trabajan juntas en jerarquías temporales para obtener un objetivo global. Por tanto, es posible suponer que los beneficios aportados por cada uno de estos paradigmas (holones y agentes) en la representación de Sistemas de Fabricación pueden ser aplicados en conjunto para la integración de una arquitectura que dé soporte a la simulación de una Planta de Fabricación con resultados aún más favorables en comparación con las propuestas actuales. Esta es la hipótesis global que guía el desarrollo del trabajo presentado en esta memoria de tesis doctoral.

\subsection{Motivación}

Algunos investigadores han estado aplicando la tecnología de agentes en la integración de la fabricación empresarial, la colaboración, la planificación de 
procesos de fabricación, la programación para el control de planta, el manejo de materiales y la gestión de inventarios, así como la implementación de nuevos tipos de sistemas de fabricación tales como los Sistemas de Fabricación Holónicos (Deen 2003). Muchos investigadores trabajan en la fabricación basada en agentes enfocándose principalmente en la investigación básica dirigida a la mejora de la racionalidad o inteligencia de los agentes de software y en el desarrollo de mecanismos de negociación y coordinación más eficientes y efectivos. Mientras que esta clase de investigación es importante y todavía necesaria, algunos investigadores (Shen et al. 2001) consideran que el trabajo futuro de Investigación y Desarrollo (R\&D) debe centrarse en la integración de los sistemas de planificación y de programación basados en agentes con los sistemas existentes utilizados en empresas de fabricación. Entre los sistemas más utilizados en las empresas de fabricación se encuentran los sistemas ERP (Enterprise Resource Planning) y MRP (Materials Resource Planning). Los investigadores del área consideran que solamente cuando se logren y validen tales integraciones en escenarios industriales, la tecnología de agentes será aplicada ampliamente en la industria de la fabricación.

Identificar una herramienta de simulación que sea capaz de ejecutar tareas de simulación independientemente de la naturaleza de la tarea no es complicado. Sin embargo, resulta difícil encontrar una herramienta que pueda ejecutar "inteligentemente" la simulación de tareas cada vez más complejas. La dificultad radica en integrar en la herramienta el conocimiento necesario del sistema original y que a la vez actúe como un asistente que proporcione consejos y guíe al usuario durante la simulación. Por tanto surge la necesidad de definir una Arquitectura de un Entorno de Simulación Inteligente que además permita en el futuro la optimización de las tareas y proporcione soluciones enfocadas a un conjunto de objetivos definidos para un Sistema de Fabricación Inteligente.

Con dicha necesidad en mente, se pretende integrar la aplicación exitosa del paradigma de los Sistemas Multiagente en el ámbito de los Sistemas de Fabricación Inteligentes mediante el establecimiento de las bases para la resolución de las necesidades en el ámbito de la Simulación de Sistemas de Fabricación Inteligentes. Asimismo, tales necesidades incluyen los requisitos de la nueva era de la fabricación como factor clave.

Finalmente, dentro del Grupo de Investigación se han realizado trabajos relacionados con metodologías y desarrollo de Sistemas de Fabricación (ANEMONA) (Botti \& Giret. 2008), pero no se cuenta con un entorno donde se puedan 
probar tales desarrollos. En consecuencia, se pretende que la Arquitectura del Entorno de Simulación permita además llevar a cabo tales pruebas.

\subsection{Objetivo}

El objetivo de este trabajo es definir y construir una Arquitectura para un Entorno de Simulación de Sistemas de Fabricación soportado por agentes que mejore las principales funciones relacionadas con la nueva era de fabricación que las herramientas actuales de simulación ofrecen. Además, se pretende que integre características adicionales tales como flexibilidad en el diseño, modificación y simulación de modelos, proactividad en el momento de toma de decisiones mediante procesos de negociación entre los elementos que integren el modelo, retroalimentación automática del sistema a partir de procesos de aprendizaje basado en los resultados obtenidos en simulaciones previas, optimización automática de recursos acorde al estado actual del entorno durante la simulación de un modelo que representa a una planta de fabricación.

Así mismo, los objetivos complementarios para el logro de este objetivo global se detallan a continuación:

a) Revisión de las bases teóricas de la Simulación. Para identificar los conceptos clave del proceso de simulación (conceptualización y desarrollo de modelos, diseño de experimentos, simulación y análisis de resultados) y las características de funcionalidad de un entorno de simulación (técnicas para el control de la simulación y gestión de eventos, control de tiempo, presentación de resultados, etc.).

b) Revisión de las bases teóricas de la Simulación basada en Agentes. Tomando como base los resultados obtenidos en el objetivo $a$ ), se identificarán las necesidades de la Simulación soportada y basada en Agentes que deberán ser consideradas en la propuesta. Asimismo se identificará el alcance de la aplicación de la simulación basada en agentes obtenido hasta el momento.

c) Análisis de Estándares de Simulación de Sistemas de Fabricación. Este análisis comprende la identificación de los elementos básicos de un Sistema de Fabricación, su funcionalidad, necesidades, etc., y permitirá la identificación de los estándares a considerar durante la Simulación de un Sistema de Fabricación.

d) Análisis de las herramientas actuales de Simulación de Sistemas de Fabricación. Permitirá visualizar la aplicación de las herramientas de simulación 
de Sistemas de Fabricación y su alcance con respecto a los requisitos de la nueva era de la fabricación. Asimismo, permitirá identificar las características de posibles mejoras en los procesos de simulación que puede aportar el paradigma de Sistemas Multiagente. Para conseguir este objetivo, se plantea realizar una evaluación extensiva de las actuales herramientas de simulación más representativas donde sea posible observar sus debilidades relacionadas con los requisitos de la nueva era de la fabricación.

e) Análisis de los requisitos de la Simulación de Sistemas de Fabricación Inteligentes. Este análisis permitirá determinar las técnicas existentes o proponer técnicas alternativas basadas en Inteligencia Artificial que aporten mejoras en los procesos de Simulación de Sistemas de Fabricación. En dicho análisis se identificarán los siguientes elementos esenciales: a) un Modelo que proporcione la representación de partes o elementos específicos del equipo de fabricación y su control, b) un Modelo de las instalaciones de la planta de producción para emular el equipo físico, c) una interfaz para la ejecución y d) la visualización gráfica del comportamiento global del sistema.

f) Plantear una propuesta de Arquitectura de un Entorno Simulación y un Metamodelo soportados por agentes para Sistemas de Fabricación Inteligentes. La propuesta integrará los resultados obtenidos en los objetivos del a) al e). Los objetivos previos permitirán definir dos elementos: el marco teórico de la Arquitectura del Entorno de Simulación Soportado por Agentes, y la definición de un Metamodelo que aporte la flexibilidad necesaria en la definición de un Sistema de Fabricación Inteligente.

g) Desarrollar un prototipo del Entorno de Simulación. Se validarán tanto la Arquitectura del Entorno de Simulación y el Metamodelo del Sistema de Fabricación (resultados obtenidos en el objetivo $f$ ) mediante el desarrollo e implementación de un prototipo.

h) Validar la propuesta mediante casos reales. Con el prototipo obtenido en el objetivo $g$ ) se validará la funcionalidad de la Arquitectura del Entorno de Simulación y el Metamodelo mediante la aplicación del prototipo a casos de estudio reales. 
Finalmente, se pretende que una vez validada la propuesta, tanto la Arquitectura como el Metamodelo, permitan sentar las bases para su futura integración con Sistemas ERP.

\subsection{Estructura del Trabajo}

La memoria está organizada en dos partes. En la Primera Parte, se presenta el Marco Teórico del Trabajo que incluye el Estado del Arte de las principales áreas que dan soporte a la propuesta. Como resultado de esta primera parte se identificarán los marcos teóricos que dan soporte a la simulación, la simulación basada en agentes y la simulación de Sistemas de Fabricación. Asimismo, se presentarán las oportunidades de mejora al aplicar el paradigma de agentes. La primera parte de la memoria comprende los Capítulos 2, 3 y 4.

En el Capítulo 2 se plantean las bases de la Simulación como herramienta de emulación de procesos del mundo real. Se detallan las características que lo hacen viable para llevar a cabo dicha tarea, algunas de las ventajas y desventajas asociadas a su aplicación, los aspectos vitales dentro de la definición de un Modelo de Simulación, así como las áreas de aplicación donde es posible visualizar resultados favorables.

En el Capítulo 3 se presentan las bases teóricas referente a los Agentes y los Sistemas Multiagente así como las áreas de aplicación donde es posible visualizar el éxito de su aplicación. Asimismo, en este capítulo exponemos el rol de la Simulación soportada por Agentes, algunas de sus características principales así como las áreas de aplicación donde es posible observar la explotación de las características inherentes a los Sistemas Multiagente.

En el Capítulo 4 se plantean los estándares existentes referentes a la Simulación de la Fabricación así como el alcance de los mismos. En este capítulo también se exponen los requisitos que exige la nueva manufactura a fin de que las herramientas permitan plasmar con mayor exactitud el entorno de la fabricación y los escenarios ante los que se ven expuestos. Uno de los aspectos más relevantes es la definición de las bases para la estructuración del Modelo de Simulación. Asimismo, se presentan aspectos relativos a la Fabricación Ágil y Fabricación Virtual, los Sistemas de Fabricación Inteligentes y los Sistemas Holónicos de Fabricación. En este capítulo se hace referencia a una revisión de algunas de las herramientas de simulación más relevantes con el fin de detectar posibles 
debilidades y oportunidades de mejora mediante la aplicación de los Sistemas Multiagente.

En la Segunda Parte de la memoria se presenta el Marco de la Propuesta donde se presentan las principales aportaciones del trabajo. Estas incluyen la Arquitectura del Entorno de Simulación, el Metamodelo para la definición de un Sistema de Fabricación soportada por Agentes, el Desarrollo e Implementación del Prototipo del Entorno de Simulación, las Bases y Resultados de la Evaluación del Trabajo, así como las conclusiones y trabajo futuro. Esta Parte comprende los Capítulos 5 al 12.

En los Capítulos 5 y 7 planteamos la Arquitectura de la propuesta de Simulación de un Sistema de Fabricación Inteligente asistida por Agentes. Para ello se establecen las características básicas que deberá poseer. En base a dichas características se definen los agentes que integran el Simulador y las características asociadas a cada uno. Posteriormente se definen las funciones que cada uno desempeñará a lo largo del proceso de Simulación. Asimismo se expone la secuencia de ejecución de cada uno, detallando los escenarios que se presentarán y los agentes que deberán intervenir en cada uno de ellos. Además, se presentan las principales características con respecto a la configuración de la simulación de un modelo (animación gráfica del Sistema de fabricación, control de la velocidad y sincronización de relojes, condición de parada). Finalmente se presenta el comportamiento de un Sistema de Fabricación genérico y su interacción con el Entorno de Simulación.

En el Capítulo 6 planteamos las bases para la integración de los elementos de un Sistema de Fabricación Inteligente además de los elementos requeridos para su Simulación mediante el uso de un Metamodelo de Simulación para la Fabricación Inteligente asistido por Agentes. Para ello, se establecen las características básicas que deberá poseer y las normas de modelado contempladas. Teniendo en cuenta dichas características se definen los agentes que integran el Simulador y las características asociadas a cada uno. Posteriormente se definen las funciones que cada uno de los agentes desempeñará a lo largo del proceso de la Simulación. Asimismo se expone la secuencia de ejecución de cada uno, detallando los escenarios que se presentarán y los agentes que deberán intervenir en cada uno de ellos.

En el Capítulo 8 detallamos la implementación de la propuesta expuesta en los Capítulos 5, 6 y 7. En este proceso se utiliza la metodología ANEMONA 
(Botti \& Giret. 2008).

En el Capítulo 9 presentamos las bases que se aplican para la evaluación de la etapa del modelado y la simulación del modelo. Asimismo se presentan las bases para la evaluación de los Resultados obtenidos, los cuales incluyen los Modelos, las Configuraciones de Planta y el análisis de datos de producción (tiempos muertos, uso de recursos,etc.).

En el Capítulo 10 exponemos un caso de estudio real relacionado con la fabricación metal-mecánica. En este capítulo presentamos los resultados obtenidos a partir del uso del prototipo así como el reflejo del comportamiento de los Sistemas Multiagente ante tal caso.

En el Capítulo 11 presentamos los resultados de la aplicación de las bases presentadas en el Capítulo 9 para la Evaluación de la Implementación y de la Funcionalidad del Entorno de Simulación.

En el Capítulo 12 presentamos las conclusiones de la tesis, las aportaciones del trabajo de investigación acorde a los objetivos de la misma, las líneas de trabajo futuras y finalmente se presentan las publicaciones que se han elaborado durante la realización de la presente tesis. 


\section{Parte I}

Marco Teórico. Estado del Arte. 



\section{Introducción}

En esta primera parte de la memoria de tesis se presenta el Marco Teórico, que incluye el Estado del Arte de las distintas áreas que dan soporte a esta propuesta. Cada una de dichas áreas aportan las bases teóricas que se consideran en la tesis. Las áreas clave que hemos identificado incluyen (Figura 1.1):

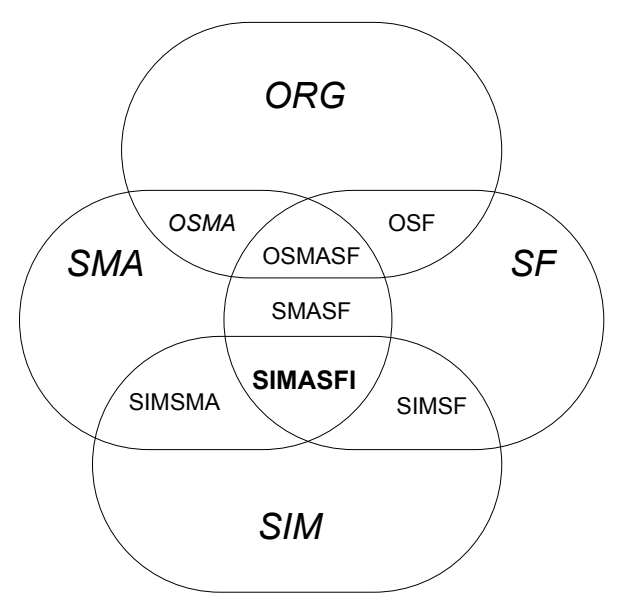

Figura 1.1: Identificación de Áreas Clave y sus Relaciones

Marco Teórico de la Simulación (SIM). En el Capítulo 2 se incluye la identificación de las características de un Modelo de Simulación, herramientas de simulación, tipos de simulación, control de tiempos de simulación.

Paradigma de los Sistemas Multiagente (SMA). En el Capítulo 3 se presenta esta área, la cual estudia el modelado de un Agente y de un Sistema Multiagente. Además se toma en consideración la estructura organizacional de un Sistema Multiagente, sus técnicas y protocolos de comunicación, técnicas de negociación y aprendizaje entre otras. 
Marco Teórico de los Sistemas de Fabricación (SF). En el Capítulo 4 se incluye la identificación de la Estructura de una Planta de Fabricación. La estructura a la vez se relaciona con la descripción organizacional de su estructura, normas, reglas de interacción, comportamiento de sus elementos, acciones, etc. Al mismo tiempo se identifican las necesidades relacionadas con la nueva era de la fabricación.

Marco Teórico de la Teoría de Organizaciones (ORG), que incluye la identificación de las características de una Organización, tipos de estructuras, normas, funcionalidad, entorno, objetivos, procesos de toma de decisiones, etc. Las aportaciones de la Teoría de las Organizaciones se incluyen a lo largo de los capítulos 2, 3 y 4, explicando cómo afecta dicho marco a cada una de las tres áreas.

Al mismo tiempo se identifican las relaciones entre estas áreas, las cuales incluyen :

- Simulación mediante Sistemas Multiagente (SIMSMA). En la Sección 3.5 se estudian los avances respecto a los modelos de simulación basados en agentes, áreas de aplicación y herramientas disponibles.

- Simulación de Sistemas de Fabricación (SIMSF). En la Sección 4.1 se identifican las bases para la Simulación de un Sistema de Fabricación, las características que debe poseer y los resultados que se espera de dicha simulación.

- Aplicación de Sistemas Multiagente en los Sistemas de Fabricación (SMA$S F$ ). En la Sección 4.7.3 se presentan las aplicaciones del paradigma de Sistemas Multiagente (SMA) en la resolución de problemas relacionados con los Sistemas de Fabricación (SF), tales como planning y scheduling. En la Sección 4.3 se observan los beneficios de la aplicación de la inteligencia artificial en problemas específicos de fabricación. Al mismo tiempo se identifican las oportunidades de mejora relacionadas con los requisitos de la nueva era de la fabricación.

- Simulación de Sistemas de Fabricación Inteligente dirigida por Agentes (SIMASFI). En el Capítulo 4 se presentan los avances respecto a la Simulación basada en agentes y las aportaciones que hasta el momento se han obtenido mediante la aplicación de la Simulación basada y soportada por agentes en la Simulación de Sistemas de Fabricación Inteligentes (SIMASFI). 
- Teoría de las Organizaciones aplicada al paradigma de Sistemas Multiagente (OSMA). En el Capítulo 3 se incluye como parte de la definición de un Sistema Multiagente, la definición de una Estructura de Organización Virtual, la cual considera aspectos básicos de la Teoría de Organizaciones (ORG).

- Teoría de las Organizaciones aplicada al Marco Teórico de los Sistemas de Fabricación (OSF). En la Sección 4.6 se observa como dentro de la definición de un Sistema de Fabricación se incluye la definición de un estructura organizacional la cual se basa en la aplicación de los principios de la Teoría de Organizaciones (ORG).

- Teoría de las Organizaciones aplicada a los Sistemas de Fabricación basados en el paradigma de Sistemas Multiagente (OSMAF). En la Sección 3.5.2 se puede apreciar que tanto los Sistemas de Fabricación (SF) como los Sistemas Multiagente (SMA) se consideran sistemas que requieren la definición de una estructura organizacional de sus elementos la cual se basa en los principios de la Teoría de las Organizaciones (ORG).

En resumen, la primera parte de esta memoria describe la aplicación de Sistemas Multiagente en los Sistemas de Fabricación (SMASF), la Simulación de Sistemas de Fabricación Inteligente dirigida por Agentes (SIMASFI) y la Teoría de las Organizaciones aplicada a los Sistemas de Fabricación basados en el paradigma de Sistemas Multiagente (OSMAF). De esta manera se pretende definir las bases de la propuesta y las aportaciones que ésta ofrece dentro del marco de la SIMASFI. 



\section{Capítulo 2}

\section{Simulación}

Tanto las industrias como las organizaciones han empleando la simulación como una herramienta que les permite prever el futuro basado en la historia de sus comportamientos. Sin embargo, la interpretación de dicha información es el elemento clave para su uso apropiado en la representación del futuro.

Como lo definió Banks en (Banks 1998) la simulación es ”... la imitación de la ejecución de un proceso o sistema del mundo real a través del tiempo. La Simulación implica la generación de una historia artificial de un sistema y la observación de dicha historia artificial para plasmar deducciones concernientes a las características operacionales del sistema real que se representa. La Simulación es una metodología de solución de problemas indispensable para la solución de muchos problemas del mundo real. La Simulación se utiliza para describir y analizar el comportamiento de un sistema, realizar preguntas del tipo -qué sucede si...- relacionadas con el mundo real, y ayudar en el diseño de sistemas reales. Tanto los sistemas existentes como los conceptuales pueden modelarse con la simulación".

Entre los conceptos subyacentes con respecto a la simulación se encuentran: el sistema real, el modelo del sistema, las variables de estado del sistema, entidades y sus atributos, el listado de procesos, y las actividades que ejecuta cada entidad. El modelo se considera como una representación del sistema real, las variables de estado son la colección de toda la información necesaria para definir que sucede dentro de un sistema en un determinado momento. Asimismo, una entidad representa un objeto que requiere una definición explícita, una entidad puede ser dinámica o estática; a su vez una entidad tiene atributos que le describen y puede tener atributos que solo le pertenezcan a ella (variables locales). Las entidades se gestionan mediante la asignación de recursos que le 
proveen servicios, esto se realiza vinculándolos a eventos específicos. Finalmente, las actividades se consideran como un periodo de tiempo cuya duración se conoce por anticipado, de este modo, cuando la duración inicia, su final puede ser programado.

El objetivo de este capítulo es la revisión de las bases teóricas referentes a la Simulación, sus características, los conceptos clave de un modelo de simulación así como la identificación de las posibles áreas de aplicación.

\subsection{Modelos y los Modelos de Simulación}

Banks plantea que un modelo es una representación de un sistema actual (Banks 1998). De tal manera, que el modelo final debería ser lo suficientemente complejo para responder a determinadas preguntas, pero no tan complejo como para no comprenderlo. "El modelado es la herramienta principal para el estudio del comportamiento de grandes sistemas complejos... Cuando modelamos sistemas, generalmente nos interesamos en su comportamiento dinámico. Típicamente, ubicamos el modelo en algún punto inicial y lo observamos delimitando una ruta en el futuro" (Simon 1990). La Simulación incorpora este concepto debido a que este implica representar desde fuera un modelo de un sistema comenzando con el estado del sistema en un punto inicial en el tiempo y evaluando las variables en el modelo a lo largo del tiempo para establecer el desempeño dinámico del modelo del sistema. Cuando el modelo es una representación válida del sistema, se obtiene información valiosa acerca del desempeño dinámico del sistema.

Por tanto al estudiar un sistema, el primer paso es la elaboración de un Modelo. Fishman (Fishman 1978) plantea que el modelo puede ser una representación formal de la teoría o una explicación formal de la observación empírica, pero que sin embargo, a menudo es una combinación de ambas. Asimismo, entre los propósitos de un modelo encontramos: que favorece una mejor comprensión del sistema, acelera el análisis, constituye un sistema de referencia para probar la aceptación de las modificaciones del sistema, es más fácil de manipular que el sistema mismo, hace posible controlar más fuentes de variación que lo que permitiría el estudio directo de la misma.

De esta manera, se puede concluir que un modelo es una representación simplificada del sistema en un instante de tiempo o espacio concreto realizada para comprender el sistema real.

Los Modelos de Simulación de Sistemas pueden ser clasificados como: de cambios discretos, de cambios continuos, o modelos combinados (Pritsker 1998). 
En la mayoría de las simulaciones, el tiempo es la principal variable independiente.

Un modelo discreto tiene variables dependientes las cuales cambian solamente en distintos puntos en el tiempo simulado, conocidos como Tiempos de Eventos. Un modelo continuo tiene variables dependientes que son funciones continuas de tiempo. Finalmente, en un modelo combinado las variables dependientes de un modelo pueden cambiar discretamente, continuamente o continuamente con saltos discretos sobrepuestos. El aspecto más importante de la simulación combinada surge de la interacción entre variables que cambian discreta y continuamente.

\subsection{El Modelado de un Sistema y el Modelado de una Organización}

Por un lado, en la Teoría de Sistemas (Bertalanffy 1986) un Sistema puede ser considerado como un conjunto de entidades que relacionadas entre sí ordenadamente contribuyen a determinado objetivo. Un Sistema puede recibir flujos de su entorno, cambiando o ajustando su comportamiento o su estado según las entradas que recibe. Por otro lado, acorde con la Teoría de Organizaciones, según Peiro (Peiro 1991) una organización consiste en una serie de individuos que realizan unas actividades o funciones específicas y diferenciadas. Además, estos individuos se estructuran siguiendo unas pautas y reglas determinadas que les permitan alcanzar los fines u objetivos de la organización. Los fines deben ser objetos de conocimiento común, que cumplan principalmente las funciones de guiar los esfuerzos de los miembros en vistas a su consecución.

De este modo, es posible identificar la coincidencia entre las características que describen a una Organización y las características asociadas a un Sistema. Por tanto, las normas que se aplican para el Modelado de una Organización se pueden aplicar en el Modelado de un Sistema.

En el Modelado de una Organización (o de un Sistema) no solo se debe considerar la integración de todos los elementos que lo componen sino sus relaciones, objetivos y funcionalidad tanto interna como con su entorno. Tales relaciones se ven sujetas a reglas que controlan su comportamiento. Por tanto, los factores a considerar en el modelado de una organización son los siguientes:

Estructura (Peiro 1991, Hodge, Anthony \& Gales 2003). Comprende todos aquellos elementos que persisten en la organización, independientemente de cuáles sean los individuos finales de la misma en cada momento. 
Vendrá definida en base a los roles, sus agrupaciones, dependencias y sus patrones de interrelación.

Funcionalidad (Peiro 1991). Especifica cuáles son los objetivos globales de la organización; los servicios y funcionalidades que ofrece; los objetivos que persiguen los distintos componentes de la organización y qué tareas y planes deben seguir para alcanzarlos.

Normatividad (Wagner \& Hollenbeck 2004). Determina el conjunto de normas y acciones definidas para controlar los comportamientos de los miembros de la organización. Se incluyen aquí tanto las normas sobre la actuación (obligaciones, permisos y prohibiciones), como las sanciones y recompensas a efectuar sobre sus miembros. La normalización proporciona los estándares y procedimientos para ayudar a los miembros de la organización a determinar cómo realizar sus tareas. Existen cuatro tipos de normalización: de tareas, de resultados, de habilidades y de comportamientos. La normalización de tareas implica la especificación de las tareas concretas y los procedimientos de trabajo que los empleados deben seguir para cumplir con sus responsabilidades. En la normalización de resultados, se definen formalmente los objetivos de los resultados o las metas de rendimiento esperadas, por lo que los miembros de la organización tienen mayor flexibilidad sobre la funcionalidad de las tareas a realizar, siempre que sus resultados sean acordes a las metas especificadas. En la normalización de habilidades, se indican las cualificaciones, conocimientos y habilidades necesarias para desarrollar las tareas de forma competente. Finalmente, en la normalización de los comportamientos, los miembros de un grupo u organización comparten un conjunto de creencias sobre la aceptabilidad de determinados tipos de comportamiento, como por ejemplo la calidad en los procesos de trabajo.

Dinamicidad (Peiro 1991). Especifica cómo evoluciona la organización a lo largo del tiempo, indicando el modo en que los agentes entran y salen de la organización de forma dinámica; adoptan determinados roles en función de sus capacidades y habilidades; y participan en aquellas unidades o agrupaciones de la organización en las que sean admitidos.

Entorno (Wagner \& Hollenbeck 2004, Hodge et al. 2003). Comprende tanto los recursos de los que depende la organización, como los proveedores de dichos recursos y los clientes o beneficiarios de la existencia de la organización. 


\subsection{La Teoría del Modelado y la Simulación}

El proceso de simulación conlleva la integración de entidades básicas que aportan o ejecutan actividades clave. Tales entidades interactúan desempeñando actividades fundamentales para el correcto Modelado de un Sistema. Aunque existen diferentes propuestas para la definición de un Modelo de Simulación, la mayoría se basan en los pasos en un Estudio de Simulación propuestos por Banks, Carson y Nelson en (Banks, Carson, Nelson \& Nicol 2001).

Para la identificación de las entidades que participan en el Estudio de Simulación, hemos optado por la Teoría del Modelado y Simulación (TMS) (Zeigler, Praehofer \& Kim 2000), donde el marco del Modelado y la simulación esta definido por cuatro entidades clave (Fig. 2.1):

Sistema original. Se refiere a la fuente ya sea real o artificial del cual se obtienen los datos.

Base de Datos del Comportamiento. Es la colección de datos recogidos.

Marco Experimental. Es el que especifica las condiciones bajo las cuales el sistema es observado o con las cuales se realizan los experimentos.

Modelo. Son las instrucciones para la generación de datos.

Simulador. Se refiere al dispositivo computacional que genera (representa) el comportamiento del modelo.

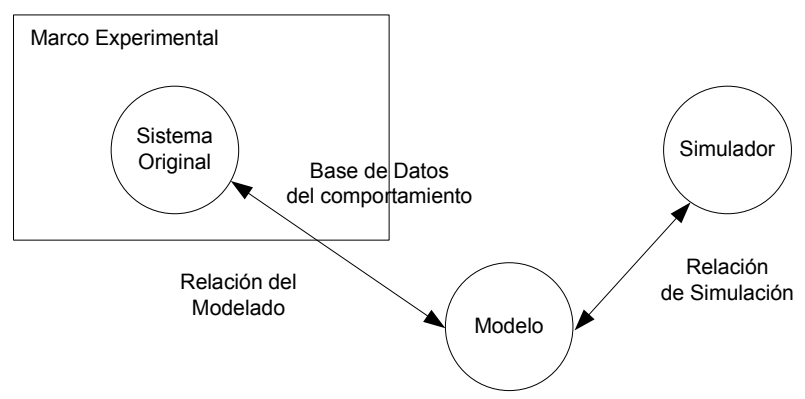

Figura 2.1: Entidades del Marco para el Modelado y la Simulación.

Tales entidades son consideradas como la base del planteamiento de nuestra propuesta presentada en los Capítulos 5 y 7 . 


\subsection{Etapas de un Estudio de Simulación}

Un proyecto de simulación es un proceso de pasos interpretativos, de desarrollo y analíticos (Musselman 1994). Entre las propuestas (Pritsker, Sigal \& Hammesfahr 1989, Banks et al. 2001, Law \& Kelton 1991) se observa que tales pasos son intrínsecos para todos los proyectos de simulación. De forma genérica todas incluyen la formulación del problema, conceptualización del modelo, recolección de datos, construcción del modelo, verificación, validación, análisis, documentación e implementación.

La literatura especializada referente a la Simulación generalmente recomienda seguir un proceso de nueve a doce pasos en el desarrollo de modelos de simulación. Una de las propuestas que describe mas detalladamente las actividades a realizar en cada uno de dichos pasos es la propuesta en (Banks et al. 2001) que incluye los siguientes pasos (Ver Fig. 2.2):

1. Formulación del Problema.

2. Definición de objetivos y plan completo del proyecto.

3. Conceptualización del Modelo.

4. Recolección de datos.

5. Traducción del Modelo en formato informatizado.

6. Verificación de Código.

7. Validación del Modelo.

8. Diseño de experimentos a ejecutar.

9. Ejecución y análisis de los resultados.

10. Documentación y reportes.

11. Implementación. 


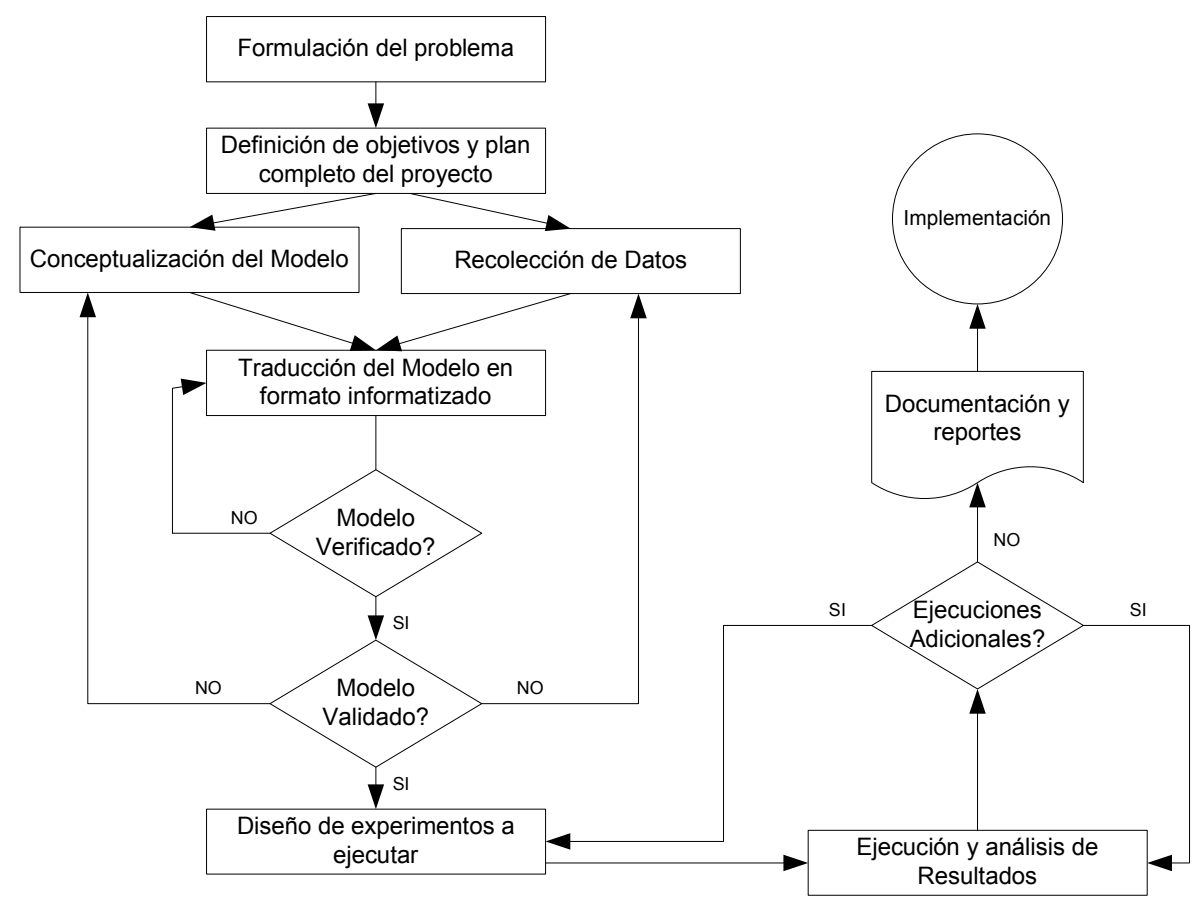

Figura 2.2: Etapas de un Estudio de Simulación.

Cabe señalar que esta propuesta de acuerdo con Mc Lean y Leong (MCLean $\&$ Leong 2002b) tiene una debilidad, esto es, la propuesta a menudo deja un trabajo considerable y posiblemente demasiada responsabilidad creativa al analista de simulación. Al aplicar esta propuesta, el proceso de modelado y la simulación tal vez son más un arte que una ciencia. Las simulaciones a menudo parten de cero de tal manera que la habilidad del analista puede destacar significativamente en la calidad de los resultados obtenidos. Existe una mínima oportunidad para que el analista pueda construir partiendo del trabajo de otros, puesto que cada simulación se construye como una solución personalizada para un problema definido único. Los datos de entrada de otras aplicaciones de programas de fabricación a menudo no tienen el formato requerido para la simulación, así que muchas veces los datos deben ser resumidos, reformateados, y/o traducidos. Además, la presión de la administración de la fabricación para obtener resultados rápidamente, puede tener un impacto negativo en la ejecución del análisis de la simulación y la calidad de los resultados obtenidos. Simplificando dicho diagrama, las principales actividades a considerar dentro del Estudio pueden ser resumidas como se muestra en la Figura 2.3. 


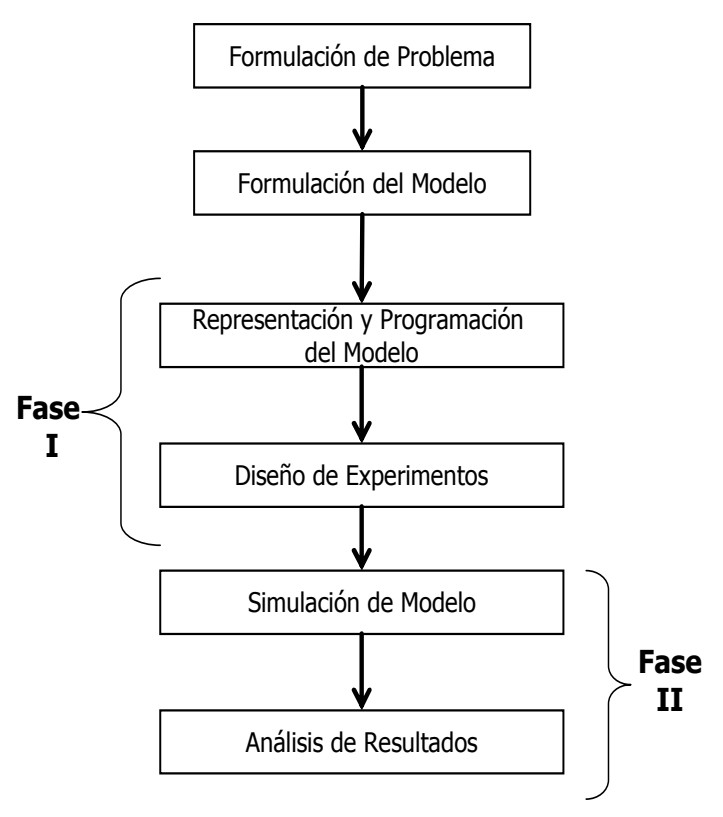

Figura 2.3: Actividades globales del Estudio de Simulación.

Algunos autores tal como Law (Law \& McComas 1999) sugieren que existen algunas ideas o técnicas importantes para decidir el nivel apropiado del detalle del modelo de simulación, para aplicar un modelo de validación, y para el desarrollo de un modelo con alta credibilidad. Los factores que se mencionan se describen a continuación:

- Establecer definitivamente los aspectos a ser considerados y las mediciones de ejecución para la evaluación del diseño del sistema desde el principio.

- Recolectar información de la distribución del sistema y los procesos operativos basado en conversaciones realizadas con los Expertos en el Tema (SME).

- Delinear toda la información y resúmenes de datos en un "documento de suposiciones", el cual se convierte en la principal documentación para el modelo. 
- Interactuar con el administrador regularmente para asegurarse de que el problema esta siendo resuelto correctamente para incrementar la credibilidad del modelo.

- Llevar a cabo una inspección estructurada apriori (antes de desarrollar cualquier programa) del modelo de simulación conceptual plasmado en el documento de suposiciones, así como tener reuniones con los expertos en el tema, administradores, etc.

- Emplear el análisis de sensibilidad para determinar factores del modelo.

- Simular la fabricación existente (si ésta existe) y comparar las mediciones de ejecución del modelo con las mediciones equivalentes del sistema actual.

\subsubsection{Formulación del Problema, Objetivos y Plan de proyec- to}

Cada estudio de simulación comienza con la formulación de un problema. Si la formulación es realizada por aquellos que tienen el problema (cliente), el analista debe asegurarse que el problema se entiende claramente. Si la formulación del problema la prepara el analista de simulación, es importante que el cliente lo entienda y esté de acuerdo con la formulación. Por tanto se sugiere que el analista prepare un conjunto de supuestos en los cuales esté de acuerdo el cliente. Incluso con todas estas precauciones, es posible que el problema requiera ser reformulado conforme el estudio de simulación avance.

Los objetivos indican las preguntas que serán contestadas por el estudio de simulación. El plan del proyecto debería incluir la formulación de varios scenarios que serán investigados. Los planes para el estudio deben ser indicados en términos de tiempo que será requerido, personal, hardware y software si el cliente desea ejecutar el modelo y guiar el análisis, etapas de la investigación, resultados de cada etapa, costo del estudio y procedimientos de contabilidad si existen.

Musselman (Musselman 1994) por su parte propone un procedimiento para la formulación del Modelo al revés, es decir, definir preguntas clave basadas en objetivos bien definidos. Los objetivos establecen los criterios contra los cuales deben ser consideradas todas las decisiones del modelado. Por tanto la formulación abarca cinco fases: 1) Comprender el entorno, 2) Identificar y establecer el orden de prioridad de las preguntas clave, 3) Definir las salidas del Modelo 
necesarias para contestar a las preguntas clave, 4) Limitar el alcance del Modelo y restringir el detalle, y 5) Especificar los Resultados del Modelo. La Figura 2.4 muestra las 5 fases así como las actividades a realizar en cada una.

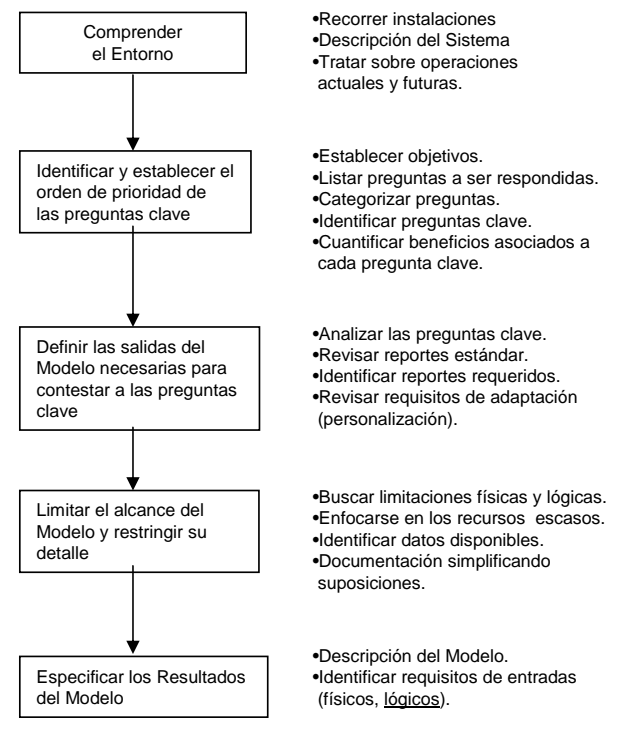

Figura 2.4: Procedimiento para la Formulación del Modelo.

\subsubsection{Conceptualización del Modelo}

La Teoría del Modelado y Simulación (TMS) proporciona las bases del formalismo para la representación de Sistemas Dinámicos. Los principales aspectos de esta teoría son:

Niveles de especificación del Sistema. Estos son los niveles en los que se divide el Sistema. En cada nivel se describe, en diferente grado de abstracción, como se comporta el sistema y los mecanismos utilizados en cada nivel para trabajar de una forma específica.

Formalismos de la especificación del Sistema. Estos son los tipos de estilos de modelado, continuos o discretos, que los modeladores pueden utilizan para la construcción de modelos de Sistemas.

La teoría de Sistemas distingue dos aspectos en un Sistema: la estructura y el comportamiento. Visto como una caja negra, el comportamiento externo de un sistema es la relación que se define entre las entradas que recibe y salidas 
que genera con el tiempo. El comportamiento de entrada/salida consiste en las tuplas de datos (entrada y salida correspondientes) generados por el sistema real o modelo. La estructura interna de un sistema incluye su estado y el mecanismo de transición de estado (éste es el elemento que, determina cómo las entradas influyen en el estado actual y en los siguientes estados) así como el mapeo de estado del sistema al generar las salidas. De esta forma, al conocer la estructura del sistema es posible (analizar, simular) deducir su comportamiento.

Existen dos conceptos relevantes con respecto a la estructura, la descomposición y la composición. En la descomposición se plantea cómo un sistema puede ser separado en componentes del sistema. Mientras que la composición plantea como los componentes de un sistema pueden ser unidos para integrar un sistema más grande. Las diferencias entre los sistemas descompuestos y los que no han sufrido una descomposición tienen que ver con los distintos niveles de la especificación de sistemas. De esta forma aquellos sistemas con un mayor nivel de especificación proporcionan más información con respecto a la estructura del sistema.

Una vez identificado tanto la estructura (enfoque de descomposición) como el comportamiento del sistema, es necesario integrarlos para representarlos coherentemente en un Modelo.

Aunque no se han establecido principios globales para el modelado de la simulación, el Modelado se considera un arte (Morris 1967) y una actividad creativa (Evans 1991). Por tanto el contar con principios básicos para el modelado resultan una herramienta útil al abordar un proyecto de simulación (Pritsker 1998). Pritsker propone que tales principios deben incluir tres aspectos:

- La conceptualización del modelo requiere conocimiento del sistema, juicio de ingeniería y herramientas de construcción de modelos. El modelador debe entender la estructura y las reglas de operación de un sistema y por tanto tener la habilidad para extraer la esencia del sistema sin incluir detalles innecesarios.

- El secreto para convertirse en un buen modelador es la habilidad para remodelar, esto es, la construcción del modelo debería ser un proceso iterativo ya que éste continuamente es refinado, actualizado, modificado y extendido.

- El proceso de modelado es evolutivo debido a que durante el proceso de modelado deja ver gradualmente información importante. 


\subsubsection{Desarrollo de un Modelo de Simulación}

En el desarrollo de un Modelo de Simulación validado es necesaria la participación de tres entidades básicas: el Sistema del Mundo Real que se estudia, el Modelo teórico del Sistema y su representación basada en computadora, y el Programa de Simulación (Vincent 1998). Asimismo, la actividad relacionada con la definición del modelo teórico del Sistema del Mundo Real se referencia como Modelado de Simulación, y la actividad donde se obtiene la representación basada en computadora se denomina Programación de la Simulación (Ver Fig. 2.5).

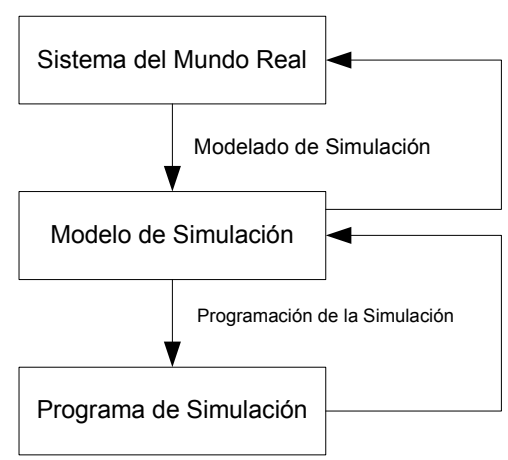

Figura 2.5: Flujo del Desarrollo de un Modelo de Simulación

\subsubsection{Recolección de Datos. El Modelado de las Entradas pa- ra la Simulación}

Una de las actividades clave en el Desarrollo de un Modelo de Simulación son los datos que se obtienen del sistema real. Los datos de entrada al sistema deben combinar la experiencia, teorías relevantes (tales como la referente a procesos estocásticos) y los datos obtenidos. A este proceso se le puede denominar "Modelado de las entradas de la Simulación" (Ver Fig. 2.6). A menudo, el objetivo del Modelado de las entradas de la Simulación es proporcionar un modelo que sea razonable dados los objetivos de la simulación. El aspecto más difícil del modelado de los datos de entrada es la generación de datos de suficiente calidad, cantidad, y variedad para realizar un análisis razonable. 


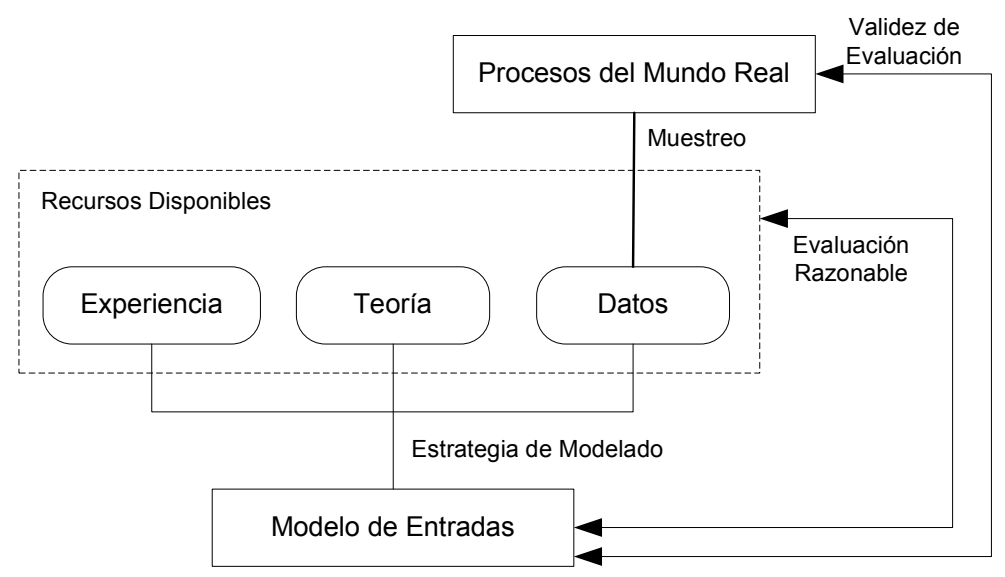

Figura 2.6: Modelado de las entradas de la Simulación

En esta etapa deben tratar de evitarse situaciones que afecten este proceso tales como: tiempo reducido para la recolección, poco personal para su recolección, gran cantidad de información a procesar, etc.. Algunos de los problemas que pueden presentarse con los datos recopilados son: a) los datos son recopilados con insuficiente precisión, e incluso son redondeados al entero más cercano, dejando a un lado los valores reales observados, b) las tablas pueden contener valores de más de un proceso, los cuales no están documentados, c) los datos deben ser representativos de diferentes procesos del mundo real y no de un caso específico.

\subsubsection{Traducción del Modelo en formato Informatizado. La Simulación de Modelos.}

Una simulación es una manipulación de un modelo de forma que funcione en el espacio o en el tiempo para comprimir éste, permitiendo percibir las interacciones que no serían fácilmente apreciables por su separación en el tiempo o el espacio. El Modelado y la Simulación es una disciplina para la consecución de un nivel de comprensión de la interacción entre las partes del sistema y del sistema como un todo. El nivel de comprensión que se puede obtener mediante esta disciplina sería difícil de conseguir utilizando otra disciplina (Law \& Kelton 1991).

De acuerdo con Heim (Heim 1997), los modelos de simulación poseen el potencial para brindar la mayor parte de la información requerida para reducir 
el riesgo asociado al diseño, análisis y operación de sistemas complejos para la realización de un producto. Pero debido a que abarca tanto los modelos integrados como el ámbito para reflejar adecuadamente la ejecución interdependiente de los componentes del sistema, son costosos y difíciles de construir.

Según Davis (Davis 1999) la utilidad de los modelos de simulación y su análisis deriva en su incorporación dentro de estudios más comprensibles. Esto es, un modelo o estudio de simulación es de utilidad limitada. La primera razón para simular un sistema es que no se puede obtener una expresión analítica para la respuesta basada en el sistema mientras que ésta opera bajo un conjunto específico de entradas y valores para un diseño crítico de los parámetros del sistema. De tal manera que, si es posible especificar una solución analítica, entonces los analistas de sistemas no deberían hacer simulaciones. Por otro lado, en muchos casos donde las expresiones analíticas han sido especificadas para pronosticar la ejecución del sistema, usualmente existe la simplificación de suposiciones que degradan de manera significativa la exactitud (precisión) del modelo. Es por ello, que los verdaderos beneficios de la solución propuesta solo se pueden analizar mediante estrictos análisis de simulación.

\section{Tipos de Modelos de Simulación con respecto al tiempo.}

Los modelos pueden clasificarse de diversas maneras (Fishman 1978).

- Un modelo de simulación estática (simulación de Monte Carlo) es una representación de un sistema en un instante de tiempo determinado.

- Una simulación dinámica es una representación de un sistema cuando evoluciona con el tiempo.

- Un modelo de simulación determinista no contiene variables aleatorias.

- Un modelo de simulación estocástica contiene una o más variables aleatorias.

Al permitir que el constructor del modelo emplee el conocimiento abstracto de los modelos existentes, la integración del modelo reduce el esfuerzo en la programación, simplifica la validación del modelo e incrementa la gama de opciones de diseño que pueden ser considerados. La integración de modelos también es una estrategia efectiva para el desarrollo de modelos grandes, debido a que la interoperabilidad brinda la selección del lenguaje y es un entorno más apropiado para el diseño de cada elemento. 


\section{Aproximaciones para un estudio de Simulación.}

Existen cuatro enfoques que son equivalentes y deben proporcionar los mismos resultados al realizar una Simulación (Balci 1988):

- Aproximación de método orientado a eventos. Este enfoque considera los diferentes eventos y cómo afectan al sistema.

- Aproximación de método orientado a actividades. Este considera las actividades y determina qué puede empezar o terminar en cada avance de reloj.

- Aproximación de método orientado a procesos. Este enfoque considera una entidad y la secuencia de eventos que se producen a su paso por el sistema.

- Aproximación de método de tres fases. Este enfoque considera una asignación de recursos disponibles a actividades en el instance en que se realiza el avance de tiempo.

Actualmente la mayoría de las propuestas de Simulación están orientadas a eventos y a los procesos.

\section{Aproximación de Simulación basada en Eventos.}

En este tipo de simulación, los eventos son aquellos que pueden cambiar el estado del sistema. Bajo dicho esquema tanto los tiempos de llegada como los tiempos de servicio distribuidos estadísticamente se producen aleatoriamente. Se utiliza un sistema de colocación en cola (la cual es una estructura de datos) de los eventos que esperan por el servicio y finalmente se eliminan dichos eventos para ser servidos.

Los elementos que intervienen en la Simulación basada en eventos se listan a continuación:

a. Reloj de Simulación. Es aquel que mantiene el tiempo simulado.

b. Lista de Eventos. Contiene una lista ordenada de los eventos siguientes.

c. Rutina de Inicialización. Prepara el sistema para la simulación.

d. Rutina de Evento. Su función es actualizar el estado del sistema y la generación de eventos futuros.

e. Rutina de Temporización. Avanza el reloj hasta el instante del evento.

f. Rutinas de variables aleatorias. Estas rutinas generan variables de distribuciones de probabilidad. 
g. Estado del Sistema. Se refiere a los datos almacenados en colas, listas de eventos, etc., que permiten identificar el estado actual del sistema.

h. Almacenamiento de estadísticas. Se almacenan los datos de ejecución de la simulación.

La Figura 2.7 presenta la interrelación entre estos elementos durante la simulación orientada a eventos.

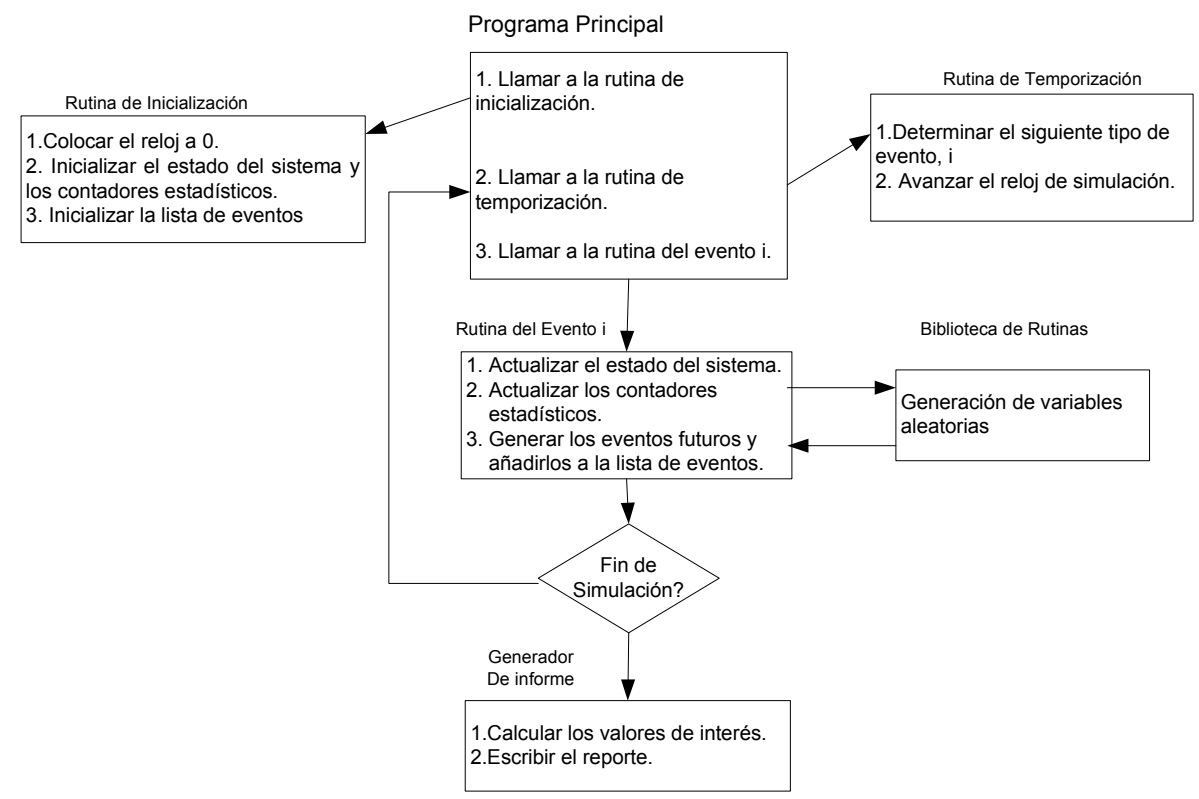

Figura 2.7: Aproximación Orientada a Eventos.

\section{Aproximación de Simulación basada en Procesos.}

Un proceso es una secuencia de eventos ordenada temporalmente. En este enfoque en vez de modelar el cambio de estado del sistema, se describe el progreso de un trabajo a través del sistema. Como los trabajos llegan en momentos diferentes, el comportamiento del sistema está descrito por una serie de procesos (uno por cada entrada), algunos de los cuales pueden sobreponerse. En este caso puede transcurrir en el modelo en contraste con los demás enfoques, los cuales proporcionan solamente variación instantáneas. Para resolver los conflictos entre los procesos se sirve de preposiciones de "espera" y "retraso" tanto en los contextos condicionales e incondicionales. De esta manera, las proposiciones incondicionales "esperar" se relacionan al enfoque de programación temporal de 
eventos mientras que las proposiciones condicionales corresponden en gran parte a la comprobación lógica del enfoque del examen de actividad. En la Figura 2.8 se describe el flujo de control de éste tipo de aproximación. Así, mientras que en el diagrama de flujo del método de programación temporal de eventos se representa un evento, en el de interacción de proceso se abarcan varios eventos.

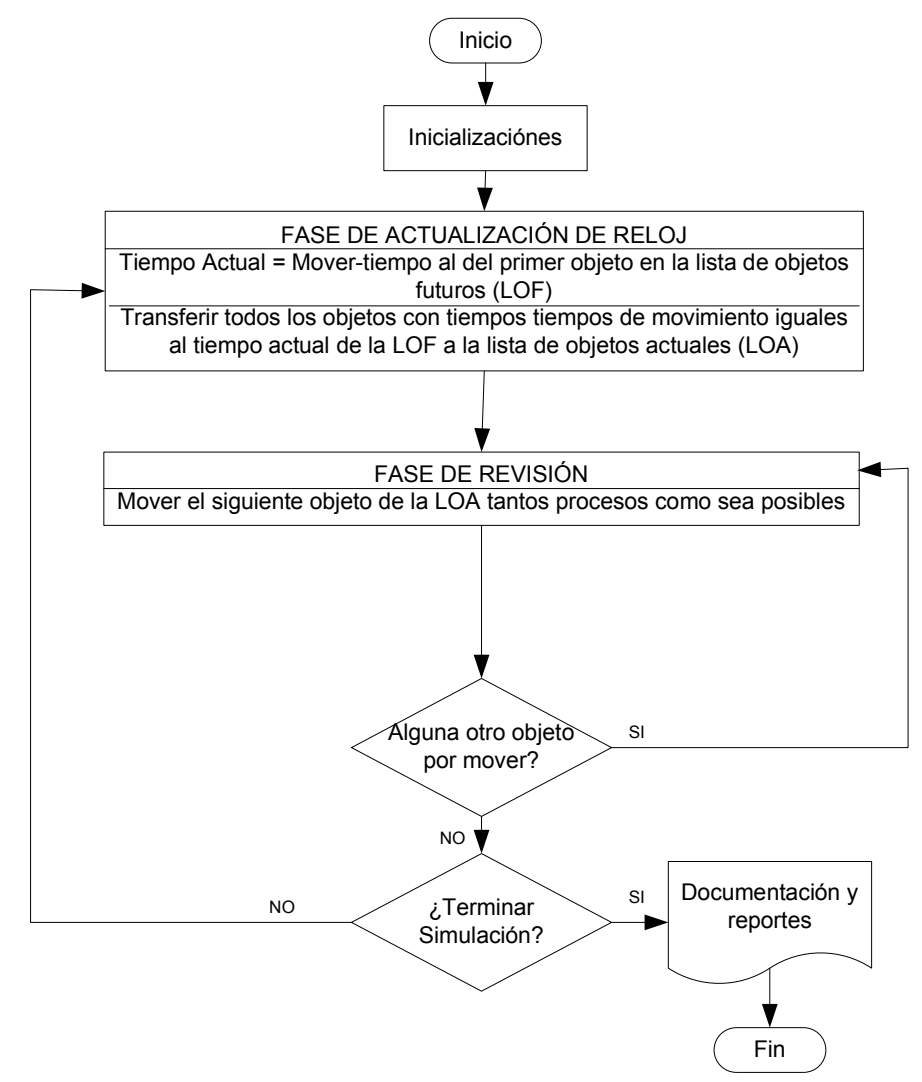

Figura 2.8: Aproximación Orientada a Procesos.

\section{Aproximación de Simulación basada en Actividades.}

Una actividad es una serie de operaciones que transforman el estado de una entidad, además de que se conoce la duración de la actividad por anticipado. Este enfoque también es conocido como la aproximación de dos fases. El rastreo de las actividades genera un programa de simulación compuesto de módulos independientes esperando a ser ejecutados. El rastreo se realiza en lapsos de tiempo fijos en los cuales se verifica si un evento ha sucedido o no. Si un evento 
aparece, el estado del sistema se actualiza. Por lo tanto siempre que se avanza el tiempo hasta el siguiente evento de la cola de eventos, y entonces se examinan todas las actividades para determinar cuál puede iniciar o terminarse (Figura 2.9) en ese momento.

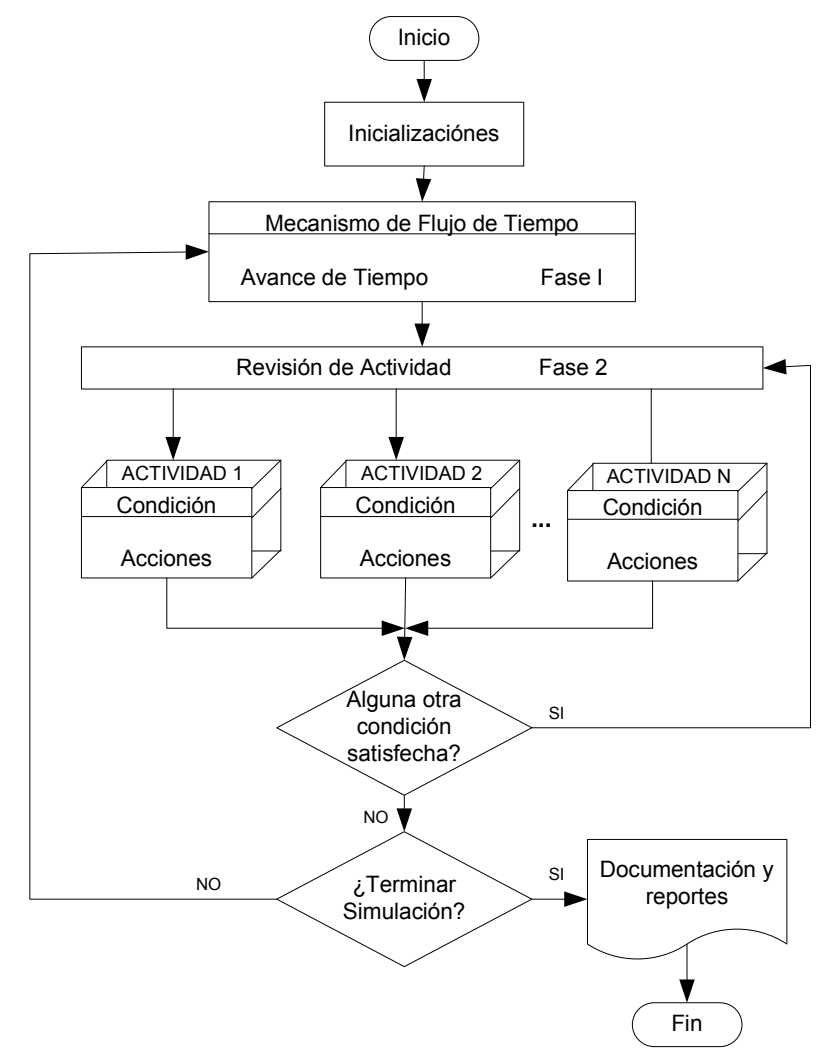

Figura 2.9: Aproximación Orientada a Actividades.

\section{Aproximación de Simulación en Tres Fases.}

En esta aproximación de estructura de modelado de simulación, el avance de tiempo se realiza hasta que se presenta un cambio en el estado del sistema o hasta que algo suceda. El sistema es examinado para determinar todos los eventos que tienen lugar en ese instante de tiempo. Solamente cuando todos los recursos están por liberarse, tiene que ser liberada su reasignación en nuevas actividades que inicien en la tercera fase de la simulación. En resumen, la primera fase se refiere al avance de tiempo. La segunda es la liberación de aquellos recursos programados para terminar sus actividades en este instante. Finalmente, la tercera 
fase es la inicialización de actividades dado el estado general de la disponibilidad de los recursos (Figura 2.10).

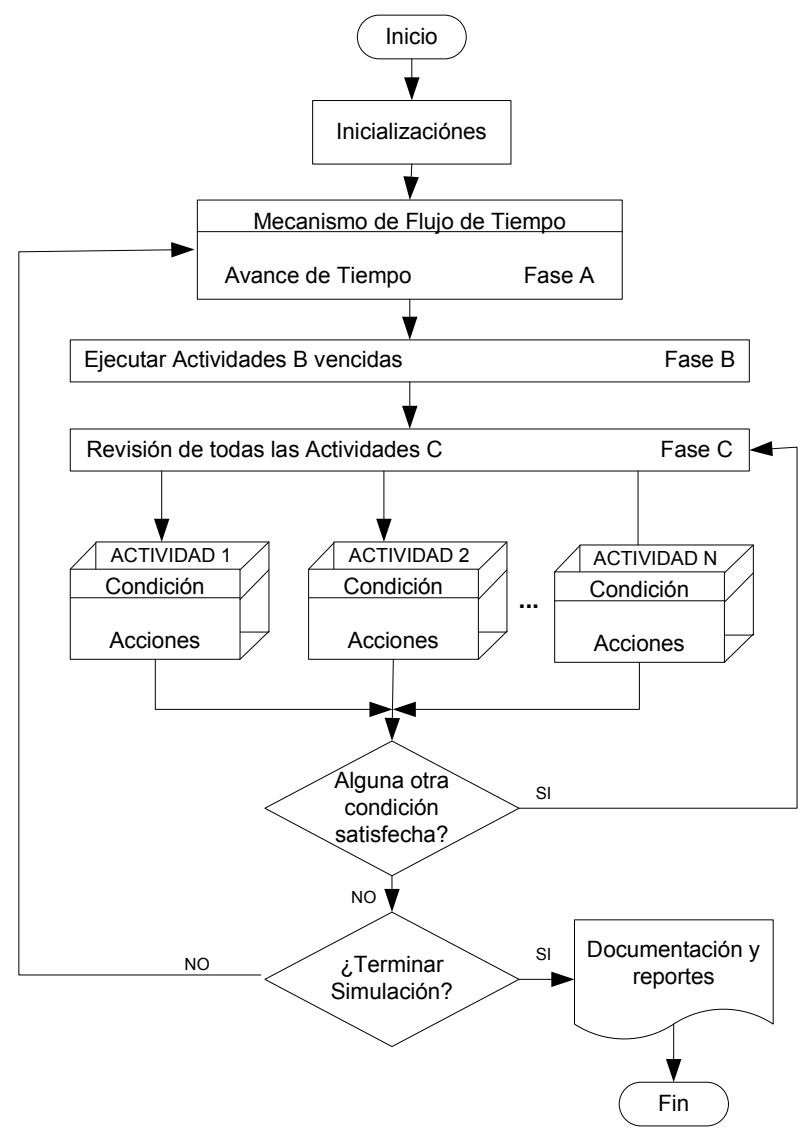

Figura 2.10: Aproximación en Tres Fases.

\section{Manipulación del Tiempo.}

Existen diversas propuestas para el manejo del tiempo durante la simulación de un modelo las cuales se utilizan de acuerdo al concepto que se desee aplicar como elemento clave para identificar el control del tiempo de la Simulación de un modelo.

Un aspecto fundamental de un sistema dinámico es el paso del tiempo. El tiempo se concibe como aquel que opera de forma independiente, donde todo los cambios dinámicos se ordenan siguiendo dicho flujo de tiempo. La base temporal se define como una estructura: 


$$
\text { time }=\{T,<\}
$$

donde:

$T$ es un conjunto y $<$ es una relación de ordenación de los elementos, además es transitiva, irreflexiva y antisimétrica.

La relación de ordenación permite utilizar los términos tales como pasado, futuro y presente. Si $t$ es interpretada para funcionar como tiempo presente. entonces el conjunto de $T_{t)}=\tau \mid \tau \epsilon T, \tau<t$ denota el pasado y el conjunto $T_{(t}=$ $\tau \mid \tau \epsilon T, t<\tau$ denota el futuro. El pasado y el futuro son disjuntos, tal como es requerido en un mundo donde cada momento cuenta y no puede ser recapturado en el futuro una vez que ha pasado. El conjunto de $T_{t \mid}=\tau \mid \tau \epsilon T, \tau \leq t$ denota el pasado incluyendo el presente y el conjunto $T_{\mid t}=\tau \mid \tau \epsilon T, t \leq \tau$ denota el futuro incluyendo el presente. A menudo esto no es crítico si se negocia con intervalos abiertos y cerrados. En este caso, $T_{t>}$ significa tanto $T_{t)}$ como $T_{t \mid}$. Asimismo, $T_{<t}$ denota $T_{(t}$ como $T_{\mid t}$. De esta forma, el conjunto $T_{\left[t_{1}, t_{2}\right)}=\tau \mid \tau \epsilon T, t_{1} \leq \tau<t_{2}$ denota el intervalo de tiempo $\left[t_{1}, t_{2}\right)$ desde el instante de tiempo inicial $t_{1}$ (incluido en el intervalo) y el tiempo final $t_{2}$ (excluido). Análogamente se definen los intervalos $T_{\left(t_{1}, t_{2}\right)}$ o $\left(t_{1}, t_{2}\right)=\tau \mid \tau \epsilon T, t_{1}<\tau<t_{2}, T_{\left(t_{1}, t_{2}\right)}$ o $\left[t_{1}, t_{2}\right]=$ $\tau\left|\tau \epsilon T, t_{1} \leq \tau \leq t_{2}, T_{\left(t_{1}, t_{2}\right]} \mathbf{o}\left(t_{1}, t_{2}\right]=\tau\right| \tau \epsilon T, t_{1}<\tau \leq t_{2} . T_{<t_{1}, t_{2}>} \mathbf{0}<t_{1}, t_{2}>$ se refiere a los intervalos mencionados.

Para el intervalo $<t_{1}, t_{2}>, t_{1}$ es denominado el tiempo inicial o de inicio y $t_{2}$ es el tiempo final o de finalización. La base de tiempo puede contener un elemento mínimo $t_{0}$ el cual sería el límite inferior, por lo tanto todos los elementos $t \epsilon T$ son mayores que $t_{0}$. Si no existiera un límite inferior, entonces $-\infty$ denota un límite inferior ficticio. Si una base de tiempo no tiene un límite superior, el límite superior ficticio es denotado por $\infty$. Obsérvese, que $-\infty$ y $\infty$ no se incluyen en la base de tiempo. El símbolo de infinito satisface $\infty+t=\infty$ para cualquier $t \epsilon T$.

De esta forma, mediante la base de tiempo es posible describir cómo es que se presentan los comportamientos a lo largo del tiempo.

Tipos de Mecanismos de Avance de Tiempo.

Considerando el mecanismo de avance de tiempo se pueden distinguir (Banks et al. 2001): Síncrona en el tiempo y Orientada a Eventos.

a. Mecanismo de Avance de Tiempo Síncrona. Se pueden observar características tales como: a) intervalos de tiempo siempre iguales, y b) el reloj de 
simulación avanza un intervalo en cada ciclo. Sin embargo, este mecanismo tiene un problema en cuanto a que si los retardos de tiempo son cortos y largos a la vez, los ciclos de reloj muy pequeños pueden utilizar mucho tiempo computacional.

En este mecanismo es posible observar características específicas tales como:

a. Evalúa el modelo e intervalos de tiempo,

b. Cuantifica el tiempo considerando dos situaciones:

- Los eventos no simultáneos pero próximos en el tiempo serán tratados como simultáneos, y

- Hay que elegir cuidadosamente los intervalos de tiempo para separar los eventos (lo que puede conducir a ejecuciones de simulación demasiado largas).

c. Las condiciones lógicas se implementan fácilmente, y

d. Los cálculos no están sincronizados con los eventos.

b. Mecanismo de Avance por Eventos. En este mecanismo se observan las siguientes características: a) los eventos se producen en cualquier instante, b) existe una única lista de eventos planificados, c) los eventos se mantienen en orden temporal, d) el sistema sólo calcula en los momentos en los que se producen eventos, e) el reloj de simulación avanza hasta el instante del siguiente evento, y f) los cálculos están sincronizados con los eventos.

\section{Reloj Maestro y Eventos.}

Una de las propuestas estándar utilizadas por Morgan (Morgan 1984) para establecer una secuencia de tiempo es la utilización de un "reloj maestro" el cual almacena el tiempo actual donde los cambios en el tiempo no son unidades constantes, sino que controla dichos cambios en base al siguiente evento. El periodo de tiempo hasta el siguiente evento se obtiene mediante una referencia a una "lista de eventos" la cual proporciona los posibles eventos que podrían presentarse a continuación, y también determina cuando deberán ocurrir. En este caso se utiliza una cola basada en la filosofía "primero en entrar- primero en salir" (FIFO). Esto es hasta que el primer elemento de la cola es atendido y liberado, el siguiente elemento espera a ser atendido. Esta propuesta se puede observar mediante un diagrama de flujo que describe su ciclo básico (Ver Fig.2.11). 


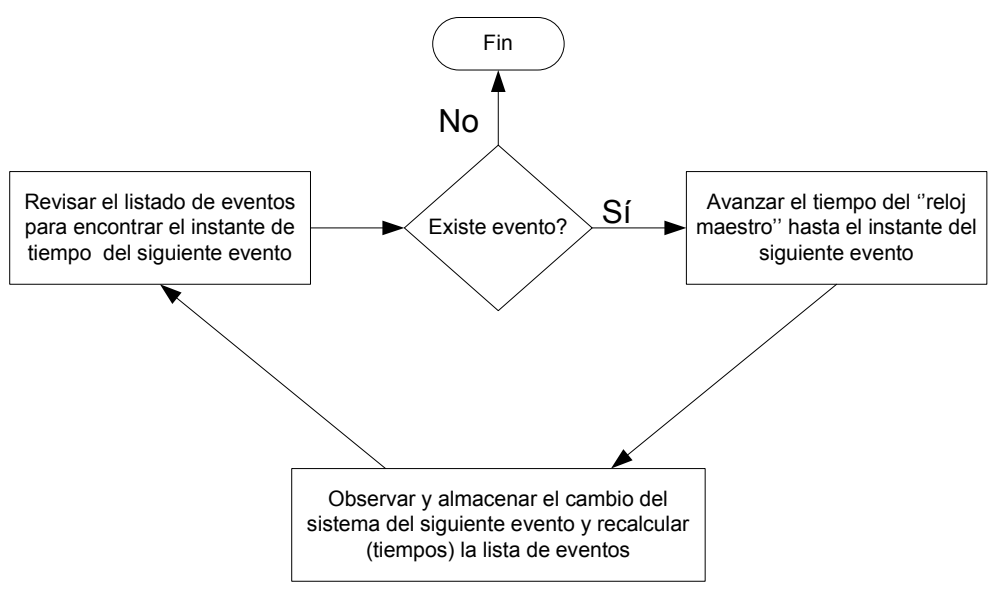

Figura 2.11: Control de Tiempo basado en Lista de Eventos

\section{El estado de la Simulación y el Tiempo.}

El estado del sistema generalmente se expresa como una función de tiempo. Dos conceptos de tiempo se manejan en la simulación de un sistema (Hamilton \& Tumay 1997): Tiempo de Simulación y Tiempo de Ejecución. El tiempo de simulación es el periodo de tiempo simulado por el modelo. El tiempo de simulación generalmente se establece en cero al iniciar la ejecución de la simulación y actúa como un contador del número de las unidades del tiempo de la simulación. La duración de la ejecución de una simulación es la diferencia entre el tiempo inicial establecido en el reloj y el tiempo final del reloj al terminar la simulación. El tiempo de ejecución es el tiempo que toma la computadora para simular el periodo de tiempo.

El tiempo de ejecución está influenciado por la complejidad del modelo de simulación el número de eventos simulados, así como por la duración de la ejecución. En muchos casos, el tiempo de ejecución es significativamente diferente del tiempo de simulación.

Elección del mecanismo de avance de tiempo.

La elección del mecanismo adecuado no es una tarea sencilla y depende de cinco aspectos clave:

- El propósito del modelo.

- La precisión de los resultados a obtener. 
- El tiempo de procesador (CPU) preciso para cada mecanismo.

- Las necesidades de memoria.

- El esfuerzo de programación en cada caso.

Por tanto, considerando el tiempo de ejecución, la elección del método más adecuado depende de tres aspectos:

- El número de entidades capaces de generar eventos significativos para el sistema.

- El número de eventos generados por cada entidad por periodo de tiempo (densidad de eventos).

- La longitud o tiempo medio de un evento (desde que comienza hasta que finaliza el cambio).

Por otra parte, resulta importante considerar que las necesidades de memoria y el esfuerzo de programación no parecen un factor determinante, por lo que la decisión dependerá del sistema a simular.

Por tanto, se elegirá un mecanismo determinado siempre y cuando en el sistema a simular se presenten las características listadas en las Tabla 2.1.

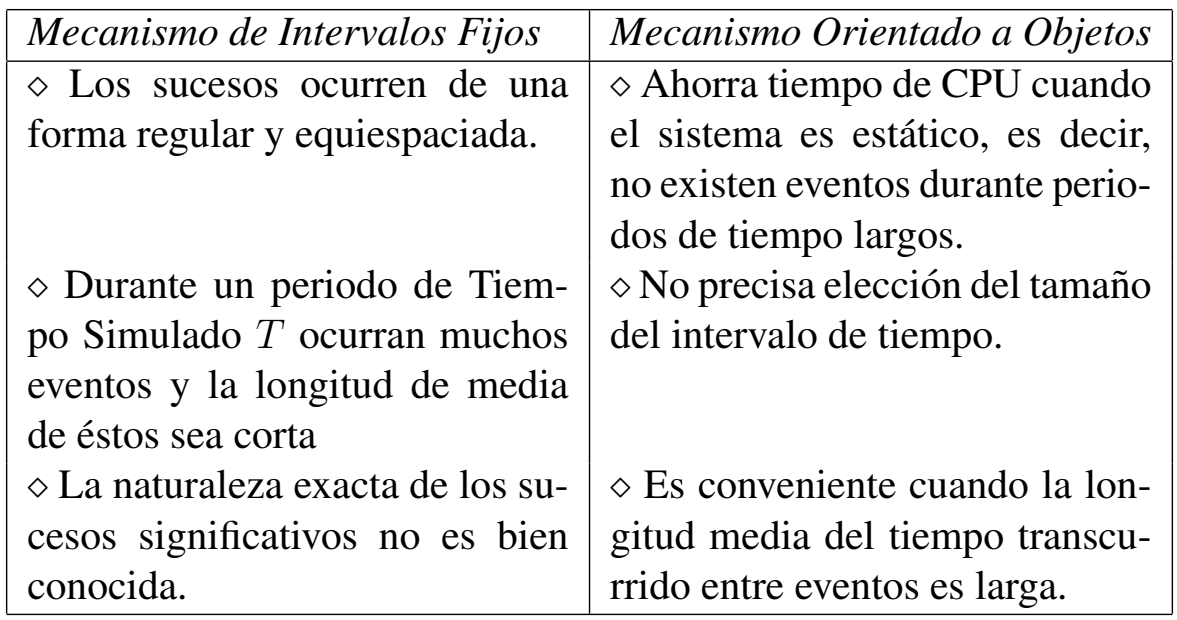

Tabla 2.1: Tipos de Mecanismos de Control de Tiempo

\section{Definición de Condición de Parada.}

Cada simulación debe tener un evento de parada, el cual define cuanto tiempo se ejecutará la simulación (Banks et al. 2001). Generalmente existen dos formas para detener una simulación: 
1. En el periodo de tiempo inicial 0, se define un evento de parada de simulación en un periodo de tiempo $T_{E}$ ubicado en el futuro. Por tanto, antes de iniciar la simulación, se conoce que la simulación se ejecutará dentro del intervalo de tiempo $\left[0, T_{E}\right]$. Por ejemplo, simular una planta de fabricación durante $T_{E}=40$ Horas.

2. La duración de $T_{E}$ se determina por la misma simulación. Generalmente, $T_{E}$ es el tiempo en el que ocurre algún evento especificado $E$. Por ejemplo, $T_{E}$ es el tiempo del evento 100 en un centro de trabajo específico. $T_{E}$ es el tiempo en el cual el centro de distribución embarca la última caja de las órdenes del día.

\subsubsection{Verificación de Código del Simulador.}

Balci en (Balci 1998) indica que es altamente recomendado que la verificación se realice como un proceso continuo. Incluso cuando se utiliza un controlador de ejecución, o depurador, es muy recomendable como soporte en el proceso de verificación. El aseguramiento de la calidad total incluye la medición y valoración de una variedad de características de calidad tales como exactitud, eficiencia en la ejecución, mantenimiento, portabilidad, reusabilidad y usabilidad.

La verificación del modelo se ocupa de la construcción de un modelo correcto. La exactitud al transformar una formulación del problema en la especificación de un modelo o la exactitud para convertir una representación del modelo de un micro-diagrama de flujo a un programa de software ejecutable.

\subsubsection{Validación del Modelo.}

Antes de asumir que un modelo de simulación puede ser utilizado para la toma de decisiones, se debe comprobar su consistencia con el sistema verdadero, en la forma en que existe y antes de que se haga algún cambio. El éxito de esta validación establece la base para la confianza en los resultados que genera el modelo bajo nuevas condiciones. Si un modelo no puede reproducir el comportamiento del sistema sin sufrir algún cambio, difícilmente se puede esperar que produzca resultados representativos al hacerle cambios (Fishman 1978).

Naylor en (Naylor 1971) indica que tanto la importancia relativa del tema así como el establecimiento de criterios universalmente aplicables, son necesarios para aceptar un modelo de simulación como una representación válida de un sistema real. 
Esto es, suponiendo que se tienen los datos de entrada y salida de un sistema, al integrar los datos de entrada en el modelo de simulación parece lógico que los resultados de la simulación deberían ser semejantes a los que genera el sistema original. Esta es una forma sencilla de validar el modelo. Los aspectos que se deben, sin embargo, considerar al evaluar los resultados obtenidos, es que los valores que se han introducido en el sistema pueden ser los valores promediados de un estudio anterior y es factible un margen de "error" el cual se puede considerar como "aceptable" durante la simulación.

\subsubsection{Diseño de Experimentos.}

En cuanto al diseño de experimentos existen varias propuestas. Fishman en (Fishman 1978) presenta dos opciones: a) la técnica de reducción de varianza, la cual permite obtener por el mismo coste, resultados con mayor exactitud estadística, y b) tomar como entrada una secuencia de experimentos que se tenga en mente, llamada disposición mental, la cual ofrece una mayor exactitud estadística y facilita su análisis estadístico.

Un componente clave en el diseño de experimentos se refiere a la selección de las condiciones de operación o reglas de decisión y los valores de los parámetros que se van a especificar en un conjunto de experimentos que según se supone, van a revelar cambios de los procesos del resultado, si acaso existen. Si suponiendo que la entrada de un experimento consiste en $j$ parámetros de entrada (llamados factores) y que se espera ejecutar experimentos con cada factor en $N$ diferentes niveles. Cada combinación de niveles recibe el nombre de tratamiento, de tal manera que se tienen $j^{N}$ tratamientos experimentales. Así el número de tratamientos experimentales en dicho experimento sería factorial completo, lo cual puede volverse muy costoso para la etapa de validación. Por tal razón Fishman presenta técnicas alternativas las cuales pueden ser llamadas "Planes de muestreo" que comprenden una "disposición experimental".

Los principales objetivos de la disposición experimental son: a) La comparación de resultados experimentales en condiciones alternativas de operación, y b) La detección y estimación de la relación funcional que existe entre los factores cuantitativos de entrada y los resultados experimentales.

\subsubsection{Análisis de los Resultados de una Simulación.}

El análisis de los resultados tiene varios objetivos, entre los cuales se encuentran: a) la identificación de comportamiento no deseado, lo cual reduce la 
probabilidad de una mala especificación del modelo, b) comprobar la consistencia entre el mundo real y el modelo de simulación que lo representa.

Entre las opciones de análisis de resultados Fishman en (Fishman 1978) propone analizar el resultado en forma tal que revele la influencia de ciertos errores y limite los intervalos sobre los cuales pueden analizarse los resultados numéricos sin un error substantivo adicional. En este caso, los intervalos de confianza para los parámetros de la población proporcionan una seguridad de cuán representativos son los parámetros correspondientes de la muestra. Estudiar las implicaciones de las condiciones predominantes y calcular los intervalos de confianza son, en realidad, medidas de la calificación, aunque la acción tomada para combatir cualesquiera implicaciones se deben considerar como de carácter preventivo.

\section{Técnicas de análisis para Simulación Estática.}

Cuando la evolución en el tiempo no tiene importancia, entonces las consideraciones estadísticas resultan apropiadas. Los experimentos de muestreo diseñados para conocer las propiedades de los estimadores estadísticos, pertenecen a la categoría de la simulación estática (Fishman 1978).

En la literatura especializada un gran grupo de investigadores ha presentado propuestas específicas para este tipo de análisis, tales como Thoman, Bain y Antle (Thoman, Bain \& Antle 1969) quienes estudiaron las propiedades estadísticas del estimador de máxima posibilidad de los parámetros de la distribución de Weibull en muestras pequeñas. Otros estudios relacionados tienen que ver con el uso de Redes PERT, el estudio de distribuciones de muestreo, y la estimación de varios descriptores de muestreo (p.ej. media, mediana, moda, varianza, asimetría, cuantil, etc.).

\section{Técnicas de análisis para Simulación Dinámica.}

Tal y como lo expresa Fishman en (Fishman 1978), en la simulación dinámica se presta mayor atención al comportamiento promedio del sistema así como a la relación entre eventos en los procesos de interés. Ciertos procesos son de interés de acuerdo al objetivo de la simulación. La media o valor promedio de un proceso sirve generalmente como el descriptor matemático, y es esta cantidad la que a menudo se espera medir durante el curso de un experimento. Asimismo hace énfasis en la diferenciación entre dos tipos de medias:

a. Estado estable. Sea $X(t)$ un proceso estocástico con covarianza estacionaria que se ha estado desarrollando durante un periodo largo y supóngase que se 
observa este proceso en un instante seleccionado arbitrariamente $s$, durante su evolución. Entonces $E[X(s)]$, el valor esperado del proceso en el tiempo $s$ es su medio en el estado estable. El estado estable proporciona un panorama global de sus procesos respectivos.

b. Media condicional. Supóngase que $X(t)$ es un proceso que inicia su evolución en el tiempo $t_{0}$ con un valor $X\left(t_{0}\right)$, especificado arbitrariamente, entonces $E\left[X(s) X\left(t_{0}\right)\right]$ es la media condicional del proceso. El término condicional implica que la experiencia es una función de la condición inicial $X\left(t_{0}\right)$.

Sin embargo, independientemente del interés del investigador ya sea en el estado estable o la media condicional, las condiciones iniciales desempeñan un papel importante en las consideraciones del análisis de los resultados.

\section{Apariencia del Modelo durante la Simulación.}

La apariencia de la animación proporciona una herramienta de análisis adicional (Sadowski 1989). La animación es una excelente herramienta para el análisis de cuellos de botella y para la identificación de la causa de un comportamiento extraño en el sistema. La mayoría de los sistemas son muy dinámicos y cambian rápidamente con el paso del tiempo. Lo que parece ser un problema el día de hoy puede haber sido provocado por un fallo en el equipo hace varios días. Un cuidadoso análisis estadístico no debe ser sustituido u omitido por la mera observación de la animación gráfica del comportamiento de un modelo.

De esta forma, tanto la ejecución de la simulación como su respectivo análisis se utilizan para estimar una medición de la ejecución de los escenarios que están siendo simulados.

\subsubsection{Documentación y Reportes.}

La documentación resulta muy útil no solo durante la creación inicial. Si un modelo de simulación va a ser utilizado nuevamente, es necesario entender como opera el modelo de simulación. Esta actividad estimula la confianza en el modelo y con ello el cliente puede tomar decisiones basadas en sus análisis. Si el modelo es modificado, esto debe ser documentado detalladamente.

Por otra parte, el resultado de todos los análisis debería ser informado (uso de reportes) clara y concisamente. Esto le permitirá al cliente la revisión de la formulación final, las alternativas que pueden ser consideradas, el criterio mediante el cual los sistemas alternativos fueron comparados, los resultados de los experimentos y las recomendaciones del analista. 


\subsubsection{Implementación.}

El analista de simulación actúa como un periodista mas que como abogado (Banks 1998). Si el cliente ha estado involucrado a lo largo del periodo del estudio, y el analista de simulación ha seguido todos los pasos rigurosamente, la probabilidad de una implementación exitosa aumenta.

La ingeniería de software es una disciplina que se traslapa y está entrelazado con la simulación (Hamilton \& Tumay 1997). En la implementación de un modelo de simulación el modelado del sistema es la clave para el diseño de la simulación.

\subsection{Ventajas e Inconvenientes del Uso de la Simu- lación}

El uso de herramientas de simulación trae consigo algunas ventajas y desventajas asociadas a los entornos donde se apliquen. De acuerdo con Pegden (Pegden, Shannon \& Sadowski 1995) existen ciertas ventajas y desventajas asociadas a la utilización de la simulación. Entre ellas, algunas de las ventajas obtenidas al aplicar la simulación son:

- La nuevas normas, procedimientos operativos, reglas de decisión, flujos de información, procedimientos organizacionales, etc., pueden ser analizados sin interrumpir el sistema.

- Los nuevos diseños, planos físicos, sistemas de transporte, etc., pueden ser evaluados sin asignar recursos para su adquisición.

- Las hipótesis en relación a cómo o por qué ocurren ciertos fenómenos, pueden ser analizadas en cuanto a su viabilidad.

- El tiempo puede ser compactado o extendido permitiendo acelerar o ralentizar el fenómeno que se está investigando.

- Se pueden obtener interpretaciones referentes a la interacción de las variables.

- Se pueden obtener interpretaciones referentes a la importancia de las variables durante la ejecución del sistema. 
- Se puede llevar a cabo análisis de cuellos de botella indicando dónde, tanto el trabajo en proceso, la información, los materiales, etc. están siendo excesivamente aplazados (detenidos).

- Un estudio de simulación puede ayudar en la comprensión de cómo funciona el sistema, en vez de cómo los individuos piensan que el sistema funciona.

- Las preguntas sobre "qué pasaría si?" pueden responderse. Este aspecto es particularmente útil en el diseño de nuevos sistemas .

Por otro lado, algunos de los inconvenientes referentes a la utilización de la simulación de diferentes entornos son:

- La construcción de modelos requiere capacitación especial. La construcción es un arte que se aprende con el paso del tiempo mediante la experiencia. Sin embargo, si dos modelos son construidos por dos individuos competentes, los modelos pueden tener similitudes, pero es improbable que sean el mismo.

- Los resultados de la simulación pueden ser difíciles de interpretar. Debido a que la mayoría de las salidas de la simulación son esencialmente variables aleatorias (los resultados usualmente están basados en entradas aleatorias), puede ser difícil determinar si la observación es el resultado de las interrelaciones del sistema o del azar.

- El modelado de la simulación así como su análisis puede consumir tiempo y ser costoso. Escatimar en los recursos para el modelado y el análisis puede traer como resultado un modelo de simulación o un análisis que no es suficientemente apropiado.

- La simulación es aplicada en algunos casos cuando es posible obtener una solución analítica, o incluso preferible. Esto es particularmente cierto en la simulación de algunas líneas de espera, donde se tienen disponibles modelos de espera.

\section{6. Áreas de aplicación}

La simulación puede ser vista como una herramienta útil para desempeñar tareas específicas. Como sugiere Banks et al en (Banks et al. 2001), la simulación puede ser aplicada para los siguientes propósitos: 
1. La simulación permite el estudio de y la experimentación con las interacciones internas de un sistema complejo, o de un subsistema con un sistema complejo.

2. Se pueden observar y simular los cambios informacionales, organizacionales y del entorno, así como el efecto de estas alteraciones en los comportamientos del modelo.

3. El conocimiento obtenido en el diseño de un modelo de simulación puede ser de gran valor para la propuesta de mejoras en el sistema que se este investigando.

4. Mediante el ajuste de las entradas en la simulación y sus resultados, es posible detectar valiosas percepciones respecto a las variables clave de un proceso y sus interacciones.

5. La simulación puede usarse como un dispositivo pedagógico para reforzar las metodologías de soluciones analíticas.

6. La simulación puede usarse para experimentar con los nuevos diseños o normas antes de su implementación, así como para prepararse para lo que pueda suceder.

7. La simulación puede usarse para verificar soluciones analíticas.

\subsection{Conclusiones}

En este capítulo se han identificado las bases de la Teoría para el Modelado de un Sistema, los elementos clave en un Modelo de Simulación y las etapas que incluye un estudio de Simulación. Asimismo, se han presentado las ventajas e inconvenientes derivados de la aplicación de la simulación en general y las áreas donde ha sido aplicada.

Como resultado de esta revisión ha sido posible identificar las características esenciales que se deben considerar para la definición de un entorno de simulación así como las funcionalidades que dicho entorno debe incluir. Asimismo, se toman en consideración las etapas que incluye un estudio de simulación para el desarrollo de nuestra propuesta. En particular se enfatiza la contribución de la simulación en la resolución de problemas específicos de los sistemas de fabricación. 


\section{Capítulo 3}

\section{Los Sistemas Multiagente y la Simulación}

El paradigma de agentes y sistemas multiagente constituye actualmente un área de creciente interés dentro de la Inteligencia Artificial, entre otras razones, por ser aplicable a la resolución de problemas complejos no resueltos de manera satisfactoria mediante técnicas clásicas (Julian \& Botti 2003). Numerosas aplicaciones basadas en este nuevo paradigma vienen siendo empleadas en infinidad de áreas (Jennings \& Wooldridge 1998a), tales como control de procesos, procesos de producción, control de tráfico aéreo, aplicaciones comerciales, gestión de información, comercio electrónico, aplicaciones médicas, juegos, etc.

El objetivo de este capítulo es resumir las bases teóricas relacionadas con los Agentes y los Sistemas Multiagente, conceptos básicos, las características que se utilizan para la definición de agentes, y las áreas donde se ha aplicado la Teoría de Sistemas Multiagente hasta el momento. Asimismo se describe la relación entre los Sistemas Multiagente y su exitosa aplicación en la Simulación de Sistemas. De esta manera, se presenta la aplicación del paradigma de agentes en el diseño de un modelo de simulación y el tratamiento de un entorno de simulación. Finalmente se describen las áreas de aplicación del paradigma de agentes entre las cuales se incluye la Simulación.

\subsection{Agentes y Sistemas Multiagente}

Un agente inteligente es un proceso computacional capaz de realizar tareas de forma autónoma y que se comunica con otros agentes para resolver problemas mediante cooperación, coordinación y negociación. Habita en un entorno 
complejo y dinámico con el cual interactúa para conseguir un conjunto de objetivos (Wooldridge 2002a). En otras palabras, un agente es una entidad activa que lleva a cabo procesos cognitivos como respuesta a las percepciones que recibe del entorno donde se encuentra, de tal manera que responde actuando sobre el mismo.

Al igual que con el concepto de Agente para la definición de Sistemas Multiagente también encontramos algunas variantes como las que se mencionan a continuación:

- Un Sistema Multiagente es diseñado e implementado como varios agentes interactuando entre sí, para así lograr una funcionalidad deseada.

- Su aspecto clave reside en que realiza operaciones muy simples que permiten resolver problemas complejos, el intercambio de información entre agentes es el factor que hace posible obtener buenas soluciones (Pérez, 2004).

- Los Sistemas Multiagente son sistemas computacionales en los cuales dos o más agentes interactúan o trabajan en grupo para ejecutar un conjunto de tareas o para satisfacer algún conjunto de objetivos (Lesser 1999).

- Un Sistema Multiagente se concibe como una sociedad, similar a una sociedad humana, consistente de entidades que poseen características similares a los humanos, tal como movilidad, inteligencia, habilidad de comunicación, etc. (Mouratidis, Giorgini \& Manson 2003).

- Los Sistemas Multiagente son sistemas con un número variable de entidades autónomas que interactúan, y que se comunican entre ellas mediante protocolos flexibles y complejos.... Los sistemas Multiagente usualmente son sistemas continuos con un bajo acoplamiento entre los componentes individuales del sistema (Lind 1999).

- Los Sistemas Multiagente, son sistemas distribuidos con un número de agentes heterogéneos autónomos individuales que interactúan y operan en un entorno. Los agentes pueden ser físicos o lógicos (Amigoni \& Somalvico 2003).

- Un Sistema Multiagente está compuesto de un número de agentes inteligentes que interactúan (Weiss 1999, Wooldridge 2002b), donde un agente 
inteligente, puede ejecutar actividades inferenciales, que puede ser implementado como un programa software, como una computadora dedicada, o como un robot dedicado (Russell \& Norvig 1995).

\subsection{Comunicación entre agentes}

En un Sistema Multiagente un agente debe prever las acciones de los otros agentes en su propia tarea de planificación y cómo puede influir en las acciones de otros agentes en beneficio de sus propios objetivos. Los efectos de las acciones de otros agentes sobre un agente dado pueden o no ser favorables e incluso ir en contra de los objetivos del agente. Por tal motivo, resulta importante definir cómo los agentes se comunicarán durante las interacciones en las que participen.

Por otra parte, en un sistema multiagente se pueden presentar situaciones de cooperación o de competición. En el primer caso, los agentes serán capaces de combinar esfuerzos para obtener soluciones a problemas que el propio agente, por sí mismo, no es capaz de encontrar (Cooperative Multiagent Systems, CMAS). En el segundo, los agentes intentan obtener soluciones para sus propietarios (Self-Interested Multiagent Systems, SMAS). En este último, las estrategias de negociación juegan un papel importante para la resolución de conflictos, asignación de tareas y toma de decisiones.

En los Sistemas Multiagente se pone énfasis en la coordinación, de tal manera que la solución de problemas distribuidos resulta adecuada siempre y cuando los agentes que lo integran son capaces de comunicarse y cooperar con otros.

Cuando dos agentes intentan interactuar, se presenta la necesidad de establecer normas y un canal de comunicación adecuado, para ello se requiere definir un lenguaje y el significado de los símbolos que lo componen, tener un mecanismo de comunicación para intercambiar mensajes, asignar turnos para hablar, definir los tipos de procesos de negociación, etc, para que estos puedan coordinarse.

Se dice que dos agentes han establecido un acto comunicativo (acto de habla) cuando un agente realiza acciones que tienen como objetivo modificar la estructura cognitiva de otro agente.

\subsubsection{Métodos de Comunicación.}

La comunicación es la base de la coordinación y está formada por los protocolos de comunicación y el resultado de los métodos de comunicación. Entre los métodos de comunicación se diferencian los Sistemas de Pizarra y los Sistemas de Mensaje-Diálogo. En la propuesta, estos métodos se utilizarán en diferentes 
niveles de comunicación para la coordinación de las actividades de los agentes que integren en el Entorno de Simulación (Capítulos 5 y 7).

En los sistemas de Pizarra, la pizarra es una estructura de datos que es usada como mecanismo general de comunicación entre las múltiples fuentes de conocimiento y es gestionada y arbitrada por un controlador (Hayes-Roth 1985, Nii 1986b, Nii 1986a, Jagannathan, Dodhiawala \& Baum 1989). De esta manera, la pizarra es un área de trabajo común a los agentes donde poder intercambiar información, datos y conocimiento.

Los Sistemas de Mensajes por otra parte permiten la implementación de estrategias de coordinación complejas. En este método los mensajes que intercambian los agentes pueden ser utilizados para establecer comunicaciones y mecanismos de cooperación utilizando protocolos definidos. Debido a que el contenido del mensaje tiene un formato libre, estos permiten establecer una comunicación muy variada. En este sistema un agente emisor, transfiere un mensaje específico a otro agente, el receptor. Los mensajes son intercambiados directamente entre dos agentes. No se utiliza memoria, ni otros agentes son capaces de leer el mensaje si no va dirigido a ellos. Sin embargo, de ser necesario es posible utilizar el "broadcasting" como una excepción, en la cual el mensaje es enviado a todos los agentes del sistema o a un grupo especificado. Para permitir que los mensajes sean utilizados para implementar estrategias de cooperación se deben definir dos aspectos: 1) definir el protocolo de comunicación que especifique el proceso de comunicación, el formato de los mensajes y la elección del lenguaje de comunicación; y 2) todos los agentes asociados deben conocer la semántica del lenguaje de comunicación.

De esta forma, la clase más sencilla de interacción entre agentes es aquella en la que un agente reacciona a los efectos del entorno generados por los demás agentes a medida que éstos ocurren, en este caso se habla de agentes reactivos.

\subsubsection{Detección de Situaciones de Cambios de Estado.}

Carrascosa en (Carrascosa 2004) resalta la importancia de la detección de situaciones de cambio significativas, en las que el agente que no es capaz de detectar una situación significativa de cambio, no podrá realizar las acciones oportunas que le permitan adaptarse a dicho cambio. De tal manera que una situación significativa de cambio será aquella frente a la cual el agente no podrá responder de la misma forma que lo estaba haciendo hasta ahora. En el trabajo de Carrascosa se propone la especificación de un proceso de meta-razonamiento que se encarga de implementar la adaptación mediante un Lenguaje de Control. El mecanismo que utiliza se basa en eventos definidos por el usuario como determinantes 
para el sistema. Para ello, especifica un conjunto de reglas de meta-razonamiento las cuales se dividen en dos grupos: reglas críticas y reglas opcionales. En nuestra propuesta se pretende controlar el comportamiento de los agentes que permiten visualizar el resultado de las interacciones de un Sistema Multiagente externo al Sistema (Capítulo 5 y 7). De tal manera que en nuestra propuesta se hace uso de las bases teóricas de la definición de un Agente ARTIS (Russell \& Norvig 1995) el cual es un agente que además de ser autónomo, reactivo, pro-activo y tiene continuidad temporal, de forma opcional puede incluir otras características tales como comunicación con otros agentes y comportamiento social.

Para la detección de cambio se plantea el uso de un Módulo de Control el cual funciona basando su información en un blackboard tradicional. Este módulo controla cómo y cuándo se van a ejecutar el resto de las partes.

\subsubsection{Negociación entre agentes.}

De acuerdo con Rizo et. al. (Rizo, Llorens \& Pujol 2002), la negociación es importante en la modelización de sistemas multiagente. Se puede considerar como un proceso de comunicación entre un grupo de agentes para cumplir un contrato mutuamente aceptado. La negociación en los sistemas multiagente se observa desde varios puntos de vista. Por una parte, tanto la asignación de subproblemas como la asignación de recursos puede contemplarse como una negociación; por otro lado, también deben existir negociaciones entre agentes individuales para desarrollar una cooperación en beneficio del sistema. La negociación puede ser competitiva o cooperativa, según el comportamiento de los agentes individuales.

Existen varias situaciones diferentes a la hora de entablar una negociación:

- Ninguno de los agentes obtiene beneficio a través de la negociación, puesto que cada agente sigue su propio objetivo y no existen dependencias directas entre ambos objetivos.

- Al menos uno de los agentes logra el objetivo de forma más rápida o con menos esfuerzo.

- Existen situaciones reales de conflicto entre los agentes, debido a recursos, logros de objetivos similares, etc.

Una clasificación interesante, desde el punto de vista individual del agente, divide las cuatro posibles formas de actuar en la negociación: a) cooperación 
simétrica, b) compromiso simétrico, c) cooperación/compromiso no simétrico y d) conflicto.

En las estrategias de negociación, actualmente están tomando relevancia los algoritmos genéticos (Holland 1992), puesto que cada agente podría generar un número aleatorio de estrategias de negociación y aplicarlas, utilizando posteriormente aquellas que den mejor resultado. Sin embargo, el sistema podría requerir de un número grande de estrategias de negociación, con lo que puede resultar inviable. También está creciendo el uso de la teoría de juegos (von Neumann \& Morgenstern 1947, Weibull 1995, Smith 1976) en el ámbito de la negociación, asumiendo negociaciones más bien agresivas. Los conceptos claves de teoría de juegos aplicada a la negociación incluyen: a) función de utilidad, b) espacio de transiciones, y c) estrategias y protocolos de negociación.

Las características más importantes en una negociación son el lenguaje utilizado por los agentes que participan, el protocolo de negociación y el proceso de decisión o estrategia que cada uno de los agentes utiliza para determinar su posición, concesiones y criterios para llegar a un acuerdo. Un mecanismo de negociación debe incluir características tales como: eficiencia, estabilidad, simplicidad, distribución y simetría.

Este planteamiento de negociación da pie a la posibilidad de procesos de negociación automáticos los cuales pueden ahorrar tiempo a los Usuarios (humanos) además de realizar contratos más beneficiosos (Rosenschein \& Zlotkin 1994). De esta forma, para la negociación es posible aplicar distintas técnicas tales como: votación, subasta, pacto, mecanismos de mercado, contratación y coaliciones.

\section{Procesos de Negociación.}

En los Sistemas Multiagente (SMA) los agentes son diseñados, por lo general, individualmente para actuar de acuerdo a sus intereses (agentes autointeresados, maximizadores de su propia utilidad) y no al interés común (Bond \& Gasser 1988, Zlotkin \& Rosenschein 1991, Rosenschein \& Zlotkin 1994). En estos sistemas las estrategias no podrán ser explícitamente impuestas desde fuera, sino que será necesario diseñar protocolos de negociación que permitan a los agentes seleccionar una determinada estrategia no por imposición, sino porque ésta es la óptima para cada uno de ellos y no habría ninguna razón para adoptar cualquier otra.

Los agentes autointeresados requieren estrategias en equilibrio o estables, para llevar a cabo una negociación, ya que ningún diseñador obtendrá mayores beneficios construyendo un agente que use otra estrategia distinta, si sabe que 
los otros agentes están usando estrategias en equilibrio. De modo que lo interesante sería encontrar un modelo de negociación no cooperativo, caracterizar sus estrategias en equilibrio y relacionar las soluciones resultantes con una o varias soluciones cooperativas, ya que éstas son las que obtienen resultados más beneficiosos (Belmonte, Pérez \& Triguero 2007).

El problema es que encontrar estrategias en equilibrio no es un proceso mecánico (Kraus 1997). Generalmente cada agente debe hacer una estimación acerca de cuáles serán las estrategias que estarán en equilibrio. Para ello intentará adivinar el conjunto de acciones que serán usadas en cada estado de la negociación. Sin embargo, el número de posibles ofertas que un agente podría proponer es muy elevado, así como el número de posibles combinaciones de acciones con que los otros agentes le podrían responder (suponiendo información completa). Por todo ello, calcular las estrategias en tiempo real puede ser un problema inviable. Una posible solución sería identificar las estrategias en equilibrio antes de que se lleve a cabo el proceso de negociación, y posteriormente insertarlas en los agentes (Belmonte et al. 2007). Entre los modelos de mecanismos de negociación cuyos resultados en equilibrio coinciden con algunas de las soluciones clásicas cooperativas (tales como la solución de Nash (Nash 1950) y el valor de Shapley (Shapley 1953)) se encuentran la Estrategia de Zeuthen (Zeuthen 1930), el Modelo de Ofertas Alternantes de Rubinstein (Rubinstein 1982), y el Mecanismo de Negociación Bipersonal (Mas Colell 1997).

Por otro lado, el problema que se debe afrontar al implementar agentes en el mundo real no es tanto el de la incertidumbre como el de la limitación de recursos (Belmonte et al. 2007). Por ello, los agentes reales a lo más que pueden aspirar es a una "racionalidad acotada" (Simon 1955) y el investigador y el diseñador se ven obligados a formalizar y emplear una definición de racionalidad apta para situaciones en las que los cálculos y análisis son costosos o limitados.

Una línea teórica en la que se ha trabajado intensamente es la modelización de agentes mediante autómatas finitos que disponen de un número limitado de estados (Neyman 1985, Rubinstein 1986) o de máquinas de Turing (Binmore 1987, Binmore 1988). Por ejemplo, Neyman modeliza cada jugador $i$ mediante un autómata finito determinista $A_{i}$ provisto de una función adicional de acción, que para cada estado produce una "acción" $a_{i}$ del jugador $i$, y cuya entrada está constituida por las acciones $\left(a_{1}, \ldots, a_{i-1}, a_{i+1}, \ldots, a_{n}\right)$ de los restantes jugadores. El autómata juega un juego iterado, donde su estado en la repetición $t$ está determinado por su estado en la repetición anterior $t-1$ y por las acciones de los restantes jugadores en la misma repetición $t-1$. Este formalismo 
permite expresar en forma computacionalmente acotada distintas estrategias para el juego iterado, cuyo número está relacionado con el número de estados del autómata.

En este trabajo los agentes que participan en el proceso de negociación utilizan esta última propuesta de definición de estados de autómata finitos (Capítulo 7).

\section{Protocolos de Comunicación.}

Los protocolos de comunicación están especificados en diferentes niveles: 1) el nivel inferior especifica el método de conexión, 2) el nivel medio especifica el formato o sintaxis de la información que es transferida, y 3) el nivel superior especifica el significado o semántica de la información. La semántica se refiere tanto a la esencia del mensaje como al tipo de mensaje. Para la definición de dichos protocolos, se requiere un lenguaje de intercomunicación entre los agentes autónomos distribuidos (Agent Communication Language, ACL). Actualmente, existen dos propuestas que se siguen para el desarrollo de la mayoría de los sistemas de agentes: el lenguaje KQML (Finin, Fritzson, Mckay \& McEntire 1994) y el propuesto por la organización FIPA (FIPA 2001).

En nuestra propuesta se ha optado por la utilización del Lenguaje FIPA debido a su mayor grado de estandarización con respecto al lenguaje KQML (Capítu$\operatorname{los} 5,6$ y 7$)$.

\section{Protocolos de Interacción.}

Los protocolos de interacción entre agentes (Agent Interaction Protocols, AIP) generalmente son complejos. Un protocolo de interacción entre agentes describe un patrón de comunicación como una secuencia permitida de mensajes entre agentes y las restricciones del contenido de dichos mensajes.

Para la representación de los protocolos se puede utilizar AUML (Agent Unified Modeling Language) (Odell, Parunak \& Bauer 2001, AUML 2007). Este lenguaje es una extensión de UML para describir a los agentes. Se estructura en tres capas y extiende las siguientes representaciones:

Plantillas y paquetes. Estos representan el protocolo completo.

Diagramas de Secuencia y colaborativos. Los cuales presentan la dinámica entre agentes (inter-agent). 
Diagramas de Actividad y gráficas de estado. Los cuales permiten presentar las dinámicas intra e inter agentes.

\subsection{El Proceso de Aprendizaje en los Sistemas Mul- tiagente.}

Una de las características clave asociadas a la Teoría de Sistemas Multiagente, se refiere a la posibilidad de aprender a partir de la experiencia. Por tanto, resulta importante presentar las bases del proceso de aprendizaje que utilizan los agentes. En nuestra propuesta se considera incluir dicha característica como parte de la definición de un Sistema de Fabricación Inteligente (Capítulo 7).

A pesar de que los agentes que integran un Sistema Multiagente pueden ser programados con comportamientos diseñados previamente, a menudo es necesario que los agentes aprendan nuevos comportamientos en linea, de tal manera que la ejecución de un agente o de un sistema multiagente mejore gradualmente (Stone \& Veloso 2000, Sen \& Weiss 1999). Esto es común debido a que la complejidad del entorno hace que el diseño previo de un agente sea difícil o incluso imposible (Busoniu, Babuska \& DeSchutter 2008).

Entre las técnicas empleadas para el proceso de aprendizaje de un Agente, se encuentra el Aprendizaje por Refuerzo. Un agente que emplea el aprendizaje por refuerzo (AR), aprende de las interacciones de prueba y error en un entorno dinámico. En cada paso del tiempo, el agente percibe el estado completo del entorno y ejecuta una acción, lo cual provoca que el sistema cambie a un nuevo estado. El agente recibe una señal de recompensa escalar que evalúa la calidad de esta transición. La retroalimentación es menos informativa que en el aprendizaje supervisado donde el agente debería descubrir las acciones correctas por si mismo sin la retroalimentación de su ejecución (Cherkassky \& Mulier 1998). De esta forma, la simplicidad y generalidad de esta propuesta hace del Aprendizaje por Refuerzo atractivo para el aprendizaje multiagente. Sin embargo, existen cuestiones a considerar tales como la dificultad en la definición de un objetivo de aprendizaje para el aprendizaje multiple de los agentes, y la coordinación de los comportamientos entre ellos y sus resultados. Además, la escalabilidad de los algoritmos a problemas de tamaño real, actualmente problemático en el aprendizaje de agentes individuales, es una razón incluso mucho más importante para considerar la posibilidad del Aprendizaje por Refuerzo en Sistemas Multiagente 
(ARSM) (Busoniu et al. 2008).

En el ARSM, el sacrificio entre la exploración-explotación en linea de los algoritmos AR para encontrar un equilibrio entre la explotación del conocimiento actual del agente y la exploración de las acciones disparadas por la información recibida que ha sido elegida para mejorar dicho conocimiento. Los agentes exploran para obtener información pero no solo del entorno, sino también de otros agentes. Sin embargo, demasiada exploración puede desestabilizar la dinámica de aprendizaje de otros agentes, de tal manera que la tarea de aprendizaje es más difícil para el agente explorador. La elección de las acciones de los agentes deben ser mutuamente consistentes para mantener su intención.

En el aprendizaje por refuerzo de un agente individual, el entorno del agente se describe mediante un proceso de decisión de Markov. Un proceso de decisión de Markov finito es una tupla $\langle X, U, f, p\rangle$ donde $\mathrm{X}$ es el conjunto finito de estados del entorno, $U$ es el conjunto finito de acciones de agentes, $F: X x U x X \rightarrow[0,1]$ es la función de probabilidad de la transición de estado, y $\rho: X x U x X \rightarrow \mathcal{R}$ es la función de recompensa.

La señal de estado $x_{k} \in X$ describe el entorno en cada paso del tiempo discreto $k$. El agente puede alterar el estado cada instante del tiempo al tomar acciones $u_{k} \in U$. Como resultado de la acción $u_{k}$, el entorno cambia su estado de $x_{k}$ a alguna de $x_{k+1} \in X$ de acuerdo a las probabilidad de transición de estado dadas por $f$ : la probabilidad de terminar en $x_{k+1}$ dado que la $u_{k}$ que se ejecuta en $x_{k}$ es $f\left(x_{k}, u_{k},\right), x_{k+1}$. El agente recibe una recompensa escalar $r_{k+1} \in \mathcal{R}$, de acuerdo con la función de recompensa $\left.\rho: r_{k+1}=\rho\left(x_{k}, u_{k},\right), x_{k+1}\right)$. Esta recompensa evalúa el efecto inmediato de la acción $u_{k}$.

El comportamiento de un agente se describe mediante su política $h$ la cual especifica cómo el agente elige sus acciones en un estado dado. La política puede ser estocástica, $h: X x U \rightarrow[0,1]$, o determinística $h: X \rightarrow U$. Una política se denomina estacionaria si no cambia con el tiempo. El objetivo del agente es maximizar en cada paso del tiempo k, el retorno descontado previsto:

$$
[H] R_{k}=E\left\{\sum_{j=0}^{\infty} \gamma^{j} r_{k+j+1}\right.
$$

donde $\gamma \in[0,1)$, es el factor de descuento, y la expectativa se toma sobre las transiciones de estado probabilísticas. La cantidad de $R_{k}$ compacta, representa la recompensa acumulada por el agente a largo plazo. La tarea del agente es, sin 
embargo, maximizar su ejecución a largo plazo, mientras solo se recibe la retroalimentación de su ejecución inmediata de un paso.

La aceleración que aporta el ARSM se debe a la computación paralela cuando los agentes explotan la estructura descentraliza de la tarea. Compartir la experiencia puede auxiliar a los agentes con tareas similares para aprender mejor y rápidamente. Cuando uno o más fallan en un sistema multiagente, el resto de los agentes pueden ejecutar sus tareas. Esto implica que ARSM sea inherentemente robusto. Asimismo, por diseño, la mayoría de los sistemas multiagente permiten la fácil inserción de nuevos agentes en el sistema, conduciendo a un alto grado de escalabilidad.

La generalización del proceso de decisión de Markov para el caso de los Sistemas Multiagente es el juego estocástico. Un juego estocástico es una tupla $<X, U_{1}, \ldots, U_{n}, f, \rho_{1}, \ldots, \rho_{n}>$ donde $n$ es el número de agentes, $X$ es el conjunto discreto de estados del entorno, $U_{i}, i=1, \ldots, n$ son los conjuntos discretos de las acciones disponibles para los agentes, obteniendo como resultado el conjunto de acciones conjuntas $U=U_{1} x \ldots x U_{n}, f: X x U x X \rightarrow[0,1] \mathrm{y}$ $\rho_{i}: X x U X X \rightarrow \mathcal{R}$ que es la función de probabilidad de la transición de estado.

Entre los dominios en los cuales se ha aplicado esta técnica se encuentra el Control Distribuido (Stephan, Debes \& Gross 2000, Wiering 2000, Bakker, Steingrover \& Schouten 2005), Equipos de Robots (Bowling \& Veloso 2002, Mataric 1996, Fernandez \& Parker 2001, Ishiwaka, Sato \& Kakazu 2003), Automatización de Comercio (Tsu \& Soo 2001, Lee \& Oo 2002, Tesauro \& Kephart 2002, Raju, Nrahari \& Ravikumar 2003) y Gestión de Recursos. Debido a que éste último es el dominio que utilizaremos (Capítulos 6 y 7), a continuación se detalla dicha aplicación.

$\mathrm{Al}$ aplicar este paradigma para la gestión de recursos en una Planta de Fabricación, los agentes forman un grupo de agentes cooperativos, que pueden actuar como:

a) Gestores de Recursos (Crites \& Barto 1998). Cada agente gestiona un recurso, y los agentes aprenden cómo gestionar el mejor servicio ante las solicitudes para optimizar una cierta métrica de la ejecución.

b) Clientes de Recursos (Schaef, Shoham \& Tennenholtz 1995). Los agentes o clientes aprenden como seleccionar al mejor recurso de tal manera que se optimiza una determinada métrica.

Las métricas de ejecución incluyen el promedio de tiempos de procesamiento de trabajos, mínimo tiempo de espera por recursos, utilización de recursos y equidad en el servicio a clientes. 


\section{4. Áreas de Aplicación de los Sistemas Multiagen- te}

Los Sistemas Multiagente son un paradigma poderoso para la computación del siglo XXI (Lesser 1999), en el cual las redes de interacción, el tiempo real, y los agentes inteligentes parecen integrar el trabajo de las personas y las máquinas. Por su parte, los agentes adaptan dinámicamente su solución a problemas ajustándose de manera efectiva. Tales ajustes se basan en el uso de patrones, configuración de los recursos y las habilidades e información de que dispone cada uno de ellos. Algunas de las aplicaciones más representativas de los Sistemas Multiagente se pueden agrupar en las seis categorías globales listadas a continuación:

\section{Aplicaciones Distribuidas}

Algunos de los ejemplos de dominios de aplicación distribuidas que han empleado el enfoque de Sistemas Multiagente incluyen:

1. Evaluación de Situaciones Distribuidas, la cual enfatiza cómo los agentes (distribuidos con diferentes esferas de conocimiento y control tales como segmentos de red) deberían compartir sus interpretaciones locales para llegar a explicaciones y respuestas consistentes y comprensivas. Por ejemplo, diagnóstico de red (Sugawara \& Murakami 1992, Oates et al. 1997), recopilación de información en internet (Decker, Pannu, Sycara \& Williamson 1997) y redes de sensores distribuidos (Mason \& Jonhson 1989, Carver \& Lesser 1991).

2. Programación y Planificación Distribuida, la cual enfatiza cómo los agentes (asociados con cada célula de trabajo) deberían coordinar sus programas para evitar y resolver conflictos sobre los recursos, para así maximizar la salida del sistema. Por ejemplo, programación de fábrica (Neiman et al. 1994, Panurak 1987, Sycara et al. 1991), control de redes (Alder, Davis, Weihmayer \& Worrest 1989) y entornos inteligentes (Huberman \& Clearwater 1995, Boman, Davidsson, Skarmeas \& Clark 1998).

3. Sistemas Expertos Distribuidos, los cuales enfatizan cómo los agentes comparten información y realizan negociaciones para la obtención de soluciones colectivas (diseños) basados en su experiencia y criterios de solución. Por ejemplo, ingeniería concurrente (Lander 
\& Lesser 1997) y la restauración de servicios de red (Cockburn \& Jennings 1996, Jennings 1994).

\section{Aplicaciones Industriales}

La tecnología de Sistemas Multiagente ha demostrado su utilidad en el modelado de los Sistemas de Fabricación, entre los ejemplos de dichas aplicaciones podemos mencionar: CIIMPLEX (Peng, Finin, Labrou, Chu, Long, Tolone \& Boughannam 1998), HOLOS Architecture (Rabelo \& Camarinha-Matos 1994), MASSIVE (Rabelo, Camarinha-Matos \& Afsarmanesh 1999), MethaMorph I (Maturana \& Norrie 1996), MethaMorph II (Shen et al. 1998), DEDEMAS (Tonshoff, Seilonen, Teunis \& Leitao 2000), CORTES (Sadeh \& Fox 1989), IFCF (Lin \& Solberg 1992), YAMS (Parunak 1998), MASCADA (Valckenaers et al. 1999), Production System (Bussmann \& Schild 2001), PABADIS (Sauter \& Massotte 2001), Chilled Water System Automation (Maturana \& et. al. 2002), FactoryBroker (Colombo et al. 2006), Shop Floor engineering (Barata \& CamarinhaMatos 2003) y Daimler Chrysler (Bussmann \& Schild 2000)). Por otra parte las características propias de los agentes (proactividad, reactividad y sociabilidad) han permitido aplicar esta tecnología a la Integración de la Empresa y la Administración de la Cadena de Suministro.

\section{Aplicaciones en Medicina}

Entre las características comunes de los problemas en el campo médico y que se consideran más relevantes se encuentran (Moreno 2005):

- Es habitual que el conocimiento necesario para solucionar un problema esté distribuido en diferentes lugares.

- La solución de un problema depende de la coordinación de las tareas a realizar por diferentes individuos con diversas habilidades y competencias, usualmente sin la supervisión de un coordinador único centralizado.

- Los problemas del ámbito médico son habitualmente complejos, y no es fácil aplicar las técnicas habituales de Ingeniería del Software para solucionarlos.

Algunos ejemplos de las aplicaciones de Sistemas Multiagente son: planificación de actividades sobre los pacientes (Hannebauer \& Müller 2001), 
gestión de trasplantes de órganos y tejidos (Moreno \& Bocio 2001), gestión de salud dentro de una comunidad (Mouratidis, Manson, Giorgini \& Phil 2002), acceso a información médica (MARVIN (Baujard, Baujard, Aurel, Boyer \& Appel 1998)) y sistemas de ayuda a la decisión (GUARDIAN (Larsson \& Hayes-Roth 1998)).

\section{Aplicaciones en Recuperación de Información}

Se han hecho algunas propuestas de clasificación de este tipo de agentes, tales como la de Caglayan (Caglayan \& Harrison 1997) que los divide en agentes de escritorio, agentes Intranet y agentes Internet; Klush (Klush 2001) los divide en agentes cooperativos o no-cooperativos, adaptativos, racionales y móviles, o Julián et al. (Julián, Rebollo \& Carrascosa 2001) que los dividen en agentes de búsqueda, agentes de filtrado y agentes de monitorización. Entre los ejemplos de este tipo de aplicación encontramos a Letizia (Lieberman 1995), Amalthea (Moukas \& Maes 1998), WebMate (Chen \& Sycara 1998), Citeseer (Bollacker, Lawrence \& Giles 1998), InfoSpider (Menczer \& Belew 2000), Expertfinder (Vivacqua \& Lieberman 2000) y Granite Nights (Grimes, Chalmes, Edwards \& Preece 2003).

\section{Aplicaciones en Comercio Electrónico}

Una de las razones más importantes que presenta el comercio electrónico (Noriega, Rodríguez-Aguilar \& Sierra 2005) es que sus problemas son complejos tanto teórica como técnicamente, suscitando innovaciones y desarrollos que pueden ser resueltos mediante la aplicación de la tecnología de agentes. Entre los ejemplos asociados al comercio electrónico podemos mencionar MASFIT (Proyecto-Europeo 2000) y COMPRANET (Jaso 2004).

\section{Telecomunicaciones}

Las redes de telecomunicaciones (Garijo 2005) constituyen el entorno natural para que los agentes desplieguen sus capacidades. Desde principios de los 90 se ha desarrollado un amplio abanico de aplicaciones (Albayak \& Garijo 1998, Albayak 1999).

Entre algunos de los ejemplos más representativos citaremos: a) la gestión de redes donde MAITE (Arauz, Banerjee \& Krishnamurthy 2001) se desarrolló como una herramienta de apoyo a la operación y mantenimiento de la red de centrales IBERCOM. En dicha herramienta los agentes residen en un nodo, reciben las alarmas a través del sistema de gestión e interactúan 
con los nodos de la red a través de él, b) el desarrollo de servicios de telecomunicaciones donde podemos mencionar el asistente personal desarrollado por Garijo et al. (Garijo, Tous, Corley \& Tesselaar 1998) para la ejecución de trabajo cooperativo entre un viajero frecuente y la organización de sesiones de trabajo con sus colegas en distintas localidades. El agente personal de comunicación (APC) es un agente cognitivo que se registra automáticamente con un servicio de directorio que permite la localización del usuario, y le pregunta los datos para establecer la sesión de trabajo; con dichos datos establece contacto con posibles proveedores de servicios de vídeo-conferencia y les pide sus precios. Los agentes de los proveedores -agentes proveedores de servicios (SPA)- pueden llevar a cabo negociaciones con los clientes. Así sucesivamente se establecen otros enlaces con el resto de los elementos necesarios tales como los Agentes proveedores de Red (NPA) para llevar a cabo la sesión bajo las mejores condiciones posibles dentro de los parámetros negociados, c) en la mejora de procesos de negocio donde el proyecto EURESCOM EU P815 "Communications Management Process Integration using software Agents" (Eurescom 2001), permite realizar la gestión de proyectos de red inteligente. Su arquitectura está formada por tres capas comunicadas mediante un entorno de procesamiento distribuido, la capa de agentes contiene un gestor que crea y supervisa los agentes del sistema, y dos tipos de agentes: el asistente del gestor del proyecto PMA y el asistente del desarrollador PDA. En ésta, los agentes se comunican con una capa intermedia para controlar el plan del proyecto y el acceso a las herramientas así como el flujo de resultados.

\subsection{La Simulación y los Sistemas Multiagente}

La Simulación es utilizada en dos categorías de aplicaciones (Yilmaz \& Tuncer 2007): 1) para obtener experiencia (simulación para entrenamiento) con los siguientes tipos de usos: virtuales y simulaciones constructivas, y 2) para realizar experimentos (simulación en áreas diferentes al entrenamiento). La simulación también puede ser percibida como una infraestructura que soporta actividades del mundo real, una actividad de cómputo, un actividad basada en un modelo, una actividad de generación de conocimiento y una actividad de procesamiento de conocimiento.

Los tipos de problemas que son resueltos mediante sistemas de simulación, están requiriendo más y más soluciones abiertas, adaptativas y flexibles. Por 
ejemplo, en problemas científicos más realistas, la naturaleza del problema cambia y la simulación se amplía. Los parámetros iniciales, así como los modelos pueden ser irrelevantes bajo condiciones emergentes. Los modelos relevantes requieren ser identificados e instanciados para continuar la exploración. Sin embargo, la exploración manual a menudo no es efectiva y realista en cuanto al coste dentro del espacio de estados de un problema grande. Contemplar la incertidumbre es fundamental para analizar los fenómenos de evolución complejos. La adaptabilidad en simulaciones es necesaria para evolucionar sistemas de forma flexible. La utilización de agentes se defiende y han sido aplicados en numerosos dominios de aplicación (Parunak 1999) para eludir las limitaciones de los métodos convencionales.

Tomando en consideración las oportunidades de aplicación que ofrecen las áreas de la Simulación, los Agentes y los Sistemas Multiagente, el objetivo de esta sección es la presentación de los requisitos básicos de un Sistema de Simulación basado en Agentes y la Simulación soportada por Agentes así como los beneficios que ha aportado la Simulación basada en Agentes en diferentes áreas de aplicación.

La Simulación dirigida por Agentes consiste en tres distintas áreas que pueden agruparse en dos categorías: a) el uso de la Simulación para Agentes, y b) el uso de Agentes para la Simulación. En la primera se encuentra la Simulación de Agentes; y en la segunda, la Simulación Basada en Agentes y la Simulación Soportada por Agentes (Fig. 3.1) (ADS 2008, Yilmaz \& Tuncer 2007).

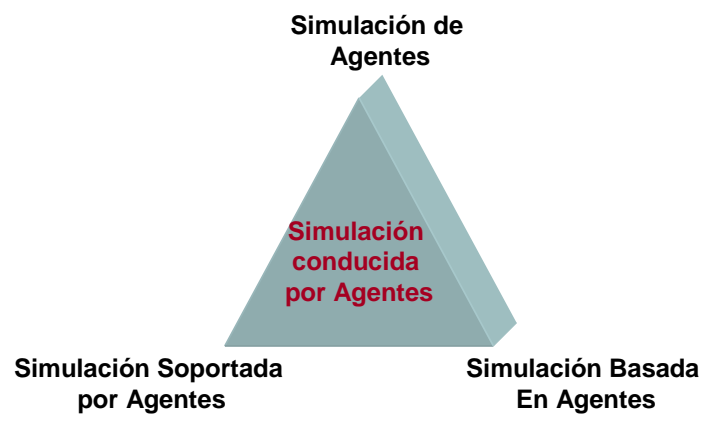

Figura 3.1: Simulación Dirigida por Agentes

La Simulación de Agentes es un área ampliamente extendida, donde se utiliza la simulación para representar sistemas de agentes. En la actualidad la simulación de agentes se ha aplicado en áreas tales como: aplicaciones de ingeniería (ingeniería eléctrica, mecatrónica, robótica, software, logística), de gestión y 
economía (e-commerce, economía), en sistemas sociales y comportamiento humano (negociación, teoría de organizaciones, comportamiento humano/psicológico), entornos (ecosistemas) y militar.

La Simulación Basada en Agentes intenta facilitar dos tareas (Bazzan et al. 2005): comprender el sistema y hacer predicciones cualitativas y cuantitativas en relación al futuro. Un modelo de simulación basado en agentes consiste de un conjunto de agentes los cuales encapsulan el comportamiento de varios individuos. La ejecución del modelo emula el comportamiento global del sistema. La Simulación Basada en Agentes utiliza los agentes para la generación del comportamiento del modelo en un estudio de simulación.

La Simulación Soportada por Agentes utiliza los agentes en un estudio de simulación con los siguientes propósitos:

- Para proveer asistencia computacional para las funciones de interfaces finales o internas;

- Para procesar elementos de un estudio de simulación de forma simbólica (por ejemplo, para verificaciones de consistencia y fiabilidad incorporada);

- Para proveer habilidades cognitivas a los elementos de un estudio de simulación, tales como aprendizaje o comprensión de habilidades.

La Simulación Basada en Sistemas Multiagente (MASB) emerge como un soporte ante la alternativa de la simulación de sistemas complejos (Drogoul et al. 2003). Contrario a la simulación numérica, donde el conocimiento solo puede ser representado por variables y relaciones entre variables, la MASB permite un amplio rango de representaciones: fórmulas, reglas, heurísticas, procedimientos, etc.

De tal manera que en nuestra propuesta se aplica por una parte la simulación basada en agentes para la definición del modelo de simulación (Capítulo 6) y para los elementos se integran las habilidades cognitivas a los elementos del modelo, características de la simulación soportada por agentes (Capítulos 5,6 y 7).

\subsubsection{El Proceso de Diseño de Simulación basada en Agentes.}

Entre las propuestas para el proceso de diseño de la simulación donde se puede aplicar el paradigma de agentes se encuentra la de Drogoul (Drogoul 
et al. 2003). Drogoul et.al presentan un modelo para el diseño de una simulación. Dicho modelo parte de la propuesta de Fishwick (Fishwick 1997) y de las modificaciones a dicho modelo realizadas por Gilbert y Troitzch (Gilbert \& Troitzch 2005). En dicho modelo existen dos actores que interactúan para generar una simulación y sus contribuciones. Tales actores incluyen: a) un Sistema Objetivo, el cual caracteriza el fenómeno para predecir o la teoría que requiere ser explicada. En ésta se incluye a los expertos de un dominio en particular o de un tema específico, quienes reciben el nombre de temáticos; b) debido a que la simulación se ejecuta en computadora, se requiere de expertos en ciencias de la computación para la construcción de programas: los científicos de la computación y c) una tercera comunidad, que participa en el proceso de la construcción de simulaciones (diseño, construcción, ejecución y análisis), los modeladores.

Los expertos de un dominio (denominados temáticos), definen la intención del proceso de simulación (p.ejem., la asociación entre el sistema objetivo y la aplicación de la simulación). Sin embargo, poseen como mucho una idea superficial de lo que es posible o no definir en sus modelos. Estos expertos manejan tres tipos de datos del sistema objetivo: a) teorías y supuestos (lo que conocen o estiman), b) observaciones (lo que ven o analizan), y c) preguntas (lo que quieren comprender) agrupadas en tres categorías: predictivas, especulativas y preguntas teóricas (Troitzsch 2000).

Los temáticos que se interesan por la simulación multiagente comparten el mismo perfil: generalmente tienen dos niveles de conocimiento al mismo tiempo, denominados micro y macro conocimiento. El macro-conocimiento es un conjunto de conocimiento "global" respecto al sistema objetivo, por lo general obtenido a partir de las observaciones. El micro-conocimiento es el conocimiento "local" respecto a las "particularidades" sin las cuales el sistema objetivo no existiría, éste se compone de observaciones y suposiciones. La mayoría de los temáticos se adentran en el diseño de simulaciones multiagente precisamente porque les interesa vincular estos dos niveles.

En un formalismo basado en agentes, el micro-conocimiento se traduce como el modelo del dominio, el cual contiene agentes provistos con comportamientos y datos obtenidos de observaciones relevantes, suposiciones y teorías. Generalmente, éste se define utilizando un lenguaje específico del dominio. El macro-conocimiento se utiliza posteriormente para proporcionar escenarios y métricas para la simulación. En el modelo del dominio, el temático manipula agentes "reales" (p.ejem., agentes que pueden ser observados y analizados en el sistema objetivo). 
La especificación de los temáticos no permite realizar su transcripción directa a una solución operacional debido a la diferencia semántica. Por tanto, el modelo del dominio tiene que ser traducido en algo más formal que pueda ser eventualmente implementado por un científico de la computación. Ésta es la función del modelador. Su rol es aclarar los conceptos y eliminar las ambigüedades mediante la especificación de un modelo de diseño. En el proceso de simulación, se puede decir que este modelo es para una implementación basada en agentes lo que un diagrama UML es para un programa orientado a objetos.

El modelador manipula "agentes conceptuales" (p.ejem., agentes que constituyen un refinamiento formal de un agente previo). Sus propiedades se expresan empleando conceptos de los sistemas multiagente tales como (Jennings 2000): modelo de comportamiento, comunicaciones, tipo de entorno, etc.

El diseño del modelo probablemente es el modelo más difícil de definir, debido a que depende de la información proporcionada por el temático y algunas de las restricciones inherentes a la implementación elegida. Su construcción difícilmente es directa debido al número de iteraciones entre los dos niveles (Rasmussen \& Barret 1995).

Finalmente, el científico de computación, no solamente tiene como objetivo escribir un programa de cómputo, sino también propone un modelo que pueda ser discutido con el modelador. Sin este modelo, sus propuestas y elecciones pudieran no recibir ninguna retroalimentación de los otros dos roles.

Darle forma al modelo operacional es una operación que inmediatamente precede a la construcción de un sistema de cómputo. En este modelo, el científico de computación puede manipular agentes computacionales. La semántica asociada al modelo operacional por lo tanto está restringida a las posibilidades de la implementación. Este modelo a menudo se anula y se reemplaza directamente por la implementación, debido a que al científico de computación, en muchas instituciones se le sigue considerando como un simple técnico. Desde el punto de vista de la simulación global, el modelo operacional, permite a los modeladores y a los científicos de computación comprender, e incluso cambiar, lo que va a ser implementado.

En particular, mediante la especificación de propiedades técnicas tales como la distribución de los agentes, las técnicas de planificación de tiempo utilizadas, etc. y mediante su integración en un modelo global, se facilita la comparación 
entre diferentes modelos o ayudan a comprender el rol de las características específicamente computacionales en la aparición de estructuras en una simulación (Axtell 2000a).

Aunque en el proceso de diseño se asocia en cada modelo (de dominio, de diseño y operacional) a un rol determinado, es posible que una misma persona lleve a cabo dos roles diferentes. Por ejemplo, un experto del dominio puede ejecutar el rol de temático y el de modelador si el dominio le permite realizar una transcripción cuasi-formal sin ser ambigüa. Al mismo tiempo, el científico de cómputación puede ejecutar el rol de modelador si su conocimiento sobre el dominio del sistema objetivo se lo permite.

En el proceso completo descrito en la Figura 3.2, las capas horizontales corresponden a los diferentes roles. Este proceso consiste en una sucesión de modelos que proceden del entorno real al entorno de simulación. Este diagrama sirve de guía para los diseñadores de simulaciones multiagente, aunque puede utilizarse como un marco para la Simulación Basada en Sistemas Multiagente (MASB).

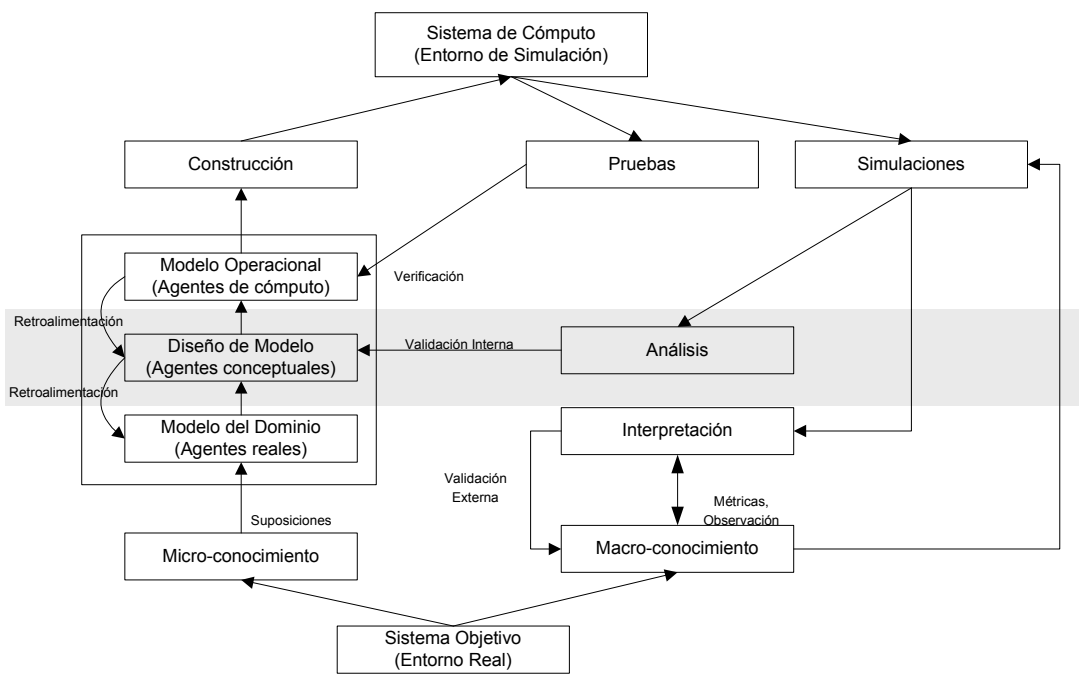

Figura 3.2: Proceso de Diseño Completo.

De esta forma, los "agentes" utilizados para configurar cada modelo (el modelo de dominio, de diseño y operacional) pueden ser definidos de forma diferente (debido a la diferencia entre la semántica, y los lenguajes, utilizados por los diferentes roles): por ejemplo, aunque los agentes descritos en el modelo de 
dominio servirán como las bases para los agentes que poblarán el modelo operacional, probablemente estén menos formalizados. Idealmente, un paralelo podría ser plasmado entre el uso de agentes en MASB y el uso de objetos en la programación orientada a objetos. Los agentes pueden encontrarse en el modelo del dominio como metáforas de "individuos" autónomos, proactivos, comunicándose e interactuando entre ellos en un sistema objetivo. Asimismo, pueden encontrarse en el modelo del diseño, como un soporte conceptual para formalizar la definición de tales individuos en términos de comportamientos, modelos de comunicación, etc.

Una de las partes más difíciles, al diseñar un MASB, es la formalización de los comportamientos de agentes después de que han sido definidos por el temático. La reciente aparición de la simulación participativa permite a los expertos e inexpertos la interacción para definir estos comportamientos, mediante juegos de roles, o siendo inmersos como "agentes humanos" en la ejecución de una simulación (Barreteau, Bousquet \& Attonaty 2001). De esta manera, a los agentes se les proporcionan habilidades (u observaciones) de aprendizaje de sus "Usuarios". Este tipo de procedimientos de aprendizaje está siendo investigado activamente en varias líneas de Inteligencia Artificial bajo los nombres de: Autonomía Ajustable (Chalupsky, Gil, Knoblock, Lerman, Oh, Pynadath, Russ \& Tambe 2001), Aprendizaje por Imitación (Dautenhahn 1996), Aprendizaje por Demostración (Gaussier, Moga, Banquet \& Quoy 1998), Aprendizaje Social (Gaussier et al. 1998), Aprendizaje situado interactivamente (Hughes 2000), Refuerzo de Aprendizaje (Ishiwaka et al. 2003, Bakker et al. 2005, Bowling \& Veloso 2002, Busoniu et al. 2008), etc. Una de sus principales ventajas es que el agente puede aprender su comportamiento "en situaciones" y de forma progresiva y que el experto realmente se involucra en el proceso (Hanneman \& Patrick 1997).

En cuanto a los Sistemas Distribuidos, se debe considerar que el entorno computacional se encuentra físicamente distribuido entre varias estaciones en red. Como tal, los problemas asociados con este escenario se reflejan en la necesidad de mantener un estado de coherencia global y reducir las disparidades entre las partes de la simulación que se ejecutan en diferentes máquinas, balancear la carga entre los nodos de la red, y la necesidad de la tolerancia ante los fallos de un elemento individual.

Debido a la inherente distribución de un Sistema Multiagente, éste puede 
guiar estos problemas en el nivel del sistema, mediante el uso de agentes móviles (Michel, Bommel \& Ferber 2002). De esta forma, se permite una distribución transparente para el usuario. Asimismo, la distribución no solamente es dinámica, sino que se adapta a las particularidades de la simulación. Los niveles de abstracción que se manejan en la simulación (agentes, grupos, comunidades) pueden responder a los niveles de distribución que se presentan en las redes. Esto permite que durante el desarrollo sea más fácil dar mantenimiento y comprender los sistemas de simulación distribuidos (Michel et al. 2002).

\subsubsection{Tratamiento del Entorno en la Simulación Multiagente.}

Los entornos reales en los que los agentes operan, son inherentemente dinámicos, esto es, el entorno cambia superando el control de los agentes. En la Simulación multiagente, la operación del mundo real con el paso del tiempo se imita mediante la ejecución de un modelo multiagente, p.ejem. un modelo compuesto de un número de agentes situados en un entorno simulado (Helleborg, Vizzari, Uhrmacher \& Michel 2007). Los agentes representan los actores originales que están presentes en el sistema del mundo real. El entorno simulado representa el entorno real en donde los actores del sistema del mundo real se encuentran situados. Los agentes, así como el entorno, son elementos esenciales de un modelo para una simulación multiagente (Klugl, Fehler \& Herrler 2005). En consecuencia, tanto los agentes como el entorno, deben ser soportados explícitamente en el desarrollo del modelo multiagente.

Recientes investigaciones proponen que el entorno en la simulación multiagente comprende dos elementos: el entorno simulado y la simulación del entorno (Klugl et al. 2005). El entorno simulado es parte del modelo y representa el entorno real en donde están los agentes. El entorno de simulación por otra parte, es la infraestructura para ejecutar la simulación. Entre los trabajos de investigación relativos a los entornos en la simulación multiagente podemos encontrar: AGRE (Ferber, Michel \& Baez 2005), la cual soporta estructuras sociales y espaciales de un entorno; MMAS (Bandini, Manzoni \& Vizzari 2005), que manipula un modelo multicapas del entorno, en él cada capa representa una estructura conceptual o espacial del entorno real; MIC (Gouaïch, Michel \& Guiraud 2005), que soporta la interacción entre agentes mediante la interacción entre objetos y la interacción entre espacios; ELMS (Okuyama, Bordini \& daRochaCosta 2005), el cual es el lenguaje de descripción del entorno que soporta explícitamente percepción e interacción específicas de agentes cognitivos. 
Por otra parte, para ejecutar un modelo, los entornos de simulación generalmente requieren el modelo que será descrito en un formalismo de simulación en particular. Un formalismo de simulación proporciona conceptos para un modelo específico así como para su evolución con el paso del tiempo. Por ejemplo, en los simuladores de eventos discretos se soporta la ejecución de modelos descritos en términos de conceptos de estado y eventos. Los simuladores híbridos soportan modelos definidos en términos de variables de estado, ecuaciones, eventos en el tiempo, y eventos de estado. En consecuencia, un modelo que se describe en términos de los conceptos de un dominio específico, debe ser traducido en los conceptos de un formalismo de simulación antes de que pueda ser ejecutado en un entorno de simulación específico (Helleborg et al. 2007). De esta forma la estructura del entorno simulado se expresa mediante:

a) Entidades y Propiedades del Entorno. Las entidades del entorno representan entidades caracterizadas por su sola existencia en el entorno real. El entorno real generalmente contiene numerosas entidades de diferentes tipos que pueden ser incorporadas como entidades del entorno en el entorno simulado, de tal manera que se pueden definir como sigue:

$$
E=\left\{e_{1}, e_{2}, \ldots, e_{n}\right\}
$$

donde:

$E$ es el conjunto de entidades del entorno.

Las entidades del entorno se pueden dividir en un conjunto de subconjuntos separados, donde cada subconjunto agrupa entidades del mismo tipo.

$$
\operatorname{Part}_{E}=\left\{E_{1}, E_{2}, \ldots, E_{n}\right\}
$$

donde:

Part $_{E}$ es una partición de las entidades del entorno.

con:

$$
\begin{aligned}
E_{i} & \subseteq E \\
E & =\cup_{i=1 \ldots k} E_{i} \\
E_{i} & \cap E_{j}=\phi, \Lambda_{i} \neq j
\end{aligned}
$$


Mientras que una propiedad del entorno es una cantidad distribuida que representa una métrica, siendo una característica global del entorno real (p.ejem. temperatura, humedad).

b) El Estado de los Integrantes del Entorno. El estado del entorno simulado esta implícitamente definido por el estado de todos sus integrantes. El estado de un integrante del entorno simulado puede ser visto como una lista de valores que definen su estado en cualquier momento.

c) Los Agentes plasmados como Entidades del Entorno. La representación es clave para describir la relación entre los agentes y el entorno (Brooks 1991). Los agentes se plasman como entidades del entorno. Una entidad del entorno representa un elemento tangible por medio del cual un agente existe en un determinado entorno. De tal manera que si $\mathrm{Ag}=$ $\{a g 1, a g 2, \ldots, a g N\}$ es un grupo de agentes que viven en un entorno simulado, la representación de los agentes como agentes del entorno se define mediante una función de representación (Embody) que asocia un agente a una entidad del entorno que representa el agente.

$$
\begin{aligned}
\text { Embody } & : A g \rightarrow E \\
\text { Embody }(a g) & =e^{A}
\end{aligned}
$$

De esta forma al vincular una entidad a un agente, se determina la forma en la que el agente puede afectar el entorno y viceversa.

\subsection{3. Áreas de aplicación de la Simulación guiada por Agen- tes.}

De manera más específica, algunas de las áreas donde se ha aplicado la Simulación guiada por Agentes obteniendo resultados favorables han sido:

\section{Simulación Social.}

La Simulación Social Basada en Agentes (ABSS) según Davidsson (Davidsson 2000) se caracteriza por una intersección entre tres áreas científicas denominadas, Computación Basada en Agentes (área de investigación dentro de la Ciencia Computacional), las Ciencias Sociales (basada particularmente en las interacciones entre entidades sociales) y la Simulación por computadora (estudio de diferentes técnicas para la simulación de fenómenos en una computadora). 
De tal manera que la intersección entre las ciencias sociales y la computación basada en agentes da origen a los Aspectos Sociales de los Sistemas de Agentes (SAAS) los cuales incluyen el estudio de normas, instituciones, organizaciones, cooperación, competición, etc. A las actividades asociadas a la intersección entre la simulación por computadora y la computación basada en agentes, se les denomina Simulación basada en Multiagentes (MABS). Esta se encarga del estudio del uso de la tecnología de agentes para la simulación de cualquier fenómeno en una computadora. Finalmente, a la intersección entre las ciencias sociales y la simulación por computadora se le denomina Simulación social (SocSim), y corresponde a la simulación de fenómenos sociales en una computadora utilizando alguna técnica de simulación la cual comúnmente utiliza modelos simples de la simulación de entidades sociales.

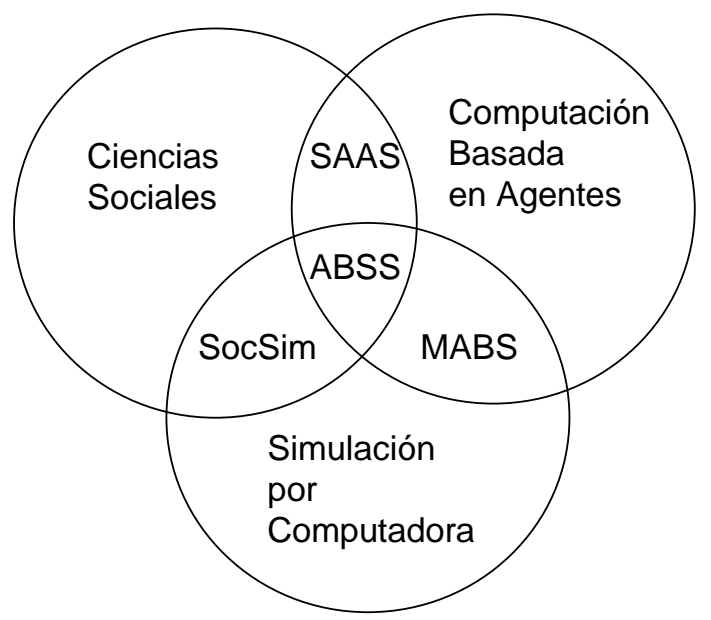

Figura 3.3: Áreas que intervienen en la simulación social

En (Axtell 2000b) se plantean varios motivos por los cuales emplear la computación basada en agentes en las ciencias sociales, y se argumenta que dependiendo de la complejidad del modelo social, existen tres usos distintos de la simulación basada en agentes: modelos con agentes como simulaciones clásicas (cuando los modelos pueden formularse y resolverse completamente), agentes artificiales como complemento a la formulación de teorías matemáticas (para modelos parcialmente resolubles), y computación con agentes como herramienta de análisis (cuando los modelos son intratables o probablemente sin solución). 
Entre algunos de los ejemplos de Simulación Social encontramos: a) la evaluación del impacto de la utilización de pronósticos de clima entre agricultores de pequeñas fincas (Ziergovel, Bithell, Washington \& Downing 2005) donde el modelo combina características de decisión cualitativa junto con información cuantitativa medioambiental, b) simulación de entornos para redes interorganizacionales (SNET) (Gans et al. 2005), la cual está basada en una combinación de dos formalismos: $i^{*}$ (un lenguaje para el modelado gráfico originalmente utilizado para describir requisitos iniciales) para modelar la red estáticamente, y ConGolog (un lenguaje de programación de alto nivel basado en la lógica) para simular dinámicamente la red y así analizar aspectos dinámicos como por ejemplo la confianza, c) simulación de gestión de procesos de negocio ARIS (IDS-scheer 2003), la cual permite identificar cuellos de botella y pasos innecesarios en cadenas de procesos dentro y a través de organizaciones con el objetivo de planificar los recursos y la reingeniería de procesos de negocio y d) modelado de grupos de redes económicas (Bentley, Lake \& Shennan 2005), donde se buscan patrones que emerjan por si mismos bajo un importante y amplio rango de parámetros considerando las condiciones iniciales. Para lograrlo, cada agente representa un nodo de la red el cual produce y consume sus propias combinaciones de dos productos distintos reproduciendo patrones que generalmente se observan en redes sociales del mundo real, incluyendo cualidades de agrupamiento (clustering) que pueden ser cruciales en la dinámica de la industria.

La simulación social también ha sido aplicada en temas relativos a la representación de la confianza como el capital relacional de los agentes (Castelfranchi et al. 2006). En este trabajo se parte de una red de dependencia clásica (en la cual las necesidades, objetivos, habilidades y recursos se encuentran distribuidos entre los agentes) con socios potenciales, en la que se introduce el análisis de lo que significa que un agente sea de confianza y cómo esta condición podría ser utilizada estratégicamente por el agente para el logro de sus objetivos, esto es, el porqué esta representa una forma de poder. Por tanto, proponen un estudio que intenta entender a la confianza como el capital de los individuos; cómo se construye, gestiona y almacena dicho capital; y en particular cómo este capital es el resultado de los objetivos y creencias de otros. Asimismo, también se han realizado estudios referentes al modelado de sistemas complejos, mediante el estudio de sistemas sociales y las interacciones entre los agentes que lo representan (Sansores \& Pavón 2006) y propuestas para el metamodelado para la Simulación de Sistemas Sociales basados en Sistemas Multiagentes (Pavón, Sansores \& Gómez 2008). En este trabajo se describe una nueva propuesta para el estudio de sistemas adaptativos complejos en las ciencias sociales mediante 
el modelado basado en agentes (MBA), modelando y simulando dichos sistemas como sociedades inteligentes (Capítulos 5, 6 y 7). Esta técnica se enfoca en cómo las interacciones locales entre los agentes generan estructuras emergentes más grandes y socialmente globales además de sus patrones de comportamiento.

\section{Diseño de Plantas de Fabricación.}

El desempeño de operaciones libres de riesgos en instalaciones de trabajo según Gabbar en (Gabar et al. 2003) es un factor crítico para satisfacer el incremento en los retos del mercado. Los ejercicios de diseños seguros incluyen valoraciones cuantitativas donde la velocidad de propagación, probabilidades, y riesgo pueden ser calculados mediante un simulador dinámico.

Asimismo, se plantea la división de una planta de fabricación como una solución útil y complementaria para la mejor ejecución de la simulación. Mediante la división del modelo del diseño de la planta en pequeñas partes es posible mejorar la ejecución de experimentos durante la simulación de plantas complejas. La división de plantas complejas es necesaria para analizar y comprender el comportamiento de cada parte por separado o analizar varias partes simultáneamente.

Entre los ejemplos de aplicación de simulación en el diseño, podemos encontrar el diseño de plantas químicas (Gabar et al. 2003) donde, basado en un Sistema Multiagente los agentes inteligentes se desarrollan para realizar la distribución de las tareas de simulación entre diferentes sesiones de nodos de simulación. Para ello se hace uso de agentes mediadores para habilitar la comunicación entre los diferentes nodos de simulación mediante mecanismos de contratos o un broker.

\section{Modelado de Sistemas Complejos.}

Un sistema complejo posee características básicas inherentes al gran número de interacciones y comportamientos de los elementos que lo componen. Una de las definiciones que lo describen con mayor detalle es la propuesta por Moriello en (Moriello 2003), que lo describe como "los sistemas complejos se caracterizan fundamentalmente en que su comportamiento es imprevisible. Sin embargo, complejidad no es sinónimo de complicación: este vocablo hace referencia a algo enmarañado, enredado, de difícil comprensión. En realidad, y por el momento, no existe una definición precisa y absolutamente aceptada de lo que es un sistema complejo, pero pueden darse algunas peculiaridades comunes. En primer término, está compuesto por una gran cantidad de elementos relativamente 
idénticos. En segundo lugar, la interacción entre sus elementos es local y origina un comportamiento emergente que no puede explicarse a partir de dichos elementos tomados aisladamente. Por último, es muy difícil predecir su evolución dinámica futura; o sea, es prácticamente imposible vaticinar lo que ocurrirá más allá de un cierto horizonte temporal”.

Tomando en consideración las propiedades de los sistemas complejos propuestas por Pavard en (Pavard \& Dugdale 2001) que indican que: a) es fundamentalmente no determinístico y no tratable, b) es posible su descomposición, y está limitada funcionalmente, c) la representación y naturaleza de la información es distribuida, y d) comprende propiedades emergentes y autoorganización. Además es necesario considerar la idea que plantean E. Alonso y D. Kudenko (Alonso \& Kudenko 2001), de que los agentes necesitan grandes cantidades de conocimiento del dominio en sistemas de múltiples agentes complejos y dinámicos. Una aproximación lógica es, por tanto, que los Sistemas Multiagente son un candidato idóneo para diseñar e implementar tales sistemas.

Un ejemplo reciente de la aplicación de los Sistemas Multiagente en sistemas complejos es el manejo de Bases de Datos donde una de las aplicaciones se refiere a las extracción de reglas de bases de datos complejas (Ryoke \& Nakamori 2005). La base de datos compleja se considera compuesta por atributos nominales y números mixtos y algunas estructuras heterogéneas. Las estructuras heterogéneas a menudo se presentan en diferentes dimensiones con diferentes características. Por tanto, la selección de objetos tal como el componente de estructuras locales y la selección de atributos para la expresión de la regla deberían considerarse en conjunto en el modelado del sistema. Los agentes identifican el modelo lineal así que el error no se extiende más allá que el parámetro dado.

\section{Simulación de Mercados.}

La simulación basada en agentes permite la simulación de micro y macro economías y sociedades. En la microeconomía es posible la construcción de mecanismos de subastas, por otra parte, el fenómeno de la macroeconomía requiere el modelado de un amplio rango de fenómenos y eventos que típicamente son asumidos con las bases de las microeconomías sintéticas. 
Entre los ejemplos relacionados con este tipo de simulación podemos mencionar: a) simulación del Mercado Laboral (Chaturvedi et al. 2005) para el estudio de problemas críticos que surgen en el mercado laboral mediante el modelado de de una economía laboral existente programando el comportamiento del agente a un micro nivel, observando el comportamiento a macro nivel que emerge, y calibrando el sistema para así emular la economía real en direcciones específicas, b) simulación de mercados financieros (Raberto et al. 2001) el cual muestra características de comercio reales y considera el hecho de que son finitos los recursos de los agentes de tal manera que puede ser posible llevar a cabo experimentos computacionales utilizando varios tipos de agentes artificiales y c) simulación de bolsas de valores artificiales (Cincotti, Focardi, Marchesi \& Raberto 2003) con un monto finito de dinero y valores.

\section{Simulación Espacial.}

El modelado de problemas basado en agentes actualmente se aplica en varias escalas espaciales. Deadman y Gimblett (Deadman \& Gimblett 1999) realizan investigación sobre las interacciones entre las personas y el entorno utilizando modelos basados en agentes en los cuales las personas simuladas deciden sobre la ruta a elegir durante recreaciones de viajes en un área forestal. Itami y Gimblett (Itami \& Deadman 2000) desarrollaron RBSIM2 el cual es una herramienta de simulación por computador, integrada con un Sistema de Información Geográfica (GIS) para ser utilizada como una herramienta de evaluación para gestión de parques. Asimismo, Deadman (Deadman 1999) intentó reproducir un fenómeno hallado durante experimentos típicos reunidos de acuerdo al escenario de "la tragedia de los comunes". Mientras que Benenson (1998), simuló la dinámica en patrones de residencia en una gran ciudad utilizando Sistemas Multiagente. Batty, Desyllas \& Duxbury (2003) utilizan agentes para simular el movimiento de un gran número de personas en un carnaval y desfiles en escalas pequeñas de escalas espaciales. Finalmente Ligtenberg et al. (2004) simulan escenarios espaciales basados en el modelado del tomador de decisiones multiactor dentro de un proceso espacial.

\section{Simulación de Transporte y Gestión de Tráfico.}

Los sistemas de transporte pueden contener millones de entidades "inteligentes" que requieren ser simuladas y/o controladas (Bazzan et al. 2005). Con el fin de concebir diseños de sistemas de transporte y tráfico más eficientes, se 
incluyen dispositivos de control, técnicas para la optimización de las redes existentes así como sistemas de información; los escenarios de transporte y tráfico son extraordinariamente atractivos para la tecnología multiagente en particular.

Hasta el momento la Simulación basada en Multiagentes ha sido aplicada en la solución de problemas relacionados con la programación de transportes (Davidsson, Henesey, Ramstedt, Tornquist \& Wernstedt 2005) tal como la asignación de tareas a vehículos, con enfoques distribuidos en los cuales se incluyen procesos de negociación, protocolos de contratos, y algunas veces se basan en mercados, de esta manera los agentes representan roles tales como una compañía, un camión, un cliente, etc. También se incluye la gestión y el control de tráfico aplicados a la congestión en carreteras. Por último en cuanto al modo de transporte, los agentes se han utilizado para intentar incrementar la eficiencia de las operaciones de terminales de contenedores.

Entre algunos de los ejemplos más recientes relacionados con esta área encontramos el diseño de dos Sistemas de Toma de Decisiones (Ossowski, Hernández, Belmonte, Fernández, García-Serrano, Perez-de-la Cruz, Serrano \& Triguero 2005) utilizando una arquitectura abstracta para la gestión del tráfico del mundo real aplicados en un ejemplo de tráfico en un área de Bilbao. Así como la gestión de escenarios de la flota de autobuses en un poblado de Málaga. La simulación del tráfico aplicado al fenómeno "Paradoja Braess" (Bazzan \& Klügl 2005) en el cual establece que el agregar un nuevo camino a una red de tráfico podría no reducir el tiempo total del recorrido mediante la implementación de un simulador microscópico donde se reemplazaron las funciones de coste por la del tiempo real de ida y vuelta percibido por cada conductor; y la conducción cooperativa (Hallé \& Chaib-draa 2005) donde se hace uso de comunicaciones para guiarse por si mismos los vehículos cooperativos en un Sistema de Autopista Automatizado. En dicho sistema se modelan secciones de coches como agentes software autónomos utilizando una arquitectura de agentes guiados jerárquicamente, donde la arquitectura está basada en tres capas (orientación, gestión, y control de tráfico).

\section{Simulación de Sistemas de Fabricación.}

Durante los últimos años, los paradigmas en la ingeniería de la fabricación han ido cambiando hacia los sistemas de fabricación basados en agentes (Calderón \& García 2005). La estrategia planteada es la utilización de sistemas 
de control altamente distribuidos basados en agentes para el control de un sistema de gestión de la fabricación adaptable de forma automática a las exigencias de los pedidos. Mediante la negociación entre los distintos agentes del sistema de fabricación y las reglas de distribución del trabajo establecidas, se pretende atender, de forma inmediata, la demanda de producción exigida por los pedidos a medida que éstos se produzcan.

Los Sistemas de Simulación de Plantas de Fabricación basados en Agentes tienen requisitos específicos que se deben cubrir. Según Marik et al. (Marík et al. 2005) los requisitos son:

- Para que la simulación tanto de las instalaciones de fabricación/procesos del sistema puedan ser controlados por agentes, es necesario poseer:

1. Un buen modelo de las instalaciones de fabricación/procesos controladas y una herramienta de simulación para ejecutar este modelo (esta herramienta proporcionará la emulación del entorno físico de fabricación).

2. Un entorno de ejecución apropiado para ejecutar los agentes y modelar sus interacciones (plataformas de agentes tal como JADE (JADE 2005), JACK (Busetta, Rönnquist, Hodgson \& Lucas 1999), A-GLOBE (Sislak, Rehak \& Pechoucek 2005), etc.)

- Los algoritmos controlados por agentes para la simulación por lo general son reutilizados (90 - 100\%) para el control de la vida real del equipo de fabricación. Por tanto, el papel de control de agentes también es (en la fase de simulación) emulada. Éste es el por qué de que la simulación deba ser guiada por otro sistema basado en agentes, usualmente organizado como una interacción apropiada entre dos emulaciones.

- Es necesario contar con las siguientes dos interfaces de ejecución:

1. Una interfaz entre el control de agentes y la emulación de las instalaciones de fabricación/procesos controladas (para la fase de simulación).

2. La interfaz para unir el control de agentes con las instalaciones físicas de la fabricación/procesos controladas (para el control de la vidareal), 
Debido a que este último tema forma parte de los temas elementales de este trabajo, la Simulación de Sistemas de Fabricación es desarrollada como un capítulo posterior (Capítulo 4) donde se presenta con detalle todos los aspectos que le rodean.

\subsection{Entornos de Simulación de Sistemas Multiagen- te}

Actualmente se cuenta con entornos para la simulación de Sistemas Multiagente, entre algunas de las herramientas más representativas se encuentran Repast, MASON y NetLogo. Estas herramientas proporcionan entornos para el modelado de Sistemas Multiagente y su simulación, sin embargo, cada una incluye ciertas características que las distinguen.

REPAST. Es un entorno para el modelado basado en agentes que utiliza un enfoque orientado a objetos. Este entorno incluye un planificador de eventos discretos de multihilos concurrentes (Repast 2004). Para el desarrollo de modelos utiliza el lenguaje java, groovy(lenguaje dinámico para la máquina virtual de java) y diagramas de flujos. El entorno de ejecución de modelos es gráfico, incluye la trazabilidad de los resultados y herramientas gráficas, así como conexiones automatizadas para una variedad de herramientas externas opcionales (Rstatistics environment, VisAD, We$\mathrm{ka}$, Matlab e iReport). El entorno ofrece la definición flexible de espacios jerárquicos anidados, así como el modelado y visualización de entornos en dos dimensiones. Asimismo, incluye: a) librerías de algoritmos genéticos, redes neuronales, regresión y generación de números aleatorios y b) un marco automático para la simulación Monte Carlo que soporta múltiples modos de optimización de resultados.

MASON. Es un entorno de simulación multiagente de eventos discretos desarrollado en java (Luke, Cioffi-Revilla, Panait \& Sullivan 2004). El entorno ofrece suficiente funcionalidad para cubrir necesidades de simulación sencillas. MASON incluye librerías de modelos y una suite opcional de herramientas de visualización en dos y tres dimensiones. Sus simulaciones tienen especial énfasis en la simulación de "enjambres" que se caracterizan por contener un gran número de agentes (por encima de un millón de agentes) e interacciones, donde se tiene una visualización y modificación ocasional. El entorno no proporciona herramientas para la construcción de 
modelos simplificados para programadores inexpertos; ni tampoco incluye características de dominios específicos tales como modelos físicos, sensores de robots, gráficas, e importación de datos de sistemas de información geográficos.

NetLogo. Es un entorno de programación de modelos para la simulación natural y social de fenómenos (Tisue \& Wilensky 2004). Mientras que el entorno de desarrollo está basado en java y los modelos se pueden generar como un applet, el lenguaje de programación de los modelos basados en agentes está basado en logo y está orientado a procesos. Los agentes de NetLogo se ubican en una red (grid) la cual permite el diseño de modelos simples que contemplan un espacio limitado. En NetLogo el tiempo es discreto, así, los agentes realizan acciones solamente en ciertas marcas en el tiempo por lo que no existen eventos entre dichas marcas de tiempo.

\subsection{Conclusiones}

Como resultado de la revisión de los principios del paradigma de agentes, los Sistemas Multiagente y su aplicación en el área de la Simulación, es posible concluir que la aplicación de la Teoría de los Sistemas Multiagente proporciona un alto grado de flexibilidad para su manejo durante el proceso de simulación de comportamientos complejos. Nuestra hipótesis es que la aplicación de la teoría de los Sistemas Multiagente en el proceso de Simulación, dará como resultado un Entorno de Simulación Inteligente que permita la aparición de escenarios que alteran las condiciones iniciales (de la simulación) acorde a los eventos generados por el comportamiento complejo real de estos sistemas, permitiendo de esta manera una simulación más próxima a los requisitos de la nueva fabricación. 



\section{Capítulo 4}

\section{Simulación de Sistemas de Fabricación y los Sistemas Multiagente}

La Fabricación Estratégica es un concepto introducido por Steve Brown quien lo describe como : "viendo las capacidades de las operaciones de producción como un núcleo de competencia, con una visión de negocios a largo plazo y estando completamente consciente de todas las oportunidades del mercado. La planificación de estrategias es útil para superar a los competidores mediante sectores específicos en los cuales es posible competir, mientras que se evitan premeditadamente aquellos en los que no es posible, y se entablan asociaciones verticales y horizontales" (Brown 1996). De este modo, basado en las características de la Fabricación Estratégica, tales como las innovaciones del producto, control de materiales, tecnología de procesos, y recursos humanos McLean y Leong (MCLean \& Leong 2002a) sostienen que la simulación de la Fabricación debería ser considerada como un componente clave de la Fabricación Estratégica.

Las aplicaciones de la simulación en fabricación incluyen (MCLean \& Leong 2002a): el modelado y verificación de procesos de fabricación discretos y continuos, programación fuera de línea de robots y otras maquinarias, selección de ubicaciones, planificación de planta, visualizaciones del sistema y procesos, análisis ergonómicos, evaluación del algoritmo de planificación y reglas de despacho, e ingeniería de procesos de negocio.

El objetivo de este capítulo es la identificación de los requisitos que son considerados como la base para la Simulación de Sistemas de Fabricación y las 
mejoras aportadas por la aplicación de la Tecnología de Sistemas Multiagente incluidas en la propuesta descrita en los Capítulos 5, 6 y 7. Dichos requisitos incluyen la consideración de los estándares propios de las Herramientas de Simulación y los requisitos actuales de los Sistemas de Fabricación. Para ello se describen las características propias de la Simulación del proceso de Fabricación y las aspectos relacionados con el modelado de un Sistema de Fabricación. Para la identificación de las mejoras aportadas por la Tecnología Multiagente se parte de una revisión de las Herramientas de Simulación actuales que han sido aplicadas en la Simulación de Sistemas de Fabricación. Finalmente, se presentan algunos ejemplos de aplicaciones basadas en agentes para el modelado de Sistemas de Fabricación.

\subsection{Simulación de Sistemas de Fabricación}

Un aspecto relevante en cuanto al objetivo que persigue el uso de Simulación en la Fabricación es que esta técnica se adapta a las necesidades básicas del entorno, tales como:

- La motivación que tienen los fabricantes para mantenerse competitivos.

- El alto nivel de automatización que se aplica en la fabricación.

- Las tendencias tales como la fabricación just-in-time (justo a tiempo) requieren ser evaluadas.

- Los Sistemas de Fabricación están bien definidos.

- Los Sistemas de Fabricación y de gestión de materiales generalmente son muy complejos para otras técnicas analíticas.

La simulación permite responder a preguntas que están relacionadas con las necesidades de los Sistemas de Fabricación, tales como:

- ¿Cuándo debería ser adquirida una nueva pieza del equipo?

- ¿Cuánto personal será necesario el próximo mes para cumplir con las órdenes?

- ¿Puede ser aceptada una nueva orden sin retrasar otro trabajo?

- ¿Cómo operará la planta en los próximos cinco años?

- ¿Cómo se puede reducir el inventario en proceso y el ciclo de vida mientras aumenta el rendimiento? 
- ¿Dónde aparecen cuellos de botella que dan origen al retraso de órdenes?

Asimismo McLean (MCLean \& Leong 2002a) considera que las aplicaciones de la simulación de fabricación incluyen: modelado y verificación de procesos de fabricación tanto discretos como continuos, programación fuera de línea de robots y otra maquinaria, elección de ubicaciones, planificación de planta, visualización del sistema y sus procesos, análisis ergonómico de tareas manuales y distribución del área de trabajo, evaluación de los algoritmos de planificación y reglas de despacho, e ingeniería de procesos de negocio.

De manera genérica, se pueden identificar seis tipos de Sistemas de Fabricación (Harell \& Tumay 1996): Taller de proyectos, Taller de trabajo, Fabricación celular, Sistemas de Fabricación Flexible, Taller de flujo en lotes y Sistemas de flujo en líneas (líneas de ensamble, producción o transporte). Por otra parte, Rohrer diferencia dos tipos de Fabricación (Rohrer 1998): Fabricación Discreta y Batch. La fabricación discreta involucra piezas individuales o partes. La fabricación en batch, por otro lado, se aplica al trabajo en proceso que puede ser manipulado como un fluido o un bloque sólido.

Aunque existen distintos tipos de Sistemas de Fabricación, se pueden identificar algunos elementos comunes en todos ellos. Estos elementos básicos deberían ser la base para los datos de entrada utilizados en un Modelo de Simulación (Tabla 4.1).

\begin{tabular}{|c|c|c|c|}
\hline Producto & Recursos & Demanda & Control \\
\hline Partes/piezas & $\begin{array}{l}\text { Distribución de Equi- } \\
\text { po }\end{array}$ & Ordenes de Clientes & Gestión de Almacén \\
\hline Rutas & Número de máquinas & Fecha de Inicio & Control de Inventario \\
\hline $\begin{array}{l}\text { Tiempos de Pro- } \\
\text { cesamiento }\end{array}$ & Tiempo de Paro & $\begin{array}{l}\text { Fecha de Vencimien- } \\
\text { to }\end{array}$ & $\begin{array}{l}\text { Control de Planta de } \\
\text { Fabricación }\end{array}$ \\
\hline $\begin{array}{l}\text { Tiempos de Con- } \\
\text { figuración }\end{array}$ & $\begin{array}{l}\text { Mantenimiento Pre- } \\
\text { ventivo }\end{array}$ & $\begin{array}{l}\text { Inventario en Proce- } \\
\text { so }\end{array}$ & $\begin{array}{l}\text { Rastreo de Inventario } \\
\text { en Proceso }\end{array}$ \\
\hline $\begin{array}{l}\text { Lista de Materia- } \\
\text { les }\end{array}$ & $\begin{array}{l}\text { Areas de almacena- } \\
\text { miento }\end{array}$ & & PLCs \\
\hline Rendimiento & $\begin{array}{l}\text { Herramientas / Equi- } \\
\text { pos }\end{array}$ & & Reglas de estación \\
\hline \multirow[t]{2}{*}{ Retrabajos } & $\begin{array}{l}\text { Clasificación de tra- } \\
\text { bajos }\end{array}$ & & \\
\hline & $\begin{array}{l}\text { Calendarios de tur- } \\
\text { nos }\end{array}$ & & \\
\hline
\end{tabular}

Tabla 4.1: Elementos de un Sistema de Fabricación.

Cada uno de los elementos comunes desempeñan actividades clave para la operación de un sistema, tal que, sin uno de ellos no es posible completar el ciclo 
completo de fabricación. En consecuencia, dichos elementos deben ser incluidos como base del modelado de un Sistema de Fabricación.

Producto. Un producto puede ser una parte, un lote, o productos que pueden ser fabricados. Los productos pueden proceder de procesos de fabricación previos en un momento determinado. Los productos tienen rutas que definen la secuencia de procesamiento. Las rutas definen cómo es el flujo del producto a través del proceso de fabricación. Para cada operación, el tiempo de procesamiento y configuración determinan por cuánto tiempo una máquina u operario ejecutará una tarea dada. Estos tiempos pueden ser determinísticos (constantes) o estocásticos (aleatorios), y pueden ser determinados por la relación máquina/parte (el término de parte se refiere a piezas compradas o materias primas) o solamente por la máquina. La lista de materiales define los subensambles que comprende el ensamble del producto final. Dichos subensambles deben estar disponibles para poder continuar. Los subensambles también tienen su propia secuencia de procesamiento, éstos deben ser producidos o comprados con suficiente anterioridad para el ensamble final. La producción y el retrabajo se encuentran en varias operaciones de fabricación. Debido a las imperfecciones en el proceso de fabricación, ocasionalmente, los productos tienen que ser considerados como desperdicios o pueden ser "retrabajados". Ambos factores afectan el rendimiento global de la operación, así como otras métricas de rendimiento.

Recursos. Los recursos se utilizan para fabricar productos. Los Recursos incluyen, entre otros, máquinas y recursos humanos así como herramientas y accesorios, sistema de gestión de materiales, areas de almacenamiento, etc. La distribución del equipo afecta el flujo de las operaciones, y si se toma en consideración la gestión de materiales, la distribución debe ser incluida en la simulación. Las máquinas tienen tanto fallos aleatorios como una programación de mantenimiento preventivo. Por tanto, existen periodos en los que las máquinas no están disponibles, aunque otras máquinas permanezcan trabajando. El reenrutamiento del trabajo durante periodos de inactividad puede tener un efecto mayor en la operación y debe ser incluida en el modelo de simulación. Los recursos humanos poseen diferentes niveles de habilidad y trabajan de acuerdo con calendarios de turnos. De tal manera que, es importante modelar los turnos y tiempos de inactividad cuando no todas las áreas en las instalaciones de la fábrica tienen el mismo calendario. Si todas las máquinas y operarios inician y terminan sus actividades al mismo tiempo, los efectos de la preparación son 
insignificantes, y no es necesario modelar los turnos.

Demanda. La demanda en un Sistema de Fabricación está definida por las órdenes de los clientes. Los clientes generalmente ordenan cantidades específicas de productos y definen una fecha de entrega específica. El fabricante es responsable de determinar cuándo iniciar la fabricación de los productos así como las fechas de vencimiento que puede cumplir. La Simulación puede ayudar a determinar la fecha más lejana de inicio que le permitirá cumplir con las fechas de vencimiento.

En la operación de la fábrica, siempre existe trabajo en proceso. La mayoría de los modelos de simulación asumen que las instalaciones se inician vacías y paradas (detenidas). A menudo, los modelos de simulación necesitan ejecutarse durante un cierto periodo de tiempo para inicializar el inventario en proceso (IEP). Si el modelo es grande, el tiempo de inicialización puede ser muy largo, y esto hace necesario encontrar una forma para ejecutar rápidamente una simulación del modelo. La respuesta a ello es inicializar los datos del inventario en proceso obteniendo los datos de una fuente externa al modelo.

Control. Los sistemas de control basados en computadora ayudan a tomar decisiones en cuanto a cómo un producto se debe enrutar, recopilar información acerca del estado actual del producto, o mantener un nivel apropiado de inventario. Estos sistemas de control interactúan con la simulación en dos formas. Primero, proporcionan datos de entrada que serán utilizados en la simulación. Segundo, estos sistemas a menudo toman decisiones operativas que deberían ser representadas en una simulación. La replicación de algoritmos de control en un modelo de simulación es uno de los enormes cambios a los que se afrontan los constructores de modelos. Las decisiones de programación local también son denominadas "reglas de estación". Para cada centro de trabajo, la regla de estación realmente es la respuesta a la pregunta ¿qué tengo que hacer a continuación?. Las reglas de la estación pueden ser tan simples como el primero que entra el primero que sale (FIFO), o la regla puede utilizar un árbol de decisiones muy complejo con muchos pasos. La definición de procesos inteligentes para la toma de decisiones en el nivel de las máquinas ha sido el tema de diversos estudios, los cuales se han enfocado en la obtención de la mejor forma de tomar decisiones programadas localmente. En la simulación es importante representar lo que sucede en el mundo real con un alto grado de exactitud. 
Con respecto a las reglas de estación para la toma de decisiones, entre las opciones expuestas, a continuación presentaremos las bases para la definición de un Sistema de Colas.

\subsubsection{Sistemas de Colas y su Simulación.}

Por una parte la Simulación a menudo ha sido utilizada para el análisis de modelos de colas (Banks et al. 2001). En un típico modelo de cola, los clientes llegan en diferentes tiempos y se agregan a la cola, o línea de espera, eventualmente se atienden y finalmente dejan la cola. El término "cliente" se refiere a cualquier tipo de entidad que puede ser vista como una solicitud de "servicio" de un sistema. Por tanto, muchas instalaciones de servicio, sistemas de producción, instalaciones de reparación y mantenimiento, sistemas de comunicación y computación, y sistemas de gestión y transporte de materiales pueden ser vistos como sistemas de cola.

Los elementos clave de un sistema de colas son los clientes y los servidores. El término “cliente” puede referirse a personas, máquinas, camiones, pallets, órdenes, es decir, cualquier cosa que llegue a una instalación y solicite un servicio. El término "servidor" puede referirse a recepcionistas, mecánicos, máquinas automáticas de almacenamiento, empaquetadoras automáticas, es decir, cualquier recurso (persona, máquina, etc.) que proporcione el servicio solicitado.

De esta forma, es posible proponer que en una Planta de Fabricación, las Tareas (consideradas como "clientes") asignadas a los Recursos - operarios y máquinas - (considerados como "servidores"), puedan ser gestionadas mediante la definición de un Sistema de Colas.

Un Sistema de Colas se describe por la población que la solicita, la naturaleza de las entradas, el mecanismo de servicio, la capacidad del sistema, y la disciplina de la cola. Por tanto, a continuación se describe cómo se integra un Sistema de Colas aplicado a una Planta de Fabricación.

Población solicitante. La población la integran los clientes potenciales. La población puede ser finita o infinita. Supongamos un ejemplo de una Planta de Fabricación, donde se tienen cinco máquinas y tres Ordenes de Producción solicitan la ejecución de seis tareas. Las tareas son los "clientes" que solicitan ser atendidas. Las máquinas son los "servidores" quienes "sirven" a las tareas de acuerdo a su capacidad disponible. Por tanto, en el ejemplo la población es finita y consiste de las seis tareas. En cambio, 
si se desconoce el número de Ordenes que pueden solicitar tareas, entonces la población se vuelve infinita. La principal diferencia entre modelos con población finita e infinita es cómo se define el tiempo de llegada. En un modelo con población infinita, el tiempo de llegada no se afecta por el número de clientes. Cuando el proceso de llegadas es homogéneo durante un periodo de tiempo, el tiempo de llegada generalmente se asume que es constante. Por otro lado, para modelos con población infinita, el tiempo de llegada depende del número de clientes que se estén atendiendo o esperando.

Capacidad del Sistema. En muchos sistemas de cola existe un límite de clientes que pueden estar en la línea de espera del sistema. Un cliente que llega y encuentra que el sistema está lleno, no entra y regresa inmediatamente a la población solicitante.

El proceso de llegadas. El proceso de llegadas para modelos generalmente se caracteriza en términos de tiempos de inter-llegada de clientes sucesivos. Las llegadas pueden ocurrir en tiempos programados o en tiempos aleatorios. En los tiempos aleatorios, los tiempos de inter-llegada generalmente se caracterizan por una distribución de probabilidad. Además, los clientes pueden llegar uno a la vez o en batch (grupo). El batch puede ser de un tamaño constante o aleatorio. El proceso de llegadas de Poisson ha sido exitosamente utilizado como modelo de llegada de solicitudes u órdenes. De esta forma, si $A_{n}$ representa el tiempo de inter-llegada entre el cliente $n-1$ y el cliente $n$ ( $A_{1}$ es el tiempo de llegada del primer cliente), entonces para el proceso de llegada de Poisson, $A_{n}$ está exponencialmente distribuido entre $1 / \lambda$ unidades de tiempo. La tasa de llegada es de $\lambda$ clientes por unidad de tiempo. El número de llegadas en un intervalo de tiempo de longitud $t$, se dice que $N(t)$, tiene una distribución de Poisson con $\lambda t$ clientes.

Comportamiento de la Cola o Disciplina de la Cola. El comportamiento de la cola se refiere a las acciones de los clientes mientras que están esperando a ser atendidos. En algunas situaciones, existe la posibilidad de que los clientes se vayan si consideran que la linea de espera es muy larga. Es posible que después de colocarse en la cola observen que se mueve lentamente y decidan abandonarla, o se muevan entre las líneas de espera si consideran que se mueven lento.

Tiempos de Servicio y el mecanismo de servicio. Los tiempos de servicio de llegadas sucesivas se denotan por $S_{1}, S_{2}, S_{3}, \ldots$, donde éstos pueden ser constantes o con duración aleatoria. En el último caso, se caracterizan por 
una secuencia de variables aleatorias independientes e idénticamente distribuidas. Algunas veces, los servicios pueden ser idénticamente distribuidos para todos los clientes de un determinado tipo o prioridad, mientras que los clientes de diferentes tipos pueden tener distribuciones de tiempo de servicio completamente diferentes. Además, en algunos sistemas, el tiempo de servicio depende de la hora del día o de la longitud de la línea de espera.

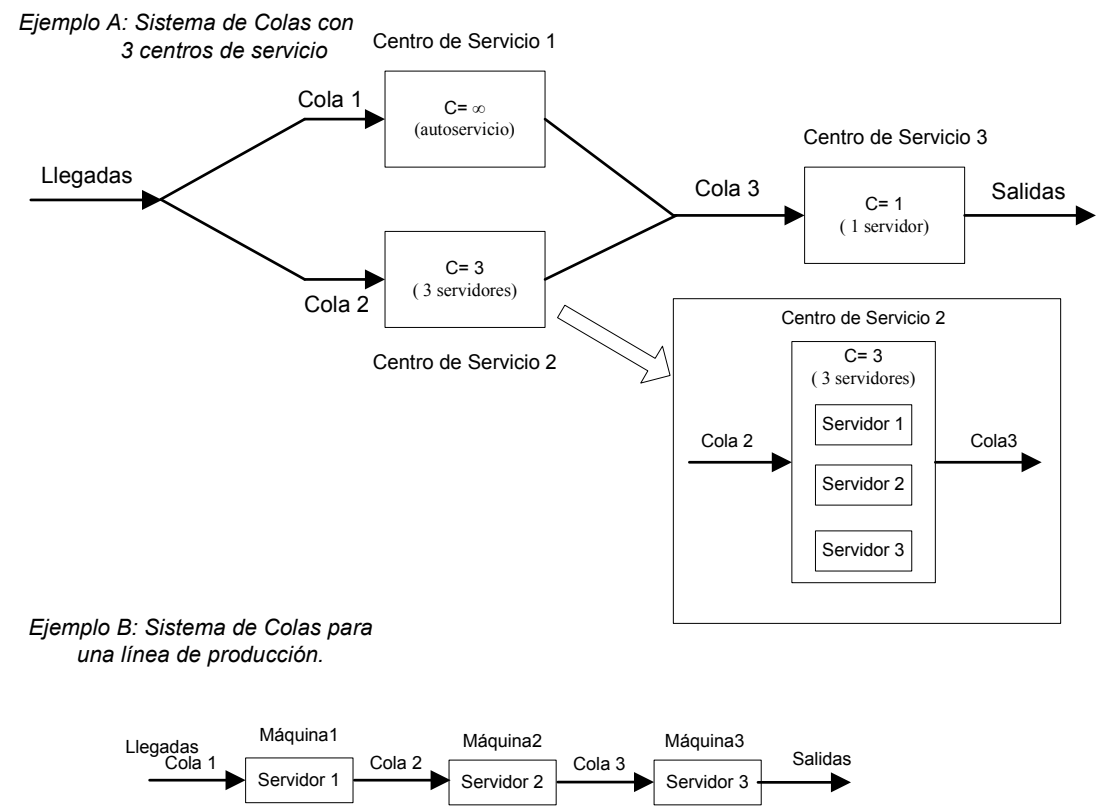

Figura 4.1: Ejemplos de Sistemas de Colas.

Por tanto, un sistema de colas consiste de un número de centros de servicio y colas interconectadas. Cada centro de servicio consiste de un número de servidores, $c$, que trabajan en paralelo; esto es, cuando se alcanza el principio de la línea, un cliente toma el primer servidor disponible. Los mecanismos de servicios paralelos pueden ser un único servidor $(c=1)$, tener múltiples servidores $(1<c<\infty)$ o tener servidores ilimitados $(c=\infty)$. En la Figura 4.1 se presentan dos ejemplos de Sistemas de Colas. En el ejemplo A se observa un sistema con tres centros de servicio con diferentes características. El centro de servicio 1 y 2 ofrecen colas paralelas que ofrecen diferentes características para las llegadas al sistema. En el centro de servicio 1 el número de servidores es infinito, es decir, es un centro de autoservicio. En el centro de servicio 2 se 
cuenta con 3 servidores, los cuales integran un subsistema que a su vez ofrecen servicios en paralelo. En el centro de servicio 3 se cuenta con un único servidor. Por otra parte, en el ejemplo B, se presenta un sistema de colas para una línea de producción integrada por 3 centros de servicio cada uno con un único servidor (máquina 1 , máquina 2 y máquina 3 ).

\section{Simulación de Sistemas de Colas.}

Para la simulación de sistemas de colas es necesario comprender los conceptos de estado, eventos y reloj de simulación (Banks et al. 2001). El estado del sistema es el número de unidades en el sistema y el estado del servidor, puede ser ocupado o detenido. Un evento es un conjunto de circunstancias que provocan un cambio instantáneo en el estado del sistema. En un sistema de colas con un canal único existen solamente dos posibles eventos que pueden afectar el estado del sistema. Estos son la entrada de una unidad al sistema (el evento de llegada) o la finalización de un servicio de una unidad (el evento de salida). El sistema de colas incluye el servidor, la unidad que está siendo atendida (si se está atendiendo una unidad), y las unidades en la cola (si alguna unidad esta esperando). El reloj de simulación es utilizado para realizar un seguimiento simulado de tiempo.

Si una unidad ha terminado el servicio, la simulación procede como se muestra en la Figura 4.2. Observe que el servidor solamente tiene dos posibles estados: éste está ocupado o detenido.

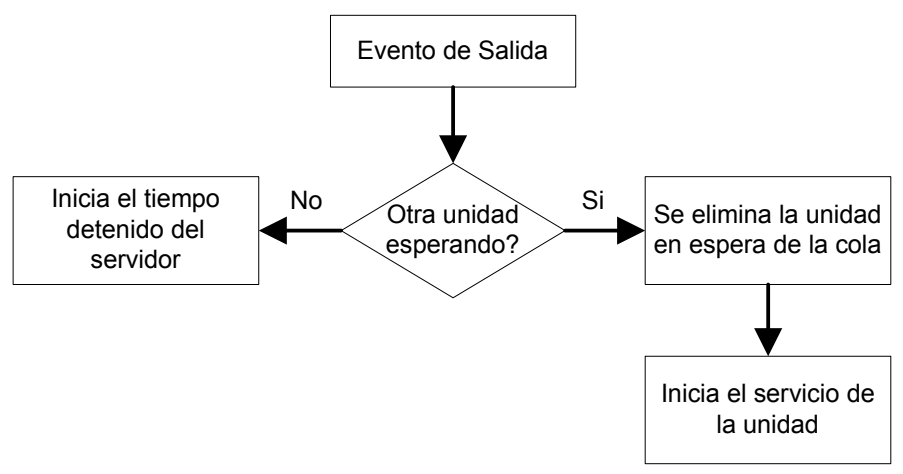

Figura 4.2: Diagrama de Flujo de servicio finalizado

El evento de llegada aparece cuando una unidad entra en el sistema. En la Figura 4.3 se presenta el diagrama de flujo para el evento de llegada. La unidad puede encontrar el servidor ya sea ocupado o detenido; por tanto, la unidad inicia 
el servicio inmediatamente o entra a la cola del servidor. La Tabla 4.2 muestra el curso de acción que la unidad puede seguir al encontrar el servidor.

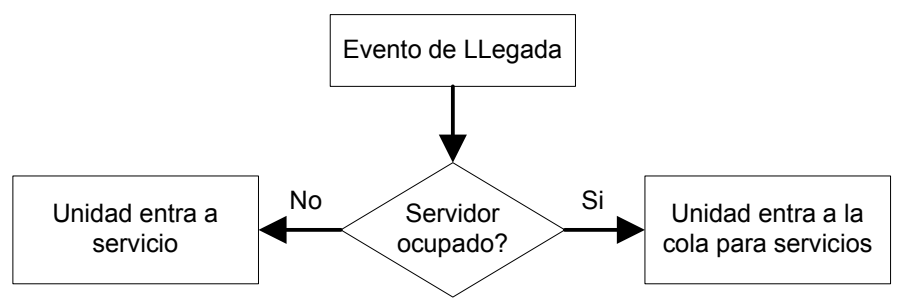

Figura 4.3: Diagrama de Flujo de cuando una unidad entra al sistema.

\begin{tabular}{|c|c|c|c|}
\hline & \multicolumn{2}{|c|}{ Estado de la cola } \\
\hline & & No vacía & Vacía \\
\hline Estado del & Ocupado & Entra a la cola & Entra a la cola \\
\hline Servidor & Detenido & Imposible & Entra a servicio \\
\hline
\end{tabular}

Tabla 4.2: Acciones potenciales de la unidad a su llegada.

Después de que el servidor finaliza un servicio, éste puede estar ocupado o permanecer ocupado con la siguiente unidad. Si la cola no está vacía, otra unidad entrará al servidor y éste estará ocupado. Si la cola está vacía, el servidor estará detenido después de finalizar el servicio. Es imposible para el servidor estar ocupado si la cola está vacía cuando se finaliza un servicio. Similarmente, es imposible para el servidor quedarse detenido después de finalizar un servicio si la cola no esta vacía.

Los tiempos de simulación de llegadas y salidas se calculan en una tabla de simulación personalizada para cada problema. En la simulación, los eventos generalmente aparecen en tiempos aleatorios, la aleatoriedad imita la incertidumbre del mundo real. En la propuesta esta técnica es utilizada por las entidades que integran el Sistema de Fabricación (operarios, máquinas, herramientas, etc.) durante su Simulación (Capítulos 6 y 7).

\subsection{Estándares de Simulación y Fabricación}

El desarrollo de la tecnología de simulación así como los estándares de interfaces de soporte han sido identificados repetidamente por la industria como 
una prioridad en la investigación que promete grandes retribuciones. Un estudio establece que "el Modelado y la Simulación (M\&S) están emergiendo como tecnologías clave para dar soporte a la fabricación en el siglo 21, y ninguna otra tecnología ofrece mas que una fracción del potencial de M\&S para la mejora de productos, perfeccionamiento de procesos, reducción referente al ciclo de tiempo del diseño-a-fabricación, así como la reducción en los costes de producción del producto" (IMTR 1998).

El Consejo Nacional de Investigación de Estados Unidos de América (NCR) (MCLean \& et. al. 2003), repetidamente ha identificado a la simulación y el modelado como un área de investigación de alta prioridad. En un estudio realizado en 1995, la NCR menciona: "Últimamente, las capacidades del modelado y la simulación resultados de la investigación esquematizada deberían ser capaces de soportar la configuración y construcción de una fábrica real con un alto nivel de ejecución (en múltiples dimensiones), además de plantear cómo operar de la mejor forma una vez que ha sido construida. Una demostración concreta de estas habilidades debería ser la creación de una plataforma capaz de comparar los resultados de las operaciones reales de una fábrica con los resultados de la simulación de las operaciones de la fábrica usando aplicaciones de tecnología de información...". Para que el modelado y la simulación sean útiles en cuanto a las necesidades de fabricación, se destacan dos áreas de investigación con especial atención: el desarrollo de la tecnología de la información para manejar los modelos de simulación de forma útil y oportuna, así como la recopilación de conocimiento referente a la fabricación que debe ser reflejado en los modelos"(NCR 1995).

La Simulación de la Fabricación así como los Programas de Visualización en el Instituto Nacional de Estándares y Tecnología (NIST) están enfocados en la aceleración del desarrollo de estándares para las librerías del Modelo de Simulación y los datos. Asimismo, el programa de simulación del NIST se centra en la definición de normas y experimentos estándar para la simulación que permitirán a la industria de la fabricación hacer más eficaz su simulación. El Departamento de Seguridad Nacional (DHS) de USA también ha reconocido el valor de la experiencia de NIST en la simulación y esta dándole el apoyo adicional al programa para dar orientación en las normas y en las pruebas para aplicaciones de modelado, simulación y análisis del DHS (NIST 2007).

Los tres principales componentes del programa incluyen: a) marcos de trabajo y arquitecturas, b) modelos de datos y normas y c) prototipos de simulación y sistemas de pruebas. 
a) Marcos y arquitecturas. NIST ha desarrollado los marcos y arquitecturas de integración distribuidas tanto para aplicaciones de seguridad de la producción y la nación. Los marcos y arquitecturas han establecido la dirección para la estandarización, creación de prototipos y las actividades de las pruebas para la interfaz de NIST. La Organización de Normas de Interoperabilidad de Simulación (SISO) aborda la arquitectura de modelización y simulación de NIST para la gestión de incidentes durante la formación (entrenamiento). Esta parte del programa tiene como fecha de finalización contemplada el año 2010.

b) Modelos de datos y las normas. NIST ha proporcionado liderazgo y conocimientos técnicos para la Organización de Normas de Interoperabilidad de Simulación (SISO) para desarrollar un modelo de datos de simulación del núcleo de la producción (CMSD). El CMSD ofrece interfaces de datos neutrales para integrar aplicaciones de software de plantas de fabricación con los simuladores de producción. Ahora se está ampliando el CMSD a la dirección flujo en plantas de fabricación simulación, diseño de planta y otros tipos de datos. Un número importante de organizaciones (los fabricantes, proveedores de software, las instituciones de investigación y agencias gubernamentales) ha apoyado y participado en la validación de las especificaciones y han proporcionado aportaciones técnicas y revisiones. Esta parte del programa tiene como fecha de finalización contemplada el año 2010.

c) Sistemas de pruebas y prototipos de simulación. Los ingenieros y científicos de NIST involucrados en el programa de simulación utilizan tecnología de simulación para obtener experiencia de primera mano con los problemas que enfrentan los usuarios industriales, para validar soluciones de normas y para establecer la interoperabilidad y otras capacidades de las pruebas. Un enfoque importante es el desarrollo de instalaciones de pruebas de una interoperabilidad nueva, dinámica, basada en simulación para la producción de aplicaciones de software. NIST ha elaborado un número de simulaciones para apoyar la interoperabilidad de la base de simulación pruebas, incluyendo una cadena de suministro automotriz, una planta de montaje final de un vehículo y diversas operaciones de plantas. Las interfaces que hasta ahora han sido incorporadas de estas simulaciones incluyen el Modelo de Datos de Simulación de Fabricación Principal SISO y la Especificación de apoyo a la visibilidad de inventario del Grupo de Aplicaciones A Abiertas (OAGIS). 
El trabajo futuro se centrará en la integración de aplicaciones de software de producción con una planta de la máquinas virtuales para apoyar la validación y las pruebas de interoperabilidad. Esta parte del programa tiene como fecha de finalización contemplada el año 2011.

Como parte de la estrategia de uno de los programas de NIST, McLean y Leong (MCLean \& Leong 2002b) están desarrollando estándares de datos para la simulación de fabricación. Los estándares de simulación son útiles para acelerar el proceso de modelado y reducir sus costes. De acuerdo con McLean y Leong, algunos de los factores que deberían ser considerados para la creación de una estructura uniforme de un modelo se refiere a la clasificación de los aspectos relacionados con la simulación de fabricación y sus niveles de abstracción. Éstos factores además son considerados durante la definición de los elementos que deben ser integrados en el diseño del modelo de simulación. Tales factores se describen a continuación:

\section{a) El sector del mercado industrial:}

El sector del mercado industrial probablemente es el factor más importante a considerar en el desarrollo de un sistema de clasificación de tipos de simulación de fabricación. Este hecho se basa en que el sector es el que identifica los productos finales que tienen que ser fabricados. Tanto la jerarquía de las organizaciones, los sistemas, y procesos a menudo son únicos para cada uno de los sectores de fabricación. De esta forma, los modelos y los datos requeridos para la simulación de un caso de estudio se determina primero por el sector y a continuación por el nivel jerárquico de fabricación.

\section{b) Niveles de modelado jerárquico}

El nivel jerárquico de la organización de fabricación, sistema o proceso es el segundo atributo de la estructura de clasificación de la simulación. Las descomposiciones de las actividades difieren de las jerarquías en cuanto a que las actividades o funciones sólo pueden ser identificadas en cada nivel de la estructura. Diferentes industrias tienen diferentes números de niveles, agrupación de elementos, y convenciones de asignación de nombre en sus descomposiciones.

Debido a que una descomposición en particular no es necesariamente correcta o incorrecta para todas las industrias, el esquema de clasificación jerárquica de la simulación debe considerar variaciones en las jerarquías 
a través de las industrias. La estructura propuesta por McLean y Leong contiene una meta-jerarquía que puede ser empleada para relacionar varias jerarquías y modelos utilizados en diferentes sectores del mercado. Debido a que no existe un acuerdo universal entre los diferentes sectores de fabricación, los mismos nombres de los niveles pueden utilizarse por diferentes industrias en el mismo o diferente meta-nivel.

Debido a que existen importantes diferencias en la naturaleza de los modelos y los datos requeridos para simular cada nivel se aplican divisiones en la meta-jerarquía de fabricación. Por tanto, la meta-jerarquía de simulación del nivel más alto al más bajo se describe a continuación:

Economía. Representa múltiples mercados en una región geográfica de interés. Los modelos de este tipo pueden incluir modelos de mercado de fabricación como un elemento. Estos modelos pueden ser desarrollados por economistas o investigadores en el gobierno federal, estatal o regional. Los factores en este tipo de modelos pueden incluir comportamientos esperados por el cliente, coste del dinero, mano de obra, materiales, estado de la economía nacional, etc. Las salidas de estos models pueden ser utilizadas como entradas en el desarrollo del pronóstico del mercado.

Mercado. Los modelos de este nivel corresponden a sectores individuales, grupo de sectores, o subdivisiones de sectores del esquema de sectores del mercado industrial. Los analistas de simulación pueden requerir el modelado de los sectores del mercado para el pronóstico de demanda, precios, etc.

Cadena de suministro. En este nivel múltiples empresas trabajan en cooperación para entregar productos finales. Algunos ejemplos de los elementos funcionales de una cadena de suministro pueden incluir, redes, distribuidores, almacenes, plantas de ensamble final, y minoristas. Típicamente, algunos elementos de una cadena de suministro cruzan los limites de la empresa. Los diseñadores de modelos de simulación de cadenas de suministro pueden interactuar con diseñadores de otras empresas que utilizan diferentes simuladores para complementar su modelo e integrar características adicionales necesarias para interactuar con sus modelos.

Empresa. En este modelo jerárquico, el nivel de empresa define los límites de la corporación. Una empresa puede localizarse en una o más instalaciones y descomponerse de forma organizacional en múltiples 
departamentos. Típicamente una cadena de suministro debería estar compuesta por múltiples empresas.

Instalaciones. Cada una de las instalaciones en una empresa puede requerir ciertos departamentos, equipo, etc. debido al hecho de que es un sitio único. Los bienes que se mueven entre las instalaciones pueden implicar importantes cuestiones de transporte. La distribución de operaciones de producción en múltiples instalaciones pueden requerir duplicación de operaciones de soporte, equipo, etc.

Departamento. Una instalación está típicamente compuesta por múltiples departamentos tales como unidades organizacionales que llevan a cabo diferentes procesos del negocio. Los departamentos pueden estar ubicados en múltiples instalaciones. Los departamentos pueden descomponerse en departamentos más pequeños. Los ejemplos de departamentos incluyen: ingeniería, producción, finanzas y ventas.

Línea, área o células. Este nivel es una agrupación física de estaciones y/o equipo con el objetivo de la fabricación de un producto. Una familia de productos, o para ejecutar un conjunto similar de procesos. Las líneas, áreas y células pueden descomponerse en líneas, áreas o células más pequeñas. Las unidades en este nivel pueden ser utilizadas en múltiples departamentos y/o instalaciones.

Algunos ejemplos de una línea de producción pueden incluir líneas para ensamblar herramientas eléctricas, electrodomésticos, y automóviles. Una célula puede agrupar estaciones que producen una familia de de partes similares, tal como, cuerpos de válvula. Un área puede ser un área de soldadura donde son llevados a cabo una variedad de operaciones de soldadura.

Estación. Las estaciones on lugares donde el trabajo es llevado a cabo por operadores o robots. Una estación puede incluir uno o más piezas de equipo, operadores, areas de almacenamiento intermedio, etc.

Equipo. Algunos ejemplos de tipo de equipo incluyen herramientas de máquinas manuales o controladas por computadora, robots, vehículos guiados automáticamente (AGV), grúas, bandas transportadoras, sistemas de almacenamiento y recuperación, etc.

Dispositivos. Los dispositivo generalmente son elementos por separado de sistemas del nivel de equipos, incluyendo varios sensores y actuadores. Tanto el compartimiento de una máquina de herramienta o un efector de robot son ejemplos de dispositivos. 
Procesos. El nivel más bajo es el proceso de fabricación físico, por ejemplo trabajar a máquina, fundir a troquel, o el ensamble mecánico. Este es el nivel donde la física, cinemática, química, etc. de los procesos de fabricación se representa.

\section{c) El caso de estudio de simulación}

Los casos de estudio son enfocados hacia el análisis y mejora de la eficiencia y efectividad de las organizaciones de fabricación, sistemas y procesos. Los estudios a menudo modelan algunos aspectos de las operaciones actuales y validan el efecto de algunos cambios hipotéticos en dichas operaciones. La ejecución de los sistemas actuales y los propuestos son evaluados de acuerdo con un conjunto de métricas. Si la simulación valida que pueden esperarse suficientes mejoras, entonces los cambios propuestos son implementados.

Los objetivos del caso de estudio definen las razones para llevar a cabo la simulación. Algunos ejemplos de objetivos de estudio pueden ser la evaluación de la mejor ubicación para una nueva planta, crear una mejor distribución para una instalación existente, determinar el impacto de la introducción de una nueva máquina en la capacidad de la planta de producción o evaluar algoritmos de planificación alternativos.

Los casos de estudio individuales pueden ser utilizados como bloques modulares y plantillas para la solución de problemas de fabricación más complejos. Por ejemplo, un problema real de fabricación puede incluir aspectos tales como la selección de ubicación y distribución de una planta. El caso de estudio resultante para la simulación puede ser construido mediante el ensamble de modelos y datos de dos tipos de casos de estudio diferentes. El analista debería enfocarse en asignar cualquier objetivo o pregunta a un sólo tipo de caso de estudio. La principal razón para esta regla es el evitar la proliferación infinita de casos de estudio definidos de forma personalizada como se hace en la práctica de la industria actual.

Por otra parte, diferentes casos de estudio pueden utilizar lo mismos modelos, datos de entrada y salida.

Algunos ejemplos de los tipos de casos de estudio que se pueden utilizar para la simulación de fabricación incluyen: pronóstico de mercado, selección de ubicación, procesos de negocio, planificación, distribución de planta, bienes de equipo, fuerza de trabajo, mezcla de productos, balanceo de líneas, estimación de costos, validación de procesos, análisis de 
tolerancia, análisis de ergonomía, instrumentación, inventario, manejo de materiales y mantenimiento.

\section{d) Modelos y Datos}

Tanto el modelo como las interfaces de los datos de entrada y salida pueden estandarizarse para un determinado sector del mercado industrial, el nivel de modelado jerárquico y el caso de estudio para la simulación. Los datos requeridos dependen del detalle y el nivel de complejidad del caso de estudio para la simulación y los objetivos del análisis.

Los datos de la simulación pueden dividirse en cuatro grupos principales: modelos, transacciones, entradas y salidas. Las transacciones que manipulan datos que son transferidos entre modelos de simulación distribuidos, son entradas de una simulación y salidas de otra. Los reportes de salida de una simulación pueden ser utilizados como entradas para ejecutar una simulación diferente. Los modelos son ciertamente entradas de una simulación y también pueden ser generados como salidas.

Hasta el momento los formatos de los datos que han sido desarrollados han sido divididos en los siguientes grupos:

General y Miscelánea: La estructura de las "Revisiones" proporciona un mecanismo para identificar las versiones de los subconjuntos de datos, fechas de revisión, y el creador de los datos. Las estructura de las "Unidades de Medición" especifica las unidades utilizadas en el archivo tales como longitud, peso, velocidad, etc. La estructura de las "Referencias" identifica archivos electrónicos y documentos en papel que dan soporte y además definen los elementos de datos contenidos dentro de la estructura de los datos de simulación. La estructura de las "Distribuciones Probabilísticas" define distribuciones estadísticas que son utilizadas para variar los tiempos de procesamiento, tiempo de paro y reparación, disponibilidad de recursos, etc.

Estructuras organizacionales: La estructura "Departamental" define los departamentos dentro de la organización, sus relaciones, y los rangos y empleados de cada departamento. El "Directorio de la Organización" se utiliza para mantener los datos organizacionales y la información de los contactos de clientes y proveedores. En este directorio se realiza una remisión de los datos de piezas, órdenes, y órdenes de compra a organizaciones y contactos. 
Especificaciones de procesos y productos: La estructura de las "Piezas" proporciona elementos para sus especificaciones, agrupa códigos de tecnología, clientes y proveedores; así como ligas a listas de materiales, planes de procesos, dibujos, modelos de piezas, y otras referencias. La estructura del "Grupo de Listas de Materiales" proporciona referencias cruzadas de los piezas y las cantidades requeridas en un lista de materiales jerárquica. Dicha estructura también es utilizada para definir estructuras de ensamble de piezas y herramientas. La estructura de los "Planes de Procesos" define las hojas de ruta, hoja de operaciones y los programas de equipos que están asociados como la producción y las actividades de soporte. Las hojas de ruta y operaciones corresponden al nivel del trabajo y la tarea en la jerarquía de trabajos. Los planes definen los pasos, reglas de precedencia entre los pasos, y los recursos asociados con la producción de piezas y la ejecución de actividades de soporte.

Operaciones de producción: La estructura de los "Calendarios" identifica los programas de los turnos, paros, y vacaciones que son considerados como periodos de tiempo. La estructura de un "Trabajo" especifica la jerarquía de los artículos a ser procesados, por ejemplo, órdenes, trabajos, y tareas. Las reglas de precedencia definidas en los planes de procesos son transferidas a los trabajos de artículos asociados. La programación de datos y asignación de recursos para cada trabajo del artículo se mantienen en la estructura, así como otros datos. Se hace una referencia cruzada entre los trabajos y las tareas y sus respectivas rutas y operaciones. La estructura de las "Órdenes de Compra" identifica ordenes internas y externas que han sido creadas para satisfacer los requisitos del inventario de piezas.

Definiciones de recursos: La estructura de los "Recursos" describe todos los recursos que pueden ser asignados para trabajar en las instalaciones, su estado, asignaciones programadas para trabajos de artículos específicos, eventos significativos y los niveles de utilización. Los tipos de recursos actualmente disponibles incluyen: estaciones y equipos, grúas, empleados, herramientas y juego de herramientas, enseres (aparatos) y juego de enseres. Las configuraciones estándares también son definidas. La estructura de las "Definiciones de las Operaciones" especifica los tipos de operaciones que se pueden llevar a cabo en una estación particular o un grupo de estaciones dentro de la instalación. La estructura del "Inventario" identifica la ubicación de piezas, materiales, herramientas y el inventario de enseres (aparatos). 
Distribución: La estructura de la "Distribución" define la ubicación de los puntos de referencia dentro de un lugar o instalación, límites de las áreas, trayectorias, recursos, y objetos de las piezas. La estructura contiene apuntadores de referencia hacia archivos gráficos externos que pueden utilizar estándares gráficos apropiados para definir con más detalle estos elementos.

Los elementos descritos anteriormente para los modelos y los atributos de los datos para la estructura de la simulación no está completa. Sin embargo, inicialmente está enfocada en las definiciones de los tipos de datos del taller de máquinas y pequeñas líneas de ensamble. Los autores de esta propuesta se encuentran desarrollando tipos de datos para el manejo de lotes y otros tantos, aunque también mencionan que los tipos de datos descritos proporcionan una funcionalidad considerable.

Por su parte, Stadtler y Kilger en (Stadtler \& Kilger 2002) indican que el modelo de una planta de fabricación tiene que incorporar todos los detalles necesarios del proceso de producción para determinar los tiempos de finalización de órdenes de producción (solicitadas por clientes), las entradas necesarias con respecto al material y recursos con cuellos de botella potenciales.

Asimismo, con respecto al nivel de detalle, el modelo puede ser descrito bajo ciertas limitantes, por ejemplo, limitándose a describir las operaciones que se ejecutan en los cuellos de botella potenciales. Por otra parte el modelo puede ser definido basado en la asociación de datos. Para ellos se dividen entre datos estructurales y datos dependientes de una situación específica.

Los datos estructurales incluyen: ubicaciones, partes, lista de materiales, rutas asociadas a instrucciones operativas, recursos de producción, especificación de proveedores, matrices de configuración y calendarios. La lista de materiales generalmente se describe en un solo nivel. De esta forma, cada parte esta vinculada a sus componentes predecesores inmediatos.

El consumo de recursos por producto puede obtenerse de la ruta y las instrucciones operativas. Tanto el número de productos por orden así como el consumo por producto son necesarios para secuenciar y programar ordenes individuales. Así es como el concepto de Modelo de Proceso de Producción (PPM) surge de la combinación de dichas representaciones. Un PPM incluye al menos una operación mientras que cada operación consiste de una o varias actividades. Una operación siempre esta asociada con un recurso. Las actividades pueden necesitar material y generar algún material como salida. Asimismo, se debe especificar en que momento se necesita un material y cuando un material generado esta disponible. La secuencia técnica de actividades dentro de una operación - también 
denominada relación- puede representarse mediante arcos. Como se realiza en un proyecto, la planificación de actividades pueden relacionarse mediante

- relaciones fin-inicio, fin-fin, fin-inicio e inicio-inicio junto con

- distancias de tiempo máximas y mínimas.

Esto permite el modelado preciso de restricciones de actividades incluyendo la ejecución paralela de actividades. El tiempo, así como los requisitos de recursos y material de una orden pueden ser derivadas mediante la asociación de los PPMs y los denominados arcos de vinculación (pegging arcs). Los arcos de vinculación conectan el material de salida de un PPM con el respectivo material de entrada de el PPM sucesor.

Por otra parte los datos dependientes de una situación específica varían de acuerdo a la situación actual de la planta de fabricación. Estos integran inventarios iniciales, incluyendo trabajos en proceso, configuración del estado de los recursos y conjunto de ordenes a procesarse dentro de un intervalo de tiempo. Los procedimientos operacionales que especifica el Usuario pueden consistir de: reglas de tamaño de lotes, reglas de prioridad o selección de rutas. Aunque las reglas para la construcción de tamaño de lotes debería basarse en la situación actual de la producción, en los Sistemas de Planificación Avanzada a menudo se requiere predefinir reglas simples. Si existen rutas alternativas a para realizar la producción de una orden, entonces se debería esperar que el sistema elija la mejor. Sin embargo, se sabe "por experiencia" que el Usuario tiene que elegir su ruta "preferida". Algunas veces es posible tener una lista de calificaciones para las rutas alternativas como una entrada predefinida.

En la propuesta, con respecto a los niveles de abstracción que se consideran para el Modelado del Sistema de Fabricación, se incluyen los últimos niveles de la metajerarquía presentada en este capítulo (líneas, estación, equipo, dispositivos y procesos). Mientras que para la definición de cada uno de ellos se aplican los formatos de los datos referentes a: estructuras organizacionales, especificaciones de proceso y productos, operaciones de producción, definiciones de recursos y su distribución física (Capítulo 6). Asimismo, para la definición de modelos se considera la asociación de los datos a los elementos del Sistema de Fabricación basándose en los conceptos de datos estructurales y datos dependientes de una situación durante la definición y validación de la propuesta (donde se aplicará a casos de estudio) (Capítulos 7 y 10). 


\subsection{Requisitos Actuales de Fabricación y la Simu- lación con Agentes}

Los requisitos establecidos por la "próxima generación de Sistemas de Fabricación" presentados por Shen (Shen \& Norrie 1999, HMS 2004) plantea nueve aspectos fundamentales a considerar:

- Integración de la Empresa. Para lograr la competitividad global y la respuesta rápida al mercado, la empresa de fabricación individual o colectiva debería integrarse con su sistema de administración (compra, órdenes de trabajo, diseño, fabricación, planificación y asignación de recursos, control, transporte, recursos, personal, materiales, calidad, etc.) y sus socios a través de una red

- Organización Distribuida. Para una integración efectiva de la empresa a través de organizaciones distribuidas, serían necesarios sistemas distribuidos basados en el conocimiento para enlazar la administración de demandas directamente a los recursos, la planificación de capacidades y asignación de recursos.

- Entornos Heterogéneos. Tales sistemas de fabricación necesitarían acomodar software y hardware heterogéneos tanto en sus entornos de fabricación como en sus entornos de información.

- Inter-operabilidad. Entornos heterogéneos de información pueden utilizar lenguajes de programación diferentes, representar los datos con diferentes lenguajes y modelos de representación, y operar en diferentes plataformas de procesamiento. Los sub-sistemas y componentes de tales entornos heterogéneos deberían inter-operar de una manera eficiente.

- Cooperación. Las empresas de fabricación deberían cooperar con sus proveedores, socios, y clientes para el suministro de materiales, fabricación de partes, comercialización del producto final, etc. Tal cooperación debería ser eficiente y de respuesta rápida.

- Integración de Humanos con el software y el hardware. Las personas y los computadores necesitan estar integrados para trabajar de manera colectiva a varios niveles de desarrollo en el proceso de fabricación y en todo el ciclo de vida del producto, con acceso rápido al conocimiento y la información. Fuentes de información heterogéneas deben ser integradas para soportar 
estas necesidades y para mejorar las capacidades de decisión del sistema. Se requieren entornos de comunicación bi-direccional para permitir comunicación efectiva y rápida entre los humanos y los computadores para facilitar así su interacción.

- Agilidad. Se debe prestar considerable atención en reducir el tiempo del ciclo del producto para ser capaces de responder a los deseos de los clientes de manera más rápida. La fabricación ágil es la habilidad de adaptarse rápidamente a entornos de fabricación de cambio continuo y no anticipado, y, además, es el componente clave en las estrategias de fabricación para la competitividad global. Para lograr la agilidad, las utilidades de fabricación deben ser capaces de re-configuración rápida e interacción con sistemas y socios heterogéneos. Idealmente, los socios se contratan de manera dinámica sólo para el tiempo requerido para completar una tarea específica.

- Escalable. Un sistema de fabricación es escalable cuando recursos adicionales pueden ser incorporados dentro de la organización cuando estos sean necesarios. Esta capacidad debería estar disponible en cualquier nodo de trabajo en el sistema y en cualquier nivel dentro de los nodos. La expansión de recursos debería ser posible sin alterar los enlaces previamente establecidos en la organización.

- Tolerancia a Fallos. El sistema debería ser tolerante a fallos tanto a nivel de sistema como a nivel de sub-sistema para detectar y recuperarse de fallos a cualquier nivel, y, así, minimizar el impacto que estos fallos pudieran tener en el entorno de trabajo.

\subsubsection{Posibilidades de mejora mediante MAS con respecto a los Requisitos de Fabricación}

Un aspecto importante de los Sistemas de Control basados en Agentes es que a diferencia de los clásicos Sistemas de Fabricación Integrados por Computadora centralizados (CIM), es que no existe una unidad de toma de decisiones central (Vrba \& Marík 2005). En los enfoques (CIM) tradicionales, el conocimiento relativo a todos los posibles estados e interacciones de varias partes en el sistema de control así como los programas se encuentran concentrados en un controlador central. Esto permite definir el comportamiento global deseado del sistema de control exactamente en el periodo del diseño. Por el contrario, en el caso 
de los agentes con solamente un control parcial y la observación del entorno, los programas se construyen dinámicamente en tiempo de ejecución mediante negociaciones complejas. La simulación basada en agentes proporciona una evaluación cualitativa más parecida a un sistema de control de agentes. Este tipo de simulación permite el diseño de un modelo que representa la complejidad y el dinamismo del entorno del mundo real, además de que proporciona mejoras en cuanto a la flexibilidad y robustez de los procesos de fabricación. Por otra parte, el futuro del sector de la fabricación tendrá que apegarse a los cambios de la denominada "nueva fabricación" donde los sistemas de fabricación tendrán que satisfacer requisitos fundamentales (Shen \& Norrie 1999, HMS 2004).

Para detectar las posibles debilidades y posibilidades de mejora mediante la aplicación de los Sistemas Multiagente, se considera como base un reporte técnico sobre la revisión de herramientas de simulación (Ruiz, Giret \& Botti 2006a) bajo el criterio de clasificación expuesto en la Sección 4.7.1. En la revisión se incluyeron los requisitos de la nueva fabricación para así determinar qué herramientas de simulación consideran dentro de sus funcionalidades la integración de los requisitos mencionados. Al mismo tiempo, en dicha revisión se observa que aunque las herramientas incluyen algunos de los requisitos de la nueva fabricación, ninguna abarca todos los requisitos establecidos. Una de las principales debilidades detectadas es que en la mayoría de las herramientas no se ofrecen propiedades relacionadas con la inteligencia artificial tales como la autonomía, proactividad y flexibilidad.

De este estudio comparativo y de la revisión del estado del arte podemos derivar la siguiente hipótesis: si un Entorno de Simulación integra autonomía, proactividad y flexibilidad como parte de sus funcionalidades, entonces dicho entorno realizará una simulación de fabricación que permita integrar un mayor número de variantes mejorando la representación del sistema real en comparación con las herramientas que no las ofrezcan y por lo tanto ofrecerá resultados más fiables.

Algunas de las oportunidades de mejora asociadas a los requisitos elementales de la nueva fabricación (Ruiz, Giret \& Botti 2006b) que pueden ser resueltos mediante la aplicación de la Tecnología Multiagente se listan a continuación:

Integración de la Empresa: Algunas de las herramientas actuales están enfocadas en procesos específicos de un sistema de fabricación tal como líneas de ensamble y flujo de materiales. Solo algunas de ellas incluyen el ciclo de vida completo de un producto. La Teoría de Sistemas Multiagente, 
nos permite simular el comportamiento global de cada entidad combinando comportamientos simples y complejos en diferentes niveles incluyendo elementos físicos (humanos, maquinaría, espacios físicos de la planta de producción, etc.) y reglas globales involucradas en el ciclo de vida del producto.

Organización Distribuida: Las herramientas de simulación actuales, generalmente están basadas en modelos pre-establecidos. Las herramientas de simulación incorporan algoritmos complejos para la optimización de procesos, sin embargo, la base de datos del sistema permanece centralizada en puntos específicos de la estructura de la organización del sistema. La tecnología Multiagente, permite distribuir y limitar el alcance de los agentes de acuerdo al estado actual del sistema.

Entornos Heterogéneos: Generalmente cada herramienta de simulación está desarrollada en un lenguaje de programación específico el cual puede ser propietario o comercial tal como C o Fortran. Debido a las limitaciones de cada lenguaje de programación solo es posible proporcionar interfaces de integración específicas. La tecnología Multiagente permite simular cualquier entorno a pesar de las restricciones físicas o el programa propietario de cada equipo o maquinaria.

Inter-operabilidad: La mayoría de las herramientas de simulación proporcionan interfaces entre ellas y otros programas mediante archivos formateados mediante herramientas CAD, archivos de texto, interfaces DLLS y ODBC, etc. La tecnología Multiagente permite la comunicación con cualquier tipo de lenguaje de programación sin limitaciones.

Cooperación: Los Sistemas Multiagente ofrecen una característica relevante en cuanto a cooperación se refiere, es decir, los agentes que integran un sistema poseen además de objetivos en común, objetivos globales lo cual favorece el que el nivel de confianza para trabajar en grupos y cooperar en el desempeño de tareas sea una característica única en los Sistemas Multiagente.

Estructura Abierta y Dinámica: Algunas de las herramientas de simulación actuales ofrecen algunas posibilidades para realizar modificaciones "onthe fly" sin interrumpir el funcionamiento del sistema. Algunas herramientas emplean capas de programación integradas en estructuras jerárquicas, sin embargo, la adición o eliminación de un elemento no es una tarea fácil 
para el usuario. Una de las ventajas de la utilización de agentes, es que los agentes pueden ser agregados o eliminados automáticamente sin interrumpir la operación del sistema de acuerdo con las necesidades del estado actual del sistema.

Integración de humanos con el software y el hardware: Las herramientas de simulación actuales permiten incorporar el elemento humano pero están limitadas en cuanto a los atributos que se permiten parametrizar. La teoría Multiagente permite incorporar características asociadas a comportamientos complejos de las interacciones humanas con el software y hardware del sistema.

Agilidad: Las herramientas de simulación actuales se basan en modelos donde la complexidad de cada modelo y sus algoritmos pueden variar, sin embargo, los eventos se especifican por anticipado, incluyendo planes de acción alternativos. La tecnología Multiagente gracias a sus características asociadas a la autonomía y proactividad permite detectar y ajustar el sistema automáticamente. Esta opción permite la creación y control de la ejecución de planes y estrategias cuando un evento inesperado se presenta durante la simulación. Este comportamiento del sistema puede implementarse mediante grupos de agentes emergentes que ejecutan procesos complejos.

Escalable: Los Sistemas Multiagente permiten agregar funcionalidades específicas mediante la integración de comportamientos adicionales a los agentes existentes o en su caso agregar agentes nuevos para satisfacer nuevos requisitos sin corromper la estructura y funcionamiento actual del sistema.

Tolerancia a Fallos: Las herramientas de simulación están basadas en modelos $y$ algunos de ellos proporcionan un optimizador adicional que se basa en algoritmos complejos. Cuando se presenta un fallo es necesario corregir el modelo ya sea deteniendo el sistema o mientras el sistema sigue su ejecución. La tecnología Multiagente, gracias a las características propias de los agentes (autonomía y proactividad), permite responder y ejecutar automáticamente planes alternativos apropiados sin interrumpir o detener la operación global del sistema.

En las herramientas actuales parece no ser una tarea sencilla realizar las modificaciones necesarias para que puedan incluirse los requisitos de la "nиеva fabricación”. Sin embargo, la utilización del paradigma de Sistemas Multiagente es una alternativa viable que por su naturaleza permite en un principio ofrecer dicha integración en un Entorno de Simulación Flexible. 


\subsection{Enfoque Holónico para Fabricación}

Los Sistemas Holónicos son un enfoque que ha sido propuesto para resolver los nuevos requisitos de la fabricación, por tanto, en esta sección se presentan las bases de dicho enfoque.

El enfoque de los Sistemas Holónicos de fabricación fué definido inicialmente utilizando como elemento base el concepto de holon de Koestler (Koestler 1989). Después se desarrollo en el contexto de la fabricación por Suda (Suda 1990) y Christensen (Christensen 1994) con el fin de proveer una base o capacidad de "plug and play" para desarrollar y operar un sistema de fabricación en la fábrica del futuro. El más reciente propósito de este enfoque es el desarrollo de una arquitectura para sistemas de fabricación altamente descentralizados, construidos de una mezcla de módulos de componentes estandarizados, autónomos, cooperativos e inteligentes, y así implementar entornos rápidamente cambiantes (Christensen 2002).

La naturaleza de los Sistemas Holónicos de Fabricación (HMS) impone requisitos nuevos y únicos en la arquitectura del sistema de producción, definida como la estructura y relaciones entre las unidades funcionales inteligentes en un sistema, posiblemente incluyendo las interfaces del sistema con su entorno (Christensen 2002).

Los Sistemas Holónicos de Fabricación (HMS), como uno de los nuevos paradigmas en fabricación, consisten de agentes u holones autónomos, inteligentes, flexibles, distribuidos, y cooperativos (P. Valckenaers, Bonneville, Van Brussel, Bongaerts \& Wyns 1994). En un HMS cada actividad de un holón es determinada por medio de la cooperación con otros holones, en oposición a ser determinada por mecanismos centralizados. En este tipo de sistemas, los agentes inteligentes llamados "holones" tienen tanto una parte física como una parte software. Un holón puede ser parte de otro holón. Una de las características más prometedoras del enfoque holónico es que éste representa la transición entre los sistemas jerárquicos y heterárquicos (P. Valckenaers et al. 1994, Bongaerts, Monostori, McFarlane \& Kádár 1998). De esta forma, el sistema se conduce hacia el paradigma de la "fabricación ágil", donde los componentes de sistemas de producción son reprogramados y reconfigurados constantemente y se encuentran al mismo tiempo dentro de un sistema de fabricación con un volumen denso de información.

La funcionalidad de la implementación de este tipo de componentes incluye las características necesarias para habilitar a los componentes de fabricación físicos de una planta de fabricación para iniciar "negociaciones" y mutuamente "coordinar" su comportamiento, gestionando la producción. Sus características 
se presentan a continuación (Colombo et al. 2006):

- Capacidad para asignar, unir, abandonar y participar en relaciones de cooperación para el control de la ejecución de tareas de fabricación.

- Capacidad para razonar sobre las tareas de fabricación y sus relaciones para controlar, adquirir y compartir el conocimiento relacionado con este razonamiento.

- Capacidad para proporcionar comandos de control apropiados y funciones para modificar dinámicamente las aplicaciones existentes y ejecutar nuevas tareas o recuperarse de situaciones anormales.

La nueva generación de sistemas de fabricación definida como Sistemas de Fabricación Inteligentes (IMS), tiene una característica en particular, sus recursos tienen que ser capaces de tratar tanto procesamiento de conocimiento de procesos de fabricación y equipo, como requisitos de procesamiento de material. El IMS consiste de un control distribuido y entidades que colaboran dinámicamente para satisfacer tanto objetivos locales como globales (Shen \& Norrie 1999).

\subsubsection{Fabricación Virtual y los Sistemas Holónicos de Fabri- cación}

La Fabricación Virtual (VM) proporciona la habilidad para el análisis del diseño del producto y los procesos son el testeo real de los productos físicos. En un entorno de Fabricación Virtual, las modificaciones en el diseño y los planes de diseño desde diferentes aspectos de la fabricación pueden ser fácilmente realizados con el propósito de obtener un diseño libre de errores antes del comienzo de la producción real.

La Fabricación Virtual proporciona cuatro elementos fundamentales en la fabricación (Lin, Minis, Nau \& Regli 1994) : 1) la Fabricación Virtual puede utilizarse para probar los escenarios de producción, obteniendo como resultado un fuerte sistema de "pre-producción" (p.ejem. sistemas que son desarrollados y verificados pero que nunca experimentan ejecuciones de producción reales), 2) la Fabricación Virtual puede soportar la generación de costos estimados de producción y programación más fiables debido a que los modelos se basan en procesos reales, no solo parámetros, 3) el Modelado y la Simulación (M\&S) puede mejorar significativamente la flexibilidad de la producción, y por tanto, 
reducir los "costos fijos", y 4) tanto las predicciones de costes fiables, riesgo y programación pueden mejorar sustancialmente el proceso de toma de decisiones.

El concepto de la Fabricación Virtual (VM) fue propuesto para integrar diferentes formas de fabricación virtual y hacer un modelo computacional equivalente a un Sistema de Fabricación Real (RMS) desde el aspecto de la información que contiene (Onosato \& Iwata 1993). En una extensión al concepto de la Fabricación Virtual (Iwata \& Onosato 1995), el Sistema de Fabricación se descompone en cuatro Subsistemas de Fabricación: Sistema Físico Real (RPS), Sistema de Información Real (RIS), Sistema Físico Virtual (VPS), y Sistema de Información Virtual (VIS). La Fabricación Virtual permite la simulación de procesos de fabricación sin utilizar las instalaciones reales, y de esta forma acelerar el diseño y rediseño de Sistemas de Fabricación Real (Ver Fig. 4.4).

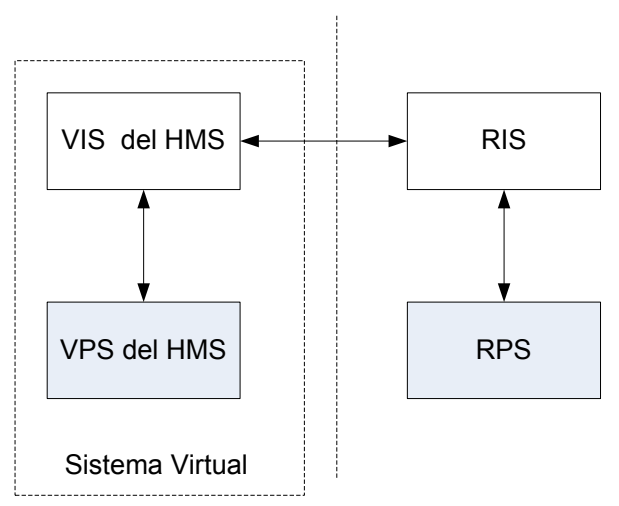

Figura 4.4: Holonificación de un Sistema de Fabricación mediante la teoría de Fabricación Virtual.

Una característica fundamental del concepto de Fabricación Virtual es que ésta ejecuta el mapeo de uno a uno entre el Sistema Real y el Virtual. Esto es, el Sistema de Información Virtual y el Sistema Físico Virtual intentan simular el Sistema de Información Real y el Sistema Físico Real respectivamente, tan exactamente como sea posible.

De esta manera, la principal contribución de la Fabricación Virtual con relación a los Sistemas Holónicos de Fabricación (HMS), es la separación mediante el mapeo uno a uno de los elementos para controlar un Sistema de Fabricación (centralizado / jerárquico) de una forma holónica. En este enfoque, suponiendo que existe una unidad de control central en el Sistema Tradicional, los requisitos básicos para la "holonificación" de este sistema se traducen en: a) la habilidad de 
comunicarse con el mundo exterior, b) la transferencia de información de control a los recursos, c) la captura de la información del estado, y su transferencia a la unidad central, d) la interrupción de la operación de los recursos en periodos dados, y e) el paro o modificación de procesos iniciados previamente. Como resultado de la aplicación de este enfoque, es posible obtener un HMS que contempla las bondades de un Sistema de Fabricación Virtual.

Asimismo, las técnicas de simulación pueden contribuir en la elaboración de nuevos algoritmos, reducir el riesgo en inversiones y a desarrollar el Sistema de Información Virtual de un HMS mediante el concepto de Fabricación Virtual.

La Fabricación virtual incluye la simulación de un producto y los procesos involucrados en su fabricación. La tecnología de simulación permite optimizar los factores clave que afectan directamente a la rentabilidad de los productos fabricados tales como la forma final, niveles de esfuerzo residual, y durabilidad del producto. Éstos afectan directamente la rentabilidad, mediante la reducción del coste de producción y utilización de material.

Más aún, la Fabricación Virtual, también reduce el costo de utillaje (elementos de montaje), elimina la necesidad de múltiples prototipos físicos, y reduce el desperdicio de material. Las pequeñas mejoras en la fabricación tienen efectos importantes en términos de coste y calidad. La Fabricación Virtual utiliza la computadora para simular la realización de un producto y los procesos necesarios para su fabricación. Asimismo, utiliza tecnologías de análisis de elementos finitos no lineales para proporcionar información detallada de un producto, tal información es utilizada para la optimización de factores tales como esfuerzo residual, fabricación, y estimaciones del ciclo de vida del producto. La razón de la aplicación de la Fabricación Virtual mediante la simulación se debe a la naturaleza de la simulación que es la búsqueda de información más detallada. Cada simulación actúa como un punto de ventaja desde el cual se puede obtener una mejor visión de las posibilidades actuales. De esta forma las simulaciones se refinan hasta que en un momento determinado puede ser posible obtener una solución óptima. La necesidad de obtener soluciones rápidas, más definidas y en menor tiempo, y con mayor exactitud, hacen de la simulación de la Fabricación Virtual una herramienta útil en el área de la fabricación.

Para la simulación virtual de un entorno de fabricación es necesario realizar actividades específicas que permitan obtener la información necesaria para representar las características del entorno de fabricación. Según Caputo et. al. las acciones incluyen (Caputo, DiGironimo \& Marzano 2006): 
1. Recolección de Datos. Incluye distribución de planta, modelos 3D, fotografías, secuencia de operaciones, disponibilidad, productividad, etc. Los datos predictivos de fiabilidad y mantenimiento $(\mathrm{R} \& \mathrm{M})$ que continuamente se respaldan mediante la monitorización y verificación.

2. Simulación de Células de Trabajo. Para lo cual se utilizan herramientas de software. El software modular es crucial en esta etapa, para permitir a los diseñadores trabajar en paralelo y reducir el tiempo de trabajo. La evaluaciones ergonómicas de células de trabajo manuales y simulaciones robóticas de células de trabajo automizadas también se incluyen. Hasta que no se termina de simular todas las células de trabajo, éstas pueden ser representadas como una "caja negra" con un ciclo de tiempo asignado, tasa de fallos, disponibilidad, etc. Como resultado se obtienen los modelos de cada célula.

3. Integración. En esta etapa se contempla integración de cada máquina y estación en la herramienta de simulación. En este momento la simulación está completa, y los datos de salida pueden ser analizados. En esta etapa es posible el uso de herramientas de Realidad Virtual (guantes, cascos, gafas,etc.) que faciliten la interacción de los seres humanos con el entorno virtual.

4. Análisis de Resultados. Debe ser verificado que cada parámetro asignado, tales como eficiencia técnica y disponibilidad, se ajustan con los requisitos del cliente. Si los datos son satisfactorios, la simulación completa puede ser presentada al cliente. Si no, se sigue el flujo de datos en reversa para que los ingenieros puedan cambiar los parámetros y por tanto corregir el problema.

5. Presentación. Se presenta el trabajo completo al cliente.

La Fabricación Virtual por tanto se define como un entorno de fabricación sintético (una mezcla de objetos reales y simulados, actividades y procesos) integrado, utilizado para mejorar la exactitud y validación en todos los niveles de decisión y control (Lin et al. 1994).

\subsubsection{Sistemas Holónicos de Fabricación y los Sistemas Mul- tiagente}

La integración tanto de los "Sistemas Holónicos de Fabricación (HMS)" y el paradigma de los "Sistemas de Fabricación Orientados a Agentes" actualmente se presentan como la base de un Sistema de Fabricación Inteligente (IMS). 
La investigación de los Sistemas Holónicos (HS) está motivada en las tareas flexibles de fabricación. Por consiguiente, está orientada hacia los estándares de comunicación de bajo nivel y el comportamiento de bajo nivel. Por otra parte, la investigación en el área de los Sistemas Multiagente (MAS) está motivada en la programación de sistemas inteligentes distribuidos. Se centra en el comportamiento social de entidades inteligentes y se ocupa principalmente de la investigación de modelos de comportamiento, estrategias de cooperación y coordinación, optimización del desempeño de tareas, aprendizaje a partir de las propias experiencias, creación de coaliciones, etc. En resumen, a diferencia de MAS, que es un enfoque software amplio que puede ser utilizado además para el control inteligente distribuido, un HS es, por definición, un enfoque específico de fabricación para sistemas de control distribuidos (Botti \& Giret 2003). En los sistemas Holónicos de Fabricación, los agentes se utilizan para modelar holones los cuales son entidades software y hardware (Deen 1994, Christensen 1994, Hasegawa, Gou, Tamura, Luh \& Oblak 1994, Biswas, Bagchi \& Saad 1995). Asimismo, Gaud (Gaud 2007) propone la definición del modelado de Sistemas Multiagente Holónicos para representar sistemas de propósito general .

Un ingeniero puede diseñar un Sistema de Fabricación desde diferentes niveles de abstracción (mecanismos, máquinas, lineas de producción), por tanto, los elementos de un nivel pueden ser agrupados para observar su comportamiento desde un nivel superior. Un agente abstracto (Giret \& Botti 2004b) implica una noción de modelado que permite modelar Sistemas Multiagente utilizando diferentes niveles de abstracción. Por tanto, un agente abstracto puede puede representar un agente atómico o un Sistema Multiagente. Por otro lado, un holón se refiere a un sistema (o fenómeno) que es un todo por si mismo o forma parte de un sistema más grande. Esto puede ser visto como un sistema que está dentro de otro sistema. Por tanto, en este trabajo un agente y un holón serán considerados como nociones similares y se utilizarán de forma intercambiable (Giret \& Botti 2004a).

Además, un HMS puede considerarse, desde el punto de vista de la arquitectura software, como un tipo particular de comunidad de agentes con estructura y comportamiento muy relacionados a las tareas de fabricación (Rannanjarvi \& Heikkila 1998).

\subsubsection{Arquitectura de Fabricación basada en Agentes}

Para la aplicación del paradigma de agentes, es necesario tomar en cuenta las principales cuestiones con respecto a las arquitecturas de fabricación basadas 
en agentes que se deben considerar, las cuales se agrupan en cinco áreas (Kádár, Monostori \& Szelke 1998):

Estructura de Agentes. La estructura interna de agentes y el nivel de su autoencapsulación.

Comunicación. Incluye los protocolos de comunicación, el cual es el lenguaje de intercambio común.

Formación de Grupos. Integra la negociación, protocolo de comunicación apropiado, persuasión de las máquinas para participar en un grupo, sistemas de premios/penalizaciones, mecanismos de mercado, función objetivo del agente.

Reconfigurabilidad. Se refiere a la propiedad de los Sistemas Abiertos (adición, eliminación, sustitución de máquinas, etc.)

Escalabilidad. Implica el potencial conveniente para aumentar el alcance del sistema (Van Brussel, Valckenaers, Wyns, Bongaerts \& Detand 1996).

Optimización global versus local. Se refiere a cómo es posible lograr la optimización global con agentes autointeresados que persiguen sus propios objetivos; cuál es la proporción óptima entre la jerarquía y heterarquía en una situación dada; cómo ajustar el sistema; y cómo conseguir el aprendizaje del sistema al nivel de los agentes.

Un aspecto fundamental es la definición de una arquitectura HMS, la cual requiere la aplicación de una metodología apropiada en la que se respete los principios básicos tales como la autonomía, la cooperación y la flexibilidad. En la literatura especializada, existen dos familias de propuestas de control de fabricación: el control jerárquico y el heterárquico (Dilts, Boyd \& Whorms 1991). Entre las arquitecturas más representativas encontramos INTERRAP (Muller 1997), PROSA (Brussel, Wyns, Valckenaers, Bongaerts \& Peeters 1998), Arquitectura basada en Agentes y bloques funcionales (Fletcher, Garcia-Herreros, Chritensen, Deen \& Mittmann 2000), y MetaMorph II (Maturana \& Norrie 1996). Sin embargo, basándonos en una comparación de las características estructurales de Arquitecturas de Referencia compatibles que permiten el control jerárquico y heterárquico presentada por Van Brussel et al. en (Brussel et al. 1998) es posible observar ciertas características importantes que la Arquitectura de Referencia PROSA posee. Estas características son: a) cubre todos los aspectos de las arquitecturas de control jerárquicas y heterárquicas, b) cubre todas las funciones 
relevantes y c) puede incorporar un amplio rango de algoritmos de control de enfoques centralizados y distribuidos.

\subsubsection{Arquitectura de Referencia PROSA}

En esta sección se hace referencia a la descripción de los tipos de holones (Producto, Recurso, Orden, y Staff) que ofrece la Arquitectura de Referencia PROSA. La Arquitectura de Referencia PROSA (Brussel et al. 1998) introduce importantes innovaciones: la estructura del sistema está separada del algoritmo de control, los aspectos logísticos están separados de los técnicos, y permite la incorporación de algoritmos de control híbridos más avanzados (Bongaerts, Monostori, McFarlane \& Kádár 1998, Bongaerts, Van Brussel \& Valckenaers 1998).

En la Arquitectura PROSA (Brussel et al. 1998) el modelo se compone por tres holones básicos: holón de Recurso, holón de Producto, y holón de Orden. Cada uno de los holones es responsable de un aspecto del control de fabricación, el cual puede ser logístico, de planificación, o de capacidades de recursos. Adicionalmente la arquitectura preveé la posibilidad de brindar holones de Staff para ayudar a los holones básicos y proporcionarles la información suficiente para la correcta toma de decisiones en la resolución de problemas. Los tres holones básicos cubren todas las funciones críticas de fabricación: operaciones de programación, control de niveles de inventario, coordinación de máquinas para ejecutar operaciones, planificación de mantenimiento, monitorización, desarrollo de procedimientos de control, planificación de procesos, diseño de producto, y control de calidad (Lin \& Solberg 1994, Biemans 1989). Cada uno de los holones básicos es responsable, respectivamente, de un aspecto del control en la fabricación: a) logística interna, b) plan de fabricación, y c) manejo de recursos. Para asistir, con conocimiento experto, a los holones básicos se pueden agregar holones "Staff".

La estructura del sistema completo de fabricación es una holarquía dual descompuesta en una sub-holarquía de asignación de recursos (órdenes de trabajo, recursos y staff) y una sub-holarquía de control de proceso (producto y la parte de control de proceso de los recursos). Una de las técnicas de coordinación y de control de la holarquía se puede implementar utilizando conceptos inspirados en el comportamiento social de insectos, más específicamente hormigas (Cicirello \& Smith 2001). Se distinguen tres categorías diferentes de dominios de cooperación: uno para conocimiento de producción, uno para procesamiento, y uno para ejecución de proceso. El protocolo de comunicación utilizado es el Contract Net. 
Cuando una orden de trabajo ingresa al sistema, el holón de orden de trabajo se convierte en el administrador de la tarea, la divide en sub-tareas, anuncia cada sub-tarea, luego selecciona las ofertas de fabricación más apropiadas y asigna la tarea. El administrador de la tarea controla el progreso del contrato, y en caso de que el contratado falle en la culminación de la tarea, es libre de re-asignar la tarea.

En consecuencia, cada uno de los holones debe tener asociado un conjunto de características que permitan describirlo internamente y externamente, le permitan comunicarse con otros holones y ejecutar sus tareas apropiadamente.

\section{Holón de Producto.}

Un holón de Producto mantiene el conocimiento de proceso y de producto para asegurar la fabricación correcta del producto. Actúa como un servidor de información para los demás holones del HMS. Un holón de Producto almacena información acerca del estado del proceso de los componentes del producto durante la fabricación. Asimismo, almacena información sobre configuración, diseño, plan de proceso, lista de materiales, procedimientos de aseguramiento de calidad, etc., sobre el ciclo de vida del producto. Como tal, éste contiene el "modelo del producto" del tipo de producto, no el "modelo del estado del producto" de una instancia de producto física. Por tanto, la representación de un holón se puede realizar mediante el diseño de un modelo que lo describa adecuadamente. Cuando se modela un Holón de Producto, éste debe contener el nivel de detalle adecuado que le permita abarcar todas sus características funcionales.

Por otra parte, el modelo del estado del Producto contiene información en cuanto a las dimensiones actuales, calidad, ubicaciones, superficie, orientación, etc. Éste se actualiza con información reciente de un producto a medida que recorre un proceso de producción. En consecuencia, la información del estado del producto se refiere a la información del producto y representa el estado de la parte de un producto o del producto completo en algún momento. Andersen y Mortensen (Andreasen \& Mortensen 1998) sugieren una definición y estructuración del tipo de parámetros que pueden asignarse a un producto. Un prerequisito para calificar la información del estado del producto es que sea almacenada en un repositorio donde exista un "cliente". Esta es una idea en la cual siempre existirá un cliente que hará algo con el producto o la parte del producto.

Asimismo, el componente de producto u holón de producto también contiene una parte física y una de control. La parte física puede incluir materia prima y 
partes. Una parte de control puede contener el control de enrutamiento, el control de proceso, y la información para la toma de decisiones y producción (Chirn \& McFarlane 2000).

\section{Holón de Recurso.}

Los objetos físicos de una planta de fabricación se pueden clasificar en dos grupos generales de acuerdo con sus propiedades. Uno es el recurso que realiza las operaciones de fabricación y el otro es el producto que recibe los tratamientos de fabricación. El componente de recurso u holón de Recurso es un componente del sistema autosuficiente (autónomo) el cual ejecuta operaciones de trabajos en proceso (WIP), tales como fabricación, ensamble, transporte, y evaluación. Además de las partes físicas visibles, un componente de recurso contiene una parte de control invisible la cual ejecuta sus operaciones, la toma de decisiones y cuenta con la habilidad para comunicarse y hacer uso de su base de datos (Chirn \& McFarlane 2000). El holón de Recurso contiene los métodos para asignar los recursos de producción, así como el conocimiento y los procedimiento para organizar, utilizar y controlar los recursos durante la producción. Asimismo, ofrece su capacidad de producción y funcionalidad a otros holones del entorno (Brussel et al. 1998). Un holón de Recurso es una abstracción que puede representar una fábrica, un planta, máquinas, cintas, componentes, materia prima, herramientas, herramientas, áreas de almacenamiento de material, personal, energía, etc.

\section{Holón de Orden.}

Un holón de Orden representa una tarea en el sistema de fabricación. Es responsable de realizar correctamente el trabajo asignado y a tiempo. El holón de Orden manipula el producto físico que será producido, el modelo de estado del producto, y todo el procesamiento de la información logística asociada al trabajo. Este tipo de holón posee un cierto comportamiento de control para gestionar las actividades a través de la fábrica. Un holón de orden puede representar órdenes de clientes, órdenes internas para mantener en inventario, órdenes para realizar prototipos, órdenes de mantenimiento y reparación de recursos, etc. A menudo, el holón de Orden puede ser considerado como una pieza de trabajo con un cierto comportamiento de control para gestionarse y moverse en la fábrica, p.ejem. para negociar con otras partes y recursos para la producción. El holón de Orden usualmente realiza tareas asignadas a un despachador, monitor de progreso, y un programador a corto plazo (Brussel et al. 1998). 
Un holón de Orden de producción contiene los atributos y métodos que son generales para todos los holones de orden. Este holón de Orden se puede especializar en un holón de orden de producto y un holón de orden de paquete (Larsen, Sorensen \& Langer 2001). El holón de orden de producto representa las entidades más básicas de una orden dentro de un sistema de fabricación que puede ser caracterizado como autónomo y cooperativo, y con ello capaz de controlar su propia producción. El holón de orden de paquete representa la agregación de varios holones de orden. Estos holones de Orden se pueden relacionar de tal manera que ellos poseen el mismo plan de procesos o que todos ellos son partes que deberán ser ensambladas. El holon de orden de paquete se especializa en un holón de orden de ensamble. El holón de orden de ensamble agrupa holones de orden que están reunidos físicamente mientras que el holón de orden de paquete agrupa holones de orden que no lo están.

\section{Holón de Staff.}

Los holones de Staff permiten asistir a los holones básicos (Holón de Producto, Orden, y Recurso) a realizar su trabajo. Los holones de Staff ayudan, basándose en las soluciones de problemas anteriores de los holones básicos, y les proporcionan suficiente información para que puedan tomar la decisión correcta para solucionar el problema. Los holones básicos son los responsables de la toma de decisiones, y el holón de Staff se considera como un experto externo que proporciona consejos (Brussel et al. 1998).

El concepto de holón de Staff permite la presencia de elementos y funcionalidad centralizados en la arquitectura. El holón de Staff es útil para problemas que son muy difíciles de resolver de manera distribuida, y que permite una migración asegurada de sistemas de control de planta jerárquica a un arquitectura holónica.

\subsection{Características de la Simulación de Fabrica- ción}

De acuerdo con Miller (Miller \& Pegden 2000), la simulación de fabricación debe incluir los siguientes elementos: 
Características de Modelado de Fabricación: Existe un número de características comunes que deben ser incluidas en el modelo de simulación: recursos, manejo de materiales, lógica de control, memoria intermedia (memoria local) de lógica de estaciones de trabajo, plantas de procesos y órdenes, datos centralizados de recursos, estaciones de trabajo y trabajos.

Programación: La simulación puede ser utilizada sobre bases operacionales para producir programas de producción para la planta de producción. Cuando la simulación se emplea de esta forma, la simulación es un Programador de Capacidad Finita (Finite Capacity Scheduling -FCS-). La simulación brinda un método simple para la generación de programas para la planta de producción. En la programación basada en simulación, existen dos tipos de reglas de decisión que pueden aplicarse cuando se programa cada paso del trabajo: una regla de selección de operación, y una regla de selección de recurso.

A pesar del hecho de que el proceso de estandarización de la simulación de fabricación sigue en progreso (IMTR 1998), existen conceptos básicos que deben ser considerados en la simulación de fabricación tal como la optimización en la utilización de recursos, la planificación de procesos e información.

\subsection{Modelo de Simulación de Fabricación}

La integración de un modelo de acuerdo con (Heim 1997) se utiliza para unir modelos funcionales de componentes individuales y así crear redes de modelos que puedan compartir datos y coordinar sus actividades. Los modelos individuales incluyen componentes tales como: celdas de trabajo, máquinas, o subsistemas de manejo de material que representan porciones de un sistema más grande. Los modelos individuales pueden ser también programas que evalúen la ejecución del sistema, tales como un modelo de planificación de capacidad, donde se compare la ejecución de configuraciones de producción alternativos representados por modelos independientes. Pueden ser creados diferentes diseños del sistema mediante la modificación de los parámetros de los modelos participantes o cambiando los elementos del modelo que representan a los componentes del sistema de fabricación.

La identificación de los elementos que integran un Sistema de Fabricación y los diferentes niveles de abstracción donde se ubica cada uno de ellos permite 
describir sus comportamientos basándonos en sus funciones, relaciones e interacciones y traducirlos en un modelo funcional. Debido a que el modelo incluye elementos de un determinado nivel de abstracción del Sistema de Fabricación, el modelo permitirá la simulación del comportamiento del Sistema de Fabricación en dicho nivel. Por tanto, aplicando la meta-jerarquía de fabricación del nivel más alto al más bajo que describen McLean y Leong en la Sección 4.2 es posible identificar los elementos que deben ser considerados en la definición de un modelo para la representación de un Sistema de Fabricación.

Los modelos de simulación generalmente se utilizan para diseñar plantas de fabricación individuales o células. Las entidades, atributos y relaciones de los elementos de un Sistema de Fabricación forman un modelo de representación en un cierto nivel de instanciación. Sin embargo, a fin de definir el nivel de conceptualización del Sistema de Fabricación se requiere el diseño de un metamodelo que permita definir instancias de modelos que representen Sistemas de Fabricación más extensos y complejos que contemple un rango amplio de variantes. De este modo, el metamodelo aporta una mayor flexibilidad en el diseño de Sistemas de Fabricación.

De acuerdo con Yu y Popplewell (Yu \& Popplewell 1994) la simulación frecuentemente se utiliza en el diseño de sistemas de fabricación. Mientras tanto las técnicas de metamodelado han madurado de tal forma que pueden proporcionar una extensión más versátil para la simulación, y una visión más detallada en cuanto al modelado de sistemas. Más aún, la relativa simplicidad computacional de los metamodelos ofrece la posibilidad de modelar sistemas más extensos.

\subsection{Herramientas de Simulación para Sistemas de Fabricación}

Debido que no existen criterios estándares para la simulación de fabricación, existen diversos puntos de vista referentes a cómo un sistema de fabricación debe descomponerse para simularlo apropiadamente. Las herramientas de simulación simulan diferentes niveles de abstracción de los sistemas. 


\subsubsection{Clasificación de Herramientas de Simulación}

Algunas herramientas simulan procesos de negocios, otras simulan elementos físicos, mientras que otras combinan ambos tipos de simulación. Por ejemplo, Banks en (Banks et al. 2001) emplea un nivel de abstracción elevado para clasificar los programas de simulación en 5 categorías: Programas de propósito general, Programas orientados a Fabricación , Programas de Ingeniería de Procesos de Negocio, Programas de Planificación basados en simulación, Animadores y Programas de Soporte de Simulación. El enfoque propuesto por Klingstam (Klingstam \& Gullander 1999) clasifica la simulación de la fabricación en dos grupos que permiten agrupar indicando su funcionalidad como: Simulación de Eventos Discretos (DES) y Simulación Geométrica (GS).

Basándonos en nuestro principal objetivo que se refiere a la Simulación de áreas referentes a la Fabricación y a la amplia variedad de programas disponibles, solo mencionaremos herramientas utilizadas en la Simulación de Fabricación enfocadas a la Tecnología de Ingeniería de Producción Asistida por Computadora (CAPE).

\section{Simulación de Eventos Discretos para Sistemas de Fabricación.}

La Simulación de Eventos Discretos simula el comportamiento de las entidades cuando aparece un evento en un momento determinado (Hoeger \& Jhones 1996). Las entidades son los componentes del sistema que se describen como variables. El instante en el que las variables cambian es denominado un evento. Cuando se presentan los eventos estos guían la simulación. Así mismo, el tiempo en un sistema de eventos discretos no avanza linealmente pero si lo hace a intervalos irregulares (Geuder 1995). Las principales áreas de la simulación de eventos discretos son: simulación de flujo de materiales, análisis de sistemas de fabricación, y simulación de flujo de información.

Entre los ejemplos de este tipo de Herramientas de Simulación de Eventos Discretos (DES) (Ruiz et al. 2006a) actualmente podemos encontrar los programas listados en la Tabla 4.3. 


\begin{tabular}{|l|l|}
\hline Programas DES & Compañia \\
\hline Arena & Systems Modelling \\
AutoMod & Brooks Automation (AutoSimulations) \\
DE3 & BYG Systems \\
Dosimis3 & Simulations Dientleistungs Zentrum GmbH \\
eM-Plant & UGS \\
Enterprise Dynamics(Taylor ED) & Incontrol Enterprise Dynamics \\
Extend & Imagine That \\
Factor/Aim & Symix(Pritsker) \\
FlexSim(TaylorII) & Flexsim Software Products, Inc. \\
GPSS/H & Wolverine Software \\
G2 Rethink & Gensym \\
Micro Saint & Micro Analysis and Design \\
MMS & nHance Technologies \\
ProModel & Prouction Modelling of Utah \\
Quest & Delmia Corp.(Deneb Robotics) \\
Schedula & Codework \\
Ses/Workbench & HyPerformix \\
ShowFlow & Incontrol Simulation Software B.V. \\
SIMAS II & CIMPACT Sarl \\
SimBax & AICOS Technologies AG \\
SimFlex & Flextronics \\
em-Plant(Simple++) & UGS \\
Simprocess & CACI Products Company \\
SIMUL8 & SIMUL8 Corporation Products \\
SLX & Wolverine Software \\
Spar & Clockwork Solutions \\
Witness & Lanner Group \\
\hline
\end{tabular}

Tabla 4.3: Programas de Simulación de Eventos Discretos

\section{Descomposición de Simulación de Eventos Discretos.}

La descomposición en simulación resulta un elemento clave en el momento de la implementación de un Sistema de Simulación de Eventos Discretos distribuidos. De acuerdo con Rigther (Righter \& Walrand 1989), existen seis enfoques para la descomposición de simulación: Compiladores simultáneos, Experimentos distribuidos, Funciones de lenguaje distribuidos, Eventos Distribuidos y Componentes de Modelo distribuidos, y Enfoque Combinado (p.ej. Funciones distribuidas con eventos distribuidos y elementos distribuidos). 
a. Compiladores simultáneos. Este método utiliza un compilador simultáneo para localizar secuencias de código en una simulación secuencial que puede ser procesada simultáneamente en procesadores separados o en un multiprocesador.

b. Experimentos distribuidos. En este enfoque se ejecutan replicaciones independientes de la misma simulación en $\mathrm{N}$ procesadores y promedia los resultados. Para ello no se requiere de coordinación entre procesadores a excepción del momento en que se realiza el promedio.

c. Funciones de lenguaje distribuidos. Este enfoque trata de la asignación de tareas de soporte de simulación (p.ej. generación de variables aleatorias, procesamiento de un conjunto de eventos, etc.) a varios procesadores individuales.

d. Eventos Distribuidos. Este enfoque se basa en la programación de eventos asociados a un evento evento global incluso cuando la lista de eventos está distribuida. Los protocolos que mantienen la consistencia son necesarios debido a que hoy en día los eventos procesados pueden afectar el siguiente evento de la lista. Por lo tanto, las dependencias de los eventos deben conocerse antes de la programación. Este enfoque es apropiado cuando se tiene un reducido número de procesos o cuando los componentes del sistema requieren un monto considerable de información global.

e. Componentes de Modelo distribuidos. La descomposición de un modelo de simulación en componentes ligeramente vinculados hace posible que a los componentes se les asigne un proceso, donde varios procesos pueden ser ejecutados en el mismo procesador. Este enfoque explota la inherente simultaneidad en el modelo pero requiere de una sincronización cuidadosa.

Con respecto a la sincronización de los procesos, en este enfoque generalmente se controla mediante el pase de mensajes. Las primitivas de sincronización necesarias para la simulación basada en mensajes son (Bagrodia, Chandy \& Misra 1987):

- Crear y terminar procesos,

- Enviar mensajes a procesos,

- Esperar mensajes y/o tiempo de simulación para avanzar. 
En una topología estática, tal como un sistema de colas, una buena descomposición se refleja en la asignación de un proceso a cada estación y tener el movimiento de los clientes representado por el pase de mensajes.

En una topología dinámica en cambio, los procesos pueden ser utilizados para representar los componentes que están interactuando. En esta topología, los componentes pueden moverse en cualquier dirección y pueden interactuar con el resto de los componentes. En una variante más eficiente de este método el espacio físico es dividido entre regiones o sectores a los que se les asigna un proceso. En este caso, los mensajes se utilizan para describir las interacciones entre los componentes en diferentes sectores o el movimiento de componentes de un sector a otro (Pooch \& Wall 1993).

Por lo tanto, este procedimiento es especialmente apropiado para sistemas que requieren un mínimo de la información global y control, de tal manera que intenta minimizar la necesidad de sincronización. Tales sistemas a menudo funcionan en un modo altamente asíncrono (Righter \& Walrand 1989).

f. Enfoque Combinado. Este enfoque se refiere a la combinación de los enfoques anteriores.

\section{Simulación Geométrica para Sistemas de Fabricación.}

La Simulación Geométrica (Ruiz et al. 2006a) se lleva a cabo con anterioridad y en algunos casos se puede decir que ejecuta una simulación continua. Los sistemas de simulación geométrica simulan la geometría de un elemento o un sistema de fabricación completo, usualmente en tres dimensiones. El ingeniero de producción construye un sistema de fabricación virtual el cual incluye tanto la maquinaria y equipo como el control lógico para la simulación de las funciones de fabricación . Este tipo de simulación también es denominada "fabricación virtual". Sus áreas de aplicación son: visualización en 3D, simulación de sistemas de fabricación geométrica, programación fuera de línea robots y detección de colisiones. La mayoría de los simuladores geométricos han sido desarrollados explícitamente para la simulación y programación fuera de línea de robots.

Algunos ejemplos de Herramientas de Simulación Geométrica se listan en la Tabla 4.4. 


\begin{tabular}{|l|l|}
\hline Prgramas GS & Compañía \\
\hline AMESim & Imagine \\
CimStation Robotics(CSR) & Applied Computing \& Engineering LTD \\
CMMSimulator & Applied Computing \& Engineering LTD \\
Delmia & Delmia Corp. \\
FoCs & alphaWorks-IBM \\
GRASP & BYG Systems \\
HCADWin & NeM \\
IGrip & Delmia(Deneb Robotics) \\
ITI-SIM & ITI GmbH \\
ProDyn & Ingenious Inc. \\
em-WorkPlace PC(RobCad) & UGS \\
SIMPLORER & Ansoft Corporation \\
Softmachines & Applied Computing \& Engineering LTD \\
Universal Mechanism 2.0 & Universal Mechanism Software Lab \\
\hline
\end{tabular}

Tabla 4.4: Programas de Simulación Geométrica

Debido a que existen programas de simulación que proporcionan tanto la oportunidad de realizar la Simulación de Eventos Discretos como la Simulación Geométrica (de eventos continuos) en la Tabla 4.5 se presenta un grupo adicional de este tipo de herramientas a las cuales hemos denominado de Simulación Mixta.

\begin{tabular}{|l|l|}
\hline Software Simulation & Company \\
\hline AweSim & Symix Systems(Pritsker) \\
SIMSCRIPT II.5 & CACI Products Company \\
3DCreate & Visual Components(AMTRI) \\
\hline
\end{tabular}

Tabla 4.5: Programas de Simulación Mixta

Un segundo enfoque situado en un nivel de abstracción similar es el propuesto por Law en (Law \& McComas 1997) quien clasifica a las herramientas de simulación en dos grupos: Lenguajes y Simuladores.

- Lenguajes. Un lenguaje de simulación es un programa de propósito general por naturaleza (refiriéndose a las aplicaciones que permite desarrollar) y donde el desarrollo del modelo se realiza mediante "programación". Su principal desventaja es que requiere un experto en programación. Un lenguaje de simulación orientado a la fabricación es aquel donde el modelo construido está orientado específicamente a la fabricación y el manejo de materiales. 
- Simuladores. El Simulador orientado a la fabricación es aquel que está diseñado para el modelado de sistemas de fabricación específicos. Este tipo de programas posee dos características principales: su orientación está dirigida a la fabricación y se requiere una programación reducida o nula para construir el modelo (en relación con los lenguajes de simulación).

\subsubsection{Características de las Herramientas de Simulación}

Debido a la existencia de una amplia variedad de criterios aplicados para la evaluación de programas de simulación, es importante destacar las características más representativas de este tipo de herramientas.

Algunos investigadores tal como Banks (Banks 1991, Banks, Aviles, McLaughlin \& Yuan 1991, Banks 1996, Banks et al. 2001), Breedam (Breedam, Raes \& Velde 1990), Davis (Davis \& Williams 1994), Holder (Holder 1990), Kochlar (Kochhar 1989), Law (Law \& Haider 1989, Law \& Kelton 1991, A.M.Law \& McGomas 1992), Mackulak (Mackulak, Savory \& Cochran 1994), Hlupic (Hlupic 1997, Hlupic \& Paul 1995a, Hlupic \& Paul 1995b), Kuljis (Kuljis 1996), Nikoukaran (Nikoukaran, Hulpic \& Paul 1999), y Baldwin et al. (Baldwin, Eldabi, Hlupic \& Irani 2000) han propuesto varios criterios para la evaluación de programas de simulación desde distintos puntos de vista y niveles de abstracción.

Por otra parte Nikoukaran (Nikoukaran \& Paul 1999) ha reunido varios criterios de evaluación de programas de simulación con el objetivo de identificar sus características mas relevantes. Dichas características incluyen: Clase de Programa, Tipo de Simulación, Enfoque, Enfoque de modelado, Clases de Computadoras, Compatibilidad, Sistema Operativo, Despliegue de Animación, Librería de Iconos, Modo de ejecución de Animación, Distribución de la Pantalla, Tamaño del Modelo, Modos de introducción de datos, Desarrollo del Modelo, Reportes Personalizados, Reportes Estándar, Creación de archivos, Mantenimiento de la Base de Datos, Atributos, Variables globales, Asignación de ruta (enrutamiento) condicionado, Velocidad de Ejecución del Modelo, Replicas independientes, Capacidad de restauración, Capacidad de examinar la ejecución posteriormente, Periodo de preparación, Análisis de datos de entrada, Distribuciones estándar, Intervalos de confianza, Gráficas de negocios, Presentación dinámica, Generadores de números aleatorios, Análisis de resultados, Depurador interactivo, Revisión de errores en línea, Ayuda en línea, Programación, Interface, Soporte a Cliente, Discos de demostración, Documentación, Pedigree, Costo, y Características de fabricación. 
De tal forma que utilizando estos criterios se evaluaron las herramientas citadas en el apartado anterior. De esta evaluación se puede concluir que la mayoría de las herramientas actuales incluyen, en mayor o menor medida, las características necesarias para ofrecer las funcionalidades de un Simulador. Sin embargo, tales herramientas tienen limitaciones para poder ofrecer la flexibilidad y escalabilidad necesaria para adaptarse a los cambios continuos asociados a las necesidades de la nueva era de la fabricación.

\subsubsection{Elementos clave de las Herramientas de Simulación ba- sada en Agentes para la Fabricación}

Desde la perspectiva de los diseñadores de sistemas de control basados en agentes, la simulación parece ser una herramienta indispensable para sintonizar y validar los algoritmos controlados por agentes antes de ser aplicados a las instalaciones actuales (Panurak \& VanderBok 1997). De máxima importancia resulta el estudio del comportamiento emergente, particularmente la detección y análisis de los patrones de comportamiento inesperados y sospechosos (Vrba \& Marík 2005). Es aparente, que la realización de pruebas en el entorno real de fabricación es simplemente inconcebible o podría ser al menos extremadamente costoso y, quizá peligroso. La solución más directa es simular los procesos/instalaciones de fabricación. Así, para los propósitos de la simulación basada en agentes, es necesario contar con los siguientes componentes:

- Modelo del Sistema de control basado en agentes que proporcione la representación de partes/componentes particulares de equipo de fabricación y su control.

- Modelo de las instalaciones de fabricación para emular físicamente el equipo de fabricación

- Interface de ejecución.

- Visualización para la visualización gráfica del comportamiento global del sistema. 


\subsubsection{Entornos de Simulación de Fabricación basadas en Agen- tes}

Los Sistemas Multiagente han demostrado su utilidad en el modelado de Sistemas de Fabricación, entre algunos de los ejemplos más relevantes podemos mencionar a CIIMPLEX (Peng et al. 1998), HOLOS Architecture (Rabelo \& Camarinha-Matos 1994), MethaMorph I (Maturana \& Norrie 1996), MethaMorph II (Shen et al. 1998), DEDEMAS (Tonshoff et al. 2000), CORTES (Sadeh \& Fox 1989), IFCF (Lin \& Solberg 1992), YAMS (Parunak 1998), MASCADA (Valckenaers et al. 1999), Production System(Bussmann \& Schild 2001), PABADIS (Sauter \& Massotte 2001), Chilled Water System Automation (Maturana \& et. al. 2002), FactoryBroker (Colombo et al. 2006), Shop Floor Engineering (Barata \& Camarinha-Matos 2003) y Control distribuido de sistemas de fabricación flexibles (Araúzo 2007).

Estas herramientas se basan en el aprovechamiento de las características de los Sistemas Multiagente para la definición del Modelo de Simulación de los Sistemas de Fabricación. Sin embargo, estos Simuladores no se han enfocado en abarcar todas las funcionalidades y características propias de un Simulador de Modelos que pueden ser explotadas mediante la aplicación de la Teoría de los Sistemas Multiagente.

\subsection{Conclusiones}

Como resultado de esta revisión, es posible observar que las características de los agentes en un sistema Multiagente resultan clave en la resolución de la problemática que surge al intentar simular comportamientos complejos presentes en una planta de fabricación actual.

Por un lado, se observa que los elementos de un Sistema de Fabricación adoptan comportamientos complejos que se adaptan conforme las necesidades de su entorno. Por otro lado, se observa que los Sistemas Multiagente ofrecen la posibilidad de representar un entorno complejo y dinámico, el cual cambia continuamente en respuesta a las acciones de sus elementos internos y externos.

Al mismo tiempo se observa que los Entornos de Simulación de Sistemas de Fabricación Comerciales aunque permiten la simulación de modelos de Sistemas de Fabricación complejos, tienen una limitada flexibilidad para la adaptación de modelos acorde a las necesidades de la nueva era de la fabricación. Este tipo de entornos, al estar basados en la simulación de Sistemas de Fabricación convencionales, no permiten el uso de técnicas de negociación, cooperación y 
optimización asociadas a la Teoría de Sistemas Multiagente. Asimismo, tanto sus interfaces como su uso están orientados a los Usuarios finales expertos en el dominio de la fabricación.

Por su parte, en los Entornos de Simulación de Sistemas Multiagente se observa que están orientados a la simulación y análisis llevados a cabo por Usuarios pertenecientes al ámbito académico-científico. En comparación con los Entornos Comerciales, este tipo de entornos facilita su adaptación para el diseño de modelos complejos de organizaciones, puesto que permite el uso de técnicas asociadas a la Teoría de Sistemas Multiagente. Estos entornos tienen ciertas limitaciones con respecto al tipo de agentes que se integran en un mismo modelo y su representación gráfica no es fácilmente comprensible para los Usuarios finales. Asimismo, el diseño del modelo, en algunos casos, tiene que ser realizado por un programador experto ya que los entornos no están preparados para Usuarios principiantes en Tecnología de Sistemas Multiagente que desean aplicarlos a otras áreas. En este tipo de entornos el Usuario aplica un nivel de abstracción bajo para el diseño y análisis de los modelos.

Finalmente, como resultado de las revisiones presentadas en la primera parte de la memoria, se han identificado tres premisas: a) las oportunidades de mejora mediante la aplicación de la Teoría de los Sistemas Multiagente detectadas como resultado de la evaluación de las herramientas de simulación actuales, b) el éxito de la aplicación de la Teoría de los Sistemas Multiagente para resolver problemas asociados a Sistemas de Fabricación y c) la posibilidad de mejora de la Simulación de Sistemas de Fabricación mediante la aplicación de la Teoría de los Sistemas Multiagente, para ofrecer la flexibilidad y adaptabilidad necesarias con el fin de integrar los requisitos de la nueva fabricación.

Basándonos en las tres premisas previas, proponemos la integración de las características de los Entornos de Simulación de Sistemas Multiagente y los de Simulación de Sistemas de Fabricación donde se incluyan sus ventajas, en un entorno que pueda ser utilizado por Usuarios finales no expertos en Sistemas Multiagente. El entorno estará soportado por la Teoría de Sistemas Multiagente y será aplicado para la Simulación de Modelos de Sistemas de Fabricación Inteligentes.

Cabe enfatizar la idea de Shen et al. (2001) quienes consideran que el trabajo futuro de Investigación y Desarrollo (R\&D) debe centrarse en la integración de Soluciones basadas en Sistemas Multiagente con los Sistemas utilizados en los Sistemas de Fabricación (ERP -Enterprise Resource Planning- y MRP -Materials Resource Planning-). Por tanto, nuestra propuesta considera como un primer paso, el acercamiento de las técnicas basadas en agentes para la Simulación de 
Sistemas de Fabricación. Tales mejoras van dirigidas a la integración de racionalidad o inteligencia de los agentes software (mecanismos de negociación y coordinación más eficientes y efectivos) durante los procesos de Simulación del comportamiento, tanto del Entorno de Simulación como del Sistema de Fabricación que se simula. En tal acercamiento nuestro objetivo es la definición de un entorno que pueda ser gestionado por Usuarios que son expertos en el dominio de la fabricación pero que aún desconociendo la Tecnología de los Sistemas Multiagente puedan aprovechar los beneficios que ésta ofrece.

De este modo, el objetivo principal de esta tesis es contribuir en la integración de las características de la Simulación de Sistemas de Fabricación y la Simulación soportada por Agentes en un Simulador de Sistemas de Fabricación Inteligentes. Para ello, se contempla la Definición de una Arquitectura de Simulación soportada por agentes que permita el diseño y simulación de Modelos de Sistemas de Fabricación Inteligentes. La arquitectura incluirá mejoras en los procesos de Simulación de Sistemas de Fabricación orientadas a cubrir los requisitos de la nueva fabricación. Asimismo, para el modelado se contemplará la definición de un Metamodelo de Sistemas de Fabricación soportado por agentes que sea flexible. El metamodelo permitirá el diseño de modelos simples, y al mismo tiempo, gracias a su flexibilidad será posible definir los comportamientos complejos de sus elementos (operarios, máquinas, herramientas, staff).

Nuestra propuesta considera el uso de la Tecnología de los Sistemas Multiagente puesto para definir entidades inteligentes que van adquiriendo conocimiento que se utiliza en procesos de toma de decisiones. Con ello, se pretende mejorar la funcionalidad de un entorno de simulación y aportar la flexibilidad necesaria para la adaptación de modelos como respuesta a los cambios del entorno (propios de un Sistema de Fabricación). Además se contempla la integración de los requisitos de la nueva era de la fabricación factor clave en los Sistemas de Fabricación actuales. Asimismo, en nuestra propuesta, gracias a la Simulación Inteligente soportado por agentes, será posible representar al mundo real incorporando el control del tiempo y sus cambios con mayor detalle, flexibilidad y autonomía. 


\section{Parte II}

\section{Marco de la Propuesta.}





\section{Introducción}

En la segunda parte de esta memoria se presenta el Marco de la Propuesta, que incluye la Arquitectura del Entorno de Simulación soportada por Agentes y el Metamodelo que permite la definición de Sistemas de Fabricación inteligentes con un amplio rango de variantes. Las principales características que se consideran para esta parte incluyen:

- Explotación de Flexibilidad.

- Distribución de Control.

- Emulación de comportamientos complejos.

- Gestión de elementos necesarios e innecesarios acorde al estado actual del sistema.

- Características de SMA (proactividad, reactividad, autonomía, flexibilidad).

- Integración de Modelos de Sistemas de Fabricación Inteligentes.

Tomando en consideración las etapas incluidas en el Diagrama de Estudio de una Simulación (Figura 4.5), la propuesta se centra en actividades específicas que incluyen: a) asistencia durante la representación y programación del modelo, b) aportar flexibilidad para plantear las hipótesis y el diseño de experimentos, c) la simulación del modelo, d) proporcionar información para el análisis de resultados, y e) la validación de los resultados contra la hipótesis. 


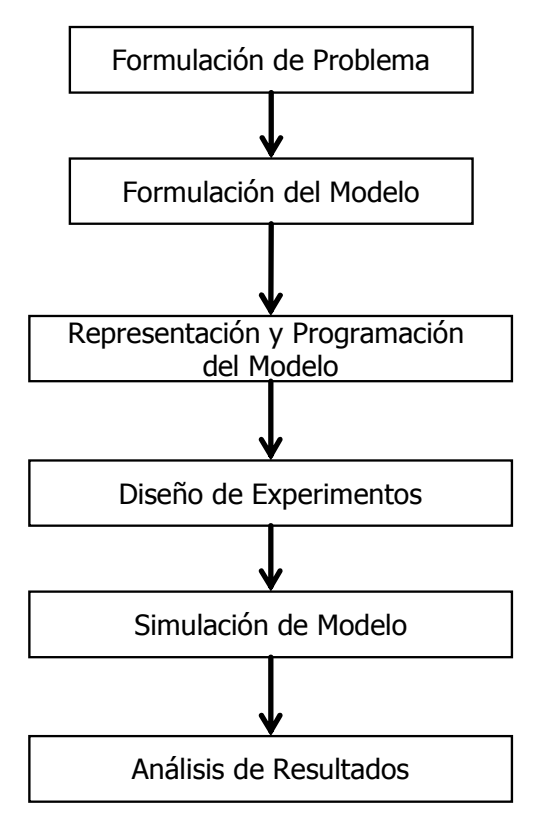

Figura 4.5: Diagrama de Estudio de una Simulación.

De este modo, basándonos tanto en el marco teórico de la Simulación (Capítulo 2), la Simulación y los Sistemas Multiagente (Capítulo 3) y la Simulación de Sistemas de Fabricación soportada por Agentes (Capítulo 4), en la propuesta para la Simulación de un Sistema de Fabricación Inteligente se contempla la definición de dos elementos básicos (Figura 4.6):

a. Definición del Metamodelo de Sistema de Fabricación (MMSF). La definición de un Metamodelo para el diseño de Modelos de Sistemas de Fabricación que ofrezca la flexibilidad necesaria para el diseño de experimentos mediante la Creación de Modelos acorde a las necesidades del Usuario.

b. Definición de Arquitectura para Entorno de Simulación (ESSA). Dicha arquitectura permite la definición de un Entorno que facilite la Creación de un Modelo de un Sistema de Fabricación Inteligente y su Simulación siendo soportada por Agentes. De esta forma se diseña un Sistema Multiagente que de soporte a la funcionalidad de un Entorno de simulación. 


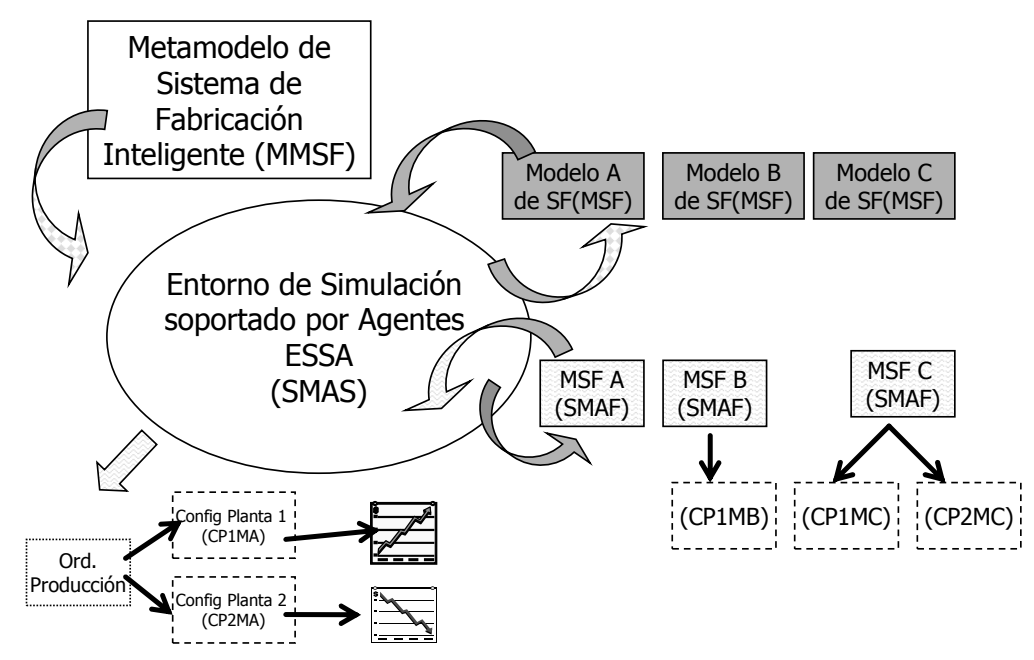

Figura 4.6: Elementos de la Propuesta.

Tanto el Metamodelo (MMSF) como la Arquitectura del Entorno de Simulación (ESSA), dan soporte a la ejecución de actividades clave para la Simulación de Modelos (Figura 4.6). Tales actividades incluyen:

1. Creación de un Modelo de Sistema de Fabricación Inteligente. En esta actividad, partiendo del diseño del Sistema de Fabricación definido por el Usuario, se crea el Modelo de Sistema de Fabricación (MSF). El MSF internamente se traduce como un Modelo de Agentes en el cual cada uno de los agentes integra el comportamiento de cada uno de los elementos definidos en el Sistema de Fabricación original. Para ello se hace uso del Metamodelo (MMSF) definido previamente. Dicho Metamodelo sirve como base para la definición del Modelo de Sistema de Fabricación Inteligente (MSF). De este modo, el comportamiento de cada uno de los agentes que integran el modelo de agentes se ve afectado por los atributos asociados a los elementos del sistema original durante la Creación del Modelo.

2. Creación de Sistema Multiagente de Fabricación. Partiendo del Modelo de Sistema de Agentes (MSF) que representa al Modelo de Sistema de Fabricación (MSF), se genera un nuevo Sistema Multiagente que lleva a cabo las actividades de un Sistema Multiagente de Fabricación Inteligente (SMAF). El Sistema Multiagente de Fabricación (SMAF) por tanto establece contacto con el Sistema Multiagente del Entorno de Simulación (ESSA) para que éste simule y muestre gráficamente al Usuario los resultados de sus interacciones internas. 
3. Gestión de Órdenes de Producción. Asimismo, durante la Simulación del Sistema Multiagente de Fabricación (SMAF), como proceso clave, se presenta la gestión de Órdenes de Producción. Las Órdenes de Producción son asociadas a Configuraciones de Planta aplicables en el Modelo que se está simulando en el Entorno de Simulación (ESSA). En esta actividad se integra la posibilidad de aplicar técnicas de negociación basadas en el paradigma de agentes para la asignación de tareas.

En los Capítulos 5 y 7 se presenta en detalle la arquitectura que da soporte al entorno de simulación y en el Capítulo 6 se presenta el metamodelo para Sistemas de Fabricación Inteligente, así como sus relaciones.

Asimismo, para la validación tanto de la arquitectura como del metamodelo propuesto surge la necesidad de desarrollar un prototipo. Por tanto, la definición del mismo se presenta en el Capítulo 8. En el Capítulo 11 se presentan los resultados de la Evaluación del prototipo.

Por otra parte, se requiere la evaluación de los resultados, la definición de métricas asociadas a la evaluación de modelos, configuraciones y datos de producción. Tales métricas así como los resultados obtenidos se presentan en los Capítulos 9 y 10.

Finalmente, en el Capítulo 12 se incluyen las conclusiones, las principales aportaciones de este trabajo, las líneas de investigación de trabajo futuro, así como las publicaciones científicas donde se han presentado las diferentes etapas de esta propuesta. 


\section{Capítulo 5}

\section{Modelo de la Arquitectura del Entorno de Simulación para Sistemas de Fabricación}

La definición de la Arquitectura del Entorno de Simulación considera el marco teórico referente a la Simulación (Capítulo 2), la aplicación de los sistemas Multiagente para la Simulación (Capítulo 3) y la Simulación de Sistemas de Fabricación Inteligente (Capítulo 4).

De este modo, siguiendo el Diagrama de Estudio de Simulación (Figura 5.1), la definición de la Arquitectura del Entorno de Simulación enfoca su atención en actividades específicas agrupándolas en dos fases globales. La arquitectura del entorno propuesta en la Fase I, ofrece soporte a las actividades de representación y programación de Modelos así como el diseño de experimentos, y en la Fase II, se contempla la Simulación del Modelo y el Análisis de Resultados. 


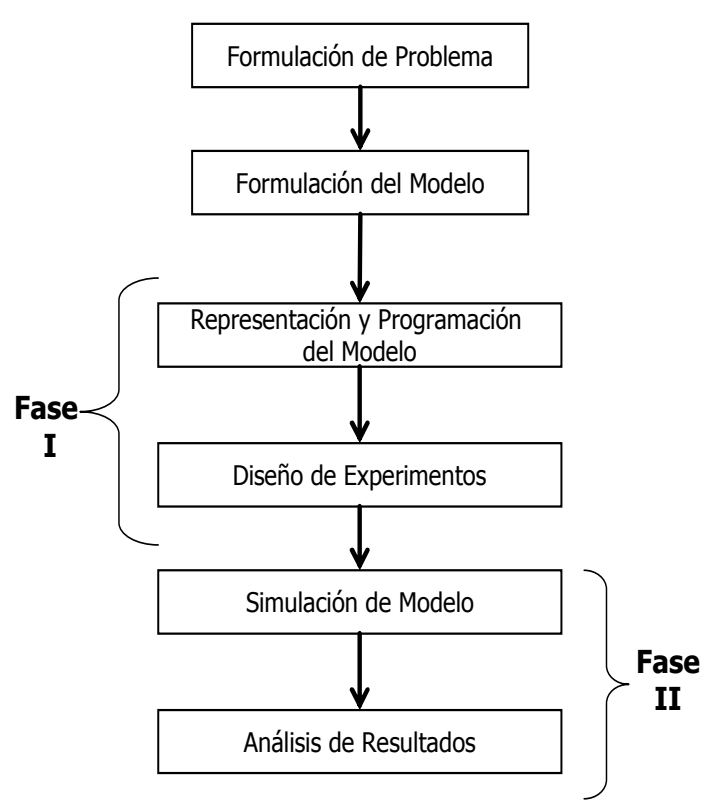

Figura 5.1: Actividades a las que dará soporte la Arquitectura del Entorno de Simulación.

La Arquitectura del Entorno de Simulación permite especificar cómo se descomponen los agentes en un conjunto de módulos que interactúan entre sí para lograr la funcionalidad requerida. Como se indicó en el Capítulo 3, la definición del entorno en la simulación multiagente comprende dos elementos: el entorno simulado y la simulación del entorno. Por tanto, en este capítulo presentamos el Modelo Teórico que describe el sistema multiagente que de soporte a la simulación del entorno con respecto a la Fase I.

En este capítulo se presentan las bases de la Arquitectura del Entorno de Simulación que integra las ventajas de la aplicación de los tecnología de los Sistemas Multiagentes para la Simulación de Sistemas de Fabricación. Para ello se toman en consideración: a) los requisitos actuales de la nueva era de los Sistemas de Fabricación y b) las oportunidades de mejora (identificadas en el Capítulo 4) que aporta la tecnología de Sistemas Multiagente con respecto a las actuales herramientas de Simulación para Sistemas de Fabricación. 


\subsection{Identificación y Definición de Agentes para crear y simular la Planta de Fabricación}

La consideración de las posibilidades de mejora que aporta la utilización de Sistemas Multiagente mencionadas en la Sección 4.3.1 ha dado paso al planteamiento de una alternativa de desarrollo de un Simulador basado en Agentes el cual permita resolver las debilidades de las herramientas actuales. Las principales características de la propuesta se encuentran relacionadas en la explotación de la flexibilidad, distribución de control, emulación de comportamientos complejos, y la gestión de elementos necesarios e innecesarios de acuerdo al estado actual del sistema, las cuales son características propias de un Sistema Multiagente.

El principal objetivo de este trabajo es plantear una arquitectura para un entorno de simulación que sirva como una alternativa que permita la gestión de los requisitos de la nueva manufactura de forma sencilla para el usuario. Partiendo de las etapas que comprenden un Estudio de Simulación (presentadas en el Capítulo 2), en este trabajo tales etapas se agruparon en dos fases principales: el Modelado del Sistema y la Simulación del Modelo (Fig. 2.1). En la Fase I Modelado del Sistema se agrupan las etapas de Conceptualización del Modelo, Recolección de Datos, aspectos relacionados con la Traducción del Modelo en formato informatizado, la verificación, y validación del Modelo. En la Fase II Simulación del Modelo, también se integran parte de los aspectos relacionados con la Traducción del Modelo en formato informatizado, la verificación, validación, documentación y reportes, y el análisis de resultados.

En la propuesta se contemplan dichas actividades considerando un enfoque diferente con respecto a la distribución y control de las mismas. Esto es, se propone la construcción de una Plataforma de Agentes que proporcione los servicios necesarios para la definición tanto del Modelo del Sistema de Fabricación como de la Simulación del Modelo. El conjunto de agentes aporta funcionalidades específicas que tienen por objeto mejorar el funcionamiento de los esquemas que aplican los simuladores actuales mediante la explotación de las habilidades que brindan los agentes (distribución de control, flexibilidad, proactividad, negociación).

La Arquitectura para el Entorno de Simulación se representa mediante un Sistema Multiagente (SMA) que interactúa con un Sistema de Fabricación el cual también está representado por otro Sistema Multiagente. Ambos Sistemas 
Multiagente intercambian información con el objetivo de que el entorno de Simulación represente gráficamente el comportamiento del Sistema de Fabricación simulado.

\subsubsection{Características contempladas en la propuesta.}

Antes de proponer la definición del esquema básico del Entorno de Simulación es necesario identificar las características que se contemplan en la Arquitectura. Para tal efecto, basándose en el listado de características mencionadas en la Sección 4.7.2, se han seleccionado aquellas características que se encuentran relacionadas directamente con el funcionamiento del Entorno de simulación. Por el momento, no se consideran las características complementarias pero no de menor importancia, tales como proporcionar el soporte al usuario o contar con un disco de demostración. Las características seleccionadas incluyen: 1) Mantenimiento de la Base de Datos, 2) Despliegue de Animación, 3) Modo de ejecución de Animación, 4) Modos de introducción de datos, 5) Creación de archivos, 6) Interface, 7) Análisis de datos de entrada, 8) Capacidad de examinar la ejecución posteriormente, 9) Revisión de errores en línea, 10) Velocidad de Ejecución del Modelo, 11) Características de fabricación, 12) Librería de Iconos, 13) Desarrollo del Modelo, 14) Réplicas independientes, 15) Reportes Personalizados, 16) Reportes Estándar, 17) Gráficas de negocios, 18) Presentación dinámica, 19) Programación, y 20) Análisis de resultados.

Por tanto, en la definición de la Plataforma de Agentes las características seleccionadas se traducen en actividades que son desempeñadas por un responsable, el cual es un agente. De este modo, cuando un agente desempeña un grupo de actividades se considera que un agente esta desempeñando un rol. Debido a la dinamicidad y flexibilidad propias de los agentes, un agente puede desempeñar varios roles dependiendo de las condiciones de su entorno. Asimismo, un agente puede cambiar su rol activo para actuar adecuadamente ante las acciones de otros agentes.

\subsubsection{Identificación de Roles}

Cabe resaltar que aunque los roles identificados en un principio desempeñan funciones asociadas a cada una de las etapas, se pretende ofrecer la posibilidad de que puedan desempeñar actividades en ambas etapas como consecuencia de los posibles escenarios que aparecen durante la ejecución de la simulación. En 
este apartado se presentan los roles identificados en las dos Fases a fin de observar sus interacciones.

Con respecto a la Fase I El Modelado del Sistema, los roles identificados surgen como resultado de la necesidad de integrar aspectos clave de las funcionalidades asociadas a la Representación del Modelo y que al mismo tiempo permitan el Diseño de los Experimentos.

Para dar solución a la debilidad señalada por Mc Lean y Leong (MCLean \& Leong 2002b) con respecto a la considerable responsabilidad creativa asociada al analista durante las primeras etapas del estudio de simulación (Banks et al. 2001) (Formulación y Representación del Modelo, Figura 5.1), en la propuesta, se incluye la asistencia al diseñador durante la Creación de un Modelo sin necesariamente partir de cero. Para ello, se contemplan dos alternativas: a) la reutilización de modelos creados previamente y b) el uso de plantillas asociadas a un tipo de escenario específico (corte, soldadura, acabados, etc.). Por tanto, se observa la necesidad de contar con un rol o agente que agrupe las funcionalidades necesarias para el proceso de creación del Modelo del Sistema de Fabricación: el Asistente de Modelado.

Por otra parte, con respecto a los datos de entrada, se contempla la funcionalidad de lectura de datos de entrada, su traducción y asociación a los elementos correspondientes. De tal manera que se requiere un rol o agente que proporcione las funcionalidades para la importación de datos de entrada al sistema: el Gestor de Importaciones. Sin embargo, no basta con traducir los datos en información útil para el sistema si estos no se verifican y analizan, es por ello que se requiere un rol o agente que realize dicha tarea: el Verificador.

Siguiendo con las etapas que conlleva el Modelado del Sistema, en cuanto a la Conceptualización del Modelo, resulta necesario aportar la funcionalidad tanto para la asociación de la información analizada y la definición del comportamiento externo de cada uno de los elementos que lo integran. De tal manera, surge la necesidad de un rol o agente que permita la gestión y definición del comportamiento de dichos elementos: el Gestor de Iconos. Asimismo, este rol tiene que ver con la definición de la estructura del sistema. La estructura, de acuerdo con el paradigma de los Sistemas Multiagente, permite la definición de los distintos niveles de especificación (niveles de modelado jerárquico presentados en el Capítulo 4). En cada nivel (centros de trabajo, equipo, dispositivos y procesos), se integra un mayor grado de información que detalla la estructura 
interna del sistema permitiendo la identificación del estado del sistema y los mecanismos de transición.

Como se mencionó en el Capítulo 2, entre las aproximaciones para realizar el estudio de simulación, se encuentra la aproximación orientada a Eventos, en la cual se consideran los diferentes eventos y cómo afectan al sistema. Este enfoque parece el más adecuado puesto que en un Sistema de Fabricación la posibilidad de que se presenten eventos de manera aleatoria es factible. Al mismo tiempo, los eventos desencadenan la ejecución de actividades específicas que alteran el estado del sistema y sus componentes. De tal manera que se requiere un rol o agente que gestione tales eventos: el Generador de Eventos.

Con respecto a la Fase II Simulación del Modelo, los roles identificados surgen como resultado de la necesidad de integrar aspectos clave de las funcionalidades asociadas a la Simulación del Modelo y el Análisis de Resultados.

Para la Simulación del Modelo resulta imprescindible la consideración de tres aspectos fundamentales: la detección de eventos generados, la velocidad a la cual se generan y su respectiva visualización. En este momento se hace necesaria la interacción con el rol o agente detectado anteriormente, el Generador de Eventos. En consecuencia, resulta importante la visualización del comportamiento definido en cada elemento, identificando así la necesidad de un rol o agente que controle las actividades relacionadas con la animación de los elementos que integran el Modelo: el Gestor de Animación.

Un evento que aparece en el tiempo de forma continua o aleatoria da origen a un cambio de estado del sistema el cual tiende a reaccionar ante su aparición. Basándonos en la aseveración planteada por Hamilton (Hamilton \& Tumay 1997) quien indica que el Estado del Sistema generalmente se expresa como una función de tiempo, el concepto de Tiempo de Simulación (periodo de tiempo simulado por el modelo) tiene gran relevancia para la generación de eventos y su animación. Esto es, durante la Simulación, la aceleración del tiempo conlleva a la velocidad a la cual se generan los eventos y su respectiva visualización. De ahí que se identifique la necesidad de un rol o agente que controle la velocidad de generación de los eventos y el correspondiente control del tiempo de simulación: el Controlador de Velocidad. 
La animación gráfica de la simulación del modelo no solo tiene como propósito la visualización del comportamiento sino que tiene que ver con una herramienta útil para la etapa de análisis de los Resultados de la Simulación. Como lo indica Sadowski (Sadowski 1989), la animación es una excelente herramienta para el análisis de cuellos de botella y para la identificación de la causa de un comportamiento extraño en el sistema.

Se debe considerar que la animación gráfica es una herramienta de análisis adicional a otros métodos tales como la presentación documentada del análisis del comportamiento de los elementos del sistema de fabricación ( máquinas, herramientas, operarios, etc.) como resultado de la simulación los cuales proporcionan datos estadísticos de mayor control. Para tal efecto se identifica la necesidad de dos roles: el Analizador de Datos de Salida, y un rol o agente que controle la creación, integración de datos en formatos estadísticos y generación de informes para su posterior análisis: el Generador de Reportes.

Debido a que los resultados de una simulación pueden ser analizados por herramientas especializadas en el análisis estadístico, resulta factible que se integre la funcionalidad de la exportación de los resultados al Gestor de Importaciones y se convierta en un Gestor de Importaciones/Exportaciones.

La identificación de todos estos roles o agentes y sus funciones requieren de un medio que les permita comunicarse con el Usuario final, una interfaz. Las interfaces permiten al usuario comunicarse en un lenguaje común con el entorno de simulación. Por lo tanto, surge la necesidad de una agente que además de proporcionar el medio de comunicación entre el Usuario y los agentes internos, también ofrezca la posibilidad de escalar las posibilidades de comunicación con los mismos: el Gestor de Interfaces.

Partiendo de la identificación inicial de los roles necesarios para abarcar las funcionalidades propias de un Entorno de Simulación, de acuerdo con el paradigma de agentes, cabe señalar la posibilidad de asociar tales roles no solo a un agente sino a más de un agente de acuerdo al escenario en el que se encuentre el sistema.

Finalmente, entre las ventajas de la aplicación del paradigma de Sistemas Multiagente se encuentra la posibilidad de crear y/o eliminar instancias que son necesarias o no de acuerdo al estado actual del sistema con el fin de optimizar recursos físicos del equipo de cómputo donde se ejecuta la simulación. Las funciones de monitorización del estado aparecen como elemento clave durante ambas etapas, de tal manera que surge la necesidad de un rol que coordine las 
actividades de los agentes a un nivel superior: el Sincronizador.

De esta forma, para la primera fase del planteamiento se han identificado los siguientes roles o agentes:

- Gestor de Importaciones/Exportaciones

- Verificador

- Generador de Eventos

- Asistente de Modelado

- Gestor de Iconos

- Gestor de Animación

- Controlador de Velocidad

- Generador de Reportes

- Gestor de Sincronización

- Gestor de Interfaces

Una vez identificados los roles, en la Tabla 5.1 se muestran las características que han sido asociadas a cada uno de ellos identificándolas por la numeración asignada en la Sección 5.1.1.

\begin{tabular}{|l|l|}
\hline Rol & Características \\
\hline Gestor de Importaciones/Exportaciones & 4,5 \\
Verificador & $1,4,7,13$ \\
Generador de Eventos & 4,11 \\
Asistente de Modelado & $11,13,19$ \\
Gestor de Iconos & 2,12 \\
Gestor de Animación & $2,3,8,9,14,18$ \\
Controlador de Velocidad & $2,3,10$ \\
Analizador de Datos de Salida & $1,16,17,20$ \\
Generador de Reportes & $15,16,17$ \\
Gestor de Sincronización & \\
Gestor de Interfaces & 6 \\
\hline
\end{tabular}

Tabla 5.1: Características de los Roles asociados al la funcionalidad del Entorno de Simulación 
Una vez identificados los roles o agentes así como las características que deben poseer, se deben describir estas últimas. Las características se detallan mediante la definición de actividades específicas que llevan a cabo como respuesta a su percepción del estado actual del sistema. En la siguiente sección, se presenta la definición de las funciones de cada uno de los roles.

\subsection{Funciones de Roles}

Las acciones realizadas por cada uno de los roles o agentes planteados en la sección anterior, se traducen en objetivos que deberán lograr cada uno de acuerdo al estado actual del sistema mediante funciones específicas. Las funciones incorporadas en cada rol o agente son:

\section{Gestor de Importaciones/Exportaciones:}

Las funciones del Analizador de Importaciones/Exportaciones son las siguientes: a) Importar información del entorno real a simular relacionada a equipos o bases de datos. b) Traducir la información en una estructura apropiada para el Sistema. c) Traducir la información importada de acuerdo a la información requerida por los agentes y el modelo de los recursos. d) Exportar información a un archivo con una estructura genérica para ser utilizada por otro(s) programa(s).

\section{Verificador:}

Las funciones del Verificador son las siguientes: a) Verificar que el contenido de los datos importados sea adecuado. b) Verificar que los elementos integrados en el modelo sean los apropiados. c) Verificar que las instancias asociadas a los elementos del modelos sean las adecuadas al tipo de elemento.

\section{Generador de Eventos:}

La función del Generador de Eventos es generar información adicional al estado inicial del sistema. La información es utilizada para introducir datos durante la simulación tales como: ruidos (cambios de temperatura, cambio de equipos), agregar nuevos equipos o recursos humanos. El usuario puede interactuar con este rol.

\section{Asistente de Modelado:}

Las funciones del Asistente de Modelado son las siguientes: a) Construir el modelo de simulación utilizando las librerías de iconos. b) Ofrecer esquemas funcionales básicos para definir el modelo. c) Crear agentes mediante 
la definición de su ontología, comportamiento, protocolos de comunicación, etc. d) Editar un modelo predefinido. e) Verificar que el Modelo de Simulación no contenga errores. La especificación del Modelo de Simulación se basa en la arquitectura de referencia Prosa (Brussel et al. 1998) utilizando los conceptos de holones de orden, recursos, productos y staff.

\section{Gestor de Iconos:}

Las funciones del Gestor de Iconos son las siguientes: a) Agregar iconos desde un archivo externo. b) Crear nuevos iconos. c) Eliminar iconos. d) Editar iconos predefinidos. e) Asociar datos a cada uno de los atributos de un icono acorde a: comportamiento, ontología, y protocolos de comunicación. Dichos atributos han sido definidos previamente de acuerdo a su naturaleza (producto, orden, recurso o staff). f) Proporcionar iconos cuando el Asistente de Modelado los solicite.

\section{Gestor de Animación:}

Las funciones del Gestor de Animación son las siguientes: a) Animar los iconos de un Modelo de Sistema de Fabricación. b) Controlar la secuencia de la animación de los iconos acorde los eventos que se simulen. c) Verificar las opciones de animación de iconos.

\section{Gestor de Velocidad:}

Las funciones del Gestor de Velocidad son las siguientes: a) Especificar la velocidad de ejecución de la Simulación del Modelo. b) Acelerar o disminuir la velocidad de la simulación. c) Configurar el reloj global. El Usuario especifica la velocidad de ejecución de la simulación.

\section{Analizador de Datos Salida:}

Las funciones del Analizador de Datos de Salida son las siguientes: a) Analizar los resultados obtenidos después de la simulación de un modelo para que pueda retroalimentar al Sistema Multiagente. b) Proporcionar la información analizada asociada a los tipos de iconos correspondientes.

\section{Generador de Reportes:}

Las funciones del Generador de Reportes son las siguientes: a) Crear plantillas de los reportes de negocio y las gráficas. b) Editar las plantillas de los reportes y las gráficas. c) Generar reportes y gráficas basados en los resultados de la simulación.

\section{Gestor de Interfaces:}

La función del Agente de Gestión de Interfaces es proporcionar módulos 
para crear o modificar las interfaces actuales. Esta característica permite agregar y adaptar a una interfaz nuevos requisitos de una forma sencilla sin afectar la estructura actual del entorno de simulación.

Finalmente, las funciones del Gestor de Sincronización son las siguientes: a) Revisar continuamente el estado actual del sistema, b) Solicitar la creación o eliminación de agentes específicos acorde al estado actual del sistema, c) Supervisar la velocidad de animación del modelo, d) Verificar animación de iconos del Modelo y e) Monitorizar y mantener la configuración de la simulación del Modelo.

\subsection{Integración de Roles en la Simulación}

Una vez definidas las funciones, es posible asociar cada uno de los roles a las Fases de la Simulación donde participa activamente. En la Tabla 5.2 se observa un grupo de roles que participa en ambas fases, así como aquellos roles que sólo intervienen en la Fase II.

\begin{tabular}{|c|c|}
\hline $\begin{array}{c}\text { Fase I } \\
\text { Creación del Modelo de Simulación }\end{array}$ & $\begin{array}{c}\text { Fase II } \\
\text { Simulación del Modelo }\end{array}$ \\
\hline \multicolumn{2}{|c|}{ Asistente de Modelado } \\
\hline \multicolumn{2}{|c|}{ Gestor de Iconos } \\
\hline \multicolumn{2}{|c|}{ Verificador } \\
\hline \multicolumn{2}{|c|}{ Generador de Eventos } \\
\hline \multicolumn{2}{|c|}{ Sincronizador } \\
\hline \multicolumn{2}{|c|}{ Gestor de Importaciones/Exportaciones } \\
\hline \multicolumn{2}{|c|}{ Gestor de Interfaces } \\
\hline & Gestión de Animación \\
\hline & Gestión de Velocidad \\
\hline & Generador de Reportes \\
\hline & Analizador de Datos de Salida \\
\hline
\end{tabular}

Tabla 5.2: Fases de Simulación y los Roles que actúan en cada una

El Gestor de Interfaces tiene relación con ambas fases puesto que su principal función es agregar funcionalidad a las interfaces que se utilizan para llevar a cabo las funciones de los agentes.

El siguiente paso es el planteamiento del esquema de las principales interacciones entre los agentes que integran cada una de las fases, en la Figura 5.2 se muestran los conjuntos de roles y las relaciones entre ellos. 

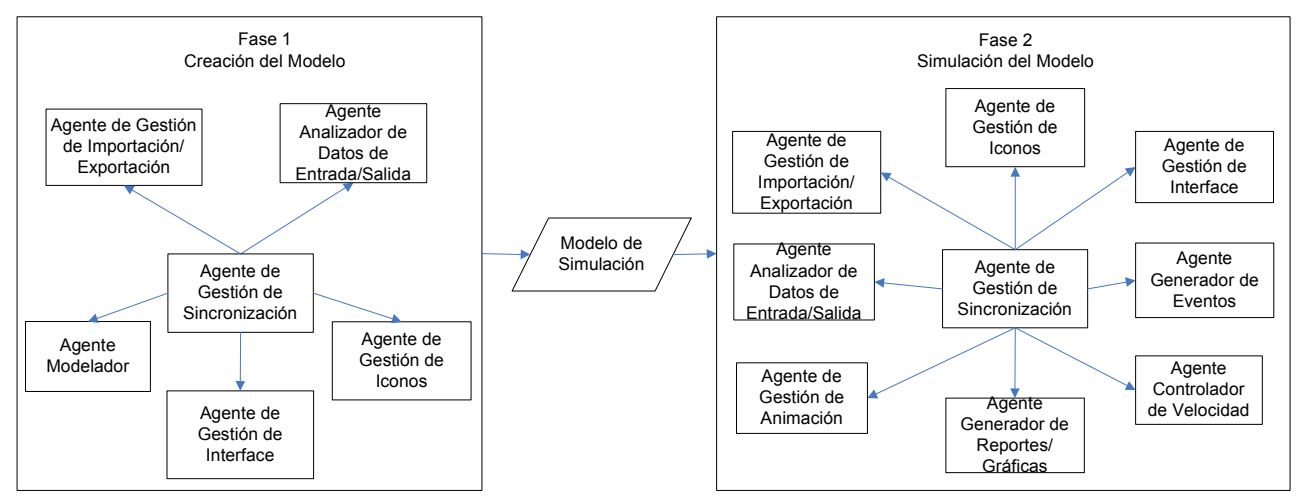

Figura 5.2: Esquema Global de la Secuencia de Simulación y sus Agentes o roles

En la siguiente sección se plantea la secuencia de ejecución inicial que describe las principales interacciones entre ellos.

\subsection{Secuencia de Ejecución}

En esta sección se plantea la Secuencia de Ejecución de cada uno de los conjuntos de agentes incorporados en las fases de la simulación como lo muestra la Figura 5.2. Sin embargo es importante resaltar que la ejecución de los agentes y sus respectivas funciones en cada fase están sujetas a las necesidades del sistema en base a su estado actual y las condiciones del entorno.

En la “Primera Fase”, siguiendo el Diagrama de Estudio de Simulación en cuanto a las actividades de Representación y Programación del Modelo (Figura 5.1), el Gestor de Importación/Exportación proporciona información del mundo real. El Verificador analiza la información importada y el Gestor de Iconos la asocia a los iconos correspondientes. Esta información es incluida en los elementos que integran el sistema para ser utilizados posteriormente por el Asistente de Modelado. El Asistente de Modelado proporciona esquemas de procesos funcionales básicos para incluir elementos que están agrupados en librerías de iconos que son administradas por el Gestor de Iconos. El Modelo de Simulación puede ser editado por el Usuario; esta funcionalidad le permite al Usuario programar el comportamiento que desee que lleve a cabo cada uno de los elementos para así definir los detalles de los atributos del Modelo de Simulación. El Gestor de Iconos permite al Usuario agregar iconos en cualquier momento. Una vez que el Modelo esta terminado el Verificador revisa el modelo en busca de posibles 
errores antes de ponerlo a disposición de su uso para la Fase II.

Una vez que se tiene un "Modelo de Simulación" definido en la primera Fase, en la "Segunda Fase", siguiendo el Diagrama de Estudio de Simulación en cuanto a las actividades de Diseño de Experimentos y Simulación del Modelo (Figura 5.1), el Gestor de Sincronización (basado en los requisitos del sistema que van acorde al estado actual del sistema y su entorno) controla la simulación del modelo, este agente está a cargo de la generación y eliminación de instancias de los Agentes que ejecutan los roles de Gestor de Velocidad, Gestor de la Animación, Generador de Reportes, Generador de Eventos, y Analizador de Datos de Salida. Antes de iniciar la animación del modelo, el Gestor de Velocidad marca el ritmo de tiempo durante la animación. El Gestor de la Animación lleva a cabo la animación en base al ritmo establecido, y finalmente, el Analizador de Datos de Salida analiza los resultados y el Generador de Reportes en base a las plantillas establecidas y los resultados de la simulación, genera los reportes y/o gráficas para su análisis posterior. El Generador de Eventos genera información adicional que el Gestor de Sincronización incluye en la animación del modelo de simulación. Finalmente, siguiendo el Diagrama de Estudio de Simulación en cuanto a las actividades de Análisis de Resultados (Figura 5.1), el Analizador de Datos de Salida analiza los resultados de la simulación cuando ésta finaliza y los facilita para su revisión. Finalmente dicha información es reutilizada por los agentes del sistema.

Cada uno de los conjuntos de agentes (o roles de agentes) desempeñan funciones específicas de acuerdo al escenario actual del sistema. Los escenarios activos son el resultado de la identificación de los posibles estados del sistema. En la siguiente sección se describe en detalle las actividades de los elementos que participan en cada uno de los estados del sistema que activan escenarios específicos.

\subsection{Definición de Escenarios}

De acuerdo con la secuencia general de cada una de las Fases de la Simulación descrita en la Sección 5.4, es posible identificar los escenarios asociados a cada una. Asimismo, tanto la generación como la eliminación de instancias de agentes (o roles ejecutados por agentes) está sujeta al escenario actual del sistema. 
En la Figura 5.3 se muestra el Esquema Global de los escenarios que se presentan a lo largo del proceso de Simulación en sus dos fases.

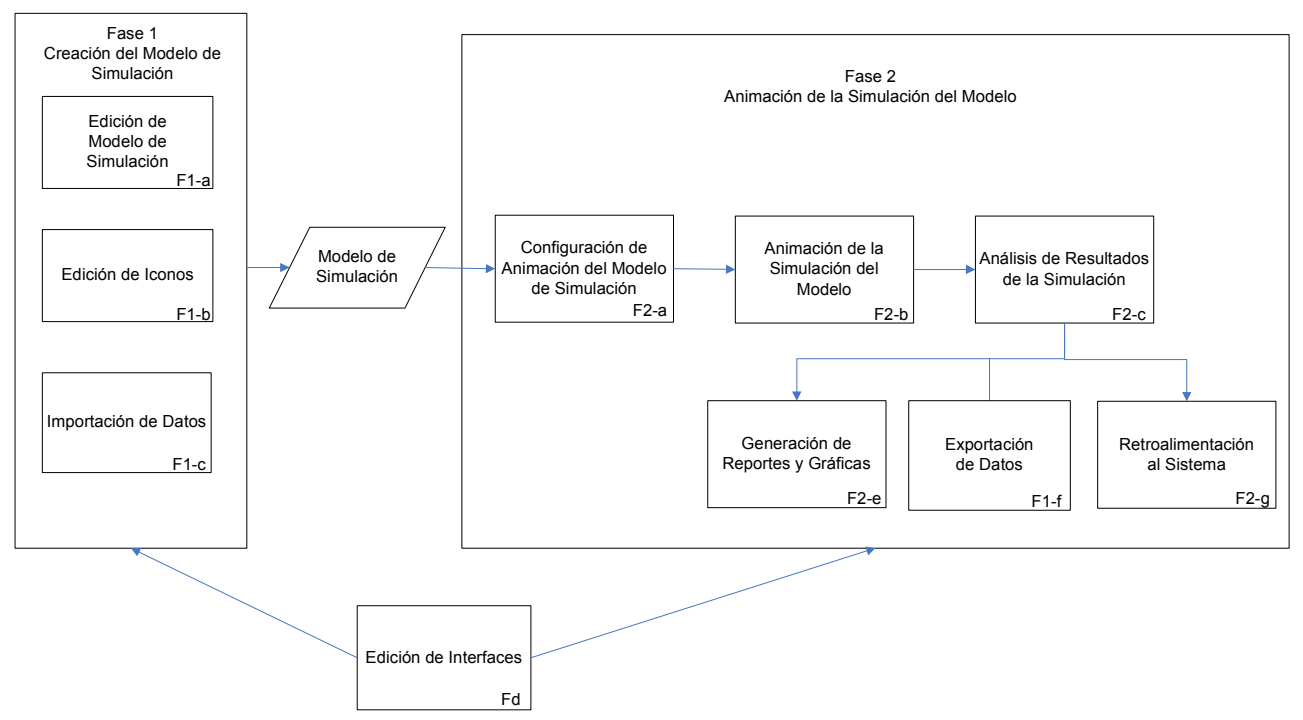

Figura 5.3: Esquema Global de Escenarios y sus Agentes (roles)

Los escenarios de cada una de las Fases a su vez están relacionadas con las Etapas que guían un Estudio de Simulación. De esta forma, en la Fase I se incluyen tres escenarios: a) Edición del Modelo, b) Edición de Iconos e c) Importación de Datos.

En el escenario de la Edición del Modelo se crea/modifica el Modelo de un Sistema de Fabricación el cual será simulado en la segunda fase. En el escenario de Edición de Iconos se crean/editan los iconos asociados a elementos específicos que representan elementos del modelo (máquinas, herramientas, operarios, staff). Finalmente en el escenario de Importación se transfiere y traduce la información obtenida del Sistema de Fabricación al Entorno de Simulación.

La Fase II, por su parte, incluye seis escenarios principales: a) Configuración de la Simulación, b) Simulación del Modelo, c) Análisis de los Resultados de la Simulación, d) Generación de Reportes y Gráficas, e) Exportación de Resultados y f) Retroalimentación del Sistema.

En el escenario de la Configuración de la Simulación del Modelo, el Usuario puede especificar la velocidad de simulación, tipo de animación (para cada uno 
de los elementos) y los eventos a considerar durante la simulación. En el escenario de la Simulación del Modelo se ejecuta como tal la simulación/animación del comportamiento del modelo de acuerdo a la configuración previamente establecida. Después de que finaliza la Simulación, en el escenario de Análisis de los Resultados de Simulación los datos generados durante la simulación se organizan y traducen en información útil. En ese momento, a petición del Usuario pueden aparecer dos escenarios: a) Generación de Reportes y Gráficas y b) Exportación de Resultados. En el escenario de Generación de Reportes y Gráficas a petición del Usuario los datos analizados se integran en reportes y gráficas de negocio. En el Escenario de Exportación de resultados los resultados de la simulación pueden exportarse para su análisis externo. Finalmente, el escenario Retroalimentación del Sistema se activa automáticamente integrando los resultados como parte de la base de conocimiento de los elementos del sistema.

Con el objeto de clarificar el propósito de cada escenario, en algunos de los escenarios se identifican subescenarios útiles para seguir las etapas del estudio.

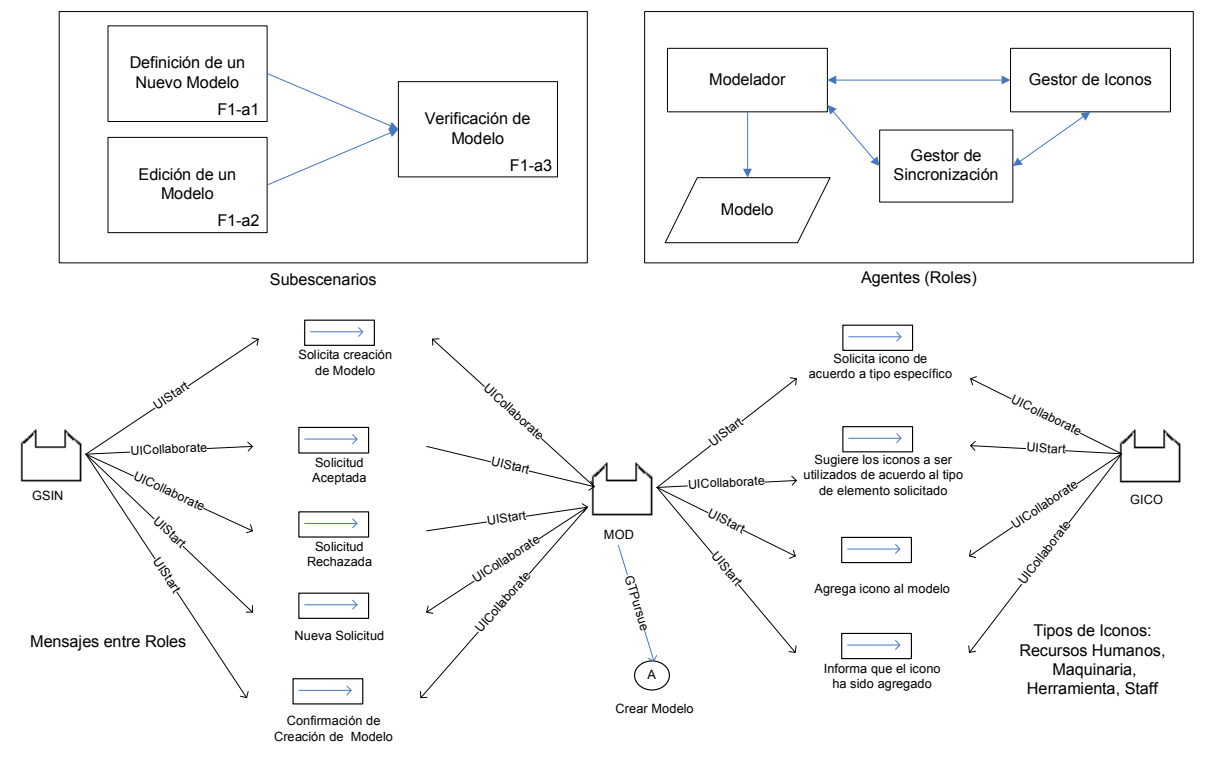

Figura 5.4: Subescenarios, Roles e Interacciones entre Agentes (roles) del Escenario de Edición del Modelo

La Figura 5.4 muestra las principales interacciones entre los agentes en los subescenarios identificados en el escenario Edición del Modelo (Fla), estos son denominados Definición de un Nuevo Modelo (F1-a1), Edición de un Modelo (F1-a2) y Verificación del Modelo (F1-a3). En la Figura 5.4 también se muestran 
los principales mensajes entre los agentes que se presentan. En estos escenarios los agentes ejecutan las siguientes tareas:

- En el escenario (F1-a1), el Gestor de Sincronización coordina al Modelador, quien permite al Usuario crear un nuevo modelo añadiendo iconos contenidos en la Librería de Iconos proporcionada por el Gestor de Iconos. El Modelador también permite al Usuario delimitar los atributos (parámetros) de todos los iconos. Finalmente, el Modelador almacena el modelo creado.

- En el escenario (F1-a2), el Gestor de Sincronización coordina al Modelador, quien permite al Usuario editar un modelo previo utilizando iconos contenidos en la Librería de Iconos proporcionada por el Gestor de Iconos. Finalmente, el Modelador almacena el modelo editado.

- En el escenario (F1-a3), el Modelador verifica el modelo, si este no contiene errores (falta algún dato en los parámetros), éste le informa al Gestor de Sincronización para proceder con la Simulación del Modelo. Por el contrario, en caso de que se encuentren errores, el Modelador se lo informa al Usuario y regresa al escenario (F1-a2) para corregirlo. Cuando el Modelo es correcto, el Gestor de Sincronización actualiza el estado del sistema y activa el escenario Configuración de Simulación (F2a).

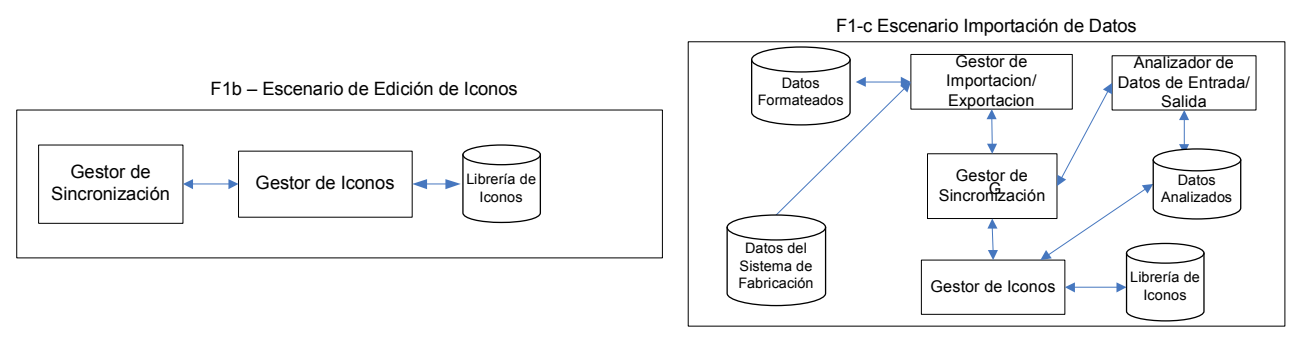

Figura 5.5: Agentes (roles) en los Escenarios Edición de Escenarios e Importación de Datos(F1c)

La Figura 5.5 muestra las principales interacciones entre los agentes en el escenario Edición de Iconos (F1b). En este escenario, los agentes ejecutan las siguientes tareas: cuando el Gestor de Sincronización detecta una petición de Usuario con respecto a la adición o modificación de un icono, el Gestor de Sincronización se lo informa al Gestor de Iconos. Así, el Gestor de Iconos permite al Usuario agregar un nuevo icono, eliminar un icono actual, o modificar los 
atributos asociados a un icono (capacidad por hora, turno, tiempos de espera, de ejecución, de cola, etc.).

La Figura 5.5 también muestra las principales interacciones entre los agentes en el escenario Importación de Datos (Flc). En este escenario, los agentes ejecutan las siguientes tareas: a) el Gestor de Sincronización coordina al Gestor de Importación/Exportación quien recibe la información del Sistema de Fabricación Real y lo importa en el sistema en el formato apropiado y b) cuando la información está formateada, el Gestor de Sincronización se lo informa al Analizador de Datos de Entrada/Salida, quien los analiza, clasifica y envía la información al Gestor de Iconos quien la asocia a los iconos correspondientes.

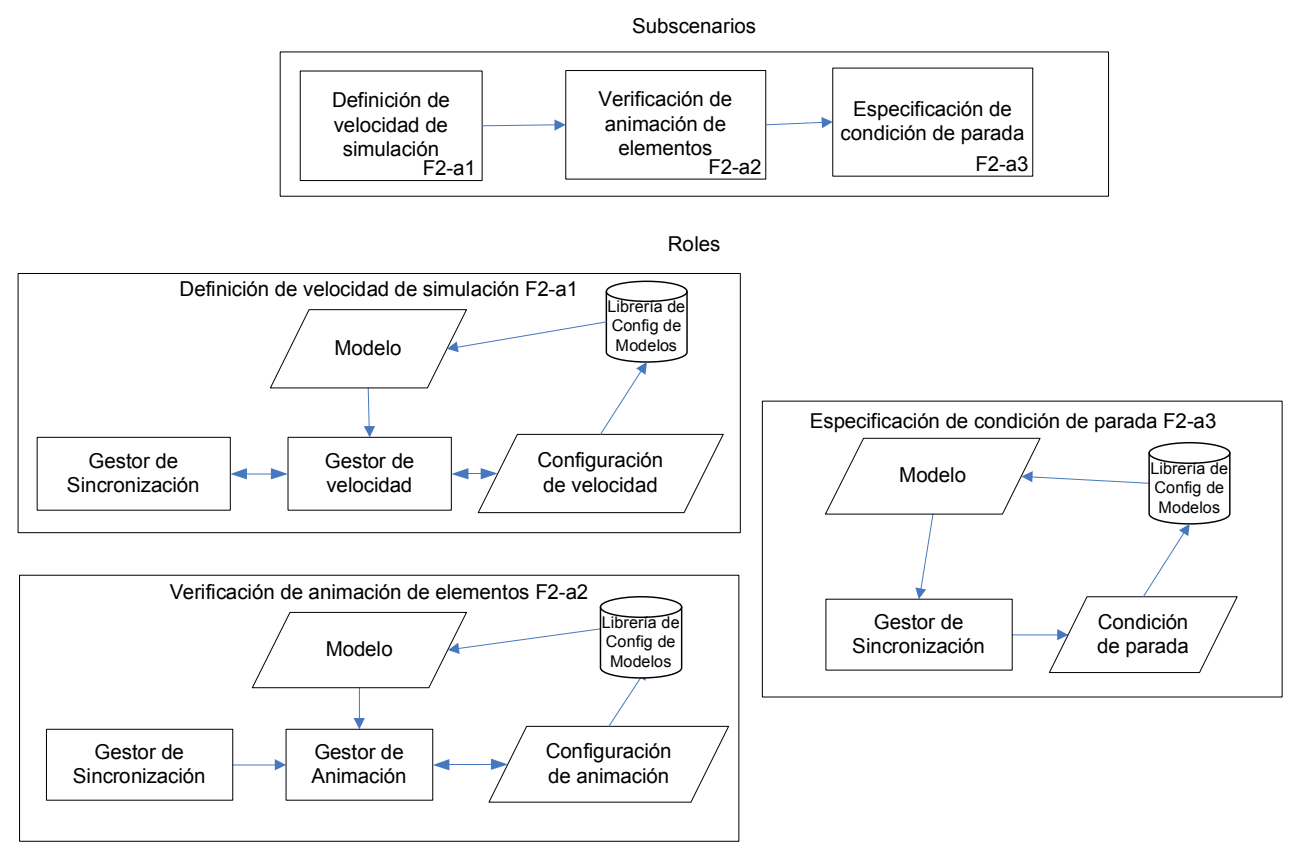

Figura 5.6: Agentes (roles) e interacciones en los Subescenarios de la Configuración de Simulación (F2a)

La Figura 5.6 muestra las principales interacciones entre los agentes en los subescenarios identificados en el escenario Configuración de Simulación del Modelo (F2a), estos son denominados Definición de velocidad de simulación (F2-a1), Verificación de animación de los elementos (F2-a2) y Determinar la condición de parada de la Simulación (F2-a3). En estos escenarios los agentes ejecutan las siguientes tareas: 
- En el escenario (F2-a1), el Usuario define la velocidad de la simulación para ejecutar la simulación. Entonces el Gestor de Sincronización recibe esta información y se la envía al Gestor de Velocidad. Esta velocidad se almacena en la configuración de la simulación.

- En el escenario (F2-a2), el gestor de Sincronización envía la configuración de velocidad al Gestor de Animación, quien verifica los atributos de los elementos para la animación gráfica de los mismos de acuerdo con la velocidad establecida.

- En el escenario (F2-a3), el Usuario determina la condición de parada de la simulación. La condición de parada indica en qué momento el Usuario desea que termine la simulación. Por tanto, puede ser expresada: a ) en tiempo ( $\mathrm{N}$ dias, $\mathrm{N}$ semanas (hasta 5), $\mathrm{N}$ meses (hasta 13) ó $\mathrm{N}$ años), b) de acuerdo a las Ordenes (al terminar una orden de producción específica) o c) cuando se obtenga una producción $\mathrm{P}$ de productos terminados. Entonces el Usuario informa al Gestor de Sincronización que la configuración esta preparada.

- Cuando la configuración está preparada, se actualiza el estado del sistema y el Gestor de Sincronización activa el escenario Animación de la Simulación del Modelo(F2b).

- El Usuario puede cambiar la configuración de la simulación en cualquier momento. Esto generalmente sucede cuando el Usuario ha observado los reportes y gráficas generadas al finalizar la simulación, sin embargo, el sistema permite al Usuario cambiar la configuración cuando la simulación se está ejecutando (on-line).
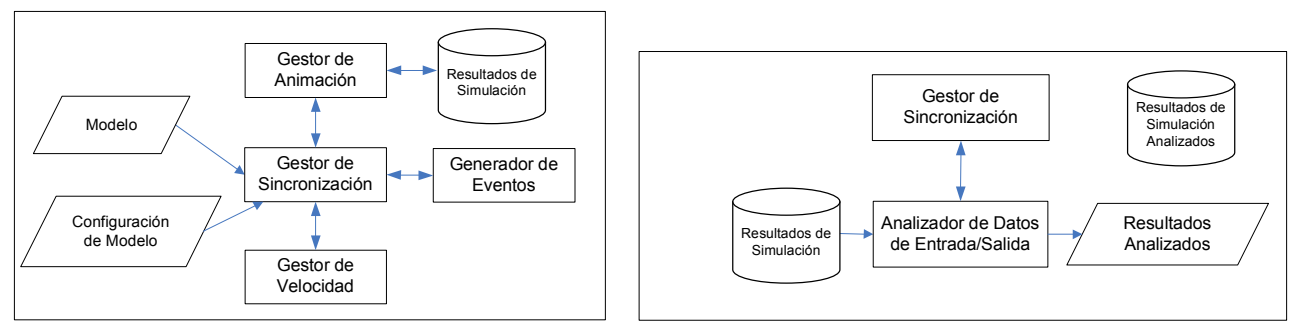

Figura 5.7: Agentes (roles) e interacciones en los escenarios de Animación (Simulación) del Modelo (F2b) y el Análisis de los Resultados de Simulación $(\mathrm{F} 2 \mathrm{c})$ 
La Figura 5.7 muestra las principales interacciones entre los agentes en el escenario Animación (Simulación) del Modelo (F2b).En este escenario, los agentes ejecutan las siguientes tareas:

a) Cuando el Gestor de Sincronización detecta que la Configuración de un Modelo está preparada, éste informa al Gestor de Velocidad la velocidad seleccionada, al Gestor de Animación los atributos activados y al Generador de Eventos el tipo de eventos a considerar. Entonces el Gestor de Sincronización monitoriza cómo el Gestor de Eventos genera eventos acorde a la velocidad que le indica el Gestor de Velocidad los cuales son animados gráficamente por el Gestor de Animación de acuerdo a la velocidad indicada.

b) Durante la Simulación del Modelo, los resultados son almacenados automáticamente por cada uno de los elementos ya sea de forma temporal o en la base de datos, generando a su vez archivos de rastreo.

c) Asimismo, durante la Simulación del Modelo, el Usuario puede solicitar un cambio en la velocidad o tipo de animación. Cuando esto sucede, el Gestor de Sincronización informa del cambio de velocidad al Gestor de Velocidad, del cambio de velocidad para la generación de eventos al Gestor de Eventos, y del cambio del tipo de animación al Gestor de Animación.

d) El Gestor de Sincronización supervisa el proceso de simulación hasta que se alcanza la condición de parada establecida por el Usuario.

e) Cuando la simulación ha terminado, se actualiza el estado del sistema y el Gestor de Sincronización activa el escenario de Análisis de Resultados de Simulación $(F 2 c)$.

La Figura 5.7 también muestra las principales interacciones entre los agentes en el escenario Análisis de Resultados de Simulación (F2c).En este escenario, los agentes ejecutan las siguientes tareas:

a) Cuando el Gestor de Sincronización detecta que la simulación ha terminado, éste se lo informa al Analizador de Datos de Entrada/Salida, quien analiza los resultados y los traduce en información útil.

b) Cuando la información ya ha sido analizada, se actualiza el estado del sistema y el Gestor de Sincronización realiza dos acciones: 1. Queda a la espera de la petición del Usuario para activar el escenario Generación de Gráficas y Reportes $(F 2 e)$ y la respectiva exportación de resultados auxiliándose del Gestor de Importaciones/Exportaciones, y 2. Activa el escenario de Retroalimentación del Sistema $(F 2 g)$. 


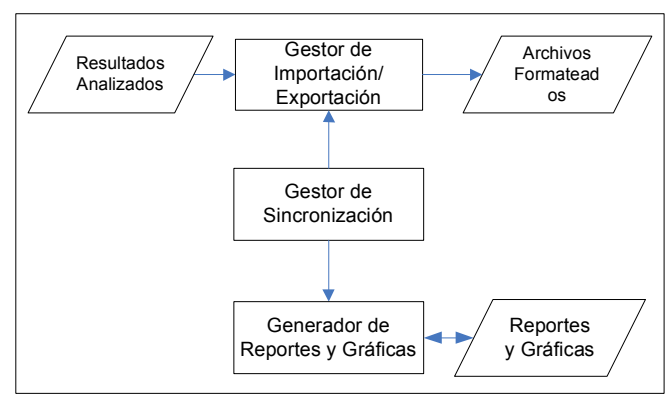

F2-e Generación de Reportes y Gráficas

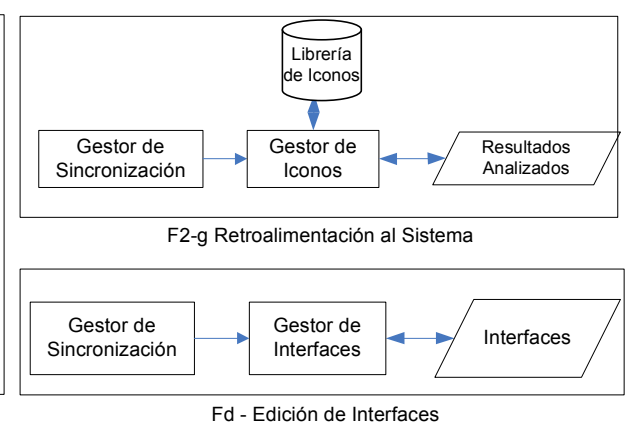

Figura 5.8: Agentes (roles) e interacciones en los escenarios Edición de Interfaces (F2d), Generación de Reportes y Gráficas (F2e) y Retroalimentación al Sistema (F2g)

La Figura 5.8 muestra las principales interacciones entre los agentes en el escenario Generación de Gráficas y Reportes (F2e). En este escenario, los agentes ejecutan la siguiente tarea: cuando el Gestor de Sincronización detecta que el análisis de resultados ha terminado se lo informa al Generador de Reportes y Gráficas quien las genera y muestra al Usuario.

La Figura 5.8 también muestra las principales interacciones entre los agentes en el escenario Retroalimentación al Sistema ( $F 2 g$ ). En este escenario, los agentes ejecutan la siguiente tarea: cuando la simulación ha terminado el Gestor de Sincronización se lo informa al Gestor de Iconos quien asocia la información analizada a cada uno de los iconos de acuerdo a sus comportamientos.

La Figura 5.8 también muestra las principales interacciones entre los agentes en el escenario Edición de Interfaces $(F d)$. Este escenario puede aparecer antes, después o durante el proceso de simulación. En este escenario, los agentes ejecutan la siguiente tarea: cuando el Gestor de Sincronización recibe una petición del Usuario de modificación o creación de interfaz para mejorar la funcionalidad del sistema, éste se lo informa al Gestor de Interfaces, quien permite al Usuario crear/modificar la interfaz indicada.

Finalmente en la Tabla 5.3 se presenta un listado de las principales acciones que llevan acabo los dos Agentes clave (el Agente Modelador -AModelador- y el Agente Simulador -ASimulador-) para el control en las dos principales fases (Creación de Modelo y Simulación de Modelo). 


\begin{tabular}{|l|c|c|}
\hline Acción & AModelador & ASimulador \\
\hline Agregar escenarios al Modelo & $\mathrm{Si}$ & $\mathrm{Si}$ \\
Agregar elementos al Modelo & $\mathrm{Si}$ & $\mathrm{Si}$ \\
Asociar instancias a elementos & $\mathrm{Si}$ & $\mathrm{Si}$ \\
Eliminar elementos del Modelo & $\mathrm{Si}$ & $\mathrm{Si}$ \\
Crear instancias en BD & $\mathrm{Si}$ & $\mathrm{Si}$ \\
Validar modelo & $\mathrm{Si}$ & $\mathrm{Si}$ \\
Crear agentes & $\mathrm{No}$ & $\mathrm{Si}$ \\
Eliminar agentes & $\mathrm{No}$ & $\mathrm{Si}$ \\
Definir condición de parada & $\mathrm{No}$ & $\mathrm{Si}$ \\
Definir velocidad de simulación & $\mathrm{No}$ & $\mathrm{Si}$ \\
Definir animación de iconos & $\mathrm{No}$ & $\mathrm{Si}$ \\
Control de reloj & $\mathrm{No}$ & $\mathrm{Si}$ \\
Control de velocidad & $\mathrm{No}$ & $\mathrm{Si}$ \\
Animación de iconos & $\mathrm{No}$ & $\mathrm{Si}$ \\
Control de ejecución de simulación & $\mathrm{No}$ & $\mathrm{Si}$ \\
Creación de traza de eventos & $\mathrm{No}$ & $\mathrm{Si}$ \\
Generación de Reportes y Gráficas & $\mathrm{No}$ & $\mathrm{Si}$ \\
\hline
\end{tabular}

Tabla 5.3: Principales Acciones del AModelador y el ASimulador

En esta sección es posible obtener una idea general de las relaciones que se establecen entre los roles o agentes en cada escenario y observar cómo cada uno de los roles o agentes que interactúan en el Entorno de Simulación se interrelacionan con el fin de lograr objetivos específicos. En las siguientes secciones se presentan las relaciones de los roles identificados y los agentes que integran la plataforma del Entorno de Simulación. Tal asociación corresponde a las actividades que los agentes deben ejecutar acorde con el escenario donde se encuentre el Sistema y las interacciones con otros agentes tanto del Entorno de Simulación como los agentes que componen el Sistema de Fabricación Inteligente.

\subsection{Las Fases de Simulación y el Agente de Sincro- nización}

El Agente de Sincronización (ASincronizador) tiene como función principal la monitorización de las actividades de los agentes y por consecuencia del estado del sistema. Este agente es quien determina qué instancias de agentes deben ser creadas de acuerdo con las solicitudes del Usuario. De tal manera que puede desempeñar dos roles: 
Generador de Eventos. De acuerdo a la recepción de peticiones del Usuario mediante interfaces genera los eventos correspondientes para que el resto de los agentes del sistema reaccionen.

Gestor de Instancias de Agentes. Este crea o elimina instancias tanto de Agentes para la Fase I, como de Agentes para la Fase II de acuerdo a la monitorización del estado del sistema (Fig.5.9).

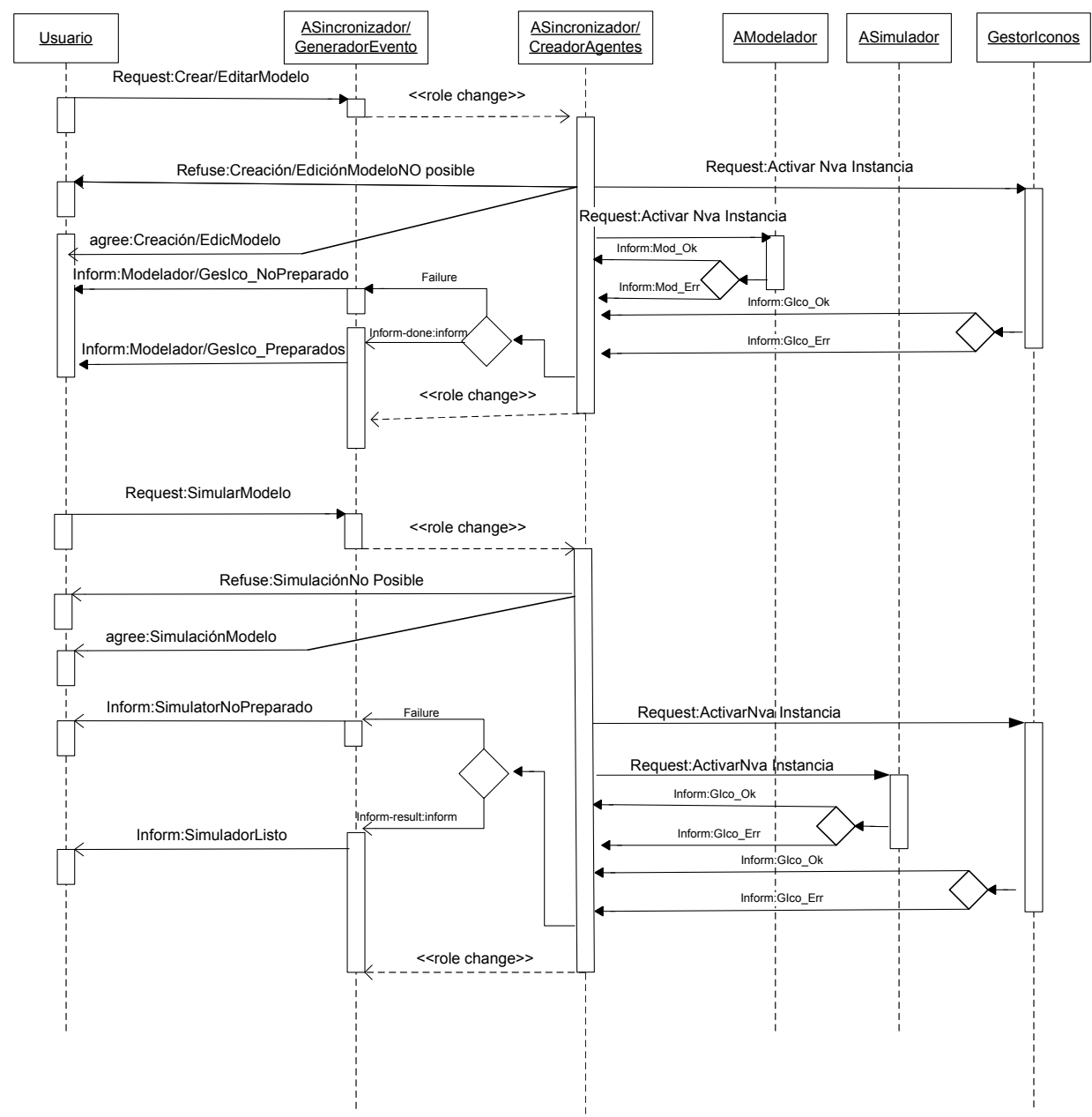

Figura 5.9: Diagrama de Cooperación entre Usuario y Agente de Sincronización

Cabe señalar que el Sincronizador permite la ejecución paralela de ambas Fases. Esto es, el Sistema ofrece la posibilidad al Usuario de crear/editar modelos 
al mismo tiempo que se ejecuta la Simulación de un Modelo que ya ha superado la Fase I. En caso de que un Modelo esté siendo Simulado y requiera ser editado para integrar nuevos elementos al Modelo, el Sistema ofrece las posibilidades tanto de agregar instancias a la Base de Datos como agregar o eliminar iconos o instancias del Modelo.

Siguiendo el principal objetivo de cada una de las Fases, se plantea la necesidad de dos agentes globales: el Agente Modelador (AModelador) y el Agente Simulador (ASimulador), responsables de las principales actividades de la Fase I y Fase II.

De acuerdo con la identificación tanto de escenarios como de roles que pueden ser agrupados en un agente presentada en las Secciones 5.1 y 5.5, en las siguientes secciones se presenta el detalle del comportamiento de dichos escenarios mediante la descripción de los estados por los que evoluciona el sistema en las Fases I y II. Asimismo, también se presentan las principales protocolos de interacción entre los Agentes (en sus roles) en cada estado. Tales protocolos permitirán visualizar de forma gráfica el comportamiento de cada uno de los elementos ante sucesos específicos.

\subsection{Fase I. Configuración preliminar del Modelo}

Las actividades preliminares al Diseño del Modelo de un Sistema de Fabricación se centran en alimentar la Base de Datos del Entorno de Simulación con los datos de las instancias reales del Sistema a representar así como con los datos de las posibles instancias que pueden ser incorporadas aunque no se cuente con ellas en el sistema real. Por lo tanto, tales actividades comprenden dos situaciones (Fig. 5.10): a) la importación de datos del Sistema Real a la base de datos del Entorno de Simulación y b) la posibilidad de agregar/modificar/editar instancias individuales de elementos (operarios, máquinas, herramientas, Staff, productos, materia prima, etc.) se tengan o no físicamente en el Sistema de Fabricación real.

Ambas opciones se asocian a un responsable del control que conlleven las tareas a realizar en cada situación. Por tanto, para la importación de datos del Sistema de Fabricación Real surge la necesidad de crear un Agente que desempeñe el rol de Gestor de Importación y Exportación, al cual se le denomina el Agente de Importación/Exportación (AImportación/Exportación). En este paso cabe señalar que la técnica empleada para la Recolección de Datos se deja en 
manos del Usuario puesto que el entorno de simulación permite: a) simular el comportamiento de la Planta de Fabricación bajo las condiciones que haya especificado el Usuario ${ }^{1}$ y b) Presentar los resultados obtenidos (partiendo de tales datos) para su evaluación. El Usuario es quien determina la técnica basándose en las consideraciones presentadas en la Sección 2.4.4.

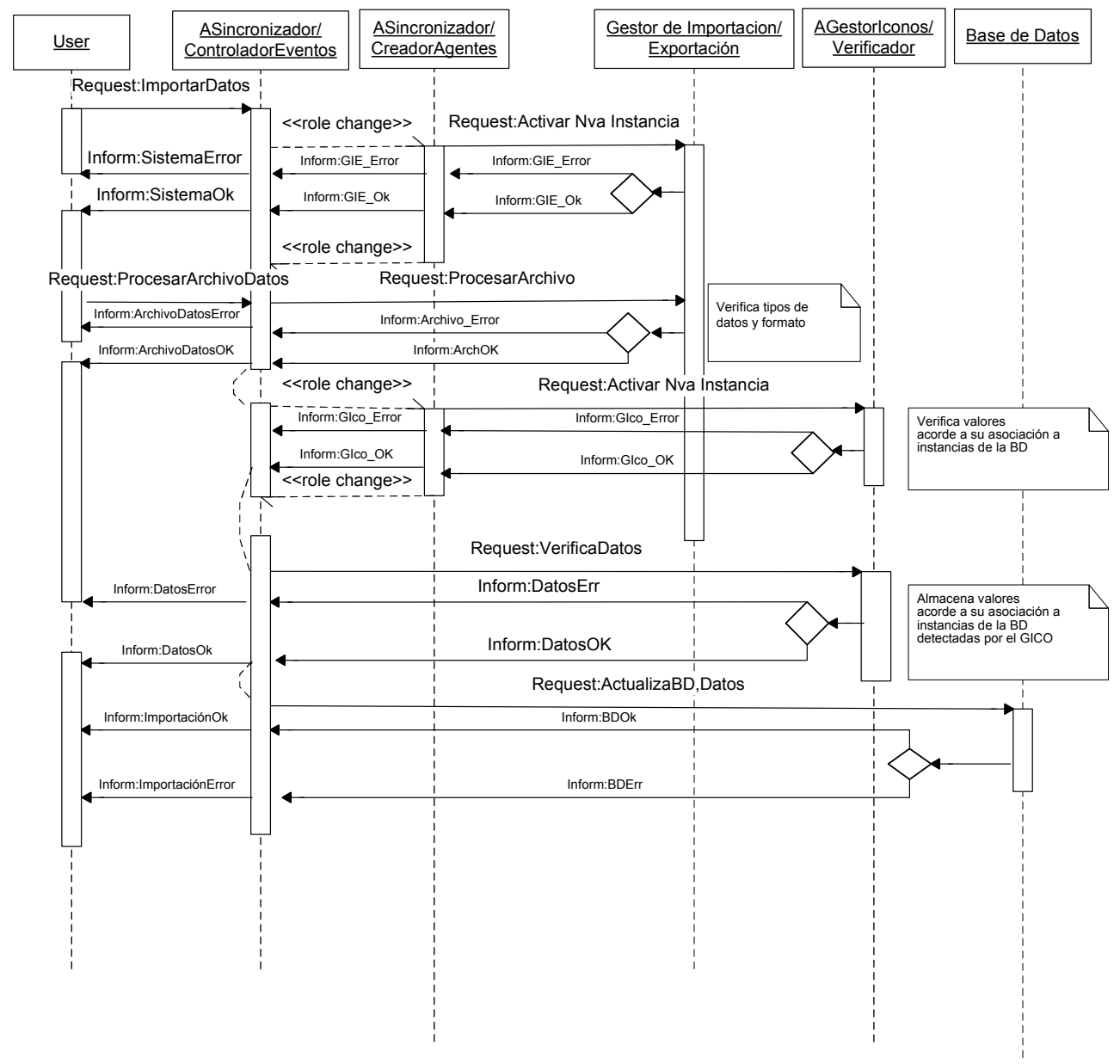

Figura 5.10: Diagrama de Cooperación entre el Gestor de Importación / Exportación, Gestor de Iconos, el Sincronizador y el Usuario durante la Importación de Datos.

${ }^{1}$ A lo largo de este trabajo se identificará como "Usuario" a la persona que realiza las funciones de diseño del modelo y ejecutor de las pruebas de simulación. 
Por otra parte, para la adición/edición de instancias surge la necesidad de crear un Agente el Agente de Gestión de Iconos (AGestorIconos) quien tendrá asociados dos roles: el de Gestor de Iconos (para la adición, modificación y eliminación de instancias en la Base de Datos mediante una interfaz) y el de Verificador (para comprobar que los datos de los atributos asociados a una instancia son correctos).

Asimismo, es aquí donde la instancia del AGestorIconos resalta la importancia de su integración en el Entorno de Simulación durante la Fase I. El principal objetivo del AGestorIconos es ofrecer los elementos acorde a las peticiones del Usuario mediante el uso de iconos que asociados a los elementos básicos para la representación de un Sistema de Fabricación, no sólo los que se refieren a las instalaciones físicas y de recursos humanos (p. ej. operarios, staff, máquinas, herramientas y su ubicación física dentro de la Planta de Fabricación) sino aquellos que se refieren a la definición de Productos, rutas, listas de materiales, etc.

Durante la Fase de Creación del Modelo, el Usuario puede actualizar las instancias de los elementos que pueden formar parte del Modelo que se esta creando. Para ello resulta importante determinar los protocolos de comunicación a seguir en las interacciones entre el Usuario y el AGestorIconos. De esta forma, dicha configuración se pone a disposición del AModelador (Fig. 5.11).

Un aspecto importante referente a la creación de instancias, es la que refiere a la creación de un Producto. Al definir una instancia de un Producto, el sistema solicita no solo los datos del producto sino que además requiere que se definan:

Lista de Materiales. Se refiere a la Lista de Materiales requeridos para su fabricación (esta lista puede incluir tanto Materia Prima como otros Productos).

Ruta de Fabricación. En la ruta se definen los procesos requeridos para la fabricación del mismo indicando el tiempo necesario para ejecutar tal proceso.

Lista de Transiciones. Una vez creada la Ruta de Fabricación, con el objetivo de describir, lo más cercano a la realidad las acciones y la secuencia de los procesos incluidos en la Ruta de Fabricación, en la lista se define la secuencia de ejecución de los procesos de la Ruta de Fabricación. La secuencia muestra qué procesos deberán ejecutarse en forma secuencial o en su caso paralelamente. 


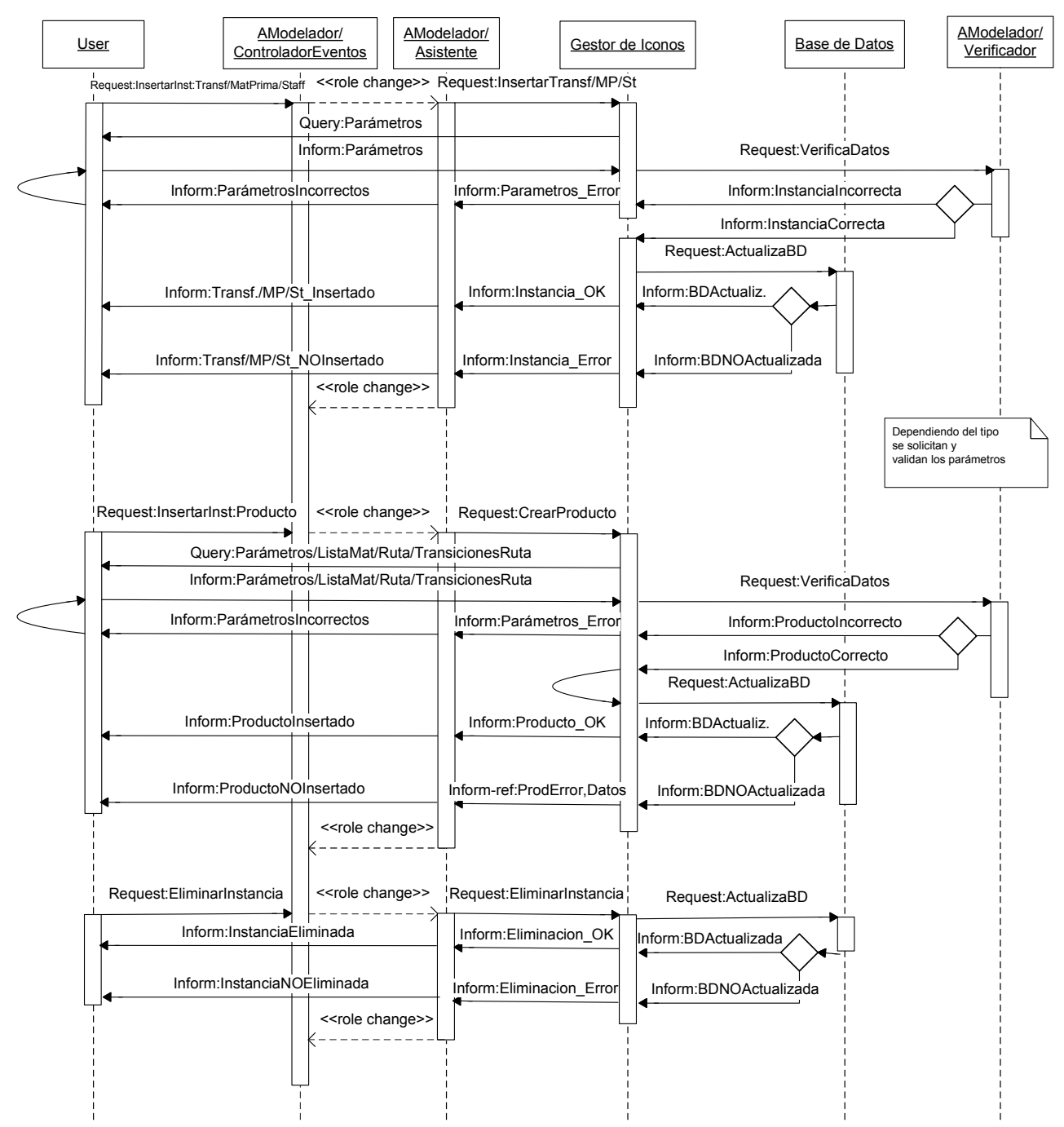

Figura 5.11: Diagrama de Cooperación entre el AGestorIconos y el Usuario durante la Creación de instancias para un Modelo.

\subsection{Fase I. Creación del Modelo}

En la Fase I Creación del Modelo, se presenta una interacción contínua entre el Usuario y el Entorno de Simulación mediante una interfaz transparente en el momento de la creación o edición de un modelo.

Para la primera Fase es necesario establecer ciertos criterios que serán útiles para el Usuario (diseñador del Modelo). Entre los criterios que involucran 
algunos de los aspectos fundamentales se encuentran:

- Proporcionar alternativas de ayuda eficaces durante el diseño del modelo.

- Integrar un nivel de detalle suficiente para integrar información básica pero suficiente para describir la situación real del Sistema de Fabricación fácilmente. En este caso las interfaces entre el Usuario y el Entorno de Simulación juegan un papel clave para el logro de una via de comunicación eficiente entre ambos.

- El diseño apropiado de una Base de Datos que permita el almacenamiento de la información que soporta la interfaz gráfica del Entorno de Simulación.

\subsubsection{Detección y Control de Cambios de Estado}

Basado en la teoría para la detección de cambios de estado presentados en la Sección 3.2; en la Primera Fase, la detección de cambios de estado del sistema se realiza mediante el método de detección de eventos.

El AModelador se encuentra sujeto en parte a las solicitudes del Usuario. Los eventos pueden ser disparados tanto por intervenciones del Usuario como por el mismo AModelador como resultado de la finalización de actividades específicas. La Figura 5.12 muestra los distintos estados del sistema que se pueden presentar durante la Fase I. Las líneas continuas identifican aquel escenario que precede a otro escenario, mientras que las líneas discontinuas aquellos escenarios que pueden aparecer opcionalmente a petición del Usuario (diseñador). En la Fase I los dos estados que dan inicio al proceso son: Creación de un Modelo y Apertura de un Modelo.

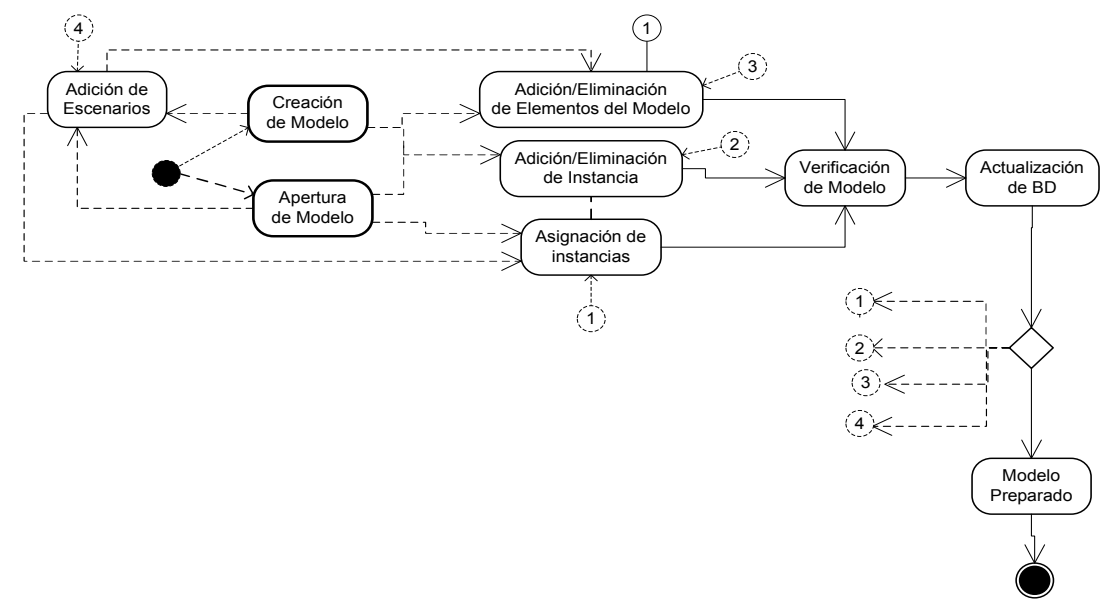

Figura 5.12: Diagrama de Estados del Sistema durante la Fase I. 
En esta Fase el AModelador tiene asociados tres roles: el de Controlador de Eventos (para gestionar las solicitudes del Usuario), el de Asistente de Modelado (para la creación y diseño del Modelo) y el de Verificador (para verificar los elementos que componen el Modelo).

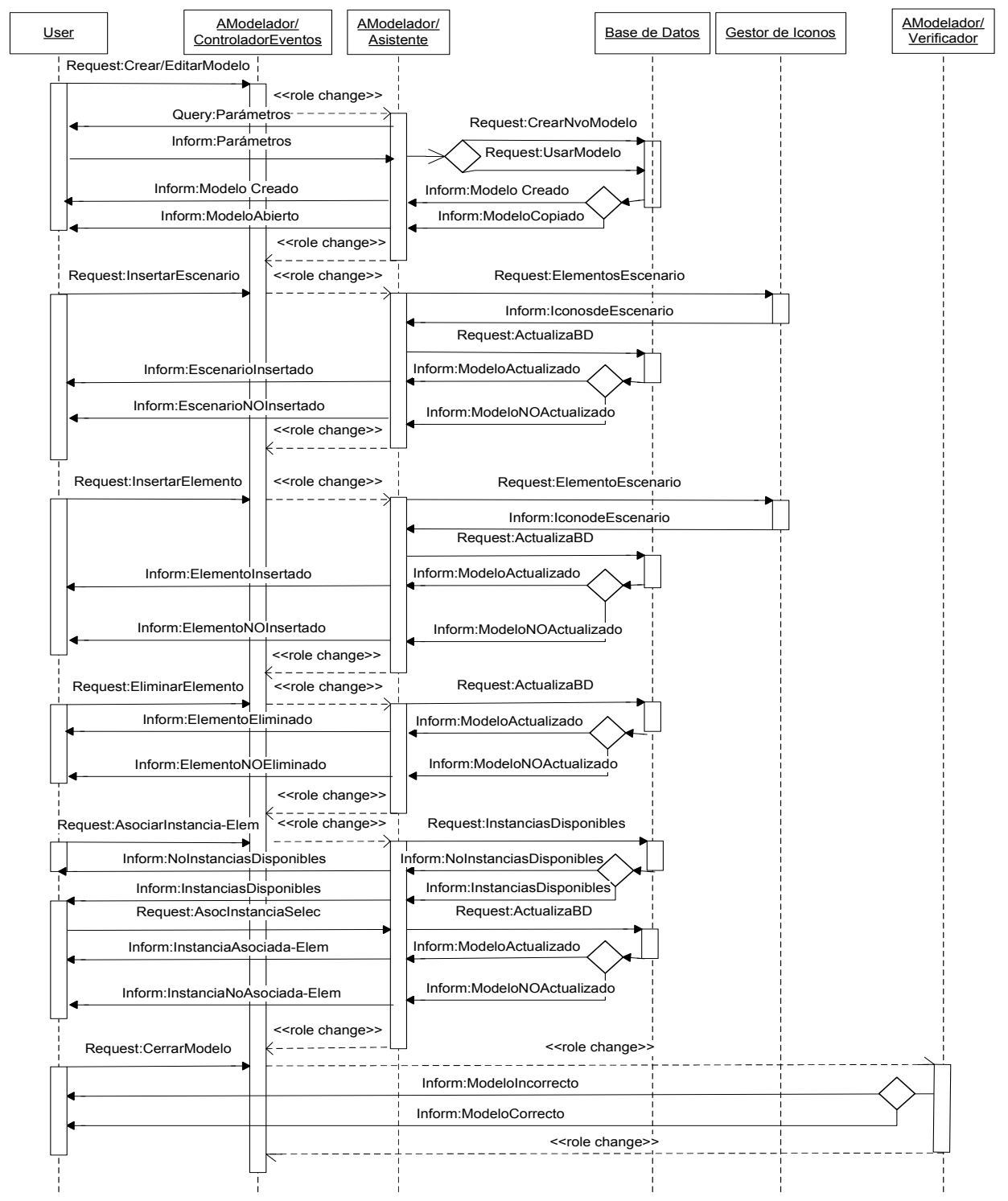

Figura 5.13: FASE I. Diagrama de Cooperación entre el AModelador(sus roles), el AGestorIconos y el Usuario durante la Creación de Modelos

Cuando el Usuario (diseñador) solicita crear un nuevo modelo, entonces el 
ASincronizador activa las instancias que ejecutan dicha tarea: el AModelador y el AGestorIconos. Durante esta etapa se consideran todos los aspectos clave con respecto a los elementos necesarios para llevar a cabo la Simulación de Sistemas de Fabricación presentados en el Capítulo 4 (Operarios, Máquinas, Herramientas, Staff y sus relaciones).

El AModelador tiene como principal objetivo proporcionar medios adecuados para que el Usuario cree un Modelo que represente la distribución física de los recursos de la planta de fabricación que desea simular.

El AModelador dá soporte a la Creación/Edición de Modelos de Sistemas de Fabricación, éste se encuentra en una constante verificación de las solicitudes del Usuario y de los mensajes que recibe de otros agentes. De esta forma, el Modelador reacciona ante la aparición de eventos específicos generados tanto por la intervención del Usuario como por eventos generados a partir del AGestorIconos. Ante dichos eventos, el AModelador cambia el rol que desempeña y por tanto las acciones e interacciones tanto con otros agentes como con el Usuario que debe ejecutar (Fig. 5.13). El AModelador puede desempeñar tres roles esenciales:

Controlador de Eventos. El AModelador se encuentra sujeto a la aparición de eventos disparados por la intervención del Usuario o como resultado de las interacciones entre éste y el AGestorIconos. De acuerdo al evento que detecta, éste determina el nuevo rol al que el AModelador debe cambiar. De esta manera su aparición es aperiódica y se mantiene vivo hasta el momento de la finalización de la Fase I para un modelo dado.

Asistente de Modelado. Cuando el Usuario solicita la creación/edición de un Modelo, el AModelador desempeña este rol a fin de que sean insertados ya sea los iconos (representación gráfica) que representan elementos del Sistema de Fabricación, o la asociación de instancias específicas a dichos iconos.

Verificador. Cuando el Usuario indica que desea terminar con la creación del modelo, el AModelador activa su rol de Verificador con dos objetivos: a) Validar que todos los elementos tengan asociada su respectiva instancia y b) Validar que cada escenario contenga los elementos apropiados (p.ej. si en un escenario de corte la máquina de corte no es automática, en el escenario se debe tener al menos un operario que la manipule).

De esta manera, de acuerdo con el estado actual del Sistema, en la Fase I 
aparecen distintos protocolos de comunicación y activación de los distintos roles que puede ejecutar un agente.

Siguiendo el Diagrama de Cooperación de Agentes en la Fase I (Fig. 5.13), en el momento en que el Usuario solicita la Creación/Edición de un Modelo, el AModelador activa un rol: Asistente de Modelado. El Asistente de Modelado en este momento actúa ofreciendo la posibilidad de indicar el tipo de industria para el cual se desea crear el Modelo y el Producto asociado a dicho modelo. De acuerdo con la selección del Usuario, este rol busca en su Base de Datos los modelos que se ajustan a dicha selección y pueden ser de utilidad al Usuario poniéndolos a su disposición. En caso de que el Usuario decida empezar de cero el diseño del modelo, entonces el $\boldsymbol{U}$ suario le asigna un identificador al nuevo modelo asociando, el tipo de industria para el cual esta dirigido, y el producto a fabricar en él. El Asistente de Modelado también permite la asociación de un nuevo producto a un Modelo ya existente. Cuando se elige un Modelo existente, se crea una copia dejando un respaldo del Modelo original.

Siguiendo el Diagrama de Estados (Fig.5.12), en ese momento el AModelador/Asistente de Modelado permite cambiar el estado del Sistema en dos sentidos (Protocolo en Fig. 5.13: a) adición de elementos individuales en un escenario del Modelo (operarios, herramientas, máquinas, staff) y b) adición de escenarios de fabricación ${ }^{2}$ de acuerdo al tipo de proceso que requiera (p.ej. para un escenario de corte, el AModelador/Asistente de Modelado agrega al Modelo un conjunto de elementos tales como operario, máquina, staff en un solo paso).

En ambos casos, cuando el estado agrega un nuevo(s) elemento(s) al Modelo, el Sistema cambia a un tercer estado: Asignación de instancias. En este estado se lleva a cabo la vinculación de las entidades del entorno real a los agentes que integran el entorno simulado tal y como se presenta en la Sección 6.3. En la asignación de instancias es posible asociar el icono de cada escenario a una instancia específica (p.ej. escenario: soldadura, icono:máquina, instancia: Pistola Kestler) la cual tiene asociados parámetros (turno, capacidad por hora, tiempo de preparación, tiempo de cola, etc.) que delimitan su capacidad para ejecutar un proceso. Cuando el Usuario solicita la asignación de una instancia, el AModelador/Asistente de Modelado le ofrece sólo aquellas instancias disponibles de

\footnotetext{
${ }^{2}$ A partir de este momento, el término de "escenario" en un modelo se utiliza para denominar a una agrupación funcional de elementos que pueden ejecutar un proceso específico (corte, laminado, soldadura, pintura, acabados, etc.) dentro de la planta de fabricación la cual representa una célula de fabricación (Sección 4.6.
} 
acuerdo al escenario (y su proceso) al que está asociado el elemento al quiere asignarle la instancia.

Durante el Modelado se definen también las relaciones entre los elementos del Sistema de Fabricación. Tales relaciones permiten asociar los Operarios a los equipos que estos manipulan. Este tipo de relaciones se utilizan más tarde en la Segunda Fase para el control de los recursos (Máquinas y Operarios) que ofrecen sus servicios durante la Simulación del comportamiento de la Planta de Fabricación ante las solicitudes de una Orden de Producción.

Los escenarios pueden ser modificados para así agregar elementos adicionales al escenario o eliminar elementos que no se consideren necesarios (p.ej. en un escenario de corte se integra una máquina y un operario por defecto, sin embargo, cuando una instancia específica de máquina cortadora opera de forma automática, no se requiere un operario que la manipule) (Protocolo en Fig. 5.13).

Cuando dichos estados finalizan el AModelador/Asistente de Modelado cambia el estado del Sistema de forma automática a un cuarto estado: Verificación del Modelo (Fig. 5.12), y cambia su rol al de Verificador (Protocolo en Fig. 5.13). En este estado el AModelador/Verificador verifica que los datos asociados a cada icono correspondan al tipo de parámetro requerido y en su caso que se cuente con todos los parámetros necesarios para poder simular el modelo en la Fase II. Al finalizar la Verificación del Modelo, el AModelador/Verificador activa un quinto estado: Actualización de la Base de Datos y cambia su rol al de AModelador/Asistente de Modelado. Durante este estado el AModelador/Asistente de Modelado se encarga de que los cambios realizados en los estados anteriores sean almacenados en la Base de Datos.

Los estados del primero al quinto pueden repetirse tantas veces como lo solicite el Usuario hasta que complete el Modelo que represente el Sistema de Fabricación Real. Una vez que el Usuario determina que el Modelo está terminado, el AModelador/Asistente de Modelado recibe la petición del Usuario de guardar/cerrar el Modelo y entonces el estado del Sistema cambia a Modelo Preparado.

Cuando el modelo está preparado, el AModelador/Asistente de Modelado lo pone a disposición del Sincronizador para ejecutar la Fase II.

Durante la Fase I es posible que el Sistema cambie a un sexto estado: Adición/Eliminación de Instancia. Este estado surge como resultado de la necesidad de agregar una nueva instancia o modificar/eliminar una instancia específica a la Base de Datos. Este estado del Sistema puede aparecer ya sea después de crear 
un módulo nuevo o de abrir un modelo ya existente o al intentar asociar una instancia específica a un Icono a petición del Usuario. Cuando esto sucede, el ASincronizador/GestordeInstancias crea y activa una instancia del AGestorIconos/GestorIconos para crear o modificar una instancia y ponerla a disposición del AModelador/Asistente de Modelado. Al finalizar esta actividad el Sistema cambia al estado de Verificación del Modelo donde el AModelador/Asistente de Modelado cambia su rol al de Verificador. El AModelador/Verificador comprueba que el Modelo no contenga errores. Si el modelo no contiene errores, el sistema cambia al estado Actualización de la BD donde el AModelador/Asistente de Modelado almacena los cambios. Por el contrario si detecta errores entonces el estado del Sistema cambia nuevamente al de Edición del Modelo donde el AModelador/Verificador le informa al Usuario los errores detectados para su corrección.

En la Figura 5.14 se observan los protocolos de comunicación que siguen los agentes que intervienen durante el Proceso de Creación del Modelo.

\subsubsection{Reglas de modelado aplicadas durante la Creación y Verificación del Modelo.}

Antes de que un Modelo se encuentre disponible para ser simulado en la Fase II, éste es verificado por el AModelador/Verificador mediante la comprobación de reglas básicas para el modelado de organizaciones. Para ello, se han considerado los factores para el Modelado relacionado con la Teoría de Organizaciones presentado en la Sección 2.2. Por tanto, en la propuesta se establecen reglas para la definición del modelo con respecto a la normalización de habilidades y comportamientos. Estas reglas básicas aseguran que el modelo cuenta con la información necesaria para ejecutar su simulación correctamente. Las reglas básicas que se verifican en cada escenario del Modelo incluyen:

1. Si de acuerdo con el Identificador de una máquina o herramienta asignada se determina que ésta NO es automática, es decir, requiere ser manipulada por un operario. Se verifica que el escenario incluya al menos un operario. (Norma de Comportamiento)

2. Si de acuerdo con el Identificador de una máquina o herramienta asignada se determina que ésta es automática, es decir, NO requiere ser manipulada por un operario. Se verifica que el escenario no incluya un operario. Si éste existe, se manda un mensaje de información al Usuario para que corrija tal evento. (Norma de Comportamiento) 


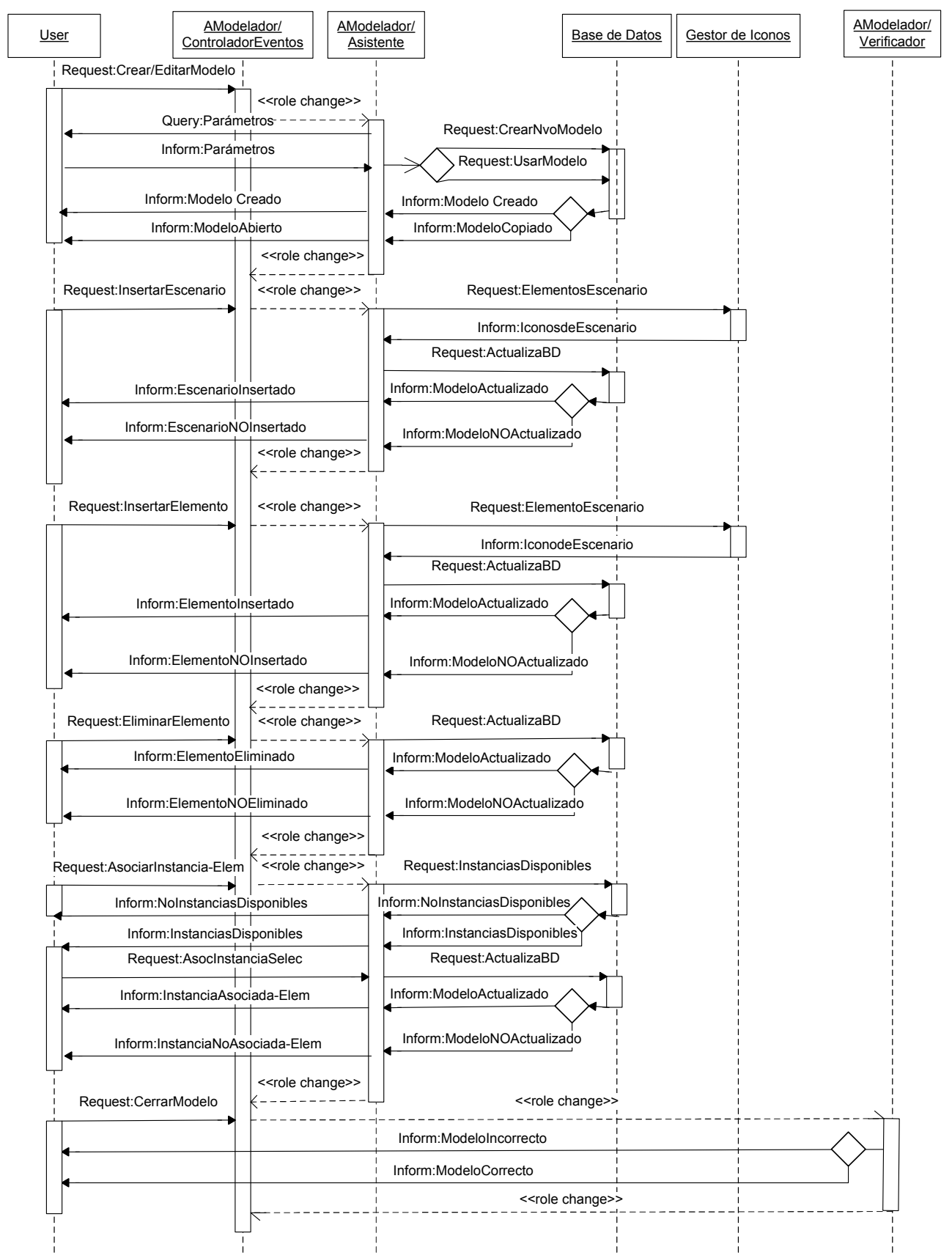

Figura 5.14: Diagrama de Cooperación entre los Agentes durante la Creación del Modelo 
3. Un Identificador de una instancia de Operario no puede estar asociado a dos iconos en el mismo modelo. (Norma de Habilidad)

4. Un Identificador de una instancia de máquinas o herramientas puede estar asociado a varios iconos siempre que la cantidad de tales recursos lo permita.

5. Todos los iconos de Operario, máquina, herramienta o Staff deben tener asociado un Identificador de instancia, que determina las características de funcionalidad que ofrecerá su agente como servicios en la segunda fase (Simulación del Modelo). En este caso se asocian características tales como el turno/horas diarias en el que están disponibles, tipo de cualificación (solo en el caso de operarios: principiante, medio o experto) y tipo de proceso que pueden ejecutar (p.ejem. corte, soldadura, pintura, lavado, y acabados). (Norma de Habilidad)

6. Cuando se define una relación, de acuerdo con el tipo de relación:

- Si la relación es de tipo "Manipula", un Operario solamente puede manipular Máquinas o Herramientas. Las Máquinas y Herramientas solo pueden ser manipuladas si estas no trabajan de forma automática. (Norma de Comportamiento)

- Si la relación es de tipo "Supervisa", un Staff solamente puede supervisar Operarios, Máquinas y Herramientas. (Norma de Comportamiento)

Durante la creación del Modelo se verifican las reglas 3 a la 6 y al finalizar un modelo se verifican las reglas 1 y 2 . Si alguna de las reglas descritas no se cumple, el Modelo no puede ser utilizado en la Fase II. Las relaciones entre la Orden de Producción y los Operarios, Máquinas, Herramientas, y Staff se controlan en la Fase II. En la Fase II, cuando una Orden de Producción se lanza, se le asocia un Producto. Entonces la Orden de Producción solicita los servicios de los Operarios, Máquinas, Herramientas, y Staff, de acuerdo a la ruta del producto. Los protocolos de negociación pueden basarse en peticiones de servicios solicitados por la Orden de Producción a los Operarios, Máquinas, Herramientas, y Staff disponibles o en solicitudes de tareas generadas por los Operarios, Máquinas, Herramientas, y Staff a las Ordenes de Producción activas. Cuando una Orden se lanza, el Staff supervisa sus actividades. 


\subsection{Conclusiones}

En este capítulo se han presentado las bases de la Arquitectura del Entorno de Simulación que dá soporte a la Primera Fase de la Simulación de una Planta de Fabricación (Creación de Modelos). El Modelo diseñado por el Usuario se traduce internamente en un Modelo de Agentes. En la Segunda Fase, el Modelo de Agentes permite la simulación de comportamientos complejos asociados a cada uno de los elementos de la Planta de Fabricación real. Asimismo, se observa la necesidad de un Metamodelo que sirva de base para la definición de Modelos de Plantas de Fabricación Flexibles e Inteligentes que permita la integración de un amplio rango de variantes. En el siguiente capítulo, se presenta el detalle de dicho Metamodelo. Mientras que, en el Capítulo 7 se presenta en detalle la segunda fase de la Simulación, donde se hace uso del Modelo generado en la primera Fase. 



\section{Capítulo 6}

\section{Modelo Teórico del Metamodelo para la Simulación de una Planta de Fabricación}

Siguiendo el Diagrama de Estudio de Simulación la definición del Metamodelo para un Sistema de Fabricación Inteligente, dará soporte a las actividades de Representación y Programación de Modelo así como el Diseño de Experimentos (Figura 6.1). El metamodelo ofrecerá la flexibilidad para la definición de modelos con un amplio rango de variantes que den soporte al diseño de experimentos.

Basado en los pasos para llevar a cabo la simulación de un sistema de fabricación, en esta sección se presenta el Modelo Teórico que describe el metamodelo que a su vez, se aplicará para la creación de modelos que permitan representar y simular plantas de fabricación con un amplio rango de variantes.

La importancia del uso de metamodelos para la definición de un Sistema de Fabricación Flexible planteada en la Sección 4.6, trae consigo la necesidad de definir el metamodelo adecuado que proporcione la flexibilidad necesaria tanto para definir la Planta de Fabricación como para su simulación soportada por agentes. La definición del Modelo para la simulación de una Planta de Fabricación constituye un elemento clave en la definición del Entorno de Simulación y la gestión de dicho Modelo a fin de simular adecuadamente el comportamiento de los elementos que lo integran. 


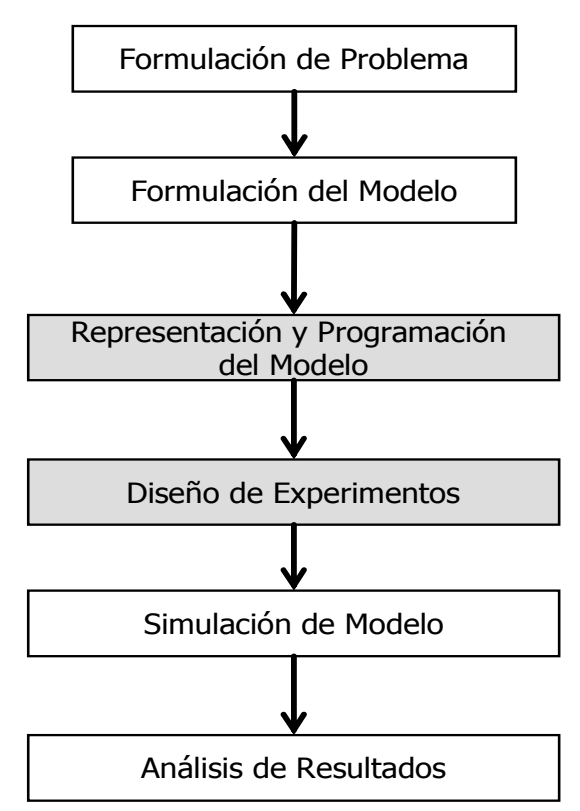

Figura 6.1: Diagrama de Estudio de una Simulación.

Como se indicó en la Sección 3.5.2 la definición del entorno en la simulación multiagente comprende dos elementos: el entorno simulado y la simulación del entorno. Por tanto, en este capítulo presentamos la descripción del sistema multiagente que da soporte a la definición del entorno simulado.

De acuerdo con la Estructura del Entorno de Simulación propuesta y su respectivo proceso presentado en las secciones anteriores, en este capítulo se presenta el metamodelo que permite representar una Planta de fabricación flexible que además integra los elementos necesarios para su simulación. El Modelo obtenido dispara la Segunda Fase (Simulación de Modelos, Capítulo 7) donde es posible observar el comportamiento de los elementos que lo integran durante la simulación de sus funciones.

En la Primera Fase, el Usuario del Sistema hace uso del Modelador para crear un Modelo que representa una Planta de Fabricación Real. En dicho Modelo se incluyen los elementos identificados mediante iconos que son proporcionados por el Gestor de Iconos. El proceso de creación del modelo es supervisado por el Agente de Sincronización hasta el momento en que es verificado y liberado para su utilización en la Segunda Fase. 
En la Segunda Fase se recibe como entrada el modelo generado en al Primera Fase. Bajo la supervisión de un Agente de Sincronización, como primer paso se establecen los parámetros que configuran la simulación de dicho modelo (velocidad, tipo de animación). A continuación se pone en marcha la simulación del modelo donde se llevan a cabo procesos de negociación entre los elementos que integran el modelo a fin de optimizar el uso de los recursos existentes. Una vez finalizada la simulación, los resultados obtenidos son analizados y presentados mediante reportes y gráficas ante el Usuario que diseñó el modelo. Dichos datos son reutilizados para ser añadidos como parte del comportamiento de los elementos que participaron en la simulación. Asimismo, pueden ser exportados para su análisis externo.

Como ya se mencionó en la Sección 4.6 debido a la importancia del uso de los Metamodelos en la definición de un Sistema de Fabricación, en esta propuesta se plantea la definición de un Metamodelo que brinde flexibilidad al diseñador para la creación de un modelo que permita representar apropiadamente el comportamiento de un Sistema de Fabricación específico y simular tal comportamiento.

Antes de establecer la propuesta del Modelo de Simulación es importante delimitar la definición de cada uno de los elementos que lo integran. Para ello, tomaremos en consideración los estándares planteados por McLean y Leong (MCLean \& Leong 2002b) y Stadtler y Kilger (Stadtler \& Kilger 2002) presentados en la Sección 4.6.

\subsection{Identificación de Elementos para el Metamo- delo}

Como se menciona en los conceptos básicos referentes a la Fabricación (Capítulo 4), el término Producción se refiere a la creación de bienes y servicios los cuales se apoyan en la Gestión de Operaciones. De esta forma nuestro objetivo es la definición de un metamodelo que contemple todos los elementos necesarios para la representación de un Sistema de Fabricación que permita simular su comportamiento lo más cercano a la realidad. 
Por un lado, el metamodelo integra las características asociadas fundamentalmente a un Sistema de Fabricación, tales como la definición de elementos básicos, estructura organizacional, rutas, productos, restricciones, sistema de gestión, uso de recursos. Por otro lado, amplía los atributos de cada elemento mediante la integración de las características asociadas a un Sistema Multiagente tales como proactividad, reactividad, distribución de control, flexibilidad y adaptación, técnicas de negociación y protocolos de comunicación, definición de comportamiento y servicios. Todo ello está dirigido a la definición de una estructura organizacional virtual, la cual, además de cubrir las necesidades cambiantes asociadas a los requisitos de la nueva era de la fabricación, amplia el alcance de su comportamiento mediante la aplicación de técnicas de inteligencia artificial (Figura 6.2).

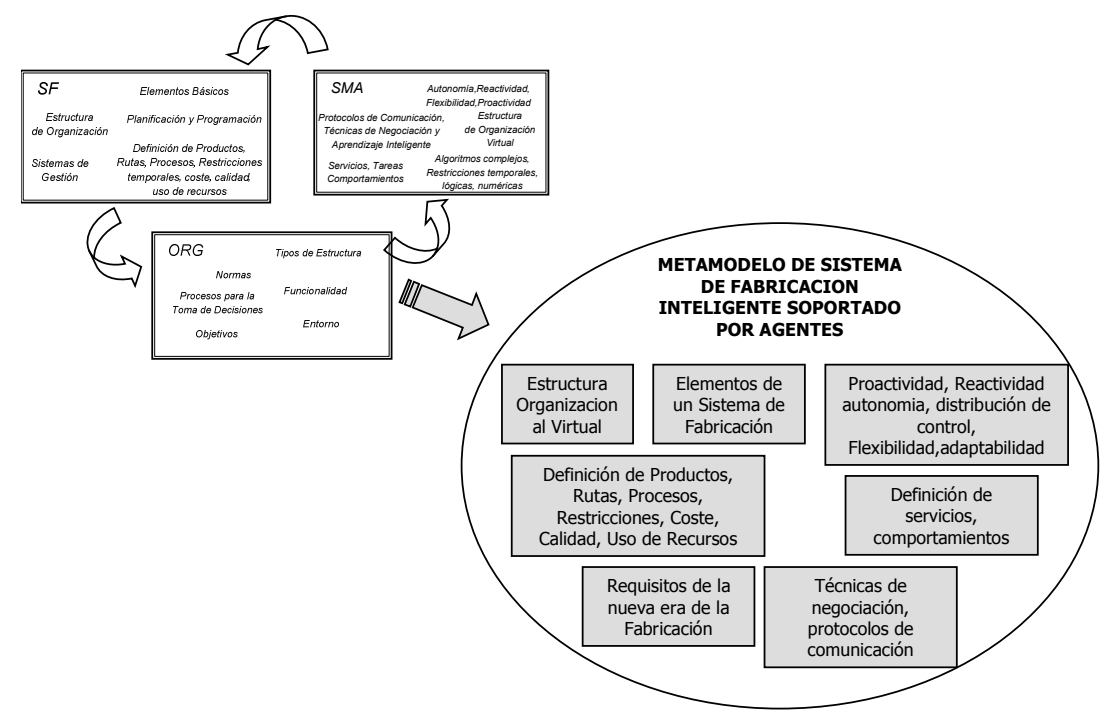

Figura 6.2: Áreas consideradas en la Definición del Metamodelo.

Para la identificación de los elementos que deben ser considerados como parte del metamodelo se han considerado estudios referentes a investigaciones relacionadas con los elementos básicos que integran un Sistema de Fabricación presentados en la Sección 4.1, así como la metajerarquía del modelo de fabricación de McLean y Leong (MCLean \& Leong 2002b) presentada en la Sección 4.2.

En la propuesta, se lleva a cabo la simulación de los tres últimos niveles de la metajerarquía: descripción de equipos, dispositivos y procesos. Dichos niveles 
permiten la definición posterior de los dos niveles superiores: estación y línea, área o célula.

En esta sección se presentan dichos niveles, la identificación de los elementos que integrarán el metamodelo a su vez está delimitada por la principal función a la cual se enfoca la simulación que en este caso es la Producción, definiendo con ello el siguiente nivel superior que es el de Departamento.

De esta forma, en el metamodelo se describirán: a) equipos tales como herramientas, robot, grúas, bandas transportadoras, máquinas, etc.; b) dispositivos tales como sensores y actuadores de los equipos, comportamiento de una máquina, y su efector; c) procesos tales como fundir a troquel, o el ensamble mecánico, en este nivel se representan procesos de física, cinemática, química, etc. Finalmente tales elementos permitirán la definición posterior de 1) estaciones que pueden incluir una o más piezas de equipo, operadores, etc.; y 2) líneas, áreas o células que agrupan estaciones y/o equipo.

De acuerdo con la literatura especializada referente a los Sistemas de Fabricación y con el fin de clarificar la utilización de conceptos asociados a los elementos que integran el metamodelo propuesto, a continuación se listan las descripciones de los elementos que utilizaremos en nuestra propuesta:

Producto: La norma ISO 9001 : 2000 define "Producto" como "resultado de un proceso", por lo que lógicamente sería aplicable, tanto a organizaciones que se identifiquen con empresas industriales, como a las que presten solamente servicios, tanto si persiguen afán de lucro como si se trata de entidades no lucrativas. En la clasificación de productos se observan:

- Productos Terminados: Los fabricados por la empresa y destinados al consumo final o a su utilización por otras empresas.

- Producto Semiterminados o Semiproducto: Se denomina semiproducto a aquellos componentes de un Producto.

- Subproducto: Es un producto secundario o incidental, generalmente útil y comercializable, derivado de un proceso de fabricación o reacción química, que no es el producto primario. A su vez se subclasifican como: Residuos y Materiales Recuperados. Los Residuos son obtenidos inevitablemente y al mismo tiempo que los productos o 
subproductos siempre que tengan valor intrínseco y puedan ser utilizados o vendidos. Los Materiales Recuperados tienen valor intrínseco, entran nuevamente en almacén después de haber sido utilizados en el proceso productivo.

Los modelos de productos se enfocan en la información que describa las características de un Producto (Magnusson \& Holm 1996). Algunos modelos describen al producto con respecto a su estructura, procesos de producción y eventualmente los recursos que realizan procesos para la producción del producto. Algunos investigadores incluso definen un Modelo de Estado de Producto (PSM) (Larsen, Bilberg \& Kirkby 1997, Larsen, Kirkby \& Verterager 1999) el cual contiene datos de la salida y los resultados de los procesos de producción. Para ello realizan la monitorización de atributos de producto predefinidos tanto de un producto y/o sus subpartes durante la producción. Otros definen la creación de un Metamodelo basándose en la morfología de los procesos de producción que se consideran como el principal estado de los eventos disparados (Larsen et al. 2001).

Un aspecto prioritario en la definición de un modelo es la posibilidad de la identificación y trazabilidad de los productos. Basándose en las normas del estándar ISO 9001-200, Fernández (Férnandez 2002) indica que en el caso de que los productos sean susceptibles de identificación y trazabilidad, la organización establecerá sistemas que desarrollen estos dos conceptos a lo largo de todos los procesos de realización. Se señala la obligación de establecer sistemas para garantizar la identificación del producto durante todas las fases de fabricación, hasta el momento de su utilización por el cliente y de las materias primas que entran en su composición. Esta identificación quedará registrada adecuadamente y debe permitir la trazabilidad del producto y de sus componentes desde el momento de su recepción en la empresa.

Materia Prima: Se conoce como materias primas a los materiales extraídos de la naturaleza que nos sirven para construir los bienes de consumo. Antes de construir o fabricar definitivamente un bien de consumo, las materias primas se transforman en un primer paso en productos semielaborados o semiterminados. El estado físico de la Materia Prima puede ser: Sólido, Granular, Líquido o Gaseoso.

Lista de Materia Prima: En la lista de materiales se enumeran los componentes, su descripción y la cantidad de cada uno que se requiere para hacer 
una unidad de producto. El plano de ingeniería nos muestra cómo hacer un producto de una lista de materiales.

Ruta: La ruta de un Producto enumera las tareas u operaciones (incluyendo el montaje y la inspección) necesarias para fabricar el componente a partir de los materiales especificados en la lista de materiales. La ruta tendrá una entrada para cada tarea que se lleve a cabo con el artículo. La ruta se puede plasmar en una hoja de ruta. Cuando una hoja de ruta incluye métodos específicos de operación y los estándares de tiempos, se la conoce como hoja de procesos. Las identificaciones documentales adoptan muchas veces la forma de una "Hoja de ruta" que es un impreso que lleva anotados los datos generales del producto (denominación, código, número de pedido, etc.) y los procesos por los que tiene que pasar, así como espacios en blanco en los que puedan anotarse los distintos pasos por los procesos de producción y control. De esta forma, en cualquier momento, la hoja de ruta indicará los procesos que ha sufrido la pieza inicial, los que le quedan por pasar y los valores o características de los parámetros de calidad o funcionamiento, obtenidos hasta aquí.

Tarea: El término Tarea se refiere a un trabajo o proceso que debe hacerse en tiempo limitado.

Transición: Una transición es la acción y efecto de pasar de un modo de ser o estar, a otro muy distinto del anterior.

Proceso de Fabricación: Un proceso de fabricación, también denominado fabricación o producción, es el conjunto de operaciones necesarias para modificar las características de las materias primas. Dichas características pueden ser de naturaleza muy variada tales como la forma, la densidad, la resistencia, el tamaño o la estética. En el ámbito industrial se suelen considerar convencionalmente los procesos elementales que se indican, agrupados en dos grandes familias: Mecánicos y Químicos. A su vez los procesos mecánicos se pueden subdividir como sigue:

Moldeo: Este tipo agrupa procesos tales como: fundición, pulvimetalurgia y moldeo por inyección.

Conformado o Deformación Plástica: Este tipo agrupa procesos tales como: laminación, forja, extrusión, esturado, conformado de chapa, y encogimiento. 
Procesos de arranque de material: Este tipo de procesos se subdivide en: Mecanizado y Electroerosión. El proceso de Mecanizado agrupa a los procesos de torneado, fresadora, y taladro.

Procesos con aporte de material: Este tipo se refiere al proceso de soldadura.

Tratamiento Térmico: Este tipo agrupa procesos tales como: templado, revenido, recocido, normalizado, cementación y nitruración.

Tratamientos Superficiales: Este tipo de proceso se refiere al Acabado el cual puede dividirse en procesos: eléctricos y abrasivos. El proceso eléctrico se refiere al electropulido y el proceso abrasivo al pulido.

Operario: Es aquella persona que manipula máquinas y/o herramientas durante procesos de fabricación de productos.

Máquina: La máquina herramienta es un tipo de máquina que se utiliza para dar forma a materiales sólidos, principalmente metales. Su característica principal es su falta de movilidad, ya que suelen ser máquinas estacionarias. Los principales tipos de máquinas son:

- Convencionales: Las típicas son el Torno, Taladro, Fresadora, y Pulidora. Dentro de las convencionales se encuentran las de vaivén: Perfiladora, Cepilladora y Sierra.

- Prensas: No realizan arranque de viruta, dan forma al material mediante el corte o cizalla, el golpe para el doblado y la presión. Suelen utilizar troqueles y matrices como útiles. Los procesos son muy rápidos y son máquinas de altos riesgo de accidente laboral. Entre las Prensas no convencionales se encuentran: Electroerosión, Arco de Plasma, Láser, Ultrasónica.

Herramienta: Una herramienta es un dispositivo que provee una ventaja mecánica al realizar una determinada tarea. La mayoría de las herramientas emplean una máquina simple, o una combinación de ellas. Las herramientas pueden ser manuales o mecánicas. Las manuales se usan con la fuerza del hombre mientras que las mecánicas se usan con una fuente de energía externa. Las herramientas se pueden agrupar en cuatro grupos:

- Herramientas de Corte: Sierra de mano, lima, broca, macho de roscar, escariador, terraja de roscar, tijera, cortafrío, buril, cincel, cizalla, tenaza 
- Herramientas para sujetar o atornillar: Llave, alicate, destornillador, tornillo de banco, remachadora, sargento.

- Herramientas de medición: Regla graduada, flexómetro, goniómetro, calibre pie de rey, micrómetro.

- Herramientas con diversas funciones: Martillo, granete, extractor mecánico, números y letras para grabar, punzón cilíndrico, polipasto, gramil, punta de trazar, compás, gato hidraúlico, mesa elevadora hidraúlica.

Mantenimiento: El mantenimiento se refiere a todas las acciones que tienen como objetivo mantener un artículo o restaurarlo a un estado en el cual pueda llevar a cabo alguna función requerida. Estas acciones incluyen la combinación de acciones técnicas y administrativas correspondientes (EFNMS 2001). Una clasificación de los tipos de mantenimiento es:

- Mantenimiento correctivo: se efectúa cuando las fallas han ocurrido; su proximidad es evidente.

- Mantenimiento preventivo: se efectúa para prever las fallas en base a parámetros de diseño y condiciones de trabajo supuestas.

- Mantenimiento predictivo: prevé las fallas en base a observaciones que indican tendencias.

Orden de Trabajo: La orden de trabajo es una instrucción para realizar una cantidad dada de un artículo determinado, normalmente dentro de una programación determinada sujeta a fechas de entrega. Los tipos de Orden de Trabajo pueden ser: Orden de Producción y Orden de Mantenimiento. La Orden de Producción se utiliza para generar Productos, mientras que la Orden de Mantenimiento se utiliza para gestionar servicios de reparación y/o mantenimiento de máquinas y/o herramientas.

Por tanto, tomando en consideración que entre los objetivos de una Planta de Fabricación está la generación de Productos, el metamodelo propuesto contempla los atributos esenciales para la definición de un Producto con respecto a su estructura y su estado.

En el metamodelo propuesto se incluye la identificación de los productos durante su estancia dentro de la Planta de Fabricación. Para ello se utiliza el semiproducto que cuenta con un identificador que permite dar seguimiento a los 
componentes que se transforman durante el proceso de fabricación hasta el momento en que se obtiene el Producto Terminado. Por tanto, también se incluye un control del producto basado en su Ruta de Fabricación.

Además de los Productos, entre los elementos que se describen en el metamodelo se encuentran: los operarios, la materia prima, la lista de materiales, la ruta, las máquinas y herramientas, y los procesos. Consecuentemente también se consideran aspectos relativos a las Órdenes de Fabricación y de Mantenimiento.

Por otra parte, no sólo se considera cuales son los elementos que deben integrar el modelo sino la estructura del Metamodelo. Para ello, se ha aplicado el Enfoque Holónico para Fabricación descrito en la Sección 4.4. Entre las razones de la elección de este paradigma es que la definición de un holón permite incluir tanto características físicas como características relacionadas con procesos de negociación y coordinación de comportamientos que se presentan durante la gestión de la producción. Por otra parte, se encuentra la facilidad para integrar los conceptos referentes a la "fabricación ágil" donde los componentes de sistemas de producción son reprogramados y reconfigurados constantemente permitiendo un volumen denso de información.

Finalmente se optó por esta filosofía debido a que como se ha mencionado, la integración tanto de los "Sistemas Holónicos de Fabricación (HMS)" y el paradigma de los "Sistemas de Fabricación Orientados a Agentes" actualmente se presentan como la base de un Sistema de Fabricación Inteligente. Además, un HMS puede considerarse, desde el punto de vista de arquitectura de software, como un tipo particular de comunidad de agentes con estructura y comportamiento muy relacionado a las tareas de fabricación (Rannanjarvi \& Heikkila 1998)".

La definición de una arquitectura HMS requiere la aplicación de una metodología apropiada en la que se respete los principios básicos tales como la autonomía, la cooperación, así como proveer una holarquía que permita contar con un sistema de holones que pueden cooperar para el logro de objetivos.

Por tanto, como se indicó en la Sección 4.4 para el diseño del metamodelo se utiliza la Arquitectura de referencia PROSA (Brussel et al. 1998). Dicha arquitectura ha sido seleccionada ya que aparece como un estándar aplicable en los Sistemas de Fabricación Holónicos. Asimismo, tal Arquitectura permite proporcionar al Usuario la flexibilidad necesaria durante el diseño del Modelo para la representación de un Sistema de Fabricación. 
En la siguiente sección podremos observar la identificación de las especializaciones de cada uno de los Holones basadas en las características y funciones que desempeñan dentro de una Planta de Fabricación.

\subsection{Definición de Taxonomía de los Holones del Me- tamodelo}

En este apartado se definen los distintos holones que integran el Metamodelo propuesto describiendo sus propiedades y sus respectivas relaciones. Debido a que las clases abstractas representan aquellas clases que no se pueden instanciar a objetos, sino sólo se pueden instanciar sus subclases, tanto la clase del holón de Recurso como el de Orden se definen como clases abstractas de las cuales se descomponen especializaciones de tipos de recursos y órdenes específicos los cuales tienen funciones y relaciones enfocadas en el logro de objetivos particulares y comunes. La descripción de cada una de las clases se explica en detalle mediante metamodelos que se presentan más adelante. En esta sección sólo se describen las metaclases que integran el modelo, en la siguiente sección se detallan las relaciones identificadas entre ellas.

La especialización separa los holones con respecto a sus características. La especialización se emplea para diferenciar entre los diferentes tipos de holones de recurso, de orden, y de producto (Brussel et al. 1998). De acuerdo con las características identificadas que deben ser asociadas a los Holones de Producto, Recurso y Orden así como los conceptos básicos de un Sistema de Fabricación mencionados en secciones anteriores, es posible identificar las especializaciones asociadas a cada uno de ellos.

Basada en la Clasificación de Productos presentada anteriormente, la Clase de Holón de Producto representa la simulación del proceso de fabricación a un Producto Terminado. Por el momento no es de nuestro interés controlar los Subproductos obtenidos durante dicho proceso. Sin embargo, para efectos de la simulación, se utiliza el concepto de Semiproducto. El Semiproducto permite controlar la trazabilidad del Producto Terminado mediante el control de su estado. El estado del Semiproducto cambia conforme evoluciona durante el proceso de fabricación hasta convertirse en el Producto Terminado. 
Teniendo en cuenta la clasificación de los grupos de Recursos que integran los objetos físicos de una planta de Fabricación descritos anteriormente, la Clase de Holón de ARecurso se compone de los recursos que realizan las operaciones de fabricación (Transformadores), y los que reciben los tratamientos de fabricación (Materia Prima). El holón de Orden representa una tarea en el sistema de fabricación, la Clase del Holón Orden incluye las denominadas Órdenes de Producción. Sin embargo, resulta importante incluir a las Órdenes que a su vez tienen una relación directa con los recursos de la Planta de fabricación, las Órdenes de Mantenimiento.

Para el caso de los Holones de Staff, estos no cuentan con especializaciones, sino que debido a que su función es la supervisión y soporte a los holones básicos, se podrán identificar holones de Staff con diferentes niveles de autoridad sobre dichos holones. Entre las principales relaciones que se identifican entre los holones básicos del metamodelo, se pueden observar en la Fig. 6.3 cómo el Holón Producto utiliza al Holón Recurso para transformar a la Materia Prima en Producto Terminado; así mismo el control de dicha transformación lo tiene el Holón de Orden el cual manipula a los Holones Producto. Finalmente el Holón de Staff cumple con la función de soporte mediante la supervisión tanto de los holones de Producto como de Recurso con el fin de dar una visión global en la resolución de problemas.

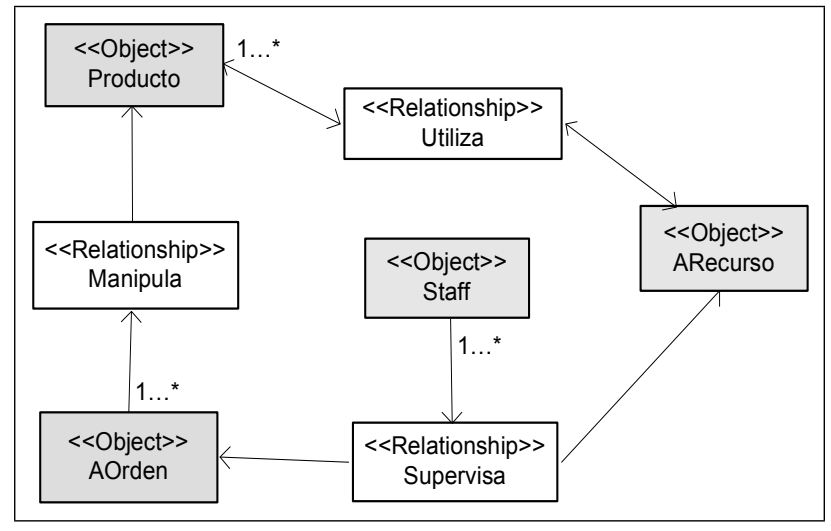

Figura 6.3: Principales Relaciones entre los Holones

Tomando en consideración las características de cada uno de los holones descritos en las secciones anteriores y como resultado de la aplicación de la Arquitectura de Referencia PROSA (Brussel et al. 1998), en la Figura 6.4 se presenta la identificación de las entidades básicas que componen el Metamodelo. El color 
gris claro representa la clases principales derivadas de la arquitectura PROSA las cuales han sido especializadas en subclases y redefinidas para ajustarlas al Entorno de Simulación soportado por agentes propuesto.

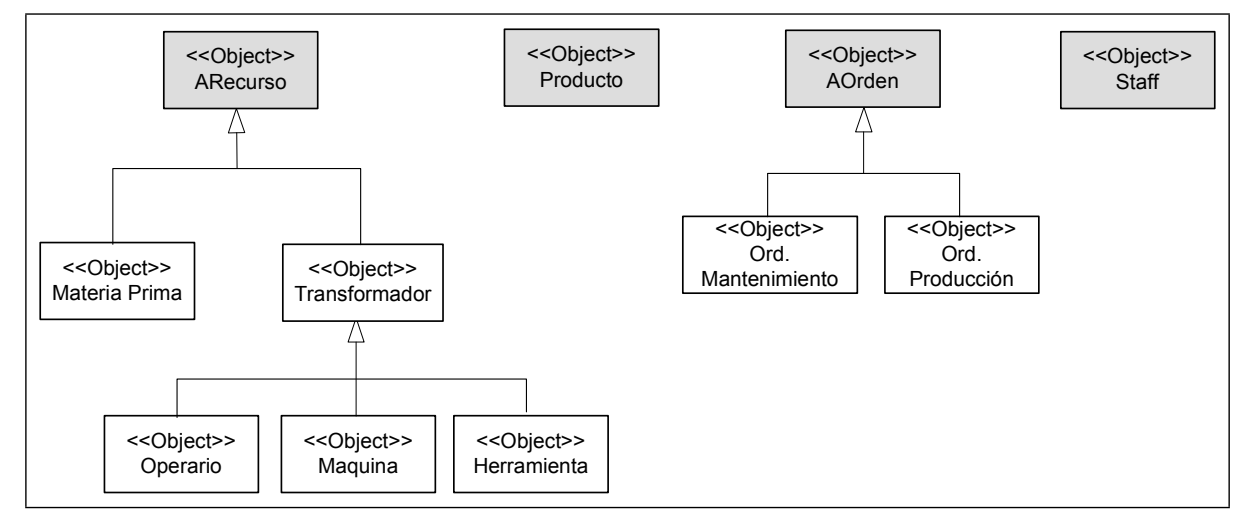

Figura 6.4: Entidades Básicas del Modelo

En las siguientes secciones se describe cada una de las clases con sus respectivas especializaciones, donde es posible observar la integración de las características ya mencionadas en cada uno de los holones que integran el Metamodelo. En cada clase se incluyen tanto propiedades y objetos asociados a sus funciones dentro de la planta de fabricación de cada tipo de holón así como las asociadas a la simulación de su comportamiento, relacionadas a la propuesta de Entorno de Simulación.

\section{Holón Producto.}

La definición del Holón Producto considera las perspectivas del Meta Estado del Producto (MEP) en base a:

- Alcance. El alcance del metamodelo está limitado a la fase de producción.

- Artefacto. Las características asociadas a las perspectivas referentes al Estado del material, la entidad del producto y el operando se incluyen como parte de las propiedades y objetos de las especializaciones de este holón.

- Evento. Cada una de las instancias en las que el artefacto se puede medir en base a la ruta que sigue el semiproducto hasta evolucionar en un Producto Terminado. 


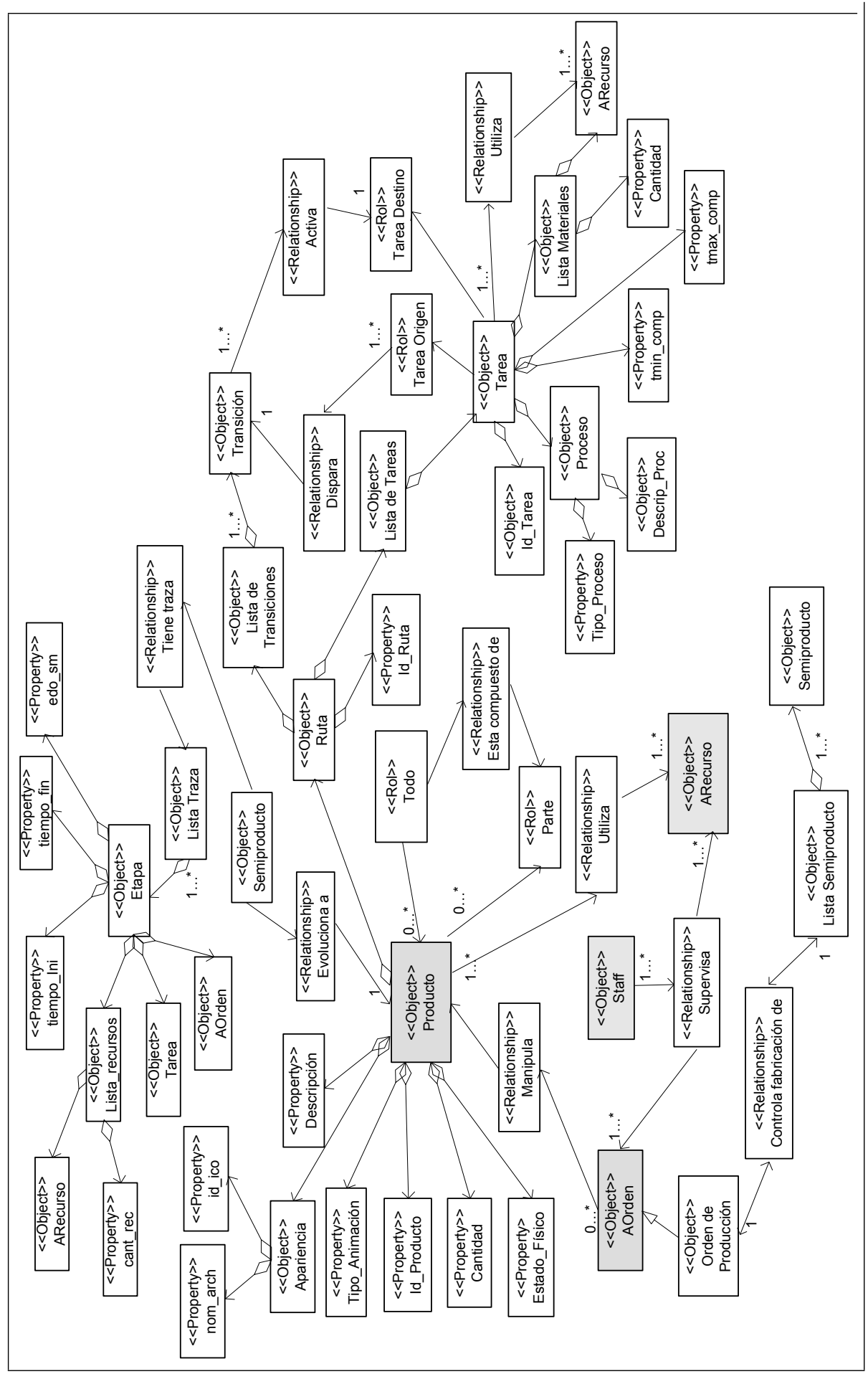

Figura 6.5: Metamodelo del Holón Producto 
Asimismo, las características asociadas a un Holón de Producto también consideran los conceptos básicos de un Sistema de Fabricación mencionados en secciones anteriores. Para efectos de la simulación del modelo el Semiproducto permite la representación simulada de una instancia física del Producto y así controlar su evolución durante el proceso de fabricación. El Holón de Producto tiene asociados tanto propiedades como objetos que permiten describir sus funciones. Tanto las propiedades como los objetos que la componen han sido definidas en base a las necesidades de sus relaciones internas y con el resto de las holones. Las relaciones identificadas se describen como sigue (Fig. 6.5):

- Durante el ciclo de fabricación los objetos de tipo Semiproducto serán los que se podrán observar como elementos tangibles ya que se utilizan para el registro de la trazabilidad del Producto durante la simulación del modelo como resultado de la ejecución de los procesos de transformación. El Producto no será posible observarlo como un elemento tangible puesto que se obtiene como resultado de la última fase del modelo que se especifique. Por lo tanto, un Semiproducto evoluciona hasta convertirse en un objeto de tipo Producto.

- A su vez un Producto puede estar compuesto de otros objetos de tipo Producto. Esta relación permite el control de Productos complejos los cuales tienen una estructura de producto asociada.

- La generación de un Producto se ve restringida a la definición de una serie de pasos que definen su fabricación, por lo que se fabrica siguiendo una Ruta.

- Una Ruta a su vez se basa en reglas que definen la secuencia de ejecución de los pasos de fabricación de un Producto por lo que una Ruta ejecuta tareas en base a una Lista de Tareas y una Lista de Transiciones.

- Una Lista de Tareas se compone de un conjunto de N Tareas. Una Tarea puede desempeñar dos tipos de roles: a) tareas que generan salidas que son utilizadas por otras tareas (Tarea Origen) y b) tareas que reciben las salidas de las tareas origen como parte de sus entradas (Tarea Destino).

- Una Lista de Transiciones se compone de un conjunto de N transiciones. La secuencia de ejecución de las tareas se basa en la aparición de una transición la cual permite vincular tareas. Una Tarea que desempeña el rol de Tarea Origen dispara una Transición la cual a su vez activa a $\mathrm{N}$ tareas que desempeñan el rol de Tarea Destino. 
- La Lista de Transiciones genera la secuencia de Tareas necesaria para la producción de un Producto la cual es registrada como parte del objeto de tipo Semiproducto.

- Un objeto de tipo Orden manipula a un objeto de tipo Producto para controlar su fabricación, por consecuencia una Orden de Producción controla la fabricación de una Lista de Semiproductos que se compone de objetos de tipo Semiproducto. En el sentido inverso, mediante dicha relación es posible consultar qué ordenes han gestionado la producción de un Producto dado, al observar el registro de la trazabilidad del producto mediante el objeto Semiproducto.

Propiedades y Objetos.

El objeto Holón de Producto se compone de cuatro propiedades:

1. Identificador del Holón Producto (id_prod). Esta propiedad permite identificar a una instancia de Producto.

2. Descripción (desc prod). Esta propiedad permite asignar un nombre a un Producto que indique su principal característica.

3. Estado Físico (edo_fisico). Esta propiedad permite conocer el estado físico que el Producto tendrá al finalizar su fabricación. El estado físico puede ser líquido, gaseoso, granular o sólido.

4. Tipo Animación (tipo_anim). Esta propiedad es utilizada sólo para efectos de la animación de los objetos de tipo Producto durante el proceso de simulación. Permitirá indicar mediante un parpadeo o un movimiento izquierda-derecha o arriba/abajo que se está realizando alguna función durante la simulación.

Las propiedades id_prod, desc_prod y edo_fisico permiten mostrar la información referente a su identificación y el estado original del producto, las cuales están asociadas a la perspectiva "Artefacto". Por lo tanto forman parte de la información necesaria para mantener la trazabilidad del producto durante el proceso de fabricación. Asimismo, el objeto Producto se compone de dos objetos: Apariencia y Ruta.

Objeto Apariencia: Esta propiedad es utilizada sólo para efectos de la animación de los objetos de tipo Producto durante el proceso de simulación. Permite asociar una imagen a un Producto. Este objeto es monitorizado 
continuamente por el Agente de Simulación quien actualiza la visualización de un Producto acorde a su estado actual.

El objeto Apariencia se compone de dos propiedades:

1. Identificador de la Imagen (id_ico). Esta propiedad permite identificar a una instancia de una imagen de un Icono asociado a un Producto.

2. Archivo de la Imagen (arch_ico). Esta propiedad permite indicar el nombre del archivo físico que contiene a la imagen que se le asocia al icono que representara al Producto.

Objeto Ruta: Contiene la descripción de cada uno de los pasos que se han de seguir a efectos de transformar los materiales de tal manera que mediante objetos de tipo Semiproducto se registre su evolución hasta la obtención del Producto. El objeto Ruta a su vez está compuesto de la propiedad:

Identificador de la Ruta (id_ruta). Esta propiedad permite identificar a una instancia de una Ruta de un Producto y así rastrearlo durante el proceso de fabricación.

Asimismo, el objeto Ruta se compone de tres objetos: Lista de Tareas, Lista de Transiciones, Configuración de Planta.

Objeto Lista de Tareas: Agrupa e indica la secuencia de las tareas necesarias para la obtención de un Producto. Por tal razón se compone de un conjunto de objetos del tipo Tarea.

Objeto Tarea: Permite indicar el detalle del proceso de transformación así como los recursos necesarios para llevarla a cabo. Esta compuesto de tres propiedades:

1. Identificador de la Tarea (id_tarea). Esta propiedad permite identificar a una instancia de una Tarea asociada a la Lista de Tareas durante el proceso de fabricación.

2. Tiempo mínimo de compleción de Tarea (tmin_com). Esta propiedad representa el Tiempo mínimo requerido para la ejecución de la Tarea.

3. Tiempo máximo de compleción de Tarea (tmax_com). Esta propiedad representa el Tiempo máximo requerido para la ejecución de la Tarea. 
Asimismo el objeto Tarea se compone de dos objetos: Proceso y Lista de Materiales.

Objeto Proceso: Permite detallar el proceso que se realiza durante la Tarea. Este objeto se compone de dos propiedades:

1. Identificador del Tipo de Proceso (tipo proc). Los Tipos de Proceso que se pueden ejecutar son de Transformación física, Cambio de Ubicación, Químico, Térmico, Mecánico, Térmico sin cambio de forma, Unión de formas, Conservación de masa con moldeo, Reducción de masa por moldeo, y Acabado superficial sin cambio de forma.

2. Descripción General del Proceso (descg proc). La Descripción General del proceso especifica el Tipo de Proceso, es decir, si es de tipo mecánico, la descripción puede ser por ejemplo "Cortar".

3. Descripción Detallada del Proceso (desc proc). La Descripción Detallada del proceso describe el nivel más bajo el Tipo de Proceso, es decir, si es de tipo mecánico, y la descripción general es "cortar", su descripción detallada puede ser "cortar 2 piezas en diagonal".

Objeto Lista de Materiales: Permite agrupar los materiales (objetos de tipo Recurso) necesarios para llevar a cabo la Tarea. Este objeto se compone de una propiedad y un objeto:

Cantidad (can_rec). Esta propiedad permite indicar la cantidad de material requerido para ejecutar la Tarea.

Objeto ARecurso: Los materiales utilizados para ejecutar una Tarea son objetos de tipo ARecurso que tienen a su vez asociadas sus respectivas propiedades. Los materiales incluyen tanto a materias primas como productos.

Objeto Lista de Transiciones: Agrupa e indica las transiciones necesarias para la obtención de un Producto. Se compone de objetos de tipo Transición.

Objeto Transición: Este elemento permite establecer la relación entre las Tareas que se ejecutan para la fabricación del objeto Producto mediante transiciones que activan o disparan Tareas, para lo cual vincula Tareas mediante tuplas (parejas). Las conexiones 


\begin{tabular}{|c|l|}
\hline T1 BEFORE T2 & Tarea T1 inicia antes que T2 \\
T1 MEETS T2 & Tarea T1 termina y T2 inicia \\
T1 OVERLAPS T2 & Tarea T2 se ejecuta antes de que termine la T1 \\
T1 DURING T2 & Tarea T1 se ejecuta mientras se ejecuta la T2 \\
T1 STARTS T2 & Tarea T1 y T2 inician al mismo tiempo \\
T1 FINISHES T2 & Tarea T1 y T2 terminan al mismo tiempo \\
T1 EQUAL T2 & Tarea T1 y T2 duran el mismo tiempo \\
\hline
\end{tabular}

Tabla 6.1: Relaciones de Tiempo entre tareas.

a su vez generan la secuencia que es registrada en el objeto de tipo Semiproducto.

Basándonos en los principios de la definición de un PPM (Modelo de Proceso de Producción - presentado por Stadtler y Kilger en la Sección 4.6-) las transiciones permiten la definición de la secuencia técnica de actividades. Y por tanto, permiten el modelado preciso de restricciones de actividades incluyendo la ejecución paralela de actividades.

Las transiciones permiten definir la secuencia de las tareas que a su vez se utilizan durante la asignación de tareas a recursos y la ejecución de las mismas. Asimismo cada una de las tareas tienen asociado un periodo de tiempo de ejecución de las mismas. Por tanto, se considera la definición de las relaciones que se pueden presentar entre intervalos de tiempo presentada por Allen en (Allen 1983). Por ejemplo, si tenemos las tareas T1 y $\mathrm{T} 2$, las relaciones de tiempo entre dichas tareas que es posible expresar mediante la definición de duración de las tareas y el uso de las transiciones se describen en la Tabla 6.1.

Las transiciones permiten identificar si una tarea puede iniciar o no dependiendo si su tarea o tareas origen han finalizado. Si embargo si la tarea o tareas origen han finalizado la tarea o tareas destino iniciarán acorde al tiempo definido para cada una de ellas en el momento en que el recurso asignado (operario,máquina,herramienta) lo tenga contemplado en su cola de trabajos.

Por tal razón se compone de un conjunto de objetos del tipo Tarea que son asociados mediante el uso de dos propiedades:

1. Tarea Origen (t_orig). Esta propiedad permite indicar la tarea origen que activa o dispara tareas Destino. Por lo tanto 
tiene asociado un objeto de tipo Tarea.

2. Tarea Destino (t_dest). Esta propiedad permite indicar la tarea destino que es activada o disparada por una Tareas Origen. Por lo tanto tiene asociado un objeto de tipo Tarea.

Debido a que el objeto Semiproducto es aquel que para efectos de simulación permite registrar su evolución dentro de la ruta real que sigue el proceso de producción de un Producto, dicha secuencia (traza) y su detalle se almacena mediante un objeto de tipo Lista Traza.

Objeto Lista Traza: Agrupa e indica el detalle de la secuencia de las Tareas llevadas a cabo para la obtención de un Producto haciendo referencia a las instancias de los elementos que participaron en cada una de ellas. Por tal razón a su vez se compone de objetos de tipo Etapa:

Objeto Etapa: Este objeto permite almacenar el detalle de cada uno de los pasos que integran la traza de los procesos de producción que se llevaron a cabo para la obtención de un Producto. Este objeto se compone de tres propiedades:

1. Estado del Semiproducto (edo_sm). Esta propiedad indica el estado del Semiproducto identificando la etapa en la que se encuentra en dicha Tarea. Sus valores pueden ser: en espera, en proceso, o terminado.

2. Tiempo de Inicio (tiempo_ini). Esta propiedad indica el instante real en que se inicia la Tarea.

3. Tiempo de Fin (tiempo_fin). Esta propiedad indica el instante real en que termina la Tarea.

4. Identificador del Recurso (id_recurso). Esta propiedad permite identificar la instancia del Recurso que se utilizo para la Tarea. Este incluye, maquinas, herramientas, y materiales.

5. Identificador del Centro de Trabajo (nom_ct). Esta propiedad permite identificar el centro de trabajo al cual se encuentra asociado el Recurso que se utilizo para la Tarea.

A su vez incluye tres objetos: Tarea, AOrden y Lista de Recursos.

Objeto Tarea: Este objeto permite identificar la instancia de la Tarea que se ejecuta como resultado de una Transición. 
Objeto AOrden: Este objeto permite identificar la instancia de la Orden que controla la fabricación del Producto.

Objeto Lista de Recursos: Agrupa las instancias de los Recursos que participaron en dicha Tarea. Por tal razón se compone de una propiedad y un objeto:

1. Cantidad (cant_rec.). Esta propiedad permite identificar la cantidad del Recurso utilizadas para la Tarea.

Objeto ARecurso: Esta propiedad permite identificar la instancia del Recurso que se utilizó para la Tarea. Por tanto incluye, operarios, máquinas, herramientas, y materiales.

Objeto Configuración Planta: Permite indicar el detalle de los recursos que ejecutarán las tareas. La configuración de planta permite al usuario basado en la Ruta definir los Recursos que desea que ejecuten las tareas. La definición de diferentes configuraciones permite observar el comportamiento de la planta y la capacidad a nivel individual de los recursos que la integran. Tales configuraciones permiten realizar análisis de diferentes escenarios para la fabricación de un Producto.

Está compuesto de tres propiedades:

1. Identificador de la Configuración (id_conf). Esta propiedad permite identificar a una instancia de una Configuración asociada a la Lista de Tareas durante el proceso de fabricación.

2. Identificador de la Tarea (id_tarea). Esta propiedad identifica a una instancia de una Tarea asociada a la Lista de Tareas a la cual se le asociara un recurso.

3. Identificador del Recurso (id_rec). Esta propiedad identifica a una instancia de un Recurso asociada a la instancia de una Tarea.

\section{Holón ARecurso.}

En la Figura 6.6 se muestra el Holón "ARecurso" donde es posible identificar dos especializaciones de los tipos de recursos Transformador y Materia Prima. 


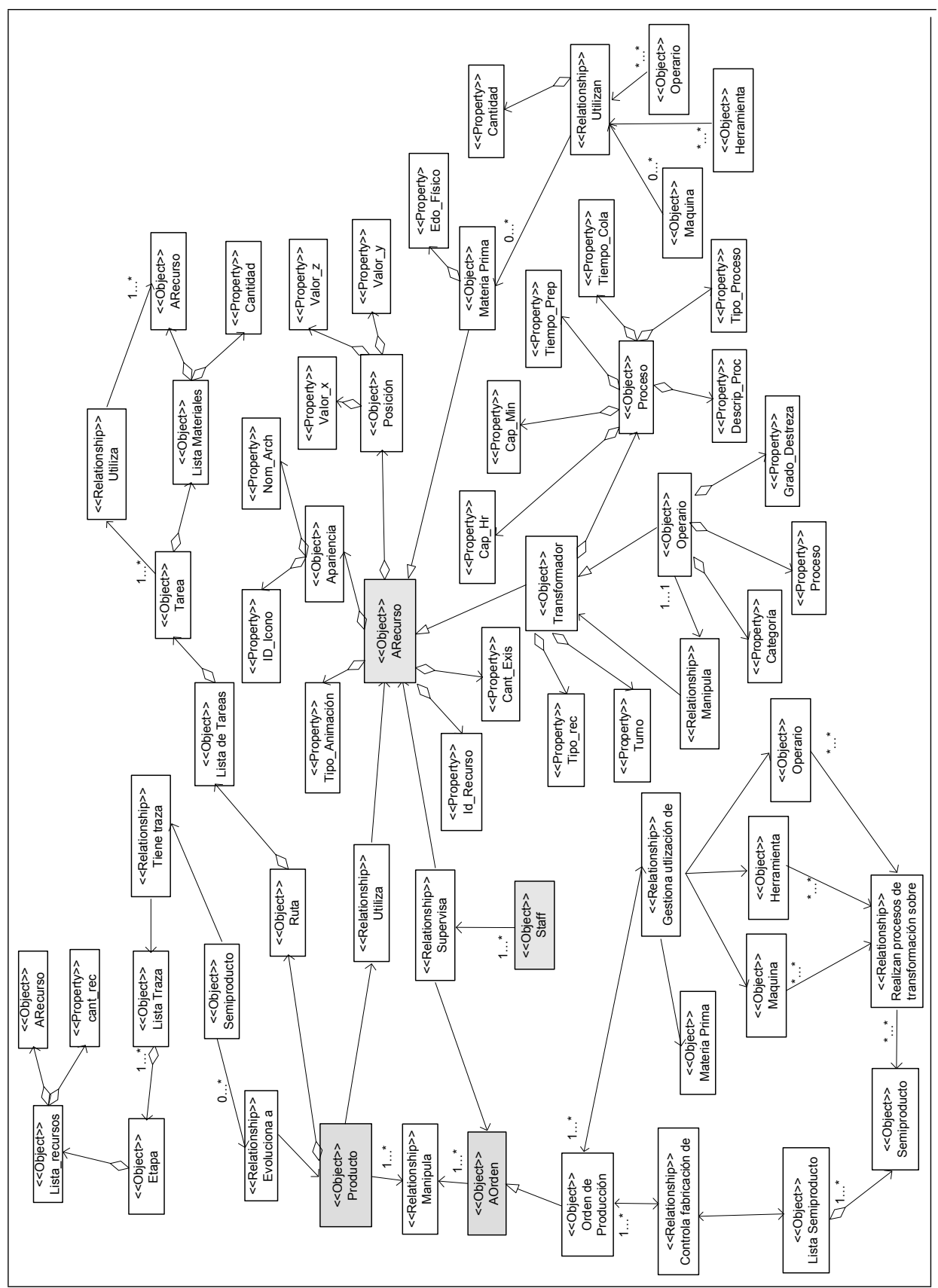

Figura 6.6: Metamodelo del Holón ARecurso

Los objetos de tipo Transformador son aquellos que realizan procesos de transformación. Por lo tanto, este objeto agrupa a las tres principales entidades 
que realizan dichos procesos Operario, Maquina, y Herramienta. El Operario se refiere al Recurso Humano que lleva a cabo procesos de transformación manual o que interactúa con otro elemento durante dichos procesos. Tanto la Máquina como la Herramienta son recursos que se utilizan durante los procesos de transformación los cuales pueden ser manipulados o no por un Operario. La Materia Prima sufre procesos de transformación mediante Máquinas, Herramientas y Operarios para la obtención de Productos.

Además de las relaciones identificadas anteriormente con respecto a la clase Producto, es posible identificar relaciones específicas entre los elementos de la misma clase Recurso que permiten describir el comportamiento de cada uno de ellos. Las relaciones identificadas se describen como sigue (Fig. 6.6):

- Aunque algunos recursos pueden realizar procesos automáticamente, en muchos casos se requiere la intervención de un operario en un mayor o menor grado. Debido a que tanto las máquinas como herramientas tendrán los mismos comportamientos, éstas se quedarán integradas dentro del Holón Transformador. Por lo tanto, surge la relación entre el holón Operario y el holón Transformador donde un Operario manipula $N$ objetos de tipo Transformador.

- Durante los procesos de transformación tanto los objetos de tipo Operario y Transformador utilizan cantidades específicas de Recursos de tipo Materia Prima y/o Producto.

- Como resultado de la ejecución de estos procesos se observa cómo los recursos Operario, Transformador realizan procesos de transformación sobre objetos de tipo Semiproducto permitiendo con ello su evolución hasta la obtención del Producto.

Propiedades y Objetos

El objeto Holón ARecurso se compone de cuatro propiedades:

1. Identificador del Recurso (id_rec). Esta propiedad permite identificar la instancia de un Recurso.

2. Número de recursos (cant_exis). Esta propiedad permite identificar la cantidad de recursos existentes de cada una de las instancias definidas.

3. Tipo Animación (tipo_anim). Esta propiedad es utilizada sólo para efectos de la animación de los objetos de tipo Recurso durante el proceso 
de simulación. Permitirá indicar mediante un parpadeo o un movimiento izquierda-derecha ó arriba/abajo que esta realizando alguna función durante la simulación.

4. Centro de Trabajo (ct_trab). Esta propiedad permite identificar el centro de trabajo al cual está asociado el recurso. Esta propiedad permite identificar agrupaciones de recursos con distintas habilidades en un mismo Centro de Trabajo. Tales agrupaciones permiten al diseñador distribuir la carga de trabajo a nivel de recursos y al mismo tiempo agrupar el trabajo por áreas físicas en una planta de fabricación.

Asimismo el objeto ARecurso se compone de dos objetos: Apariencia, Posición. Estas propiedades son utilizadas sólo para efectos de la animación de los objetos de tipo ARecurso durante el proceso de simulación.

Objeto Apariencia: Esta propiedad es utilizada sólo para efectos de la animación de los objetos de tipo Recurso durante el proceso de Simulación. Permite asociar una imagen a un Recurso. Este objeto es monitorizado continuamente por el Agente de Simulación quien actualiza la visualización de un Recurso acorde a su estado actual.

El objeto Apariencia se compone de dos propiedades:

1. Identificador de la Imagen (id_ico). Esta propiedad permite identificar a una instancia de una imagen de un Icono asociado a un Recurso.

2. Archivo de la Imagen (arch_ico). Esta propiedad permite indicar el nombre del archivo físico que contiene a la imagen que se le asocia al icono que representara al Recurso.

Objeto Posición: Permite identificar la ubicación física dentro de la planta de Fabricación del Recurso. La Posición del Recurso permitirá observar el avance físico del objeto de tipo Semiproducto. El objeto Posición se compone de tres propiedades:

1. Valor Eje $X\left(\right.$ val $\left._{-}\right)$). Valor de la ubicación física del Recurso sobre el eje de las $\mathrm{X}$.

2. Valor Eje Y (val_y). Valor de la ubicación física del Recurso sobre el eje de las $y$. 
3. Valor Eje Z (val_z). Valor de la ubicación física del Recurso sobre el eje de las z.

En el nivel de las especializaciones del objeto ARecurso, es posible identificar propiedades que permiten detallar sus funciones al definir tanto propiedades como objetos específicos que permiten delimitar su comportamiento.

Objeto Materia Prima: Se refiere a aquellos materiales que son utilizados por los recursos durante los procesos de transformación y que contribuyen en la evolución del Semiproducto, por tanto tiene asociada cuatro propiedades:

1. Estado Físico (edo_fís). Esta propiedad permite identificar el estado físico de la Materia Prima antes de sufrir algún proceso de transformación. El estado físico puede ser líquido, gaseoso, granular o sólido.

2. Cantidad Existente (qty_exis). Esta propiedad permite identificar la cantidad total existente en el inventario.

3. Cantidad Disponible (qty_disp). Esta propiedad permite identificar la cantidad que esta disponible para ser reservada para las Ordenes de Producción.

4. Cantidad Reservada (qty_res). Esta propiedad permite identificar la cantidad total que ha sido reservada para Ordenes de Producción previas.

Objeto Transformador: Este objeto se compone de dos propiedades:

1. Tipo de Recurso (tipo_rec). Esta propiedad permite identificar si el Recurso es una Máquina $(\mathrm{M})$ o una Herramienta $(\mathrm{H})$.

2. Turno (turno). Permite identificar a qué turno/horario pertenece el Recurso para así delimitar su capacidad disponible.

Asimismo, el objeto Transformador se compone de dos objetos: Proceso y Cola de Trabajos.

Objeto Proceso: Permite detallar el proceso que puede realizar un objeto Transformador. Este objeto se compone de ocho propiedades: 
1. Identificador del Tipo de Proceso (tipo proc). Esta propiedad permite identificar el tipo de proceso en general el cual describe la habilidad del Recurso. El Tipo de Proceso está asociado a procesos de tipo Transformación física, Cambio de Ubicación, Químico, Térmico, Mecánico, Térmico sin cambio de forma, Unión de formas, Conservación de masa con moldeo, Reducción de masa por moldeo, y Acabado superficial sin cambio de forma.

2. Descripción del Proceso (desc proc). La Descripción del proceso detalla el Tipo de Proceso, es decir, si es de tipo mecánico, la descripción puede ser "Cortar".

3. Capacidad por Hora (cap_hora). Indica la capacidad en unidades que puede procesar el Recurso en una hora.

4. Capacidad mínima (cap_min). Indica la capacidad en unidades que mínimamente debe procesar el Recurso en una hora para evitar la subutilización del recurso.

5. Tiempo de preparación (tmpo_prep). Esta propiedad se refiere al tiempo de preparación que requiere el Recurso antes de comenzar un Proceso.

6. Tiempo de cola (tmpo_cola). Esta propiedad se refiere al tiempo que deben esperar sus entradas antes de iniciar con el proceso en dicho Recurso.

7. Tiempo mínimo de compleción (tmin_com). Esta propiedad se refiere al tiempo mínimo necesario para que el Recurso termine un proceso.

8. Coste por hora (cte $h r$ ). Esta propiedad se refiere al coste por hora de utilización del Recurso.

Las propiedades referentes a los tiempos y la propiedad de coste por hora son útiles para una Orden de Producción al momento de valorar la asignación de Tareas. Asimismo tales propiedades son utilizadas para efectos de análisis posteriores.

Cola de Trabajos: Este objeto sólo se utiliza para efectos de simulación sin embargo los datos contenidos en el mismo son almacenados en la Base de Datos para su posterior análisis. Como se presentó en la Sección 4.1.1, un sistema de colas permite un análisis que incluye los tiempos de llegada de trabajos y entrada a la cola, tiempos de espera, etc. Tales trabajos eventualmente son atendidos y finalmente dejan la 
cola. El proceso de toma de decisión para la colocación de trabajos en la cola puede ser un proceso simple o más complejo para el cual es posible aplicar las técnicas de negociación basadas en el paradigma de agentes. La etapa de negociación de la Orden de Trabajo con los Recursos para la asignación de tareas es un aspecto clave en la definición del comportamiento de los agentes.

La toma de decisión toma en consideración los tres aspectos que considera el diseño de un FMS: maximizar el volumen de producción dentro de las restricciones de tiempo de las máquinas, mientras que al mismo tiempo se busca minimizar el coste y maximizar la flexibilidad (Sección 4.6).

Una vez que los trabajos son aceptados en la cola, el Agente de Recurso se convierte en un Servidor que atiende los trabajos siguiendo la filosofía básica de un Sistema de Colas. Es decir, el trabajo (la unidad) inicia el servicio inmediatamente si el Agente de Recurso (el servidor) está detenido. Asimismo, después de que el Agente de Recurso (el servidor) finaliza un servicio, éste puede estar ocupado o permanecer ocupado con el siguiente trabajo (unidad). Si la cola no está vacía, otro trabajo (unidad) entrará al Agente de Recurso (el servidor) y éste estará ocupado. Si la cola está vacía, el Agente de Recurso (el servidor) estará detenido después de finalizar el servicio. Es imposible para el Agente de Recurso (el servidor) estar ocupado si la cola está vacía cuando se finaliza un servicio. Similarmente, es imposible para el Agente de Recurso (el servidor) quedarse detenido después de finalizar un servicio si la cola no está vacía.

El Entorno de Simulación contempla que el Usuario pueda definir específicamente la asignación de Tareas de la Orden de Producción a un Transformador específico o en su caso la Orden de Producción aplique alguna de las técnicas de negociación basadas en el paradigma de agentes tales como el uso de subastas (Veeramani \& Wang 1997, Veeramani, Wang \& Rojas 1998), el razonamiento basado en casos (CBR) (Choy \& Lee 2000), los protocolos de tiempo de vencimiento (Anussornnitisarn, Peralta \& Nof 2001), el algoritmo de equilibrio de temperatura (Gordillo, Giret \& Botti 2006) y técnicas basadas en el comportamiento animal tales como: el uso de coordinación de colonias de insectos mediante uso de umbrales 
(Cicirello \& Smith 2001) y el sistema de ardillas (Carmorlinga \& BArker 2003, Gordillo, Giret \& Botti 2005). La Cola de Trabajos permite identificar las Tareas que tiene asignadas un recurso así como informar sobre su procesamiento a la Orden de Producción. Así mismo, este objeto permite al Transformador brindar información sobre su estado (en espera, trabajando) al Agente de Simulación quien controla el aspecto visual de los Agentes de Recurso.

En la propuesta, las propiedades de la cola de trabajos ofrecen los parámetros necesarios para la aplicación de algunas técnicas, en particular para la aplicación de la técnica de subastas y del sistema de ardillas. De tal forma que este objeto se compone de nueve propiedades:

1. Identificador de la Orden de Producción(id_po). Esta propiedad permite identificar la instancia de una AOrden aceptada por el Recurso.

2. Identificador de la Tarea(id_tarea). Esta propiedad permite identificar la instancia de una Tarea aceptada por el Recurso.

3. Estado de la Tarea(edo_tarea). Esta propiedad permite identificar si la tarea esta en espera, esta en proceso o ha sido terminada.

4. Tiempo de Inicio(tmp_ini). Esta propiedad permite identificar el momento en que el Recurso debe iniciar la Tarea.

5. Tiempo de Fin(tmp_fin). Esta propiedad permite identificar el momento en que el Recurso debe terminar la Tarea.

6. Cantidad Total de Piezas(qty_pzas). Esta propiedad permite identificar el número total de piezas que el Recurso debe fabricar.

7. Tiempo por pieza(tmp pza). Esta propiedad permite identificar el periodo de tiempo que el Recurso debe destinar a la fabricación de cada pieza.

8. Tiempo de Inicio(tmp_inir). Esta propiedad permite identificar el momento en que el Recurso inicia la tarea.

9. Tiempo de Fin(tmp finr). Esta propiedad permite identificar el momento en que el Recurso termina la tarea.

El objeto Transformador agrupa a las distintas clases de objetos que realizan procesos de transformación, que incluyen:

Objeto Operario: El objeto Operario se compone de dos propiedades: 
1. Categoría (cat_op). Esta propiedad se refiere a la clasificación que se puede asignar a los Operarios, la cual incluye tres niveles: Experto, Medio, y Principiante.

2. Grado Destreza (grado_des). Esta propiedad se refiere al Grado de destreza asociado a la categoría del Operario el cual se verá reflejado en su productividad

Objetos Maquinaria y Herramienta: Los objetos Maquinaria y Herramienta no requieren propiedades adicionales a las del objeto Transformador ya que éstas proporcionan suficiente información para detallar su comportamiento.

Las valores de las propiedades Turno, Capacidad por hora y Capacidad Mínima sólo se aplican para las especializaciones de los recursos de tipo Operario, Maquina, y Herramienta las cuales permiten controlar el scheduling ya sea de forma distribuida o centralizada. Mediante un objeto de tipo Staff es posible centralizar toda la información para controlar la capacidad global de la planta de fabricación, o en su caso permitir el scheduling distribuido mediante la negociación directa entre los recursos basados en las órdenes que se requieran fabricar. Una propiedad a resaltar es la Capacidad Mínima la cual permite optimizar el uso de los recursos evitando pérdidas debido a la subutilización del recurso.

\section{Holón AOrden.}

En la Figura 6.7 se muestra la Clase AOrden donde es posible identificar dos especializaciones de los tipos de órdenes: Órden de Producción y Órden de Mantenimiento. Estas especializaciones permiten la manipulación de los recursos en dos sentidos: la transformación de Materia Prima en Productos (Orden de Producción) y la realización de labores de Mantenimiento sobre los Recursos (Orden de Mantenimiento).

Las relaciones identificadas dentro de la clase AOrden que permiten describir el comportamiento de cada uno de los elementos que la componen se describen como sigue (Fig. 6.7): 


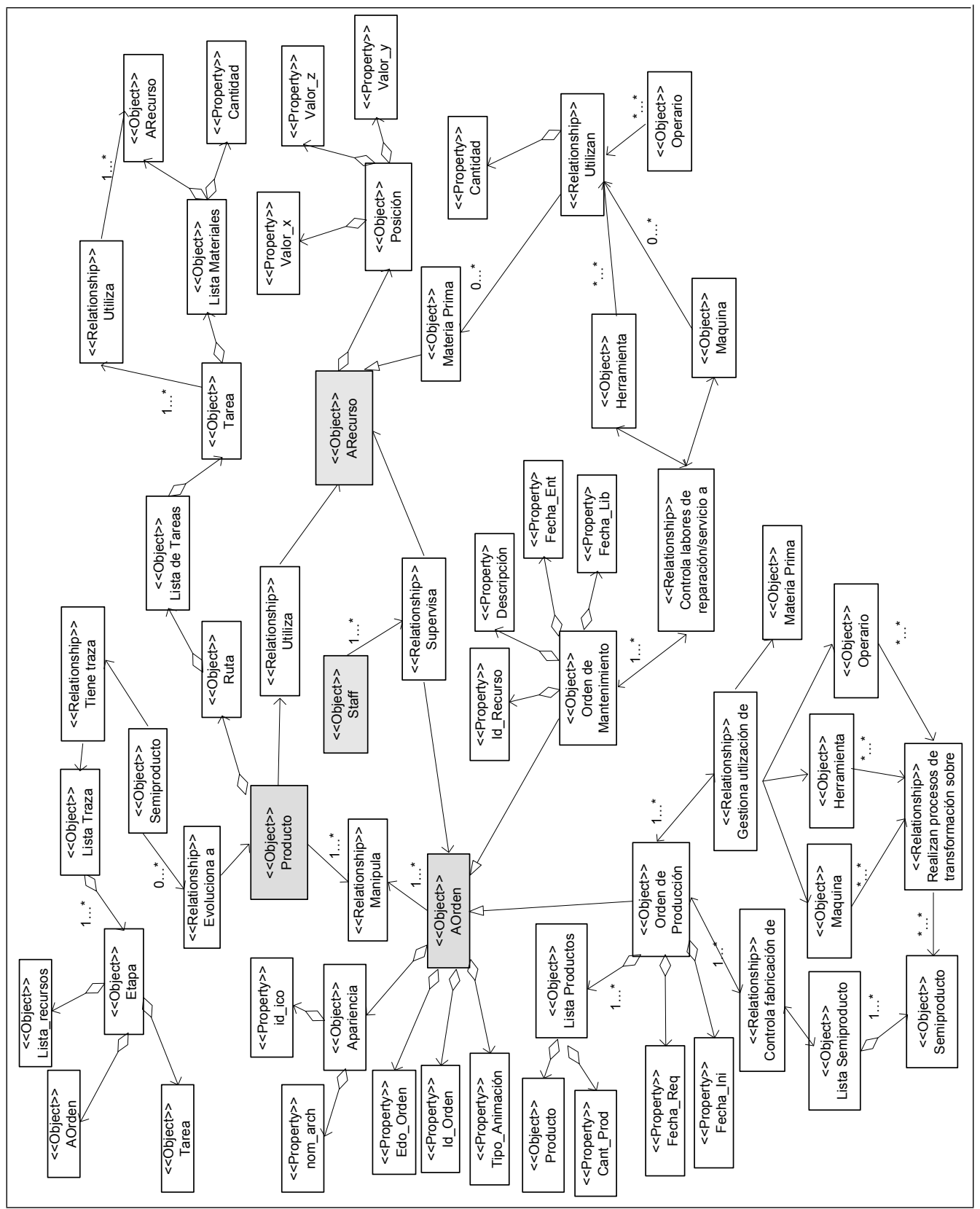

Figura 6.7: Metamodelo del Holón AOrden 
- Una Orden puede ser de dos tipos: aquellas relacionadas con la fabricación de productos Orden de Producción y las relacionadas con labores de reparación y servicio a recursos Orden de Mantenimiento.

- Una Orden de Producción controla la fabricación de objetos de tipo Lista de Semiproductos que agrupa a objetos de tipo Semiproducto los cuales permite registrar la traza generada como consecuencia de la ejecución de procesos de transformación hasta la obtención de un Producto, y gestiona la utilización de $\mathrm{N}$ recursos de tipo Operario, Máquina, Herramienta y Materiales acorde a la ruta del Producto.

- Tanto las máquinas como las herramientas deben someterse a revisiones periódicas para asegurar que el desempeño de los mismos se mantenga en un nivel aceptable durante su periodo de vida útil. Por lo tanto mediante una Orden de Mantenimiento es posible controlar labores de reparación/servicio sobre un Recurso de tipo Máquina o Herramienta.

Propiedades y Objetos

El objeto holón AOrden se compone de tres propiedades:

1. Identificador de la Orden (id_ord). Esta propiedad permite identificar la instancia de una AOrden.

2. Estado de la Orden (edo_ord). Esta propiedad permite identificar el estado de la AOrden que puede ser por ejemplo, en Espera, en Proceso o Terminado.

3. Tipo Animación (tipo_anim). Esta propiedad es utilizada sólo para efectos de la animación de los objetos de tipo Orden durante el proceso de simulación. Permitirá indicar mediante un parpadeo o un movimiento izquierdaderecha ó arriba/abajo que esta realizando alguna función durante la simulación.

Asimismo el objeto Holón AOrden se compone de un objeto: Apariencia.

Objeto Apariencia: Esta propiedad es utilizada solo para efectos de la animación de los objetos de tipo Orden durante el proceso de simulación. Permite asociar una imagen a una Orden. Este objeto es monitorizado continuamente por el Agente de Simulación quien actualiza la visualización de una Orden acorde a su estado actual.

El objeto Apariencia se compone de dos propiedades: 
1. Identificador de la Imagen (id_ico). Esta propiedad permite identificar a una instancia de una imagen de un Icono asociado a una AOrden.

2. Archivo de la Imagen (arch_ico). Esta propiedad permite indicar el nombre del archivo físico que contiene a la imagen que se le asocia al icono que representara a la AOrden.

En el nivel de las especializaciones del objeto AOrden, es posible identificar propiedades que permiten detallar sus funciones mediante la definición de sus propiedades y objetos los cuales delimitan su comportamiento.

Objeto Orden de Producción: El objetivo de una Orden de Producción es la fabricación de Productos. Por tanto, como se mencionó anteriormente permite gestionar la fabricación de objetos de tipo Producto mediante el control de la fabricación de objetos de tipo SemiProducto los cuales se agrupan en una Lista de Semiproductos. La Orden de Producción gestionará la fabricación de un Producto mediante la asignación de Tareas, incluidas en una Ruta de Fabricación, a Recursos acorde a una Configuración de Planta específica. Los Recursos verificarán la aceptación de las tareas, reservarán tiempo y colocarán las Tareas en su cola de trabajos antes de ejecutarlos. En su caso, la Orden de Producción será capaz de negociar con los Recursos disponibles la asignación de Tareas acorde a ciertos criterios, por ejemplo, asignar la tarea a aquel recurso que ofrezca un mejor tiempo de entrega, o aquel con un menor coste de fabricación.

Se compone de tres propiedades:

1. Fecha Requerida (fecha_req). Esta propiedad permite indicar la fecha para la cual se requiere tener el Producto terminado.

2. Fecha de Inicio (fecha_ini). Esta propiedad permite indicar la fecha en la cual se inicia la fabricación del Producto.

3. Fecha de Entrega (fecha_ent). Esta propiedad permite indicar la fecha en la cual se finaliza la fabricación del Producto.

Asimismo el objeto Orden de Producción se compone de un objeto: Lista de Productos.

Objeto Lista de Productos: Este objeto agrupa las instancias de los Productos cuya fabricación es controlada por el objeto Orden de Producción. Por tanto, se compone de una propiedad y dos objetos: 
Cantidad de Producto (cant prod). Esta propiedad indica la cantidad de Productos a producir en dicha Orden.

Objeto Producto: Este objeto permite identificar a una instancia de objetos de tipo Producto que se va a producir mediante el control de la Orden de Producción. En dicho objeto se integran tanto datos generales del producto como la Ruta de Fabricación y la Configuración de Planta a utilizar para la fabricación del Producto.

Objeto Lista de Estado de Producto. Esta lista permite observar en detalle el estado de la Orden de Producción con respecto al Producto. Es decir permite observar en detalle el estado de cada una de las Tareas que integra la Ruta de fabricación del Producto. De esta forma se da seguimiento e informa al Agente de Simulación mediante su objeto Apariencia. Por tanto, el Usuario puede obtener información visual y detallada del estado actual de la Orden de Producción. Este objeto se integra por seis propiedades:

1. Identificador de la Tarea(id_tarea). Esta propiedad permite identificar la instancia de una Tarea aceptada por el Recurso.

2. Estado de la Tarea(edo_tarea). Esta propiedad permite identificar si la tarea esta en espera, esta en proceso o ha sido terminada.

3. Tiempo de Inicio(tmp_ini). Esta propiedad permite identificar el momento en que el Recurso debe iniciar la Tarea.

4. Tiempo de Fin(tmp fin). Esta propiedad permite identificar el momento en que el Recurso debe terminar la Tarea.

5. Identificador del Recurso (id_rec). Esta propiedad permite identificar la instancia de un Recurso que tiene asignada la Tarea.

6. Tipo de Recurso (tipo_rec). Esta propiedad permite identificar si el Recurso es un Operario (O), una Máquina (M) o una Herramienta $(\mathrm{H})$.

En la Configuración de Planta el Usuario tiene la opción de definir específicamente la asignación de Tareas de la Orden de Producción a un Transformador específico o en su caso la Orden de 
Producción puede aplicar alguna de las técnicas de negociación basadas en el paradigma de agentes tales como subastas, razonamiento basado en casos, algoritmos de temperatura o técnicas basadas en el comportamiento animal (uso de coordinación de colonias de insectos mediante uso de umbrales y el sistema de ardillas) presentadas en la Sección 4.4.2.

Para efectos de Simulación, el control de avance de la producción de la Lista de Productos asociada a una Orden de Producción se lleva a cabo mediante la definición de un objeto Lista de Semiproductos que contiene un objeto Semiproducto por cada objeto Producto de la Lista de Productos. De esta forma es posible controlar el avance de la producción de cada uno de los objetos de tipo Producto mediante el registro de la evolución de objetos de tipo Semiproducto hasta la obtención de cada uno de los Productos.

Objeto Lista de Semiproductos: Este objeto agrupa las instancias de los Semiproductos asociadas a las instancias de Productos agrupadas en la Lista de Productos cuya fabricación es controlada por el objeto Orden de Producción. Por tanto, se compone de un objeto:

Objeto Semiproducto: Cada uno de estos objetos permite controlar los procesos de transformación que sufre al ser manipulado por objetos de tipo ARecurso - Operario, Máquina y Herramientas - hasta la obtención de un Producto. Por tanto, este objeto a la vez se compone de un objeto Apariencia y seis propiedades.

Objeto Apariencia: Esta propiedad es utilizada solo para efectos de la animación de los objetos de tipo Semiproducto durante el proceso de simulación. Permite asociar una imagen a un Semiproducto. Este objeto es monitorizado continuamente por el Agente de Simulación quien actualiza la visualización de un Semiproducto acorde a su estado actual. Es decir, el objeto Semiproducto se mantiene con la misma apariencia hasta que evoluciona a un Producto. En este momento el Semiproducto desaparece y toma la apariencia y propiedades del objeto Producto.

El objeto Apariencia se compone de dos propiedades:

1. Identificador de la Imagen (id_ico). Esta propiedad permite identificar a una instancia de una imagen de un Icono asociado a una Semiproducto. 
2. Archivo de la Imagen (arch_ico). Esta propiedad permite indicar el nombre del archivo físico que contiene a la imagen que se le asocia al icono que representara a la Semiproducto.

Asimismo, las propiedades del objeto Semiproducto son:

1. Identificador de la Orden (id_ord). Esta propiedad permite identificar la instancia de la AOrden a la que pertenece el Semiproducto.

2. Identificador de la Tarea (id_tarea). Esta propiedad permite identificar la instancia de una Tarea asociada a la etapa de evolución del Semiproducto y que está siendo procesada por un Recurso.

3. Estado de la Tarea (edo_tarea). Esta propiedad permite identificar si la tarea está en espera, está en proceso o ha sido terminada.

4. Tiempo de Inicio (tmp_ini). Esta propiedad permite identificar el momento en que el Recurso inició la Tarea.

5. Tiempo de Fin (tmp_fin). Esta propiedad permite identificar el momento en que el Recurso termina la Tarea.

6. Centro de Trabajo (ct_trab). Esta propiedad permite identificar el centro de trabajo al cual esta asociado el Recurso que esta procesando el Semiproducto.

Objeto Orden de Mantenimiento: Mediante este tipo de Orden es posible controlar las labores de mantenimiento necesarias sobre objetos de tipo Máquina y Herramienta para mantenerlas en las condiciones necesarias para su utilización durante los procesos de transformación. Cuando un Agente Transformador se ve afectado por una Orden de mantenimiento, éste deja de ofrecer sus servicios a los Agentes de Orden de Producción.

Por tanto, este objeto se compone de cuatro propiedades:

1. Identificador del Recurso (id_rec). Esta propiedad permite identificar a una instancia del Recurso a la que se le va a realizar el servicio de mantenimiento mediante la Orden de Mantenimiento.

2. Descripción del servicio (desc_serv). Esta propiedad se refiere a la descripción del detalle del servicio que se le debe realizar al Recurso. 
3. Fecha de Entrada (fec_ent). Esta propiedad permite indicar la fecha en la cual se retira el Recurso de la planta para darle el servicio de mantenimiento.

4. Fecha de Liberación (fec_lib). Esta propiedad permite indicar la fecha en la cual se libera el Recurso para su habilitación y utilización en la planta.

\section{Holón Staff.}

Finalmente los holones de tipo Staff tienen como objetivo la supervisión del cumplimiento de las procesos que controlan los holones de tipo Orden y el desempeño de los holones de tipo Recurso para asegurar el cumplimiento de las negociaciones establecidas entre ellos durante el proceso de fabricación.

En la Figura 6.8 se muestra el Holón Staff. Los holones de tipo Staff tienen como principal labor la supervisión de los holones de tipo AOrden y ARecurso puesto que son los holones que presentan un alto grado de actividad durante el proceso de fabricación de holones de tipo Producto. Dichas actividades incluyen los procesos de negociaciones que se entablan a fin de optimizar la utilización de los recursos respetando los tiempos límites para llevar a cabo las tareas.

Propiedades y Objetos

El objeto Staff se compone de cuatro propiedades:

1. Identificador del objeto Staff (id_staff). Esta propiedad permite identificar a una instancia de un objeto de tipo Staff.

2. Tipo Animación (tipo_anim). Esta propiedad es utilizada sólo para efectos de la animación de los objetos de tipo Staff durante el proceso de simulación. Permitirá indicar mediante un parpadeo o un movimiento izquierdaderecha o arriba/abajo que está realizando alguna función durante la simulación.

3. Nivel de autoridad (niv_aut). Esta propiedad se refiere al nivel de autoridad sobre los objetos de tipo ARecurso y AOrden que integran el modelo.

4. Código de implementación (cod_imp). Esta propiedad permitirá al Diseñador del Modelo indicar los algoritmos sobre los cuales actuará el objeto Staff para desempeñar sus labores de supervisión. 


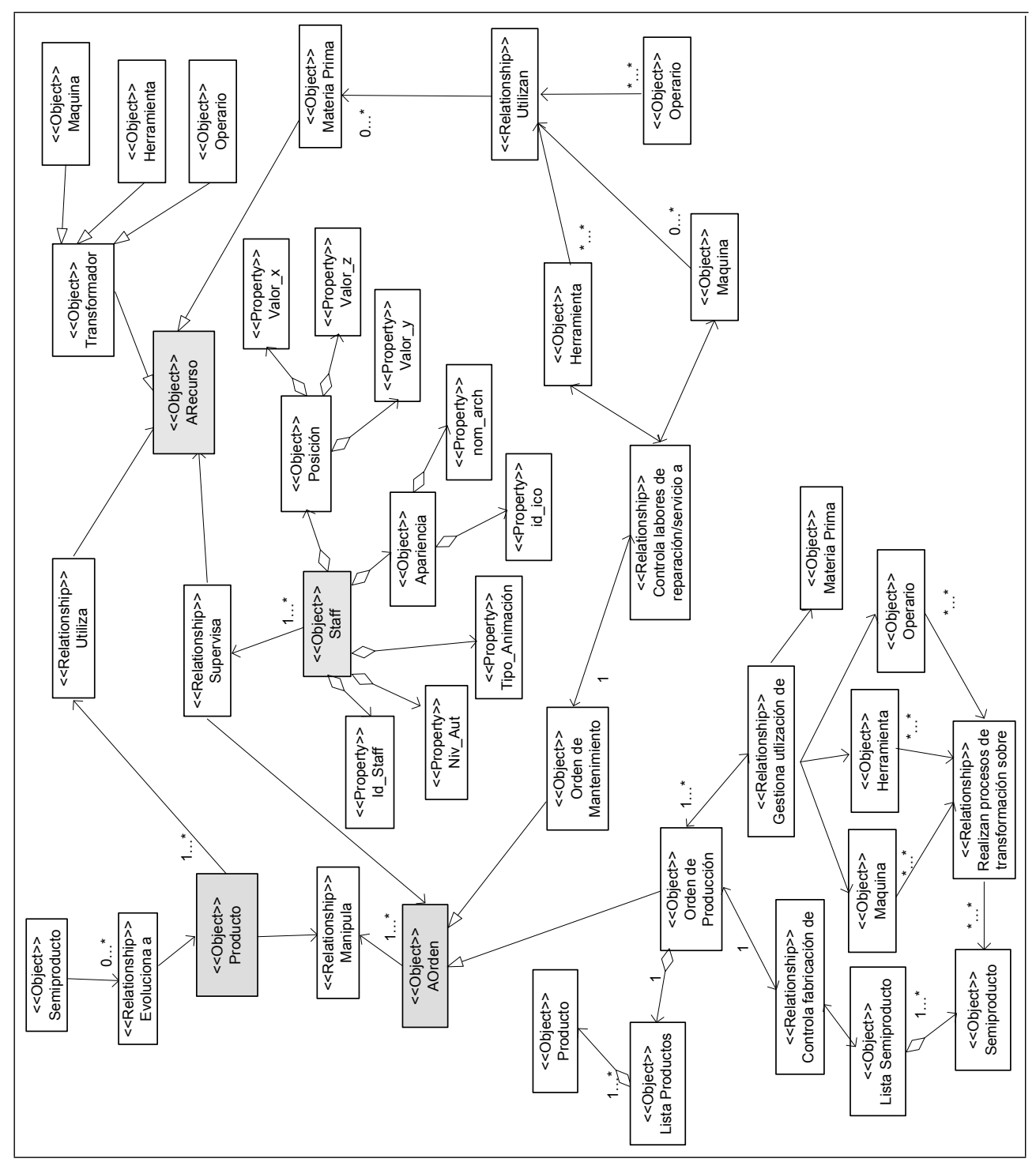

Figura 6.8: Metamodelo del Holón Staff.

Asimismo el objeto Staff se compone de dos objetos: Apariencia y Posición. Estas propiedades son utilizadas sólo para efectos de la animación de los objetos de tipo Staff durante el proceso de simulación.

Objeto Apariencia: Esta propiedad permite asociar una imagen a un objeto de tipo Staff. Este objeto es monitorizado continuamente por el Agente de Simulación quien actualiza la visualización de un Staff acorde a su estado actual. El objeto Apariencia se compone de dos propiedades: 
1. Identificador de la Imagen (id_ico). Esta propiedad permite identificar a una instancia de una imagen de un Icono asociado a objeto de tipo Staff.

2. Archivo de la Imagen (arch_ico). Esta propiedad permite indicar el nombre del archivo físico que contiene a la imagen que se le asocia al icono que representara al Staff.

Objeto Posición: Esta propiedad permite identificar la ubicación física dentro de la planta de Fabricación del Staff. La posición del Staff permitirá observar el desplazamiento físico dentro de la Planta de Fabricación del objeto de tipo Staff. El objeto "Posición" se compone de tres propiedades:

1. Valor Eje X (val_x). Valor de la ubicación física del Staff sobre el eje de las $\mathrm{x}$.

2. Valor Eje $Y\left(v_{a l} y\right)$. Valor de la ubicación física del Staff sobre el eje de las y.

3. Valor Eje Z (val_z). Valor de la ubicación física del Staff sobre el eje de las $\mathrm{z}$.

\subsection{Vinculación entre el Entorno Real y los Agen- tes del Metamodelo de Simulación}

Por otra parte, para clarificar los agentes representados como holones de Recurso (Operarios, Máquinas, Herramientas) y Staff en el Metamodelo, se han aplicado las bases para la definición del entorno de simulación mediante un formalismo de simulación (Helleborg et al. 2007) presentado en la Sección 3.5.2. El entorno de simulación del Metamodelo de la Planta de Fabricación se puede representar mediante el formalismo (utilizado en la definición de la estructura del entorno simulado) para la identificación de entidades y propiedades del entorno, así como la representación de entidades del entorno como agentes, de la siguiente forma:

Entidades y Propiedades del entorno. En la Planta de Fabricación, si por ejemplo distinguimos 5 Operarios, 3 Máquinas, 2 Herramientas, y 2 Supervisores. Las entidades del entorno quedarían expresadas como sigue:

$E=\{$ Op1,Op2,Op3,Op4,Op5,Maq1,Maq2,Maq3,Hta1,Hta2,Sup1,Sup2 $\}$

Part $_{E}=\{$ Operario,Máquina,Herramienta,Supervisor $\}$ 


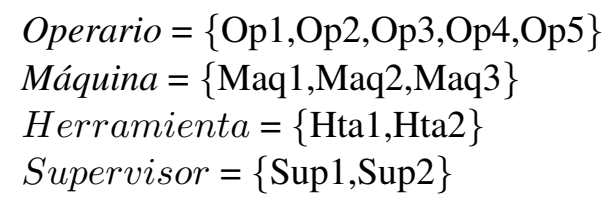

donde:

$\boldsymbol{E}$ es el conjunto de entidades del entorno.

$\boldsymbol{P a r t} \boldsymbol{E}$ es una partición en cuatro tipos de entidades.

Operario es el conjunto de operarios.

Máquina es el conjunto de máquinas.

Herramienta es el conjunto de herramientas.

Supervisor es el conjunto de supervisores.

Por el momento no consideraremos las propiedades del entorno puesto que no controlaremos aspectos relacionados a temperatura, humedad, etc.

Agentes representados como entidades del entorno. En este caso la representación de los agentes que integrarán el entorno simulado, se pueden ver expresados como sigue:

Si $A g=\{A g O p 1, A g O p 2, A g O p 3, A g O p 4, A g O p 5, A g M a q 1, A g M a q 2, A g$ Maq3, AgHtal, AgHta2, AgSupl, AgSup2 $\}$ es el conjunto de agentes del entorno simulado, se asocian tales agentes a las entidades del entorno como sigue:

$$
\begin{aligned}
\text { Embody }(\text { Juan }) & =\text { AgOp } 1 \\
\text { Embody }(\text { Pedro }) & =\text { AgOp } 2 \\
\text { Embody }(\text { PistolaKoestler }) & =\text { AgHta } 1 \\
\text { Embody }(\text { Martillo }) & =\text { AgHta } 1 \\
\text { Embody }(\text { Moldeadora }) & =\text { AgMaq } 1 \\
\text { Embody (Alvaro }) & =\text { AgSup } 1
\end{aligned}
$$

De esta forma, al asociar las entidades del entorno real a los agentes en el entorno simulado, los agentes actuarán de acuerdo a las características de la entidad que representan. Las características de cada una de las particiones (Operario, Máquina, Herramienta, Supervisor) delimitan las acciones que el agente puede llevar a cabo como resultado de sus interacciones con otros agentes. 


\subsection{Notación de los Elementos del Metamodelo}

Una vez definidos los elementos que integran el metamodelo, para efectos de la Simulación es necesaria la definición de la representación gráfica de dichos elementos. Para ello se define la especificación de una notación determinada para identificar cada una de las entidades que componen el modelo. En esta sección se presenta la notación gráfica asociada a cada una de las entidades identificadas previamente (Fig. 8.50). En la Figura 8.50 se presentan los elementos que componen esta notación. La notación se ilustra en el Capítulo 10 donde se presenta el modelado de un caso de estudio.

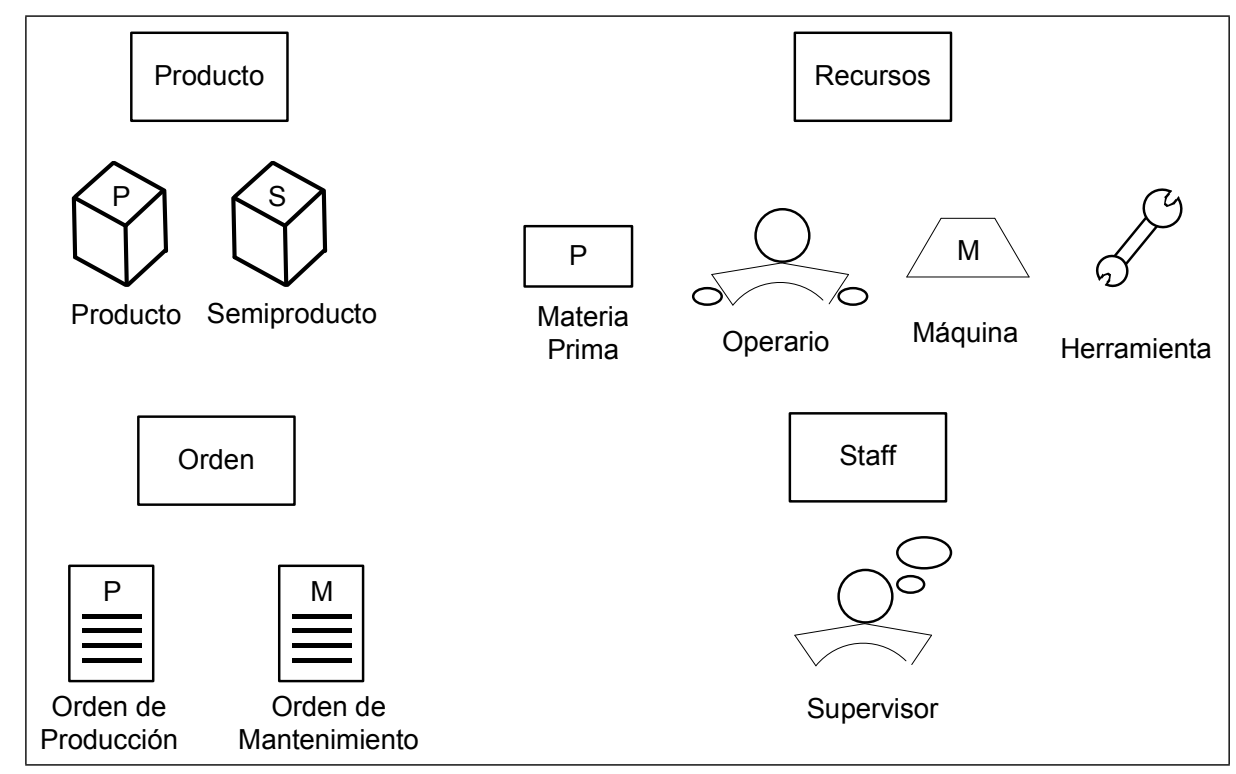

Figura 6.9: Elementos de la Notación Gráfica del Modelo a Simular.

\subsection{Interacciones entre el Metamodelo de la Plan- ta de Fabricación y el Simulador}

Aunque en la Fase de Creación del Modelo, el Metamodelo actúa como una entidad flexible que permite representar una Planta de Fabricación mediante un Modelo específico. El Modelo contiene la información que describe: por una parte la distribución física de la planta de fabricación que se está modelando, y por otra parte, se define la vinculación de los elementos de dicho modelo a las entidades que integran la planta de fabricación real y por consecuencia cada uno 
de los elementos adoptan las características de dichas entidades. Las características incluyen no sólo características internas, tales como identificador, nombre, capacidad de procesamiento, turnos, sino que además debido a la naturaleza de cada uno (Orden, Recurso, Staff) tienen relaciones preestablecidas que determinan las interacciones para las cuales están preparados.

En la Segunda Fase el Modelo obtenido se convierte en un elemento clave a partir del cual el Agente de Simulación solicita la creación de agentes que integren al nuevo Sistema Multiagente que representa a la Planta de Fabricación como una entidad activa. En ese momento la vinculación de los elementos a las entidades de la planta de fabricación real tiene una repercusión importante puesto que sus características le permitirán participar o no en las interacciones con otros agentes. Asimismo, a partir de ese momento los agentes que representan a la planta de fabricación establecen comunicación directa no solo con los agentes de la planta, sino con los agentes del Entorno de Simulación tales como el Agente de Simulación, el Agente de Resultados y el Agente de Sincronización.

\subsection{Aprendizaje por refuerzo para los Agentes del Modelo}

Considerando las bases del Aprendizaje por refuerzo en Sistemas Multiagente (ARSM) -Proceso de Decisión de Markov (presentado en la Sección 3.3)-, tal proceso de toma de decisiones aplicado por los agentes del Sistema de Fabricación, contempla el uso de tuplas que asocian determinados estados del sistema a un grupo de posibles acciones que pueden desempeñar los agentes. Tales acciones y las posibles tuplas se integran a partir tanto de la configuración inicial como de las simulaciones pasadas y las acciones ejecutadas por cada uno de los agentes.

Los agentes que representan los operarios, máquinas, herramientas y Staff actúan como Gestores de Recursos y las Ordenes de Producción como Clientes de Recursos. De tal manera que los primeros aprenden cómo gestionar el mejor servicio ante las solicitudes de las Ordenes de Producción, mientras que los agentes de Orden aprender cómo seleccionar al mejor recurso.

Como resultado de la aplicación de la estructura de la tupla que define el proceso de decisión de Markov para los Sistemas Multiagente $<X, U_{-} l, \ldots, U \_n$, $f, \rho_{1}, \ldots, \rho_{n}>$, las tuplas correspondientes a los agentes de Orden de Producción 
y los Agentes de Recursos (Operarios, Máquinas, Herramientas, Staff) se describen a continuación.

Los agentes de Orden de Producción tienen como objetivo la fabricación de un producto y su entrega en una fecha determinada. Para los agentes de Orden de Producción (como Cliente de Recurso) cada uno de los elementos de dicha tupla inicial en el Sistema Multiagente que representa a la Planta de Fabricación queda definida como sigue:

$\mathbf{n}=$ Número total de agentes de orden lanzados en la Fase II - Simulación de Modelo.

$\mathbf{X}=$ Considerando que la Orden de Producción es quien inicia los procesos de comunicación y de negociación con los Recursos, los estados del Sistema comprenden:

- Identificar tareas pendientes de asignación de recursos.

- Negociación para la asignación de tareas.

- Monitorización del progreso de la Orden de Producción.

$\mathbf{U}=$ Las acciones que puede tomar el agente de Orden.

1. Obtener la siguiente tarea para asignar recursos. Durante este proceso se verifica de acuerdo a la definición de las transiciones entre las operaciones de la ruta seleccionada. Sólo se puede avanzar en la asignación de recursos si las operaciones que preceden a la tarea actual tienen sus recursos asignados y por tanto se ha obtenido la fecha y hora inicial mínima de la siguiente tarea.

2. Identificación de agentes con servicios que incluyan un tipo de proceso específico.

3. Envío de solicitud de tarea, la cual incluye parámetros tales como: capacidad requerida en horas, fecha mínima y máxima de inicio y fin de tarea.

4. Recepción de propuestas de los agentes participantes. Esta puede ser a favor o rechazar seguir participando.

5. Selección del agente y asignación de tarea.

6. Recepción de avance en tareas asignadas. 
$\mathbf{f}=$ La función de probabilidad para la transición de estado estará dada por $f$ : $X x U x X \rightarrow[0,1]$.

$\rho=$ Sera la función de recompensa dada por $\rho_{i}: X x U x X \rightarrow \mathcal{R}$.

Por otra parte, los Agentes de Recurso tienen como objetivo el evitar los tiempos muertos de trabajo, mediante la gestión de los trabajos aceptados. Mientras que para los agentes de Recurso (como Gestores de Recurso) cada uno de los elementos de dicha tupla inicial en el Sistema Multiagente que representa a la Planta de Fabricación queda definida como sigue:

$\mathbf{n}=$ Número total de agentes de Recurso (Operarios, Máquinas, Herramientas y Staff) definidos en la Fase I - Creación del Modelo, y que se encuentran activos en la Fase II - Simulación de Modelo.

$\mathbf{X}=$ Considerando que los agentes de Recurso están a la espera de solicitudes de servicios por parte de los agentes de Orden de Producción, los estados del Sistema comprenden:

- Negociación para la aceptación de tareas.

- Informe del progreso de la Orden de Producción.

$\mathbf{U}=$ Las acciones que pueden tomar los agentes de Recurso.

1. Recepción de solicitud de tarea, la cual incluye parámetros tales como: capacidad requerida en horas, fecha mínima y máxima de inicio y fin de tarea.

2. Verificación de capacidad acorde a los parámetros recibidos.

3. Informar propuesta al Agente de Orden. Esta puede ser a favor o rechazar acorde a la capacidad disponible.

4. Recibir la confirmación de aceptación o rechazo de la propuesta.

5. Colocar la tarea aceptada en la cola de fabricación.

6. Informar el avance en tareas asignadas.

$\mathbf{f}=$ La función de probabilidad para la transición de estado estará dada por $f$ : $X x U x X \rightarrow[0,1]$.

$\rho=$ Sera la función de recompensa dada por $\rho_{i}: X x U x X \rightarrow \mathcal{R}$.

El aprendizaje obtenido por cada uno de los agentes será utilizado en las simulaciones posteriores durante los procesos de negociación. 


\subsection{Conclusiones}

Basado en las especificaciones relacionadas con los estándares de la Simulación de Sistemas de Fabricación, la Simulación soportada por el paradigma de agentes y los Sistemas Holónicos presentadas en la primera parte de la memoria, en este capítulo, se ha presentado el detalle del Metamodelo que permite el modelado de un Sistema de Fabricación Flexible e Inteligente que contempla los requisitos de la nueva fabricación. Dicho Metamodelo se utiliza como base para la Creación de Modelos en el Entorno de Simulación durante la Fase I.

Por otra parte, de acuerdo con el enfoque de PROSA, la especificación del Modelo establecido permite identificar las entidades y relaciones básicas que se distinguen entre cada uno de los elementos que la componen, permitiendo a su vez la escalabilidad y flexibilidad a fin de adaptar el modelo a las necesidades de casos de estudio específicos aportando mayor funcionalidad y adaptabilidad a las necesidades de cada uno de los escenarios para casos reales de plantas de fabricación.

En el siguiente capítulo se presenta la Segunda Fase de la Simulación. El capítulo incluye el detalle de la Arquitectura del Entorno de Simulación que da soporte a tres actividades: Simulación de Modelos, Diseño de Experimentos y Análisis de los Resultados. Asimismo, en la Segunda Fase se utilizan como datos de entrada, los Modelos generados en la Primera Fase. 


\section{Capítulo 7}

\section{Modelo de la Arquitectura del Entorno de Simulación}

Siguiendo el Diagrama de Estudio de Simulación en este capítulo se presenta la definición de la Arquitectura del Entorno de Simulación que dá soporte a las actividades de Simulación del Modelo y Análisis de Resultados incluidas en la Segunda Fase (Fase II).

Para la Segunda Fase se tiene como entrada el Modelo que representa al Sistema de Fabricación generado durante la primera fase del proceso de simulación presentada en el Capítulo 5. Internamente, el Modelo es un Modelo de Agentes el cual es monitorizado por el Entorno de Simulación para visualizar gráficamente el comportamiento de los agentes que lo componen.

\subsection{Estrategias y Protocolos de comunicación en- tre el Entorno de Simulación y el Sistema de Fabricación}

De acuerdo con los tipos de Simulación existentes, en la propuesta, la simulación se caracteriza por ofrecer una simulación dinámica que representa el sistema conforme evoluciona con el tiempo de acuerdo a la generación de eventos específicos. Una característica más del Entorno de Simulación es que permite la manipulación de un modelo de simulación determinista el cual no contiene variables aleatorias, sino que por el contrario todas las variables están controladas por parámetros que pueden ajustarse con el fin de detectar las condiciones 
sobre las cuales los elementos del sistema se comportan ante eventos específicos.

Durante la Fase II, por una parte el ASincronización es quien monitoriza el estado del sistema y por otra parte el ASimulador es responsable del control del proceso de simulación. El ASimulador es el responsable del control de las actividades que deben ejecutarse antes, y aquellas solicitudes que pueden ser atendidas durante la simulación o cuando la simulación del Modelo ha finalizado.

El ASimulador puede desempeñar dos roles esenciales:

Controlador de Eventos. El ASimulador se encuentra sujeto a la aparición de eventos disparados por la intervención del Usuario o como resultado de las interacciones entre éste y el conjunto de agentes asociados a iconos que representan el Sistema de Fabricación, Agentes de Recursos, de Orden y de Staff. De acuerdo al evento que detecta, éste determina el nuevo rol al que el ASimulador debe cambiar. De esta manera su aparición es aperiódica y se mantiene vivo hasta el momento de la finalización de la Fase II para un modelo dado.

Asistente de Modelado. Cuando el Usuario solicita la edición de un Modelo que está siendo simulado, el ASimulador desempeña este rol a fin de que sean editados ó añadidos los iconos (representación gráfica) que representan elementos del Sistema de Fabricación o sea modificada la asociación de instancias específicas a iconos del modelo. Este rol es indispensable puesto que el Usuario puede solicitar la modificación del modelo durante la fase de simulación a fin de observar el nuevo comportamiento del Sistema de Fabricación. Cuando un elemento es agregado o eliminado del Modelo, su respectivo agente es creado o eliminado. De esta forma, al nuevo agente se le asocia una instancia y al finalizar, éste se incorpora al Sistema de Fabricación de forma transparente al Usuario. El nuevo agente se encuentra preparado para participar en cualquier proceso de interacción con los agentes creados en la fase inicial de apertura del Modelo. De esta forma el Entorno de Simulación permite la escalabilidad del Sistema de Fabricación sin detener la ejecución de la simulación aportando una mayor flexibilidad al Sistema que se simula.

Asimismo, en el caso de que el Usuario requiera asociar una instancia a Agente de Recurso la cual no está registrada en la Base de Datos del sistema, este rol también genera un evento que solicita la creación de una nueva instancia. En este momento el ASincronizador recibe esa petición 
y cambia a su rol de "Creador de Agentes" para crear a su vez una instancia del Agente AGestorIconos. El AGestorIconos permite desde la Fase II agregar una nueva instancia a la Base Datos. Una vez que ha terminado, el AGestorIconos la pone a disposición del ASimulador/Asistente de Modelado quien puede hacer uso de la nueva instancia y asociarla a un icono.

Gestor de Animación. Cuando el Usuario solicita que inicie la simulación / animación de un Modelo, el ASimulador desempeña este rol a fin de que sean animados gráficamente los iconos (representación gráfica) que representan elementos del Sistema de Fabricación (Operarios, Máquinas, Herramientas, Staff, Ordenes de Producción y Mantenimiento). La principal función de este rol es ajustar la visualización gráfica de los elementos del modelo de acuerdo al comportamiento que estén desempeñando internamente como resultado de sus interacciones. Cuando los Agentes del Sistema de Fabricación cambian su estado de actividad, es decir, cambian de estar a la espera de la aceptación y ejecución de acciones de procesamiento de trabajos, o viceversa, cuando terminan los trabajos en cola y quedan a la espera de nuevos trabajos, se actualiza dicho estado en una pizarra general (black board) la cual es verificada cada determinado lapso de tiempo por el ASimulador/Gestor de Animación. Un aspecto importante de este rol es que su activación depende de restricciones temporales, que contemplan su aparición cada 5 minutos hasta el momento de la finalización de la simulación. Esta restricción también se ve afectada cuando el Usuario ajusta la velocidad de simulación.

Gestor de Velocidad. Cuando el Usuario solicita el ajuste de velocidad para la simulación/animación de un Modelo, el ASimulador desempeña este rol a fin de que el Usuario especifique la velocidad de ejecución de la simulación/animación del comportamiento de un Modelo. El objetivo de este rol es definir la velocidad global (y por lo tanto el reloj de control) de la ejecución del reloj que controla la simulación, e informar a todos los agentes que integran el modelo de fabricación, los cuales también deberán ajustar la velocidad en la cual deben ejecutar sus acciones. Ya sea antes de iniciar o durante una simulación/animación del modelo cuando el Usuario define una velocidad de ejecución, ésta es comunicada, no solo a los agentes que integran el Entorno de Simulación sino también a los agentes que actúan como elementos del Sistema de Fabricación. El Entorno de Simulación permite al Usuario ajustar la velocidad en cualquier momento. 
En la Fase II, cuando el ASimulador juega el papel de Asistente de Modelado, éste ofrece las mismas funcionalidades que el AModelador/Asistente de Modelado de la Fase I, esto es con el fin de agilizar las actividades de pruebas y verificación del Modelo bajo condiciones específicas (p.ej. durante la simulación se puede eliminar una máquina específica para identificar qué resultados se obtendrían de la operación de la planta sin tal elemento).

\subsubsection{Apertura del Modelo a Simular}

En el momento que el Usuario selecciona el Modelo a simular, el ASimulador en su rol de Controlador de Eventos ejecuta dos acciones:

a) Solicita al ASincronización/GestorInstancias la generación de las instancias de Agentes correspondientes a los elementos que incluye el Modelo seleccionado (operarios, máquinas, herramientas, staff).

b) Cambia su rol al de ASimulador/Gestor de Animación y construye gráficamente la distribución de los elementos del Modelo tal y como fue diseñado durante la fase de Creación del Modelo. En esta fase asigna una configuración de animación inicial ${ }^{1}$.

A partir de ese momento se tienen dos Sistemas Multiagente activos que interactúan representando al Entorno de Simulación y al Sistema de Fabricación (representado por el Modelo).

\subsubsection{Configuración preliminar en la Simulación del Modelo}

Entre las actividades que deben ser ejecutadas por el Usuario antes de iniciar una Simulación se encuentran:

- Determinar la condición de parada de la Simulación del Modelo.

- Establecer la velocidad de ejecución de la Simulación.

- Verificar y en su caso modificar el tipo de animación asociada a cada elemento del Modelo a simular.

- Definir el tipo de escenario de negociación entre las Órdenes de Producción y los Recursos (Operarios, Máquinas y Herramientas). En este caso, permite observar el comportamiento de la planta cuando es la Orden la

\footnotetext{
${ }^{1}$ El detalle de esta configuración se presenta en un apartado más adelante
} 
que solicita los servicios a los Recursos, o en caso contrario, lo que sucede cuando son los recursos los que solicitan Tareas a las Órdenes de Producción.

Como primer paso, al abrir el Modelo a Simular el ASincronización/GestorInstancias crea las instancias de agentes del Sistema de Fabricación.

Como se presentó en la Sección 2.4.5, en referencia al Control de la Simulación, la definición de la condición de parada es un aspecto importante que debe incluir el Entorno de Simulación. Antes de lanzar una Orden de Producción el ASimulador/Controlador de Eventos solicita al Usuario la definición de la Condición de Parada de la Simulación. Para ello tiene dos alternativas para detener la simulación: a) la simulación se detiene al finalizar una orden de producción específica, y b) la simulación se detiene en una fecha y hora específica. Por otra parte, además de incluir tal condición, el ASimulador/Controlador de Eventos permite al Usuario detener la simulación en cualquier momento bajo la responsabilidad del Usuario, con respecto a las ordenes que aún no han sido terminadas.

El ASimulador/Controlador de Eventos solicita al Usuario la definición de la velocidad para la ejecución de la animación/simulación del Modelo la cual se la comunica a su rol de Gestor de Velocidad. Cuando la velocidad se ajusta, el Gestor de Velocidad se lo informa a los agentes que integran el Sistema de Fabricación. Durante la Simulación el Usuario puede verificar y/o modificar el tipo de animación para cada uno de los elementos del modelo; tal configuración se la comunica a su rol de Gestor de Animación.

Una vez que el ASincronización/GestorInstancias ha creado las instancias de los agentes asociados al Modelo, el ASimulador en su rol de Gestor de Animación mantiene comunicación con los agentes que representan al Sistema de Fabricación, y de esta forma actualiza su representación gráfica con el comportamiento interno de cada uno de los agentes durante la simulación (ofreciendo servicios, negociando, colocando trabajos en cola y procesando trabajos).

\subsubsection{Control y Detección de Cambios de Estado}

En la Segunda Fase, la detección de cambios de estado del sistema (presentada en la Sección 3.2) se realiza mediante dos métodos: 
Blackboard ("pizarra"). En la propuesta se utiliza un blackboard común tanto al Sistema de Fabricación como al Entorno de Simulación. El Sistema de Fabricación es el responsable de alimentar el blackboard con datos referentes a los cambios de estado de sus elementos (detenido, trabajando -negociando reserva de capacidad, ejecutando trabajos aceptados-) de los agentes que lo integran (Operarios, Staff, Máquinas y Herramientas). Por otra parte, el Entorno de Simulación consulta el blackboard para detectar los posibles cambios de estado de dichos agentes y disparar un evento de actualización en la visualización gráfica de los mismos. Dicha consulta está sometida a restricciones de tiempo periódicas la cual aunque se define inicialmente, se ve alterada en el momento que el Usuario ajusta la velocidad de simulación.

Eventos. El ASimulador/ControladorEventos se encuentra sujeto a la aparición de eventos específicos que disparan una situación significativa de cambio que obliga al ASimulador a cambiar de rol y por tanto de acciones a ejecutar. Los eventos pueden ser disparados tanto por intervenciones del Usuario como por el mismo ASimulador como resultado de la consulta al blackboard.

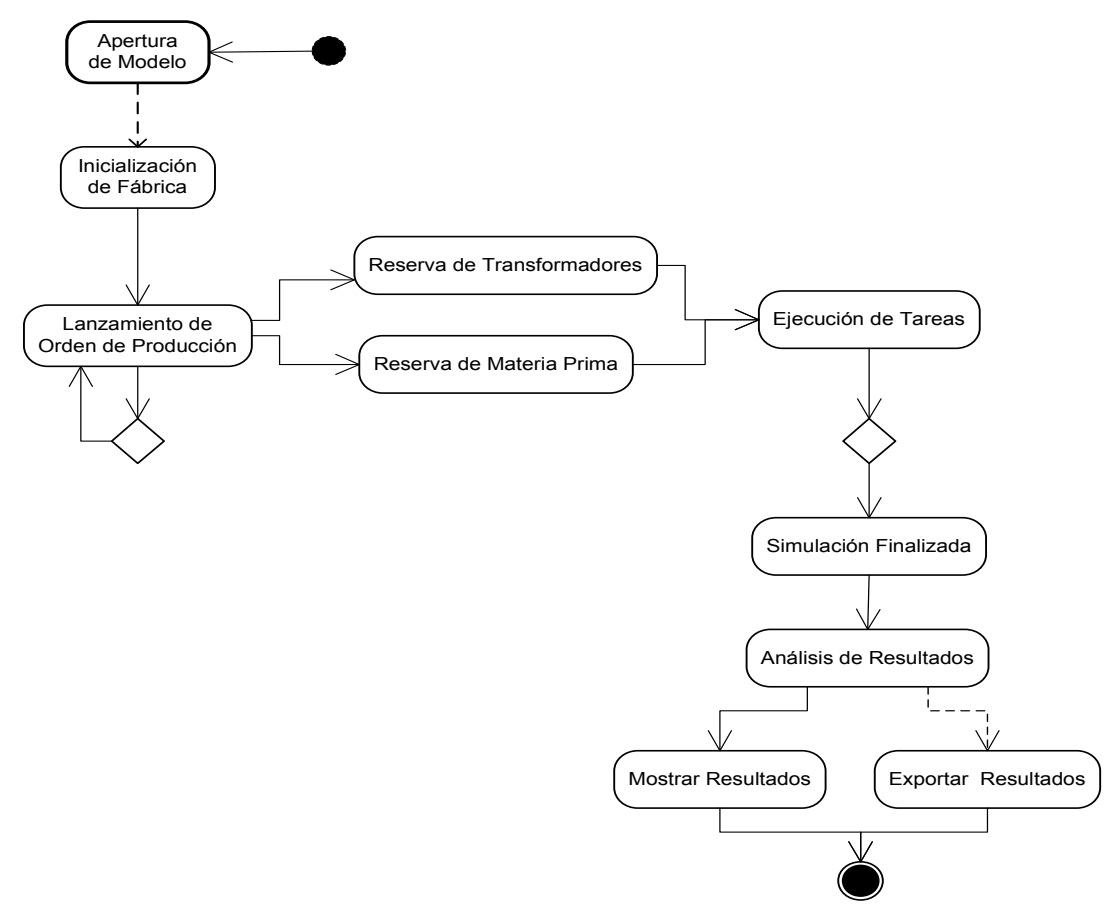

Figura 7.1: Estados del Sistema durante la Fase II 
La Figura 7.1 muestra los distintos estados del sistema que se pueden presentar durante la Fase II. Las líneas continuas identifican aquel escenario que precede a otro escenario, mientras que las líneas discontinuas aquellos escenarios que pueden aparecer opcionalmente a petición del Usuario (diseñador).

En las primeras fases de análisis se detectaron los roles requeridos a fin de proporcionar toda la funcionalidad de una herramienta de simulación en la Fase II "Simulación del modelo" (Gestor de Animación, Gestor de Eventos, Gestor de Velocidad, Controlador de Eventos,etc.). Los beneficios obtenidos al centralizar tales roles en un Agente (ASimulador) se presentan a continuación:

1. La centralización de roles permite ampliar la base de conocimiento del agente al momento de tomar decisiones y adoptar el comportamiento apropiado acorde a la situación actual del sistema.

2. Debido a que cada uno de los roles ejecutan acciones específicas y al finalizarlas no requieren permanecer vivos de forma continua sino que su activación depende de la aparición de eventos precisos.

3. La reducción de instancias que no son necesarias en todo momento, permite la optimización de recursos de memoria que deben quedar disponibles para la ejecución de procesos complejos tales como dar soporte a los recursos que desempeñan operaciones de manera continua tales como el Gestor de Animación o a los agentes que integran el Sistema de Fabricación (Orden de Producción, Operarios, Staff, Máquinas, y Herramientas).

El ASimulador da soporte a la simulación del comportamiento del Sistema de Fabricación manteniendo una constante verificación del estado del Sistema de Fabricación. De esta forma, el ASimulador reacciona ante la aparición de eventos específicos generados tanto por la intervención del Usuario como por eventos generados a partir del Sistema de Fabricación. Ante dichos eventos, el Agente cambia el rol que desempeña y por tanto las acciones que debe ejecutar (Fig. 7.2). 


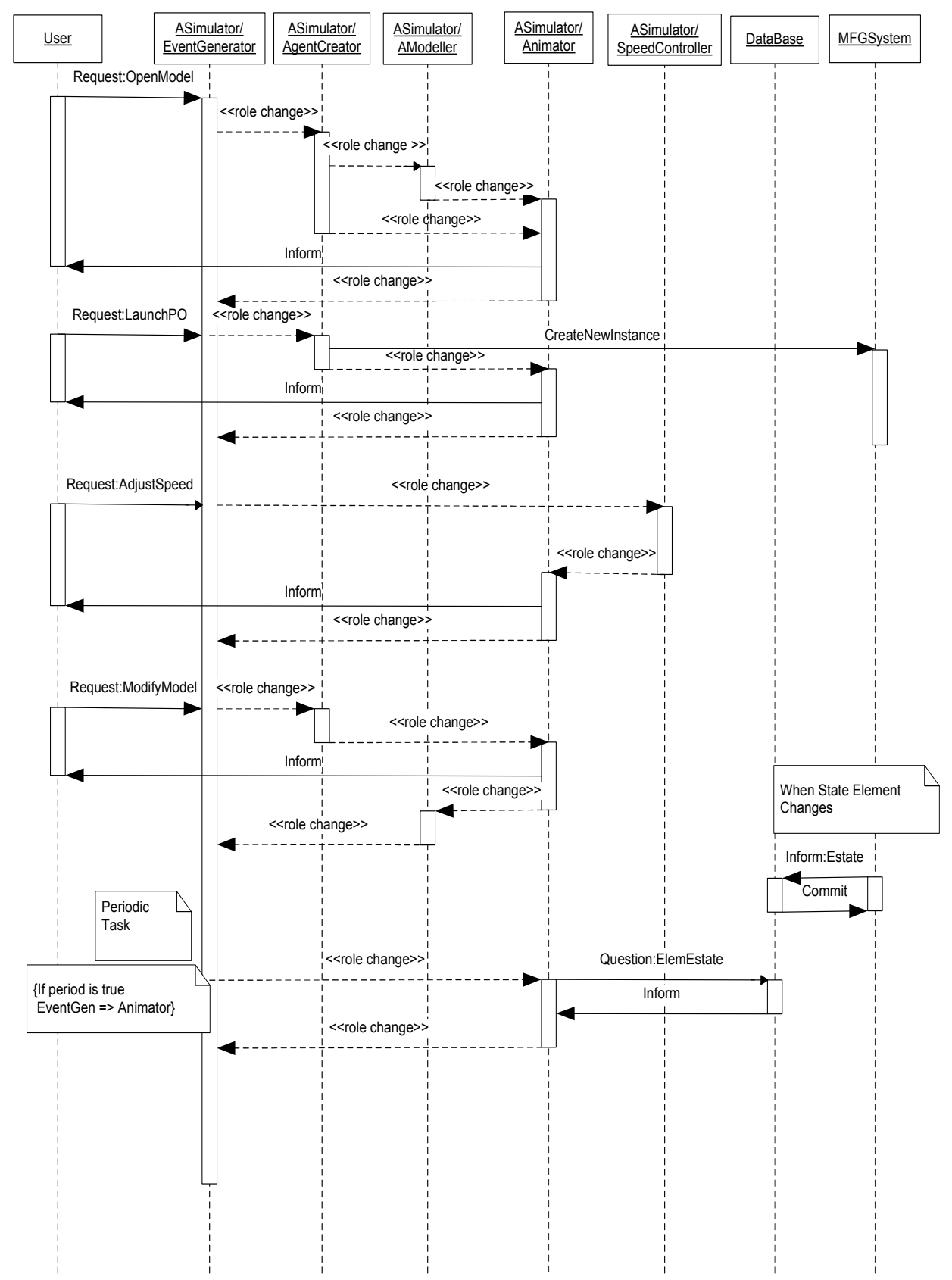

Figura 7.2: Diagrama de Cooperación entre el Agente de Simulación (sus roles) y su Entorno durante la Simulación del Modelo 


\subsubsection{Efectos de animación de Iconos}

La animación de los iconos se implementa en función del comportamiento que esté desempeñando el agente asociado al icono. Los agentes pueden estar en tres estados generales: Activo, Detenido y Muerto (Fig. 7.3). Sin embargo dependiendo del tipo de agente, mientras se encuentran en estado Activo, los agentes desempeñan distintos comportamientos en respuesta al estado general del Sistema. Tales comportamientos también pueden ser vistos como subestados los cuales requieren ser animados gráficamente.

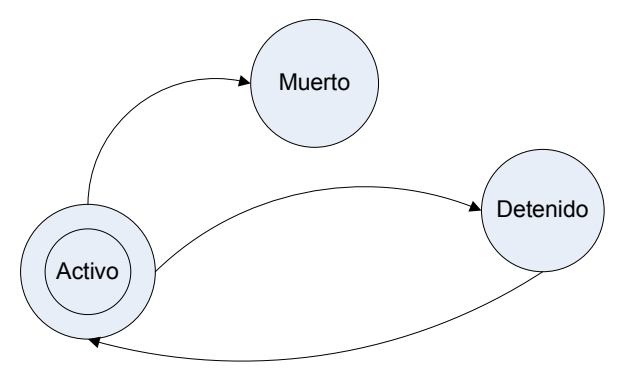

Figura 7.3: Estados generales de los Agentes

La Figura 7.4 muestra los subestados de un Agente Transformador (Operador, Máquina, y Herramienta) mientras se encuentra Activo.

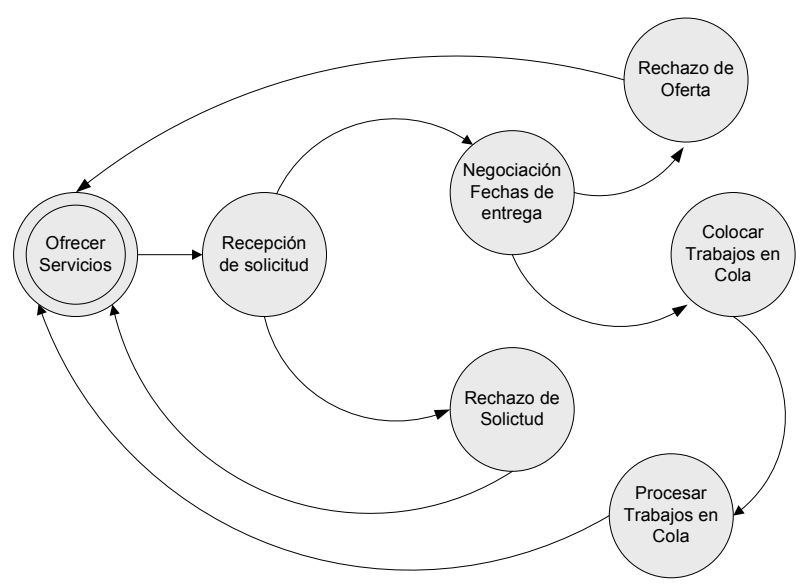

Figura 7.4: Subestados de un Transformador

La Figura 7.5 muestra los subestados de un Agente de Orden de Producción mientras se encuentra Activo. 


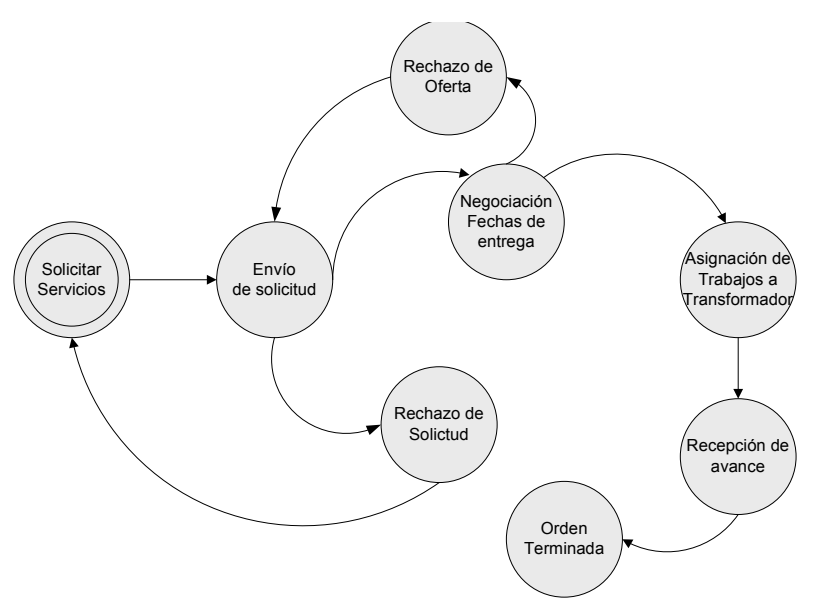

Figura 7.5: Subestados de una Orden de Producción

Los efectos de animación que se asocian a los iconos son: el Parpadeo, el Movimiento Horizontal y Vertical y el cambio de color. De acuerdo con los subestados de los agentes, la configuración de la animación incluye dos opciones: la configuración de movimiento y la configuración de color. La configuración de movimiento incluye el parpadeo, así como el movimiento horizontal y vertical. La configuración de color incluye los colores rojo, amarillo, naranja y verde. En la Tabla 7.1 se muestra la configuración inicial de tales opciones ante los subestados de un Agente Transformador durante su interacción con un Agente de Orden de Producción.

\begin{tabular}{|l|l|c|c|}
\hline \multicolumn{2}{|c|}{ Agentes } & \multicolumn{2}{c|}{ Tipo de Animación } \\
\hline Orden de Producción & Transformador & Movimiento & Cambio de Color \\
\hline Solicitar Servicios & Ofrecer Servicios & Ninguno & Rojo \\
Negociar & Negociar & Movimiento Vertical & Amarillo \\
Asignar Trabajos & Aceptar Trabajos & Movimiento Horizontal & Naranja \\
Recepción de Avance & Procesar Trabajos & Parpadeo & Verde \\
\hline
\end{tabular}

Tabla 7.1: Tipos de Animación acorde a los Subestados de los Agentes Transformadores y el Agente de Orden de Producción.

Los efectos de animación están asociados a cada uno de los iconos que representan Operarios, Máquinas, Herramientas y Staff, así como las Ordenes de Producción y Mantenimiento de manera preestablecida (Tabla 7.2). Sin embargo el Usuario antes o durante la Simulación puede cambiar dicha configuración. 


\begin{tabular}{|c|c|}
\hline Tipo Elemento & Tipo de Animación \\
\hline Operario & Movimiento \\
Máquina & Cambio de Color \\
Herramienta & Cambio de Color \\
Staff & Movimiento \\
Orden de Producción & Cambio de Color \\
Orden de Mantenimiento & Cambio de Color \\
\hline
\end{tabular}

Tabla 7.2: Tipos de Animación Asociado al Tipo de Elemento.

De esta forma, al momento en que son creados los agentes que simulan cada uno de los elementos del Modelo, todos los Agentes adoptan el estado de Ofrecer Servicios (o el estado de Solicitar Tareas cuando los Recursos solicitan tareas a las Ordenes de Producción). Cuando el estado del sistema cambia, en respuesta los agentes comienzan a ejecutar alguna acción. Tales acciones activan algunos de sus subestados cambiando así su estado de animación. Los cambios de estado de elementos del Sistema de Fabricación se informan al Sistema mediante un "blackboard" donde cada elemento (Operarios, Máquinas, y Herramientas) indica cuando tiene un cambio de estado. El Blackboard es consultado por el Gestor de Animación quien actualiza la visualización gráfica de dichos elementos. Cuando el Usuario ajusta la velocidad de la simulación, al Gestor de Animación se le informa y éste ajusta la velocidad en que se ejecuta la animación de los iconos. Esta tarea de monitorización de los cambios de estado y por tanto de animación es una tarea síncrona, la cual se ejecuta en un principio a intervalos de 3 minutos; sin embargo, cuando la velocidad se ajusta acelerando el tiempo de simulación, tal intervalo también se reduce a fin de mostrar el comportamiento de los agentes en los iconos asociados a cada uno.

Por otra parte, el Agente Gestor de Almacén no cambia su representación gráfica durante la simulación, pero desempeña tareas clave durante la simulación, tales como la reserva de materiales con los Agentes de Orden de Producción y la entrega de materiales con los Agentes Transformadores (Operarios, Máquinas y Herramientas). Los iconos de Punto de Control no tienen asociado un agente ni tampoco cambian su representación gráfica, aunque desempeñan un papel importante para la simulación puesto que sirven de apoyo a las tareas de los Agentes de Supervisión (Staff). 


\subsubsection{Control de Avance de Tiempo}

Para este apartado se han considerado los aspectos relaciones con el control del tiempo para la simulación expuestas en el Capítulo 2. Puesto que la teoría de agentes permite un manejo más complejo de control de reloj al tener el control distribuido, en la propuesta se han mezclado varios conceptos a fin de proporcionar la flexibilidad requerida para la representación de las actividades de cada uno de los Agentes del Sistema de Fabricación.

En este caso, se utiliza el mecanismo de avance de Tiempo Síncrono (intervalos fijos) puesto que las condiciones sobre las cuales se trabaja durante la simulación se adaptan a este enfoque. Esto es, la distribución del control permite agrupar los sucesos que aparecen de una forma regular y equiespaciada. Es decir, cada agente controla sus tareas las cuales son atendidas conforme a una cola de trabajos. Durante un periodo de Tiempo Simulado es posible que ocurran muchos eventos y que la longitud de la cola de trabajos sea corta en promedio.

Cuando la simulación inicia, el Tiempo de Simulación se controla en base a un reloj que hace lecturas del reloj del sistema, de tal forma que el intervalo del reloj avanza a intervalos fijos de 1000 milisegundos. En ese momento, es posible observar los comportamientos de los agentes en tiempo real y por tanto el Tiempo de Simulación es igual al Tiempo de Ejecución.

Sin embargo, este principio se ve alterado cuando el reloj requiere ser acelerado/desacelerado para reducir el tiempo de la simulación. La aceleracion / desaceleración es una condición primordial en una Herramienta de Simulación donde uno de los objetivos de su uso es la obtención de resultados en un tiempo considerablemente reducido en comparación con otras alternativas de evaluación de situaciones (p.ejem. observación directa, laboratorio de pruebas, miniplanta de fabricación). Cuando el reloj se acelera el intervalo de avance se reduce en $\mathrm{N}$ milisegundos, mientras que cuando se desacelera, el intervalo de avance se aumenta en $\mathrm{N}$ milisegundos. A partir del momento de ajuste de velocidad el intervalo de avance se modifica (aumentándolo o reduciéndolo) pero el reloj sigue avanzando a intervalos fijos.

Supongamos que las velocidades de avance son 100x, 1000x, 5000x, y 10000x milisegundos. Esto quiere decir que al acelerar la velocidad en 100x, 1000 milisegundos del Tiempo de Ejecución, representan 1100 milisegundos del tiempo de Simulación. Con la Aceleración del Tiempo de Simulación el Tiempo de Ejecución se ve considerablemente reducido (Tabla 7.3). 


\begin{tabular}{|l|c|c|}
\hline Velocidad & Tiempo Ejecución & Tiempo Simulado \\
\hline $100 \mathrm{x}$ & 1 Hora & 1,1 Hora \\
$1000 \mathrm{x}$ & 1 Hora & 2 Horas \\
$5000 \mathrm{x}$ & 1 Hora & 55 Horas \\
$10000 \mathrm{x}$ & 1 Hora & 110 Horas \\
\hline
\end{tabular}

Tabla 7.3: Velocidad y Tiempos de Ejecución / Simulación.

Por tanto, se cuenta con un Reloj Maestro (el cual es visualizado por el Usuario) que está controlado por el ASimulador/ControladorEventos quien recibe los ajustes solicitados por el Usuario y se lo informa al resto de los agentes. Un aspecto importante que se considera es el hecho de que la misma distribución del control da lugar a un control de tiempo que tiene que ser informado a los agentes activos. Cada uno de los agentes tiene que mantenerse al tanto de tareas que tienen en sus colas de trabajos pendientes, las cuales a su vez tienen intervalos de duración que generalmente son diferentes.

El mantener un solo reloj que controle el las tareas de todos los agentes no resulta coherente por dos razones: a) va en contra del principio de descentralización de la teoría de los Sistemas Multiagente y b) cada uno de los agentes llevan a cabo tareas con diferentes periodos de aparición y duración. Sin embargo, si se propone mantener un reloj maestro que informe a todos los agentes de la aceleración/desaceleración del tiempo cada vez que éste se actualiza . Por otra parte, se debe considerar que cuando el tiempo de las tareas es reducido, se debe controlar la aceleración del reloj, ya que es probable no sea posible que los agentes reciban oportunamente los mensajes de los cambios en el reloj y puedan responder en tiempo.

Por esta razón, la propuesta considera la creación de un Reloj Maestro controlado por el ASimulador/ControladorEventos y un reloj para cada uno de los agentes Transformadores (Operarios, Máquinas, Herramientas) y el Staff. Los relojes de los Transformadores se sincronizan con el Reloj Maestro ante un ajuste de velocidad, de tal manera que los agentes leen dicho reloj para informar de los eventos que tienen en su cola (p.ejem. al procesar una orden, de acuerdo con el tiempo de procesamiento establecido para una pieza, el transformador lee el reloj e indica cuando ha terminado una pieza).

En el momento que el $\boldsymbol{U}$ suario ajusta la velocidad, el Reloj Maestro se ajusta y el ASimulador/ControladorEventos lo informa a todos los agentes (Operarios, 
Máquinas, Herramientas, Staff y Ordenes de Producción) que se encuentren activos para que éstos a su vez ajusten sus relojes (Figura. 7.6). Debido a que los agentes al momento de procesar están en continua lectura de su reloj, los ajustes de reloj son considerados de forma natural, es decir, que la tarea que esté en procesamiento no se ve detenida pero sí que considera un ajuste.

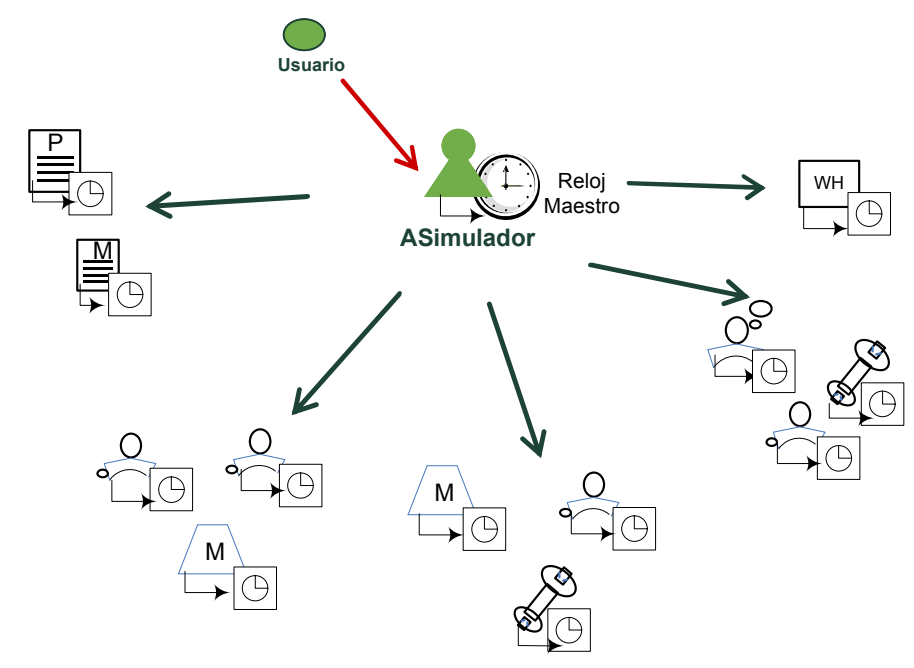

Figura 7.6: Sincronización de Relojes.

Finalmente, cuando la condición de parada ha sido establecida en referencia a una fecha y tiempo específicos, el ASimulador/ControladorEventos también está al tanto para que cuando la condición de parada es alcanzada, ésta se informe y se detenga la simulación.

Entre las ventajas de esta propuesta podemos encontrar: a) reducción en tiempo de respuesta de los agentes ante la aceleración de velocidad, b) proceso de ajuste de tiempo en línea generalizado, c) reducción de cuellos de botella por centralización de control de eventos de tiempo y d) control distribuido de tareas controladas por reloj con diferentes intervalos de ejecución.

\subsubsection{Creación de Trazas}

Resulta importante contar con un instrumento que almacene las acciones que se van ejecutando en el sistema y que a su vez se almacenen para dar seguimiento a las acciones internas entre los agentes y futuras consultas. 
Para ello, cada una de las acciones que ejecuta el Entorno de Simulación como resultado de la intervención del Usuario son registradas en un "blackboard" el cual sirve no solo para efectos de simulación sino para que el ASincronizador monitorize las acciones de los agentes. Asímismo, cada uno de los Agentes del Entorno de Simulación y los Agentes del Sistema de Fabricación van alimentando el "blackboard" con sus acciones. Por otra parte el ASimulador consulta el blackboard y va mostrando al Usuario mediante una interfaz tales acciones.

Finalmente, suponiendo que se cuenta con un Entorno de Simulación y un Modelo de un Sistema de Fabricación genérico en los cuales se busca observar bajo qué escenarios interactúan y cómo se comunican los agentes que los componen; en la siguiente sección se presentan las estrategias y protocolos básicos de comunicación que utilizan los agentes durante la simulación de un modelo.

\subsection{Estrategias y Protocolos de comunicación del Sistema de Fabricación durante la Simulación del Modelo}

El Entorno de Simulación depende de los resultados generados por las interacciones que surgen entre los elementos (agentes) que integran el Sistema de Fabricación. De tal manera que en esta sección se plantean los principales Protocolos y Estrategias que se proponen a fin de que el Entorno de Simulación se sirva de ellos para la animación del Modelo y la detección de cambios de estado. Los cambios de estado disparan acciones tales como: a) la creación de instancias de agentes requeridos (por ejemplo, cuando la simulación ha terminado de crear una instancia del Agente de Resultados, para que se le permita al Usuario generar las gráficas y reportes correspondientes) y b) la eliminación de instancias de agentes que no son necesarios (por ejemplo, cuando termina una simulación por completo es necesario eliminar todas las instancias de los agentes que integran el Modelo del Sistema de Fabricación que se simuló).

El Sistema de Fabricación tiene como objetivo la optimización de los recursos disponibles integrados en el Modelo creado durante la Fase I "Creación de Modelo”. Estos elementos (Operarios, Staff, Máquinas, y Herramientas) son puestos a disposición ante dos situaciones: a) para cubrir las necesidades del procesamiento requeridas por una Orden de Producción y b) para realizar operaciones de mantenimiento necesarias para mantener el buen funcionamiento de 
máquinas y herramientas. De esta forma cada uno de los elementos que pueden ejecutar procesos (denominados Transformadores) pueden desempeñar dos roles:

Negociador. Cuando una Orden de Producción realiza procesos de negociación para la asignación de trabajos a los recursos. Los Transformadores participan en un proceso de negociación (descrito más adelante) dirigido a la reserva de su capacidad al asignársela a la solicitud de una Orden de Producción. Cuando un trabajo es aceptado, éste se coloca en la cola de trabajos del Transformador a la espera de su ejecución.

Ejecutor. Una vez que los trabajos se encuentran en su cola de trabajos, el Transformador las ejecuta por orden de llegada. En ese momento el Recurso solicita la entrega de los materiales reservados previamente por la Orden de Producción. Conforme los trabajos son atendidos, su avance es informado a la Orden de Producción.

Asimismo, la Orden de Producción tiene asociados dos roles principales:

Negociador. Siguiendo la ruta de fabricación del Producto y su lista de transiciones, la Orden de Producción actúa como iniciador del proceso de negociación para la asignación de trabajos a los Recursos de la Planta de Fabricación y de las reservas de los materiales requeridos.

Gestor de Orden. Una vez que los trabajos han sido asignados a los Recursos Transformadores, la Orden recibe información por parte de dichos recursos sobre el avance de la producción.

En las siguientes secciones, se detalla cómo se presentan las interacciones entre agentes y cuáles son las bases sobre las cuales se plantean los comportamientos de cada uno y los procesos de negociación, estrategias y protocolos utilizados para representarlos.

\subsubsection{Simulando el Lanzamiento de una Orden de Produc- ción}

Durante la Simulación de un Modelo de un Sistema de Fabricación (contemplada en la Fase II de la propuesta) se realizan los procesos de negociación clave para la optimización de los recursos durante el lanzamiento de una Orden de Producción. En este paso el ASimulador/Controlador de Eventos recibe la 
petición del Usuario para lanzar una Orden de Producción para su fabricación. Cuando se crea el Agente Orden de Producción (AOrdenP), éste es quien inicia el proceso de asignación de trabajos a Recursos y la solicitud de los materiales necesarios.

Al lanzar una Orden de Producción, sólo se debe permitir seleccionar aquellos productos que han sido asociados previamente a un Modelo. Una vez seleccionado el producto, se elige la ruta que se utilizará, ya que se pueden haber definido más de una ruta alternativa para la fabricación del producto. A continuación, como datos básicos, se pueden definir: a) la cantidad de productos requeridos y b) la fecha en que se requiere la entrega del mismo; y como dato adicional se podría incluir la prioridad de la orden (alta, media, baja). Esta prioridad es utilizada para fines de reserva de material y reserva de capacidad para la asignación de trabajos a recursos.

Cuando se selecciona la ruta, internamente se vincula a la Orden de Producción, el listado de las operaciones (o tareas) que integran la ruta. Para validar diferentes Configuraciones de la Planta de fabricación, el Usuario puede definir qué recursos del modelo se utilizan para que ejecuten cada una de las operaciones de la ruta. De este modo, es posible seleccionar aquellos recursos que por su naturaleza pueden llevar a cabo una operación (por ejemplo, una pistola kloester sólo puede soldar). Una vez que se seleccionan, tal información queda vinculada a la orden para sus posteriores procesos de negociación y asignación de tareas.

Asimismo, cuando se selecciona la ruta, es posible obtener un listado de las configuraciones de planta previamente definidas para dicha ruta. De esta forma, el Usuario puede reutilizar una configuración utilizada previamente o crear una nueva configuración. Cuando se lanza una orden de producción de un producto, la Configuración de Planta define la asociación de las instancias de recursos a las operaciones de la ruta de fabricación seleccionada.

Para la selección de la Configuración de Planta apropiada se recurre a una evaluación. La evaluación de la configuración de la planta considera las características de los elementos seleccionados con respecto a su funcionalidad y los resultados que pueden ser obtenidos.

En la evaluación de la configuración, la propuesta contempla la evaluación de la configuración de la ruta seleccionada para el lanzamiento de un orden antes de que se ejecute su simulación. La evaluación puede ser realizada de cuatro 
formas: a) evaluar la ruta calificándola en base al tipo de elementos que integra su configuración, b) evaluar el coste de dicha configuración, c) evaluar el tiempo de procesamiento previsto con respecto al tiempo estándar y d) evaluar la configuración en base a una fórmula definida por el Usuario que integre los tres elementos anteriores.

De esta forma, el Usuario puede observar la calificación de la configuración seleccionada. En su caso, el Usuario tiene la alternativa de continuar el proceso de negociación para la asignación de las tareas con dicha configuración, solicitar al Entorno de Simulación que utilize una configuración previa o definir una nueva configuración.

\section{Bases de la estrategia para la Asignación de Tareas}

En este apartado se presenta una posible alternativa de estrategia que el Agente de Orden de Producción AOrdenP puede aplicar durante el protocolo de negociación para la asignación de Tareas a los Recursos de la planta de fabricación. La toma de decisión se basa en los tres aspectos que considera el diseño de un FMS (Sección 4.6): maximizar el volumen de producción dentro de las restricciones de tiempo de las máquinas, mientras que al mismo tiempo se busca minimizar el coste y maximizar la flexibilidad. A continuación, se presenta una estrategia orientada a maximizar el volumen de producción dentro de las restricciones de tiempo de las máquinas.

Cuando la velocidad de la simulación se encuentra dentro del rango de 100x a 1000x es posible llevar a cabo procesos de negociación que requieren un tiempo relativamente largo, tal como es el caso de las subastas. Se toma como referencia el modelo básico para el control de una planta de fabricación basado en subastas (Veeramani \& Wang 1997, Veeramani et al. 1998) (Sección 3.2.3). Aunque el modelo original plantea el proceso de una sola etapa de procesamiento, en este trabajo se plantea la ampliación del modelo para su aplicación en la fabricación de productos con más de una etapa de procesamiento. En el Entorno de Simulación se propone que los agentes de orden de producción anuncien las tareas (ofertas), mientras que los agentes de recursos (Operarios, Máquinas, Herramientas, y Staff) actúen como receptores de los anuncios de las tareas. De esta forma, el modelo básico queda como sigue:

Anuncio de Tarea. Cuando un Agente de Orden de Producción (AOrdenP) llega al sistema, siguiendo la ruta del producto que pretende fabricar, éste 
envía el anuncio de una tarea a todos los agentes de Recurso (Operarios, Máquinas, Herramientas, y Staff). Esta etapa la ejecuta en 2 fases: identificación de los agentes participantes (aquellos que tienen la capacidad para realizar dicha tarea) y envío de la solicitud de la tarea a dichos agentes.

Envío de Oferta. Cada agente de Recurso (ARecurso) evalúa su capacidad para procesar la tarea solicitada y envía una oferta al agente de Orden de Producción.

Evaluación de Oferta. El agente de Orden de Producción (AOrdenP) evalúa las ofertas, y adjudica la tarea al agente de recurso ganador. El agente ganador será aquel que ofrezca la mayor reducción en cuanto al tiempo de entrega.

Confirmación de Contrato. El agente de Recurso (ARecurso) ganador recibe la adjudicación, y envía un mensaje de confirmación del contrato al agente de Orden de Producción.

Este modelo se utiliza durante la fase de reserva de capacidad de los agentes de recursos. La etapa de procesamiento de la tarea es independiente del proceso de subasta para la asignación de dicha tarea. Aunque el procesamiento de la tarea dependerá de la cola de trabajos que tenga reservados el Agente de Recurso ARecurso. El Agente de Orden de Producción AOrdenP siguiendo la ruta de fabricación, continúa ejecutando la subasta de la siguiente tarea hasta la tarea final. A fin de coordinar dicho modelo, se aplicará el modelo del protocolo de coordinación basado en un patrón de etapas de comunicación entre agentes (Anussornnitisarn et al. 2001).

Por otra parte, cuando la velocidad de simulación supera la velocidad 1000x, entonces el proceso de negociación debe ajustarse a un tiempo más corto con respecto al tiempo requerido en una subasta. Para ello se contempla la aplicación de la técnica de Casos de Uso (Sección 7.2.2).

\section{Configuración Orden de Producción}

En la solicitud de Lanzamiento de Ordenes de Producción, el ASimulador/ ControladorEventos solicita al Usuario que defina cuatro datos básicos: 1) el Producto a fabricar (sólo se permite al Usuario elegir entre los productos asociados al Modelo abierto), 2) Ruta de Fabricación, 3) Configuración de la Planta (opcional), 4) Cantidad Requerida, 5) Fecha de Entrega, y 6) Prioridad de la 
Orden (opcional). La definición de la Configuración de la Planta es opcional, debido a que el $\boldsymbol{U}$ suario puede elegir definir la configuración de la planta (es decir, definir qué recurso específico ejecutará una tarea específica de la ruta) o permitir al Sistema que se lleven a cabo procesos de negociación de asignación de tareas con los recursos disponibles. Además, durante la solicitud de lanzamiento de Orden de Producción el Usuario puede definir el tipo de acción a tomar, cuando, durante la asignación de tareas, se presente un ciclo infinito (por ejemplo, se anuncia una tarea y ningún recurso está disponible, o cuando los recursos no tengan capacidad suficiente para cubrir el total de unidades solicitadas). Tales acciones incluyen:

- Si con el fin de optimizar la utilización de los recursos es posible distribuir la asignación del trabajo entre más de un recurso.

- En qué momento debe solicitar la intervención del Usuario a fin de evitar ciclos infinitos. Esto es, después de cuántos intentos de asignación de recursos sin éxito se debe avisar al Usuario de tal situación.

\section{Politica de Distribución de Ordenes.}

Como ya se mencionó anteriormente, antes de lanzar una Orden de Producción, es necesario que el Usuario especifique la política de distribución de órdenes, la cual permitirá la asignación completa o parcial de la cantidad de piezas que requieren ser terminadas para una operación de la ruta de fabricación:

a) Completa. En este tipo de asignación, los Recursos que participen deberán cubrir el $100 \%$ de la cantidad de piezas requerida.

b) Distribuida. En este tipo de asignación, los Recursos que participen deberán ofrecer un porcentaje mínimo de la cantidad de piezas requerida (PorD).

El porcentaje a solicitar es definido por el Usuario antes de lanzar una Orden de Producción.

Definición de Condición de Negociación Parada.

Como paso previo a la iniciación del proceso de asignación de trabajos a los recursos es indispensable que el Usuario defina una condición de parada que permita al agente de Orden de Producción $(a O P)$ determinar cuantas veces intentará asignar una tarea específica a alguno de los $\mathrm{N}$ agentes de Recurso $(a R)$ 
disponibles antes de solicitar la intervención del Usuario.

Consideraciones respecto a Tiempos de Procesamiento.

Puesto que el objetivo del Entorno es la Simulación del comportamiento de una Planta de Fabricación y la evaluación de las técnicas ya en uso, la definición de los tiempos de Fabricación y procesamiento no se generan por el Entorno de Simulación en sí, sino que se obtienen tales datos de la información proporcionada por el Usuario durante la fase de importación de datos.

Consideraciones durante la Asignación de Tareas a Recursos.

En la Fase II cuando se simula el Modelo definido en la Fase I, el Modelo se traduce en un Sistema Holónico de Fabricación (HMS). Tal Sistema toma como base la visión ideal de cómo un HMS debería operar presentada en la Sección 4.4.2. De esta forma cuando el Usuario crea una Orden de Producción, se crea un Holón de orden de producción que inicia una negociación con holones de recursos para proporcionar ciertas operaciones de fabricación. Durante el proceso de negociación, el holón de orden de producción demanda propiedades específicas de la operación, tal como alta calidad o alto rendimiento, mientras que los holones de recurso tratan de lograr su máxima utilización. De tal forma que los holones de recurso que finalmente son seleccionados para ejecutar las operaciones constituyen una configuración de planta específica.

El Entorno de Simulación considera dos posibilidades para la negociación entre el holón de orden de producción y los holones de recurso: a) la aplicación de diversas técnicas basadas en el paradigma de agentes para determinar la configuración de la planta, y b) la posibilidad de que el Usuario predefina la asignación de operaciones a los recursos.

Asimismo, para el proceso de Asignación de Tareas a Recursos, la aplicación del paradigma de agentes permite aplicar diversas técnicas (las subastas (Veeramani \& Wang 1997, Veeramani et al. 1998), el razonamiento basado en casos (CBR) (Choy \& Lee 2000), los protocolos de vencimiento de tiempo (Anussornnitisarn et al. 2001), el algoritmo de equilibrio de temperatura (Gordillo et al. 2006) y técnicas basadas en el comportamiento animal tales como: a) el uso de coordinación de colonias de insectos mediante uso de umbrales (Cicirello \& Smith 2001) y b) el sistema de ardillas (Carmorlinga \& 
BArker 2003, Gordillo et al. 2005)). El Entorno de Simulación además de permitir utilizar algunas de estas técnicas, permite al Usuario predefinir la asignación de Tareas a los Recursos incluidos en el modelo.

\section{Definir Configuración Planta.}

Cuando se crea un Orden de Producción y el Usuario decide predefinir la Asignación de Tareas a los Recursos, la definición de la configuración de planta se crea automáticamente antes de que el Agente de Orden de Producción se integre a la Planta de Fabricación. Una vez que se ha seleccionado la Ruta de Fabricación a utilizar, cuando el Usuario decide utilizar una configuración de planta previa o definir una nueva configuración, durante la definición de la Orden de Producción el sistema lleva acabo el siguiente procedimiento.

A. Selección de Tarea. El Usuario selecciona una Tarea de la Ruta de Fabricación a ejecutar. De esta forma se identifica el tipo de proceso general que requiere, el cual define el tipo de habilidad que se requiere de los agentes de recurso.

B. Selección inicial de Recursos. El sistema identifica los recursos integrados en el Modelo que poseen la habilidad identificada en la Selección de la Tarea. Posteriormente se los ofrece al Usuario quien selecciona el Agente de Recurso al que se le asignará la Tarea. Entre los datos que se ofrecen a fin de que el Usuario pueda aplicar un criterio de selección, se encuentran el centro de trabajo en el que esta asignado, y el grado de expertise (para los operarios). Tales criterios pueden servir de guía al Usuario durante la selección del Recurso más apropiado. Por ejemplo, si el objetivo del Usuario es obtener un producto con el más alto grado de calidad, los criterios le permiten observar datos tales como que un operario con un grado de destreza de "experto" garantiza un grado de calidad superior con respecto a un operario con un grado de destreza "principiante". Además es posible asociar tales grados de destreza a un porcentaje de reducción del tiempo de procesamiento con respecto al tiempo estándar.

Los Pasos A y B se repiten con todas las tareas que integran la Ruta de Fabricación seleccionada. Una vez finalizada la definición de la Orden de Producción, se crea el Agente de Orden de Producción (AOrdenP) y se integra a la Planta de Fabricación donde basado en la configuración de planta predefinida lleva a cabo el siguiente procedimiento: 
Basado en la Lista de Transiciones de la Ruta de Fabricación y en la Configuración de Planta predefinida, el Agente de Orden de Producción envía la solicitud de capacidad al Recurso asociado a la primera tarea origen.

El Recurso que recibe la solicitud, decide si puede atender a la solicitud partiendo de cuatro factores: 1) la cantidad de piezas requerida en la Orden de Producción (PzasReq), 2) el tiempo promedio de ejecución del proceso por unidad requerido por la Orden de Producción $(\operatorname{Tmp} P z a), 3)$ la fecha de entrega requerida $(F e c R e q)$ y 4$)$ la capacidad disponible de acuerdo con la fecha requerida $(C a p D R)$. En este apartado se propone una estrategia orientada a maximizar el volumen de producción dentro de las restricciones de tiempo de las máquinas.

1. Verificación de Capacidad Disponible. La capacidad disponible se calcula considerando la fecha de inicio disponible de acuerdo a la cola de trabajos aceptados previamente por el recurso, y la fecha requerida de entrega. De esta forma, de acuerdo con el tipo de Asignación de Ordenes definido por el usuario (Completa ó Distribuida), si el recurso puede cubrir al menos el porcentaje mínimo del total de unidades requeridas especificado por el usuario, entonces decide atender la solicitud y se lo comunica al Agente de Orden de Producción (AOrdenP). Esta toma de decisión se expresa mediante la siguiente ecuación.

$$
\text { PiezasD }=\left(\text { FecReq }-T C_{F f i n}\right) / T m p P z a
$$

donde:

TC Ffin, es la Fecha/hora en la que tiene programado terminar el último trabajo aceptado. En caso de que no tuviera trabajos en cola, entonces se considera la fecha/hora del momento de la solicitud.

Ca Disp, es el número de horas entre la Fecha/hora en la que tiene programado terminar el último trabajo aceptado $\left(T C_{F f i n}\right)$ y la Fecha Requerida de entrega $(F e c R e q)$ solicitada por la Orden de Producción.

PiezasD, es el número de piezas que se pueden fabricar durante el periodo de la cantidad de horas disponibles (Cap_Disp).

Si el Recurso seleccionado no tiene la capacidad suficiente para cubrir la 
solicitud, se le informa al Usuario quien tiene dos alternativas: a) Seleccionar otro Recurso y b) Permitir que el sistema negocie con un Recurso disponible aplicando la "Estrategia SelectivaII" presentada posteriormente. Posterior a la negociación con los recursos disponibles, en caso de que no sea posible atender la tarea en el tiempo solicitado, el Agente de Simulación (ASimulador) se lo informa al Usuario quien tiene dos alternativas: 1) cambiar el tiempo de entrega y volver a intentar buscar la asignación de tareas a Agentes de Recurso o 2) cancelar la Orden de Producción.

2. Verificación de posible Oferta. Basado en el tipo de distribución de órdenes (Completa /Distribuida), si el número de piezas es igual o superior al porcentaje definido por el usuario (Por D), entonces el agente de Recurso acepta o rechaza la solicitud del agente de Orden de Producción.

Casos:

a. Tipo Distribución = Completa.

Si PiezasD $>=$ PzasReq se acepta la solicitud.

b. Tipo Distribución $=$ Distribuida.

Si PiezasD $>=($ PorD $) *(P z a s R e q / 100)$ se rechaza la solicitud.

3. Responder a Ofertar servicios (propuesta). Una vez que el agente acepta la solicitud, se define la fecha de posible entrega del porcentaje de unidades acordado.

$$
\text { FecFinP }=T C_{F} \text { fin }+(\text { CantReq } * \text { TmpPro })
$$

donde:

TC_Ffin, es la fecha/hora en la que podría terminar tiene programado terminar el último trabajo aceptado.

CantReq, es el número total de Piezas Requeridas.

FecFinP, es la fecha/hora en la que el Recurso propone terminar el trabajo solicitado.

4. Aceptar trabajos. Si el agente de Orden de Producción (AOrdenP) confirma al Recurso que acepta su propuesta, el agente de Recurso (ARecurso) 
agrega el trabajo a la cola de trabajos pendientes la cual es independiente del recurso.

5. Informar del cambio de estado. El agente de Recurso ARecurso informa al agente de Orden de Producción (AOrdenP) que el trabajo ha sido aceptado y colocado en la cola de trabajos aceptados. Con ello se activa su rol de Ejecutor quien a continuación procesará el trabajo e informará del avance al agente de Orden de Producción (AOrdenP). Además informa al agente ASimulador/GestorAnimación sobre su cambio de estado con respecto a las órdenes de producción que ya ha aceptado para actualizar la visualización del icono asociado a dicho elemento en el modelo.

Los pasos del 1 al 4 se repiten hasta asignar todas las tareas de la Ruta de Fabricación. De acuerdo con la lista de Transiciones no será posible solicitar la asignación de tareas de recursos de una tarea destino hasta conocer la fecha de entrega confirmada de la tarea o tareas origen. La fecha de entrega más lejana de la tarea/s origen determina la fecha de inicio mínimo de la siguiente tarea destino.

Estrategia "SelectivaII".

En este apartado se presenta una estrategia que considera las bases de la técnica de las subastas para la asignación de tareas relacionadas con el paradigma de agentes. De tal forma, se propone que el Sistema la utilice cuando el Usuario no selecciona una configuración de planta para la asignación de las tareas a los recursos. Por tanto, al finalizar la negociación, el entorno crea automáticamente una nueva configuración de planta que se deja disponible para el lanzamiento de nuevas Órdenes de Producción.

Por un lado, cuando se crea una Orden de Producción, y se solicita que se aplique esta estrategia, el Agente de Orden de Producción AOrdenP se integra a la Planta de Fabricación. Por otro lado, se va creando la definición de la configuración de planta como resultado de las negociaciones entre el Agente de Orden de Producción (AOrdenP) y los Agentes de Recursos (ARecurso). Las negociaciones surgen durante la Simulación de la Planta conforme se les asignan las Tareas a los Agentes de Recursos.

Basado en la Lista de Transiciones de la Ruta de Fabricación y en la Configuración de Planta predefinida, el Agente de Orden de Producción (AOrdenP) 
envía la solicitud de capacidad al Recurso asociado a la primera tarea de la ruta (tarea origen).

El Agente de Orden de Producción AOrdenP, que es el iniciador del proceso que ejecuta la Asignación de tareas a Recursos (Transformadores) -Operadores, Máquinas, Herramientas- (representados por agentes que desempeñan el rol $\mathrm{Ne}$ gociador), se basa en la estrategia "SelectivaII". La asignación es controlada mediante una exploración de los $\mathrm{N}$ Recursos existentes $(n R e)$ que pueden ejecutar un proceso específico y una posterior negociación con dichos elementos. La estrategia se ejecuta en dos etapas:

A. Selección inicial de Recursos. La Orden de Producción identifica de acuerdo a la ruta de fabricación del Producto, la operación y el correspondiente tipo de proceso al que se desea asignar (reservar) los recursos necesarios. El conjunto total de los recursos puede estar integrado por subconjuntos de agentes que están preparados para ejecutar procesos específicos. De esta forma el subconjunto obtenido como resultado de la selección inicial, puede estar compuesto por 1 o N agentes de Recurso. Sin embargo, también es posible que dicho subconjunto se encuentre vacío debido a que en el modelo no se ha definido ningún recurso que esté preparado para ejecutar el proceso solicitado. En este caso se le informa al usuario de tal situación para que éste integre tal(es) recurso(s) al Modelo. En el caso de las máquinas, pueden presentarse dos situaciones: a) cuando las máquinas son automáticas éstas ofrecen sus servicios directamente a la Orden de Producción y b) cuando las máquinas requieren de un operario para poder ofrecer un servicio, la capacidad ofrecida depende de la capacidad del operario que la manipule. Esta fase se puede observar mediante la siguiente fórmula:

Si $R_{N}$ es el conjunto de todos los Recursos existentes en el modelo, el subconjunto de recursos obtenido en la Fase inicial se puede expresar como sigue:

$$
s R=\sum\left(R_{i}, P a=\operatorname{Pry} f I d<f R\right)
$$

donde:

$R_{i}$, es un recurso específico que pertenece al conjunto total de recursos disponibles.

$P a$, es el tipo de proceso que el recurso puede ejecutar. 
$\operatorname{Pr}$, es el tipo de Proceso que la Orden de Producción solicita. $s R$, es el subconjunto de recursos que pueden ejecutar el tipo proceso solicitado.

Casos:

- Si $s R=1$, se procede a la asignación directa de la tarea.

- Si $s R>1$, se procede a la segunda etapa de depuración de agentes.

- Si $s R=0$, se le indica al Usuario para que verifique el modelo y en su caso agregue al modelo un recurso que pueda atender la solicitud.

B. Selección acorde a Fecha de Inicio. Una vez identificados aquellos agentes que están preparados para ejecutar el proceso solicitado se procede a una segunda verificación. Esto es, se identifican aquellos agentes que pueden iniciar la tarea antes de la fecha requerida.

Si $s R$ es el conjunto de todos los Recursos que puede ejecutar el proceso solicitado, el nuevo subconjunto de recursos obtenido en la segunda etapa de la Fase inicial se puede expresar como sigue:

$$
s R n=\sum\left(s R_{i}, f I d<=f R\right)
$$

donde:

$s R_{i}$, es un recurso específico que puede ejecutar el proceso solicitado.

$f I d$, es la fecha en la que de acuerdo a su cola de trabajos pendientes o en ejecución puede iniciar la tarea solicitada.

$f R$, es la fecha en la que la Orden de Producción requiere se finalize la tarea solicitada.

$s R n$, es el subconjunto de recursos que pueden ejecutar el tipo proceso solicitado y que la fecha de inicio disponible es inferior o igual a la fecha requerida.

Casos:

- Si $s R n=1$, se procede a la etapa de Negociación.

- Si $s R n=0$, se le indica al usuario para que verifique el modelo y en su caso agregue al modelo un recurso que pueda atender la solicitud. 


\subsubsection{Procesos de Negociación entre Orden de Producción y Recursos}

Cuando el Usuario no ha predefinido una configuración de planta para la asignación de tareas, los procesos de negociación deben considerar factores adicionales tales como la velocidad de simulación y el número de recursos con los que se realizan las negociaciones. La velocidad de la simulación es un factor clave para la definición del protocolo de negociación que será aplicado por la Orden de Producción durante la asignación de recurso. Por lo tanto, se define que la selección del tipo de Protocolo de Negociación $(t P N)$ a utilizar se basará en el tiempo que se tiene para realizar un proceso de negociación.

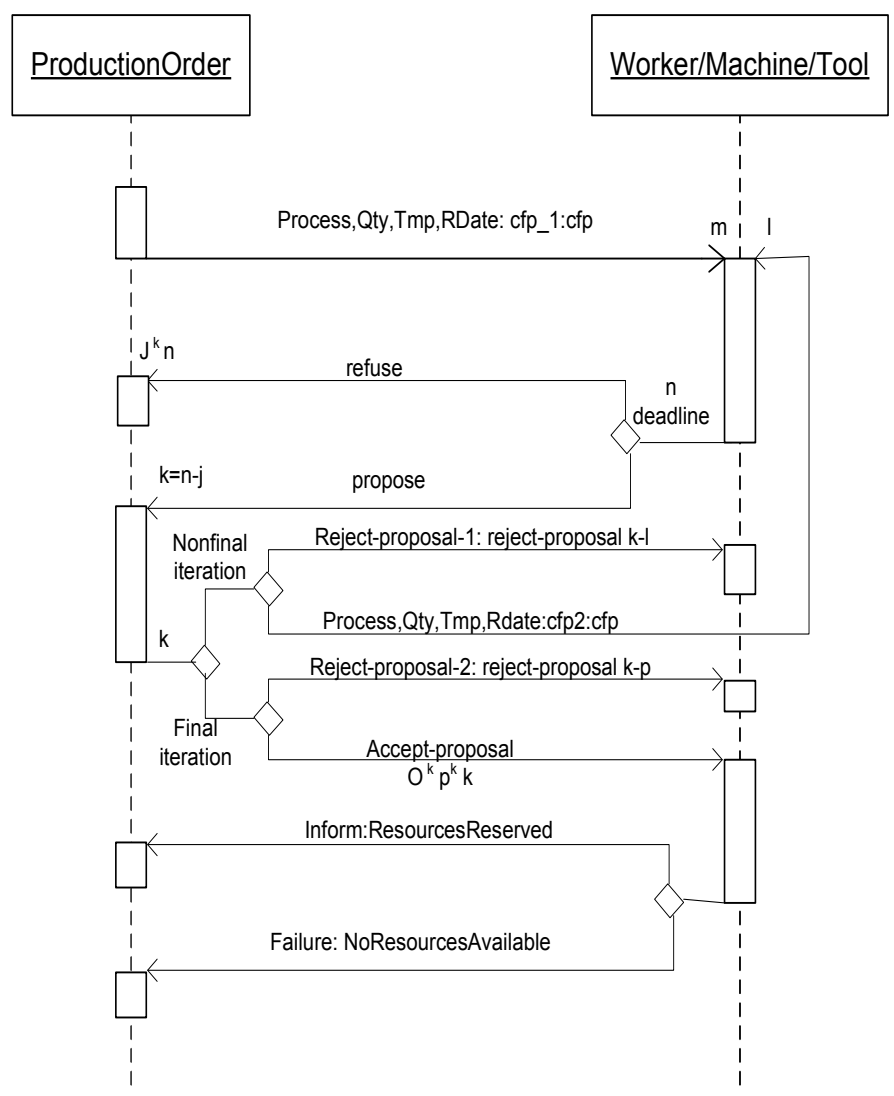

Figura 7.7: Protocolo de Negociación entre Orden de Producción y Recursos.

De esta forma, el protocolo que se utiliza para la selección y asignación de recursos es elegido considerando dos factores: a) la velocidad de simulación 
(comunicada por el Asimulador/GestorVelocidad) y b) el número de recursos obtenidos como resultado de la aplicación de la Estrategia denominada "SelectivaII" (Ver Fig. 7.7).

Tal estrategia se expresa en la siguiente tupla:

$$
t P N=(s R n, v S)
$$

Donde:

$t P N$, es el tipo de Protocolo de Negociación.

$s R n$, es el conjunto de Recursos que pueden realizar el proceso e iniciar antes de la requerida.

$v S$, es la velocidad de simulación (definida por el usuario).

De tal manera que, se realiza una estimación, la cual depende del número de agentes del conjunto $s R$ y la velocidad de simulación.

Si se define un aumento en la velocidad de hasta $50 \%$ y el número de agentes es inferior al $60 \%$ del total de Agentes de Recurso (ARecurso), el Agente de Orden de Producción (AOrdenP) utiliza un protocolo de negociación denominado "Negociador3" y los Agentes de Recursos ARecurso (Operarios, Máquinas y Herramientas) utilizan el protocolo "ParticipaR5". En el protocolo "ParticipaR5” los agentes siguen el Protocolo FIPA Contract Net (Fig. 7.8).

Por el contrario, si la velocidad supera el $50 \%$ y el número de agentes es superior al $60 \%$ del total de Agentes de Recurso, entonces el Agente de Orden de Producción utiliza la metodología de Casos de Uso denominado "UCase" para la selección de los recursos.

\section{Protocolo Negociador3}

Este protocolo lleva a cabo la negociación entre el agente de Orden de Producción (AOrdenP) con los agentes de Recursos (ARecurso) en tres pasos, tal como se indica: 


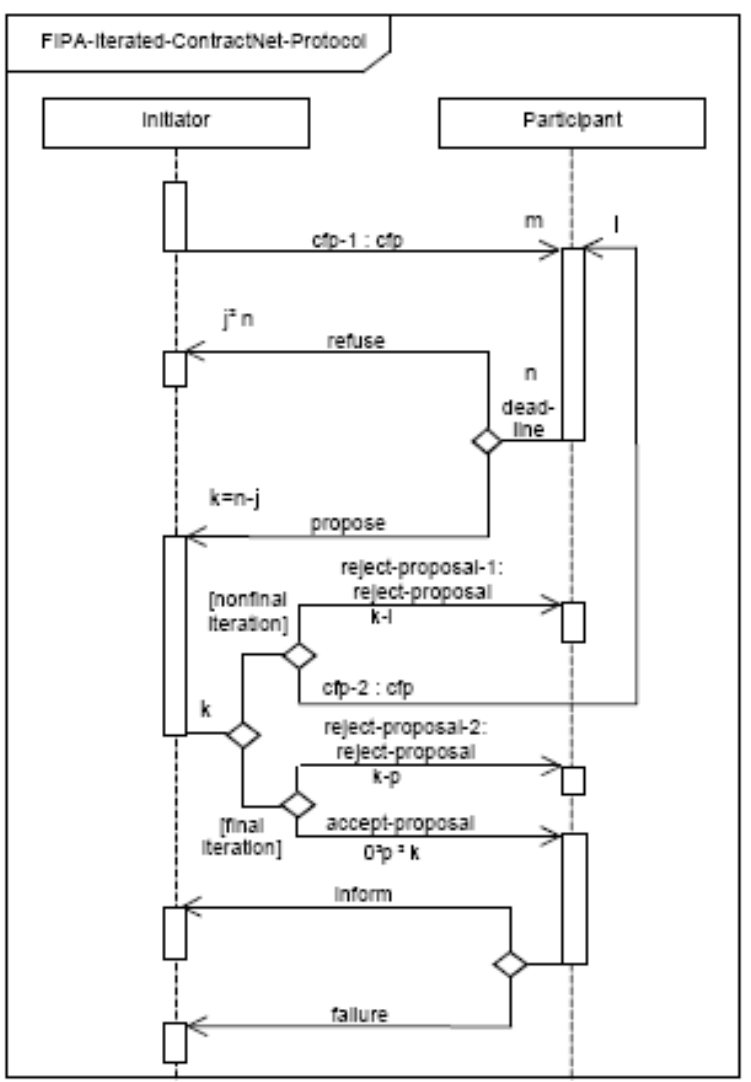

Figura 7.8: Protocolo FIPA Contract Net

1. Con el subconjunto de agentes de Recurso (ARecurso) obtenido ( $s R n$ ), se reciben las propuestas que cumplen con la Política Distribución de Ordenes (Completa/Distribuida) y verifica la cantidad de unidades y fecha de entrega propuesta.

2. Una vez recibidas las propuestas el agente de Orden de Producción AOr$\boldsymbol{d e n P}$ las evalúa de acuerdo con el Tipo de Distribución definida por el Usuario (Completa /Distribuida) y la mejor fecha de entrega posible. De esta forma, se selecciona al mejor(es) recurso(s) y acepta la(s) propuesta(s) que es el(los) Recurso(s) Ganador(es).

\section{Estrategia SelectorSub1P.}

El agente de Orden de Producción (AOrdenP) utiliza una modificación al 
método propuesto por las Subastas las cuales son mecanismos de venta o compra caracterizado por un conjunto de reglas por el que se determina la asignación de recursos y su precio en función de pujas de los participantes. A esta estrategia se le ha denominado "SelectorSub1P".

\section{Objetivos de los participantes:}

- Siguiendo esta filosofía, la Orden de Producción desempeña el papel de Vendedor el cual tiene como objetivo cumplir con la fecha de entrega del Producto Final al Cliente. Para ello, buscará la minimización del tiempo de entrega en la ejecución de cada una de las Operaciones de la Ruta de Fabricación del Producto. Además considera la posible optimización de los recursos mediante la asignación de trabajos a más de un Recurso. Así, define como base las fechas límite de inicio del proceso, y las consecuentes fechas/hora máximas de entrega de cada una de las operaciones.

- Mientras tanto, los Recursos (Operarios, Máquinas y Herramientas) desempeñan el papel de Compradores que tienen como objetivo la maximización de su capacidad diaria mediante la aceptación de trabajos que puedan ser terminados dentro de la fecha máxima de entrega definida por la Orden de Producción.

En el siguiente paso, que se refiere a la selección del "mejor recurso", se toma en consideración tanto el objetivo de la Orden de Producción (fabricar el producto respetando el tiempo de entrega, minimizar coste y tiempo de procesamiento) como la selección previa de los Recursos (los compradores) que participarán en la negociación. Entre los criterios que se pueden aplicar en las subastas, se ha utilizado la técnica de la Subasta a sobre Cerrado al Primer Precio. En la Subasta a sobre Cerrado al Primer Precio, como primer paso, todos los Recursos (los compradores) envían su propuesta a la Orden de Producción. Una vez recibidas todas las propuestas, la Orden de Producción elige al que ofrece la puja más alta. La puja más alta se puede definir en base a criterios tales como: a) aquella en que la cantidad de piezas requeridas sean entregadas en el menor tiempo posible, b) aquella en la que el coste es el más bajo y se entrega dentro del plazo máximo, c) aquella en la que se entrega en tiempo y que la cola de trabajos del recurso es la más corta, d) aquella en la que el recurso tiene el mayor grado de expertise y se entrega en tiempo, o e) una combinación de los criterios anteriores. 
Se debe considerar que existe una mayor complejidad en la identificación de la puja más alta, puesto que cuando el Usuario define una Política de Asignación Distribuida se deben evaluar las entregas parciales de los recursos y sus fechas de entrega con respecto a los recursos que ofrecen entregas completas de las unidades requeridas.

3. Cuando la Orden acepta una propuesta, ésta es informada al Recurso elegido, que confirma o rechaza la reserva definitiva del recurso.

4. Cuando el Recurso confirma que el trabajo asignado ha sido colocado en su cola de trabajos pendientes, la Orden de Producción guiada tanto por la ruta como por las transiciones definidas por el usuario al crear el Producto, busca la(s) siguiente(s) operación(es) y proceso(s) a asignar. En caso contrario, si el Recurso informa que no es posible reservar de forma definitiva el recurso (debido a que durante la espera, se ha reservado a otra orden), entonces el proceso vuelve a iniciar.

\section{Protocolo ParticipaR5.}

El Recurso que recibe la solicitud, decide si participa o no en la negociación siguiendo la estrategia de participación denominada "ParticipaR5". Esto es, decide si participa en una negociación tipo "Negociador3" partiendo de cuatro factores: 1) la cantidad de piezas requerida en la Orden de Producción (PzasReq), 2) el tiempo promedio de ejecución del proceso por unidad requerido por la Orden de Producción $(T m p P z a), 3)$ la fecha de entrega requerida $(F e c R e q)$ y 4) la capacidad disponible de acuerdo con la fecha requerida $(C a p D R)$.

Esta estrategia consta de los siguientes 5 pasos:

1. Verificación de Capacidad Disponible. La capacidad Disponible se calcula considerando la fecha de inicio disponible de acuerdo a la cola de trabajos aceptados previamente por el recurso, y la fecha requerida de entrega. De esta forma, de acuerdo con el tipo de Asignación de Órdenes definido por el Usuario (Completa ó Distribuida), si el recurso puede cubrir al menos el porcentaje mínimo del total de unidades requeridas especificado por el usuario, entonces decide participar y se lo comunica al agente de Orden de Producción. Esta estrategia se expresa mediante la siguiente ecuación:

$$
\text { PiezasD }=\left(F e c R e q-T C_{F} \text { fin }\right) / T m p P z a
$$


donde:

TC_Ffin, es la fecha/hora en la que tiene programado terminar el último trabajo aceptado. En caso de que no tuviera trabajos en cola, entonces se considera la fecha/hora del momento de la solicitud.

TC Ffin, es la fecha/hora en la que tiene programado terminar el último trabajo aceptado.

FecReq, es la fecha requerida de entrega solicitada por la Orden de Producción.

PiezasD, es el número de piezas que se pueden fabricar durante el periodo de la cantidad de horas disponibles.

De tal modo, que la capacidad disponible del Recurso se refiere al número de horas entre la fecha/hora en la que tiene programado terminar el último trabajo aceptado $\left(T C_{F} f i n\right)$ y la fecha requerida de entrega $(F e c R e q)$ solicitada por la Orden de Producción.

2. Verificación de posible Oferta. Basado en el tipo de distribución de órdenes (Completa /Distribuida), si el número de piezas es igual o superior al porcentaje definido por el usuario (PorD), entonces el Recurso propone o rechaza la solicitud de la Orden de Producción.

Casos:

a. Tipo Distribución $=$ Completa.

Si PiezasD > = PzasReq se hace una propuesta.

b. Tipo Distribución = Distribuida .

Si PiezasD $>=($ PorD $) *(P z a s R e q / 100)$ se hace una propuesta.

3. Ofertar servicios (propuesta). Una vez que el agente decide participar en la negociación, se define la fecha de posible entrega del porcentaje de unidades acordado.

$$
\text { FecFinP }=T C_{F} \text { fin }+(\text { CantReq } * \text { TmpPro })
$$

donde: 
TC Ffin, es la fecha/hora en la que podría terminar tiene programado terminar el último trabajo aceptado.

CantReq, es el número total de Piezas Requeridas.

FecFinP, es la fecha/hora en la que el Recurso propone terminar el trabajo solicitado.

4. Aceptar trabajos. Si la Orden de Producción confirma al Recurso que acepta su propuesta, el Recurso agrega el trabajo a la cola de trabajos pendientes la cual es independiente del recurso.

5. Informar del cambio de estado. El Recurso (ARecurso) informa al agente de Orden de Producción (AOrdenP) que el trabajo ha sido aceptado y que lo ha colocado en la cola de trabajos aceptados. Con ello, se activa su rol de Ejecutor que a continuación procesará el trabajo e informará del avance al agente de Orden de Producción (AOrdenP). Además informa al agente ASimulador/GestorAnimación sobre su cambio de estado con respecto a las órdenes de producción que ya ha aceptado para actualizar la visualización del icono (animación gráfica) asociado a dicho elemento en el modelo.

Posterior a la negociación con los recursos disponibles, en caso de que no sea posible atender la tarea en el tiempo solicitado, el Agente de Simulación informa al Usuario quien tiene dos alternativas: 1) cambiar el tiempo de entrega y volver a intentar la asignación de tareas a Agentes de Recurso, o 2) cancelar la Orden de Producción.

\section{Protocolo UCase.}

Este protocolo se utiliza cuando debido a la velocidad de simulación (por ejemplo, 10000x) no es posible realizar el protocolo de negociación "Negociador3" y "ParticipaR5" entonces la Orden de Producción y los Recursos optan por un proceso de negociación más sencillo el cual se basa en decisiones tomadas en simulaciones pasadas para la asignación y aceptación de trabajos. En el momento que se crea el agente de Orden de Producción, se busca el histórico de las asignaciones de trabajos asociado al producto que se pretende fabricar. En el caso de la reserva de materiales se omite el proceso de negociación con el Agente de Almacén, asumiendo que se cuenta con el inventario suficiente para cubrir la fabricación de los productos. 
El protocolo UCase utiliza para la toma de decisiones la experiencia adquirida mediante la aplicación del proceso de aprendizaje de agentes presentado en la Sección 3.3.

\subsubsection{Tratamiento de Trabajos Aceptados por los Recursos}

Finalmente cuando los trabajos solicitados por un agente de Orden de Producción (AOrdenP) han sido aceptados por ciertos Recursos (ARecurso), los Recursos informan del avance de tareas ejecutadas las cuales va almacenando en un área de blackboard. Cuando un recurso termina un trabajo éste se lo informa a la Orden de Producción.

El blackboard alimentado por los agentes del Sistema de Fabricación es consultado por el ASimulador/GestorAnimación el cual verifica el estado actual de los agentes (trabajando, ofreciendo servicios, negociando, agregando trabajos en cola) y en base a ello actualiza el icono y su comportamiento visual. Esto es, actualiza el tipo de animación que corresponde al estado de cada uno de los iconos asociados a cada uno de los elementos del Sistema de Fabricación.

El Agente de Orden de Producción (AOrdenP) se basa en la Ruta de fabricación asociada a la lista de transiciones definidas por el Usuario para determinar cuál será el siguiente trabajo a solicitar. De esta forma el agente de Orden de Producción (AOrdenP) detecta cuándo es posible la asignación de trabajos en paralelo y cuándo se seguirá una secuencia en la solicitud de los mismos.

\subsubsection{Reserva y Entrega de Materiales}

Para efectos de una representación más completa, aunque en este trabajo no se han incorporado los aspectos relacionados con el control de materiales, sí se ha considerado la creación de un Agente de Gestión de Almacén AGAlmacén que realice funciones básicas del control de un almacén de materiales y productos. Entre las consideraciones iniciales sobre las cuales este agente actúa se contempla que: a) se ha cargado el sistema con un inventario inicial y b) se ha incluido sólo una política de almacén, la denominada "Inventario Mínimo". En la política de "Inventario Mínimo" cuando la cantidad disponible se encuentra por debajo del nivel de inventario mínimo establecido, el AGAlmacén genera una Requisición de Material. En consecuencia, también se incluyen dos datos 
adicionales: la cantidad mínima a solicitar y el tiempo de entrega previsto. Por lo tanto, este agente desempeña dos roles:

Negociador. De acuerdo con las solicitudes de Material por parte del agente de Orden de Producción (AOrdenP), el AGAlmacén negocia la reserva de los materiales disponibles y en caso de que no cuente con ellos genera una Requisición de Material y así asegura que cuando el Recurso-Transformador (ARecurso) se la solicite, éste tenga la cantidad requerida disponible y sea entregada.

Despachador de Materiales. Una vez que los trabajos han sido asignados a los Recursos Transformadores (ARecurso), el AGAlmacén atiende las solicitudes de entrega de material solicitados por los Recursos.

Cuando una Orden de Producción es lanzada, el Agente de Orden de Producción (AOrdenP) a la par de que gestiona la asignación de trabajos, también gestiona la reserva de materiales con el AGAlmacén. En este paso el Agente de Gestión de Almacén desempeña el rol de Negociador.

Mientras que cuando un Recurso solicita el material reservado para una Orden de Producción, el AGAlmacén desempeña el rol de Despachador de Materiales.

\subsubsection{Simulación de Supervisión de operaciones en el Sistema de Fabricación}

Para este proceso se aplica un concepto que considera la utilización de puntos de control por centro de trabajo a los cuales se les relaciona tareas de supervisión de órdenes de producción. Generalmente, cada centro de trabajo requiere tareas de supervisión, sin embargo, es posible que se desee realizar pruebas supervisando sólo procesos clave. Entre los objetivos del control de supervisión se encuentra la monitorización e informe de las actividades de los recursos, aunque es posible que éste pueda desempeñar otras tareas complementarias como asistente de otros agentes para la toma de decisiones. En el modelado de la planta se define el número de supervisores disponibles en la planta, y dónde cada supervisor tiene asociado el tipo de proceso que puede supervisar (i.e. corte, moldeo, soldadura, etc.). Asimismo, cada uno de los centros de trabajo (células de procesamiento) tiene asociado un punto de control (representado gráficamente). De esta forma, cada vez que un supervisor acepta una propuesta se actualiza su correspondiente ruta de supervisión asociada a los puntos de control de los 
escenarios. Cuando el supervisor recorre su ruta de supervisión, éste visita cada uno de los puntos de control y registra e informa sobre el estado actual de los recursos. Las actividades del supervisor además de recorrer su ruta incluye las siguientes actividades:

- Informar si el recurso está detenido y la causa: detenido por mantenimiento, detenido por fallo, detenido sin trabajos en cola, detenido con trabajos en cola.

- Informar si el recurso está atendiendo una orden, el supervisor debe verificar el avance, mediante la solicitud de datos al recurso tales como identificador del recurso, identificador de la orden de producción, secuencia de la ruta de fabricación que está atendiendo, cantidad de piezas terminadas, cantidad de piezas faltantes, y el momento exacto de la toma de dicha información. ${ }^{2}$

- De acuerdo con los datos recopilados por el supervisor durante su recorrido, el supervisor puede incluso indicar a la orden de producción lanzar nuevamente la reasignación de tareas si considera que no es posible la recuperación de los tiempos de entrega.

Todas estas acciones son informadas tanto al Sistema de Fabricación como al Sistema de Simulación.

\subsection{Visualización de Resultados de la Simulación}

La ejecución de una simulación y su respectiva simulación constituyen una herramienta útil para la validación del modelo, sin embargo, como se mencionó en el Capítulo 2, el análisis del Modelo estaría incompleto si no se presentaran los resultados de la simulación en formatos estadísticos. Por tanto, el Gestor de Reportes y Gráficas (AResultados) es el responsable de proporcionar al usuario mediante una interfaz amigable los resultados, ya sea utilizando interfaces gráficas o documentación. En la Figura 7.9 se presentan los protocolos que se siguen para tal efecto.

\footnotetext{
${ }^{2}$ Nota: Independientemente de la supervisión, el Recurso informa constantemente del avance de las piezas conforme las termina.
} 


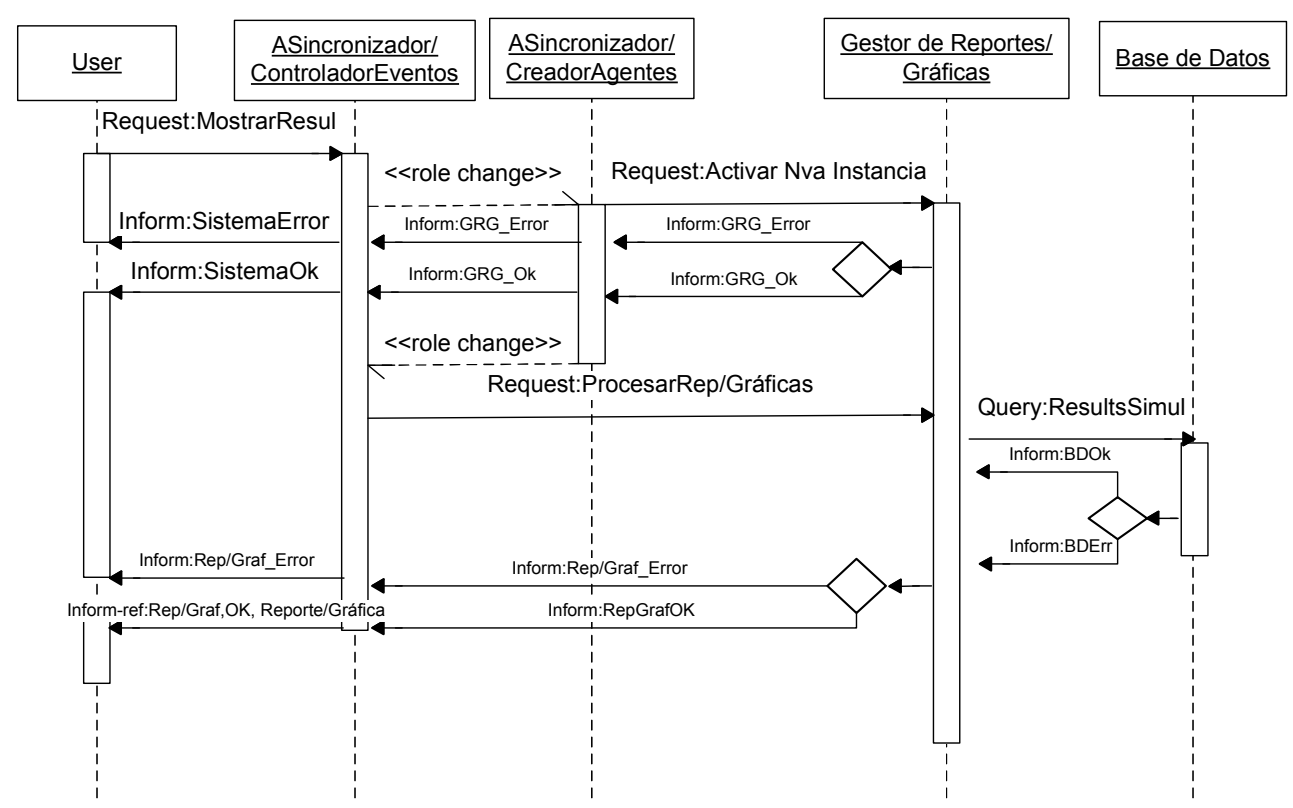

Figura 7.9: Diagrama de Cooperación entre el Agente de Reportes/gráficas y su Entorno durante la Visualización de Reportes y Gráficas

Como resultado del registro del comportamiento de los Agentes del Sistema de Fabricación es posible obtener datos estadísticos que sean de utilidad para el análisis de los escenarios simulados. Tales datos asocian los resultados del proceso de negociación siguiendo la ruta de fabricación de un Producto hasta la terminación de una Orden de Producción (Tabla 7.4).

A continuación se lista la información que puede ser obtenida partiendo de la simulación:

Rutas de Fabricación de Productos. Esta permite obtener el detalle de las rutas de fabricación de un Producto dado indicando las instancias específicas que participaron, fechas, capacidad asignada, etc.

Carga de Recursos. Este listado muestra los recursos más utilizados durante la fabricación de productos, permitiendo a la vez identificar los posibles cuellos de botella.

Carga de Centros de Trabajo. Este listado permite identificar a un nivel más alto con respecto al reporte de Carga de Recursos, el centro de trabajo que concentra el mayor número de actividades durante los procesos de fabricación, identificando los posibles cuellos de botella. 


\begin{tabular}{|c|c|c|c|c|c|c|c|c|c|c|c|}
\hline Proceso & Agente & id_Recurso & Centro Trabajo & Part 1 & & Sel & Cap.Hr & Fecha Ini & Hora Ini & Fecha Fin & Hora Fin \\
\hline 10 Ensamble de SemiRemolque & Worker_welding5( & 30 & welding -S27 Soldadura & 1 & 1 & 0 & & $10 / 03 / 2008$ & $15: 04: 19$ & $3 / 2008$ & $04: 19$ \\
\hline 10 Ensamble de SemiRemolque & Worker welding4( & 4 & welding - \$26 Soldadura & 1 & 1 & 1 & 2 & 10/03/2008 & $15: 04: 19$ & $3 / 2008$ & 7:04:19 \\
\hline 20 Lavar Remolque & Worker_finishing9! & 43 & finishing - P19 Empaque & 1 & 1 & 0 & & $10 / 03 / 2008$ & $17: 04: 19$ & 103/2008 & $: 34: 19$ \\
\hline 20 Lavar Remolque & Worker_finishing6i & 29 & finishing P14- Pintura & 1 & 1 & 0 & & $10 / 03 / 2008$ & $17: 04: 19$ & $0 / 03 / 2008$ & $17: 46: 19$ \\
\hline 20 Lavar Remolque & Worker_finishing7ı & 31 & finishing P14- Pintura & 1 & 1 & 0 & & $10 / 03 / 2008$ & $17: 04: 19$ & 3/2008 & $17: 46: 19$ \\
\hline 20 Lavar Remolque & Worker_finishing8 & 42 & finishing P17 - Lavado & 1 & 1 & 1 & 0,5 & $10 / 03 / 2008$ & 17:04:19 & $1 / 2008$ & $: 34: 19$ \\
\hline 40 Aplicar Recubrimiento Ext. & Worker_finishing8 & 31 & finishing P14- Pintura & 1 & 1 & 1 & 2 & $10 / 03 / 2008$ & $17: 34: 19$ & 008 & $19: 34: 19$ \\
\hline 40 Aplicar Recubrimiento Ext. & Worker_finishing9 & 42 & finishing P17 - Lavado & 1 & 1 & 0 & & $10 / 03 / 2008$ & $17: 34: 19$ & 2008 & $19: 34: 19$ \\
\hline 40 Aplicar Recubrimiento Ext. & Worker_finishing6 & 29 & finishing P14- Pintura & 1 & 1 & 0 & & $10 / 03 / 2008$ & 17:34:19 & 008 & 0:22:19 \\
\hline 40 Aplicar Recubrimiento Ext. & Worker_finishing7ı & 43 & finishing - P19 Empaque & 1 & 1 & 0 & & $10 / 03 / 2008$ & $17: 34: 19$ & 008 & $: 22: 1$ \\
\hline 50 Ensamble de Domo & Worker_welding4(! & 4 & welding - S26 Soldadura & 1 & 0 & 0 & & & & & \\
\hline 50 Ensamble de Domo & Worker_welding5( & 30 & welding -S27 Soldadura & 1 & 1 & 1 & 1,5 & $10 / 03 / 2008$ & $19: 34: 19$ & 08 & 21:04:1 \\
\hline 50 Ensamble de Domo & Worker_welding4(! & 4 & - S26 Soldadura & 1 & 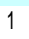 & 0 & & 2008 & 18:34:19 & 008 & 0:04:1 \\
\hline 60 Conectar Instalación Eléctrica & Worker_finishing8i & 43 & finishing - P19 Empaque & 1 & T & 0 & & $10 / 03 / 2008$ & $20: 34: 19$ & 2008 & $21: 04: 19$ \\
\hline 60 Conectar Instalación Eléctrica & Worker_finishing7ı & 31 & finishing P14- Pintura & 1 & 1 & 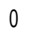 & & $10 / 03 / 2008$ & 21:04:19 & $10 / 03 / 2008$ & $21: 46: 19$ \\
\hline 60 Conectar Instalación Eléctrica & Worker_finishing8i & 42 & finishing P17 - Lavado & 1 & 1 & 0 & & $10 / 03 / 2008$ & $21: 04: 19$ & 2008 & $21: 34: 1$ \\
\hline 60 Conectar Instalación Eléctrica & Worker_finishing9 & 43 & finishing - P19 Empaque & 1 & 1 & & 0,5 & $10 / 03 / 2008$ & $21: 04: 19$ & 10/03/2008 & $21: 34: 19$ \\
\hline 60 Conectar Instalación Eléctrica & Worker_finishing6i & 29 & finishing P14-Pintura & 1 & 1 & 0 & & $10 / 03 / 2008$ & $21: 04: 19$ & $10 / 03 / 2008$ & $21: 46: 19$ \\
\hline
\end{tabular}

Tabla 7.4: Información estadística de negociaciones para una Orden.

Histórico de Carga de Recursos. Este permite observar la carga de trabajo de un Recurso específico dentro de un periodo dado. Tal información permite identificar el grado de optimización de uso de los recursos y si es viable el que siga formando parte o no de la Planta de Fabricación.

Toda esta información conforma la base para el proceso de retroalimentación del sistema. Esto es, los Agentes de Orden de Producción verifican el histórico de los Recursos que han sido seleccionados en anteriores simulaciones para la fabricación del Producto que controlan. Este conocimiento previo es útil cuando el tiempo de simulación se acelera reduciendo el tiempo disponible para el proceso de negociación con los Recursos.

\subsection{Exportación de Resultados de la Simulación de un Modelo}

De acuerdo con la secuencia de las etapas del Estudio de Simulación, la posibilidad de analizar los resultados obtenidos y poder procesarlos en herramientas especializadas es una actividad relevante para la validación del modelo. De esta forma el Agente de Importación/Exportación es el responsable de la coordinación de las actividades necesarias para exportar los resultados de forma externa al sistema. En la Figura 7.10 se presentan los protocolos que se siguen para tal efecto. 


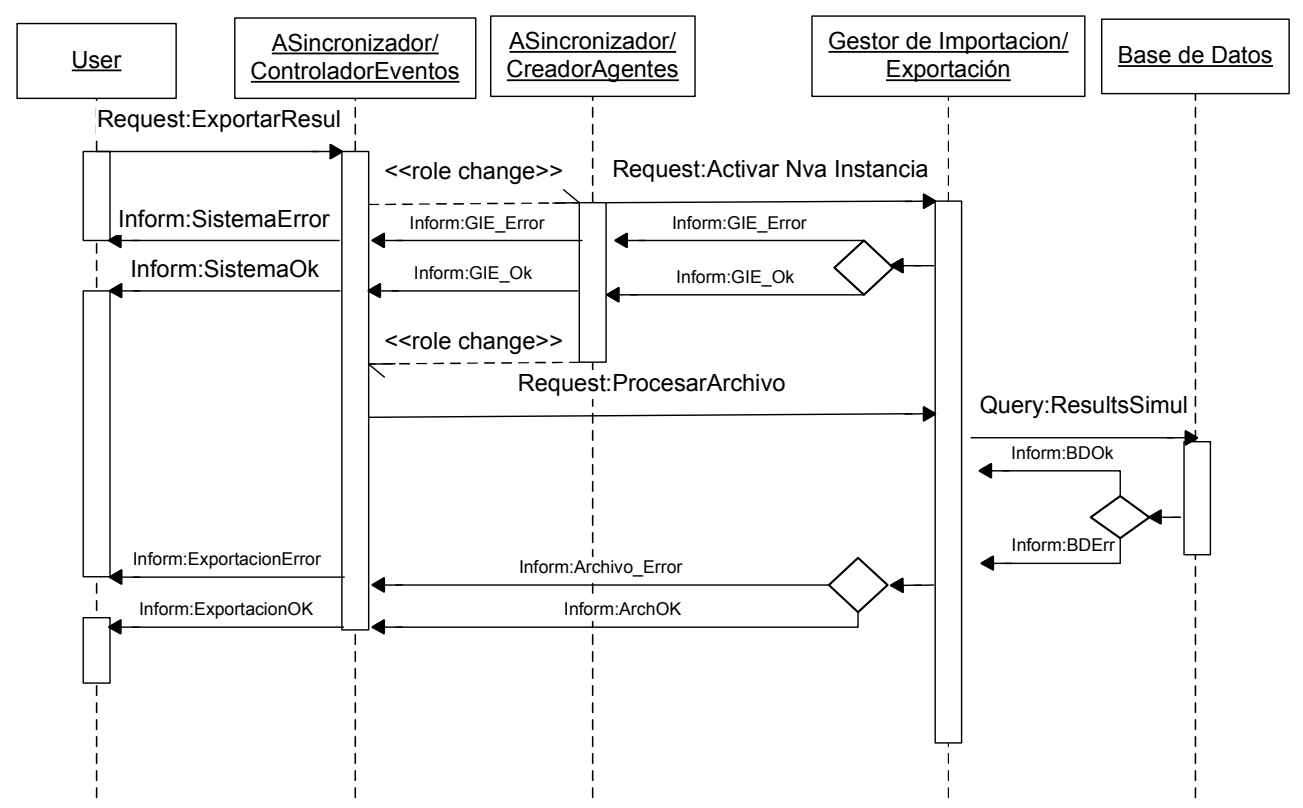

Figura 7.10: Diagrama de Cooperación entre el Agente de Importación/Exportación y su Entorno durante la Exportación de Resultados de la Simulación de un Modelo

\subsection{Conclusiones}

En este capítulo se han presentado las bases de la Arquitectura del Entorno de Simulación de Sistemas de Fabricación soportado por agentes para las actividades correspondientes a la Segunda Fase de la propuesta: la Simulación de Modelos y el Análisis de los Resultados. La Arquitectura incluye la definición de las actividades e interacciones que se presentan entre el Entorno de Simulación y la Planta de Fabricación, ambos soportados por Sistemas Multiagente. Para ello, se han presentado los protocolos de comunicación y de negociación que utilizan los agentes, así como las estrategias propuestas para la asignación de tareas durante la Simulación del comportamiento de la Planta de Fabricación. Finalmente, se ha presentado el tipo de información que se genera durante la Simulación.

Asimismo, en la arquitectura se han contemplado las posibilidades de mejora mediante la aplicación de la tecnología de Sistemas Multiagente con respecto a las Herramientas de Simulación para Sistemas de Fabricación actuales. La arquitectura propuesta se enfoca en la solución de problemas relacionados 
con la flexibilidad, control y distribución del conocimiento, simulación de comportamientos complejos, creación y eliminación de elementos automática, y la ejecución de procesos acorde a las necesidades del estado actual del sistema. Además, el entorno permite visualizar los comportamientos complejos internos de los agentes que componen la Planta de Fabricación. Como resultado de las interacciones entre los agentes, se ha presentado el tipo de información que se obtiene y que se presenta al Usuario en forma de reportes y gráficas de negocio. Asimismo, se indica cómo dicha información es retroalimentada al Sistema para su reutilización. De este modo, los agentes además de contar con la definición inicial de sus comportamientos aprenden en base a la experiencia.

En consecuencia, con el propósito de validar tanto la Arquitectura del Entorno de Simulación como el Metamodelo presentados en los Capítulos 5, 6 y 7, surge la necesidad de desarrollar un prototipo. En el siguiente capítulo se presenta la documentación que dará soporte al prototipo que se implementará. 



\section{Capítulo 8}

\section{SimIShopF: Un prototipo de Entorno de Simulación soportado por Agentes}

Basado en el Modelo Teórico de la Arquitectura del Entorno de Simulación presentado en los Capítulos 5 y 7, en este capítulo se presenta el Modelo Funcional. En dicho Modelo Funcional se muestra la relación entre el Modelo Teórico de la Arquitectura del Entorno de Simulación y su relación con el Modelo Teórico del Sistema de de Fabricación Inteligente presentado en el Capítulo 6.

Este Modelo Funcional se define siguiendo el proceso de desarrollo de la metodología ANEMONA (Botti \& Giret. 2008). En este capítulo se muestran los resultados obtenidos después de aplicar las distintas actividades de ANEMONA.

\subsection{Funcionalidad del Entorno de Simulación}

Los procesos del sistema se ilustran en la Figura 8.1. En dicha figura podemos observar un proceso Gestión de la Introducción de la Información Real del Sistema en los elementos a incluir en el Modelo, este proceso está compuesto por el Proceso 1, 2 y 3 para la importación de los datos reales del sistema de fabricación y su transformación en datos formateados acorde a la estructura de los datos que manipula el Simulador. Dichos datos se asocian a los iconos que componen la Librería de Iconos. 


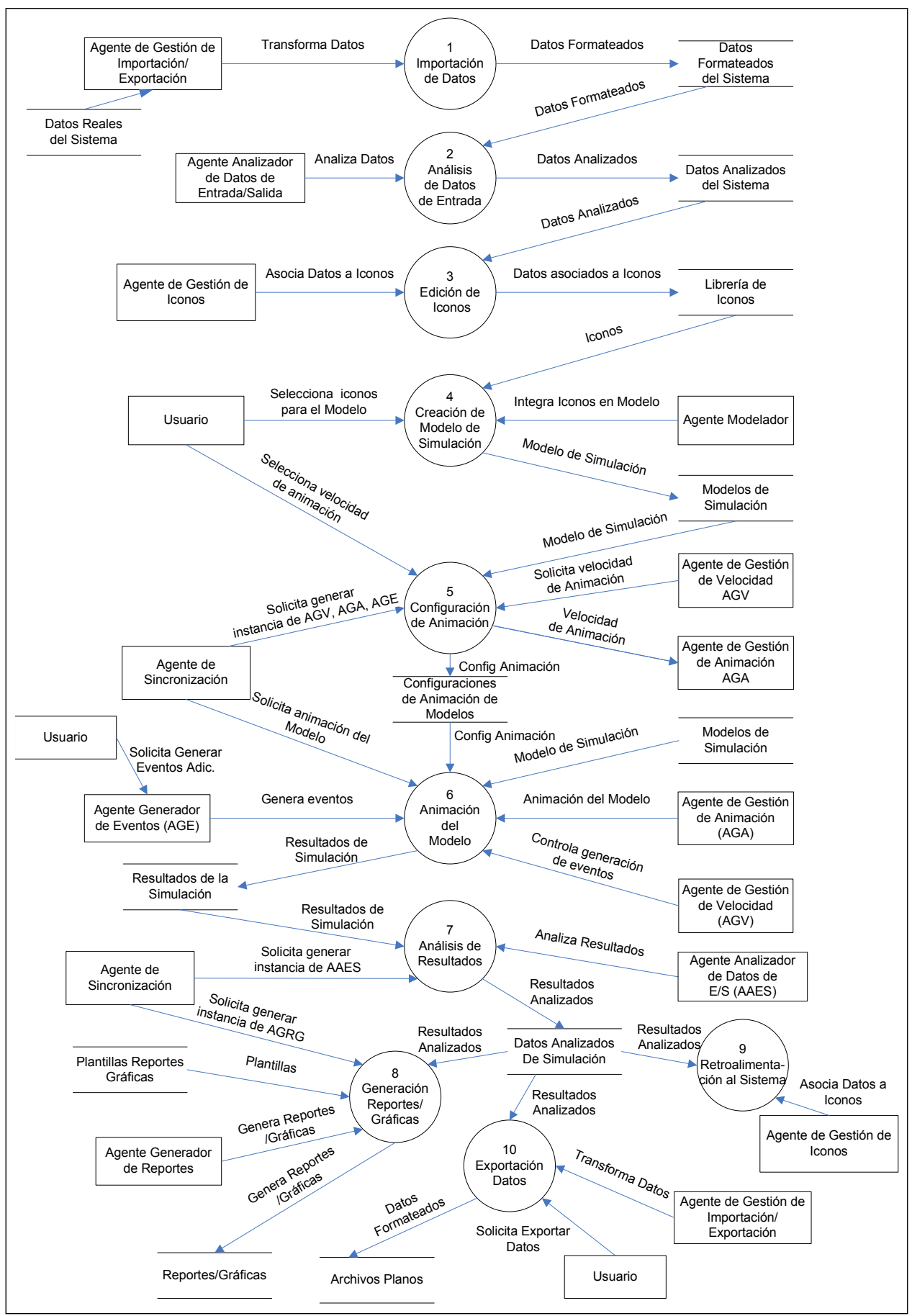

Figura 8.1: Procesos del Sistema -Parte 1- 
En el Proceso 4 - Creación de Modelo el Usuario crea el Modelo utilizando la librería de Iconos que auxiliándose por el Modelador como resultado de este proceso se obtiene un Modelo que es almacenado para su posterior Simulación.

El Gestor de Sincronización es quien a partir del Proceso 5 - Configuración de la Simulación es quien controla la generación / eliminación de instancias de las entidades que ejecutan la simulación. Durante el Proceso 5 el Usuario es quien define la velocidad de ejecución de la Simulación del Modelo así como el tipo de eventos que se considerarán, es decir, en dicho proceso se puede indicar qué tipo de ruidos adicionales o condiciones deberán considerarse durante la ejecución de la simulación. La configuración asignada a cada modelo, se almacena por separado en una base de datos de Configuraciones de Simulación de Modelos.

Basándose en la Configuración de la Simulación para un Modelo de simulación dado, en el Proceso 6 - Simulación del Modelo, el Gestor de Animación ejecuta la animación de los iconos que integran el Modelo a medida que el Gestor de Velocidad controla la solicitud de generación de eventos al Gestor de Generación de Eventos. Durante el Proceso 6 los Resultados de la Simulación generados se van almacenando para su uso posterior.

Una vez concluido el Proceso 6, el Gestor de Sincronización (GSIN) realiza el proceso de Gestión de Resultados de la Simulación el cual está compuesto por los Procesos 7, 8, y 9. El primer paso es la realización del Análisis de los Resultados de la Simulación mediante la generación de una instancia del Analizador de Datos de Entrada/Salida (ADES) los cuales son almacenados para que mediante el Proceso 8 - Generación de Reportes/Gráficas, puedan ser integrados en reportes que permitan al usuario observar el comportamiento del sistema mediante métricas específicas.

A su vez se ejecuta el Proceso 9 -Retroalimentación al Sistema - los Resultados Analizados permiten al Gestor de Iconos (GICO) retroalimentar a los iconos de la librería asociando la información analizada a sus respectivos atributos. El Proceso 10 -Exportación de Datos- es ejecutado a solicitud del usuario para exportar los Datos Formateados en archivos planos para su uso en aplicaciones externas.

En la Figura 8.2 se muestra el Proceso 11 - Edición de Interfaces. El proceso 11 permite editar las interfaces utilizadas a lo largo de los procesos descritos en 
la Figura 8.1, este proceso permite agregar funcionalidades a los atributos de los iconos, a las interfaces para la creación del Modelo y su animación, etc. Debido a que este proceso puede ejecutarse en cualquier momento no se incluye dentro de la secuencia principal del proceso de negocio.

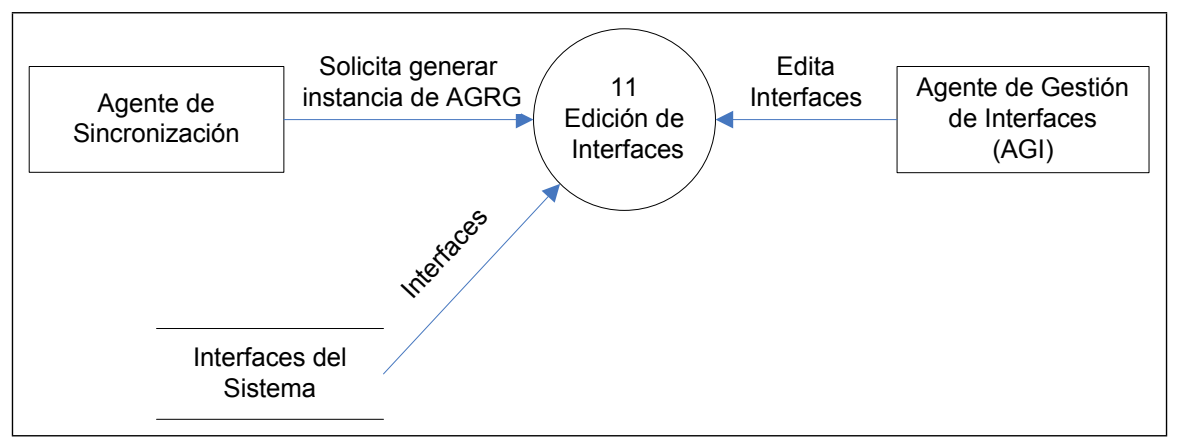

Figura 8.2: Procesos del Sistema -Parte 2-

\subsubsection{Alcance del Sistema}

Basado en la Arquitectura del Entorno de Simulación propuesta en los Capítulos 5 y 7, el Simulador basado en Agentes para Sistemas de Fabricación realizará las siguientes funciones:

- Gestionar la Importación de información proveniente del Sistema de Fabricación Real y le dará el formato adecuado, para gestionar la asociación a elementos existentes o en su caso permitir crear iconos que representan Agentes. Dichos iconos son integrados como parte de un Modelo (procesos 1, 2, 3 de la Figura 8.1) -P1-.

- Ofrecer la asistencia durante la Creación de Modelos. La asistencia durante el modelado incluye el uso de modelos previos y de plantillas para la creación de escenarios. Cada uno de los elementos del modelo tienen asociados atributos específicos que determinan sus capacidades. En base al modelo, los elementos posteriormente se traducen en agentes inteligentes (proceso 4 de la Figura 8.1) -P2-.

- Permitir establecer y controlar la configuración sobre la cual se ejecutará la Simulación del Modelo (proceso 5 de la Figura 8.1) -P3-.

- Ofrecer un control de gestión durante la Simulación del Modelo de tal manera que según el estado actual del sistema, se generen automáticamente 
la aparición o eliminación de elementos (proceso 6 de la Figura 8.1) -P4-.

- Automatizar el Análisis de los Resultados de la Simulación para a su vez transformarlos en información que permita su medición mediante el uso de Reportes y Gráficas (procesos 7, 8 de la Figura 8.1) -P5-.

- Retroalimentar al sistema en base a la información analizada y su asociación a los comportamientos de los elementos que pueden integrar un Modelo de Simulación (proceso 9 de la Figura 8.1) -P6-.

- Exportar la información analizada mediante formatos específicos para su uso en aplicaciones externas (proceso 10 de la Figura 8.1) -P7-.

- Proporcionar la gestión de edición de las interfaces que se empleen durante todo el proceso de simulación para así agregar funcionalidades al sistema (proceso 11 de la Figura 8.1) -P8-.

De este modo, una vez planteadas las principales funciones del Simulador, en la Tabla 8.1 se observan las entradas y salidas asociadas a cada uno de los procesos (identificadas como condiciones de operación en ANEMONA).

\begin{tabular}{|c||l|l|}
\hline Proceso & Entrada & Salida \\
\hline P1 & Datos Reales & Datos Formateados \\
P2 & Datos Formateados & Datos Analizados \\
P3 & Datos Analizados & Datos Asociados a Iconos \\
P4 & Iconos & Modelo \\
P5 & Modelo & Configuración de Animación \\
P6 & Configuración de Animación, Modelo & Resultados de Simulación \\
P7 & Resultados de la Simulación & Datos Analizados \\
P8 & Datos Analizados & Reportes, Gráficas \\
P9 & Datos Analizados & Datos asociados a iconos de Agentes \\
P10 & Datos Analizados & Archivos Planos de Datos \\
P11 & Interface (opcional) & Interface Mejorada \\
\hline
\end{tabular}

Tabla 8.1: Condiciones de Operación

Basado en los roles presentados en la Sección 5.1.2, en la Tabla 8.2 se definen las abreviaturas de las entidades (roles de agentes) que integran el Simulador con las cuales nos referiremos a cada uno de ellos. 


\begin{tabular}{|l|c|}
\hline Entidad & Abreviatura \\
\hline Gestor de Importaciones/Exportaciones & GIE \\
Analizador de Datos de Entrada y Salida & ADES \\
Generador de Eventos & GEVE \\
Modelador & MOD \\
Verificador & VERIF \\
Gestor de Iconos & GICO \\
Gestor de Animación & GANI \\
Gestor de Velocidad & GVEL \\
Planificador de Simulación & PSIM \\
Generador de Reportes & GREP \\
Gestor de Sincronización & GSIN \\
Gestor de Interfaces & GINT \\
\hline
\end{tabular}

Tabla 8.2: Abreviaturas de las Entidades del Simulador

Los roles identificados interactúan bajo escenarios específicos presentados previamente en la Sección 5.5, tales escenarios, siguiendo las guías de ANEMONA, se traducen como Casos de Uso (Figura 8.3).

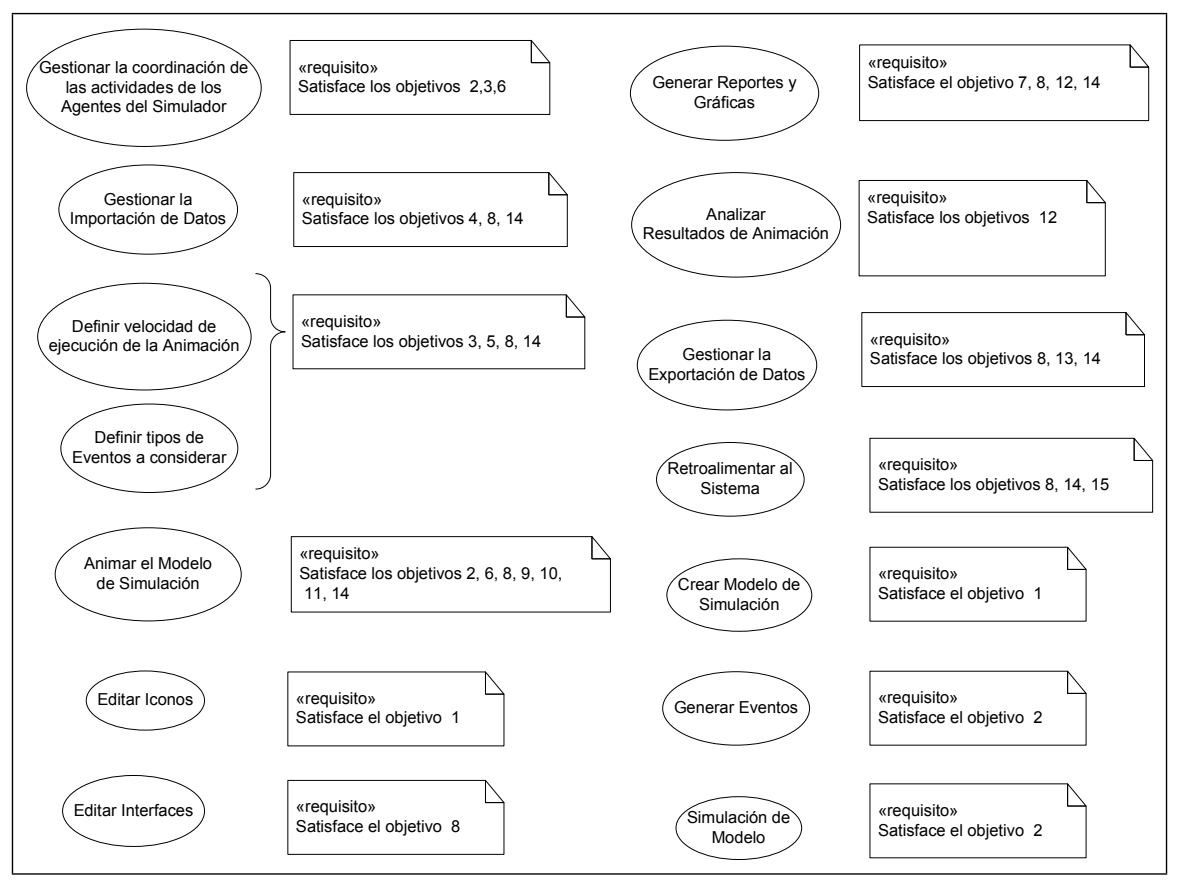

Figura 8.3: Iteración 1 - Diagrama de Casos de Uso

A continuación, siguiendo las guías HMS-CU de ANEMONA, una vez definidos los procesos y escenarios del Sistema (Simulador), se definen los objetivos 
generales que persigue el Simulador (Tabla 8.3).

\begin{tabular}{|c|l|}
\hline ID & Objetivo \\
\hline 1 & Creación de un Modelo \\
2 & Simular un Modelo de Fabricación \\
3 & Sincronizar el ciclo completo de Simulación de un Modelo de Fabricación \\
4 & Gestionar la Importación/análisis de información del / al Sistema Real para su uso en \\
& los elementos que integrarán el Modelo \\
5 & Controlar diferentes tipos de configuración para la animación de la simulación del \\
6 & modelo \\
& Controlar la sincronización de la generación /eliminación de instancias de los agentes \\
7 & Oel sistema acorde al estado del sistema \\
8 & Obtención de reportes/gráficas para la visualización de los resultados de la simulación \\
& Proporcionar interfaces que guíen al usuario durante la creación/simulación del Mo- \\
9 & Monitorizar el Estado Actual del Modelo y su Simulación para controlar la secuencia \\
10 & de la Simulación \\
11 & Realizar la Simulación del Modelo mediante la interacción de Agentes \\
12 & Almacenar los resultados obtenidos durante el proceso de Simulación del Modelo \\
13 & Permitir el análisis de los Resultados de la Simulación \\
14 & Restionar la exportación de los Resultados de la Simulación para su uso externo. \\
15 & las Simulaciones de Modelos \\
& Permitir intervención humana durante los procesos de exportación/importación y la \\
\hline
\end{tabular}

Tabla 8.3: Iteración 1 - Objetivos del Sistema

Como se observó en la Sección 5.2 los roles tienen asociadas funciones de las cuales son responsables. Así, de acuerdo al escenario (caso de uso) en el que se encuentre el sistema, los roles desempeñan funciones específicas. Como resultado de la asociación de los Roles que son responsables a cada uno de los Casos de Uso identificados (Figura 8.3), en la Figura 8.4 se muestra el Diagrama de Organización correspondiente. En el diagrama se observan las principales relaciones que existen entre las entidades (roles). Las relaciones indican qué roles son responsables de los casos de uso (Responsable), así como las relaciones de jerarquía entre ellos. En el diagrama se observan los roles que tienen una relación de subordinación (AGOSubordinación) con respecto al Gestor de Sincronización (GSIN). Asimismo, se observan relaciones del tipo Cliente-Servidor entre roles (AGOCliente-Servidor). 


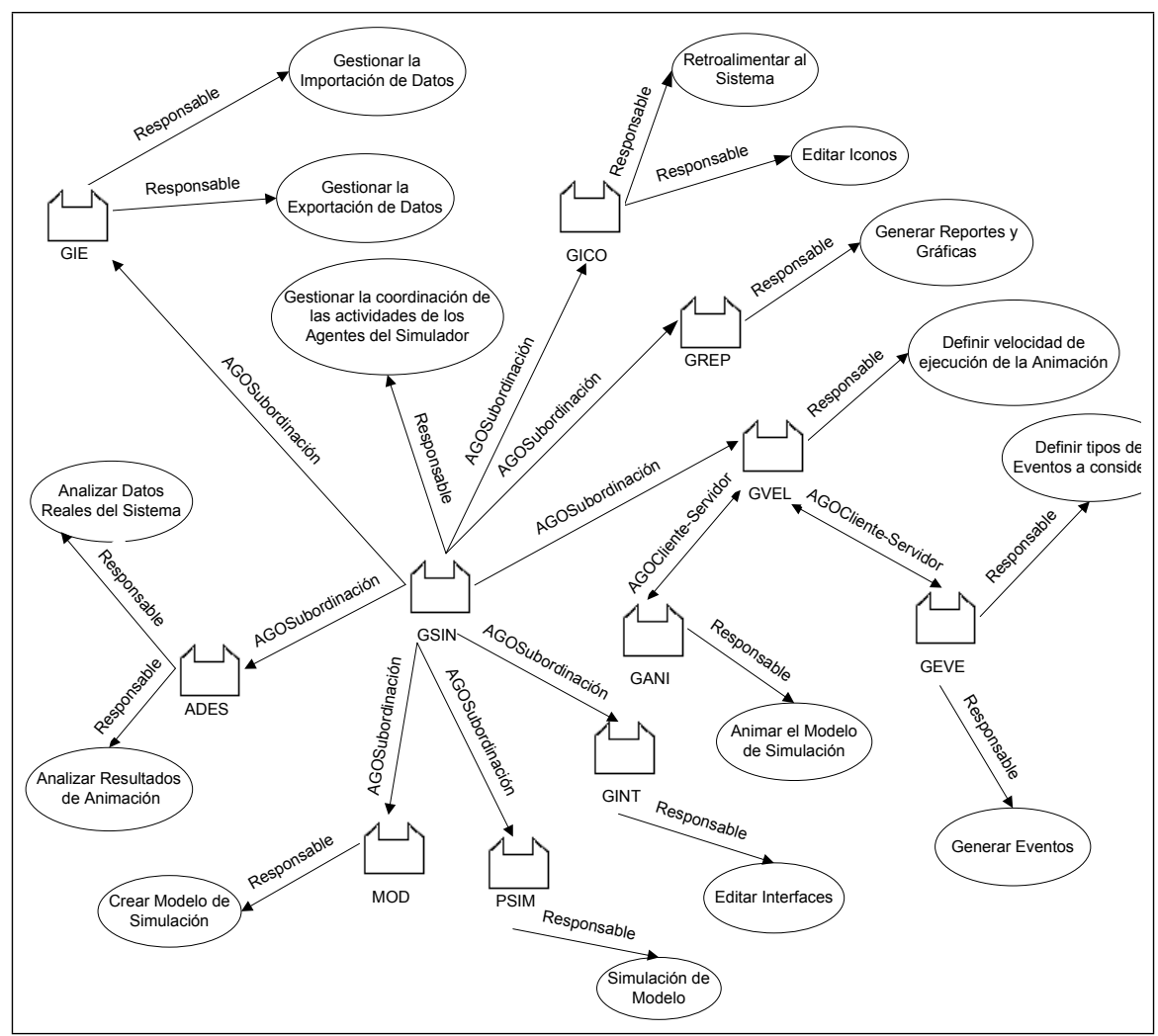

Figura 8.4: Iteración 1 - Diagrama de Organización

Acorde con la definición de la Arquitectura del Entorno de simulación (Capítulos 5 y 7), las interacciones entre los roles previamente identificadas son:

- Coordinar la Gestión de Importación de Datos. Cuando el Usuario solicita la importación de información real del sistema a modelar, el Gestor de Sincronización (GSIN) inicia un proceso de comunicación con el Gestor de Importación/Exportación (GIE) para la importacion de los datos. Después, el Gestor de Sincronización (GSIN) solicita al Analizador de Datos de Entrada/Salida (ADES) el análisis de los datos importados. Después del análisis el Gestor de Sincronización (GSIN) solicita al Gestor de Iconos (GICO) la asociación de los datos a los iconos.

- Coordinar la Creación/Edición del Modelo de Simulación. Después de la importación de datos, el Gestor de Sincronización (GSIN) requiere coordinar la Creación/Edición del Modelo de Simulación por lo que inicia un 
proceso de comunicación con el Modelador (MOD), el Gestor de Interfaces (GINT) y el Gestor de Iconos (GICO).

- Crear Modelo. El usuario crea un modelo mediante el Modelador (MOD) el cual inicia la comunicación con el Gestor de Iconos (GICO) quien proporcionará los iconos que representan cada uno de los elementos del sistema de fabricación, cuando el modelo está terminado, el Verificador (VERIF), revisa si el modelo está libre de errores e informa los resultados al Usuario, por su parte, el Gestor de Interfaces (GINT) permite realizar este proceso mediante interfaces apropiadas.

- Configurar la Simulación del Modelo. Una vez creado el Modelo, el Usuario define la configuración de la Simulación para lo cual mediante el Gestor de Sincronización (GSIN) coordina la configuración de su animación iniciando un proceso de comunicación con el Gestor de Velocidad (GVEL) para especificar la velocidad de ejecución de la simulación, el Generador de Eventos (GEVE) para definir el tipo de eventos a simular, el Gestor de Animación (GANI) para definir el tipo de animación acorde a las opciones de animación de cada uno de los elementos del modelo, y como consecuencia al Gestor de Interfaces (AINT) quien proporciona las interfaces para establecer dicha comunicación entre los agentes.

- Simulación del Modelo. Basándose en la Configuración de la Simulación establecida por el Usuario, el Planificador de Simulación (PSIM), planifica y lleva a cabo la Simulación del Modelo para lo cual inicia un proceso de comunicación con el Gestor de Velocidad (GVEL) para que indique la velocidad a la cual se generarán los eventos y su respectiva animación, el Generador de Eventos (GEVE) quien genera los eventos acorde a la velocidad que le indica el Gestor de Velocidad (GVEL), el Gestor de Animación (GANI) quien lleva a cabo la animación de cada uno de los elementos del modelo acorde a los eventos generados y el tipo de animación establecida.

- Coordinar Análisis de Simulación. Una vez terminada la simulación del Modelo, el Gestor de Sincronización coordina el análisis de los resultados iniciando un proceso de comunicación con el Analizador de Datos de Entrada/Salida (ADES), y el Gestor de Reportes y Gráficas (GREP) así con el Gestor de Interfaces (GINT) quien proporciona las interfaces apropiadas para establecer dicha comunicación.

- Coordinar Animación de Modelo. Basado en la configuración de la Animación el Gestor de Sincronización (GSIN) coordina la ejecución de la 
animación iniciando un proceso de comunicación con el Gestor de Animación (GANI) y el Gestor de Velocidad (GVEL).

- Analizar Resultados. Una vez finalizada la simulación y sujeta a la coordinación del Gestor de Sincronización (GSIN), el Analizador de Datos de Entrada/Salida (ADES) realiza el análisis de los resultados, después del análisis el Gestor de Generación de Reportes/Gráficas (GREP) genera los reportes y gráficas de negocios correspondientes para lo cual hace uso de las interfaces proporcionadas por el Gestor de Interfaces (GINT).

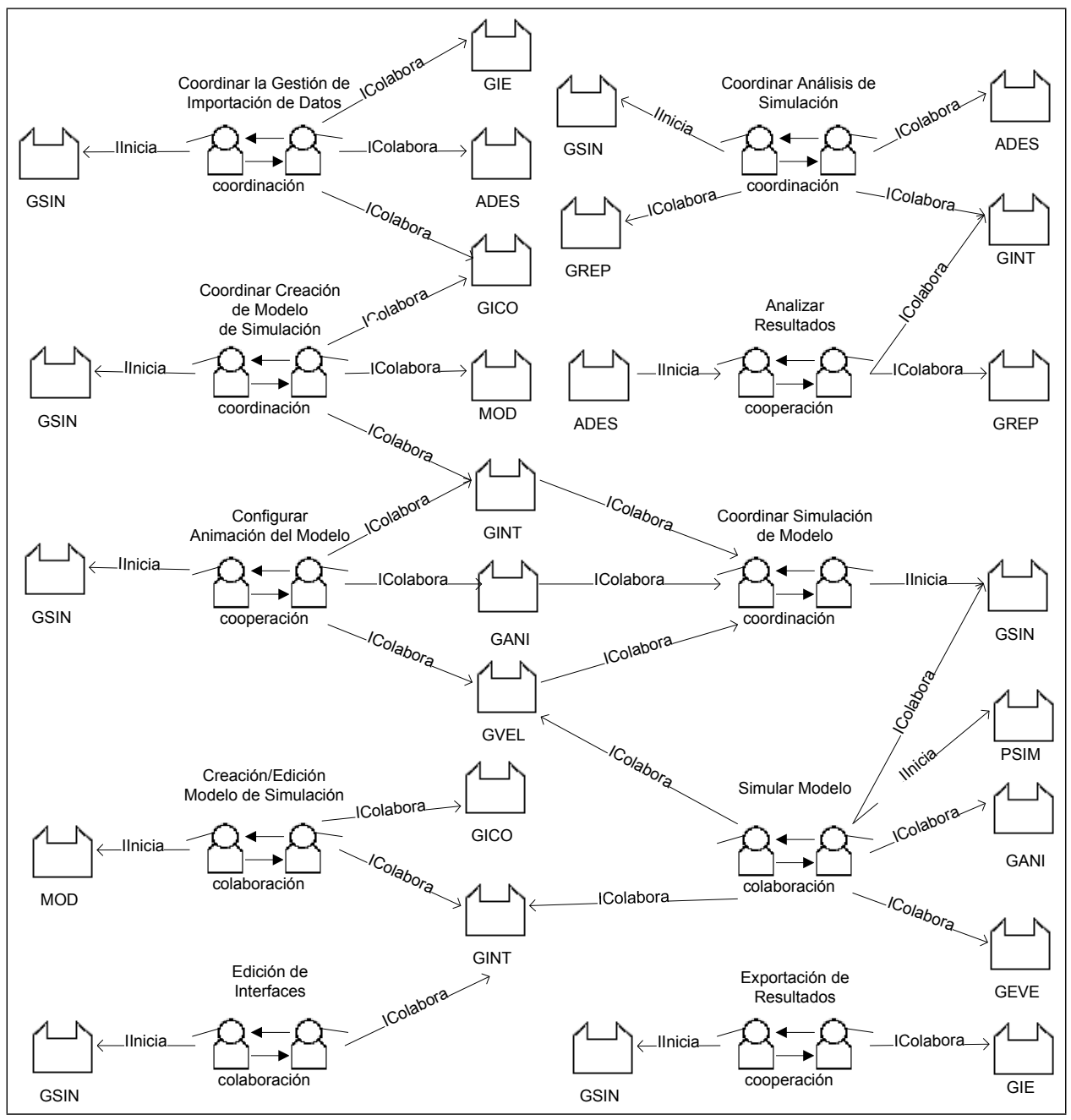

Figura 8.5: Iteración 1 - Diagrama de Interacción 
- Exportación de Resultados. El usuario tiene la opción de exportar los resultados obtenidos para lo realizar dicha petición al Gestor de sincronización quien verifica que los resultados solicitados hayan sido previamente analizados e inicia un proceso de comunicación con el Gestor de Importación/ Exportación para que genere el archivo correspondiente.

- Edición de Interfaces. Con objeto de ampliar al alcance de la funcionalidad del sistema, el usuario realiza la petición de edición de una interfaz específica al Gestor de Sincronización quien inicia un proceso de comunicación con el Gestor de Interfaces (GINT).

De este modo, siguiendo las guías de ANEMONA, mediante el Diagrama de Interacciones se presentan las interacciones previamente definidas entre los elementos del sistema (Figura 8.5).

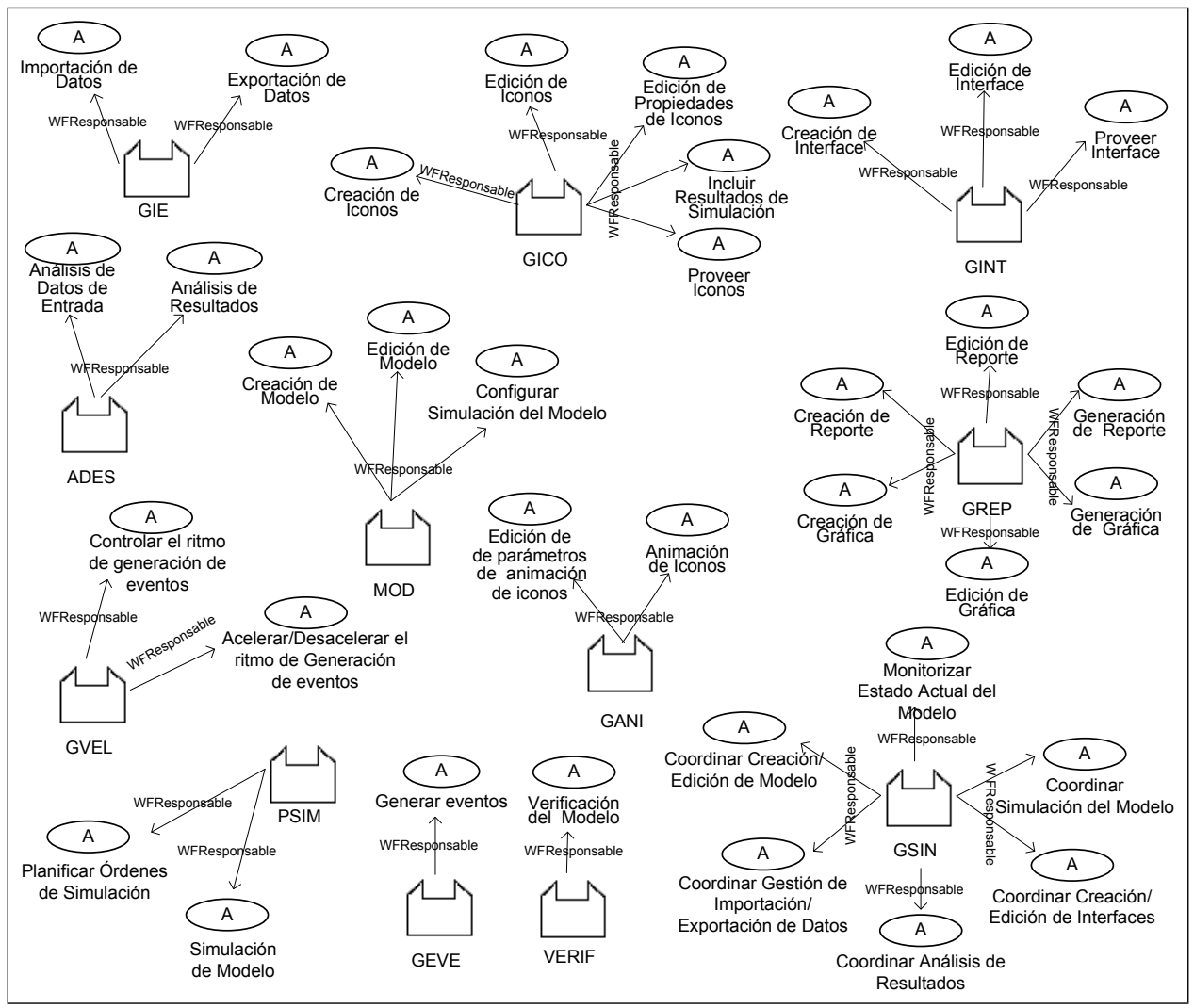

Figura 8.6: Iteración 1 - Tareas Abstractas en el Modelo de Organización 
Así, basado en el Diagrama de Interacción (Figura 8.5) de la actividad A6 de ANEMONA, se modelan las Tareas Abstractas que los Roles deben implementar para cumplir con los casos de uso de los cuales son responsables (Figura 8.6).

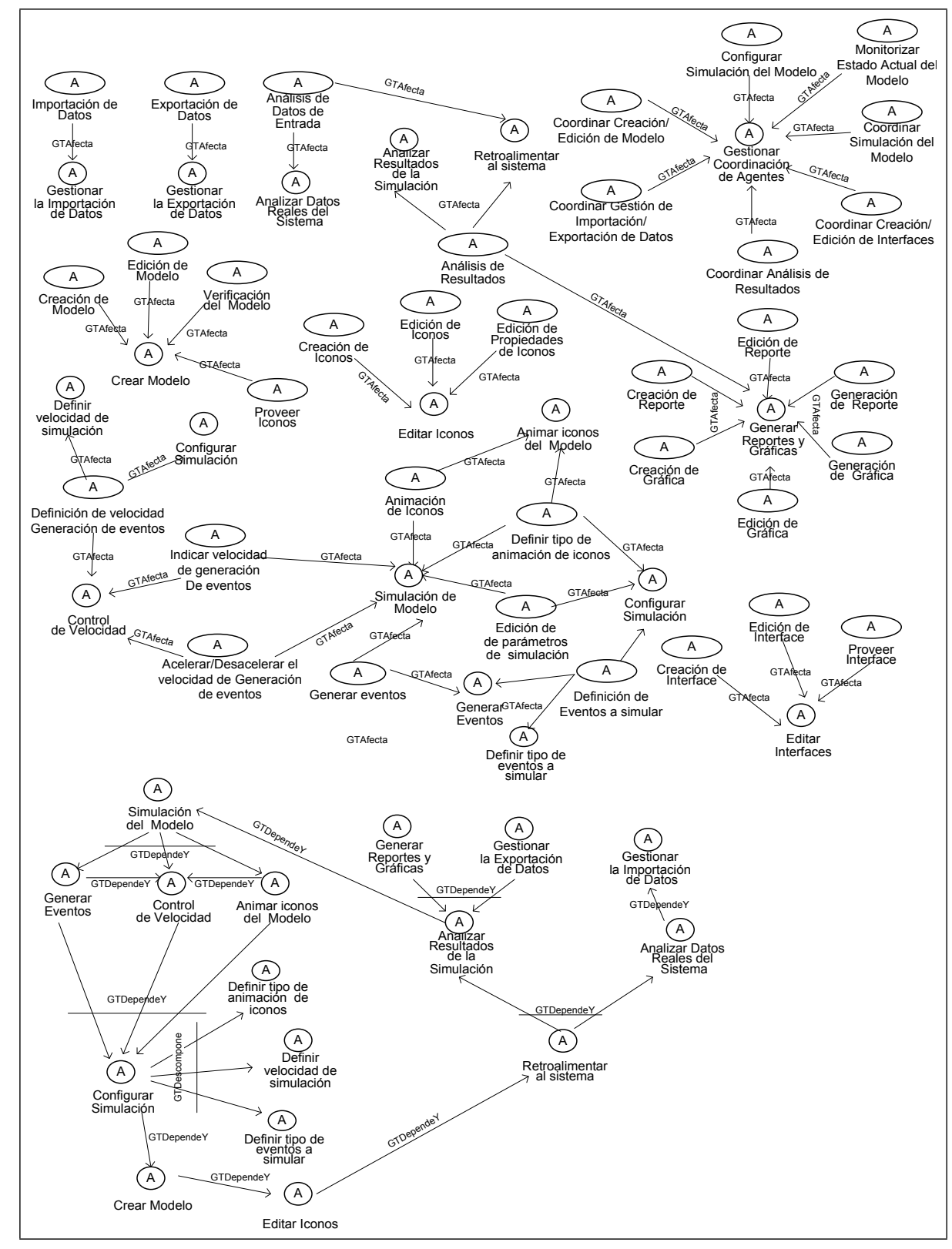

Figura 8.7: Diagrama de Tareas y Objetivos 
Como resultado de la identificación de las Tareas Abstractas (Figura 8.6) y los Objetivos asociados a los Roles responsables (Figura 8.4); siguiendo las guías de ANEMONA, el siguiente paso, es la asociación de las tareas abstractas a los objetivos que afectan, así como la dependencia entre los objetivos. Tal asociación se expresa mediante el Modelo de Tareas y Objetivos de la Figura 8.7.

\subsubsection{Los Holones}

Utilizando las Guías Prosa de ANEMONA se identifican tanto los holones como la asociación de los roles a los holones (agentes) (Figura 8.8). A continuación, se describen los holones resultado de la identificación de Agentes Abstractos de Orden, de Producto, de Recurso y Staff del Entorno de Simulación:

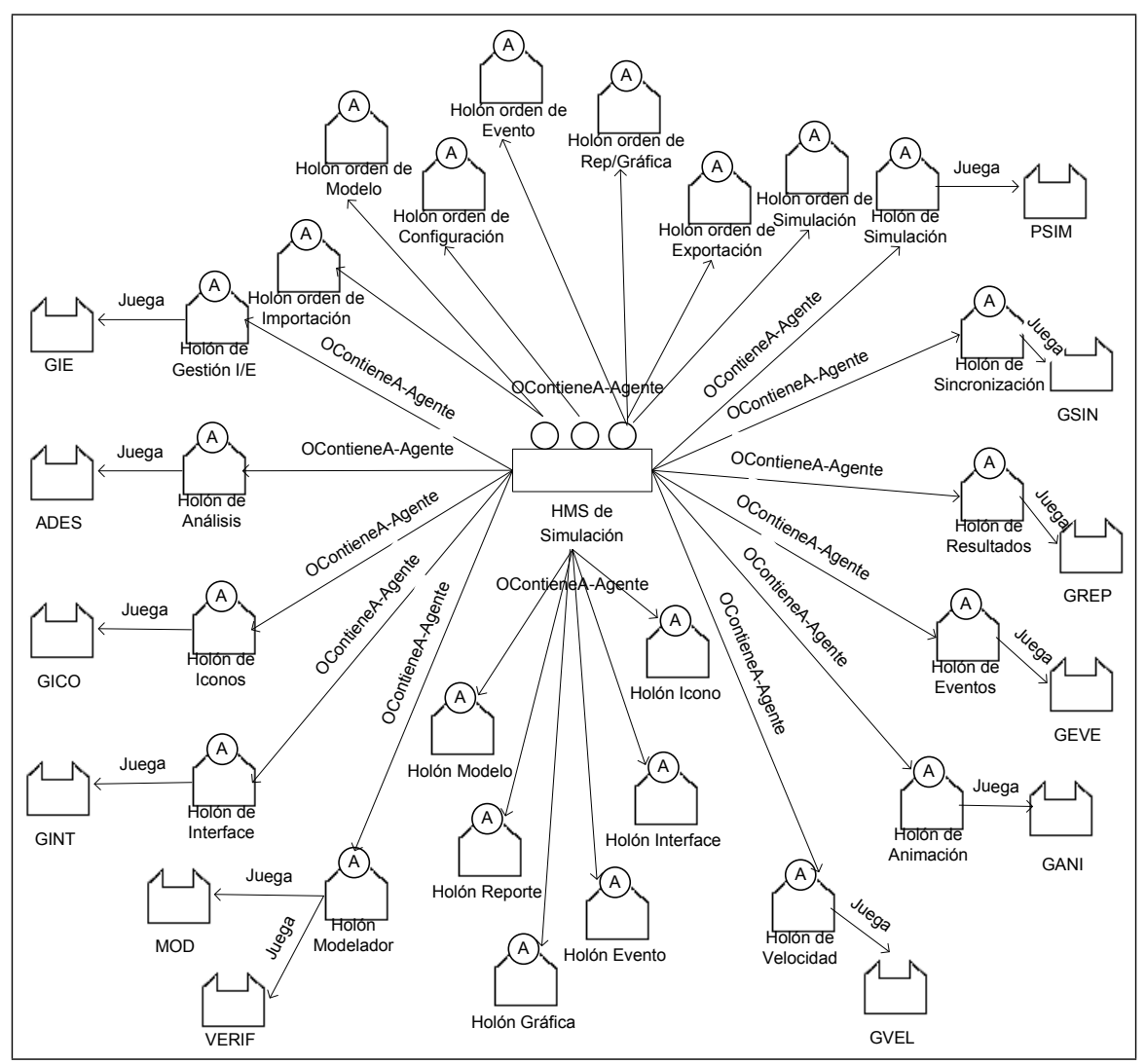

Figura 8.8: Iteración 1 - Agentes Abstractos y Modelo de Organización 
Holón de Gestión de Importacion/Exportacion. Es un agente abstracto de recurso que permite la importación de datos reales al sistema y la exportación de resultados de la simulación de un modelo en un formato apropiado. Este holón juega el rol de Gestor de Importación/Exportación.

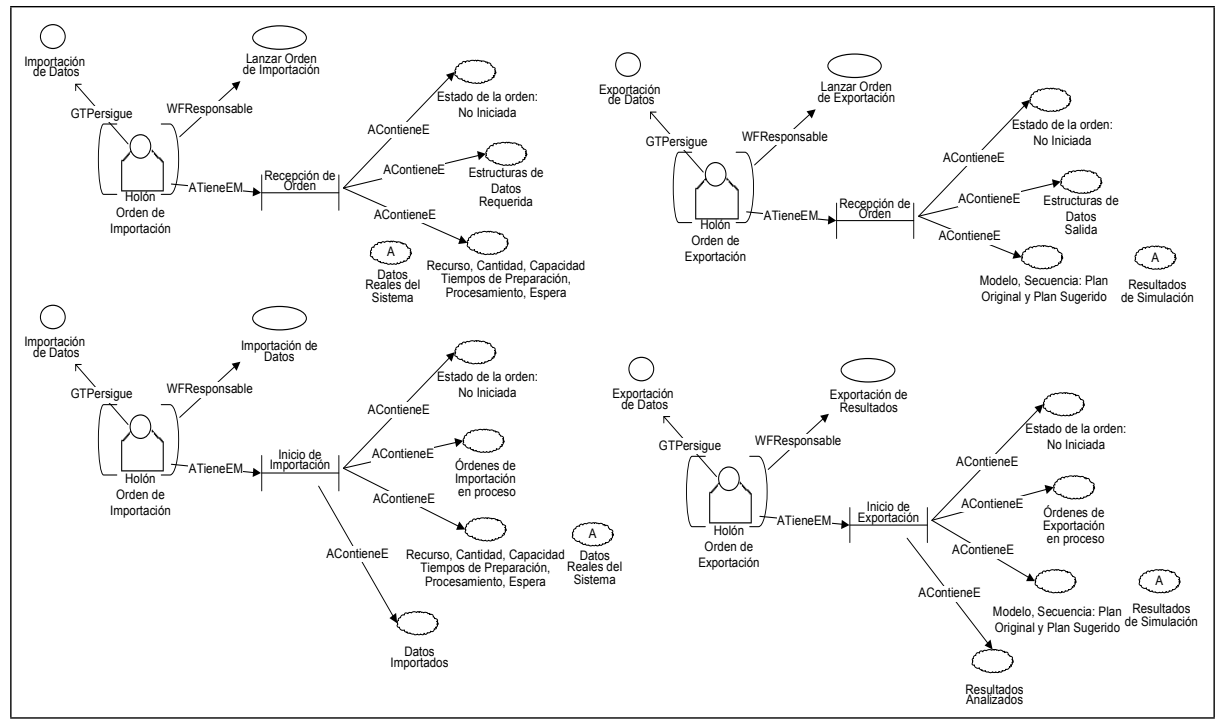

Figura 8.9: Modelo de Holon de Gestión de Importación/Exportación HGIE

En la Figura 8.9 se presenta el Modelo de Agente para el Holón de Gestión de Importacion/Exportacion.

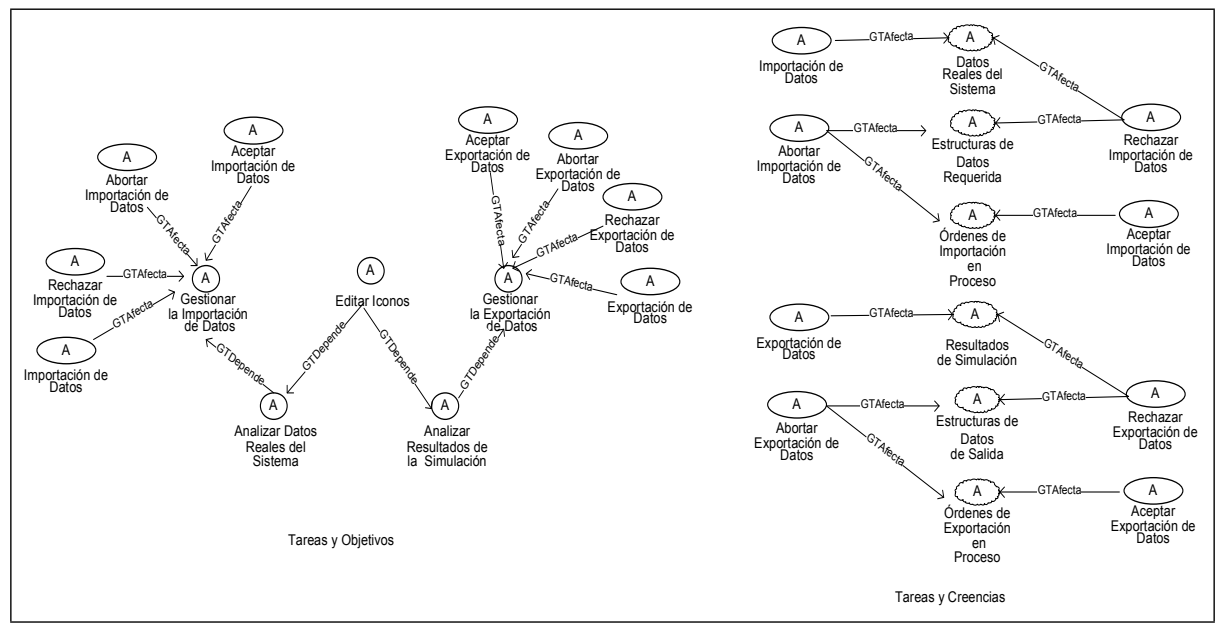

Figura 8.10: Diagrama de Tareas y Objetivos así como de las Tareas y Creencias del Holón de Gestión de Importación/Exportación 
Asimismo en dicha figura se observan los objetivos prioritarios de este agente y sus tareas globales. Además, en la Figura 8.10 se muestra el detalle de las tareas enfocadas al logro de los objetivos del holón de Gestión de Importacion/Exportacion.

Holón de Análisis. Es un agente abstracto de recurso que permite realizar el análisis de los datos reales del sistema para incorporarlos como parte de los elementos del modelo así como el análisis de los resultados de la simulación para retroalimentar al sistema. Este holón juega el rol de Analizador de Datos de Entrada/Salida.

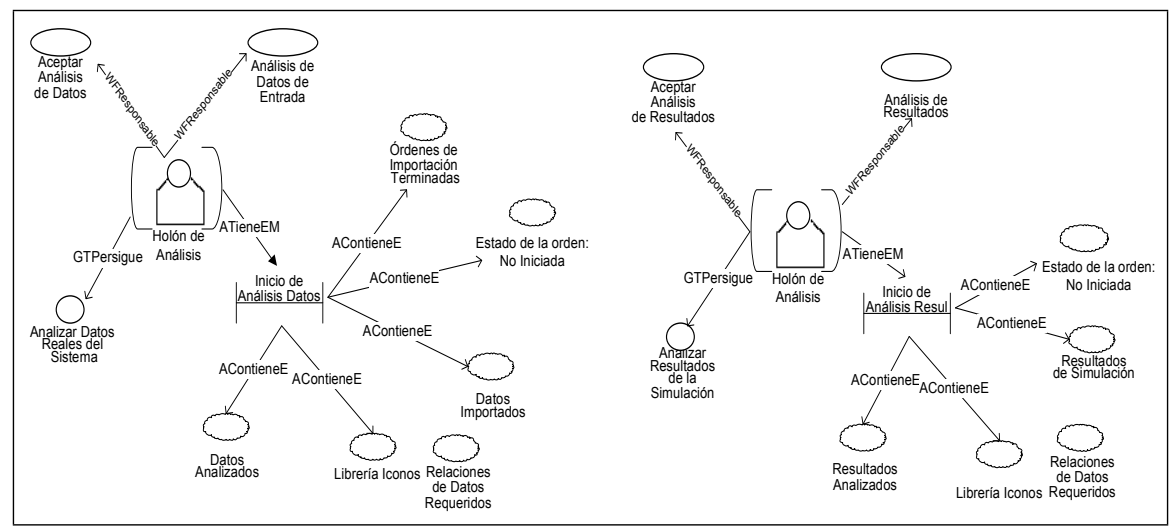

Figura 8.11: Iteración 1 - Modelo de Holon de Análisis HANA

En la Figura 8.11 se presenta el Modelo de Agente para el Holón de Análisis, en ella, se observan de forma global, sus objetivos y tareas.

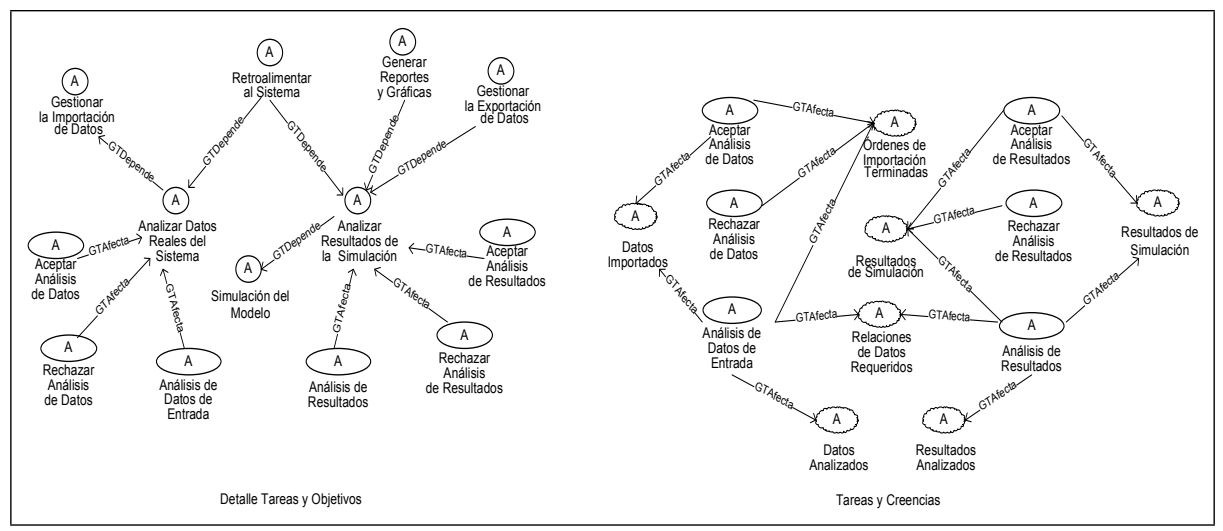

Figura 8.12: Iteración 1 - Diagrama de Tareas y Objetivos así como de las Tareas y Creencias del Holón de Análisis 
Asimismo, en la Figura 8.12 se muestra el detalle de las tareas enfocadas al logro de los objetivos del holón de Análisis.

Holón de Iconos. Es un agente abstracto de recurso que permite la gestión (creación, edición, parametrización, eliminación) de los iconos que integran el modelo. Este holón juega el rol del Gestor de Iconos.

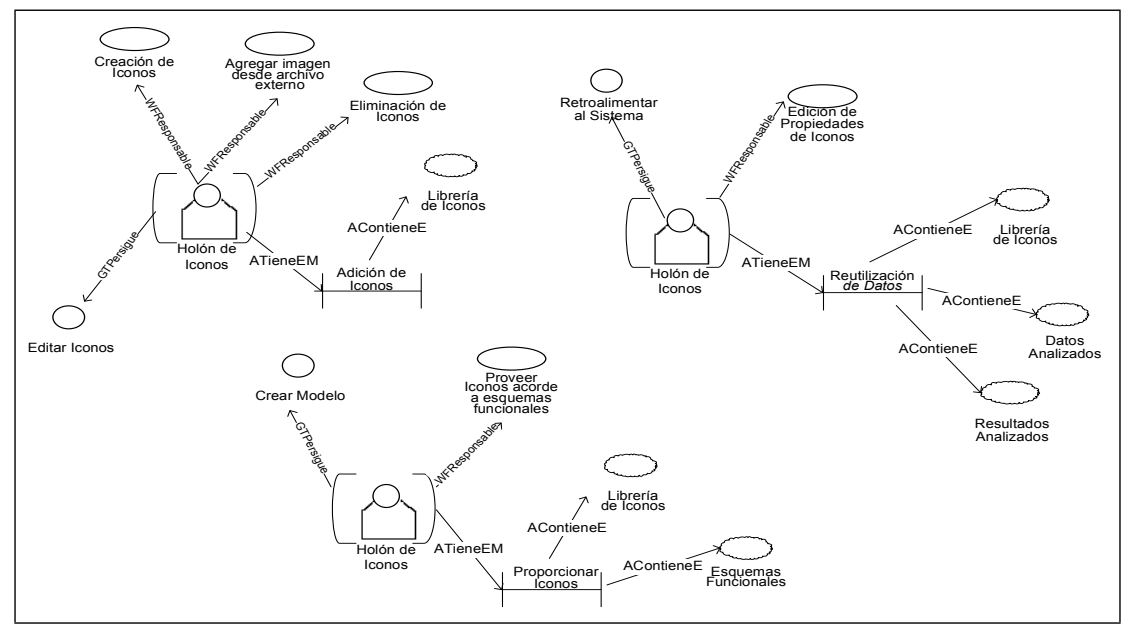

Figura 8.13: Iteración 1 - Modelo de Holon de Iconos HICO

En la Figura 8.13 se presenta el Modelo de Agente para el Holón de Iconos, en ella, se observan de forma global, sus objetivos y tareas.

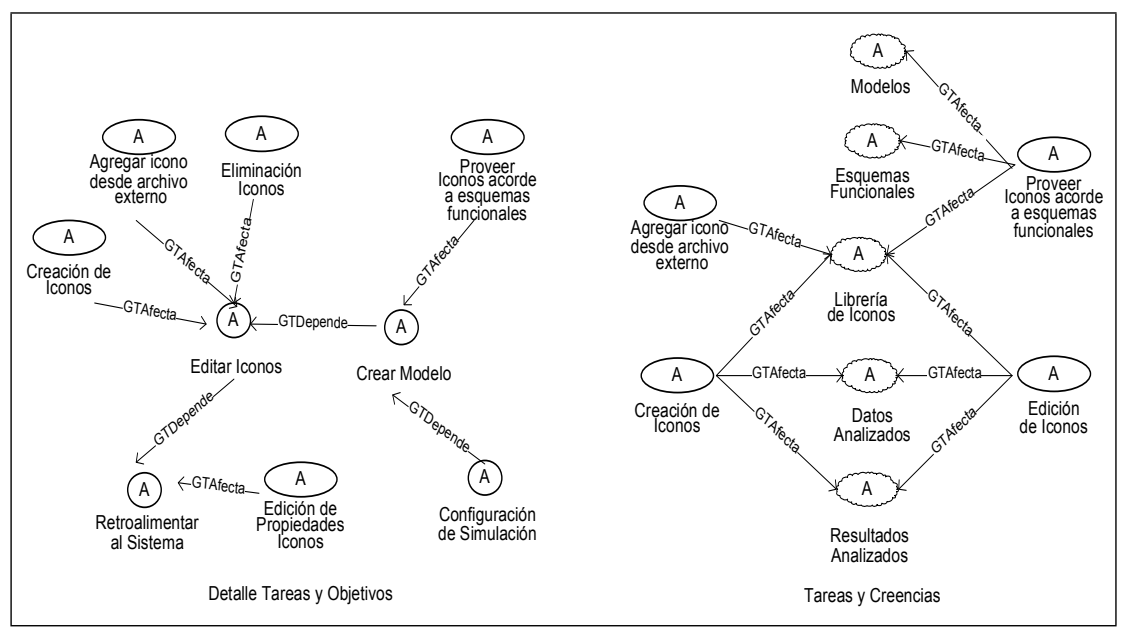

Figura 8.14: Iteración 1 - Diagrama de Tareas y Objetivos así como de las Tareas y Creencias del Holón de Iconos 
En la Figura 8.14 se muestra el detalle de las tareas enfocadas al logro de los objetivos del holón de Iconos.

Holón Gestor de Interfaces. Es un agente abstracto de recurso que permite la gestión (creación, edición, eliminación) de las interfaces que se utilizan como medio de comunicación tanto entre los roles como con el usuario. Este holón juega el rol del Gestor de Interfaces.

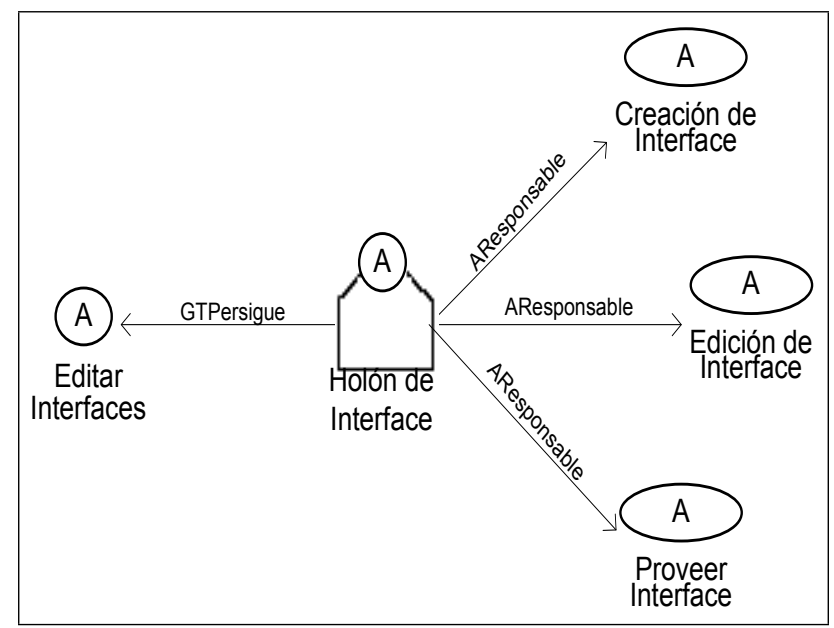

Figura 8.15: Iteración 1 - Modelo de Agente del Holón de Interface

En la Figura 8.15 se presenta el Modelo de Agente para el Holón Gestor de Interfaces, en ella, se observan de forma global, sus objetivos y tareas.

Holón Modelador. Es un agente abstracto de recurso que permite la gestión (creación, edición, verificación) de un modelo que representa al sistema real el cual está integrado por iconos. Este holón juega los roles del Modelador y del Verificador.

En la Figura 8.16 se presenta el Modelo de Agente para el Holón Modelador, en ella, se observan de forma global, los objetivos y tareas de cada uno de roles que puede desempeñar. 


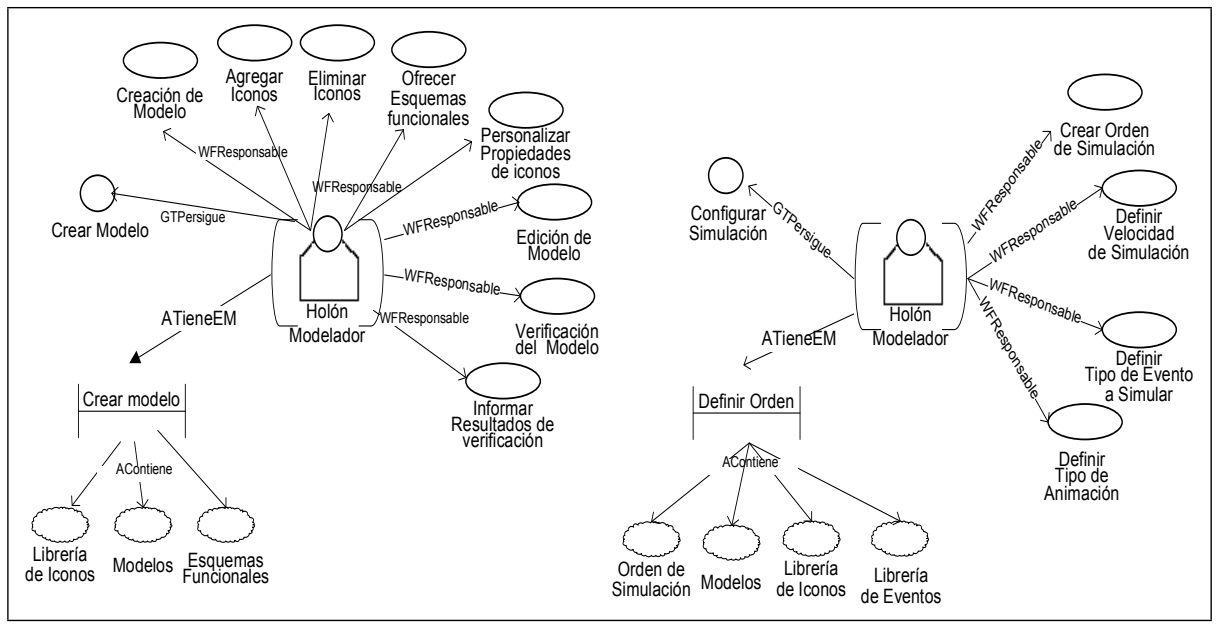

Figura 8.16: Iteración 1 - Modelo de Holón Modelador HMOD

La Figura 8.17 muestra la descomposición de objetivos y relaciones identificadas entre los objetivos y las tareas del Holón Modelador, sus objetivos tienen relación directa con objetivos prioritarios del sistema. La Creación del Modelo, es un objetivo básico para la posterior Simulación del Modelo.

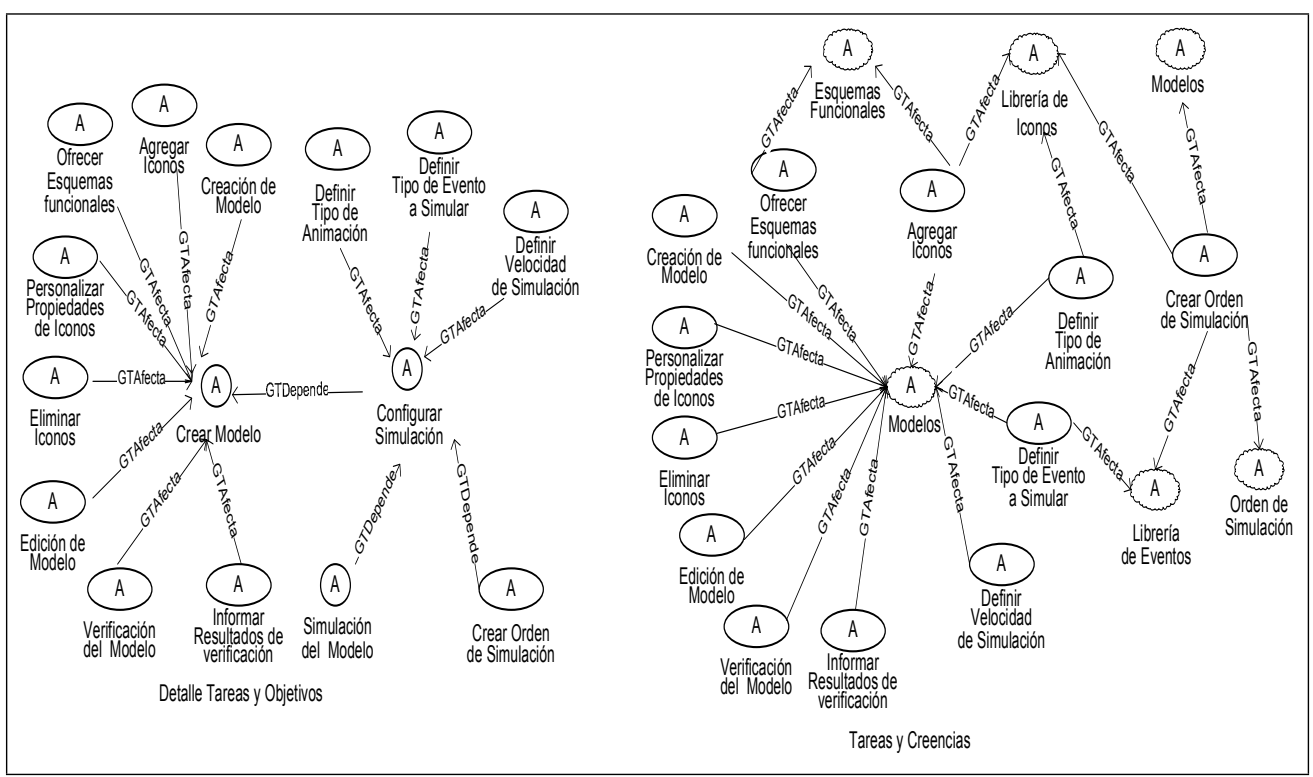

Figura 8.17: Iteración 1 - Diagrama de Tareas y Objetivos así como de las Tareas y Creencias del Holón Modelador 
Holón de Velocidad. Es un agente abstracto de recurso que permite la configuración del ritmo al cual deben generarse los eventos a animar del modelo durante un periodo establecido por el usuario. Este holón juega el rol de Gestor de Velocidad. 8.18. Mantiene el control sobre la velocidad a la que el holón Generador de Eventos dispara eventos y sobre la velocidad a la cual el holón de Animación muestra gráficamente el estado interno de los agentes. En la Figura 8.19 se muestra el detalle de las tareas enfocadas al logro de los objetivos del holón de Velocidad.

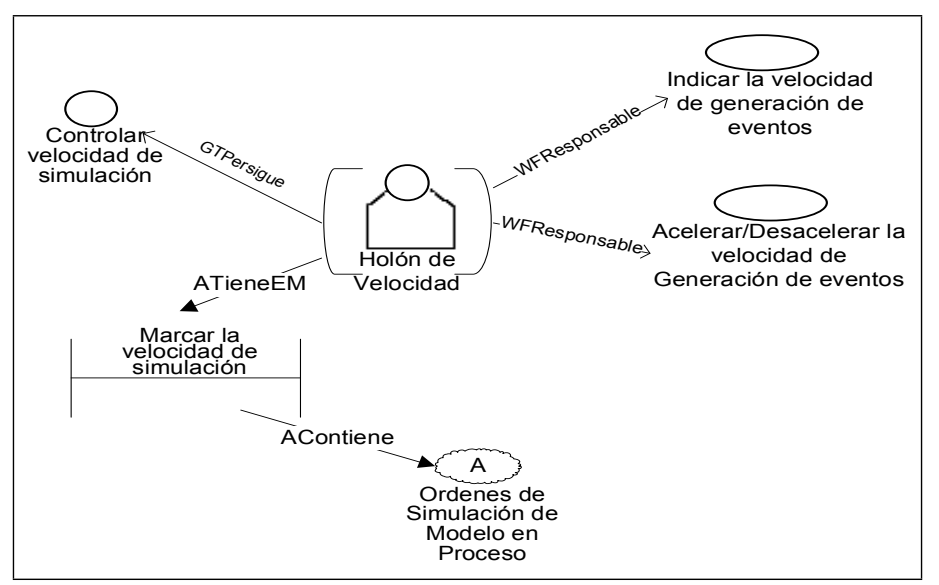

Figura 8.18: Iteración 1 - Modelo de Holón de Velocidad HVEL

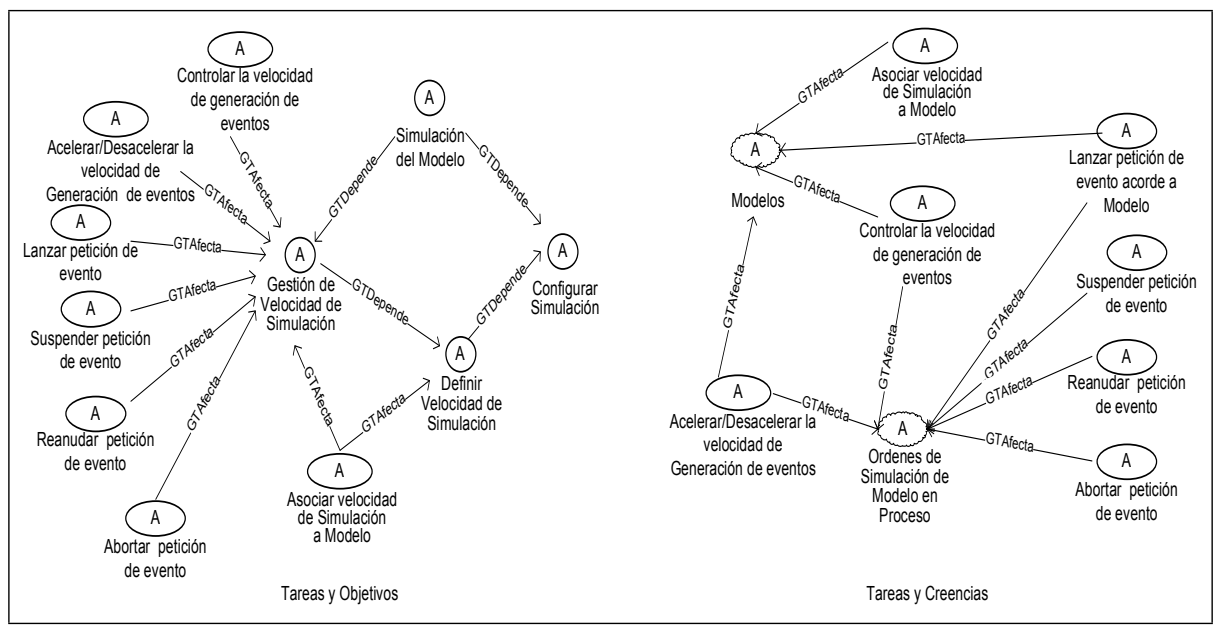

Figura 8.19: Iteración 1 - Diagrama de Tareas y Objetivos así como de las Tareas y Creencias del Holón de Velocidad 
Holón de Animación. Es un agente abstracto de recurso que permite la animación de los iconos que componen el modelo acorde al ritmo de la generación de eventos que indica el holón de Ritmo. Este agente juega el rol de Gestor de Animación (Figura 8.20). En la Figura 8.21 se muestra el detalle de las tareas enfocadas al logro de los objetivos del holón de Animación.

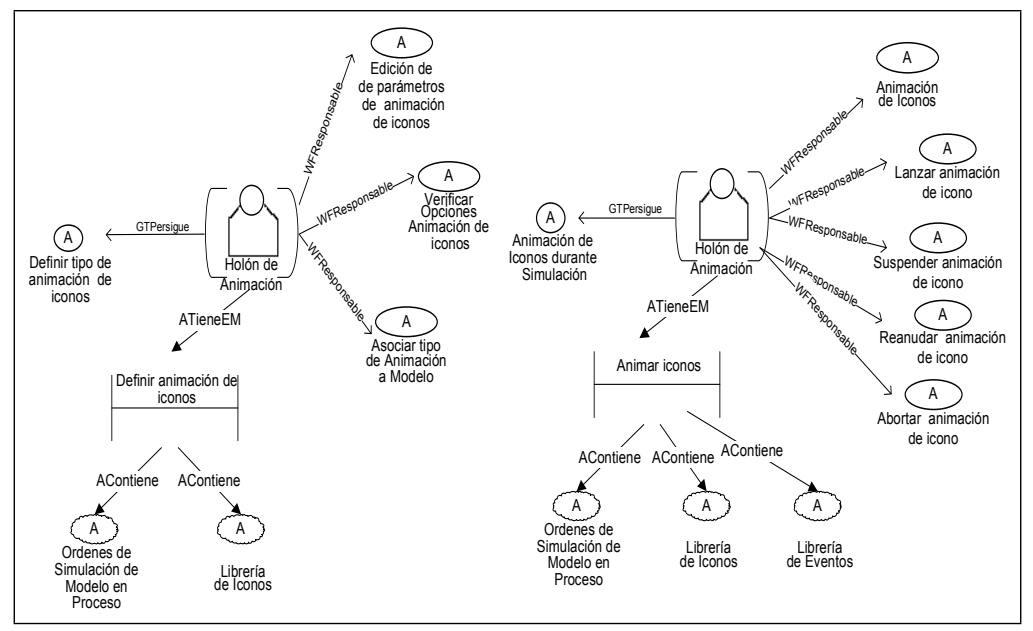

Figura 8.20: Iteración 1 - Modelo de Holón de Animación HANI

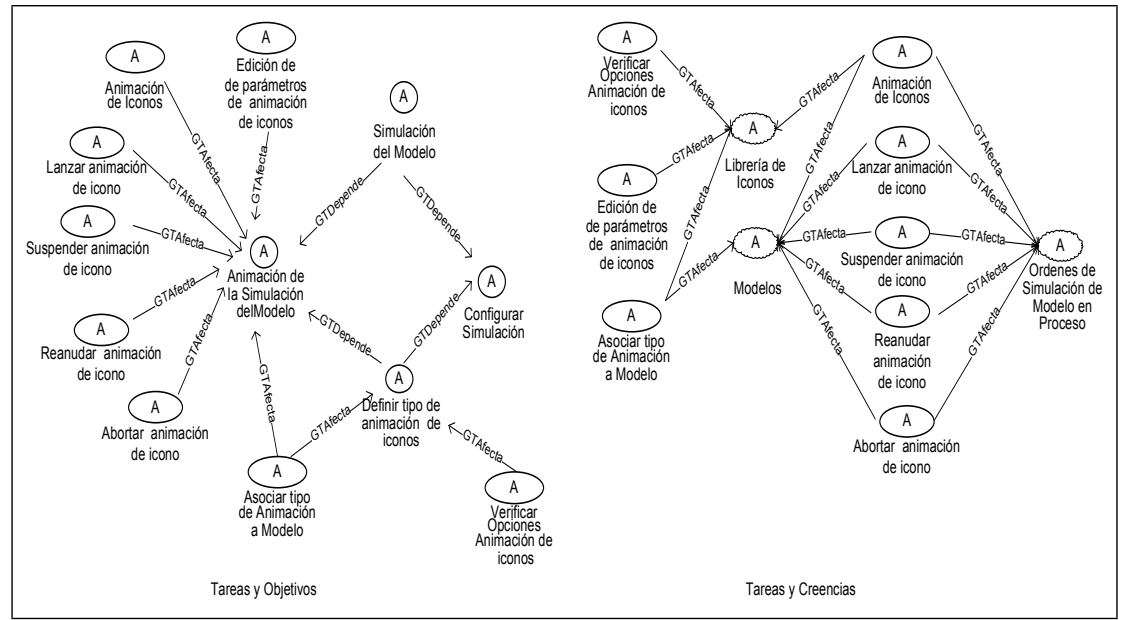

Figura 8.21: Iteración 1 - Diagrama de Tareas y Objetivos así como de las Tareas y Creencias del Holón de Animación 
Holón Gestor de Eventos. Es un agente abstracto de recurso que genera las instancias de eventos durante la simulación del modelo acorde al ritmo que marca el Holón de Ritmo. Este agente juega el rol de Gestor de Eventos (8.22). En la Figura 8.23 se muestra el detalle de las tareas enfocadas al logro de los objetivos del holón Gestor de Eventos.

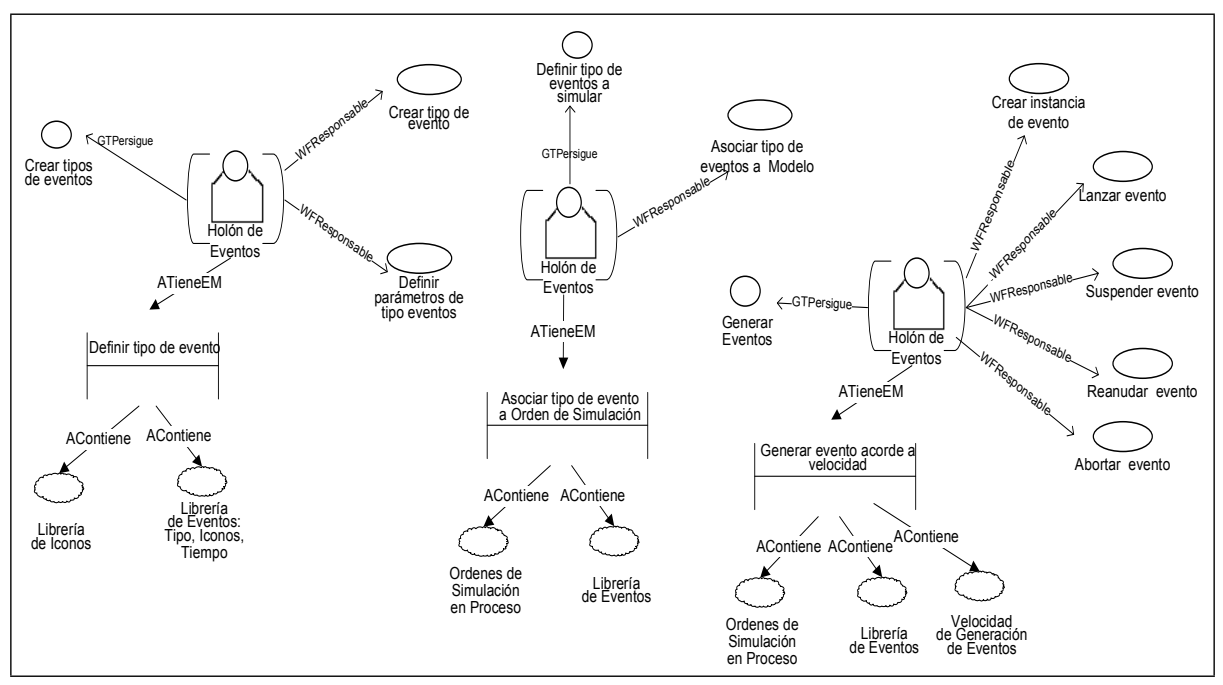

Figura 8.22: Iteración 1 - Modelo de Holón Gestor de Eventos HE-

$\mathrm{VE}$

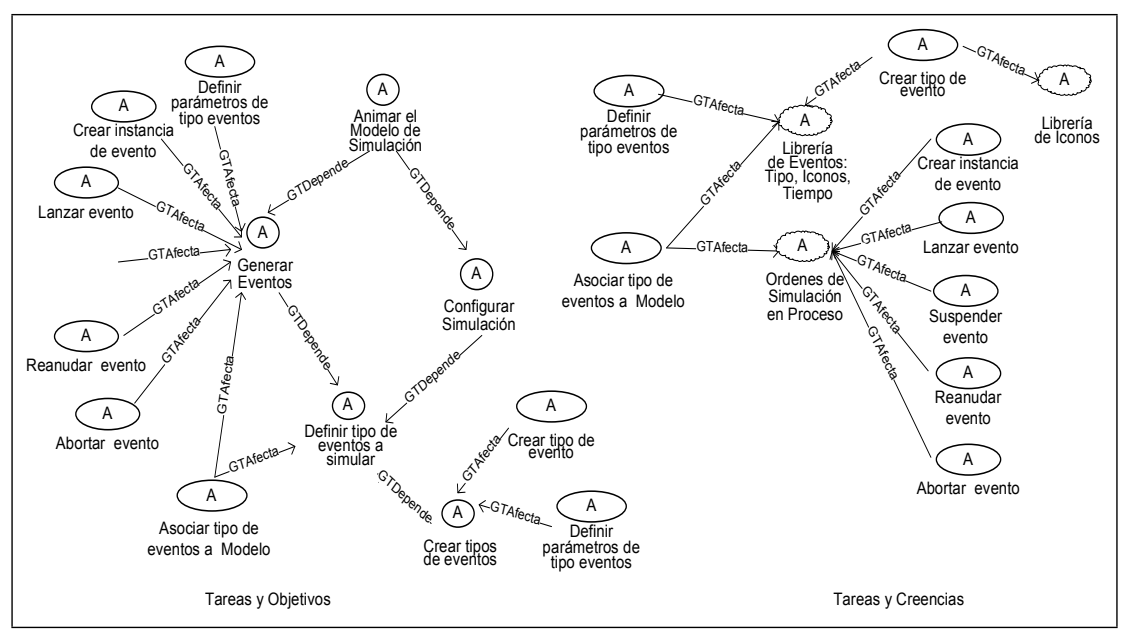

Figura 8.23: Iteración 1 - Diagrama de Tareas y Objetivos así como de las Tareas y Creencias del Holón de Eventos 
Holón de Resultados. Es un agente abstracto de recurso que permite la gestión (creación, edición, generación, eliminación, visualización) tanto de Reportes como gráficas que permitan analizar los resultados de la simulación visualmente. Este agente juega el rol de Generador de Reportes/gráficas. En la Figura 8.24 se presenta el Modelo de Agente para el Holón de Resultados, en ella, se observan de forma global, sus objetivos y tareas. Asimismo, en la Figura 8.25 se muestra el detalle de las tareas enfocadas al logro de los objetivos del holón de Resultados.

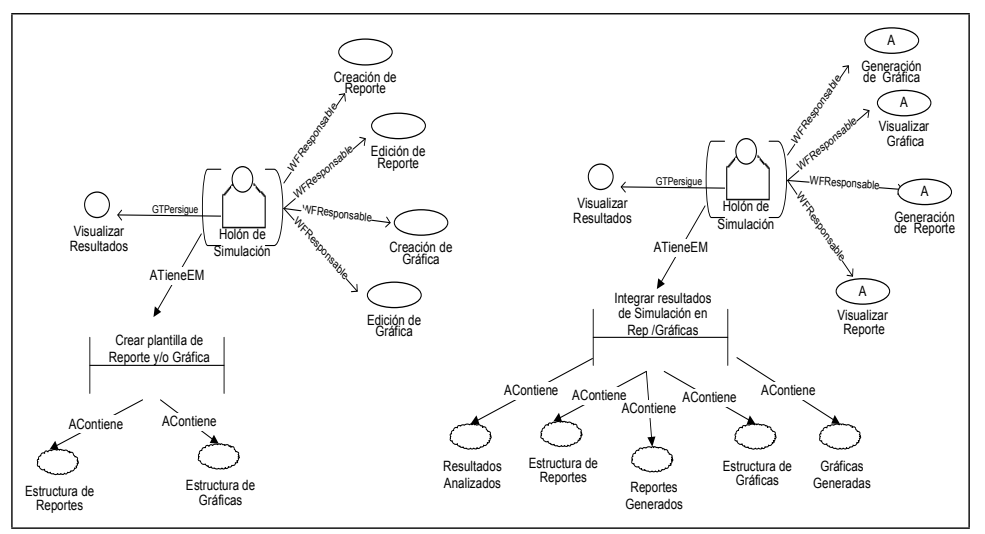

Figura 8.24: Iteración 1 - Modelo de Holón de Resultados HRES

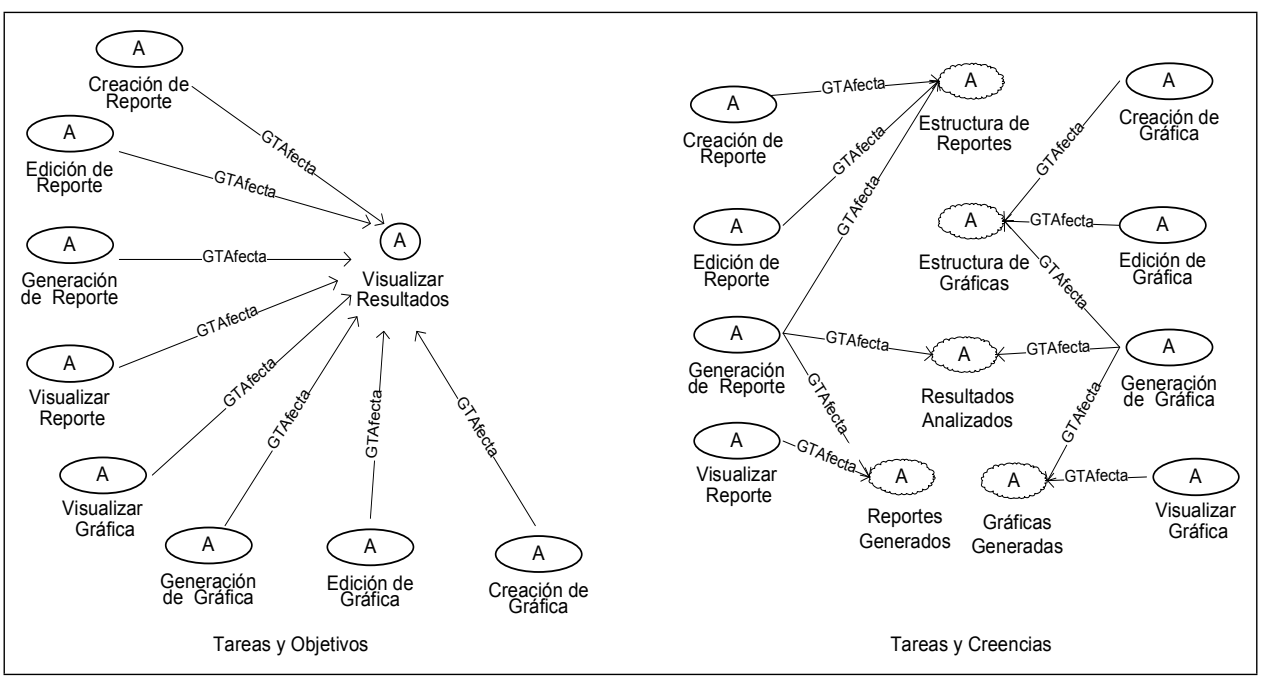

Figura 8.25: Iteración 1 - Diagrama de Tareas y Objetivos así como de las Tareas y Creencias del Holón de Resultados 
Holón de Sincronización. Es un agente abstracto de staff que está a cargo de la monitorización del estado del modelo dentro de su ciclo para generar y/o eliminar las instancias de agentes necesarios o innecesarios acorde a las necesidades del sistema y las peticiones del usuario. Este agente juega el rol de Gestor de Sincronización (Figura 8.26). Asimismo, en la Figura 8.27 se muestra el detalle de las tareas enfocadas al logro de los objetivos del holón de Sincronización.

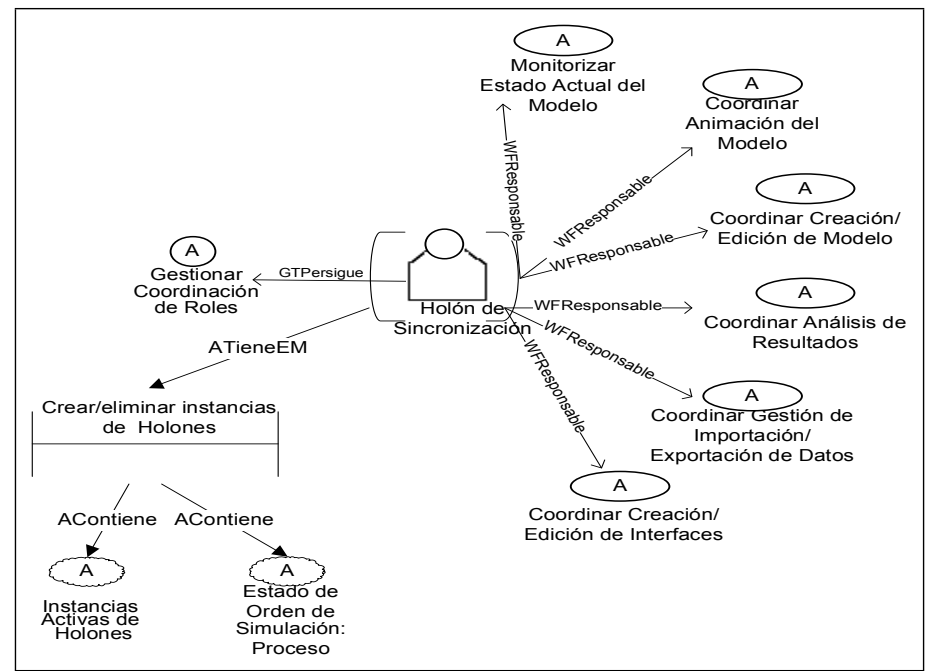

Figura 8.26: Iteración 1 - Modelo de Holón de Sincronización HSIN

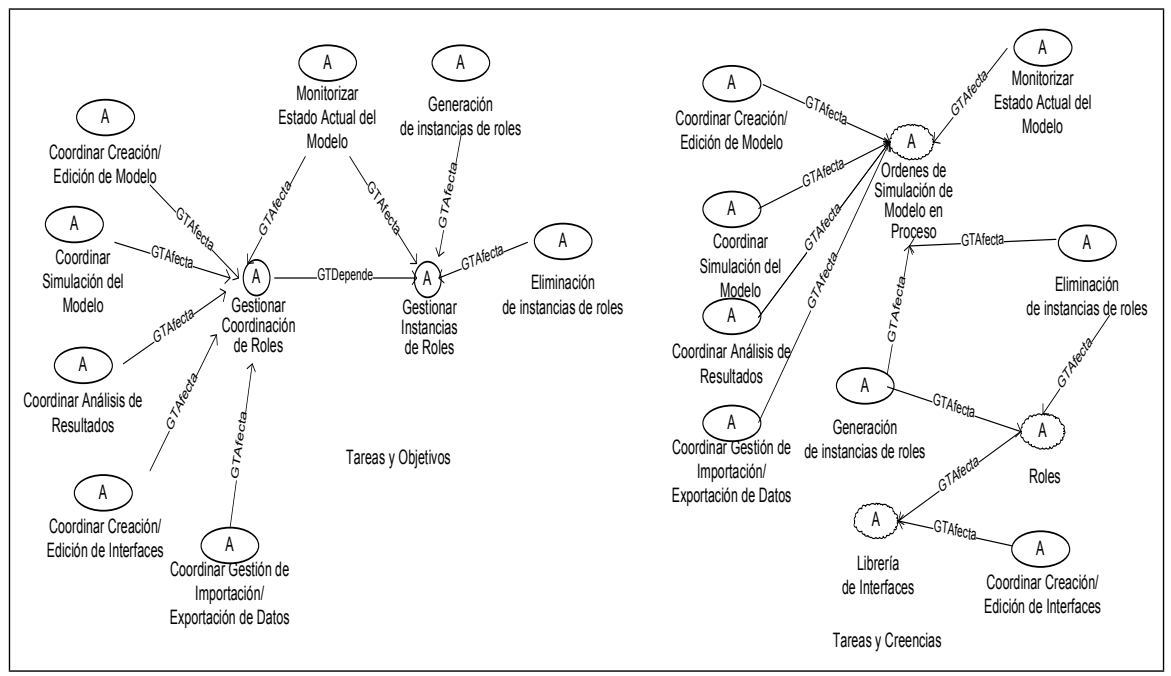

Figura 8.27: Iteración 1 - Diagrama de Tareas y Objetivos así como de las Tareas y Creencias del Holón de Sincronización 
Holón de Simulación. Es un agente abstracto de recurso que está a cargo de la planificación y simulación del modelo acorde a la configuración establecida por el usuario. Este agente juega el rol de Planificador de Simulación (Figura 8.28). Este holón tiene comunicación con los holones de Animación, de Velocidad y el Gestor de Eventos. En la Figura 8.29 se muestra el detalle de las tareas enfocadas al logro de los objetivos del holón de Simulación.

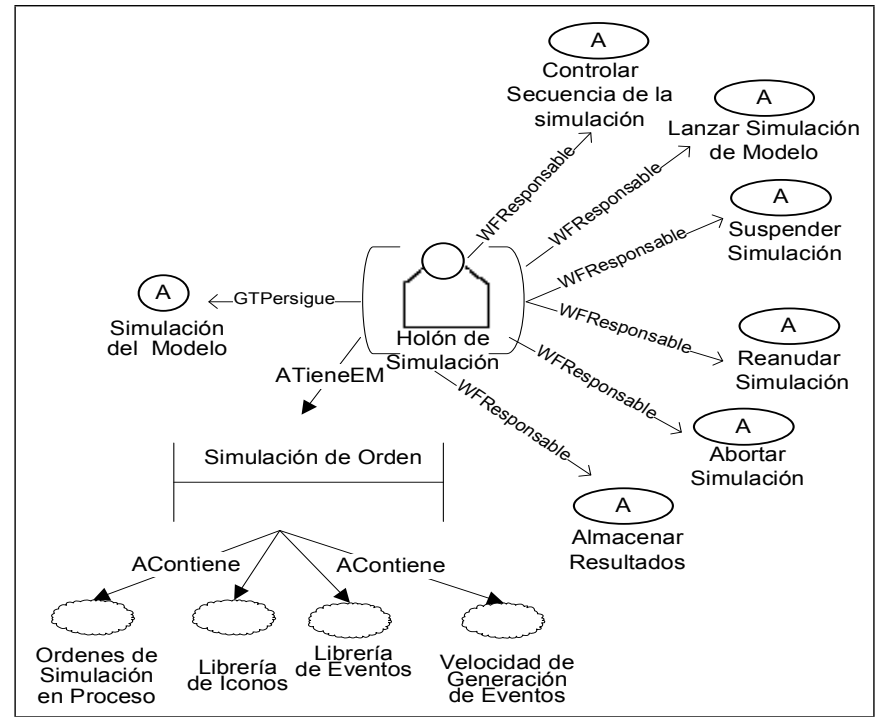

Figura 8.28: Iteración 1 - Modelo de Holón de Simulación HSIM

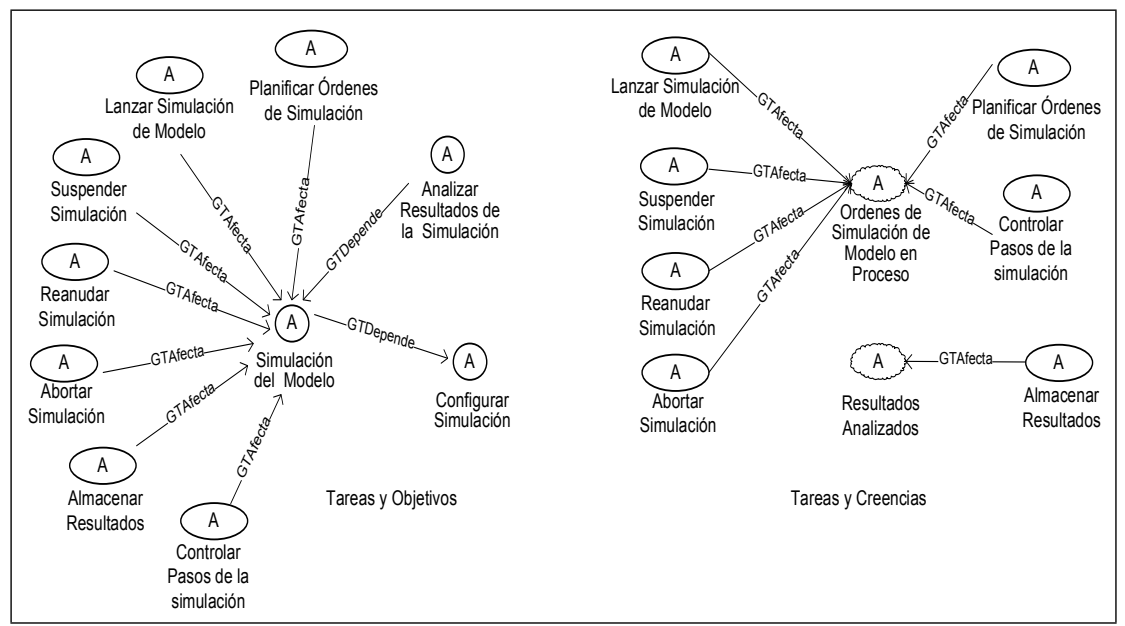

Figura 8.29: Iteración 1 - Diagrama de Tareas y Objetivos así como de las Tareas y Creencias del Holón de Simulación 
Holón Orden de Importación. Es un agente abstracto de Orden de trabajo que se genera en el proceso de Importación de Datos Reales del Sistema y es procesado por el Holón de Gestión de Importación/Exportación de Datos para introducir datos reales en un formato apropiado para su análisis (Figura 8.30).

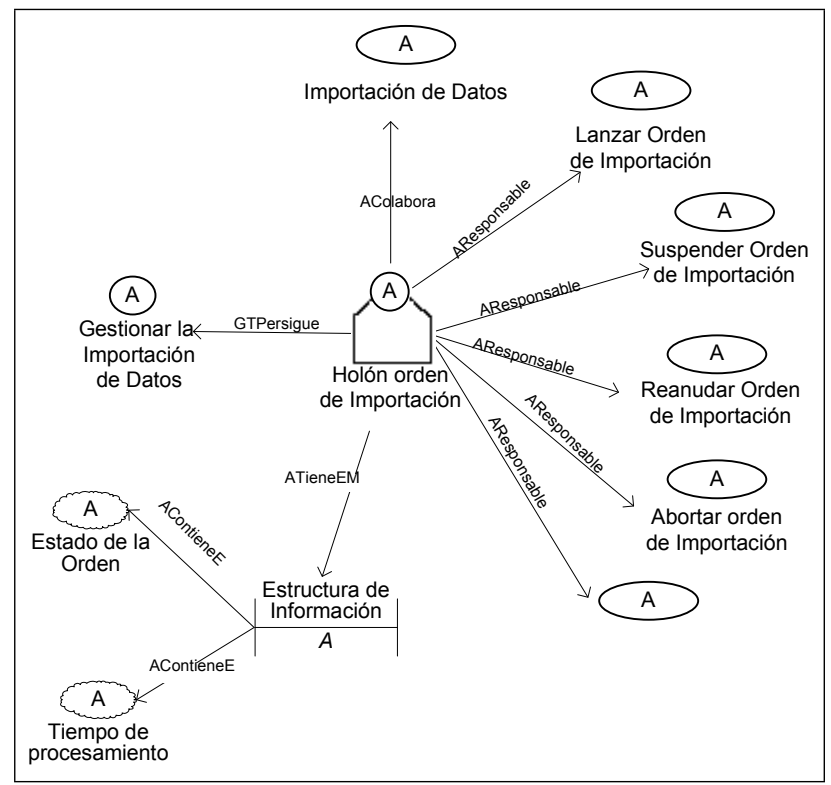

Figura 8.30: Iteración 1 - Modelo de Agente del Holón de Orden de Importación

Holón Orden de Modelo. Es un agente abstracto de Orden de trabajo que se genera en el proceso de creación de modelo y es procesado mediante el Modelador utilizando iconos asociados a partes/procesos específicos (Figura 8.31).

Holón Orden de Simulación Modelo. Es un agente abstracto de Orden de trabajo que se genera en el proceso de simulación de modelo y es procesado mediante el Simulador quien planifica la introducción de la orden en la Planta. 


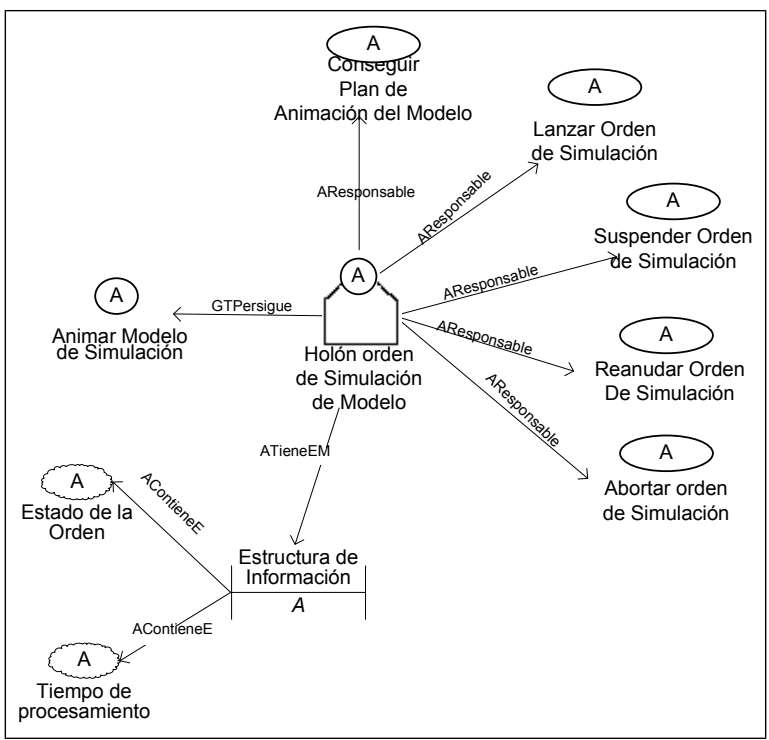

Figura 8.31: Iteración 1 - Modelo de Agente del Holón de Orden de Modelo

Holón Orden de Configuración de Modelo. Es un agente abstracto de Orden de trabajo que se genera en el proceso de Configuración del Modelo y es procesado mediante el Holón de Animación, el Holón de Eventos y el Holón de Velocidad para parametrizar la simulación del modelo (Figura 8.32).

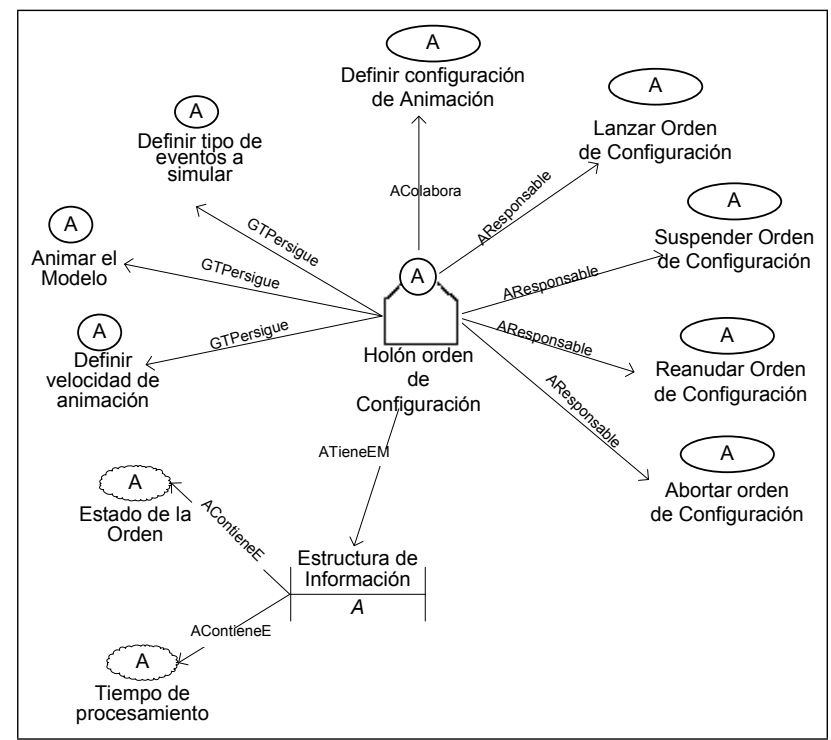

Figura 8.32: Iteración 1 - Modelo de Agente del Holón de Orden de Configuración 
Holón Orden de Evento. Es un agente abstracto de Orden de trabajo que se genera en el proceso de Generación de Eventos y es procesado mediante el Holón Eventos durante la simulación de un modelo (Figura 8.33).

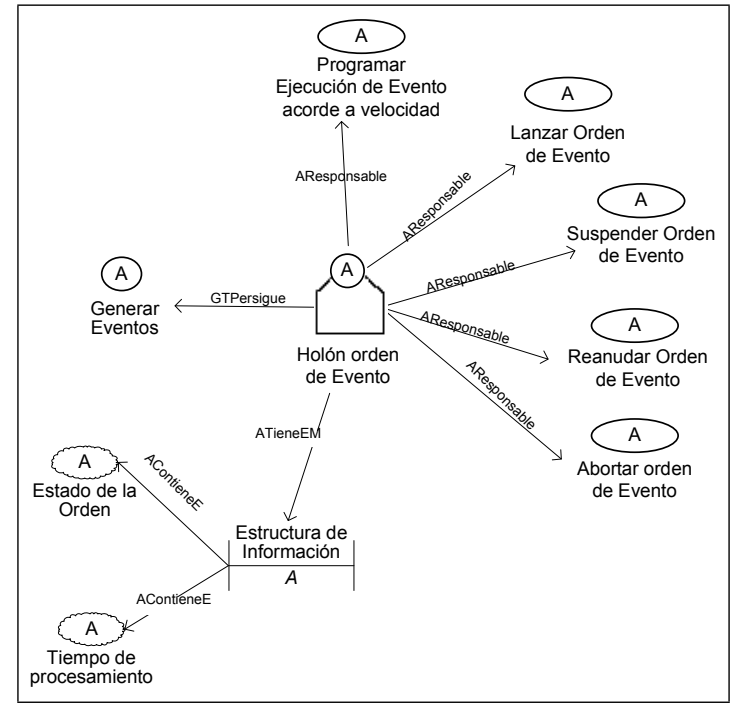

Figura 8.33: Iteración 1 - Modelo de Agente del Holón de Orden de Evento

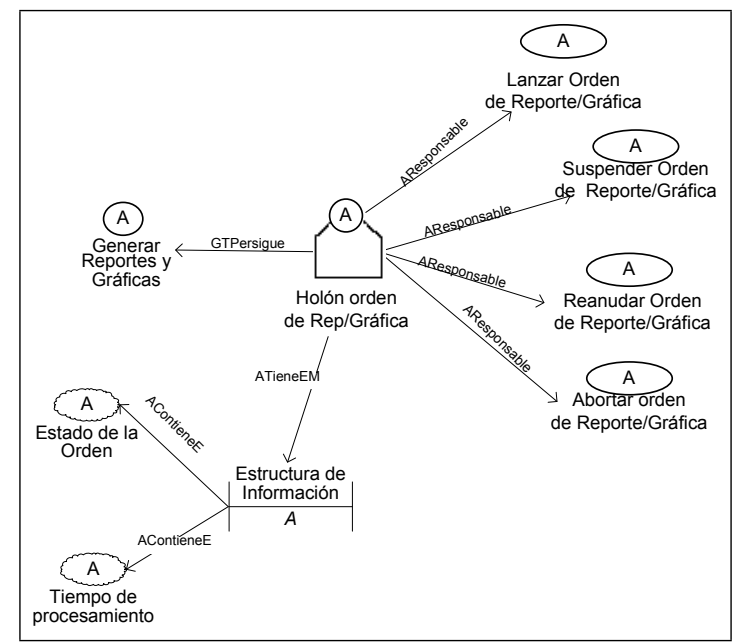

Figura 8.34: Iteración 1 - Modelo de Agente del Holón de Orden de Reporte/Gráfica

Holón Orden de Reporte/Gráfica. Es un agente abstracto de Orden de trabajo que se genera en el proceso de Creación de un Reporte o Gráfica y es procesado mediante el Holón Reportes/gráficas el cual permite el análisis 
de los resultados de la simulación acorde a Reportes o Gráficas de Negocio estándar (Figura 8.34).

Holón Orden de Exportación. Es un agente abstracto de Orden de trabajo que se genera en el proceso de Exportación de Datos Reales del Sistema y es procesado por el Holón Gestor de Importación/Exportación de Datos para introducir datos del reales en un formato apropiado para su análisis externo (Figura 8.35).

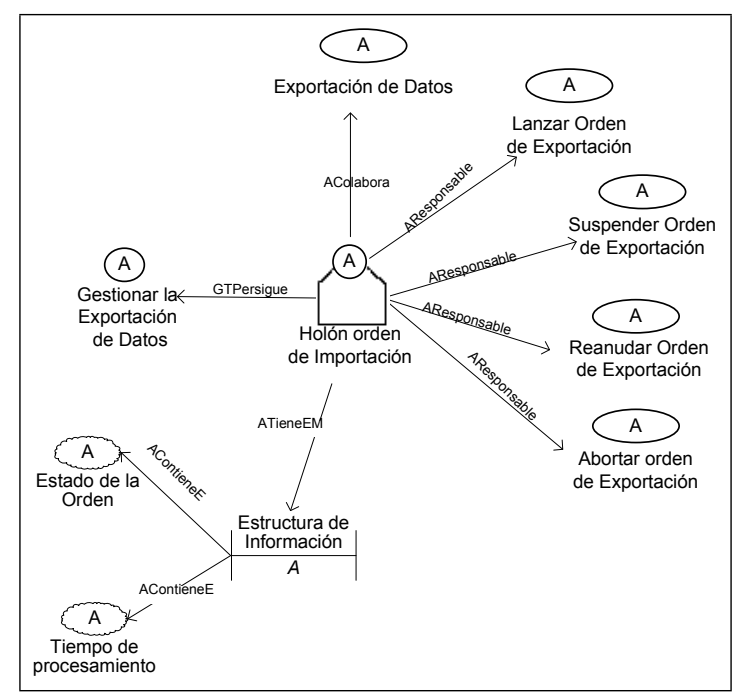

Figura 8.35: Iteración 1 - Modelo de Agente del Holón de Orden de Exportación

Holón Modelo. Es un agente abstracto de producto que mantiene la estructura del modelo de fabricación representado por iconos el cual es simulado mediante su animación (Figura 8.36).

Holón Icono. Es un agente de recurso que contiene la información relativa al elemento que representa (persona, máquina, banda transportadora, proceso, buffer, vehículo, etc.)

Holón Interfaz. Es un agente de producto que contiene la información de las interfaces que permiten establecer comunicación visual con el usuario y de forma interna entre los holones del sistema.

Holón Evento. Es un agente de producto que contiene la información de los eventos que aparecen durante la simulación a petición del Holón de de Orden de Evento. 


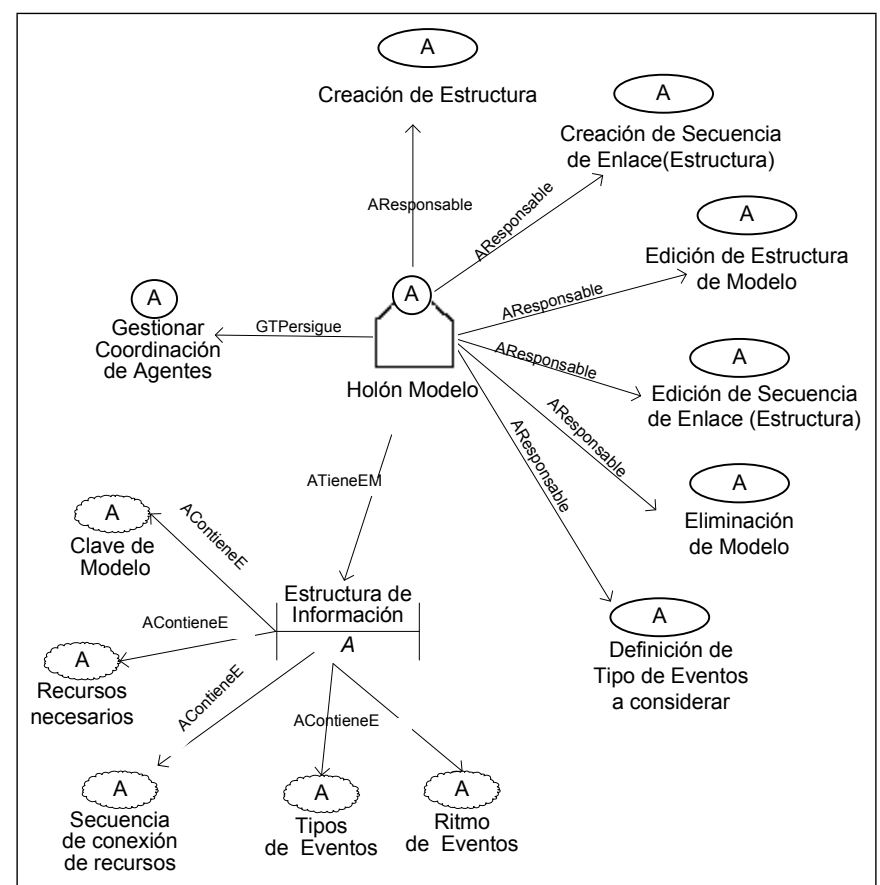

Figura 8.36: Iteración 1 - Modelo de Agente del Holón de Modelo

Holón Reporte. Es un agente de producto que contiene la información de un reporte que permite el análisis de los resultados de la simulación del modelo.

Holón Gráfica. Es un agente de producto que contiene la información de una gráfica que permite el análisis de los resultados de la simulación del modelo.

Por otro lado, para la implementación de los holones asociados al Sistema de Fabricación, se utiliza el Metamodelo para Sistemas de Fabricación presentado en el Capítulo 6. Una vez asociados los roles a los holones y en consecuencia, sus objetivos, tareas y relaciones, a continuación, basado en el Modelo de Interacciones inicial, se detallan las interacciones entre los holones. En los Diagramas de Interacciones detallados se muestran, además de las acciones y objetivos de los holones, el tipo de interacciones en cada Caso de Uso identificado. Las interacciones indican: a) qué holón inicia una interacción (UInicia) y b) qué holones cooperan entre sí (UColabora). En el Diagrama de Interacciones también se muestra la secuencia de las acciones que se llevan a cabo durante las interacciones en cada Caso de Uso. 
A continuación se presentan cinco diagramas de interacción representativos que permiten observar en detalle como se comunican los agentes del Entorno de Simulación.

Como parte de la inicialización del Sistema, en la Arquitectura del Entorno de Simulación se propone inicializar la Base de Datos a partir de la información del Sistema de Fabricación original (Sección 5.7). Para ello, el holón Gestor de Sincronización solicita al holón Gestor Importación/Exportación, la importación de los datos del Sistema original a la Base de Datos del Entorno de Simulación, una vez importada, el holón Analizador de Datos de Entrada Salida, los analiza y entrega al holón Gestor de Iconos para que los asocie al tipo de elementos apropiado (Figura 8.37).

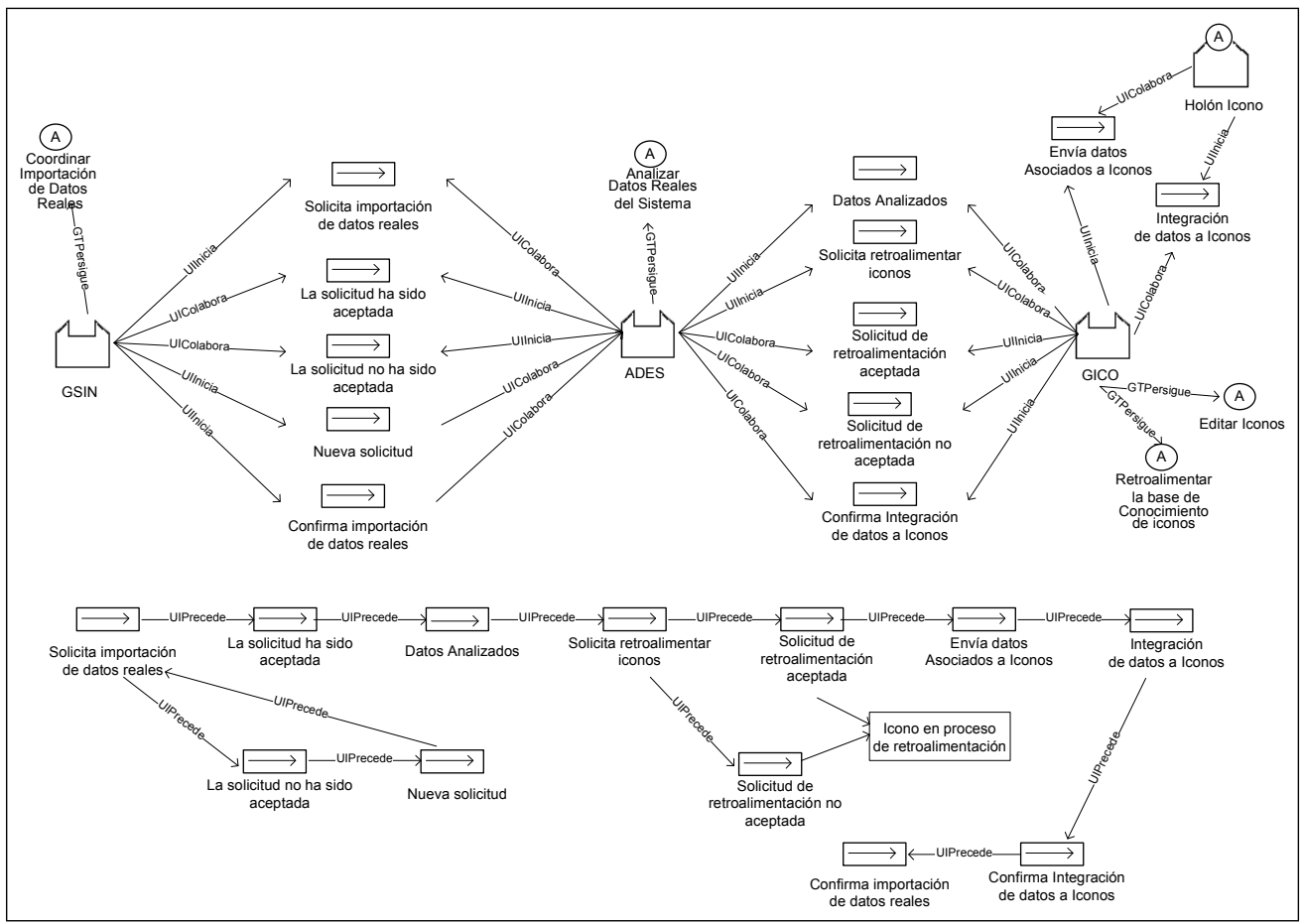

Figura 8.37: Iteración 1 - Diagrama de la Interacción Gestionar la Importación de Datos Reales

De acuerdo con los escenarios presentados en la Arquitectura del Entorno de Simulación, una vez inicializada la Base de Datos, se lleva a cabo la Creación de Modelos (Sección 5.8). En la Figura 8.38 se presenta el Diagrama de Interacciones entre el holón Gestor de Sincronización, el holón Modelador, el 
holón Verificador y el holón Gestor de Iconos. En el Diagrama se observan los objetivos que persigue cada uno durante la Creación de Modelos, así como sus acciones y su secuencia.

La secuencia incluye desde que el holón Gestor de Sincronización solicita al holón Modelador, la creación de un holón Modelo. Para la creación del holón Modelo, el holón Modelador interactúa con el holón Gestor de Iconos para integrar en el modelo holones Icono. Esta última interacción se repite hasta que el Usuario termina el diseño del modelo y entonces el holón Gestor de Sincronización solicita al holón Verificador que verifique y apruebe el Modelo (primera fase de la simulación).

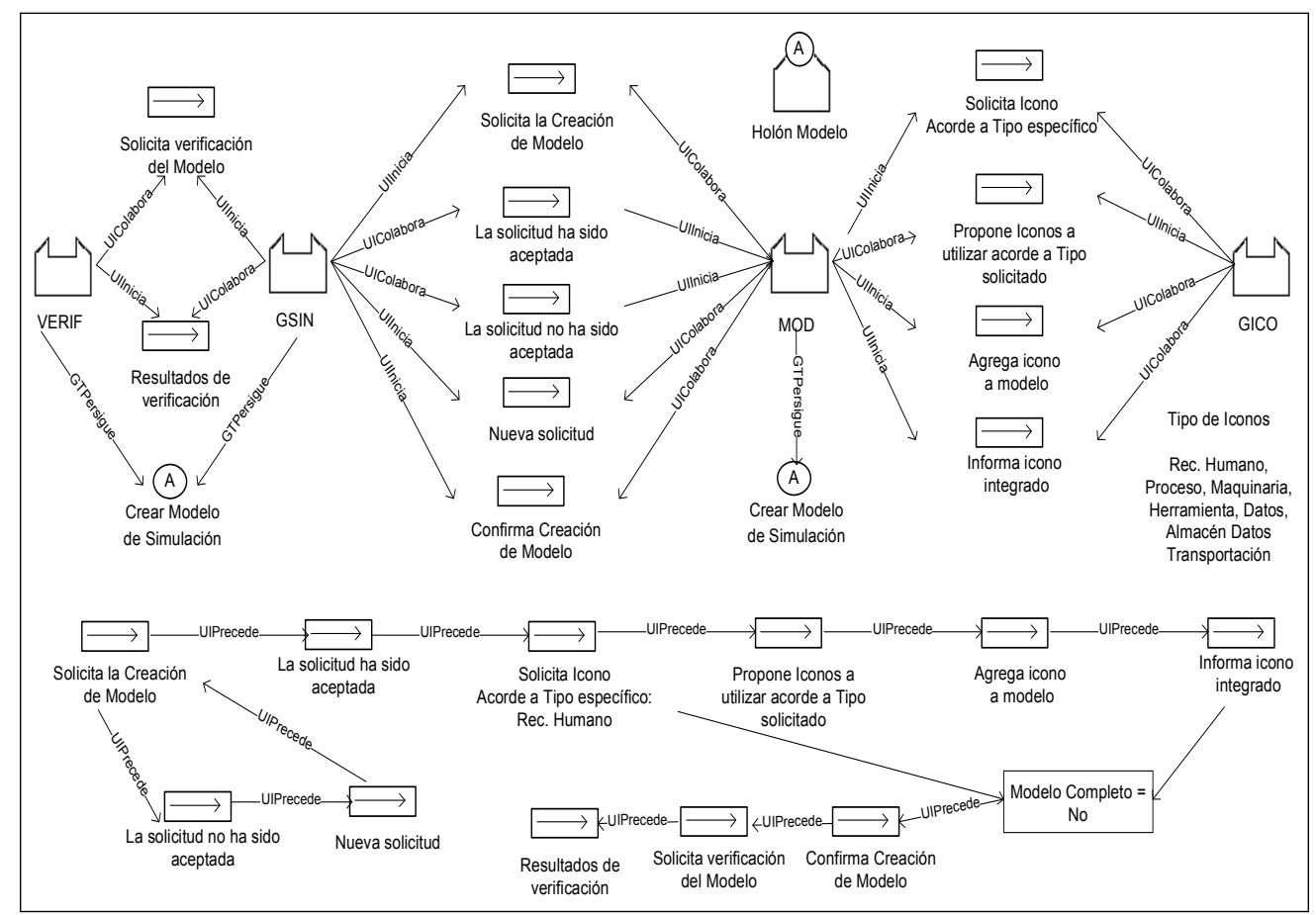

Figura 8.38: Iteración 1 - Diagrama de la Interacción Creación de Modelo

Una vez aprobado el Modelo, el holón Gestor de Sincronización pone el modelo a disposición del holón de Simulación, el holón de Animación, el holón de Velocidad y el holón Generador de Eventos para la segunda fase de la simulación. 


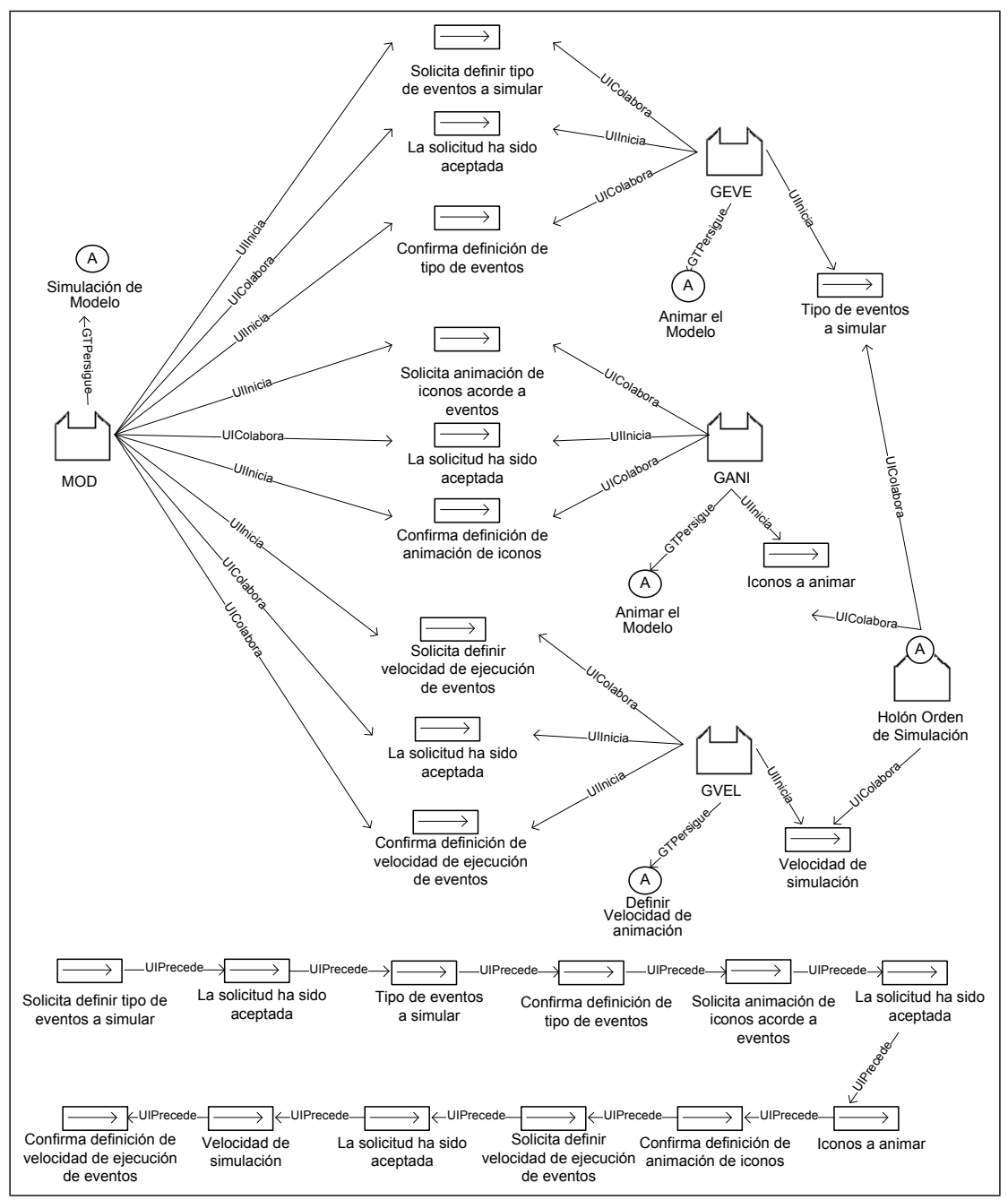

Figura 8.39: Iteración 1 - Diagrama de la Interacción Configuración de Simulación de Modelo

Una vez creado el Modelo, en la segunda fase, el escenario inicial (Caso de Uso) es la Configuración de la Simulación del Modelo (Sección 7.1.2), en él se establecen los parámetros que controlan la simulación del modelo.

En la Figura 8.39 se presenta el Diagrama de Interacciones de los holones que intervienen durante la configuración de la Simulación. En el Diagrama se observan los objetivos que persigue cada uno durante la Configuración de la Simulación de Modelos, así como sus acciones y su secuencia. 
En este Caso de Uso, el Usuario especifica los parámetros que controlarán la simulación del modelo. El holón Simulador recibe los parámetros seleccionados por el Usuario e informa: a) la velocidad al holón Gestor de Velocidad, b) el tipo de eventos a considerar al holón Generador de Eventos, c) el tipo de animación para cada icono al holón de Animación y d) el tipo de eventos al holón Orden de Simulación. Asimismo, el holón Gestor de Sincronización monitoriza que tales instancias se encuentren activas.

Los parámetros incluyen, velocidad de la simulación que a su vez controlará la generación de eventos y el tipo de animación que se utilizará para mostrar el comportamiento interno de cada uno de los elementos del Sistema de Fabricación.

Una vez que se ha creado el Modelo y se ha definido la configuración previa a la Simulación, el siguiente paso es la Simulación del Modelo (escenario presentado en la Sección 7.2). En este escenario el holón Simulador lleva a cabo, por un lado, la coordinación de las actividades del holón de Animación, el holón Generador de Eventos y el holón Gestor de Velocidad, basándose en la velocidad, tipo de animación y eventos a simular configurados.

Por otro lado, el holón Simulador monitoriza los elementos del modelo (que representa al Sistema de Fabricación) para recibir información de su estado durante la simulación. Cada vez que el holón Simulador recibe información de un cambio en el estado de los elementos del Modelo, éste informa al holón Generador de Eventos quien a su vez informa de los cambios visuales requeridos al holón de Animación. Los resultados obtenidos durante la simulación son almacenados en la Base de Datos para su posterior presentación y análisis.

En la Figura 8.40 se presenta el Diagrama de Interacciones donde se observan los objetivos que persigue cada uno de los holones, sus acciones y la secuencia que se presenta durante la Simulación de Modelos. Cuando el holón Gestor de Sincronización recibe una orden de Simulación (holón de Orden), el holón de Animación, el holón de Eventos y holón de Velocidad interactúan basándose en la configuración previamente definida.

La velocidad definida le indica al holón de Velocidad en qué momento debe solicitar al holón de eventos que genere un evento específico, tales eventos incluyen informar al holón de Animación para que éste actualize la representación gráfica del estado interno de los elementos del Modelo. 
El holón Gestor de Sincronización por su parte, verifica el estado actual del sistema hasta que se termina la simulación del modelo y se asegura que de eliminar aquellas instancias que no ya son necesarias.

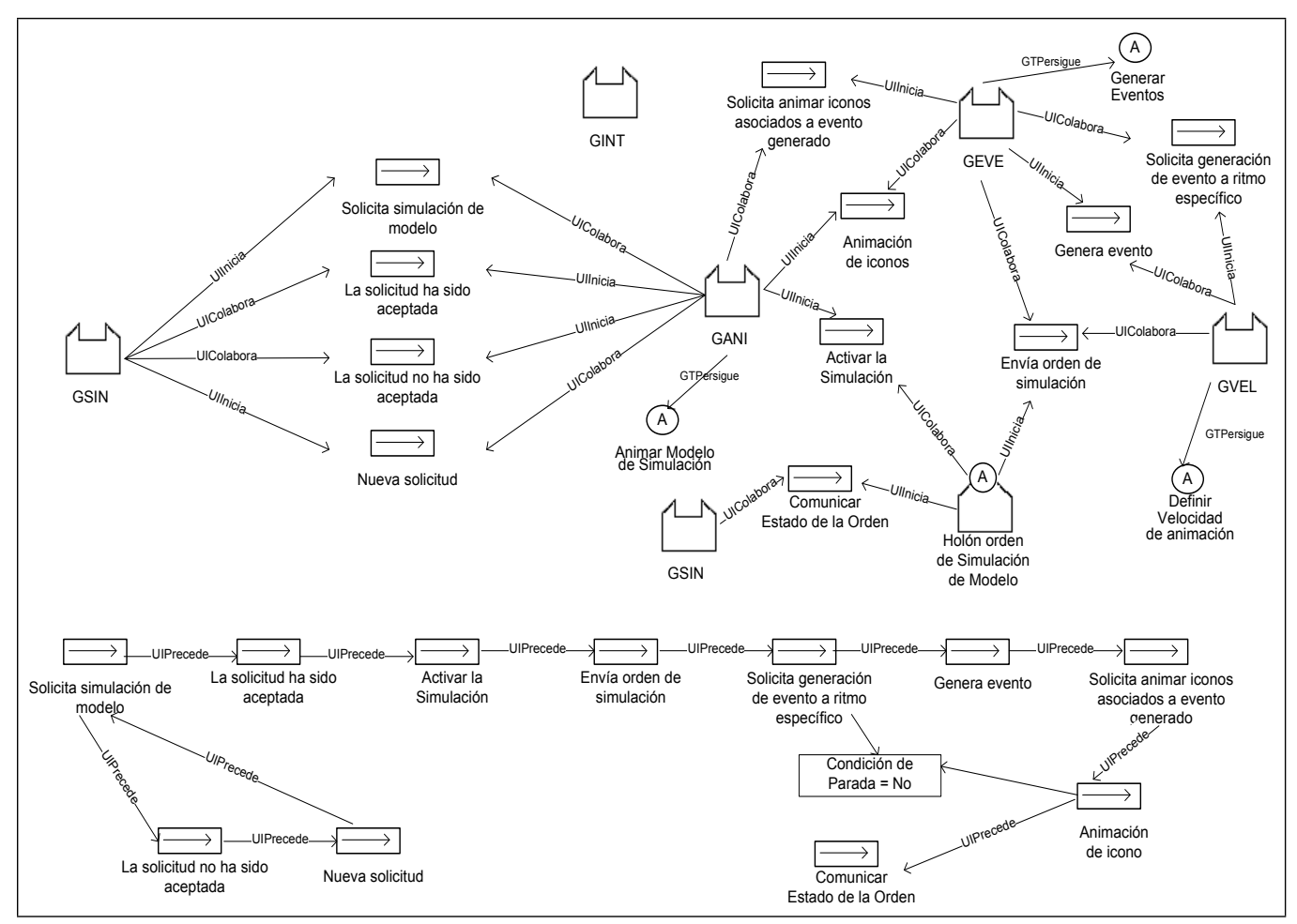

Figura 8.40: Iteración 1 - Diagrama de la Interacción Simulación del Modelo

Una vez terminada la Simulación del Modelo se procede al Análisis de los Resultados de la simulación donde intervienen el holón Analizador de Entradas/Salidas y el Gestor de Iconos.

El holón Gestor de Iconos utiliza los resultados para efectos de aprendizaje de los elementos del Modelo, los agentes aprenden en base a la experiencia adquirida durante la ejecución de la simulación.

En la Figura 8.41 se presenta el Diagrama de Interacciones que incluye las actividades que desempeñan dichos holones durante el Análisis de Resultados. 


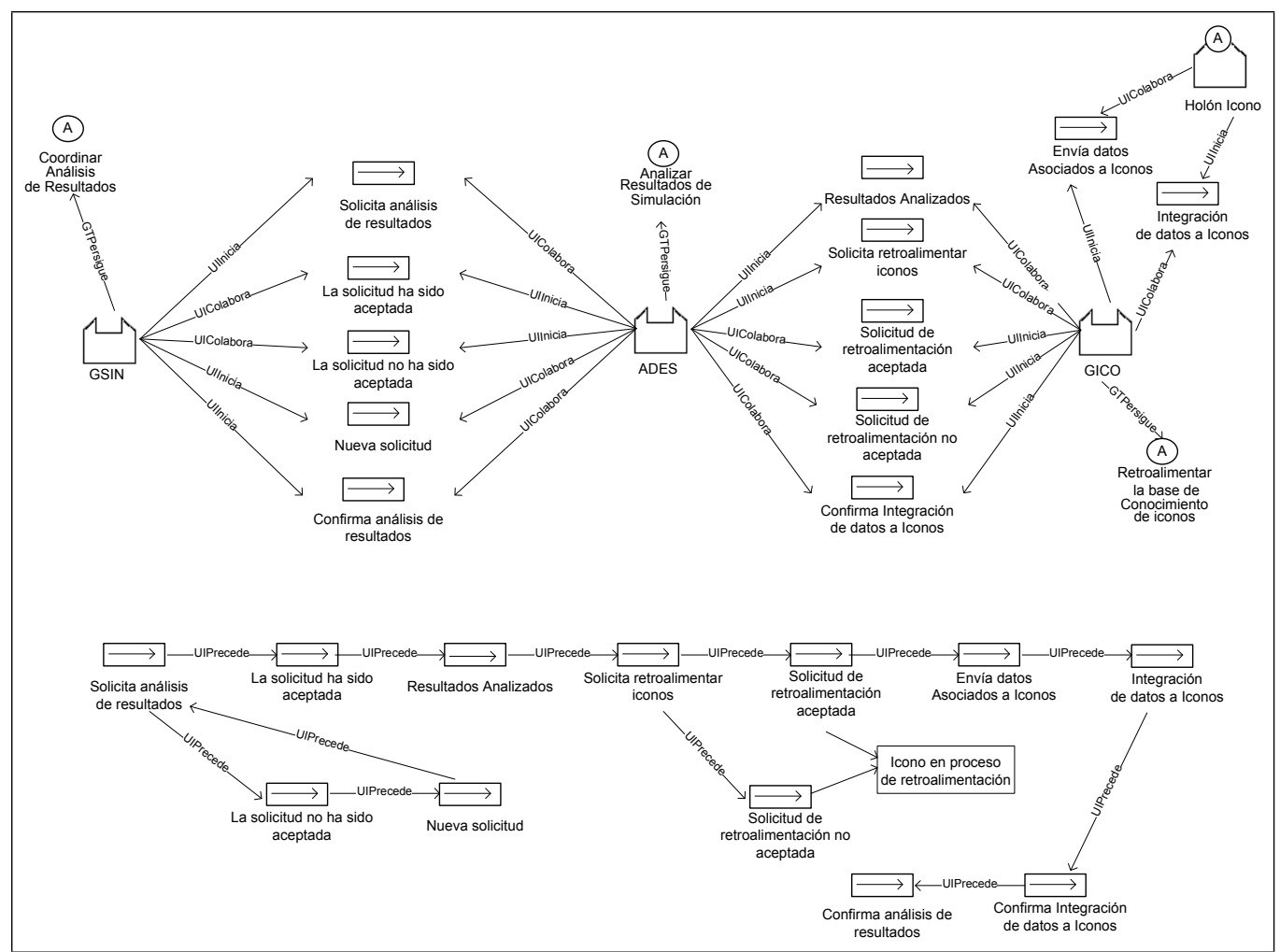

Figura 8.41: Iteración 1 - Diagrama de la Interacción Análisis de Resultados

\subsubsection{Relaciones con el Entorno}

En la Arquitectura del Entorno de Simulación propuesta en los Capítulos 5 y 7 se observa cómo el entorno tiene influencia sobre el entorno de simulación mediante el holón Gestor de Importación/Exportación. Esto se debe, por un lado, a que el holón de Importación/Exportación recibe los datos de entrada (recursos de la planta, productos, su ruta y lista de materiales), proporcionados por el Sistema Original que se va a modelar. Por otro lado, el holón de Importación/Exportación es el responsable de exportar los resultados obtenidos (almacenados en la Base de Datos del Entorno) durante la simulación de modelos para su análisis externo. En la Figura 8.42 se muestra el detalle de las entradas que el holón de Importación/Exportación percibe del entorno así como las salidas que proporciona. 


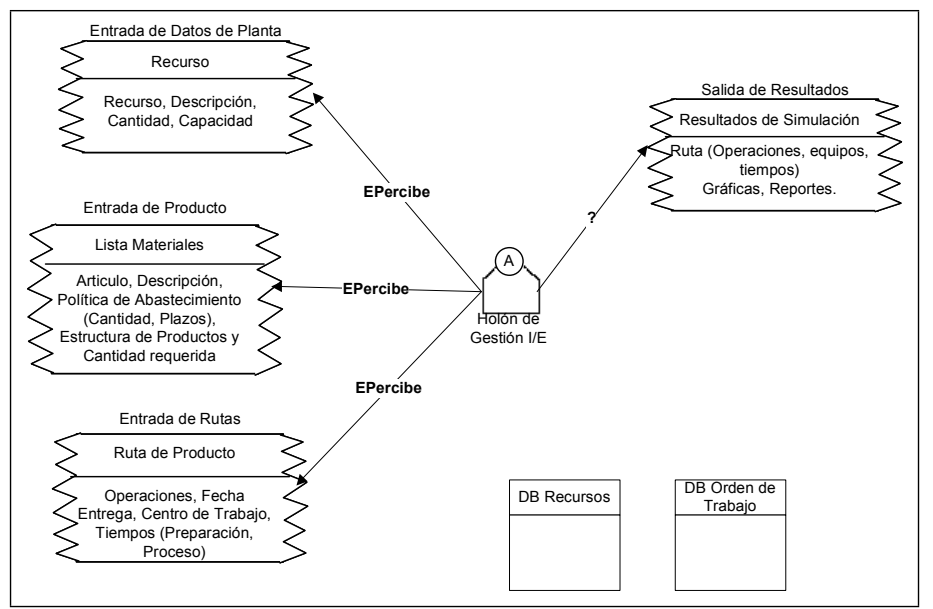

Figura 8.42: Iteración 1 - Modelo de Entorno del Holon GIE

\subsubsection{La Arquitectura del Sistema}

Una vez definido el detalle de las características de cada uno de los holones que integran el Entorno de Simulación el siguiente paso es construir la arquitectura del prototipo. Para ello, se utilizan las plantillas de Agente JADE de ANEMONA. Con respecto a los holones que permiten definir el Metamodelo del Sistema de Fabricación, se presentan dos holones básicos: el holón de Producto y el holón Transformador. Estos holones permiten inicializar y mantener la Base de Datos de instancias que pueden ser utilizadas para la definición de un Modelo.

Mediante el Holón de Producto se controlan las actividades para agregar y manipular las instancias de los productos que se pueden fabricar en el Sistema de Fabricación. En la plantilla de JADE se pueden observar los servicios que ofrece el holón de producto para detallar su estructura; éstos incluyen la definición de sus datos, su ruta (tareas y recursos que requiere) y lista de materiales (asociada a las tareas de la ruta). Asimismo el holón de Producto incluye la definición de su apariencia durante la simulación. En la plantilla también se puede observar la definición de los mensajes que el holón de producto intercambia con otros holones (tipo de mensaje, la interacción en la que participa así como el tipo de participación) (Figura 8.43). 


\begin{tabular}{|c|c|c|c|c|c|c|}
\hline \multicolumn{7}{|c|}{ 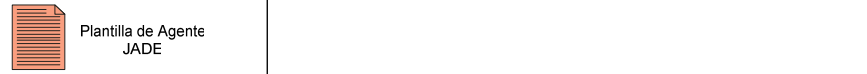 } \\
\hline 1.idAgente & \multicolumn{3}{|c|}{ Holón Producto } & & 2. Plataforma & $\begin{array}{l}\text { Importación/Exportación de } \\
\text { Datos }\end{array}$ \\
\hline \multicolumn{7}{|l|}{ 3.Servicios } \\
\hline \multicolumn{4}{|c|}{ 3.1. Nombre } & \multicolumn{3}{|r|}{ 3.2. Tipo } \\
\hline \multicolumn{4}{|c|}{ Crear Producto } & \multicolumn{3}{|r|}{ Interno } \\
\hline \multicolumn{4}{|c|}{ Crear Apariencia } & \multicolumn{3}{|r|}{ Interno } \\
\hline \multicolumn{4}{|c|}{ Crear Ruta } & \multicolumn{3}{|r|}{ Interno } \\
\hline \multicolumn{4}{|c|}{ Comunicar Resultados } & \multicolumn{3}{|r|}{ Externo } \\
\hline \multicolumn{7}{|c|}{ 4.Comportamientos } \\
\hline \multicolumn{3}{|c|}{ 4.1. Nombre } & 4.2. Tipo & & \multicolumn{2}{|c|}{ 4.3. Servicio que implementa } \\
\hline \multicolumn{3}{|c|}{ Inicialización de Producto } & Secuencial & \multicolumn{3}{|c|}{\begin{tabular}{|l} 
Crear Producto \\
Crear Apariencia \\
Crear Ruta \\
Crear Lista de Tareas \\
Crear Tarea \\
Crear Lista de Transiciones \\
Crear Transición
\end{tabular}} \\
\hline \multicolumn{3}{|c|}{ Proporcionar Tarea } & Secuencial & \multicolumn{3}{|c|}{$\begin{array}{l}\text { Solicitar Ejecución de Proceso } \\
\text { Solicitar entradas } \\
\text { Recibir entradas } \\
\text { Ejecutar Proceso } \\
\text { Comunicar Resultado } \\
\text { Solicitar siguiente Tarea }\end{array}$} \\
\hline \multicolumn{3}{|c|}{ Proporcionar Transición } & Secuencial & \multicolumn{3}{|c|}{$\begin{array}{l}\text { Recibir solicitud Tarea Destino } \\
\text { Recibir Tarea Origen } \\
\text { Vincular Tarea Origen a Tarea Destino } \\
\text { Informar siguiente Tarea Destino }\end{array}$} \\
\hline \multicolumn{7}{|l|}{ 5. Ontología } \\
\hline \multicolumn{3}{|c|}{ 5.1. Nombre } & \multicolumn{2}{|c|}{ 5.2. Ontología Base } & \multicolumn{2}{|r|}{ 5.3. Esquemas } \\
\hline \multicolumn{3}{|c|}{ Holón Producto } & & & \multicolumn{2}{|c|}{$\begin{array}{c}\text { Id_producto, Descripción, Estado, Tipo } \\
\text { Animación, id_icono, arch_ico, Id_ruta, } \\
\text { id_tarea,tmi_tmi_compleción, } \\
\text { tmax_compleción, tipo_proceso, descripción } \\
\text { proceso, Cant_recursos, Recurso, Lista } \\
\text { Transiciones }\end{array}$} \\
\hline \multicolumn{3}{|c|}{ Recursos } & & & \multicolumn{2}{|c|}{$\begin{array}{l}\text { Id_Recurso, Proceso, Capacidad min, } \\
\text { Capacidad Max }\end{array}$} \\
\hline \multicolumn{3}{|c|}{ Tarea } & & & \multicolumn{2}{|c|}{ Id_Tarea, Proceso, Producto, Tiempo } \\
\hline & & & & & Tarea & Origen, Tarea Destino \\
\hline 6.Comunicació & & & & & & \\
\hline 6.1. Men & & 6.2. Tiрo & & Inte & acción & 6.4 Tipo Participación \\
\hline Solicita crear & ucto & request & & $\operatorname{ar} \mathrm{P}_{1}$ & ducto & Colaborador \\
\hline Comunicar Prod & Creado & inform & & $\operatorname{ar} \mathrm{Pr}$ & ducto & Iniciador \\
\hline Solicita Pr & & request & Crear $\mathrm{O}$ & end & Fabricación & Colaborador \\
\hline Enviar Datos & ucto & agree & Crear O & den d & Fabricación & Iniciador \\
\hline Comunica envío & oducto & inform & Crear $\mathrm{O}$ & lend & Fabricación & Iniciador \\
\hline Producto no & & agree & Crear $\mathrm{O}$ & lend & Fabricación & Iniciador \\
\hline 7. Tiene $\mathrm{p}$ & te proces & miento físics & & & & \\
\hline
\end{tabular}

Figura 8.43: Plantilla JADE- Producto

Por su parte, el Holón Transformador es aquel que lleva a cabo procesos de transformación sobre materiales con el fin de fabricar un holón de producto determinado. Por tanto, en la plantilla se incluyen los servicios que permiten actualizar el contenido de los atributos de la instancia, así como aquellos que permiten responder a solicitudes de ejecución de procesos para una Orden de Producción específica (Figura 8.44). Con respecto a los mensajes se observa 
que participa en dos tipos de interacción clave, la creación de modelos (primera fase del proceso de simulación) y la ejecución de procesos (segunda fase de simulación).

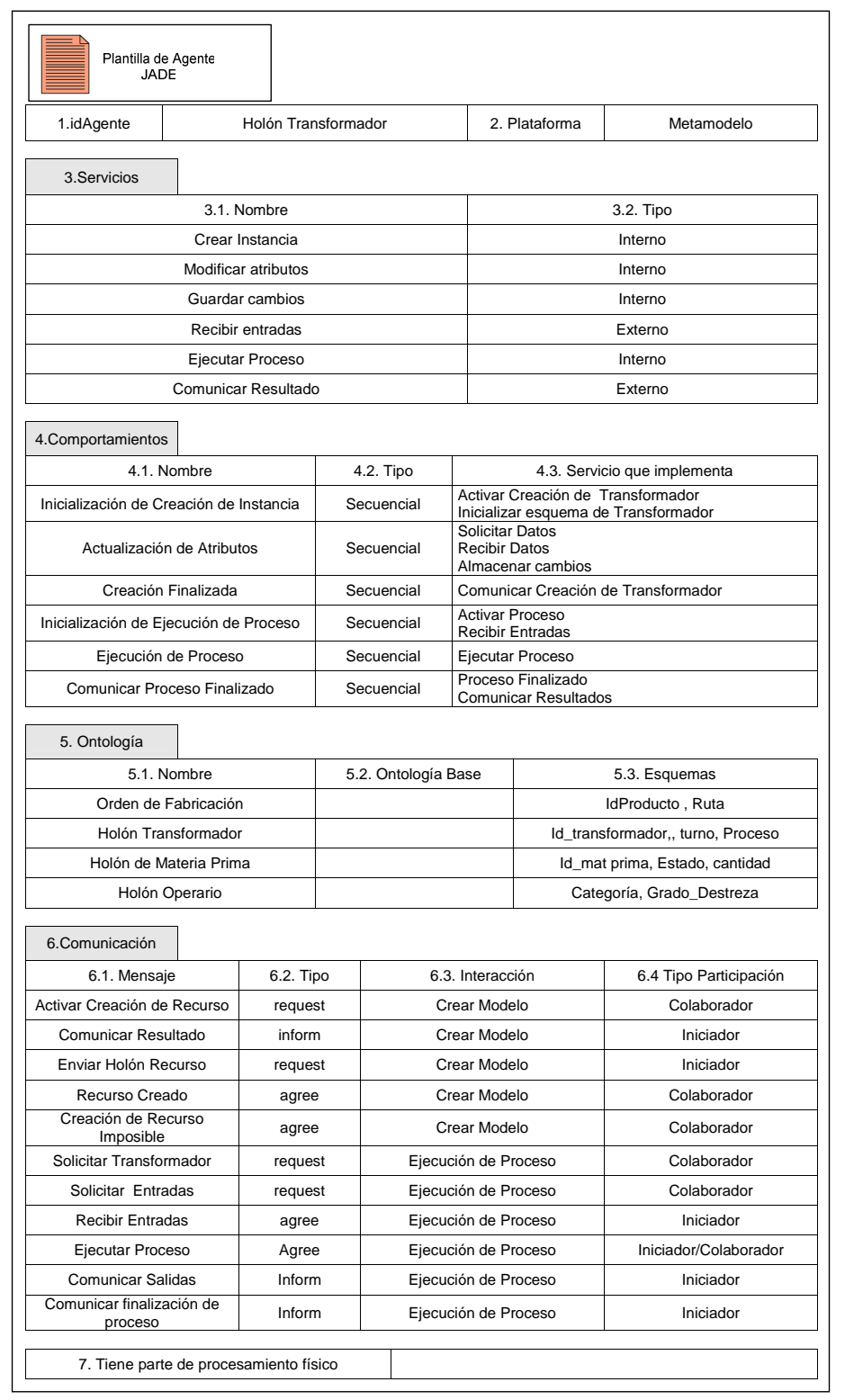

Figura 8.44: Plantilla JADE- Transformador

Con respecto al Entorno de Simulación, a continuación se presentan la definición de los holones Modelador y de Simulación. 
En la primera fase de la Simulación de Modelos, el holón Modelador ofrece dos servicios básicos, la creación del modelo y la verificación del mismo. En cuanto a la creación del modelo, los comportamientos que incluyen la adición / eliminación de iconos, conllevan internamente un mayor detalle de actividades para permitir que los iconos mantengan una distribución física y se agrupen en unidades organizativas tales como estaciones de trabajo. En cuanto a la verificación, el holón Modelador aplica las reglas de modelado (presentadas en el Capítulo 5) a fin de que el modelo se simule apropiadamente (por ejemplo, que todos los iconos tengan asociados los atributos de una instancia).

\begin{tabular}{|c|c|c|c|c|c|c|c|}
\hline \multicolumn{8}{|c|}{$\begin{array}{l}\text { Plantilla de Agente } \\
\text { JADE }\end{array}$} \\
\hline 1.idAgente & \multicolumn{4}{|c|}{ Holón Modelador } & \multicolumn{2}{|c|}{ 2. Plataforma } & Simulador \\
\hline \multicolumn{8}{|l|}{ 3.Servicios } \\
\hline \multicolumn{5}{|c|}{ 3.1. Nombre } & \multicolumn{3}{|r|}{ 3.2. Тipo } \\
\hline \multicolumn{5}{|c|}{ Lanzar Creación de Modelo } & \multicolumn{3}{|r|}{ Interno } \\
\hline \multicolumn{5}{|c|}{ Crear Modelo } & \multicolumn{3}{|r|}{ Interno } \\
\hline \multicolumn{5}{|c|}{ Lanzar Verificación de Modelo } & \multicolumn{3}{|r|}{ Interno } \\
\hline \multicolumn{5}{|c|}{ Verificar Modelo } & \multicolumn{3}{|r|}{ Interno } \\
\hline \multicolumn{8}{|c|}{ 4.Comportamientos } \\
\hline \multicolumn{3}{|c|}{ 4.1. Nombre } & \multicolumn{2}{|c|}{ 4.2. Tipo } & \multicolumn{3}{|c|}{ 4.3. Servicio que implementa } \\
\hline \multicolumn{3}{|c|}{ Recepción de Nombre de Modelo } & \multicolumn{2}{|c|}{ Secuencial } & \multicolumn{3}{|c|}{\begin{tabular}{|l|l|} 
Activar \\
Inicializar \\
\end{tabular}} \\
\hline \multicolumn{3}{|c|}{ Inicio de Adición de Iconos } & \multicolumn{2}{|c|}{ Secuencial } & \multicolumn{3}{|c|}{$\begin{array}{l}\text { Solicitud de tipo de icono } \\
\text { Elegir entre opciones de iconos de tipo solicitado } \\
\text { Añadir a Modelo el icono asociado } \\
\text { Definir atributos de icono } \\
\text { Almacenar cambios }\end{array}$} \\
\hline \multicolumn{3}{|c|}{ Fin de Adición de Iconos } & \multicolumn{2}{|c|}{ Secuencial } & \multicolumn{3}{|c|}{ Comunicar actualización de modelo } \\
\hline \multicolumn{3}{|c|}{ Recepción de Modelo } & \multicolumn{2}{|c|}{ Secuencial } & \multicolumn{3}{|c|}{ Recibir modelo } \\
\hline \multicolumn{3}{|c|}{ Inicio de Verificación de Modelo } & & encial & \begin{tabular}{|l} 
Valida \\
Mode \\
Model
\end{tabular} & $\begin{array}{l}\text { datos en atri } \\
\text { correcto } \\
\text { incorrecto, } r\end{array}$ & $\begin{array}{l}\text { tos básicos de iconos } \\
\text { uiere completarse }\end{array}$ \\
\hline Fin de Veri & ón de $\mathrm{M}$ & & & encial & Comu & car resultac & de validación \\
\hline 5. Ontología & & & & & & & \\
\hline & mbre & & & Dntologí & Base & & 5.3. Esquemas \\
\hline Orde & Modelo & & & & & & e, Producto, Cantidad \\
\hline 6.Comunicacic & & & & & & & \\
\hline 6.1. Mer & & 6.2. T & & & 3. Intera & & 6.4 Tipo Participación \\
\hline Solicita Tipo & cono & reque & & & ción de & lodelo & Iniciador \\
\hline Confirma Icon & adido & Infor & & & ción de & Iodelo & Colaborador \\
\hline Integrar Icono & odelo & Infor & & & ción de & Iodelo & Colaborador \\
\hline $\begin{array}{c}\text { Agregación } \\
\text { Impos }\end{array}$ & & Infor & & & ción de & lodelo & Colaborador \\
\hline $\begin{array}{r}\text { Solicita Verif } \\
\text { Mode }\end{array}$ & & reque & & Verif & ación d & Modelo & Iniciador \\
\hline Verificación c & delo & Infor & & Verit & ación d & Modelo & Colaborador \\
\hline Comunica R & dos & Infor & & Verif & ación d & Modelo & Colaborador \\
\hline 7. Tiene $\mathrm{p}$ & de proce & miento $\mathrm{f}$ & & & & & \\
\hline
\end{tabular}

Figura 8.45: Plantilla JADE- Modelador 


\begin{tabular}{|c|c|c|c|c|}
\hline \multicolumn{5}{|c|}{ 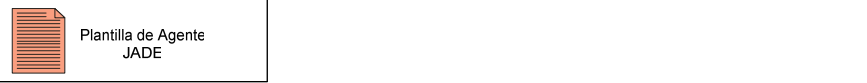 } \\
\hline 1.idAgente & Holón de Simulación & \multicolumn{2}{|c|}{ 2. Plataforma } & Simulación \\
\hline \multicolumn{5}{|l|}{ 3.Servicios } \\
\hline \multicolumn{2}{|c|}{ 3.1. Nombre } & \multicolumn{3}{|r|}{ 3.2. Tipo } \\
\hline \multicolumn{2}{|c|}{ Lanzar Configuración de Simulación } & \multicolumn{3}{|r|}{ Interno } \\
\hline \multicolumn{2}{|c|}{ Configurar Simulación } & \multicolumn{3}{|r|}{ Interno } \\
\hline \multicolumn{2}{|c|}{ Lanzar Simulación de Modelo } & \multicolumn{3}{|r|}{ Interno } \\
\hline \multicolumn{2}{|c|}{ Simular Modelo } & \multicolumn{3}{|r|}{ Interno } \\
\hline \multicolumn{5}{|c|}{ 4.Comportamientos } \\
\hline 4.1. Nombre & 4.2. Tipo & \multicolumn{3}{|c|}{ 4.3. Servicio que implementa } \\
\hline Recepción de Modelo & Secuencial & \multicolumn{3}{|c|}{$\begin{array}{l}\text { Recibir modelo validado } \\
\text { Inicializar Configuración }\end{array}$} \\
\hline Inicio de Configuración de Simulación & Secuencial & \multicolumn{3}{|c|}{$\begin{array}{l}\text { Solicitar velocidad de simulación } \\
\text { Solicitar tipo de animación } \\
\text { Solicitar tipo de evento a simular } \\
\text { Recepción de velocidad, tipo animación, tipo evento } \\
\text { Almacenar configuración } \\
\text { Configuración establecida } \\
\text { Configuración incompleta }\end{array}$} \\
\hline Fin de Configuración de Simulación & Secuencial & \multicolumn{3}{|c|}{ Comunicar configuración de modelo } \\
\hline Inicio de Simulación & Secuencial & \multicolumn{3}{|c|}{\begin{tabular}{|l|} 
Recibir modelo y configuración \\
Recibir icono y transición \\
Comunicar velocidad de simulación \\
Recepción de Evento a ejecutar \\
Solicitar ejecución de evento \\
Recibir resultados de evento \\
Almacenar resultados \\
Solicitar siguiente evento \\
\end{tabular}} \\
\hline Find & encial & Coms & ar resultad & de simulación \\
\hline 5. Ontología & & & & \\
\hline & Dntologi & Base & & 5.3. Esquemas \\
\hline Orden & & & Modelo, V & $\begin{array}{l}\text { cidad, Tipo Animación, Tipo } \\
\text { de Eventos }\end{array}$ \\
\hline 6.Comunicació & & & & \\
\hline 6.1. Men & & Intera & & 6.4 Tipo Participación \\
\hline $\begin{array}{r}\text { Solicita Simu } \\
\text { Mode }\end{array}$ & Sim & ación d & lodelo & Iniciador \\
\hline $\begin{array}{l}\text { Solicita Config } \\
\text { Simulación d } \\
\end{array}$ & Sim & ación d & lodelo & Iniciador \\
\hline Solicita Ve & Configu & ción de & imulación & Iniciador \\
\hline Solicita Tipo de & Configu & ción de & imulación & Iniciador \\
\hline Solicita Tipo d & Configu & ción de & imulación & Iniciador \\
\hline Recepción de C & Configu & ción de & imulación & Colaborador \\
\hline $\begin{array}{r}\text { Comunica Si } \\
\text { Finalize }\end{array}$ & Sim & ación d & lodelo & Iniciador \\
\hline Simulación d & Sim & ación d & Iodelo & Iniciador \\
\hline Simulación II & & ación d & Iodelo & Iniciador \\
\hline 7. Tiene $\mathrm{p}$ & & & & \\
\hline
\end{tabular}

Figura 8.46: Plantilla JADE- Holón de Simulación

En la segunda fase de la Simulación de Modelos, el holón de Simulación, controla las actividades de configuración de la animación de modelos. Asimismo, el holón de Simulación coordina y monitoriza las actividades con los holones de Animación, Velocidad y Generador de Eventos para la simulación y representación gráfica de los comportamientos internos de los holones del Sistema de Fabricación (Figura 8.46). El holón de Simulación es el que establece una 
relación continua con el Sistema de Fabricación (que también esta compuesto por holones). A su vez, el holón de Sincronización monitoriza que mientras se simula un modelo se encuentren activos las instancias asociadas al mismo, pero que cuando termina su simulación, ninguno de ellos permanezca activo.

\subsection{Implementación del Simulador}

La tecnología utilizada para la implementación del prototipo incluye: a) JAVA (Ver. 6.x) (JAVA 2005), como lenguaje de programación estándar, b) JADE (Ver. 3.4) (JADE 2005), como plataforma de desarrollo basado en JAVA, que ofrece esquemas básicos para la definición de Agentes y sus protocolos de comunicación, c) ECLIPSE (Ver. 3.2.2) (ECLIPSE 2005), como interfaz de desarrollo que permite gestionar JADE, d) PROTEGÉ-OWL (Ver. 4.x) (PROTEGE 2005), como editor de la Ontología y e) MySQL (Ver. 5.0) (MySQL 2005), que proporciona un servidor de base de datos SQL (Structured Query Language) muy rápido, multi-threaded, multi usuario y robusto, se utiliza como gestor para la Base de Datos a la cual se puede acceder mediante drivers jdbc de JAVA.

El proceso de simulación que sigue el Entorno de Simulación se divide en dos Fases globales: Creación del Modelo (Fase I) y Simulación del Modelo (Fase II). Basándonos en la documentación y análisis del Sistema así como en el modelado del sistema presentado previamente (Capítulos 5, 6 y 7), en esta sección se presenta el detalle de las actividades de los agentes internos y el aspecto del Entorno de Simulación soportado por Agentes en cada una de las fases.

\subsubsection{Fase I. Creación de Modelo}

El objetivo de la Fase I “Creación del Modelo" es la creación del Modelo que represente un Sistema de Fabricación. Nuestra propuesta incluye además de las posibilidades que aportan las herramientas actuales, los beneficios aportados por la teoría de los Sistemas Multiagente. En esta sección se presentan el detalle de las actividades realizadas por la plataforma de agentes durante la primera fase del proceso de Simulación. En esta fase se crea el modelo basado en la representación del entorno físico de la Planta y el entorno modelado del sistema multiagente que lo simulará (Figura 8.47). 


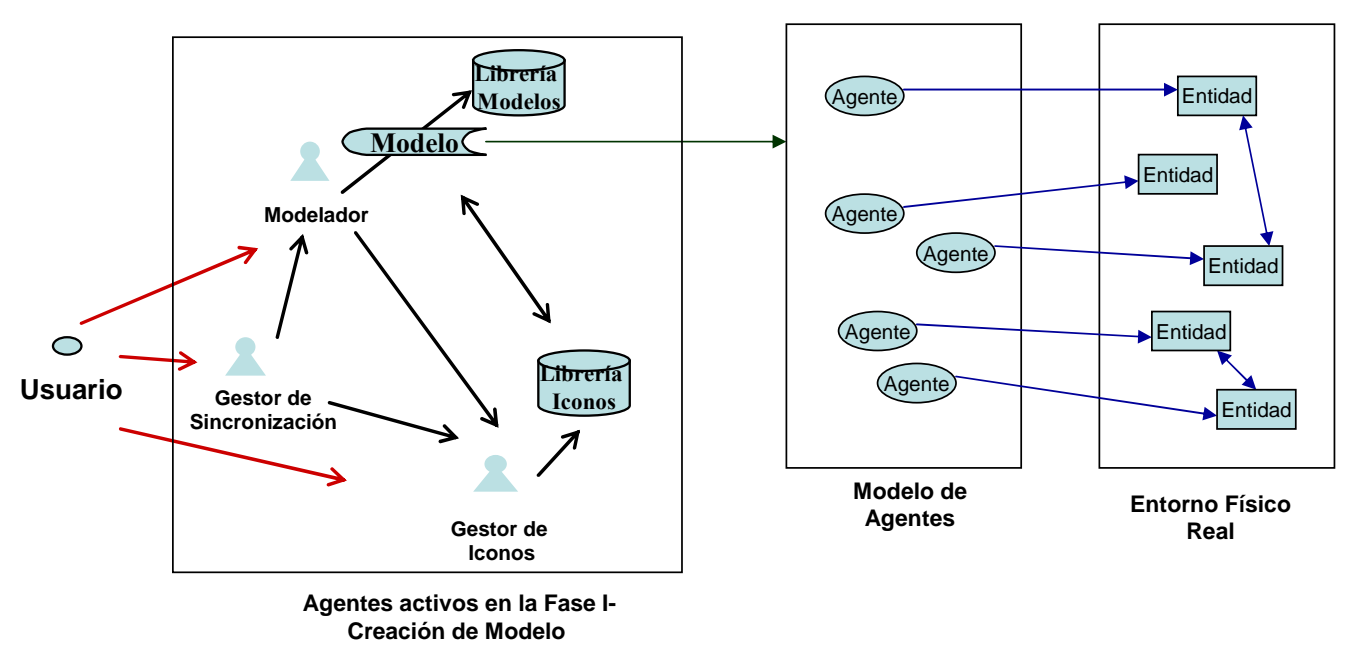

Figura 8.47: Agentes activos en la Fase I y relación entre el entorno de agentes modelado y la Planta física

\section{Pasos previos a la Creación del Modelo}

Una de las características del entorno de simulación es que el modelo se creará basado en la utilización de los recursos disponibles en la Planta de Fabricación real. Tales recursos son incluidos en la Base de Datos del Entorno. Por tanto, antes de que el Usuario cree un Modelo es necesario inicializar la Base de Datos mediante la importación de datos de la planta de fabricación real (datos referentes a Recursos Humanos, Máquinas, Herramientas, Materia Prima y Productos). Para llevarlo a cabo, el Usuario solicita la importación de dichos datos al Entorno de Simulación. Por tanto, el Agente de Gestión de Sincronización dispara la creación de una instancia del agente de Gestión de Importación/Exportación quien traduce los datos a un formato que puede comprender el entorno. Cuando los datos han sido traducidos, el Agente de Gestión de Sincronización dispara la creación de una instancia del agente de Análisis de Datos de Entrada/Salida y del Gestor de Iconos. El agente de Análisis de Datos de Entrada/Salida analiza el contenido de los datos recibidos y se los entrega al Gestor de Iconos quien crea un nuevo elemento acorde al tipo de datos recibidos y los incluye en la Base de Datos. Además, el Usuario puede agregar nuevos recursos en cualquier momento de acuerdo con las necesidades que surjan. Así, la Base de Datos proporciona instancias (operarios, máquinas, herramientas, staff) que serán asociadas a los iconos que integran el modelo creado durante la Fase I (Creación de Modelo). 
El principal objetivo de un Sistema de Fabricación es la fabricación de productos. En consecuencia, la definición de los productos es una tarea clave para la correcta simulación de su fabricación (Figura 8.48).

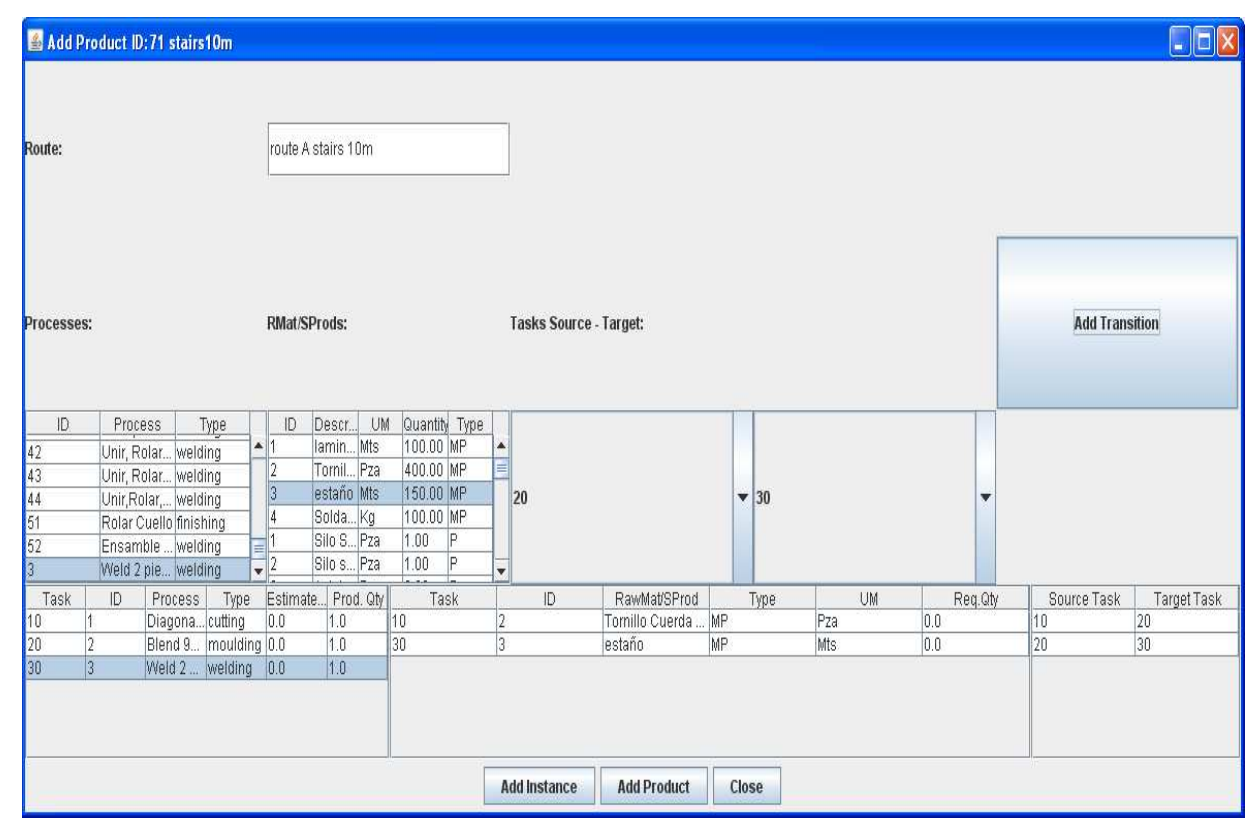

Figura 8.48: Definición de un Producto

De este modo, al crear una instancia de producto se le asocia su ruta(compuesta por tareas) y lista de materiales. Además, mediante la definición de transiciones se indica la secuencia de ejecución de las tareas permitiendo así la definición de tareas que se ejecutan de forma paralela. Asimismo, los materiales se asocian a cada una de las tareas de la ruta. Esta característica aporta una mayor flexibilidad para el diseño de los experimentos.

Las instancias de productos se utilizan cuando el Usuario crea una Orden de Producción durante la Fase II (Simulación de Modelo). De este modo, es posible representar durante la simulación complejos comportamientos que se presentan en un Sistema de Fabricación real. 


\section{Creando un Modelo}

Cuando el Usuario crea un Modelo para un producto específico el Agente de Gestión de Sincronización dispara la creación de una instancia del agente Modelador que muestra al Usuario modelos previos que puede reutilizar. Estos modelos son filtrados de acuerdo al tipo de industria (Metal-mecánica, Química, Alimenticia, Textil) y los Producto asociados previamente a dichos modelos (Figura 8.49). El Usuario puede elegir uno de ellos y reutilizarlo para un producto nuevo. Cuando el Usuario decide reutilizar un modelo previo, el sistema almacena automáticamente la versión original de dicho modelo.

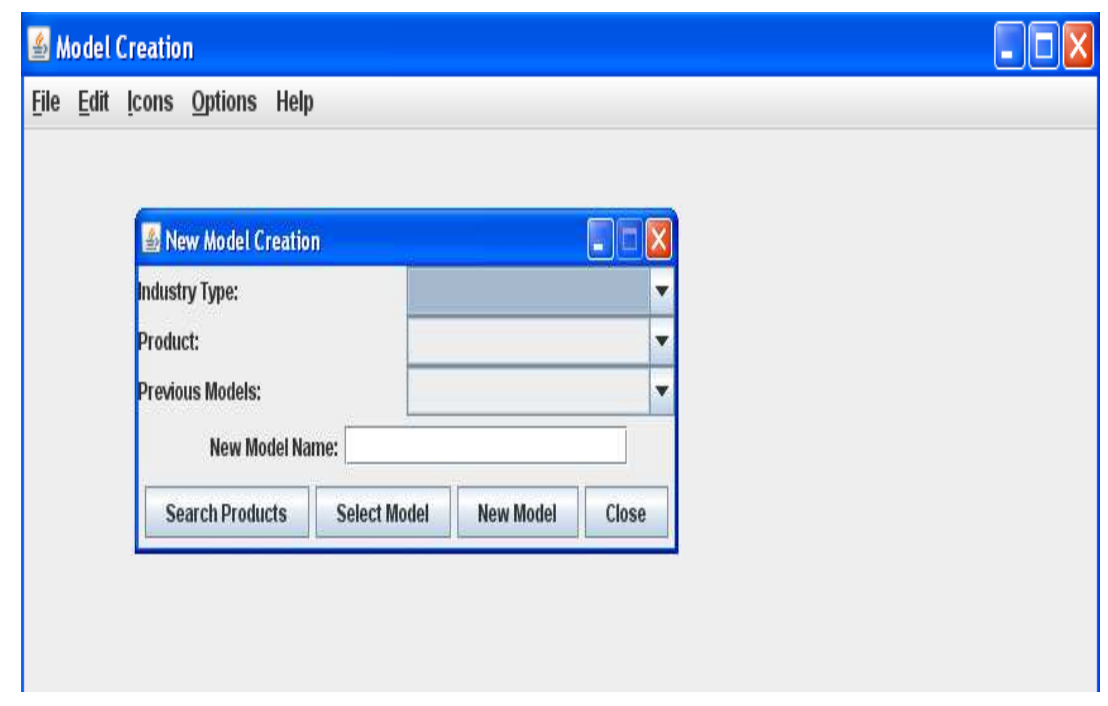

Figura 8.49: Reutilización de Modelos

\section{Representación Gráfica de Elementos}

Durante el modelado los elementos se representan gráficamente mediante iconos específicos ya presentados en la Sección 6.4. Los iconos están agrupados en cuatro categorías, Producto, Recursos, Orden y Staff (Figura 8.50). 


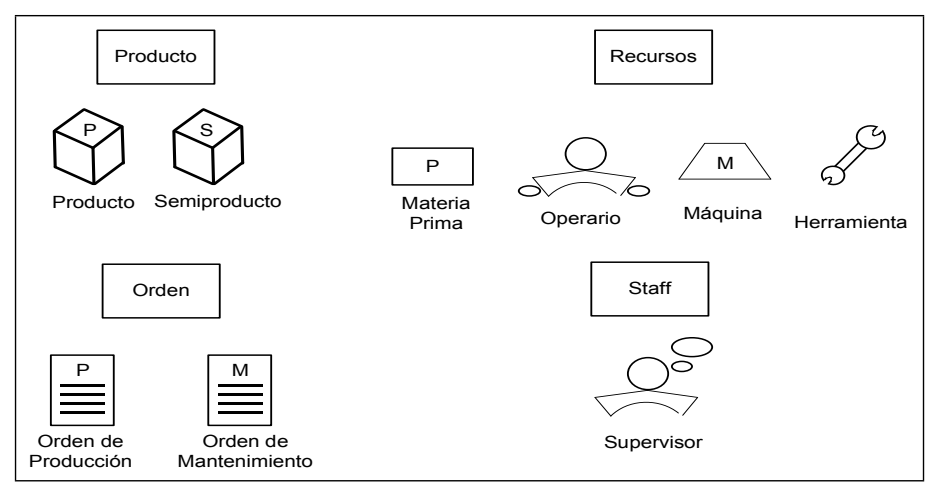

Figura 8.50: Notación Gráfica de Elementos

\section{Incluyendo Escenarios en el Modelo}

Durante la creación/modificación de un Modelo, el Agente Modelador ofrece al Usuario esquemas básicos de acuerdo al tipo de escenario que desea integrar en el modelo (por ejemplo, corte, soldadura, acabados, moldeo, térmico, químico) (Figura 8.51).

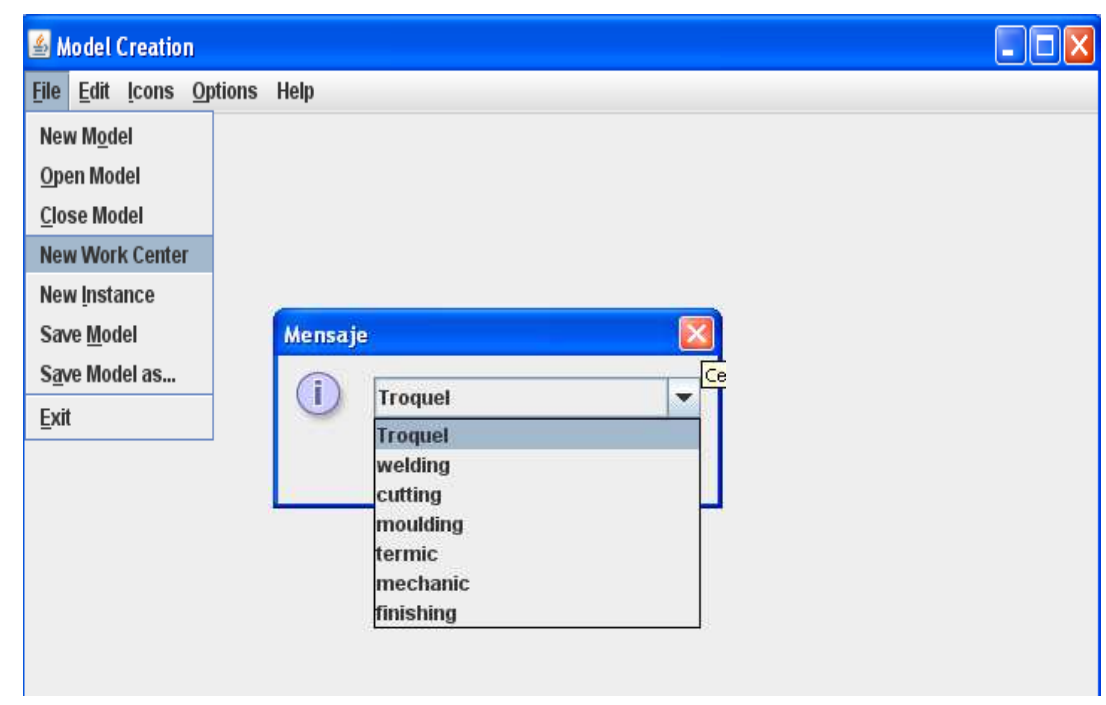

Figura 8.51: Seleccionando esquemas de escenarios

El Agente Modelador propone un esquema que incluye el tipo y cantidad mínima de elementos sugeridos (operador, máquinas y herramientas) para el tipo de escenario seleccionado así como el personal que supervise dichas actividades 
(staff). En la Tabla 8.4 se muestran los elementos sugeridos para los escenarios de corte y soldadura.

\begin{tabular}{|c|c|c|}
\hline \multicolumn{3}{|c|}{ Escenario de Soldadura } \\
\hline \multirow{3}{*}{$\begin{array}{l}\text { Recursos } \\
\text { Humanos }\end{array}$} & Requires Recursos Humanos & Yes \\
\hline & Capacidad Profesional & Soldador \\
\hline & Cantidad & 1 \\
\hline \multirow{3}{*}{ Máquinas } & Requiere Máquinas & No \\
\hline & Actividad & Soldadura \\
\hline & Cantidad & 0 \\
\hline \multirow{3}{*}{ Herramientas } & Requiere Herramientas & $\mathrm{Si}$ \\
\hline & Actividad & Soldadura \\
\hline & Cantidad & 1 \\
\hline \multirow[t]{4}{*}{ Staff } & Requiere Staff & $\mathrm{Si}$ \\
\hline & Capacidad Profesional & Supervisión \\
\hline & Alcance & Soldadura \\
\hline & Cantidad & 1 \\
\hline \multicolumn{3}{|c|}{ Escenario de Corte } \\
\hline \multirow{3}{*}{$\begin{array}{l}\text { Recursos } \\
\text { Humanos }\end{array}$} & RequiereRecursos Humanos & $\mathrm{Si}$ \\
\hline & Capacidad Profesional & Operario \\
\hline & Cantidad & 1 \\
\hline \multirow{3}{*}{ Máquinas } & Requiere Máquinas & $\mathrm{Si}$ \\
\hline & Actividad & Corte \\
\hline & Cantidad & 1 \\
\hline \multirow{3}{*}{ Herramientas } & Requiere Herramientas & No \\
\hline & Actividad & Corte \\
\hline & Cantidad & 0 \\
\hline \multirow[t]{4}{*}{ Staff } & Requiere Staff & $\mathrm{Si}$ \\
\hline & Capacidad Profesional & Supervisión \\
\hline & Alcance & Cut \\
\hline & Cantidad & 1 \\
\hline
\end{tabular}

Tabla 8.4: Esquemas Básicos acorde al tipo de Escenario

Debido a que en todos los Scenarios se requieren entradas y se generan salidas, tanto el elemento de Materia Prima como los Semiproductos se incluyen en los esquemas básicos. De esta forma, el Usuario puede crear un modelo utilizando esquemas iniciales que pueden modificarse incluyendo elementos acorde a sus necesidades. En la Figura 8.52 se muestra un modelo que incluye los escenarios de corte y soldadura de acuerdo a los esquemas sugeridos por el Modelador.

Finalmente el objetivo del Staff es asegurar que los procesos de fabricación controlados por una Orden (Orden de Producción) se cumplen así como asegurar que los Recursos (Recursos Humanos, Máquinas, Herramientas) se utilizan apropiadamente durante estos procesos.

El Staff también se asegura de que las interacciones entre los Holones de Orden y Recursos se cumplan de acuerdo con sus negociaciones previas durante los procesos de fabricación. El objetivo de las negociación es la optimización 
del uso de los Recursos durante una Tarea de acuerdo con las restricciones de la Orden (capacidad vs. tiempo y calidad).

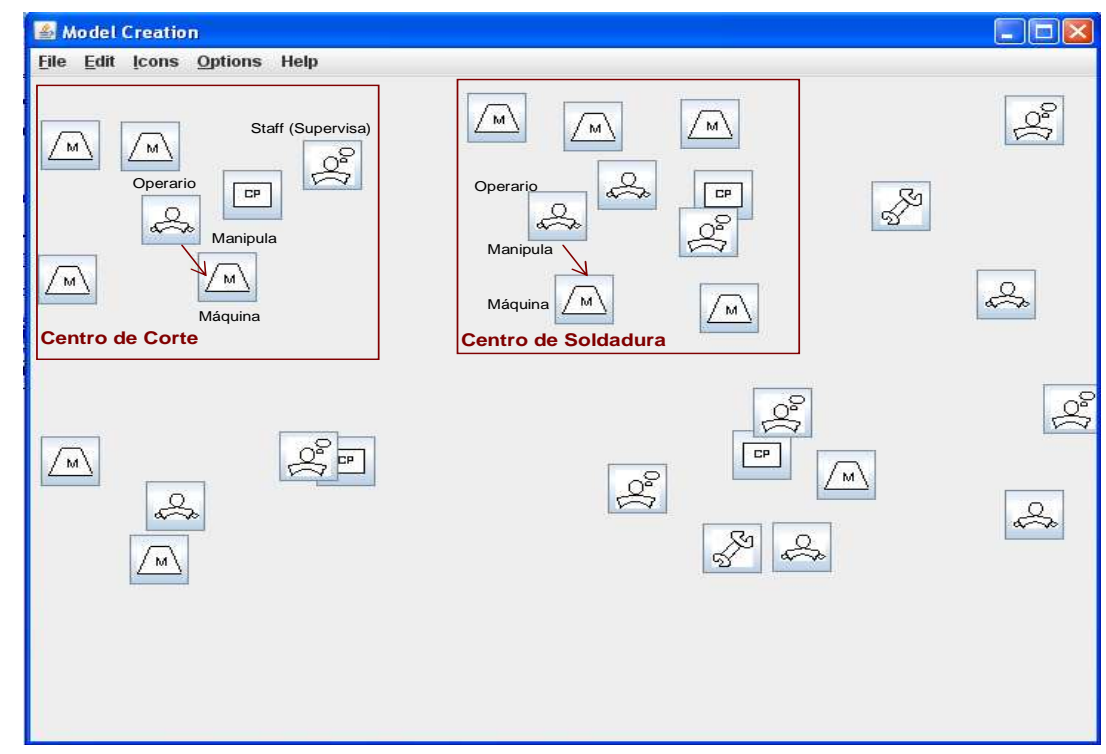

Figura 8.52: Escenarios en un Modelo

\section{Asignando Recursos en un Escenario}

Con respecto a las actuales propuestas para el diseño de un modelo en la herramienta de simulación se indican las instancias específicas de los recursos asignados en los escenarios con sus respectivos alcances y limitaciones. El Agente Modelador permite utilizar los recursos físicos disponibles. El Modelador ofrece solo aquellos recursos que pueden ejecutar la actividad indicada en el escenario (por ejemplo, Recurso: Máquina - Actividad: Corte ó Recurso:Herramienta Kostler - Actividad:Soldadura) (Figura 8.53). Entonces, el Usuario puede elegir un recurso específico de operarios, máquinas, herramientas y supervisores disponibles. El Usuario puede agregar nuevos Recursos si no quiere utilizar los disponibles. Cuando el Usuario solicita la creación de un nuevo recurso, el Agente de Gestión de Sincronización dispara la creación de una instancia del agente de Gestión de Iconos quien ayuda al Usuario para crear un recurso adecuadamente. 


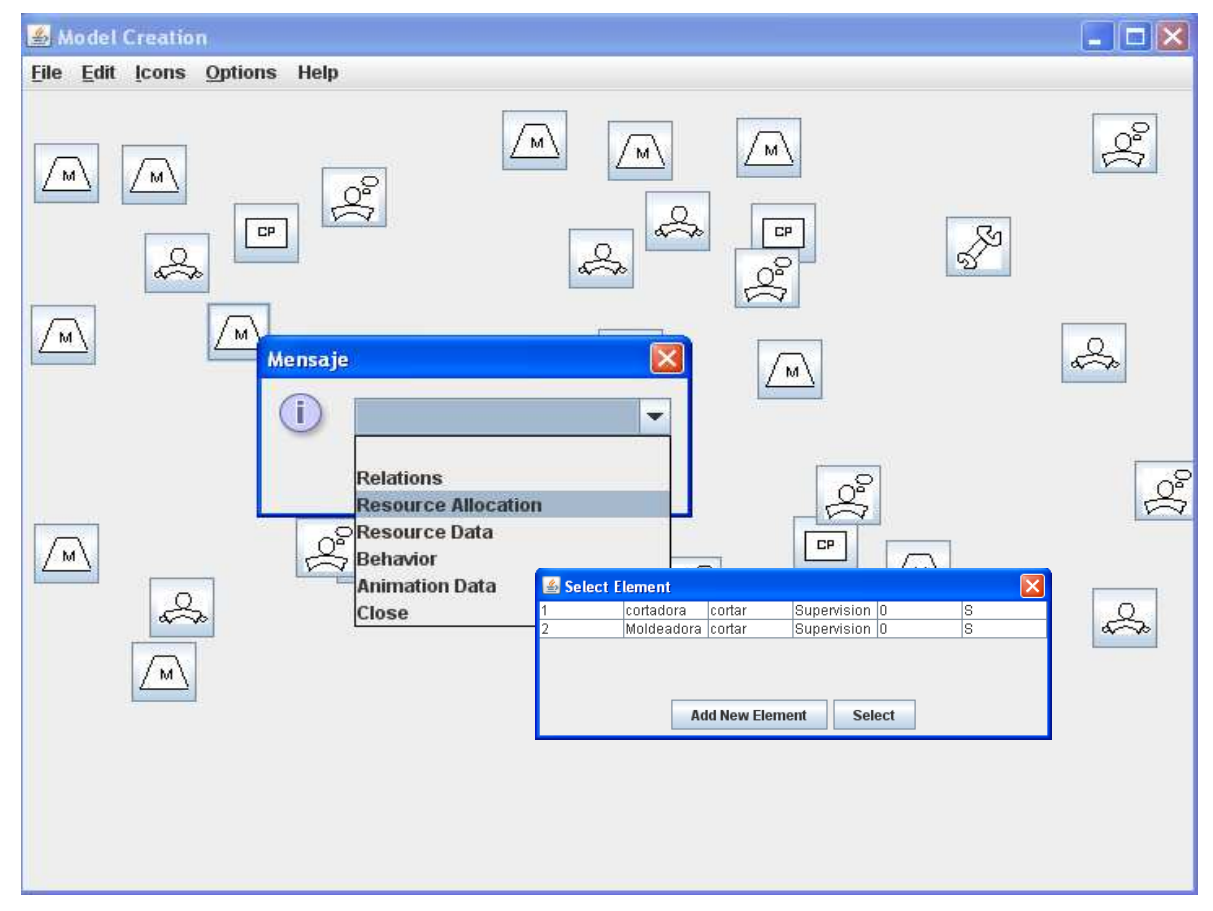

Figura 8.53: Asignación de Recursos en el Escenario de Corte

\section{Comportamiento de los Recursos}

Los elementos incluidos en el Modelo representan Agentes que tienen comportamientos específicos acorde a su tipo para ejecutar sus tareas. Los agentes que integran el modelo ofrecen servicios básicos acorde a su tipo y configuración de sus atributos. Sin embargo, el Usuario puede agregar comportamientos a fin de adaptarlos a sus necesidades. Cuando el Usuario agrega un nuevo comportamiento, debe definir algunos atributos clave tales como su tipo (Síncrono/Asíncrono), su nombre, y el código que describe su función. El Usuario debe utilizar el lenguaje Java para la edición del código (Ver Fig. 8.54).

Así, una vez que el Usuario termina un Modelo, el agente Modelador juega el rol de Verificador de Modelo quien verifica e informa al Usuario hasta que el modelo no tiene errores. Cuando el Modelo esta listo, el agente Modelador informa al Agente de Gestión de Sincronización quien inicia la Fase II (Simulación del Modelo). En la Fase II los elementos definidos en el modelo como iconos se convierten en Agentes inteligentes que interactúan para producir un Producto guiados por una Orden de Producción. 


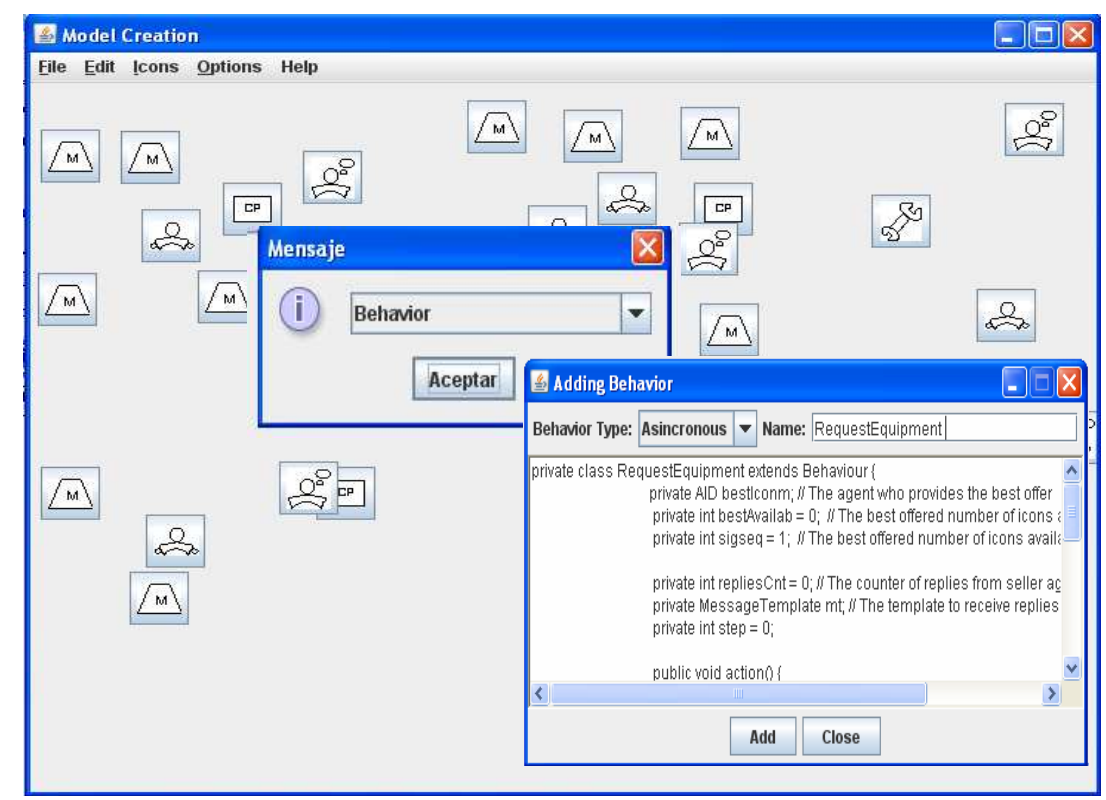

Figura 8.54: Definiendo el Comportamiento de un Recurso

\section{Verificación del Modelo}

Durante la creación del modelo se verifica que se cumplan las reglas del modelado de organización básicas planteadas en la Sección 5.8.2. Cuando el modelo se cierra el Modelador informa al Usuario sobre los errores detectados, tales como que una máquina que requiere ser manipulada no tiene asociado un operario, o que un icono no tiene asociada la instancia correspondiente, etc.

\subsubsection{Fase II- Simulación de Modelo}

Para la Simulación del Modelo, siguiendo los escenarios descritos en secciones anteriores, en esta sección se presentan las principales interfaces asociados a cada uno de los escenarios que se presentan durante la Fase II (Simulación del Modelo).

\section{Apertura del Modelo}

El modelo a simular se selecciona mediante la definición de tipo de industria, producto, y el listado de modelos asociados a dicho producto. 
En la Figura 8.55 se presenta la interfaz para la apertura y la representación gráfica del modelo seleccionado.

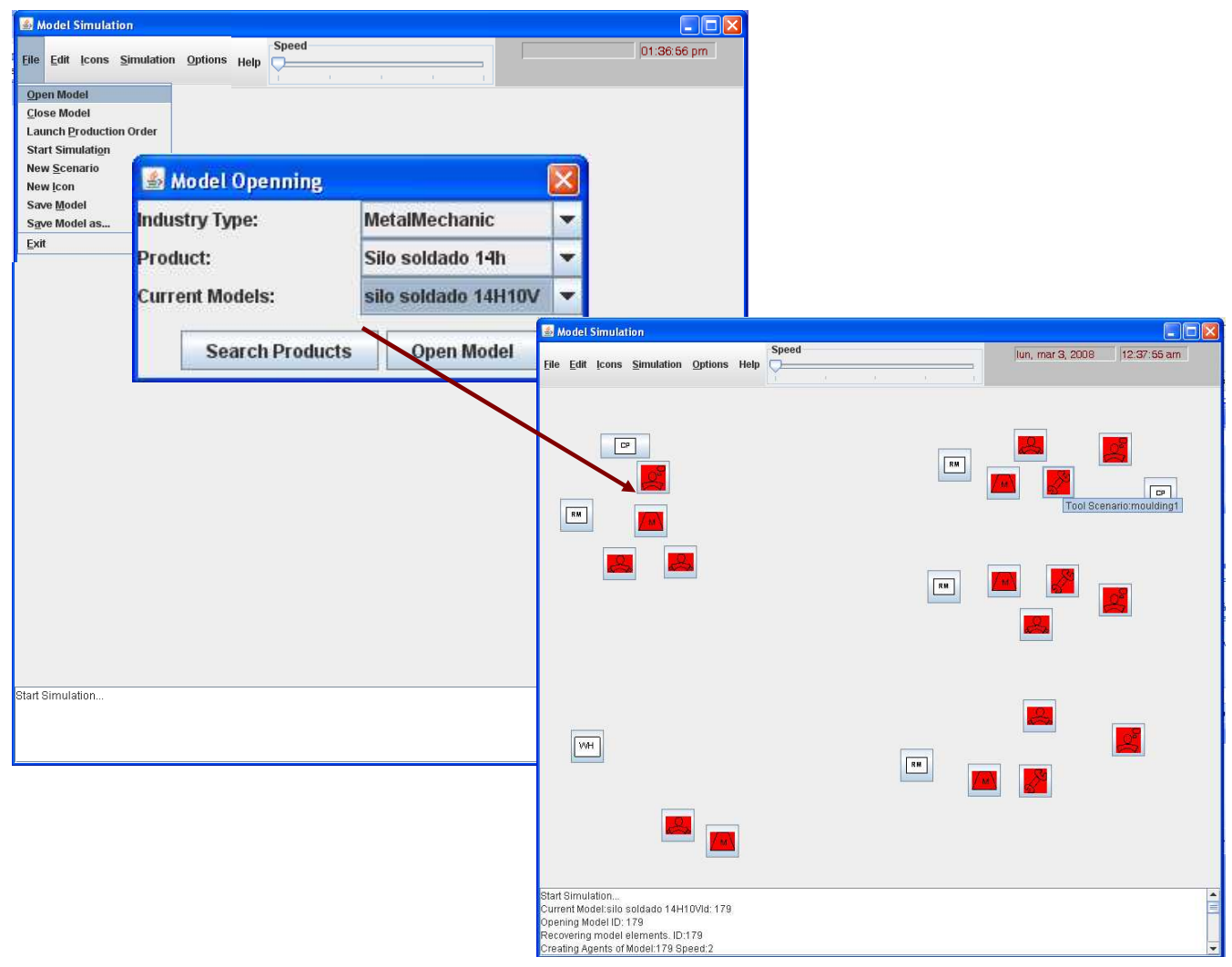

Figura 8.55: Interfaz de Apertura de Modelo y su representación gráfica

\section{Definir Condición de Parada y Velocidad}

Antes de lanzar las órdenes de producción, es necesario definir la condición de parada y la velocidad de ejecución de la simulación de los comportamientos del Sistema de Fabricación. La Figura 8.56 muestra la interfaz para la definición de la condición de parada la cual puede ser basada en una fecha y hora específica a partir del momento en que se lanza la simulación, o cuando finalice una orden específica. Aunque al principio sólo se puede definir la condición a una fecha/hora específico, la condición de parada puede modificarse durante la simulación. La opción de una orden de producción, sólo se permite seleccionar cuando al menos una orden ya ha sido lanzada a ejecución. 


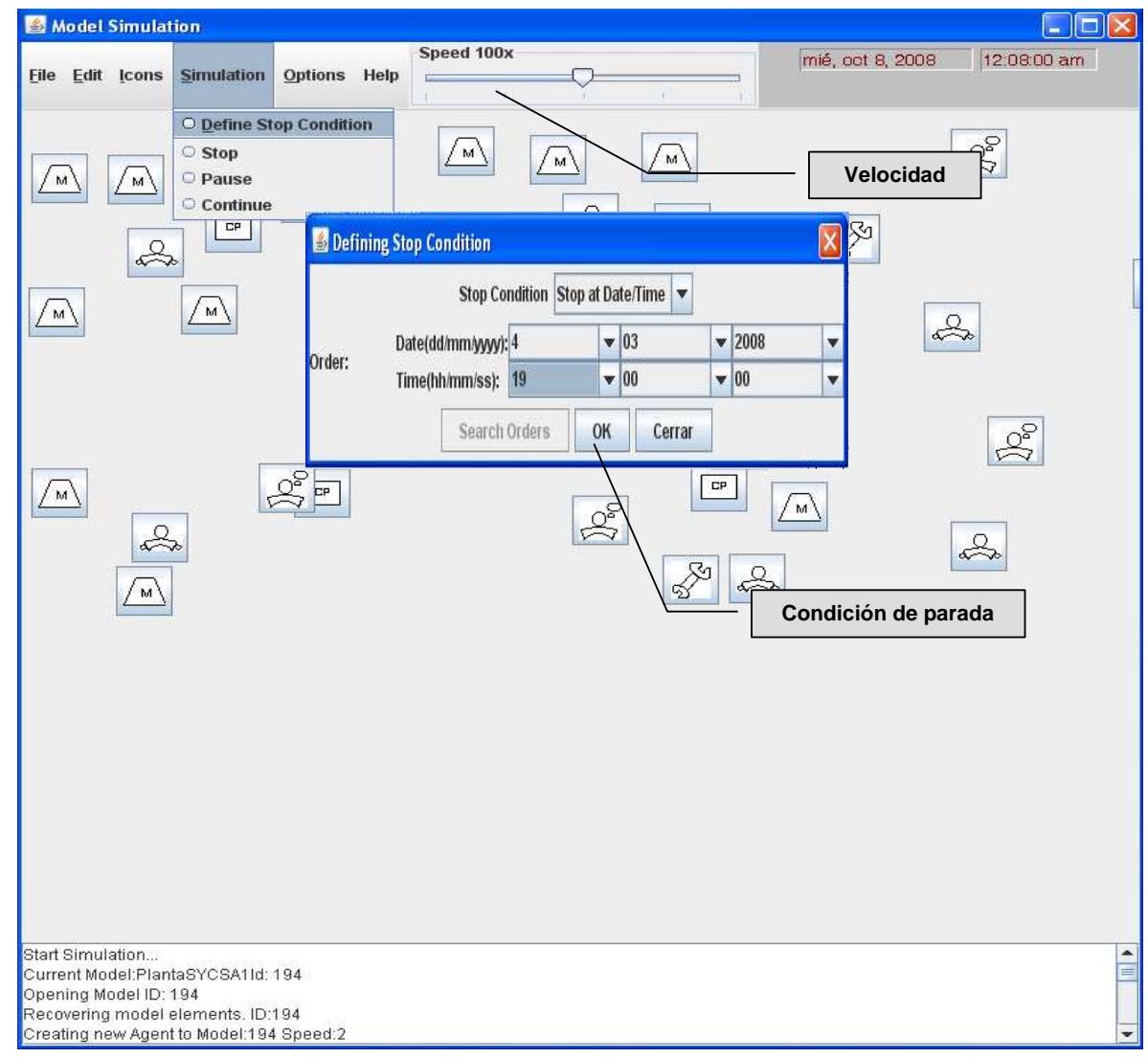

Figura 8.56: Interfaz de Definición de Condición de Parada y Velocidad

La posibilidad de ajustar la velocidad de ejecución de la simulación es un aspecto relevante para la obtención de resultados de procesos generalmente largos en el tiempo. El ajuste de velocidad permite ejecutar procesos en un tiempo reducido igualando el tiempo requerido al tiempo simulado. La Figura 8.56 muestra la interfaz utilizada por el Usuario para el ajuste de velocidad en cualquier momento durante la simulación.

\section{Lanzar Orden de Producción}

Al lanzar una Orden de Producción, sólo se permite seleccionar aquellos productos que han sido asociados previamente al Modelo abierto. Una vez seleccionado el producto, se elige la ruta que se utilizará, esto es, se pueden haber definido más de una ruta alternativa para la fabricación del producto. A continuación se define la cantidad de productos requeridos, la fecha en que se requiere la 
entrega del mismo y la prioridad de la orden (alta, media, baja). Esta prioridad es utilizada para fines de reserva de material y reserva de capacidad para la asignación de trabajos a recursos.

Al seleccionar la ruta, aparece el listado de las operaciones (o tareas) que la integran. Para efectos de validar diferentes configuraciones, se requiere que el Usuario defina qué recursos del modelo serán los que ejecuten cada una de las operaciones. De esta forma, cuando el Usuario selecciona cada una de las operaciones, en el apartado de "Recursos Disponibles" (Available Resources) aparecen aquellos recursos que por su naturaleza pueden ser seleccionados para llevar a cabo dicha operación. Una vez que los ha seleccionado, tal información queda vinculada a la orden para sus posteriores procesos de negociación y asignación de tareas.

La Figura 8.57 muestra la interfaz para lanzar una nueva orden de producción y su representación gráfica en el Modelo.

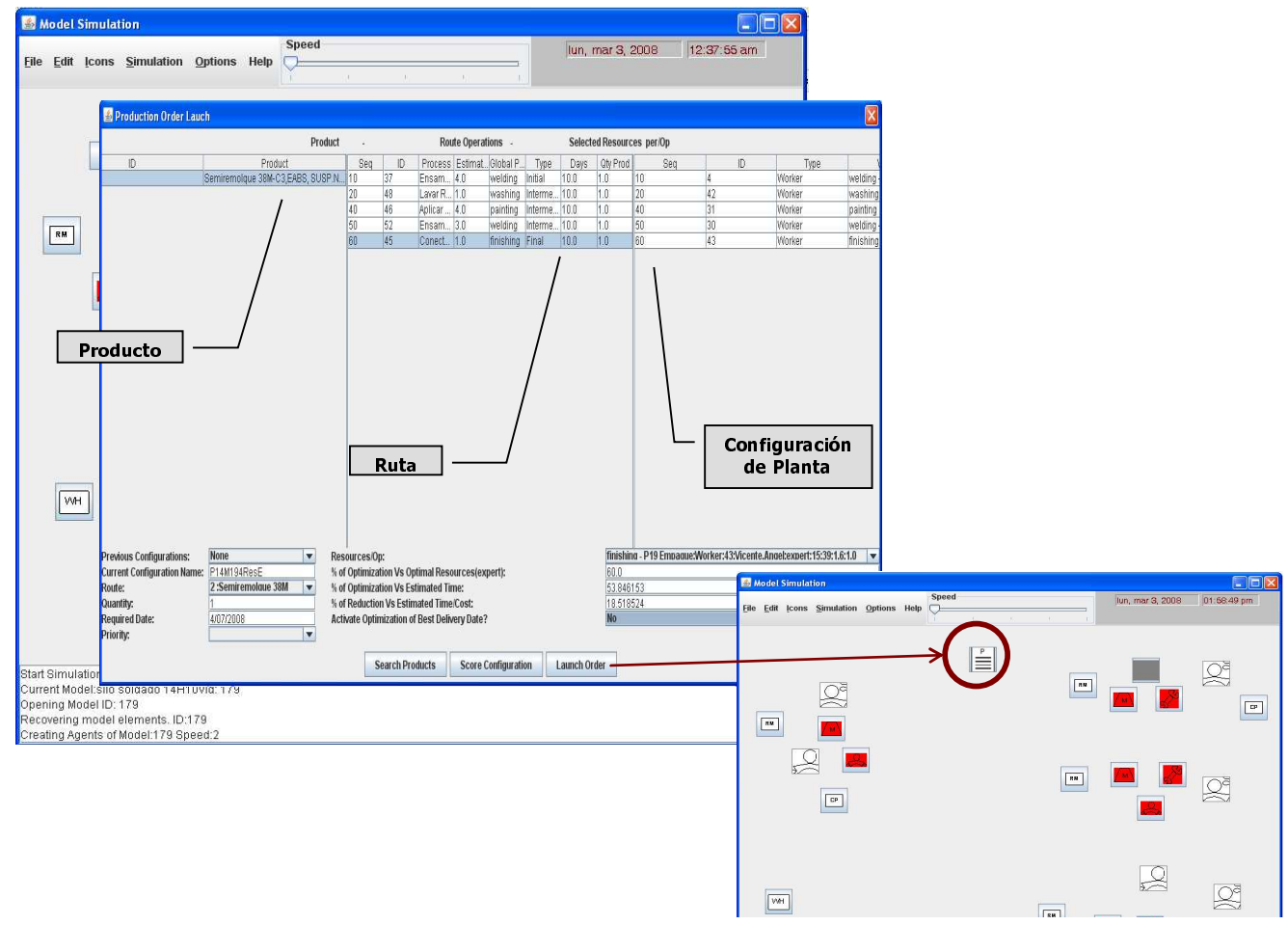

Figura 8.57: Interfaz de Lanzamiento de Orden de Producción y su representación gráfica 
Durante el lanzamiento de la Orden si el Usuario lo solicita, es posible llevar a cabo la evaluación previa de la configuración definida antes de que la orden inicie sus tareas. Para ello, el entorno ofrece cuatro diferentes alternativas: a) evaluar la ruta calificándola en base al tipo de elementos que integra su configuración, b) evaluar el coste de dicha configuración, c) evaluar el tiempo de procesamiento previsto con respecto al tiempo estándar y d) evaluar la configuración en base a una fórmula definida por el Usuario que integre los tres elementos anteriores.

\section{Trazabilidad durante la Simulación}

Durante la simulación es posible rastrear el comportamiento de cada uno de los de elementos del modelo. De este modo, se observan los datos del elemento y su estado actual. Por tanto, se pueden observar: a) cuando un recurso (staff, operario, máquina o herramienta) esta trabando, negociando o detenido, b) la operación actual y el estado de un semiproducto, c) la configuración de animación actual de cualquier elemento y d) el progreso de la producción de una orden.

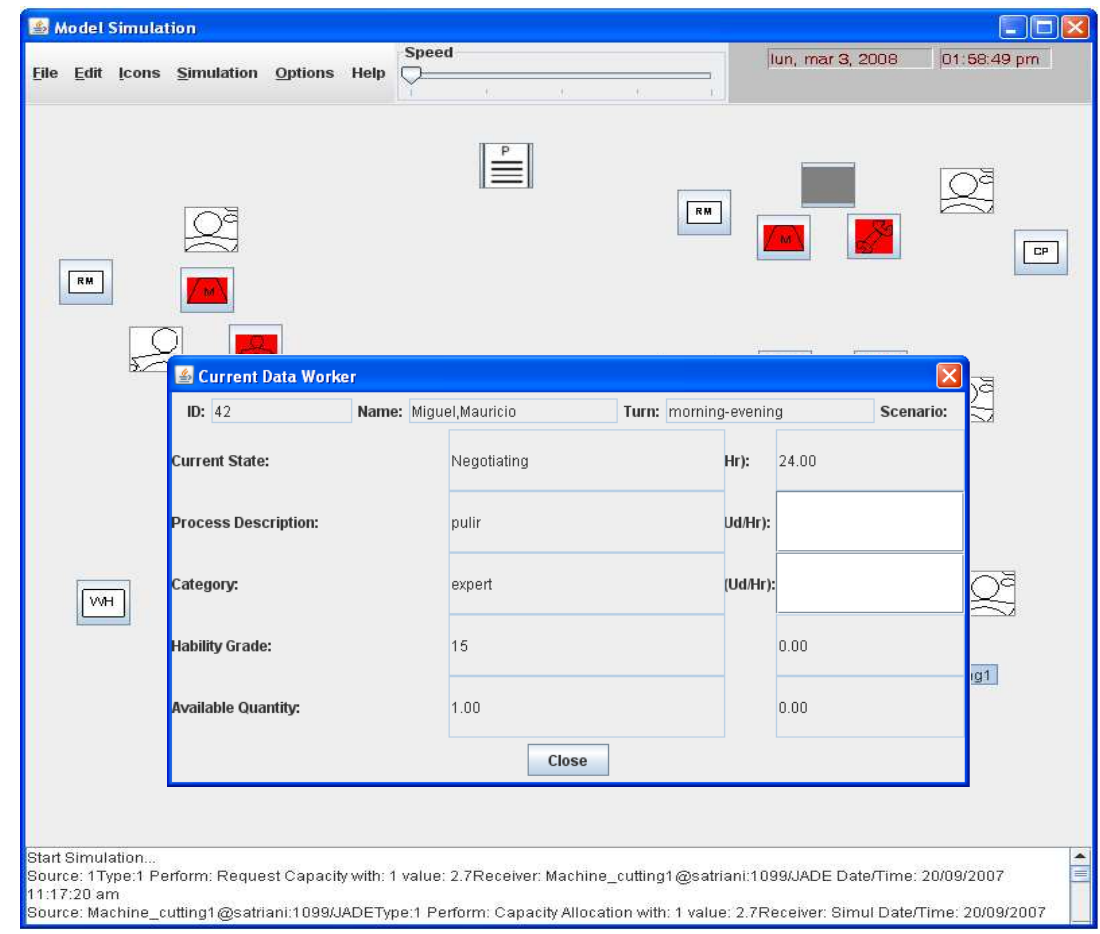

Figura 8.58: Interfaz para la Visualización de Datos de un Recurso 
Asimismo, para los recursos es posible observar sus datos que incluyen: centro de trabajo, capacidad, grado de destreza y su estado actual. La Figura 8.58 muestra la interfaz para visualizar los datos y el estado actual de un operario.

Con respecto a la animación, es posible observar y cambiar la configuración de la animación de cualquier elemento. El tipo de animación puede ser cambio de color y movimiento. Así, el tipo de animación esta relacionado con tres estados principales: detenido, negociando y trabajando. La Figura 8.59 muestra la interfaz para definir el tipo de animación de un operario.

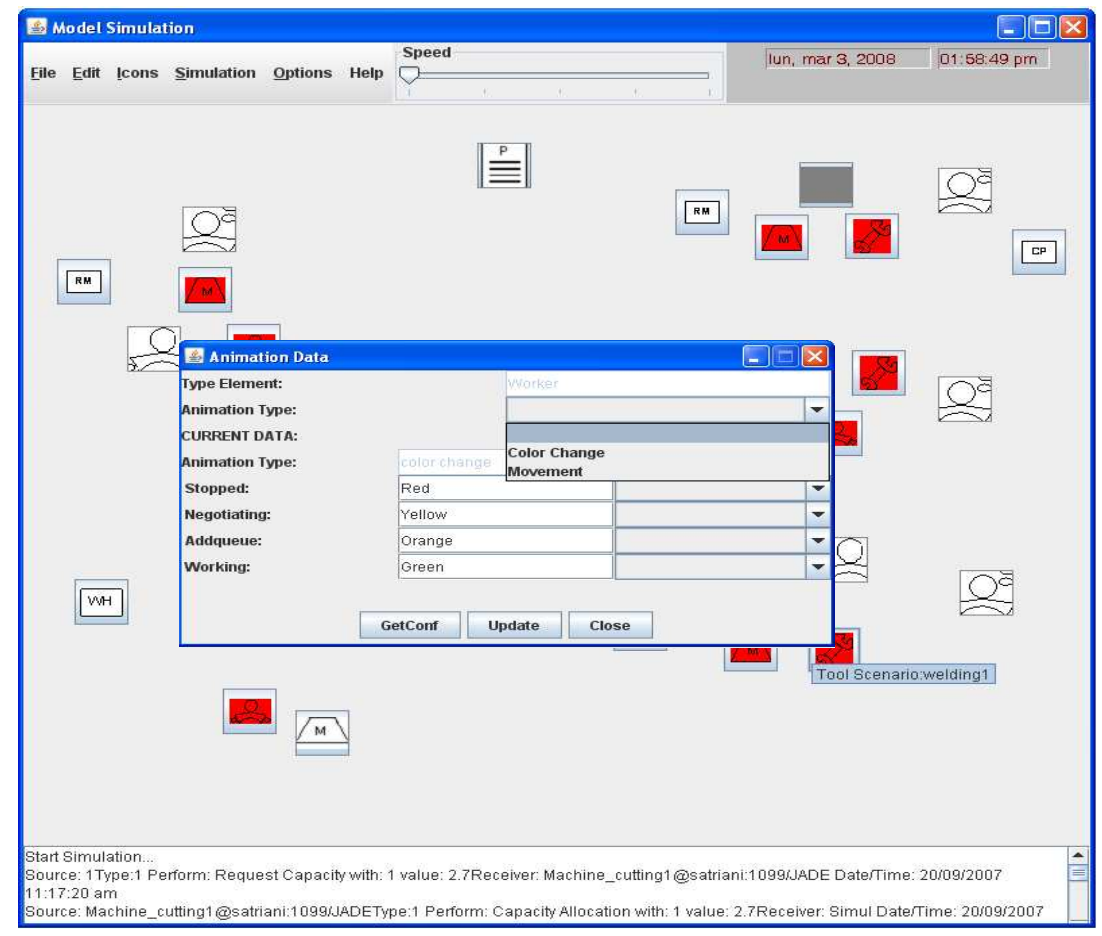

Figura 8.59: Interfaz para la Definición del Tipo de Animación de un Operario

Después de que se crea una orden de producción es posible observar su progreso. De este modo, se observa qué recurso está atendiendo una operación y su estado (en cola, en proceso o terminada). La Figura 8.60 muestra la interfaz para visualizar el progreso de una Orden de Producción. 


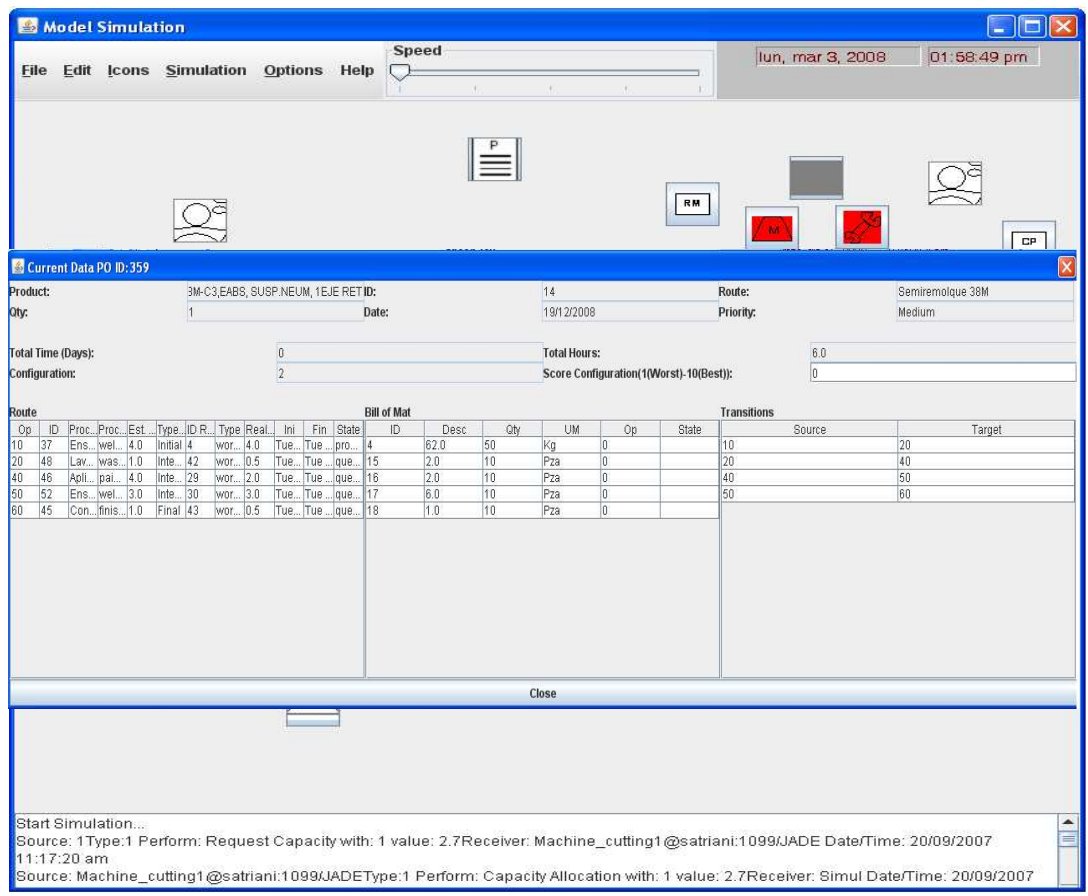

Figura 8.60: Interfaz para la Visualización del Progreso de una Orden de Producción

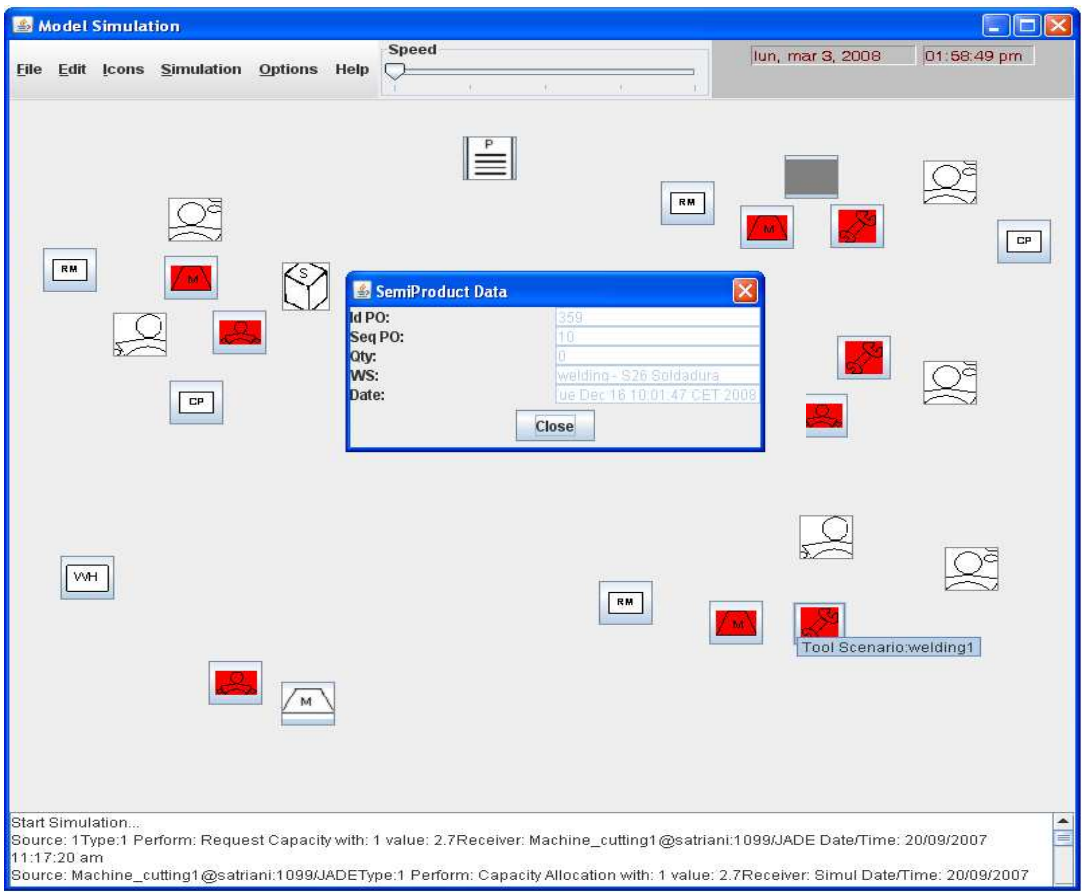

Figura 8.61: Interfaz de Visualización de Datos de un Semiproducto 
Hasta que se obtiene un producto final, durante la simulación es posible rastrear su progreso mediante el icono de un Semiproducto el cual está asociado a éste. El Semiproducto indica la ruta de operación actual y su estado. La Figura 8.61 muestra la interfaz para visualizar el estado actual de un Semiproducto.

\section{Finalización de una Orden durante la Simulación}

El Agente Simulador informa al Usuario cuando una Orden de Producción se termina. Entonces, el Usuario le asigna una calificación al comportamiento de la planta para la orden terminada que ha utilizado una configuración de la planta específica. La calificación se encuentra entre 0 (no aceptable) y 10 (aceptable).

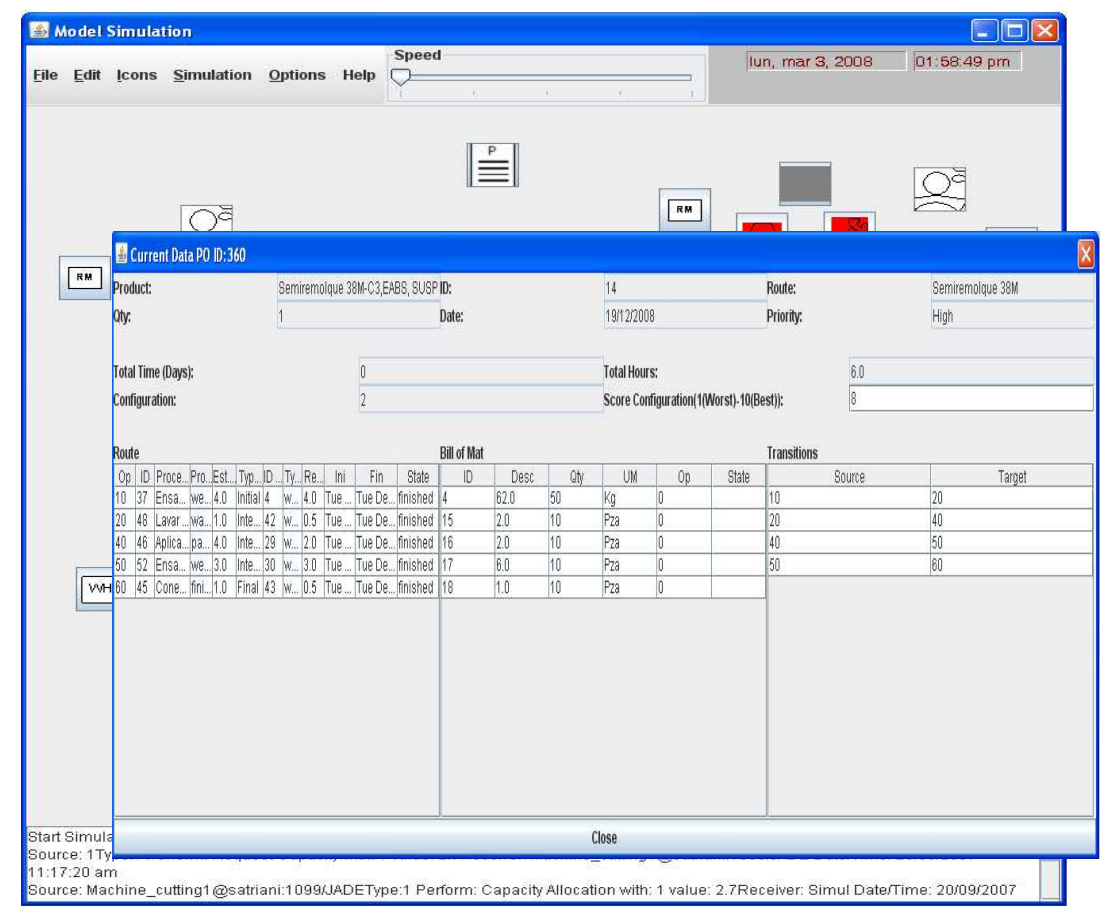

Figura 8.62: Interfaz de Visualización de finalización y calificación de Orden de Producción

La Figura 8.62 muestra la interfaz que informa al Usuario cuando una orden se termina y que se utiliza para calificarla.

Asimismo, cuando una Orden de Producción se termina, el Agente de Orden informa al Agente de Simulación que agrega un nuevo icono de Producto en la Planta. El icono de Producto indica que un Producto Final se ha terminado. 
Además, el Agente de Simulación actualiza la visualización gráfica del icono de la Orden de Producción indicando que ha sido terminada. La Figura 8.63 muestra la interfaz utilizada para observar los datos de un Producto Final. Los datos indican fecha y hora de terminación.

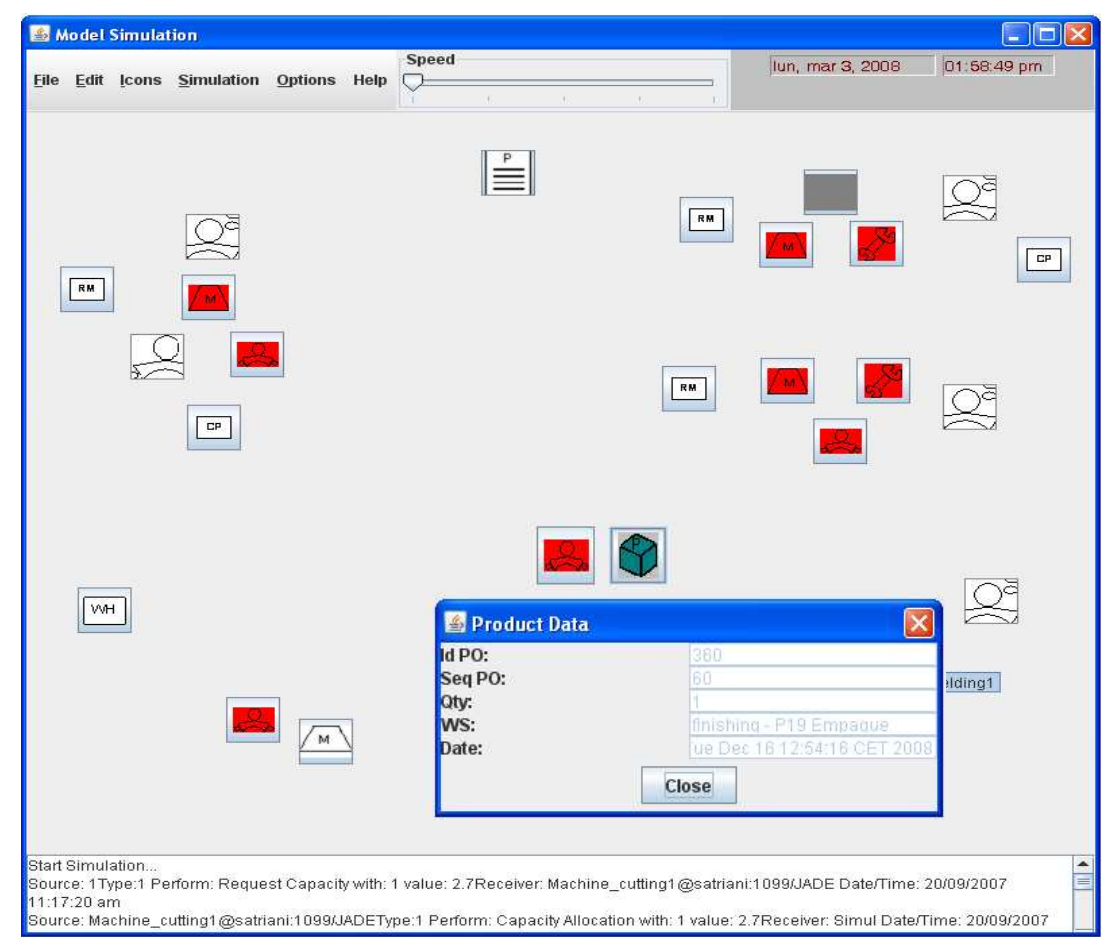

Figura 8.63: Interfaz de Visualización de Datos de Producto Final

\section{Representación Gráfica durante la Simulación}

Durante la Simulación se representan gráficamente los estados de los agentes que integran el Sistema de Fabricación (ofreciendo servicios, negociando, colocando trabajos en cola, y procesando trabajos). Las Figuras 8.64 y 8.65 muestran las diferentes representaciones gráficas de los estados de los agentes durante la simulación. 


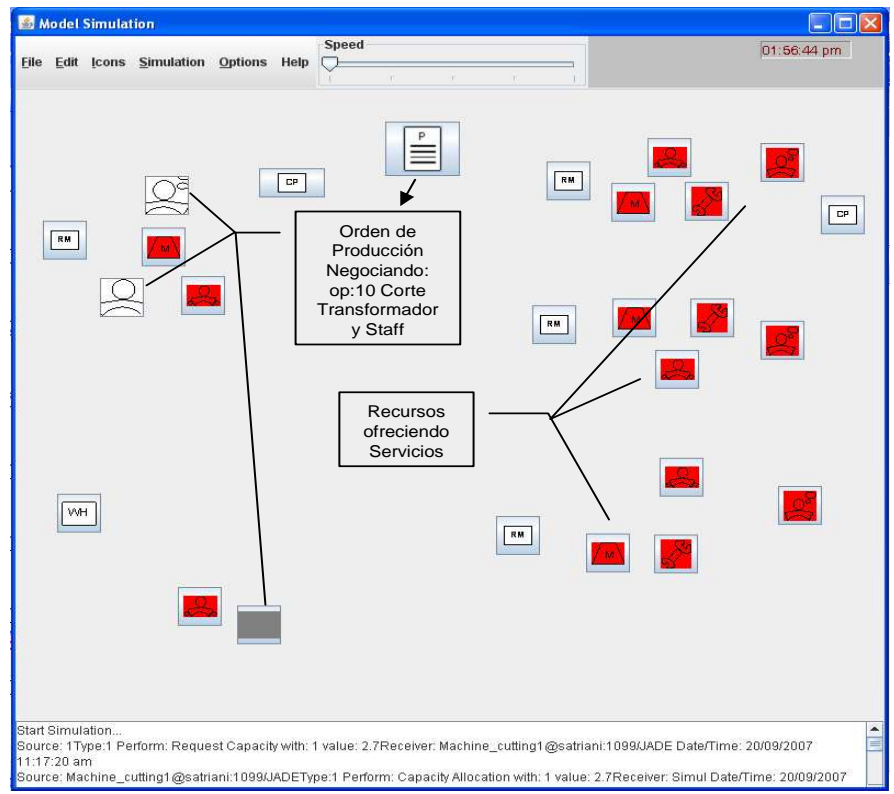

Figura 8.64: Agentes de Escenarios de Corte Negociando op 10 con la Orden de Producción

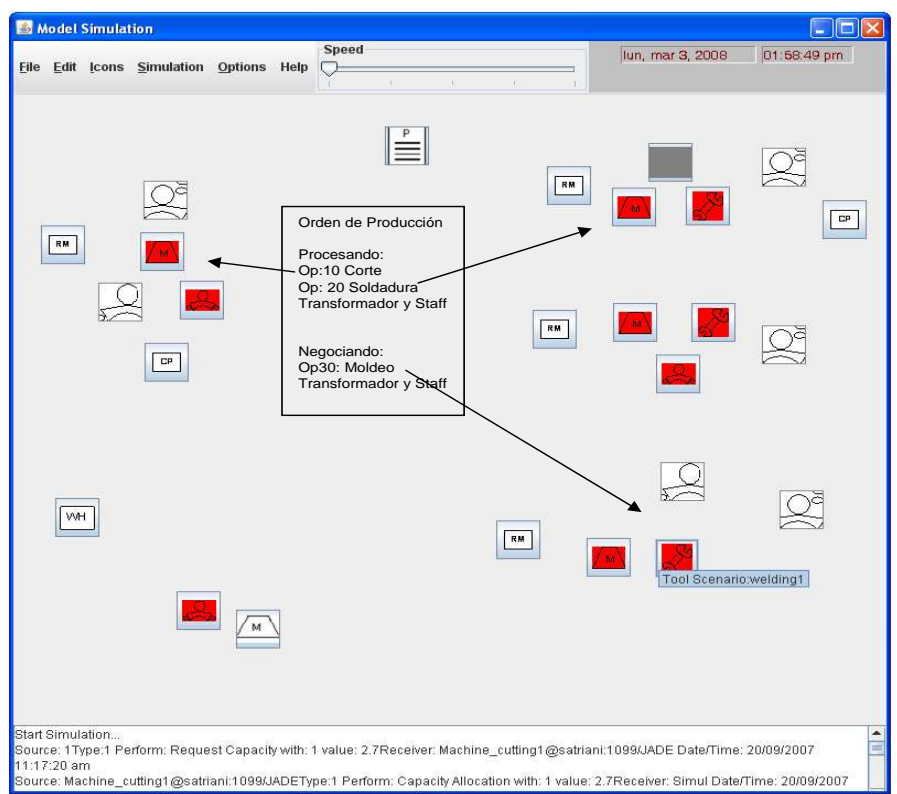

Figura 8.65: Agentes de Corte procesando op 10, Agentes de Soldadura procesando op 20 y Agentes de Moldeo negociando op 30 con la Orden de Producción 


\section{Mostrar Reportes y Gráficas}

Durante la simulación o al finalizarla, es posible observar el comportamiento de los elementos que integran el modelo mediante Reportes y Gráficas. La Figura 8.66 muestra la interfaz ofrecida y algunos de los reportes y gráficas que se pueden obtener.

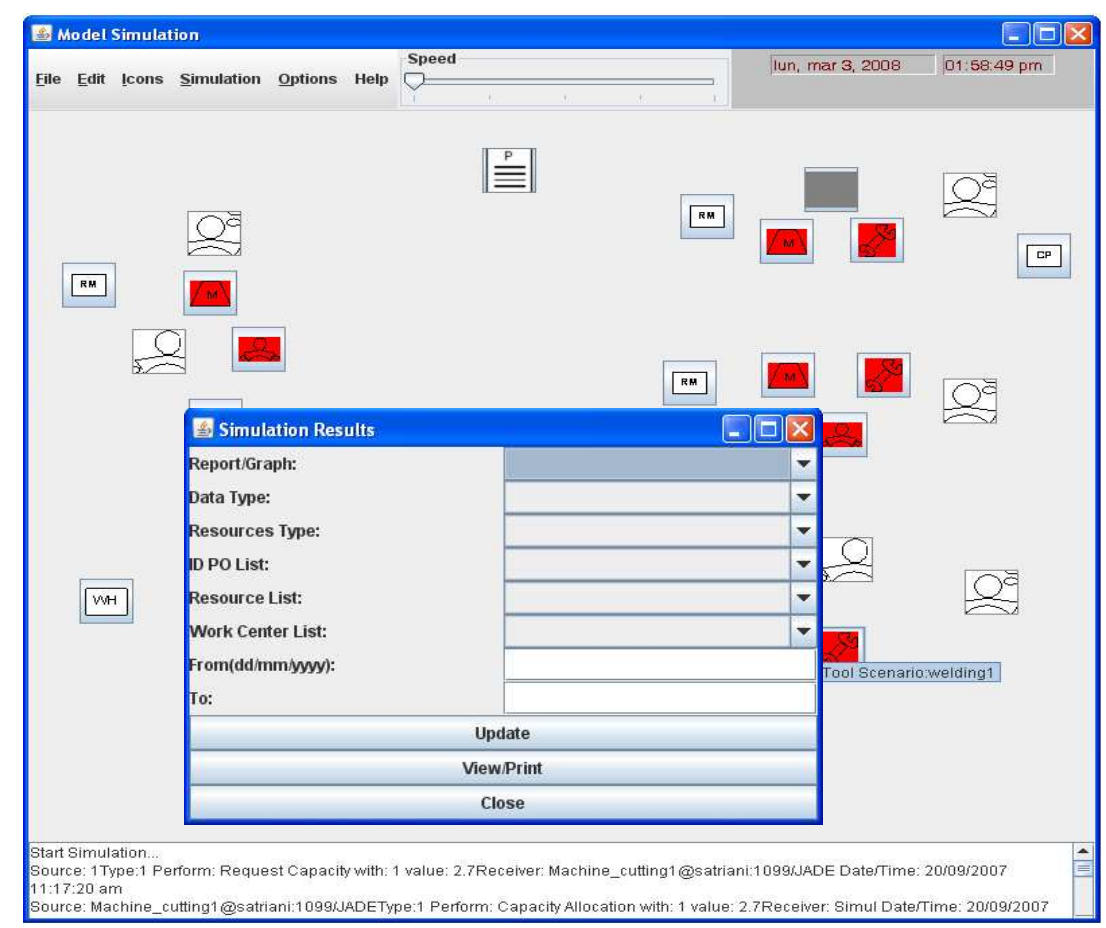

Figura 8.66: Interfaz para la solicitud de Reportes/Gráficas

\section{Control de la Simulación}

Como se indicó en la Arquitectura del Entorno de Simulación (Sección 7.2.1) se considera el Control de la Simulación en dos sentidos: la definición de la condición de parada y detener la simulación arbitrariamente.

En cuanto a la condición de parada, se ofrecen dos alternativas para detener la simulación: a) la simulación se detiene al finalizar una orden de producción específica, y b) la simulación se detiene en una fecha y hora específica. 
Por otra parte, además de incluir tal condición, el ASimulador/Controlador de Eventos permite al Usuario detener la simulación en cualquier momento bajo la responsabilidad del Usuario con respecto a las órdenes que aún no han sido terminadas.

En cuanto a detener la simulación de forma arbitraria, es posible que independientemente de la condición de parada establecida, el Usuario decida detener, pausar, o continuar con la simulación en cualquier momento, de tal manera que la herramienta incluyen tales opciones. La Figura 8.67 muestra la interfaz utilizada para tales efectos y el comportamiento de los agentes al detener la simulación.

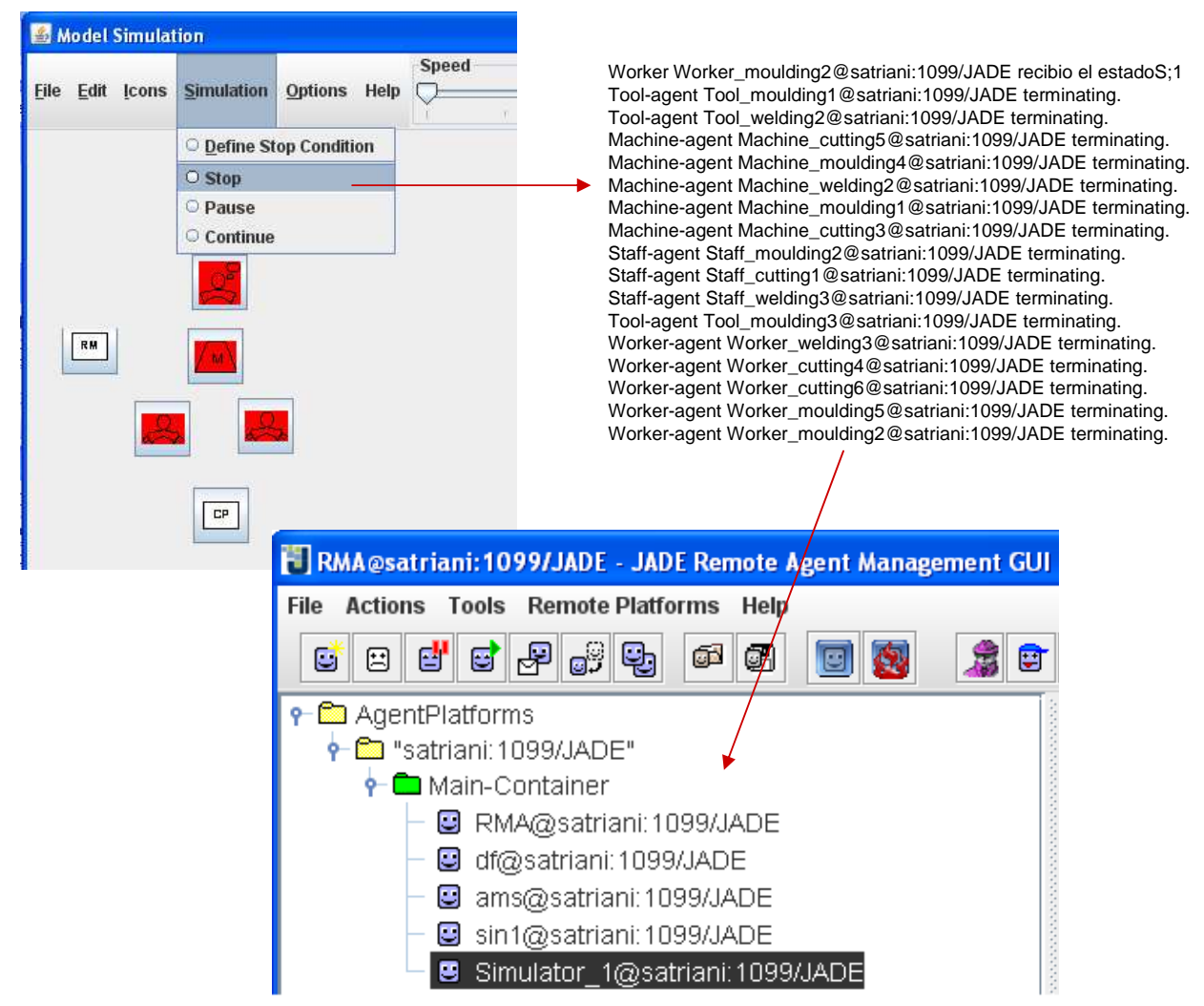

Figura 8.67: Interfaz de Control de Ejecución de Simulación y comportamiento de Agentes al detenerla 


\subsection{Ontología}

La Ontología permite la definición del lenguaje utilizado para la comunicación durante las interacciones entre los Agentes que integran el Entorno de Simulación y el Modelo del Sistema de Fabricación en las dos Fases del proceso de Simulación. A continuación se muestran ejemplos de las clases creadas mediante PROTEGÉ (PROTEGE 2005) para la definición de la Ontología (Figuras 8.68 y 8.69$)$.

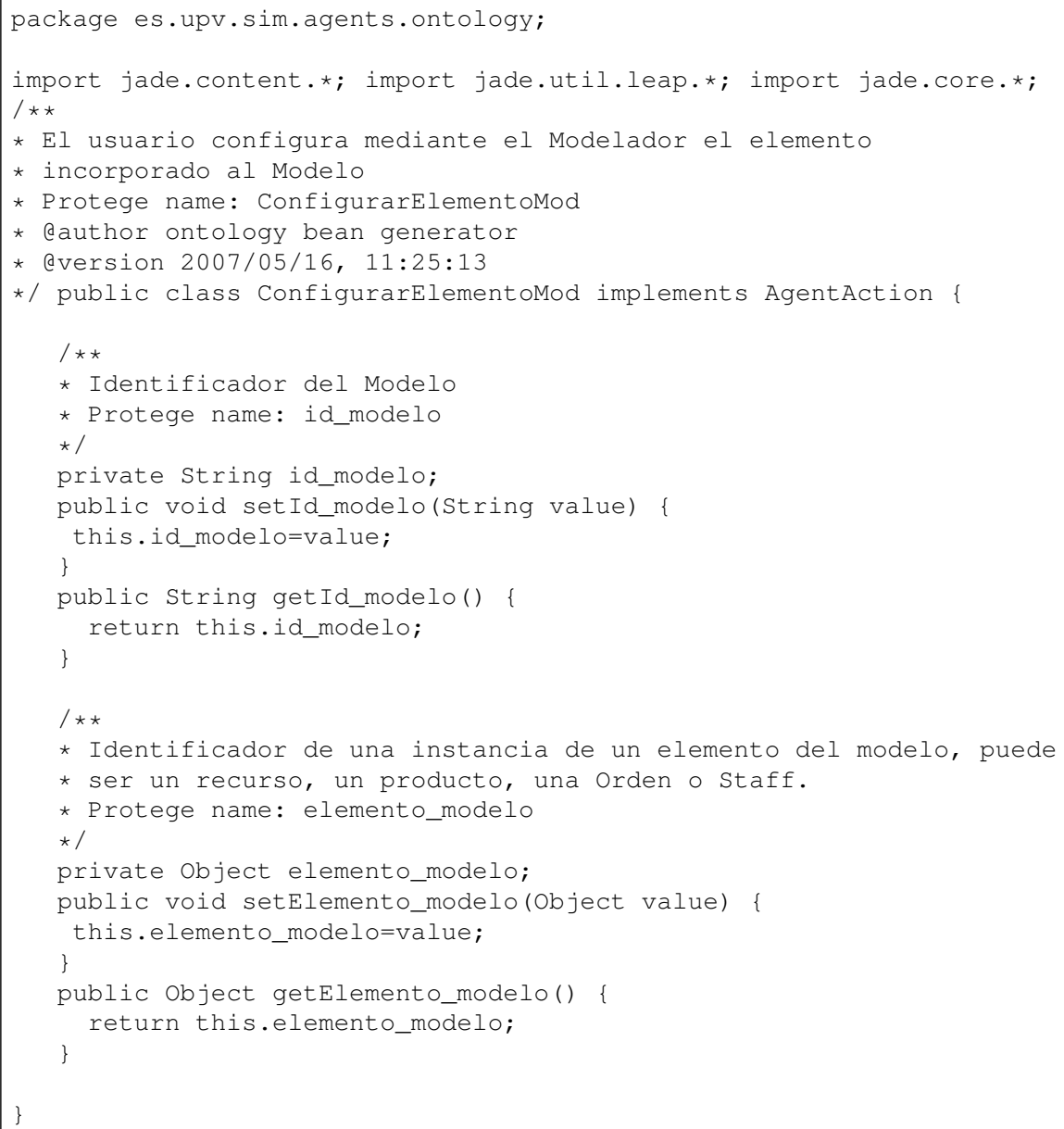

Figura 8.68: ConfigurarElementoMod 


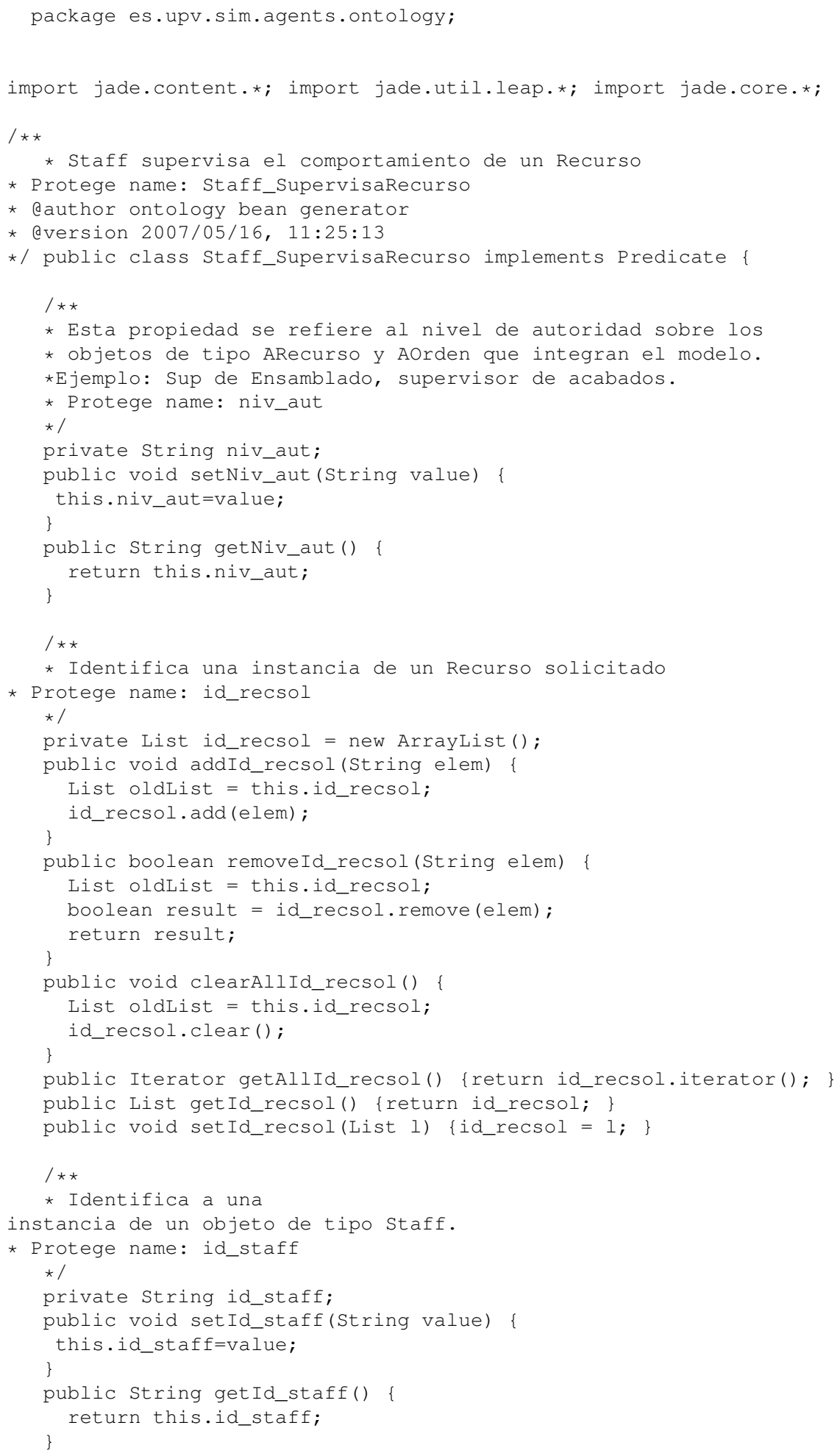

Figura 8.69: ConfigurarElementoMod 


\subsection{Conclusiones}

En este capítulo se ha presentado la documentación que da soporte a la implementación del prototipo que permitirá validar tanto la Arquitectura del Entorno de Simulación como el Metamodelo para Sistemas de Fabricación. Para ello, se aplicó la metodología propuesta en ANEMONA. Asimismo, se ha presentado la tecnología seleccionada para la implementación del prototipo. Como resultado se han presentado las interfaces más representativas del prototipo desarrollado que permiten dar seguimiento al proceso completo de simulación y la interacción entre el Entorno de Simulación y el Usuario para la creación y simulación de Modelos. En el Capítulo 9 se presentan las métricas que se aplicarán para la evaluación del prototipo así como los resultados de la evaluación global de la implementación. 



\section{Capítulo 9}

\section{Evaluación}

En este capítulo se presentan las bases para la evaluación de la Arquitectura del Entorno mediante el uso del prototipo desarrollado. Asimismo, se presentan las bases para la evaluación global de la implementación del prototipo y los resultados que genera.

En este capítulo consideramos la evaluación en tres vertientes, la evaluación de la implementación del prototipo de software para lo cual se aplicarán técnicas de Ingeniería de Software, la evaluación de la funcionalidad del entorno y la evaluación de los resultados obtenidos mediante su aplicación en un caso de estudio que incluye la evaluación de los modelos, las configuraciones y los resultados asociados a reportes de negocio (medición de uso de recursos, tiempos muertos, etc.).

\subsection{Evaluación del código del prototipo del Entorno de Simulación}

El objetivo de este trabajo es plantear la integración del paradigma de Sistemas Multiagente en un entorno de simulación para sistemas de fabricación, resaltando las ventajas de dicho paradigma ante los requisitos de la nueva era de la fabricación. Como resultado se ha obtenido un prototipo en el que se asumen dos aspectos: a) que el nivel de la interfaz gráfica no puede ser comparada con las herramientas de simulación comerciales las cuales tienen un alto grado de madurez y b) las mejoras propuestas se centran en el comportamiento interno del Entorno de Simulación. 
Sin embargo, resulta importante presentar parte de las técnicas de evaluación de software disponibles. El entorno propuesto será evaluado mediante técnicas de ingeniería de software considerando algunas de las métricas que ofrece. De forma global, las métricas que pueden ser aplicadas se pueden agrupar en dos grupos: métricas sobre el producto y métricas sobre el proceso. Entre las métricas sobre el producto encontramos las siguientes:

Tamaño del código. Se refiere a la estimación del número de líneas de código reales que pueden ser contrastadas contra una estimación y permitan servir de base para futuras estimaciones. Por una parte esta tendencia ha sido criticada puesto que estima el esfuerzo en base al número de líneas de código y que la complejidad del desarrollo no esta directamente relacionada con dicho número. Una cuestión importante al respecto se refiere a los procesos de mantenimiento posterior.

Complejidad estimada. Con el fin de superar el problema de la estimación de líneas de código, se ha puesto atención a medidas de complejidad. En este caso Albrecht (Albrecht \& Gaffney 1983) definió un método conocido como puntos de función. Este método se basa en el uso de factores de normalización dando una importancia relativa a varios requisitos funcionales. Entre las funciones básicas se consideran: entradas, salidas, consultas, ficheros de datos, e interfaces. El método define unas estimaciones de complejidad para estimar cuántos elementos de cada tipo van a ser necesarios.

Robustez. Por robustez de un programa se entiende la ausencia de fallos en su ejecución en base a sus datos de entrada durante intervalos de tiempo predeterminados.

Por otra parte las métricas sobre el proceso se enfocan en el análisis de los procesos que se realiza a partir de medidas tomadas en el desarrollo sirviendo de base para una futura mejora. Entre los elementos que se pueden medir encontramos:

Distribución de Esfuerzo. De esta forma se distribuye el trabajo para poder estimar los recursos necesarios.

Aunque para muchos investigadores es difícil desarrollar métricas directas de los factores que afectan la calidad del software, cada factor de calidad se puede obtener como combinación de una o varias métricas. Entre las métricas disponibles para determinar los factores de calidad de la herramienta encontramos: 
facilidad de auditoria, exactitud, consistencia, estandarización de datos, tolerancia a errores, eficiencia de la ejecución, facilidad de expansión, generalidad, modularidad, facilidad de operación, autodocumentación, simplicidad, facilidad de traza, etc. En el Capítulo 11 se presentan los resultados de la evaluación aplicando estas métricas en el prototipo implementado.

\subsection{Bases para la Evaluación de Funcionalidad del entorno de Simulación}

Con respecto a la evaluación de la funcionalidad del entorno de simulación, desafortunadamente no existe una metodología estándar para la evaluación de las herramientas de simulación. Sin embargo, algunos investigadores han propuesto con anterioridad diferentes enfoques de evaluación que se basan en la identificación de características que debe contener una herramienta de simulación.

Las herramientas de simulación han sido aplicadas en el área de fabricación con el fin de resolver problemas relacionados con el diseño y la operación de sistemas de fabricación debido a la complejidad que conllevan. Como resultado del énfasis en la automatización para el aumento de la productividad y la calidad así como la reducción de costos, se ha presentado un aumento de la complejidad de su tratamiento por lo que estos fenómenos sólo pueden ser analizados mediante la simulación. La evaluación de las mismas depende del nivel de abstracción que se seleccione. Sin embargo, existen dos áreas clave que son comunes en todas respecto a la funcionalidad de una herramienta y sus características: a) características asociadas a la etapa del diseño del modelo para un Sistema de Fabricación y b) características relacionadas con la simulación de dicho modelo.

Para la evaluación de los criterios relacionados con dichas áreas clave se proponen algunas técnicas tales como:

- Desarrollo paralelo con diferentes herramientas de simulación del mismo caso de estudio y el análisis de los resultados.

- Partiendo de un listado de las características ideales para una herramienta de simulación se realiza la comparación de las herramientas actuales más representativas con la nueva propuesta. Para ello se establecen métricas predefinidas asociadas a cada característica. 
La evaluación de la funcionalidad del entorno de simulación al mismo tiempo contempla dos aspectos: a) la evaluación del entorno de simulación para desempeñar tareas de creación de modelos y simulación de dichos modelos, y b) la evaluación de los modelos, las configuración de la planta de fabricación y los resultados de la simulación de dichas configuraciones. Asimismo, ambos aspectos se ven relacionados con los criterios con respecto a la satisfacción de los requisitos de la nueva era de la fabricación mediante el paradigma de los Sistemas Multiagente.

\subsubsection{Criterios de Evaluación con respecto al paradigma de Sistemas Multiagente y los requisitos de la Nueva Era de la Fabricación}

La "nueva era de la fabricación" impone un conjunto de características nuevas a un sistema de fabricación. Para satisfacer estos requisitos mediante las herramientas de simulación, nuestra propuesta utiliza técnicas Multiagente de modelado y simulación.

Distribución de Control: El control jerárquico o central es la arquitectura más común utilizada para los sistemas multiagente. Tanto el control jerárquico como el central aportan ventajas o desventajas en el control de la secuencia de operaciones y quienes las ejecutan (Krothapalli \& Deshmukh 1999).

Reconfigurabilidad: Un sistema puede utilizar bloques básicos para construir un modelo, éstos representan elementos físicos los cuales deben permitir reconfigurar los elementos para describir adecuadamente el sistema de fabricación (distribución, selección de procesos, diseño modular de equipo y herramientas, restricciones del manejo de material, etc.). Dichas configuraciones permiten el estudio y evaluación de su impacto en el momento de la simulación (Lee 1997). Tales reconfiguraciones deben ser realizadas rápidamente para observar el efecto de la misma. La reconfigurabilidad se aplica en el nivel de detalle de los elementos del modelo, sus atributos (dimensiones, geometría, materiales, subcomponentes, etc.) (Chan \& Zhang 2001).

Escalabilidad: La escalabilidad es una propiedad de los sistemas de fabricación avanzados. La escalabilidad se refiere a que en la organización pueden ser incorporados más recursos cuando sean necesarios. Esta propiedad debería satisfacerse en cualquier nodo de trabajo del sistema y a cualquier 
nivel dentro de los nodos. La expansión de recursos debería ser posible sin romper enlaces organizacionales previamente establecidos (Botti \& Giret 2003).

Flexibilidad: La flexibilidad incluye la posibilidad para hacer cambios en los equipos, algoritmos de control y estructura, los ajustes al sistema para adaptar su funcionalidad y capacidades de acuerdo con las necesidades cambiantes del mercado. También incluye la facilidad para integrar nuevos elementos o modificar elementos existentes (Botti \& Giret 2003).

Reactividad y Proactividad: Durante la fase de modelado es importante proporcionar ayuda al diseñador para que contemple aspectos relevantes de procesos que está diseñando, proporcionar soporte sobre referencias del posible impacto de las decisiones que tome durante el diseño, proporcionar posibles soluciones (basándose en modelos anteriores) (Yan, Borg \& Juster 2001). Durante la simulación estas características se observan en los procesos de negociación para la asignación de recursos del Sistema de Fabricación, a fin de optimizar su uso. Tal optimización también se refleja en la optimización del uso de los recursos físicos (memoria, espacio en disco) que este utilizando la herramienta de simulación.

Tolerancia a Fallos: El sistema deberá ser tolerante a fallos tanto a nivel de sistema como de subsistema, así como detectar y recuperarse de fallos del sistema en cualquier nivel y minimizar sus impactos sobre el entorno de trabajo (Botti \& Giret 2003).

Siguiendo el Diagrama de Estudio de Simulación (Figura 5.1 ) la última actividad que se refiere al Análisis de los Resultados permite la verificación de los resultados obtenidos durante la Simulación para ser contrastados contra la hipótesis que se planteo al diseñar el modelo y sus experimentos. De este modo, el entorno facilitará la información que permitirá al Usuario el análisis de tales resultados. 


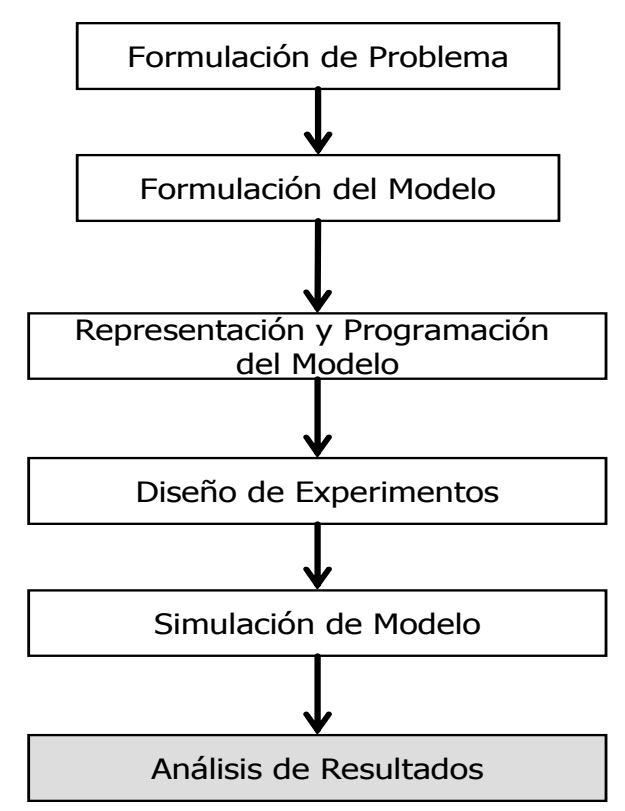

Figura 9.1: Diagrama de Estudio de Simulación.

En las siguientes secciones se presentan los criterios que se aplican para la evaluación de los distintos resultados proporcionados por el entorno.

\subsubsection{Criterios de Evaluación del Entorno de Simulación en la Fase de Modelado}

Los criterios expuestos en esta sección se aplican cuando el Usuario crea un modelo en el Entorno de Simulación. Para la evaluación de la fase de modelado, una de las metodologías más representativas es la propuesta por Eldabi y Paul (Aldabi \& Paul 2001). Esta metodología se basa en el denominado Modelado de Procesos de Negocio (MPN). La etapa de diseño del modelo es casi la misma para sistemas nuevos o aquellos donde se hace un proceso de reingeniería de sistema debido a que en los procesos de reingeniería, la organización abandona los procesos actuales y construye uno completamente nuevo. Los procesos de negocio incluyen más de un nivel de detalle. Básicamente, existen dos niveles, un nivel superior que representa los bloques de un proceso en particular y un nivel inferior que representa las operaciones cotidianas. En el nivel inferior también se incluyen detalles adicionales, tales como la ubicación física de los componentes que constituyen el proceso. De acuerdo con el enfoque del Diseño de Sistemas 
de Fabricación asistido por Computadora, el objetivo de una herramienta de simulación sería el proporcionar una plataforma que asista a los ingenieros de manufactura en el diseño de las operaciones de producción. Las dos principales características de tal plataforma son:

1. Debe ser suficientemente flexible para permitir incluir la variedad de situaciones que se presentan en el diseño de un Sistema de Fabricación, siendo sistemático, y práctico;

2. Debería presentarse a los ingenieros de tal forma que su uso sea práctico, acotado y que lo guíe durante el diseño del modelo.

El nivel superior de modelado debe ser sencillo ya que éste contiene la construcción de los bloques del sistema de fabricación sin la definición de sus detalles de operación. Por lo tanto en el siguiente listado se presentan algunas de las características a evaluar durante la fase de modelado en el nivel superior. Tanto las de nivel superior como inferior tiene una repercusión importante en el momento de la simulación así como los resultados obtenidos.

Creación de Modelado sencillo y rápido. En el nivel superior se incluyen los elementos (máquinas, operarios, herramientas, distribución física) del sistema de fabricación. Esta característica puede ser afectada por circunstancias tales como el tiempo requerido por el ingeniero para aprender a manejar la herramienta, la complejidad para incluir los elementos en el modelo, el proceso de compilación y la depuración del modelo.

Animación de bajo nivel. En este nivel la animación que se configura se caracteriza por ser una animación en 2 dimensiones que incluye cambios en el color como indicadores de cambios de estado de las entidades.

Resultados Finales. La definición de los resultados esperados permite medir el rendimiento del sistema. Estos son utilizados para comparar las diferentes configuraciones del mismo modelo.

En el nivel inferior se tienen en consideración otras características importantes.

Especificación del detalle del Modelado. En el nivel inferior se detallan los atributos de los elementos (máquinas, operarios, herramientas, distribución física) incluidos en el nivel superior del modelo del sistema de fabricación. Tales atributos incluyen: atributos de geometría, distribución física, capacidades, tiempos de espera, ejecución, preparación, etc. 


\subsubsection{Criterios de Evaluación del Entorno en la fase de Simu- lación del Modelo}

Para la evaluación de las características del entorno durante la simulación de un modelo existen diversas técnicas empleadas por investigadores a fin de establecer procedimientos comparativos. En todas las propuestas se incluye una lista de criterios referentes a las características ideales de una herramienta para efectos de la simulación y un método para evaluarlas. Entre las procedimientos propuestos para dicha evaluación se incluye asignación de valores y su normalización para asignar una puntuación, para asignar una categoría (Banks 1996). Davis y Adams incluyen además del uso de dicha puntuación, un proceso analítico de jerarquía para identificar el mejor puntuado (Davis \& Williams 1994). Otros tales como Hlupic y Paul en cambio, realizan la puntuación estimando la calidad de los simuladores, donde un 1 representa una calidad muy baja o ausencia de las característica, mientras que el 10 representa una calidad excelente (Hlupic \& Paul 1995b).

Por otra parte existen diversas propuestas en cuanto al listado de las criterios (características) más importantes en la evaluación de las herramientas de simulación. Los criterios varían en cuanto al agrupamiento de características, ya que depende del nivel de abstracción elegido para la evaluación. Entre las criterios más representativos podemos mencionar el de Law y Haider (Law \& Haider 1989), Law y McComas (Law \& McComas 1999), Kochhar (Kochhar 1989), Holder (Holder 1990), Breedam et al. (Breedam et al. 1990), Banks et al. (Banks et al. 1991), Kelton y Law (Law \& Kelton 1991), Davis y Wiliams (Davis \& Williams 1994), Banks (Banks 1996) y Maklulak (Mackulak et al. 1994). Nikoukaran en su interés por proponer una visión más amplia con respecto a las características ideales para una herramienta de simulación, agrupa los criterios propuestos por dichos investigadores haciendo énfasis en aquellas en las que coinciden (Nikoukaran \& Paul 1999) y Banks et al.(Banks et al. 2001). Además de tales características se integran aquellas que proporciona la aplicación de la Tecnología de Sistemas Multiagente (Sección 9.2.1).

De tal manera que partiendo de dicha agrupación, para la evaluación de este trabajo se propone la agrupación de ciertas características en siete categorías para identificar funcionalidades específicas de la herramienta que puedan ser medidos en el Entorno de Simulación propuesto.

Criterios del Modelado: Opciones proporcionadas para guiar al Usuario en la 
creación de un modelo, capacidad para configurar atributos de cada elemento, y opciones de reutilización de modelos. Estos criterios se evalúan en la primera fase del proceso de simulación.

Criterios de Fabricación: Tipos de equipamiento que permite incluir en el modelo: bandas transportadoras, cortadoras, soldadoras, vehículos guiados automáticamente, etc., y capacidad para integrar el recurso humano como parte de los procesos de fabricación. En la Base de Datos se almacenan los datos de los recursos disponibles en el Sistema Original, sin embargo, se permite agregar recursos adicionales durante la creación del modelo.

Criterios relacionados con la visualización: Librería de iconos, modo de ejecución de animación, tipos de animación (apariencia, movimiento, control de velocidad, etc.), tipo de interfaz (uso de ventanas, barras de herramientas), y visualización en 2 ó 3 dimensiones. En el prototipo diseñado se ofrecen interfaces sencillas que guían al Usuario durante las tareas de creación de modelos y su simulación. Asimismo, las interfaces muestran gráficamente el comportamiento interno de los elementos que integran tanto el Entorno de Simulación como el Sistema de Fabricación.

Manejo de Datos: Mantenimiento de la base de datos, generación de archivos, análisis de datos de entrada y resultados de simulación, tamaño del modelo, capacidad para reiniciar valores, capacidad de Rastreo (estado de eventos y entidades activas), y opciones para la importación de datos del sistema de fabricación real y la exportación de resultados. En el Metamodelo del Sistema de Fabricación se presenta la estructura de la Base de Datos y en el prototipo de la arquitectura se ofrecen interfaces para dar mantenimiento a la misma (importación y exportación de datos).

Presentación de Resultados: Manejo, creación y presentación de reportes y gráficas, presentación dinámica de datos en reportes y gráficas conforme el progreso de la simulación. En el prototipo se ofrece una interface que permite al Usuario visualizar los resultados en reportes y gráficas.

Depuración: Capacidad para la depuración interactiva, control de la ejecución de la simulación (detener, ejecutar hasta una condición de parada definida, ejecutar paso a paso, etc.), y verificación de los errores en línea. En la interfaz del prototipo utilizada para la simulación de modelos se incluye la opción que permite controlar la simulación mediante opciones para detenerla, pausarla, acelerarla o desacelerarla. 
Opciones de Adaptabilidad: Opciones para integrar funcionalidades adicionales o modificar la operación actual del entorno de simulación y del modelo que se simula. Estas opciones se ofrecen tanto durante la creación del modelo como durante su simulación.

\subsection{Bases para la Evaluación de Resultados obte- nidos durante la Simulación}

Los resultados obtenidos incluyen los modelos, el uso de las configuraciones de la planta y los datos de producción. Por tanto, la Evaluación de los Resultados contempla la definición de las bases para cada uno de los elementos.

\subsubsection{Criterios para la Evaluación de Modelos}

Durante la Fase de Simulación de Modelos, es posible la evaluación de distintos modelos. Esta evaluación no requiere que se hayan realizado simulaciones de órdenes de producción. Las herramientas de simulación actuales no contemplan este tipo de evaluación, sin embargo, consideramos que es importante ofrecer una evaluación global del modelo previa a su simulación.

La primera fase de evaluación, para el caso en que el modelo representa un Sistema de Fabricación real, es que el modelo genere las mismas salidas que el sistema que representa. Sin embargo cuando se trata de validar un modelo de una planta nueva, la evaluación se enfoca en la verificación de los procesos definidos y la capacidad de los recursos de la planta para darles servicio.

La evaluación de un Modelo considera las características de los elementos que lo integran con respecto a su funcionalidad y los resultados que pueden ser obtenidos. Cuando se crea el modelo, cada una de las instancias asociadas a los elementos del modelo poseen características que pueden ser evaluadas previas al lanzamiento de una orden de producción. Por tanto la evaluación de dicho modelo considera dos aspectos:

Grado de expertise (habilidad o destreza) de sus recursos. Los recursos tienen un grado de expertise clasificado en principiante, medio o experto. Dicho grado está asociado a una posible reducción en el tiempo de procesamiento de tareas. Los elementos con un grado de destreza "experto" 
pueden reducir el tiempo de procesamiento de sus tareas en un $50 \%$, mientras que un elemento con grado de destreza "medio" lo puede reducir un $30 \%$ y un "principiante" no puede reducir dicho tiempo. Para las máquinas que trabajan de forma automática también se aplica este criterio aunque el porcentaje varía. Se considera como expertas a aquellas que a criterio del Usuario tienen un mayor grado de productividad y como principiantes aquellas con menor grado de productividad.

En el caso de las herramientas y las máquinas que requieren ser manipuladas no se aplica este criterio puesto que el grado de destreza corresponde totalmente al operario que la manipula.

Número de recursos. Aquellos modelos que incluyan un mayor número de instancias con un grado de destreza de "expertos" pueden reducir los tiempos de fabricación sin disminuir el nivel de calidad del producto, en comparación con aquellos modelos con instancias que tengan grados medio o principiante.

La evaluación de un modelo considera el número de agentes y su grado de "expertise". Para ello, se supone que el grado de expertise asociado a las instancias tiene que ver no solo con la reducción de tiempos sino con la calidad del producto final. Por ejemplo, cuando una tarea se asigna a un trabajador con un grado de "experto", éste puede reducir el tiempo de procesamiento de una tarea en un $50 \%$ con una mayor posibilidad de obtener un producto de calidad óptima. Por otro lado, si la misma tarea se asigna a un trabajador con un grado de "principiante" el tiempo de procesamiento no se podrá reducir y se tiene una menor posibilidad de obtener un producto de calidad óptima.

De esta manera, los modelos pueden ser evaluados de forma global como se menciona a continuación:

- Un modelo es mejor que otro, si el primero contiene un número mayor de instancias con un grado de destreza de "experto", con respecto al segundo modelo;

- Un modelo es mejor que otro, si el primero contiene un mayor número de instancias con un grado de destreza "medio", y el segundo modelo tiene mayor número de instancias con un grado de destreza "principiante".

En un modelo, lo ideal sería que todos los elementos que lo integran tuvieran el grado de expertise más elevado (“experto"). De esta forma, se podrían tener 
una mayor seguridad en cuanto al grado de calidad del producto obtenido, mejorar los tiempos de procesamiento de las órdenes y mejorar la utilización de los recursos disponibles.

Por tanto, un modelo puede ser evaluado contra un modelo "ideal" donde el número total de recursos son de grado "experto" contra los datos reales del modelo creado. Para ello, se utilizan pesos para cada tipo de elemento. De esta forma, el peso final de cada elemento se obtiene como sigue:

- El modelo obtiene un 3 por cada recurso con un grado de destreza igual a "experto".

- El modelo obtiene un 2 por cada recurso con un grado de destreza igual a "medio".

- El modelo obtiene un 1 por cada recurso con un grado de destreza igual a "principiante".

$$
C M=\sum_{i=1}^{N} C R_{i}
$$

donde:

$C M$, es la calificación del Modelo.

$N$, es el número total de Recursos que integran el Modelo.

$i$, es el Recurso del Modelo.

$C R_{i}$, es el peso del Recurso del Recurso i.

Por ejemplo, si el número total de recursos de un Modelo es de 30 y todos fueran expertos, la calificación del Modelo Ideal $(C M I)$ sería de $30 x 3=90$. Con respecto al Modelo real creado, es posible obtener el grado de optimización con respecto al Modelo Ideal. El porcentaje del Grado de Optimización de un Modelo $(G O M)$ se obtiene aplicando la Ecuación 9.2.

$$
G O M=C M * 100 / C M I
$$

donde: 
$G O M$, es el porcentaje de optimización del Modelo.

$C M$, es la calificación del Modelo.

$C M I$, es la calificación del Modelo Ideal.

Si el $G O M$ de un Modelo es 90 indica que el grado de destreza de sus recursos se acerca a un $10 \%$ del Modelo Ideal. Entre más bajo sea el valor del GOM, el Modelo tendrá menos probabilidades de optimizar el uso de los recursos que lo integran.

Mientras que por un lado, la evaluación global del modelo indica una pauta de su comportamiento. Por otro lado, cuando se lanza una orden de producción sólo se hace uso de una parte de los elementos que integran el modelo. Cuando el Usuario solicita lanzar una orden de producción, éste puede seleccionar los elementos que integrarán una configuración específica de la planta o permitir que el Sistema seleccione la configuración adecuada. Por tanto, para que la evaluación de un modelo sea más completa, se debe considerar un nivel inferior de detalle para evaluar dichas configuraciones.

\subsubsection{Criterios para la Evaluación de Configuraciones de Plan- ta previa y posterior a la Creación de Ordenes de Pro- ducción.}

Cuando se lanza una orden de producción, el Usuario selecciona la configuración de la planta con la cual se atenderán las tareas de la ruta de la orden de producción. La evaluación de una configuración de la planta considera las características de los elementos seleccionados con respecto a su funcionalidad y los resultados que pueden ser obtenidos. Cuando se lanza una orden de producción de un producto, el Usuario puede configurar la asociación de las instancias de recursos a las operaciones de la ruta de fabricación seleccionada. Asimismo, el Usuario, previo a la confirmación de la creación de orden de producción puede solicitar que el entorno evalué la configuración de planta seleccionada.

Las herramientas de simulación actuales, no contemplan este tipo de evaluación. La evaluación de configuraciones de planta resulta clave para el diseño de experimentos, evaluaciones comparativas y reutilización de configuraciones para la validación de hipótesis. Las bases del análisis residen en tres objetivos: 
- Disminuir el tiempo de procesamiento de un producto.

- Disminuir el coste de procesamiento de un producto.

- Valorar el grado de destreza de los operarios y equipos (maquinaria y herramienta) utilizados.

Con respecto al uso de rutas alternativas, como mencionan Stadtler y Kilger en la Sección 4.6, si existen rutas alternativas para realizar la producción de una orden, entonces se debería esperar que el sistema elija la mejor. Sin embargo, indican que "por experiencia" el Usuario tiene que elegir su ruta "preferida". Al mismo tiempo indican que algunas veces es posible tener una lista de calificaciones para las rutas alternativas como una entrada predefinida. La herramienta de simulación por tanto, ofrece una alternativa para generar la calificación no sólo de una ruta si no de una configuración de planta determinada. La configuración contempla la asociación de recursos específicos a cada una de las operaciones que integran la ruta de fabricación seleccionada por el Usuario.

Por tanto, la evaluación de dicha configuración se puede llevar a cabo en dos sentidos:

Previo al lanzamiento de una Orden. Durante esta evaluación se comparan las características de los elementos elegidos durante la configuración de planta. La configuración de planta se encuentra vinculado a un producto específico. En esta evaluación se considera el número de agentes, el tipo de instancias, el grado de expertise (habilidad/destreza) de las instancias seleccionadas,etc. De tal manera que es posible identificar qué configuración puede ser mejor valorada que otra para la fabricación de un producto determinado.

En la evaluación previa, se puede evaluar la configuración de la ruta seleccionada para el lanzamiento de una orden antes de que se ejecute su simulación. La evaluación puede ser realizada de cuatro formas: a) evaluar la ruta calificándola en base al tipo de elementos que integra su configuración, b) evaluar el coste de dicha configuración, c) evaluar el tiempo de procesamiento previsto con respecto al tiempo estándar, d) evaluar en base a un promedio de calificaciones previas de la configuración definidas por el Usuario al finalizar cada orden de producción y e) evaluar la configuración en base a una fórmula definida por el Usuario que integre los tres elementos anteriores. 


\section{a) Calificando la configuración de la Ruta en base al tipo de instancias.}

El tiempo de entrega de un producto puede ser evaluado, si se considera el número de agentes y su grado de "expertise". La evaluación toma en consideración que los elementos con un grado de destreza "experto" pueden reducir el tiempo de procesamiento de sus tareas en un $50 \%$, mientras que un elemento con grado de destreza "medio" lo puede reducir un $30 \%$ y un "principiante" no puede reducir dicho tiempo. De tal manera que las configuraciones pueden ser evaluadas de forma global como se menciona a continuación:

- Una configuración es mejor que otra, si la primera contiene un número mayor número de instancias con un grado de destreza de "experto", con respecto a la segunda configuración;

- Una configuración es mejor que otra, si en la primera la orden distribuye su tareas entre instancias con un grado de destreza de "experto", y en la segunda configuración se distribuyen entre instancias con un grado de destreza "medio o principiante".

Más aún, la configuración puede ser evaluada a un nivel inferior, es decir, a nivel de operaciones de la ruta de fabricación. Para ello se utilizan pesos para cada tipo de elemento asociado a cada operación. Se ha definido un coste estándar de penalización (a menor grado de destreza mayor valor de penalización) de acuerdo al tipo de instancia. De esta forma, los pesos finales de cada operación se obtienen como sigue:

- Una operación obtiene un 1 por cada recurso con un grado de destreza igual a "experto".

- Una operación obtiene un 2 por cada recurso con un grado de destreza igual a "medio".

- Una operación obtiene un 3 por cada recurso con un grado de destreza igual a "principiante".

$$
C c p=\sum_{i=1}^{N} C O p_{i}
$$

donde: 
$C c p$, es la calificación de la configuración de la Planta.

$N$, es el número total de Operaciones que integran una ruta.

$i$, es la Operación de la Ruta.

$C O p_{i}$, es el Peso de la operación i.

De esta forma, además de calificar una Ruta asociada a un producto es posible comparar dos configuraciones tanto a nivel global como a nivel de operaciones. Dichas calificaciones servirán de retroalimentación al sistema. El Agente de Resultados toma como referencia la ruta de fabricación de un producto con la mejor calificación para compararla con una nueva configuración. En su caso, el Agente de Simulación sugiere el uso de dicha configuración cuando se lanza una orden de producción.

La mejor calificación de la ruta dependerá del número de operaciones que integre. Por ejemplo, si la ruta X contiene 6 operaciones, y la mejor calificación para cada operación es 1 (debido a que utiliza" expertos"), entonces la "calificación óptima" de la ruta X sería igual a 6. Por tanto, la mejor configuración de la ruta $\mathrm{X}$ es aquella en la que su calificación se acerque más a la "Calificación de Configuración Óptima (CCO)" de la ruta. Asimismo es posible calificar el Porcentaje de Optimización de la Configuración $(P O C)$ con respecto a la calificación óptima $(C C O)$ aplicando la Ecuación 9.4.

$$
P O C=C c p * 100 / C C O
$$

donde:

$P O C$, es el porcentaje de optimización de la Configuración.

$C c p$, es la calificación de la Configuración.

$C C O$, es la calificación Óptima de la Configuración.

Si el $P O C$ de una Configuración es 90 indica que el grado de destreza de sus recursos se acerca a un $10 \%$ de la Configuración Óptima. Entre más bajo sea el valor del $P O C$, la Configuración tendrá menos probabilidades de optimizar el uso de los recursos que la integran. 


\section{b) Calificando la configuración de la ruta de acuerdo a su coste.}

Cada uno de los recursos que integran el modelo, tienen asociado un coste por hora. De esta forma, es posible obtener el coste de una configuración antes de que se termine la simulación de la misma.

$$
C_{R}=\sum_{i=1}^{N}\left(\sum_{j=1}^{M} T m R_{i j} * C h r R_{j}\right)
$$

donde:

$C_{R}$, es el coste de la Configuración de la Ruta.

$N$, es el número total de operaciones de la ruta.

$i$, es la Operación de la Ruta.

$M$, es el número total de recursos asignados a la Operación i.

$j$, es el Recurso asignado a la Operación i.

$T m R_{i j}$, es el tiempo asignado al Recurso j en la Operación i.

$C h r R_{j}$, es el coste por hora del Recurso j.

Asimismo, el mejor coste de la ruta dependerá del número de operaciones que integre, el coste de los recursos y de la necesidades del Usuario. El Usuario puede definir un coste máximo y/o un coste mínimo permitido. Por tanto se pueden presentar dos casos:

- Si el Usuario sólo indica el coste máximo permitido. La mejor configuración de la ruta $\mathrm{X}$ es aquella en la que su coste se aleje más del coste máximo permitido de la ruta.

- Si el Usuario indica el coste máximo permitido y el coste mínimo permitido. La mejor configuración de la ruta $\mathrm{X}$ es aquella en la que su coste se aleje más del coste máximo permitido de la ruta, pero que sea mayor al coste mínimo permitido.

Es posible calificar el Porcentaje de Optimización del Coste de la Configuración (POCte) con respecto al Coste de la Configuración Estándar $(C t e C E)$ aplicando la Ecuación 9.6.

$$
\text { POCte }=100-\left(C_{R} * 100 / \text { CteCE }\right)
$$


donde:

POCte, es el porcentaje de reducción de coste de la Configuración con respecto a la Configuración Estándar.

$C_{R}$, es el coste de la Configuración.

$C t e C E$, es el coste de la Configuración Estándar.

Si el POCte de una Configuración es 30 indica que la configuración logra disminuir un $30 \%$ el coste de la configuración estándar. Entre más alto sea el valor del POCte, la Configuración ofrece más probabilidades de reducir el coste de una orden de producción.

c) Calificando la configuración de la ruta en base al tiempo de fabricación.

En este caso se puede evaluar el tiempo que llevará terminar la orden de producción. El tiempo, como se ha visto, dependerá del tipo de recursos a los que se ha asignado (principiante, medio o experto quienes pueden reducir el tiempo de procesamiento). La evaluación del tiempo se hace tomando como base el tiempo de producción estándar que tiene asociado el producto.

Por ejemplo, consideremos que el producto A tiene un tiempo de fabricación estándar de 16 horas. La mejor configuración de la ruta $\mathrm{X}$ es aquella en la que su tiempo de producción se aleje más del tiempo de fabricación estándar.

Estas tres opciones de calificación de la configuración de la ruta son aplicadas cuando el Usuario solicita lanzar una orden de producción. Para definir la configuración a utilizar, el usuario tiene tres opciones: a) seleccionar los recursos, b) solicitar que el entorno de simulación le recomiende una configuración, y c) solicitar la calificación de la configuración seleccionada.

Si el Usuario solicita que se le recomiende una configuración, el Usuario debe indicar el parámetro que utilizará el Agente de Simulación para evaluar las configuraciones previas. En su caso, el Usuario puede indicar el parámetro que utilizará el agente de simulación para identificar la mejor configuración para la ruta seleccionada y éste la ofrezca en el momento de lanzar una orden de producción. 
Si el Usuario solicita que califique la configuración seleccionada, éste debe indicar el parámetro que utilizará el Agente de Simulación para evaluar la configuración. De esta forma, el Agente de Simulación le indicará la calificación de la configuración con respecto a la mejor configuración que tenga identificada previamente. En esta opción solo se informa al Usuario la calificación de su configuración sin alterar la selección de recursos. En un momento dado, el Usuario puede decidir si cambiar su configuración o en su caso solicitar que se le recomiende una mejor configuración.

El tiempo de procesamiento esperado de una configuración se obtiene mediante la aplicación de la Ecuación 9.7.

$$
\operatorname{TmpPC}_{E}=\sum_{i=1}^{N}\left(\sum_{j=1}^{M} T m R_{i j}\right)
$$

donde:

$T m p P C_{E}$, es el tiempo de Procesamiento Esperado de Configuración de la Ruta.

$N$, es el número total de operaciones de la ruta.

$i$, es la Operación de la Ruta.

$M$, es el número total de recursos asignados a la Operación i. $j$, es el Recurso asignado a la Operación i.

$T m R_{i j}$, es el tiempo asignado al Recurso j en la Operación i.

Asimismo es posible calificar el Porcentaje de Optimización del Tiempo de Procesamiento Esperado de la Configuración (POTmEC) con respecto al Tiempo de Procesamiento de la Configuración Estándar (TmpEE) aplicando la Ecuación 9.8.

$$
P O T m E C=100-\left(\operatorname{TmpPC}_{E} * 100 / \operatorname{TmpEE}\right) ;
$$

donde:

POTmEC, es el porcentaje de reducción de Tiempo de Procesamiento Esperado de la Configuración con respecto a la Configuración Estándar. 
$T m p P C_{E}$, es el tiempo de Procesamiento Esperado de Configuración de la Ruta.

TmpEE, es el tiempo de Procesamiento Esperado de la Configuración Estándar.

Si el POTmEC de una Configuración es 30 indica que la configuración logra disminuir un $30 \%$ el Tiempo de Procesamiento de la configuración estándar. Entre más alto sea el valor del POTmEC, la Configuración ofrece más probabilidades de reducir el Tiempo de Procesamiento de una orden de producción.

Posterior al lanzamiento de una Orden. Esta evaluación considera la medición del desempeño basándose en los datos obtenidos a partir de la ejecución de una simulación de lanzamiento de una orden de producción. La evaluación del uso de una configuración se ve reflejada en datos de producción. Por tanto, el detalle de la evaluación de los resultados se presenta en una sección posterior.

\subsubsection{Aplicación de técnicas multicriterio}

Este análisis previo permite obtener una visión más clara respecto a la importancia de la definición de la configuración de la planta. Sin embargo, resulta necesario evaluar en conjunto tales atributos para identificar aquella configuración que ofrece una mejor valoración global. Con la obtención de estos criterios de evaluación es posible dirigir una segunda etapa de evaluación mediante la aplicación de la técnica de toma de decisiones basada en múltiples criterios (Gal, Stewart \& Hanne 1999).

Para ello, se aplica una técnica de evaluación multicriterio donde se normalizan los resultados obtenidos en los criterios base. De esta forma, se asignan pesos entre 0,1 y 0,9 a cada uno de los atributos de tal forma que en conjunto integren el 100 por ciento de la calificación Global de la Evaluación.

Supongamos que los pesos se asignan como sigue: para el tiempo de procesamiento es del $0,4=>40 \%$, para el coste es el $0,4=>40 \%$ y para el tipo de instancias el $0,2=>20 \%$. En la Tabla 10.15 se observa la evaluación global de las configuraciones para un caso de estudio en el que se usan estos pesos. 


\subsubsection{Criterios para la Evaluación de los Resultados relacio- nados con Datos de Producción}

En la segunda Fase de la Arquitectura del Entorno de Simulación se contempla la visualización de los resultados de la simulación de órdenes de producción (Sección 7.3). Sin embargo, aunque se contempla presentar una metodología para la definición y medición de indicadores de rendimiento de los recursos del modelo, no se pretende que ésta sea considerada como la metodología óptima para su evaluación.

Cullen en (Cullen 1999) afirma que "las numerosas dimensiones de la medición del desempeño incluye un rango de metodologías y paradigmas. Cada una tiene sus propias perspectivas [...], tienen sus propios principios internos e impone su propio discurso". Si esto es así, se pueden entender las diferencias expuestas anteriormente. El problema que surge es que la naturaleza de la medición del desempeño variará de acuerdo con la metodología empleada y al paradigma que se sustente. Otro de los aspectos que es importante distinguir es la diferencia entre estadísticas e indicadores. La cuantificación de una actividad por sí sola no brinda información sobre la calidad y el desempeño. Abbott en (Abbot 1994) afirma que "un indicador de desempeño generalmente proviene de la combinación de dos estadísticas que forman una relación". Por ejemplo, el número de horas totales de un día puede ser dividido por el número de horas netas de producción y así se genera una relación.

En la literatura especializada se han realizado numerosos estudios referentes a la identificación de indicadores de rendimiento y los factores (p.ejem. cultura organizacional, educación, formas de comunicarlos y generar reportes, revisión y actualización de los mismos, procesamiento de datos, etc.) que afectan en la definición de los mismos (Franco \& Bourne 2003). Asimismo, como resultado de dichos estudios ha sido posible identificar características clave de los indicadores de rendimiento. Entre las características se hace mención de que los indicadores deben ser: relevantes, claros y simples, precisos y exactos, medibles, consistentes, muestren mediciones de periodos de tiempo, permitan predecir futuras situaciones, y que puedan ser comparados a nivel individual y global.

Para los indicadores y sus relaciones que se utilizan para la evaluación de los resultados relacionados con los datos de producción se consideran las siguientes características: son claros, simples, fáciles de comprender y gestionar, se pueden medir, muestran el resultado de las actividades de los recursos en periodos 
específicos de tiempo y al mismo tiempo permiten observar su comportamiento histórico.

En cuanto a los indicadores que deben ser considerados en la evaluación de los Sistemas de Fabricación la literatura especializada de fabricación coincide en que que la identificación de los mismos depende del objetivo que se pretenda evaluar. Entre los diversos estudios para la evaluación de recursos y procesos de fabricación se encuentran trabajos de Mills y Bourne y de Diala et. al en (Mills \& Bourne 2005, Diala, Sidi-Ali, Abderrahman \& Habib 2007). Entre los indicadores que han sido presentados como elementos clave en la evaluación de rendimiento se incluye la eficiencia, calidad, productividad, factores de coste, utilización de recursos, tiempo de procesamiento, tiempo de entrega, etc.

Como se indica en (Bitici, Carrie \& McDevitt 1997) la estructura de un sistema de medición de rendimiento, donde el Sistema a medir produce bienes o servicios, integra indicadores que midan objetivamente el rendimiento de actividades individuales tales como: a) procesamiento de órdenes (que contempla el tiempo de procesamiento, y la exactitud del procesamiento de la orden), b) planificación de producción y c) fabricación (que incluye los plazos de entrega, tiempos de configuración, utilización, etc.). En este trabajo, hemos utilizado como referencia las fórmulas para el análisis de un Sistema de Fabricación Flexible (FMS) expuesto por Stam y Kuula (Stam \& Kuula 1991) para la identificación de los indicadores y parte de la definición de las fórmulas que permitan cuantificar los resultados y por consecuencia analizarlos.

Basándonos en estas premisas, en el Entorno de Simulación durante la evaluación posterior al lanzamiento de una orden, será posible medir los tiempos de respuesta, tiempos de procesamiento, tiempo de espera, tiempo de cola, tiempo muerto, tiempo de producción, etc., asociados a una configuración. La evaluación utiliza los resultados obtenidos en la simulación del comportamiento de la planta de fabricación ante una orden de producción. Asimismo, será posible obtener el histórico del comportamiento de los recursos de la planta de fabricación, o la comparativa entre varios recursos. Las herramientas de simulación actuales ofrecen reportes para el análisis de datos de producción.

Por su parte, el agente de Resultados puede generar una evaluación de la configuración de la planta basándose en la especificación de una fórmula definida por el Usuario que indique los parámetros a medir o en una serie de fórmulas preestablecidas. Para ello, los indicadores disponibles se describen a continuación: 
Tiempo de preparación. Es el tiempo que el recurso tarda en ponerse a punto antes de atender la primera orden de producción.

Tiempo muerto. Aquel tiempo en que el recurso no está realizando ninguna tarea.

Tiempo de Cola. Tiempo estándar que una tarea se mantiene en la cola de tareas pendientes antes de ser atendida por un Recurso.

Tiempo de procesamiento. Contrario al tiempo muerto, es el tiempo en que el recurso dentro de su turno está realizando alguna tarea.

Tiempo de Espera. Tiempo estándar que un semiproducto espera después de que ha sido atendido por un Recurso antes de pasar al siguiente proceso.

Tiempo de Fabricación. Tiempo estándar que tiene asociado un producto para su fabricación. El tiempo se basa en una ruta estándar determinada por el Usuario. La ruta de fabricación sin embargo, se elige en el momento de lanzar una orden de producción. El tiempo de fabricación incluye el tiempo de cola, tiempo de procesamiento y tiempo de espera.

Tiempo de Respuesta. Este tiempo tiene que ver directamente con el intercambio de mensajes entre los recursos. Por tanto, es el tiempo que tarda un recurso en responder ante una solicitud de disponibilidad o de asignación de reserva de tiempo para una tarea.

Además se contemplan atributos adicionales que sirven para el establecimiento de relaciones para el análisis de los indicadores.

Horas al día (HD). Número de horas totales día, que tiene un valor fijo de 24.

Horas Objetivo (HO). Este atributo lo define el Usuario ya que se refiere al nivel de horas de producción deseadas.

Horas de Paro diarias (HPD). Horas de paro asociadas a horarios de comidas o paros previstos al día.

Horas Brutas de Producción diaria (HBPD). Se refiere al número de horas al día (HD) hábiles incluyendo el número de horas de paro diarias (HPD).

Horas Netas de Producción diaria (HNPD). Se refiere al número de horas brutas de producción diaria (HBPD) menos las horas de paro diaria (HPD). 
Asimismo, también se consideran indicadores complementarios, que al mismo tiempo establecen relaciones entre los anteriores indicadores, éstos se describen a continuación:

Porcentaje de Rendimiento Bruto. Se obtiene dividiendo el número de horas objetivo (HO) entre el número de horas brutas de producción (HBP), multiplicando el resultado por 100.

Porcentaje de Paros. Se obtiene dividiendo el número de horas de paro (HP) entre el número de horas brutas de producción (HBP), multiplicando el resultado por 100 .

Porcentaje de Rendimiento. Se obtiene dividiendo el número de horas objetivo $(\mathrm{HO})$ entre el número de horas netas de producción (HNP), multiplicando el resultado por 100.

Porcentaje de Tiempo Muerto. Se obtiene dividiendo el número de horas del día (HD) entre la sumatoria del número de horas brutas de producción (HNP) más número de horas del día (HD), multiplicando el resultado por 100.

De tal manera que estos parámetros permitirán medir el porcentaje de rendimiento diario de los recursos y por ende del modelo. Esto es, el Agente de Resultados puede generar reportes que le muestren el comportamiento de la configuración de la planta de forma global o de recursos específicos de acuerdo a los parámetros indicados, o bien definir parámetros para evaluarlos.

Entre las fórmulas predefinidas que se han contemplado, se encuentran las siguientes:

Coste de Orden. El coste de la orden de producción incluye el coste de las operaciones $i$ que integran la ruta de fabricación del producto $z$. De esta forma, el tiempo total de fabricación en la operación i considera el tiempo de procesamiento asociado a cada operación $i\left(t f_{i z}\right)$ para el producto $z$ por el volumen de partes fabricadas en la operación z $\left(V_{i z}\right)$.

$$
T f_{i z}=t f_{i z} V_{z}
$$

El coste total de la Orden para el producto $z$, incluye la suma de todos los tiempos de fabricación por el coste de los recursos utilizados durante 
dicho tiempo. El tiempo de fabricación total se ve reflejado en el costo por hora de los recursos utilizados en la operación $i$. Por tanto, dicho tiempo se multiplica por el coste de la mano de obra $\left(\mathrm{Cmo}_{i z}\right)$ más el coste por hora de las máquinas $\left(C m a q_{i z}\right)$ y herramientas $\left(C h t a_{i z}\right)$ utilizadas en la operación $i$ (Ecuación 9.10).

$$
C O_{z}=\sum_{i=1}^{N}\left(T f_{i z}\left(C m o_{i z}+C m a q_{i z}+C h t a_{i z}\right)\right.
$$

Tiempo de Producción de la Orden. El tiempo de producción de una orden incluye el tiempo de preparación $(t p r)$, de cola $(t c)$, de procesamiento $(t f)$, y de espera $(t e)$ asociados a los recursos $(R)$ que se utilizan en cada operación $i$ de la ruta de fabricación de un producto $z$ por el volumen de partes procesadas en cada operación.

Sin embargo, el tiempo de producción incluye el tiempo de preparación sólo cuando es la primera orden que atiende el recurso (Ecuación 9.11).

$$
T m P_{z}=\sum_{i=1}^{N}\left(\sum_{y=1}^{R} t p r_{i y z}+t c_{i y z}+t f_{i y z}+t e_{i y z}\right)
$$

Tiempo Utilizado. Se puede identificar el tiempo de utilización de un recurso y por ende el tiempo de utilización global de la planta de fabricación. Para obtener el tiempo de utilización $(t u)$ de un recurso $x$ se suma el tiempo de procesamiento asociado a cada una de las partes $z$ por el volumen de partes $\left(V_{z}\right)$ procesadas por el recurso como se presentó en la Ecuación 9.9.

$$
t u_{x}=\sum_{i=1}^{N} T f_{i z}
$$

De tal forma que para obtener el tiempo de utilización promedio global de la planta $(T U)$ se suma el tiempo de utilización de todos los recursos de la planta y se divide por el número de recursos de la planta (R).

$$
T U=\sum_{i=1}^{R} t u_{i}
$$




\section{d) Calificando la configuración de la ruta en base a Promedio de Califica- ciones del Usuario.}

Cuando finaliza la simulación de una Orden de Producción, el Agente de Orden de Producción, informa al Usuario el resumen de la Orden, ruta, tiempos, etc. En base a dicha información el Usuario califica la configuración utilizada. La calificación utiliza el rango de 1 a 10 donde 1 indica que la configuración no ha proporcionado resultados satisfactorios acorde al criterio del Usuario, mientras que 10 indica que el Usuario considera que es una configuración con excelentes resultados. Tales calificaciones permiten obtener una visión personal del usuario en cuanto a la utilización de la configuración en diferentes escenarios de la planta.

La calificación promediada se obtiene considerando el número de órdenes en que ha sido utilizada, y la calificación que el usuario le ha asignado.

$$
C p U_{x}=\sum_{i=1}^{N} C U_{i x} / N
$$

donde:

$C p U_{x}$, es la calificación Promedio del Usuario para la Configuración $x$.

$N$, es el total de órdenes de producción donde se ha utilizado la Configuración $x$.

$C U_{i x}$, es la calificación del Usuario para la Configuración $x$ en la orden $i$.

\section{e) Calificando la configuración de la ruta en base a una fórmula definida por el Usuario.}

Las técnicas propuestas para la calificación de las configuraciones permitirán ofrecer al Usuario información más detallada del comportamiento de la planta. Tales calificaciones sirven de base para la definición de criterios que el Usuario desee aplicar para la integración de fórmulas propias para la calificación de configuraciones de la planta. 


\section{f) Comparativa de Resultados mediante alternativas de Optimización de Planta.}

Dentro de los atributos considerados en el Diseño de Experimentos, resulta importante la verificación de los resultados de dichos experimentos. El grado de optimización de la planta se evalúa comparando los resultados obtenidos al asignar tareas a los recursos mediante la asignación directa y la negociación entre la orden de producción y los recursos. Por tanto, es importante mostrar los resultados asociados a cada experimento a fin de ofrecer información útil para la toma de decisiones en la organización de la planta de de fabricación real.

De este modo, se plantea la alternativa de mostrar el estado de los recursos mediante la asignación directa de tareas y mediante la optimización por negociación basada en procesos inteligentes.

Los resultados incluyen datos tales como total de tiempo muerto, duración total de fabricación de órdenes, coste de fabricación, y tiempo de procesamiento. Asimismo se plantea la opción de visualizar la organización de las colas de trabajos que son atendidos por cada uno de los recursos que integran la planta de fabricación.

\subsection{Conclusiones}

En este capítulo se han presentado los criterios y métricas que se utilizan para la evaluación de la propuesta. Los criterios abarcan: a) la evaluación de la funcionalidad del entorno frente a las necesidades de la nueva fabricación, b) la evaluación de los modelos, c) la evaluación de las configuraciones de planta, d) la evaluación de la simulación de modelos, d) la evaluación de los resultados de la simulación y e) la evaluación del prototipo. Los criterios expuestos permiten observar las mejoras que ofrece esta propuesta.

En el Capítulo 10 se presentan los resultados de la evaluación de los modelos y configuraciones de planta, así como la evaluación de los resultados de las simulación mediante el uso del prototipo en un caso de estudio y en el Capítulo 11 se presentan los resultados de la evaluación de la implementación del prototipo y de la funcionalidad del Entorno de Simulación. 



\section{Capítulo 10}

\section{Caso de Estudio}

Acorde con los pasos a seguir en un estudio de simulación, en este capítulo se presenta el caso de estudio que se utilizó para la fase de diseño de experimentos. Asimismo se presentan los resultados obtenidos al aplicar las bases para la Evaluación de los Modelos (presentadas en la Sección 9.3.1, la Evaluación de Configuraciones (presentadas en la Sección 9.3.2) y la Evaluación de los Resultados relacionados con datos de Producción (presentadas en la Sección 9.3.4).

\subsection{Definición del Caso de Estudio}

Para la aplicación de este modelo se estableció contacto con una empresa que se dedica a la fabricación y que está enfocada a la industria metal-mecánica. La empresa tiene como herramienta de control de su proceso de fabricación un ERP robusto. En dicho ERP se tiene el detalle de los procesos, máquinas, calendarios, estructuras de producto, rutas de fabricación, tiempos e inventario. Sin embargo, el ERP no ofrece la posibilidad de realizar un proceso de simulación antes de pasar una orden de trabajo a la planta. Las necesidades que se plantean son: a) facilitar la detección de cuellos de botella, b) identificar posibilidades de mejora de procesos sin poner en riesgo el cumplimiento de fechas de entrega de órdenes reales, c) evaluación la optimización de los recursos disponibles y d) evaluar la posible adquisición de equipos y/o contratación de personal.

En el caso de estudio, la empresa se dedica a la fabricación, instalación y automatización de silos de almacenamiento. Haciendo uso del ERP mencionado, cuentan con un departamento específico que realiza el proceso de estructuración del producto a fabricar, puesto que en su mayoría se realizan pedidos a medida de las necesidades del cliente. Las áreas que comprenden sus instalaciones son: 
Corte, Doblado, Soldadura, Pintado, Secado, Embalaje, Embarque. El proceso que sigue es: partiendo de una Orden de Trabajo concertada con el cliente, ésta se controla mediante la funcionalidad de planificación del ERP. A este caso lo denominaremos a partir de ahora Planta Silos.

\subsection{Simulación de la Planta Silos.}

En esta sección se presenta las bases así como los resultados del uso del prototipo del Entorno de Simulación aplicado al Caso de Estudio relacionado con la Planta Silos.

\subsubsection{Formulación del Problema.}

Este caso está enfocado a la validación de los procesos y del uso de recursos para una nueva planta de un producto que es fabricado en otra planta real. Debido a que los datos proporcionados se enfocan a la validación de los procesos para una nueva planta de fabricación, los resultados son validados contra los resultados obtenidos en una de las plantas ya establecidas. Con ello se pretende detectar la optimización en la utilización de los recursos existentes y detectar las posibles mejoras al introducir recursos nuevos o reemplazar recursos actuales.

\subsubsection{Formulación del Modelo.}

En la formulación del Modelo se consideran dos aspectos: a) Describir la capacidad de la planta de fabricación, y b) Describir el Producto objeto de estudio que se fabricará en la planta de fabricación.

\section{Modelo de la Planta de Fabricación.}

El modelo de la planta considera aspectos relacionados a dos niveles de detalle. A nivel superior se considera el tipo y número de recursos de la planta de fabricación (operarios, máquinas y herramientas) así como su distribución física. En este nivel se consideró la integración de estos elementos en centros de trabajo. Asimismo, se identificaron todos los procesos que son llevados a cabo por dichos recursos.

A nivel inferior se consideraron las características individuales de cada uno de los recursos (capacidad de procesamiento, turnos, costes por hora, grado de 
destreza, tiempos de cola, espera y procesamiento, características de automatización de equipos, etc.). Puesto que el objetivo de una planta de fabricación es la generación de productos. En este mismo nivel se identificó la estructura de información asociada a un producto tal como su ruta de fabricación y su lista de materiales.

Con respecto al nivel superior de modelado, la creación del Modelo toma como referencia la distribución física de la planta real (Fig. 10.1). Para ello se contempla la identificación de los centros de trabajo y sus elementos, éstos incluyen tanto operarios, máquinas, herramientas y personal de supervisión (Staff).

Para la creación del Modelo se identificaron nueve centros de trabajo: H01, H21 y H24 cuyo objetivo principal son los procesos de Corte, el S26 y S27 procesos de Soldadura, P14 proceso de Pintura, P19 Empaque, P17 Lavado y AC01 de acabados y ensamble de piezas finales. Asimismo, en cada centro de trabajo se identificaron los tipos de máquinas, herramientas, operarios y necesidad de actividades de supervisión.

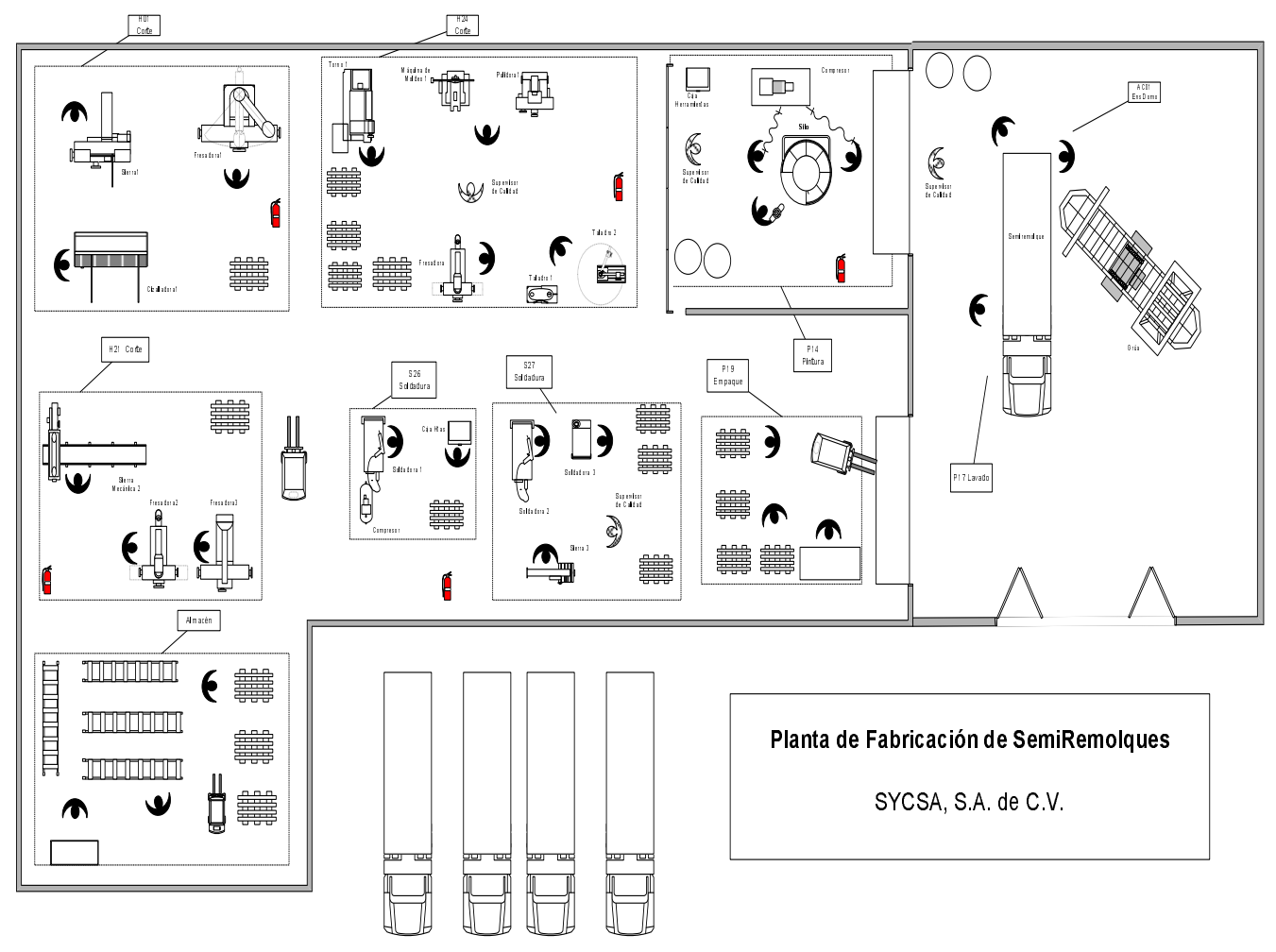

Figura 10.1: Distribución de Planta de Fabricación. 
Por otra parte, al nivel inferior de modelado, asociado a cada uno de los recursos de cada centro de trabajo se identificaron su tipo e información particular. En el caso de las máquinas se consideró el turno al que están asociadas, su capacidad diaria, tiempos de procesamiento, tipo de proceso que pueden desempeñar (en un centro de trabajo de Corte además de contar con maquinaria de corte tal como torno o cizalla, se cuenta con una máquina pulidora y una máquina de moldeo), y si la máquina puede operar de forma automática o en su caso requiere ser manipulada restringiendo su capacidad a la capacidad del operario asignado. En el caso de los operarios, estos además de indicar el centro de trabajo, turno, capacidad diaria, y el tipo de proceso que pueden desempeñar, también se considera su grado de destreza, esto es, se indicó si los operarios eran expertos, principiantes o de nivel medio. Así mismo, se consideró la posibilidad de integrar en el Modelo la identificación de una cuadrilla de empleados sujetos a un turno, horario, capacidad, que actúan como una unidad que ofrece sus servicios. En la Tabla 10.1 se muestra el detalle de los elementos que integran cada uno de los centros de trabajo ya identificados.

\begin{tabular}{|l|l|l|c|}
\hline CT/Proc.Prin./Req.P.Control & Maquinas/Autom/Proceso & Operarios/Tipo & Staff \\
\hline \multirow{3}{*}{ H01/Corte/Si } & Sierra Banda Vertical/No/Corte & Si/Op.Gral & No \\
& Cizalla Horizontal/No/Corte & Si/Op.Gral & No \\
& Fresadora Revolver/No/Corte & Si/Op.Gral & No \\
\hline \multirow{3}{*}{ H21/Corte/Si } & Sierra Mecánica/No/Corte & Si/Op.Gral & No \\
& Fresadora Horizontal/No/Corte & Si/Op.Gral & No \\
& Fresadora Vertical/No/Corte & Si/Op.Gral & No \\
\hline & Torno CNC/No/Corte & Si/Op.Gral & $\mathrm{Si}$ \\
& Fresadora Horizontal/No/Corte & Si/Op.Gral & $\mathrm{Si}$ \\
H24/Corte/Si & Maquina de Moldeo/No/Moldeo & Si/Op.Gral & $\mathrm{Si}$ \\
& Pulidora/No/Pulido & Si/Op.Gral & $\mathrm{Si}$ \\
& Taladro Estático/No/Corte & Si/Op.Gral & $\mathrm{Si}$ \\
& Taladro Giratorio/No/Corte & Si/Op.Gral & $\mathrm{Si}$ \\
\hline S26/Soldadura/Si & Pistola Koestler/No/Soldadura & Si/Op.Gral & $\mathrm{No}$ \\
\hline \multirow{2}{*}{ S27/Soldadura } & Pistola Koestler/No/Soldadura & Si/Op.Gral & $\mathrm{Si}$ \\
/Si & Soldadora Tig/No/Soldadura & Si/Op.Gral & $\mathrm{Si}$ \\
& Fresadora Horizontal/No/Corte & Si/Op.Gral & $\mathrm{Si}$ \\
\hline P14/Pintura/Si & Compresor Pintura/No/Pintura & Si/Cuadrilla & $\mathrm{Si}$ \\
P19/Empaque/Si & Sellador al Vacio/No/Empaque & Si/Op.Gral & $\mathrm{Si}$ \\
P17/Lavado/Si & Compresor Agua/No/Lavado & Si/Cuadrilla & $\mathrm{Si}$ \\
AC01/Acabados/Si & Grúa/No/Desplazamiento & Si/Op.Gral & $\mathrm{Si}$ \\
\hline
\end{tabular}

Tabla 10.1: Centros de Trabajo y Elementos Asociados.

Con respecto a los Operarios además se les asoció el grado de destreza correspondiente a sus habilidades calificadas previamente por los supervisores de la planta física (Tabla 10.2). De esta forma se ubicó al personal en las categorías de experto, medio y principiante. Con respecto a las rutas, también se identificaron los procesos globales y las tareas que se desempeñan en cada uno de los 
Centros de Trabajo definidos (Tabla 10.3).

\begin{tabular}{cllcc}
\hline ID & \multicolumn{1}{c}{ Esenario } & \multicolumn{1}{c}{ Nombre } & Categoría & Grado Destreza \\
\hline 6 & cutting -H01 Corte & Luis & experto & 15 \\
34 & cutting -H24 Corte & Pablo & medio & 10 \\
37 & cutting -H21 Corte & German & medio & 10 \\
4 & welding - S26 Soldadura & Pedro & principiante & 5 \\
30 & welding -S27 Soldadura & Carlos & principiante & 5 \\
29 & painting P14- Pintura & Joaquin,Ruben & experto & 15 \\
31 & painting P14- Pintura & Roberto & principiante & 5 \\
43 & finishing - P19 Empaque & Vicente,Angel & experto & 15 \\
51 & finishing - P19 Empaque & Raul,Sergio & principiante & 5 \\
48 & washing P17 - Lavado & Alberto,Juan & medio & 10 \\
42 & washing P17 - Lavado & Miguel,Mauricio & experto & 15 \\
\hline
\end{tabular}

Tabla 10.2: Operarios y su grado de destreza en los Centros de Trabajo asociados.

\begin{tabular}{|c|c|c|c|}
\hline ID & Descripción & Tipo & Proceso_Global \\
\hline 1 & Diagonal cut & Transformación física & cutting \\
\hline 2 & Blend 90 grades & Conservación de masa con moldeo & moulding \\
\hline 3 & Weld 2 pieces & Unión de formas & welding \\
\hline 4 & Pulir & Acabado superf. sin cambio de forma & finishing \\
\hline 5 & Mezclar & Químico & mixing \\
\hline 6 & Mover a sig ubicación & Cambio de Ubicación & movement \\
\hline 7 & Secar & Térmico & drying \\
\hline 8 & Fundir & Térmico & casting \\
\hline 10 & Elevar unidades & Mecánico & movement \\
\hline 11 & Cortar Lámina & Transformación física & \\
\hline 12 & Cortar Material & Transformación física & cutting \\
\hline 13 & Cortar Partes & Transformación física & cutting \\
\hline 14 & Cortar Partes en Cizalla & Transformación física & cutting \\
\hline 15 & Cortar Travesaños & Transformación física & cutting \\
\hline 16 & Escuadrar Partes & Transformación física & cutting \\
\hline 17 & Cortar Cono, Reducc, Brida & Transformación física & cutting \\
\hline 18 & Cortar Cuello & Transformación física & cutting \\
\hline 19 & Cortar Tensor & Transformación física & cutting \\
\hline 20 & Cortar y Doblar Partes & Transformación física & cutting \\
\hline 21 & Cortar Partes en Pantografo & Transformación física & cutting \\
\hline 22 & Hacer saques a piezas & Transformación física & cutting \\
\hline 23 & Barrenar Pieza & Transformación física & cutting \\
\hline 24 & Barrenar y Pulir & Transformación física & cutting \\
\hline 25 & Barrenar, Pulir,Doblar & Transformación física & cutting \\
\hline 26 & Cortar,Barrenar, Doblar y Pulir & Transformación física & cutting \\
\hline 27 & Doblar y Barrenar Partes & Transformación física & cutting \\
\hline 28 & Cejar Pieza & Transformación física & cutting \\
\hline & Pulir Contornos & Transformación física & cutting \\
\hline 30 & Pulir y Doblar Piezas & Transformación física & cutting \\
\hline 31 & Cortar y Ensamblar & Transformación física & welding \\
\hline 32 & Ensamble, Corte y Ensamble Domos & Transformación física & welding \\
\hline 33 & Ensamblar partes & Transformación física & welding \\
\hline 34 & Ensamblar Partes Salpicadera & Transformación física & welding \\
\hline & Ensamble de Estructura & Transformación física & welding \\
\hline 36 & Ensamble de PortaManguera & Transformación física & welding \\
\hline 37 & Ensamble de SemiRemolque & Transformación física & welding \\
\hline 38 & Ensamble de Suspensión & Transformación física & welding \\
\hline 39 & Rolar Pieza & Transformación física & welding \\
\hline 40 & Trazar, Cortar y Rolar & Transformación física & welding \\
\hline 41 & Trazo, Corte y Union & Transformación física & welding \\
\hline 42 & Unir, Rolar, Ensamblar & Transformación física & welding \\
\hline 43 & Unir, Rolar, Cerrar & Transformación física & welding \\
\hline 44 & Unir,Rolar, Cerrar, Cejar & Transformación física & welding \\
\hline 45 & Conectar Instalación Eléctrica & Mecánico & 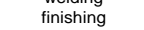 \\
\hline 46 & Aplicar Recubrimiento Ext. & Acabado superf. sin car & painting \\
\hline 47 & Aplicar Recubrimiento Int. & Acabado superf. sin cambio de $f$ & painting \\
\hline 48 & Lavar Remolque & Acabado superf. sin cambio de forma & washing \\
\hline 49 & Lavar Material & Acabado superf. sin cambio de forma & washing \\
\hline 50 & Empacar Material & Acabado superf. sin cambio de forma & packing \\
\hline 51 & Rolar Cuello & Transformación física & finishing \\
\hline 52 & Ensamble de Domo & Transformación física & welding \\
\hline 53 & Col. Access Fenos., Alinia & Acabado superf. sin cambio de forma & 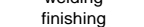 \\
\hline
\end{tabular}

Tabla 10.3: Tareas asociadas a Procesos. 
Esta información integrada en la Base de Datos del Entorno de Simulación se utiliza como soporte durante la creación del Modelo a evaluar.

\section{Productos a Fabricar.}

La fabricación de un Producto se ve sujeta a las especificaciones referentes a su ruta de producción y su lista de materiales. En la ruta de producción se indica el proceso, el tiempo requerido, y el centro de trabajo en el que se realiza la tarea. En la Tabla 10.4 se muestra la Ruta de Fabricación y la lista de materiales de la última fase de fabricación de un Silo. Con respecto a las transiciones entre las operaciones que componen la ruta, en este caso son operaciones secuenciales, sin embargo, es posible definir la ejecución de tareas paralelas.

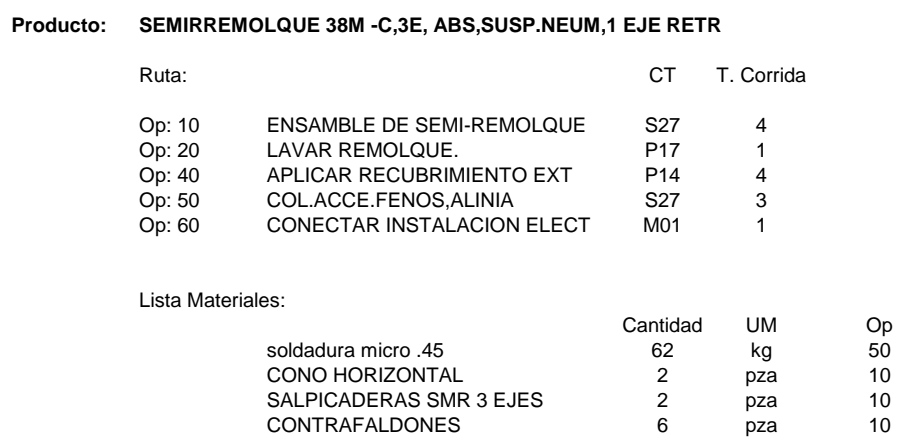

Tabla 10.4: Ruta de Fabricación y Lista de Materiales de Silo.

La información referente a la definición del Producto, su ruta de fabricación y lista de materiales se integran y almacenan en la Base de Datos de la Herramienta de Simulación. El usuario mediante interfaces puede añadir nuevos productos con sus rutas y lista de materiales, nuevos procesos y materias primas. Tal información queda disponible para ser utilizada en la simulación de lanzamiento de órdenes de producción.

\subsubsection{Representación y Programación del Modelo.}

Basándonos en la información extraída e integrada en la Base de Datos de la Herramienta, el siguiente paso es la definición del Modelo el cual incluye cada uno de los centros de trabajo con el número de elementos y los tipos correspondientes además de asignarles su instancia (Fig. 10.2). Los centros de trabajo 
pueden ser agregados mediante el uso de las plantillas donde se agregan al modelo un grupo de elementos o en su caso se puede agregar de forma individual los elementos que se deseen.

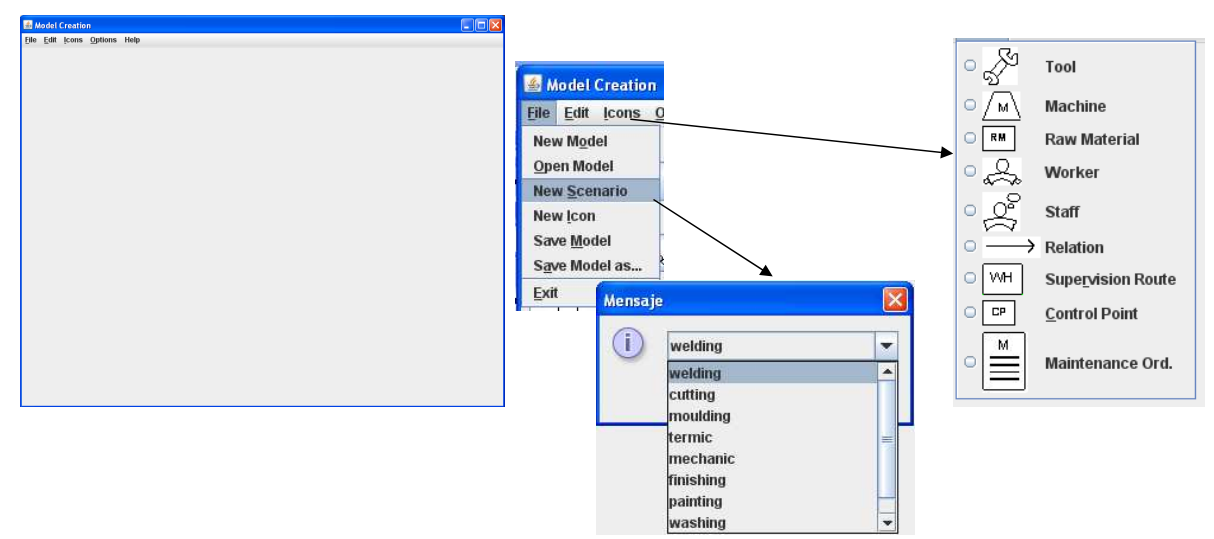

Figura 10.2: Opciones para añadir Elementos al Modelo.

Asimismo, se definen las relaciones de tipo "manipula" entre los operarios y las máquinas y herramientas de cada centro de trabajo. Tales relaciones permitirán delimitar la capacidad de las máquinas a la capacidad del operario asignado para su manipulación durante la simulación de su comportamiento (Fig. 10.3).

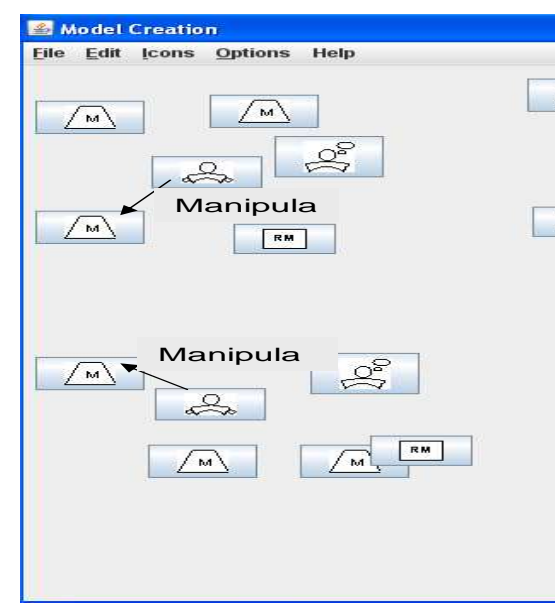

Figura 10.3: Definiendo Relaciones Operario-Máquina.

Una vez creados los iconos es posible asignarle la instancia del elemento que representan para obtener todas sus características. La Figura 10.4 muestra 
las posibles instancias de operadores que pueden ser asignadas a un icono de un centro de trabajo cuyo principal objetivo es la Soldadura. Así que es posible asignar un operario con diferentes categorías de tal manera que el rendimiento de la máquina de soldadura que manipule también se vea afectada por tal decisión.

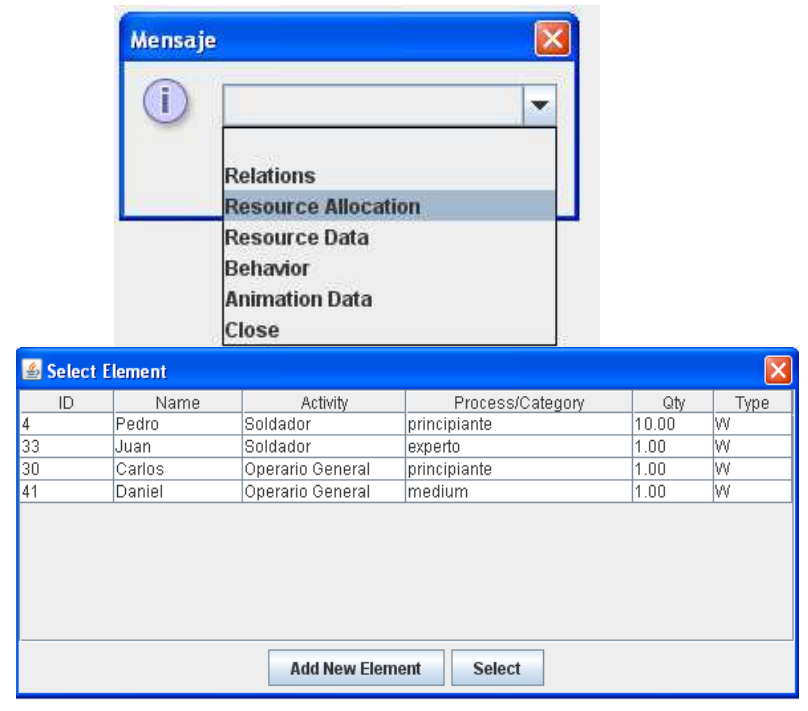

Figura 10.4: Asignando Instancia a Icono de Modelo.

Sin embargo, es posible que no se cuente con la instancia del recurso que se desea asignar durante la Simulación. Para ello, la herramienta permite desde este punto agregar una nueva instancia para su uso (Fig. 10.5).

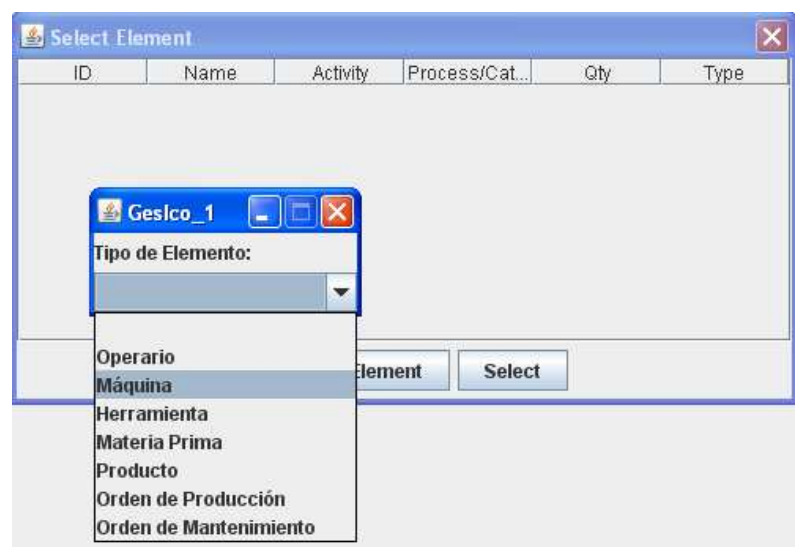

Figura 10.5: Creando Instancia en Base de Datos. 
En la Figura 10.6 se observa el modelo obtenido una vez definidos todos los elementos de la planta física. Este modelo es verificado por el Agente Modelador/Verificador para asegurar que se cumplen las reglas para el modelado de la organización previamente definidas en la Sección 5.8.2. Una vez que se valida que el modelo está libre de errores, se pone a disposición del Agente de Simulación para la Fase II.

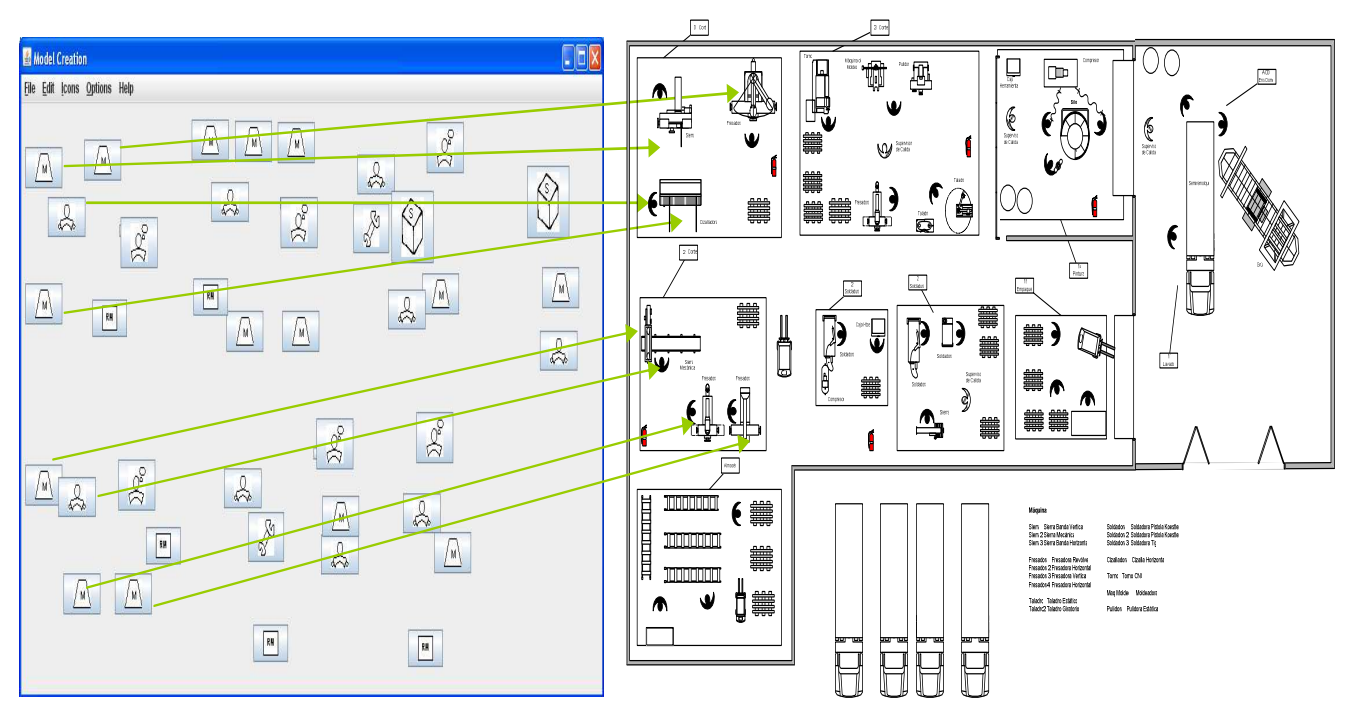

Figura 10.6: Modelo de la Planta.

Finalmente, el modelo obtenido es evaluado antes de iniciar la fase de simulación aplicando los criterios presentados en la Sección 9.3.1. Esto es, se califica el modelo basado en el grado de destreza de los operarios, máquinas, herramientas, staff y el número total de elementos que lo integran.

Para ello se identifica el número total de operarios (Tabla 10.2) que es 12 (considerando como 1 a cada uno de las dos cuadrillas con 2 miembros del mismo expertise con una capacidad global delimitada). Mientras que el número total de máquinas/herramientas es 20.

La calificación del Modelo se obtiene mediante la aplicación de la Ecuación $9.1\left(C M=\sum_{i=1}^{N} C R_{i}\right)$. Así, la calificación obtenida por el Modelo en base el grado de destreza de sus operarios se observa en la Tabla 10.5. 


\begin{tabular}{|l|c|c|c|c|}
\hline Tipo & Experto & Intermedios & Principiantes & Total \\
\hline Operarios & 4 & 3 & 5 & $4 x 3+3 x 2+5 x 1=18$ \\
Maquinas & 2 & 4 & 14 & $2 x 3+4 x 2+14 x 1=28$ \\
\hline \multicolumn{2}{l}{} & Total CM: & 46 \\
\cline { 3 - 5 }
\end{tabular}

Tabla 10.5: Evaluación de Modelo relacionado con el tipo de instancias

Se puede calcular entonces la Calificación del Modelo Ideal (CMI), donde el Total de Recursos es 32. El CMI sería $32 x 3=96$ y para el porcentaje de Optimización del Modelo (GOM) se aplica la Ecuación 9.2 (GOM = CM * 100 / CMI), donde se obtiene $46 * 100 / 96=>47,91$. Por tanto, el porcentaje de optimización de este modelo indica que el grado de optimización de los recursos que lo integran es bajo y que el tiempo de procesamiento estándar no permite ser reducido como se desearía. Además se deberían considerar alternativas para mejorar la capacitación del personal y el tipo de maquinaria que se utiliza.

\subsubsection{Diseño de Experimentos.}

Puesto que el objetivo es la validación de los procesos y del uso de recursos para una nueva planta de un producto que es fabricado en otra planta real. En los experimentos el Usuario en el momento que lanza la Orden de Producción, selecciona los recursos que desea validar al asignarles las tareas asociadas a la ruta de fabricación de un producto. De esta forma, la comparación de los experimentos incluye la validación de las diferentes configuraciones de planta seleccionadas por el Usuario para la fabricación del mismo producto.

En las configuraciones, la selección de los recursos considera la utilización de recursos con distintos grados de destreza, y la selección de procesos manuales o procesos ejecutados por máquinas. Como resultado, se podrán evaluar las configuraciones de la planta asociadas a productos específicos aplicando los criterios planteados en la Sección 9.3.2. El modelo puede ser nuevamente evaluado si este ha sido modificado en la fase de simulación aplicando el criterio planteado en la Sección 9.3.1.

Por tanto, para el producto Silo en este experimento, considerando que todas las máquinas requieren un operario, se validarán las configuraciones de los operarios seleccionados por cada operación de su ruta de fabricación. Las configuraciones se evaluarán asignando recursos de los distintos centros de trabajo de la planta disponibles para la realización de las tareas de cada operación de la ruta de fabricación. Por tanto para la fabricación de 1 producto, se presentan las configuraciones que se aplicarán en la Tabla 10.6. 


\begin{tabular}{|c|c|c|c|c|c|}
\hline \multirow[t]{2}{*}{ Configuración } & \multirow[t]{2}{*}{ Operación } & \multirow[t]{2}{*}{ Centro Trabajo } & \multicolumn{3}{|c|}{ Categoría Operario } \\
\hline & & & Principiante & Medio & Experto \\
\hline \multirow{5}{*}{ A } & 10 & S26/S27 & $\mathrm{Si}$ & \multirow{5}{*}{$\mathrm{Si}$} & \\
\hline & 20 & P17 & & & \\
\hline & 40 & P14 & $\mathrm{Si}$ & & \\
\hline & 50 & S26/S27 & $\mathrm{Si}$ & & \\
\hline & 60 & P19 & $\mathrm{Si}$ & & \\
\hline \multirow{5}{*}{ B } & 10 & S26/S27 & $\mathrm{Si}$ & \multirow{5}{*}{$\mathrm{Si}$} & \\
\hline & 20 & P17 & & & \\
\hline & 40 & P14 & & & $\mathrm{Si}$ \\
\hline & 50 & S26/S27 & $\mathrm{Si}$ & & \\
\hline & 60 & P19 & $\mathrm{Si}$ & & \\
\hline \multirow{5}{*}{$\mathrm{C}$} & 10 & S26/S27 & $\mathrm{Si}$ & \multirow{5}{*}{$\mathrm{Si}$} & \\
\hline & 20 & P17 & & & \\
\hline & 40 & P14 & & & $\mathrm{Si}$ \\
\hline & 50 & S26/S27 & $\mathrm{Si}$ & & \\
\hline & 60 & P19 & & & $\mathrm{Si}$ \\
\hline \multirow{5}{*}{$\mathrm{D}$} & 10 & S26/S27 & $\mathrm{Si}$ & \multirow{5}{*}{\multicolumn{2}{|c|}{$\mathrm{Si}$}} \\
\hline & 20 & P17 & & & \\
\hline & 40 & P14 & $\mathrm{Si}$ & & \\
\hline & 50 & S26/S27 & $\mathrm{Si}$ & & \\
\hline & 60 & P19 & $\mathrm{Si}$ & & \\
\hline \multirow{5}{*}{ E } & 10 & S26/S27 & $\mathrm{Si}$ & \multirow{5}{*}{\multicolumn{2}{|c|}{$\begin{array}{l}\mathrm{Si} \\
\mathrm{Si}\end{array}$}} \\
\hline & 20 & P17 & & & \\
\hline & 40 & P14 & & & \\
\hline & 50 & S26/S27 & $\mathrm{Si}$ & & \\
\hline & 60 & P19 & $\mathrm{Si}$ & & \\
\hline \multirow{5}{*}{$\mathrm{F}$} & 10 & S26/S27 & $\mathrm{Si}$ & & \\
\hline & 20 & P17 & \multirow{4}{*}{$\mathrm{Si}$} & & $\mathrm{Si}$ \\
\hline & 40 & P14 & & & $\mathrm{Si}$ \\
\hline & 50 & S26/S27 & & & \\
\hline & 60 & P19 & & & $\mathrm{Si}$ \\
\hline
\end{tabular}

Tabla 10.6: Configuraciones utilizadas en los experimentos.

De tal manera que tales configuraciones serán evaluadas en base a los indicadores presentados en el Capítulo 9.

\subsubsection{Simulando el Modelo.}

El primer paso es la apertura del modelo a simular, un Modelo puede estar asociado a un grupo de productos que pueden ser fabricados con los recursos de dicho modelo, es por ello que en la selección del Modelo a simular se indica el producto que se va a fabricar para visualizar los modelos que han sido asociados a tal producto (Figura 10.7). 


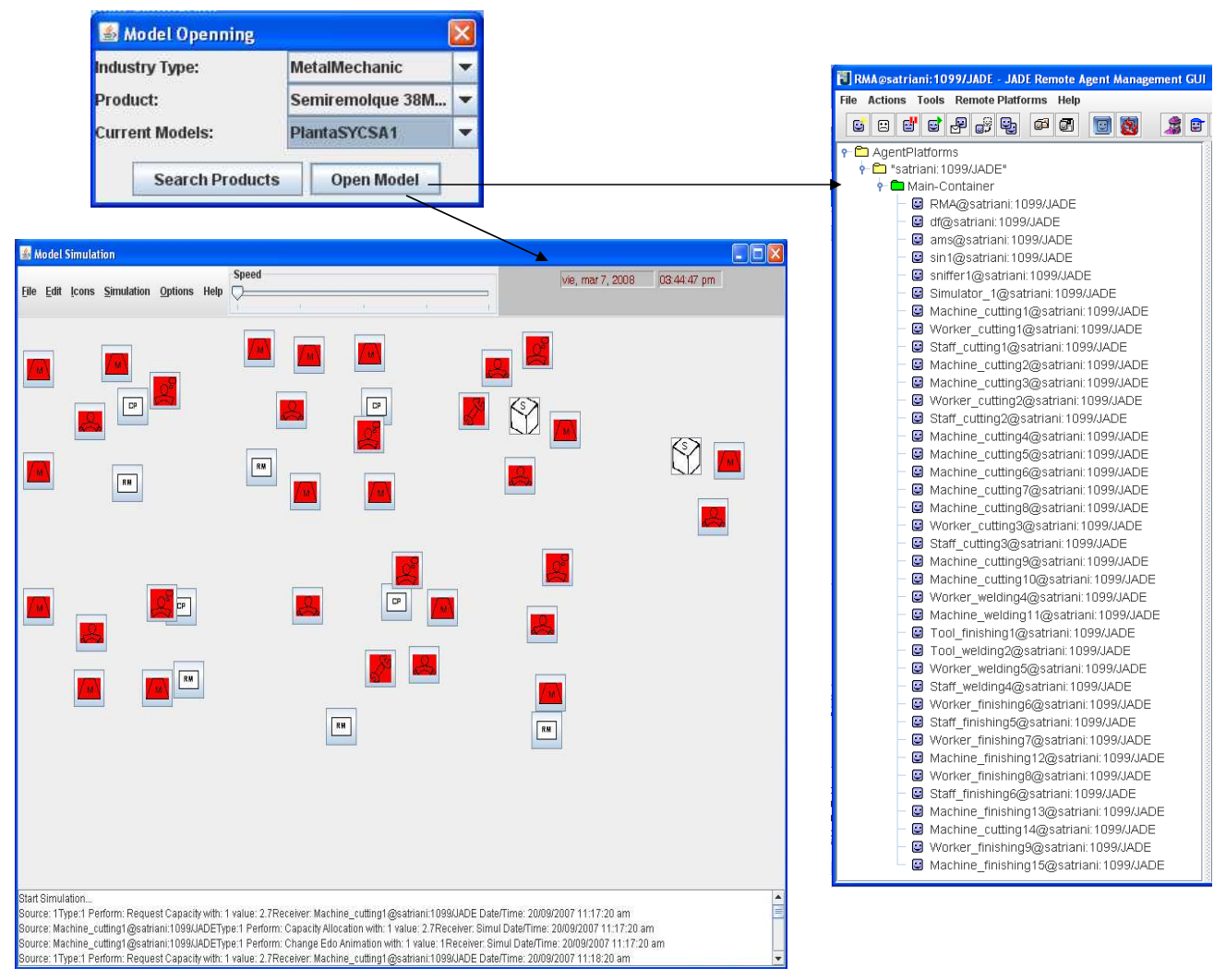

Figura 10.7: Abriendo el Modelo.

En el momento en que el Modelo se selecciona, se extrae no sólo los elementos y la distribución física que se visualiza del modelo, sino que además se crean todos los agentes relacionados a cada icono (Figura 10.8). Cada agente activado ofrece servicios acorde al tipo de elemento y las características de la instancia que representa.

Así, el Modelo de Agentes creado en la Fase I, se traduce en un nuevo Sistema Multiagente con el se comunica el Entorno de Simulación. A partir de ese momento, el Entorno de Simulación mostrará al Usuario el estado interno de cada uno de los elementos del Modelo. 


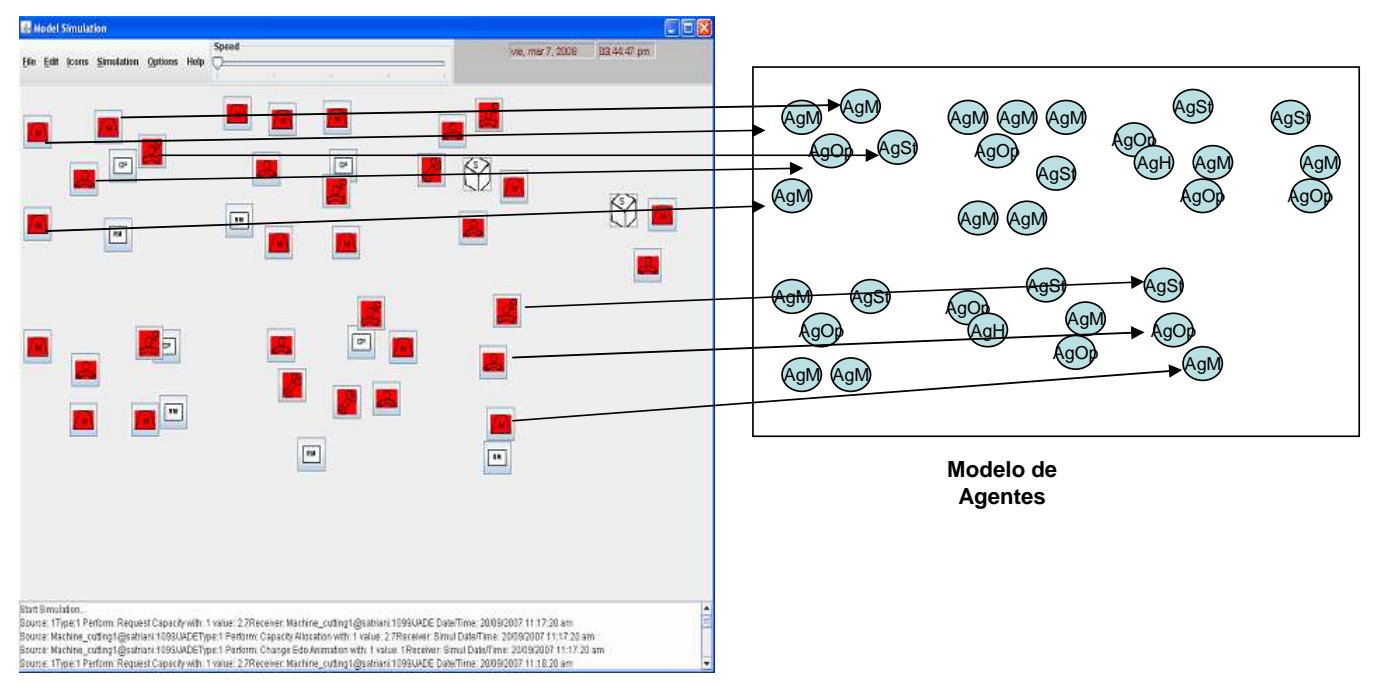

Figura 10.8: Agentes activos relacionados con el Modelo.

Antes de lanzar una Orden de Producción es necesario definir la condición de parada de la simulación, aunque la simulación puede detenerse parcial o totalmente a petición del Usuario. Además en ese momento resulta importante el definir la velocidad de ejecución de la simulación (Figura 10.11) en este caso, la simulación se ejecuta a 100x.
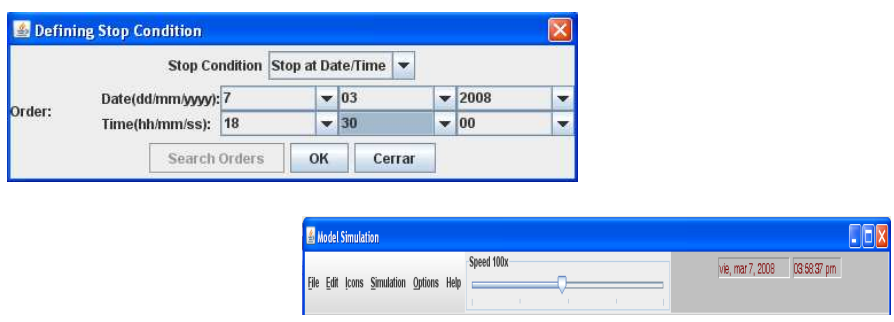

Figura 10.9: Definiendo Condición de Parada y Velocidad de Simulación.

La sincronización de los relojes es un aspecto relevante para la coordinación de las actividades de los agentes, de esta forma, al momento de ajustar la velocidad, los relojes de los agentes verifican su sincronización (Figura 10.10) puesto que todos se acelerarán o disminuirán cuando el Agente de Simulación se los indique. 

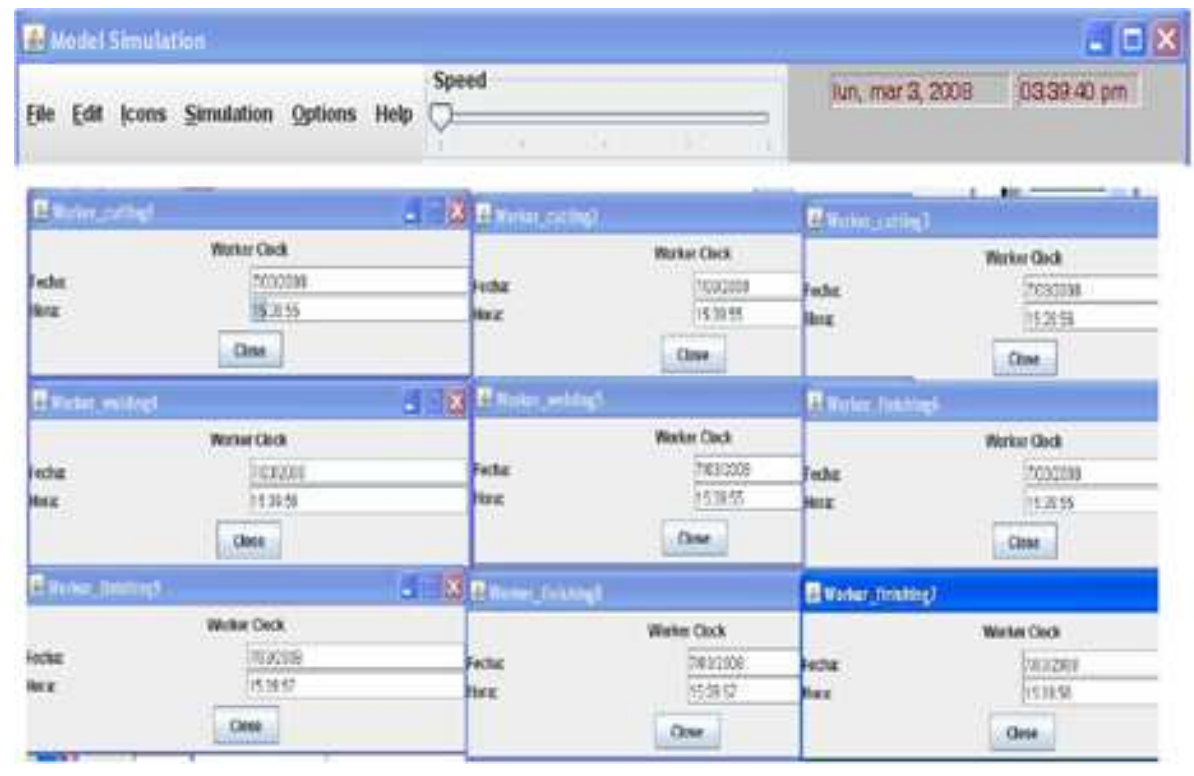

Figura 10.10: Sincronización del Reloj del Agente Simulador y los Relojes de los Agentes de la Planta de Fabricación.

\subsubsection{Lanzando a Simular una Orden de Producción}

Para lanzar una Orden de Producción a Simulación es necesario seleccionar el Producto a Fabricar, cantidad, la ruta de fabricación, fecha requerida, y su prioridad. Debido a que un producto puede tener más de una posible ruta de fabricación, en este momento es posible seleccionar una de dichas rutas para su evaluación. Los productos que se muestran en el listado de la interfaz, son aquellos que previamente han sido asociados al modelo en la Fase de Creación de Modelo (Figura 10.11).

En la Figura 10.11 se puede observar, cómo, al seleccionar la ruta, aparece el listado de las operaciones (o tareas) que la integran. Para validar diferentes configuraciones, se requiere que el Usuario defina qué recursos del modelo serán los que ejecuten cada una de las operaciones. 


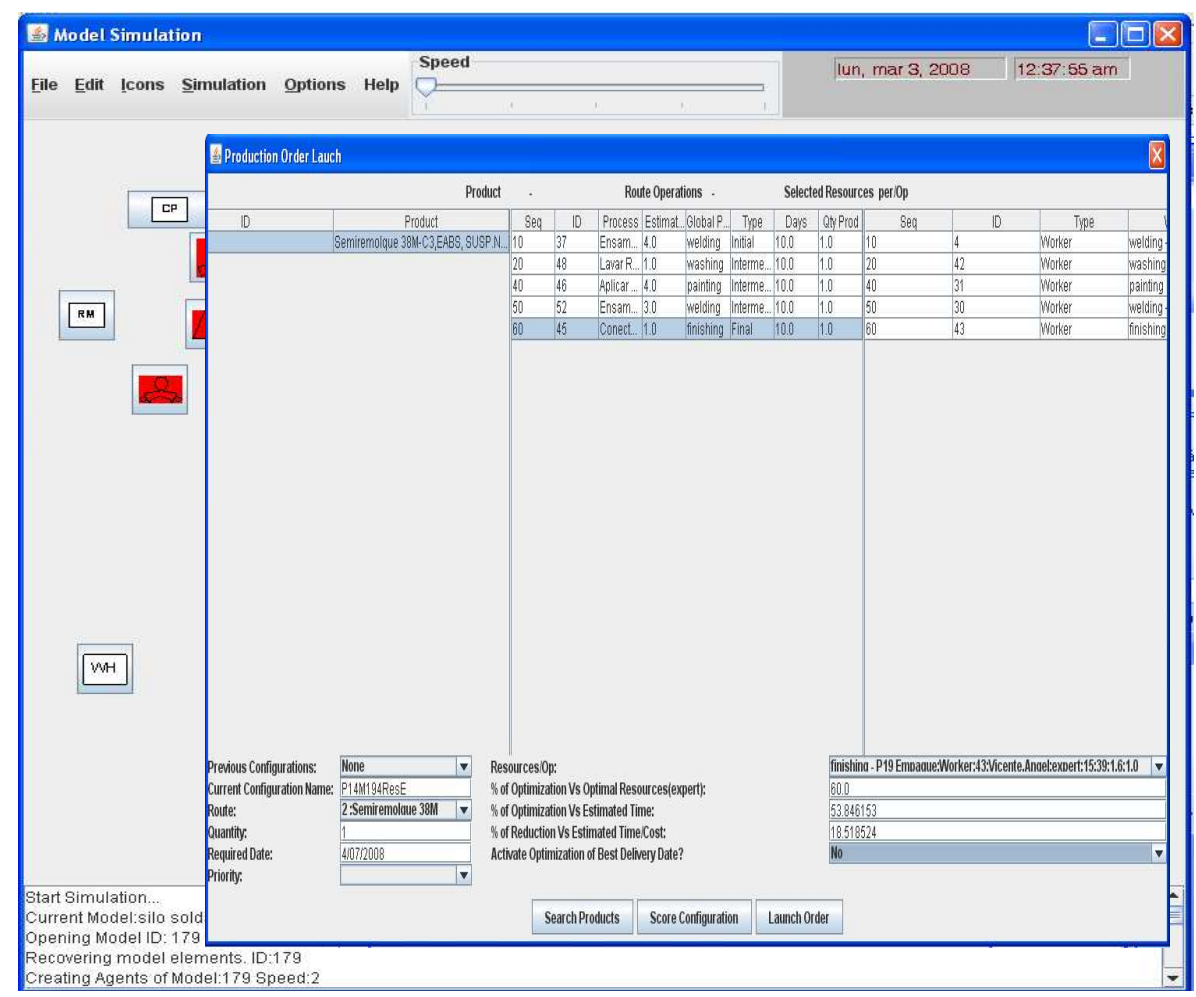

Figura 10.11: Lanzando una Orden de Producción.

De esta forma, cuando el Usuario selecciona cada una de las operaciones, en el apartado de "Recursos Disponibles" (Available Resources) aparecen aquellos recursos que por su naturaleza (p.ejem. si la operación implica el corte de partes, solo aparecerán aquellos operarios, máquinas y/o herramientas que pueden ejecutan tareas de corte) pueden ser seleccionados para llevar a cabo dicha operación.

Una vez que el Usuario ha seleccionado los recursos para cada operación, tal información queda vinculada a la orden para sus posteriores procesos de negociación y asignación de tareas.

Al lanzar la orden no sólo se crea el icono que representa los elementos del modelo, sino que se crea su correspondiente agente. El agente de Orden de Producción entonces inicia el proceso de negociación para la asignación de tareas con los Recursos de la Planta. El proceso de negociación para la asignación de las tareas a los recursos sigue los protocolos descritos en la Sección 7.2. 
En la Figura 10.12 se muestran de manera gráfica los procesos de negociación que surgen entre los agentes de la Planta de Fabricación y el Agente de la Orden de Producción.

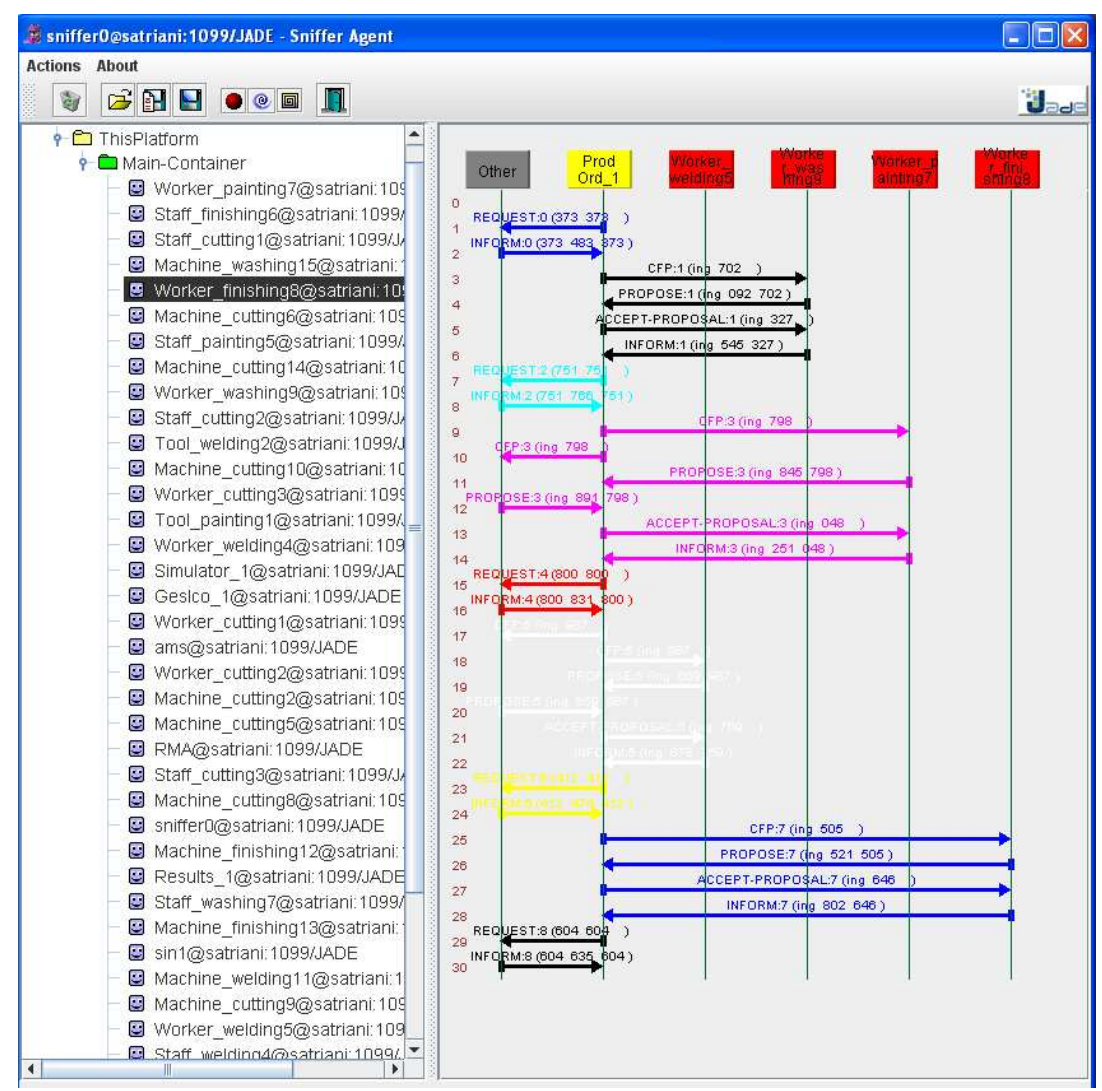

Figura 10.12: Negociación entre Agente de Orden de Producción y Agentes de Operarios.

Durante la simulación es importante visualizar el estado de los agentes, en este momento la animación gráfica de los iconos permite al usuario identificar el estado de los agentes. Como se mencionó en capítulos anteriores, la animación es una herramienta complementaria para la identificación de posibles situaciones de mejora. En la Figura 10.13 es posible observar la animación de los iconos cuando ofrecen servicios y cuando están negociando con la Orden de Producción. 


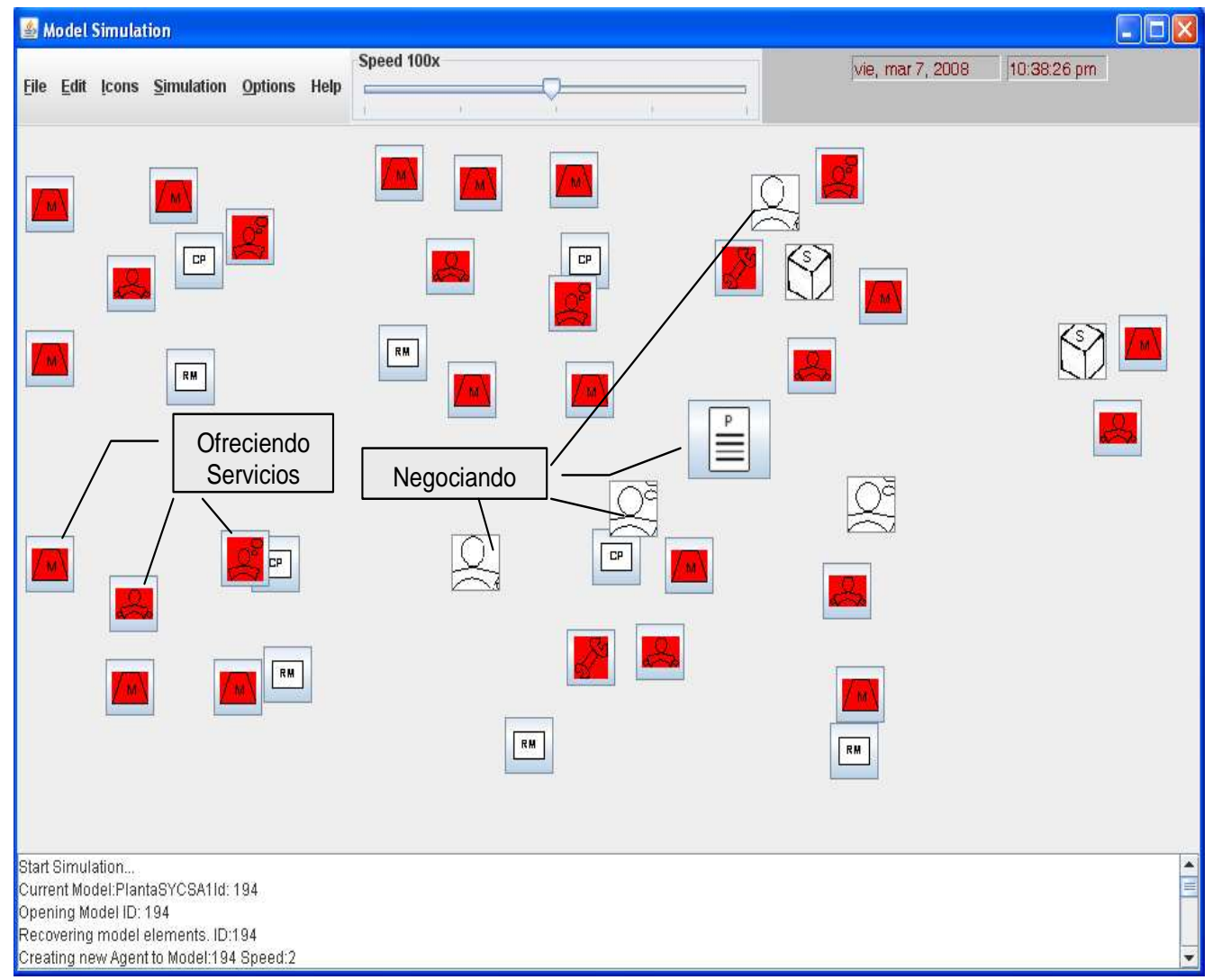

Figura 10.13: Animación Gráfica de Negociación entre Agentes.

A consecuencia del ajuste de la velocidad la cual acelera los procesos de negociación y de animación, resulta necesario que el Usuario también pueda consultar (en cualquier momento) el estado y el comportamiento del sistema y de cada uno de los agentes. Para ello, el prototipo permite observar información más detallada del estado actual del agente mediante interfaces. Y en su caso generar los reportes del comportamiento de los elementos del modelo.

En la Figura 10.14 se muestran algunas de las interfaces donde es posible observar detalles del agente, tales como su capacidad, turno, centro de trabajo, y estado actual. En la misma figura es posible observar la configuración de animación del icono, la cual puede ser modificada a solicitud del Usuario. 


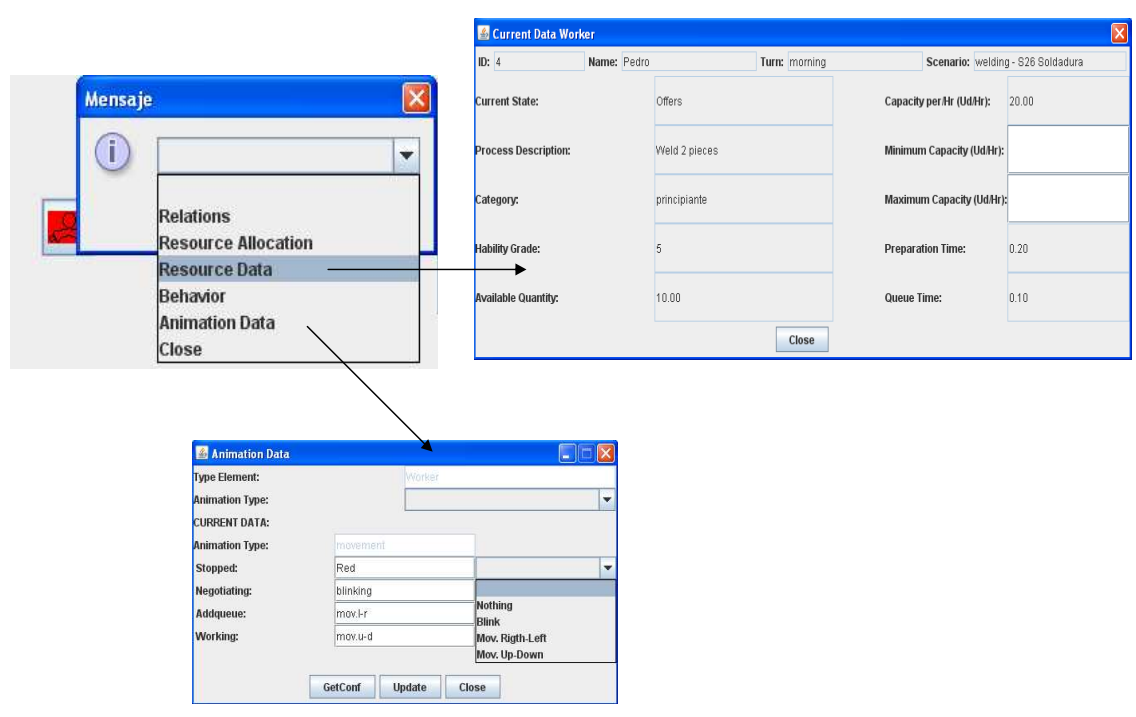

Figura 10.14: Datos del Agente y datos de su configuración de animación

A lo largo de la simulación, las negociaciones, así como las decisiones, tiempos de procesamiento son almacenados en la Base de Datos. Tal información es utilizada para extraer datos estadísticos que permitan evaluar tales resultados con respecto a los recursos.

\subsubsection{Modificando el Modelo}

En la simulación es posible la emulación de elementos que no existen en la planta física real, con el propósito de observar cómo se puede ver afectado el comportamiento de la planta ante la aparición de un nuevo elemento o en su caso ante la eliminación de un elemento actual. Para ello, en la Fase de Simulación es posible añadir/eliminar elementos individuales, tales como máquinas, herramientas, operarios a los centros de trabajo actuales o en su caso agregar o eliminar centros de trabajo. La modificación del Modelo es ejecutada en linea, es decir, no es necesario detener el proceso de simulación para agregar al nuevo agente. De esta forma, en caso de que durante la simulación se detecte un cuello de botella (p.ejem. una orden de producción no puede asignar una tarea de soldadura porque no encuentra un recurso disponible), se puede añadir un elemento (p.ejem. agregar una máquina con su respectivo operario en el centro de trabajo S26-Soldadura). Al añadir el nuevo elemento se solicita que se indique la instancia asociada a tal elemento y se crea su respectivo agente el cual pone a disposición sus servicios ante las peticiones futuras de la Orden de Trabajo. 
En la Figura 10.15 se observa como en el centro de trabajo S26-Soldadura se agregó una máquina y un operario adicional. En este caso es posible guardar esta modificación guardando el Modelo con un nuevo nombre "PlantaSycsa2".

Incluso es posible ejecutar al mismo tiempo la simulación del Modelo tal y como se definió originalmente y a la par ejecutar la simulación de dicho Modelo pero con elementos adicionales o elementos que han sido eliminados y así observar el comportamiento del sistema ante tales situaciones.

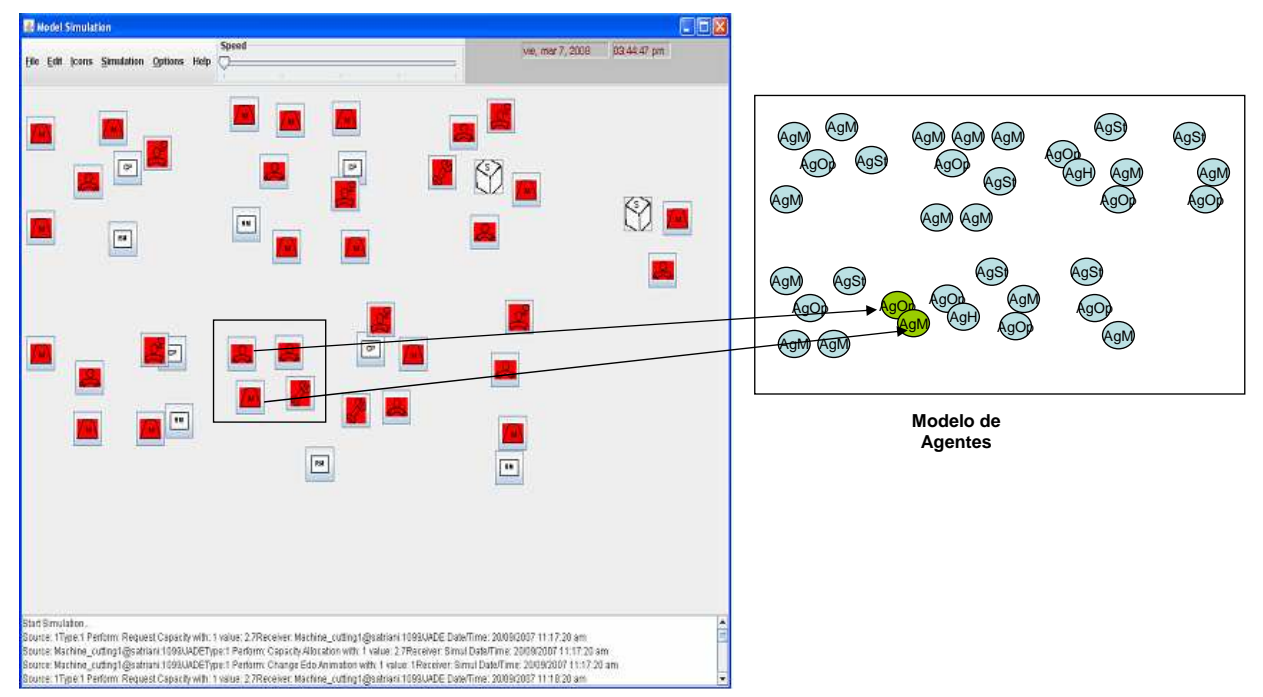

Figura 10.15: Elementos agregados en el Centro de Trabajo S26Soldadura.

\subsubsection{Deteniendo la Simulación}

Antes de lanzar la Orden de Producción hemos definido como condición de parada una fecha y hora específica, sin embargo, es posible detener total o parcialmente la simulación en cualquier momento (Figura 10.16). Cuando se detiene totalmente la representación gráfica desaparece de la interfaz, y los agentes son eliminados. Mientras que cuando se detiene parcialmente solo se detienen las tareas de los agentes sin eliminarlos permitiendo reanudar las actividades desde el punto en que se detuvieron. 


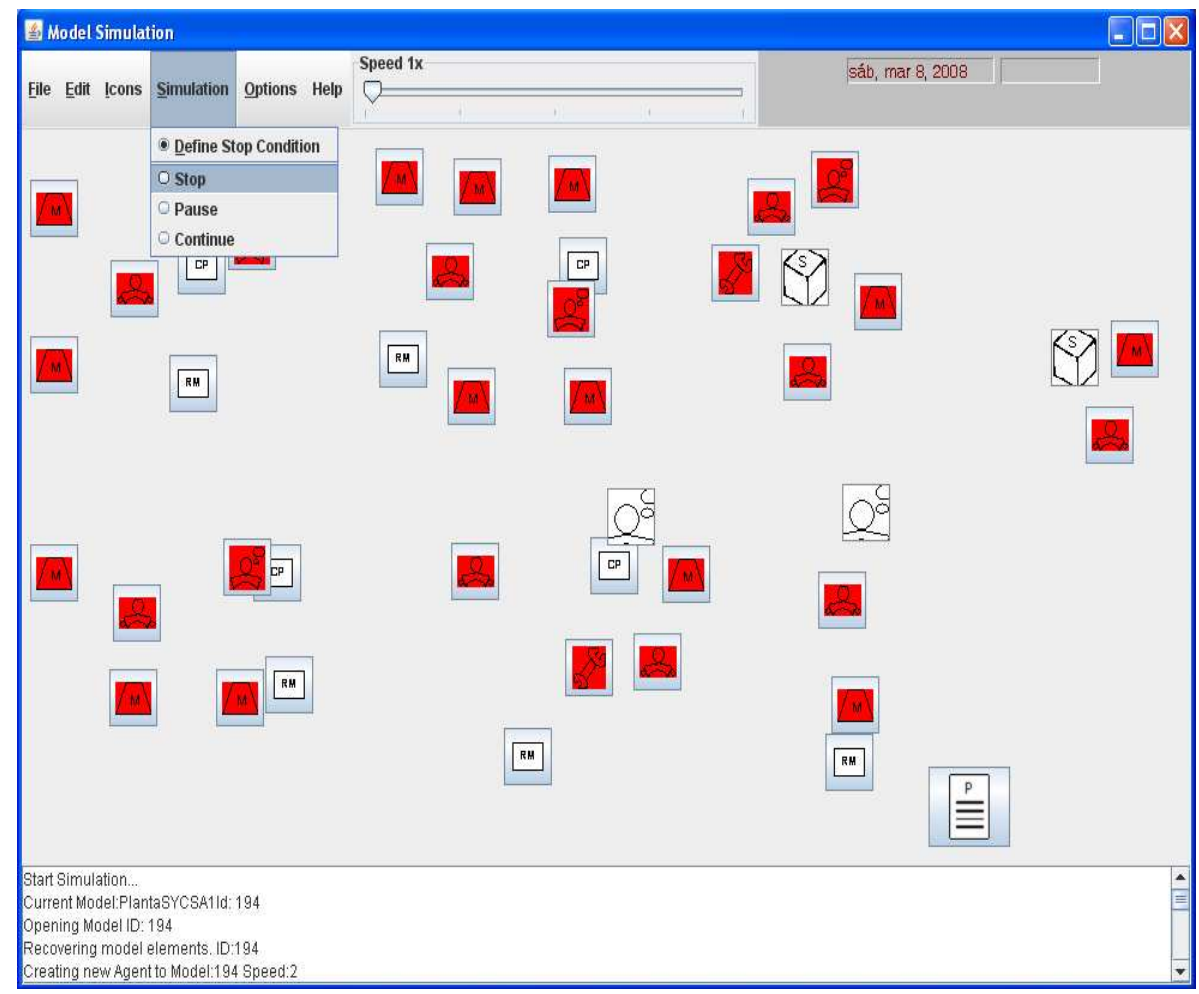

Figura 10.16: Deteniendo la Simulación.

\subsubsection{Análisis de Resultados de la Simulación}

Partiendo de la información estadística es posible obtener información que además de ser útil para la evaluación del Modelo, también lo es para retroalimentar a los agentes. La información analizada resulta de utilidad cuando debido a la aceleración del tiempo de simulación los procesos de negociación resultan costosos, en cuanto a tiempo, por lo que los agentes hacen uso de la información obtenida en simulaciones anteriores para identificar, por ejemplo, la ruta seguida con anterioridad para la fabricación de un producto dado.

En el momento que la Orden de Producción y su correspondiente agente se crean, el Agente obtiene de la Base de Datos los resultados de la información referente a simulaciones pasadas. Tal información es utilizada por el Agente de Orden de Producción para la asignación de tareas a los recursos reduciendo con ello el tiempo de negociación. En la Figura 10.17 se muestra el listado del proceso de negociación de la Orden 225 con los agentes disponibles para la fabricación 
de un Semiremolque. En la figura se observa cómo los agentes ofrecen sus servicios y deciden participar en la negociación de asignación de tareas así como los agentes que participaron en una negociación, y finalmente los agentes que procesaron la orden hasta su finalización.

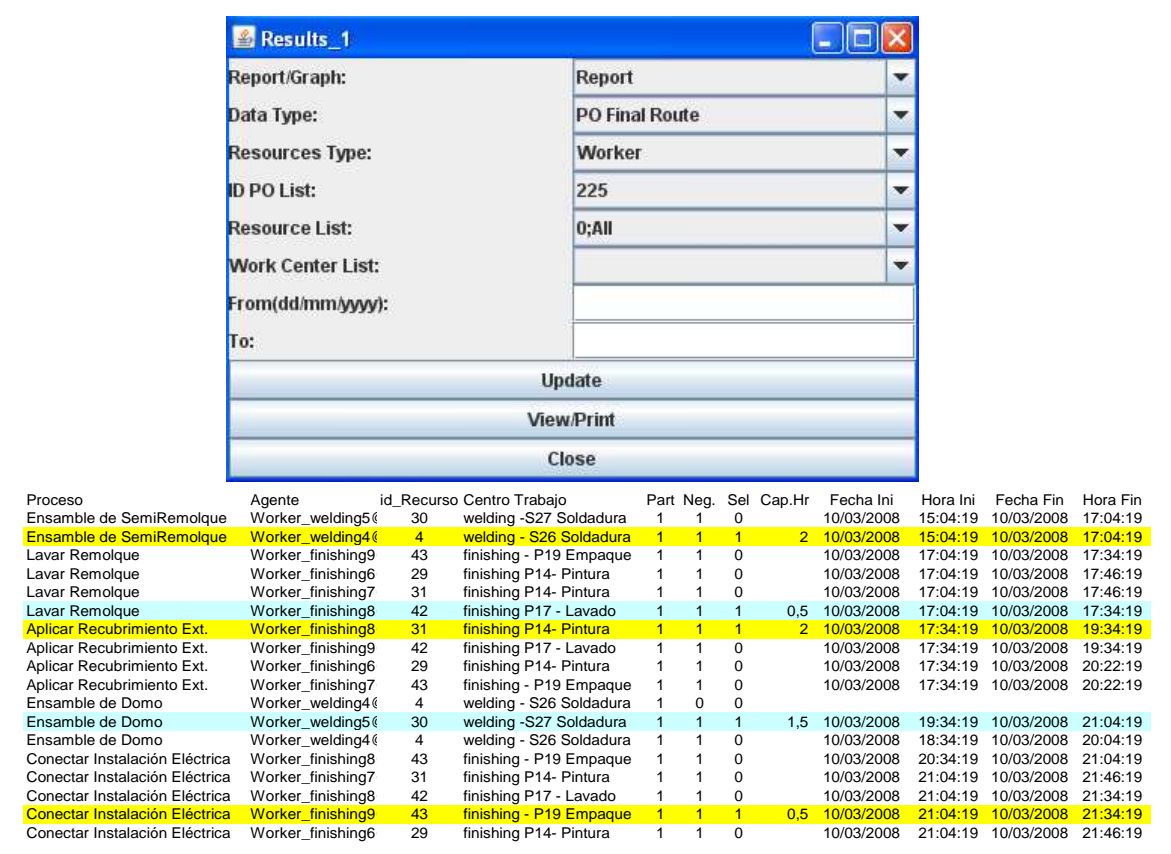

Figura 10.17: Proceso de Negociación y Ruta de Producción de Orden 225.

Este proceso que tiene una duración de 4,5 horas de tiempo de simulación, fue posible simularlo con nuestra herramienta en un tiempo de ejecución de 25 minutos basado en la aceleración de la velocidad definida en 100x.

Partiendo de los resultados estadísticos es posible identificar las tareas a las que fue asignado y el tiempo que se dedica a cada una. Este tipo de información permite al Usuario identificar las tareas que demandan una mayor carga en un recurso específico (Tabla 10.7). Este tipo de información también se puede obtener a nivel superior, esto es, identificar la carga de los Centros de Trabajo. Esta información es útil para identificar los cuellos de botella durante la Simulación. 


\begin{tabular}{clllc}
\hline ID_Recurso & Operarios & Categoria & Grado Destreza \\
\hline 43 & Vicente,Angel & expert & 15 \\
& & & \\
ID & Tarea & Capacidad Asignada (Hrs) & Tipo Proceso \\
\hline 45 & Conectar Instalación Eléctrica & 3,2 & finishing \\
46 & Aplicar Recubrimiento Ext. & 2 & painting \\
48 & Lavar Remolque & 1 & washing \\
\hline & Total & 6,2 &
\end{tabular}

Tabla 10.7: Carga de Trabajo de la Cuadrilla ID 43 por Tareas.

Aspectos tales como la identificación de cargas de trabajo por centros de trabajo asociado a Ordenes de Producción (Fig. 10.8), Rutas previas de asignación de tareas a productos, historial de asignación de carga de trabajo son algunos de los datos que utilizan los agentes para ofrecer sus servicios o reducir los tiempos de negociación y en su caso detectar recursos que se convierten en cuellos de botella y evitarlos buscando recursos alternativos. Con este tipo de análisis el sistema multiagente encuentra el soporte para el proceso de aprendizaje de los agentes.

\begin{tabular}{lr}
\hline Centro de Trabajo & Cap.Asig Hr \\
\hline welding -S27 Soldadura & 29 \\
painting P14- Pintura & 17,5 \\
finishing - P19 Empaque & 16,5 \\
welding - S26 Soldadura & 16 \\
washing P17 - Lavado & 5 \\
\hline
\end{tabular}

Tabla 10.8: Carga de Trabajo de Centros de Trabajo para el Modelo PlantaSycsa1.

Este tipo de información además permite comparar el comportamiento de diferentes Modelos ante la fabricación del mismo producto. En la Tabla 10.9 se puede observar cómo en el Modelo PlantaSycsa2 los centros de trabajo de soldadura welding-S26 y welding-S27 tienen una carga más distribuida en comparación con el posible cuello de botella del Modelo PlantaSycsa1. Esto se debe a que en el Modelo Planta Sycsa2 se agregó una máquina con su respectivo operario en el Centro de Trabajo (CT) welding-S26 Soldadura, con lo cual, el centro welding-S27 Soldadura dejó de ser un cuello de botella para rutas de fabricación con tareas de soldadura que solo podía atender el CT welding-S27. 


\begin{tabular}{lcc}
\hline Centro de Trabajo & PlantaSycsa1 & PlantaSycsa2 \\
\hline welding -S27 Soldadura & 29 & 21 \\
painting P14- Pintura & 17,5 & 17,5 \\
finishing - P19 Empaque & 16,5 & 16,5 \\
welding - S26 Soldadura & 16 & 24 \\
washing P17 - Lavado & 5 & 5 \\
\hline
\end{tabular}

Tabla 10.9: Comparativa de Carga de Trabajo en Centros de Trabajo para el Producto Semiremolque en dos Modelos de la Planta Silos.

\subsubsection{Análisis de Configuraciones de Planta}

La evaluación de las configuraciones de planta que ofrece el Sistema tienen una base específica para realizar comparativas. Las configuraciones son creadas de dos formas: a) definidas por el Usuario al crear una Orden de Producción y b) se crean como resultado de las negociaciones entre un Agente de Orden de producción y los Agentes de Recursos. Asimismo, es posible evaluar diferentes configuraciones de planta que han sido utilizadas para un mismo producto.

Este apartado se basa en los criterios de evaluación de configuraciones de planta expuestos en la Sección 9.3.2. Como se indica en dicho apartado, las bases del análisis residen en tres objetivos:

- Disminuir el tiempo de procesamiento de un producto.

- Disminuir el coste de procesamiento de un producto.

- Valorar el grado de destreza de los operarios y equipos (maquinaria y herramienta) utilizados.

Para la evaluación se han considerado 2 configuraciones seleccionadas por el Usuario (Config 2 y Config 3), una configuración obtenida mediante la negociación entre Agentes (Config 6) y una configuración estándar (Config Estd.).

Siguiendo los criterios establecidos para la calificación de la configuración de acuerdo al tipo de instancias donde se aplica la Ecuación $9.3\left(C c p=\sum_{i=1}^{N} C O p_{i}\right)$. El primer paso es definir los atributos de cada una de las configuraciones que se evaluarán respecto a dichos criterios. Con respecto al tipo de instancias, en la Tabla 10.10 se observa el tipo de instancias que se han seleccionado para cada una de las operaciones de la Ruta de Fabricación seleccionada la cual consta de cinco operaciones. 


\begin{tabular}{|c|c|c|c|c|}
\cline { 2 - 5 } \multicolumn{1}{c|}{} & \multicolumn{4}{c|}{ Configuraciones } \\
\hline Operaciones & ID 2 & ID 3 & ID 6 & Estd. \\
\hline 10 & Principiante & Principiante & Principiante & Principiante \\
20 & Principiante & Experto & Experto & Medio \\
40 & Experto & Principiante & Experto & Experto \\
50 & Principiante & Principiante & Principiante & Principiante \\
60 & Experto & Experto & Experto & Experto \\
\hline
\end{tabular}

Tabla 10.10: Tipo de Instancias Seleccionada por operación

Puesto que se valoran mejor los grados de destreza superiores de los recursos utilizados, resulta importante incluir un parámetro de valoración de la configuración por el tipo de instancias seleccionados. Asimismo, el grado de destreza de los recursos también está relacionado con los tiempos de procesamiento. De tal forma que basados en la configuración estándar, es posible la reducción o aumento del tiempo de procesamiento. En la Tabla 10.11 se observan los porcentajes que se han predefinido con respecto a la posible reducción /aumento del tiempo de procesamiento al comparar el tipo de instancias de cada configuración con respecto a la configuración estándar.

\begin{tabular}{|c|c|c|c|}
\cline { 2 - 4 } \multicolumn{1}{c|}{} & \multicolumn{3}{c|}{ Tipo Instancia en Configuración Estándar } \\
\hline Tipo Instancia Config. Selec. & Principiante & Medio & Experto \\
\hline Principiante & - & Aumento $30 \%$ & Aumento $50 \%$ \\
Medio & Reducción $30 \%$ & - & Aumento $20 \%$ \\
Experto & Reducción $50 \%$ & Reducción $20 \%$ & - \\
\hline
\end{tabular}

Tabla 10.11: Porcentajes de Reducción/Aumento de Tiempo de Procesamiento por Tipo de Instancias

Ejemplo: Si en la configuración estándar el tipo de instancia seleccionado es "Principiante" con un tiempo de procesamiento de 4 horas, y en la configuración utilizada se selecciona una instancia de tipo "Experto", el tiempo de procesamiento se reduce un $50 \%$, es decir a 2 horas. Por el contrario, si en la configuración estándar el tipo seleccionado es un "Experto" y en la configuración utilizada se selecciona una instancia de tipo "Medio", el tiempo de procesamiento aumenta un $20 \%$, es decir a 4,8 horas.

Finalmente, para efectos del análisis, las calificaciones de las instancias han sido normalizadas a un coste estándar de penalización (a menor grado de destreza mayor valor de penalización) de acuerdo al tipo de instancia. De esta forma el coste de un Experto es de 1, el de un Intermedio es 2 y el de un Principiante es 3 . 
Como resultado de la aplicación de la Ecuación 9.3 se obtiene la Calificación de la Configuración $(C c p)$ y al aplicar la Ecuación 9.4 se obtiene el Porcentaje de Optimización de la Configuración $(P O C)$. En la Tabla 10.12 se observa la valoración de la configuración de cada una de las operaciones.

\begin{tabular}{|c|c|c|c|c|c|}
\cline { 2 - 6 } \multicolumn{1}{c|}{} & \multicolumn{5}{c|}{ Configuraciones } \\
\hline Operaciones & ID 2 & ID 3 & ID 6 & Estd. & Óptima \\
\hline 10 & 3 & 3 & 3 & 3 & 3 \\
20 & 3 & 1 & 1 & 2 & 3 \\
40 & 1 & 3 & 1 & 1 & 3 \\
50 & 3 & 3 & 3 & 3 & 3 \\
60 & 1 & 1 & 1 & 1 & 3 \\
\hline Ccp: & 11 & 11 & 9 & 10 & 15 \\
POC: & 73,33 & 73,33 & 60 & 66,66 & 100 \\
\hline
\end{tabular}

Tabla 10.12: Valoración de Tipo de Instancias de Configuraciones

En la Figura 10.18 se observa la gráfica comparativa de la evaluación de las configuraciones acorde al tipo de instancias.

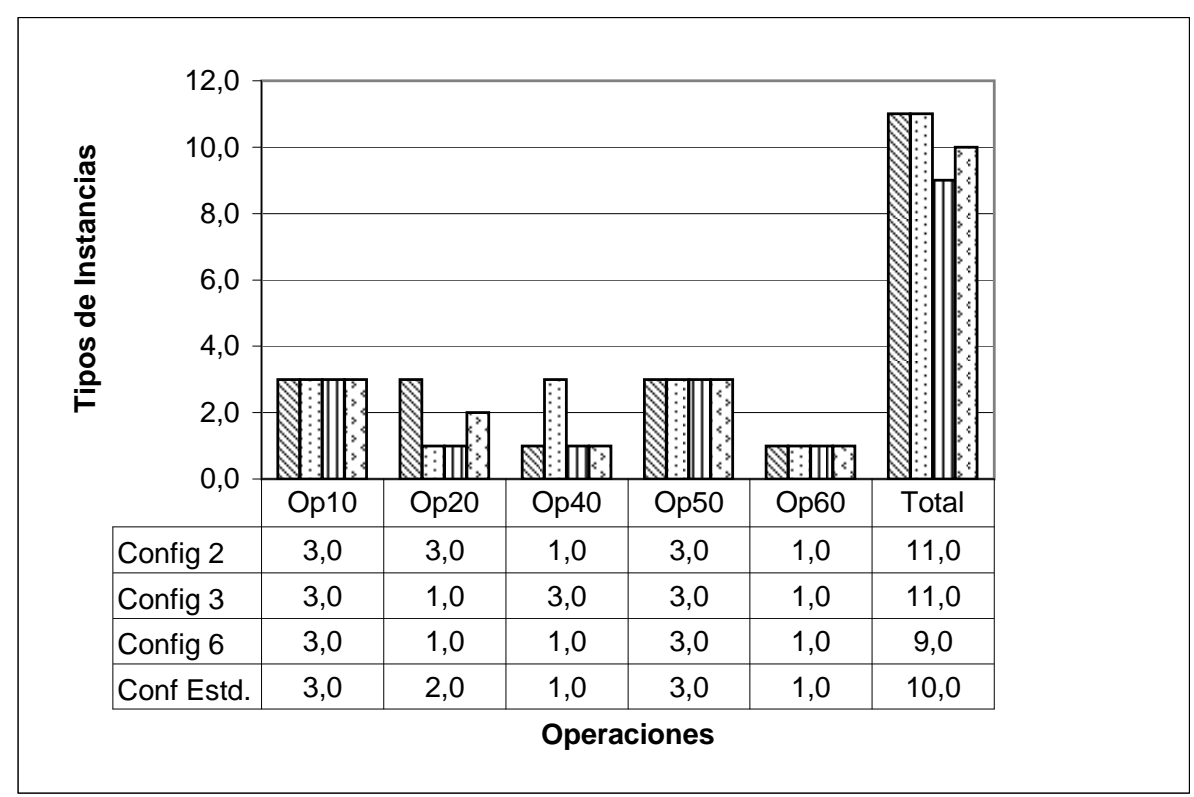

Figura 10.18: Comparativa de Configuraciones acorde al Tipo de Instancias

El tiempo de procesamiento esperado de la configuración se obtiene aplicando la Ecuación $9.7\left(T m p P_{E}=\sum_{i=1}^{N}\left(\sum_{j=1}^{M} T m R_{i j}\right)\right)$. Al aplicar la Ecuación 9.8 se obtiene el Porcentaje de reducción de Tiempo de Procesamiento Esperado de 
la Configuración (POTmEC) con respecto al Tiempo de Procesamiento Esperado de la Configuración Estándar (TmpEE).

En la Tabla 10.13 se observa el Tiempo de Procesamiento de cada una de las operaciones de la Ruta de Fabricación asignada a los recursos ( $T m R)$ así como su tiempo estándar.

\begin{tabular}{|c|c|c|c|c|}
\cline { 3 - 5 } \multicolumn{2}{c|}{} & \multicolumn{4}{|c|}{ Configuraciones (TmR) } \\
\hline Operaciones & Tmp. Est. & ID 2 & ID 3 & ID 6 \\
\hline 10 & 4,0 & 4,0 & 4,0 & 4,0 \\
20 & 1,0 & 1,0 & 0,5 & 0,5 \\
40 & 4,0 & 2,0 & 4,0 & 2,0 \\
50 & 3,0 & 3,0 & 3,0 & 3,0 \\
60 & 1,0 & 0,5 & 0,5 & 0,5 \\
\hline Total TmpPE $:$ & 13,0 (TmpEE) & 10,5 & 12,0 & 10,0 \\
POTmEC: & - & 19,23 & 7,69 & 23,07 \\
\hline
\end{tabular}

Tabla 10.13: Tiempo de Procesamiento Esperado de Configuraciones y porcentaje de Reducción respecto al Tiempo Estándar

En la Figura 10.19 se observa la gráfica comparativa de la evaluación de las configuraciones acorde al tiempo de procesamiento.

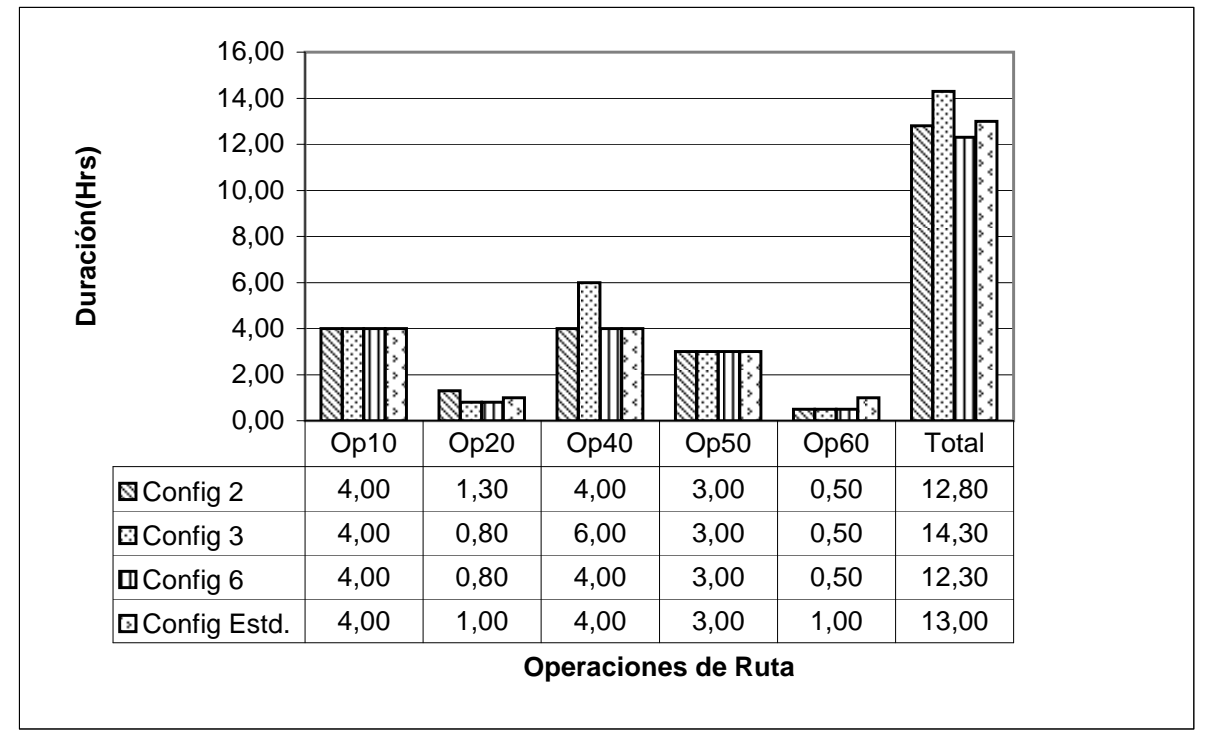

Figura 10.19: Comparativa de Configuraciones acorde al Tiempo de Procesamiento 
Con respecto al coste de la orden, éste se obtiene en función de la instancia seleccionada y el tiempo de procesamiento asignado. En la Tabla 10.14 se observa el coste de procesamiento de cada una de las operaciones. El coste se basa en los datos del tiempo de procesamiento presentados en la Tabla 10.13 para cada una de las operaciones. El coste de una configuración $\left(C_{R}\right)$ se obtiene aplicando la Ecuación $9.5\left(C_{R}=\sum_{i=1}^{N}\left(\sum_{j=1}^{M} T m R_{i j} * C h r R_{j}\right)\right)$. Al aplicar la Ecuación 9.8 se obtiene el Porcentaje de reducción de Tiempo de Procesamiento Esperado de la Configuración con respecto a la Configuración Estándar (POTmEC). Al aplicar la Ecuación 9.6 se obtiene el Porcentaje de Optimización del Coste la Configuración (POCte) con respecto al Coste de la Configuración Estándar $(C t e C E)$.

Para efectos del análisis, los costes de las instancias han sido normalizados a un coste estándar promedio por hora de acuerdo al tipo de instancia. De esta forma el coste por hora de un Experto es de 2, 4, el de un Intermedio es 1, 6 y el de un Principiante es 0,8 . Así, el coste por operación se obtiene multiplicando el tiempo de procesamiento de la operación por el coste estándar del tipo de instancia seleccionado (Tabla 10.10).

Ejemplo: Para la configuración ID 2, el coste de la operación 10 donde su tiempo de procesamiento es de 4,0 horas y el tipo de instancia seleccionada fue de un Principiante con un coste por hora de 0,8 . El coste total se obtiene multiplicando $4,0 * 0,8$ de tal forma que el coste sería igual a 3,2 .

\begin{tabular}{|c|c|c|c|c|}
\cline { 2 - 5 } \multicolumn{1}{c|}{} & \multicolumn{4}{c|}{ Configuraciones } \\
\hline Operaciones & ID 2 & ID 3 & ID 6 & Estd. \\
\hline 10 & 3,20 & 3,20 & 3,20 & 3,20 \\
20 & 1,40 & 1,92 & 1,92 & 1,60 \\
40 & 9,60 & 4,80 & 9,60 & 9,60 \\
50 & 2,40 & 2,40 & 2,40 & 2,40 \\
60 & 1,20 & 1,20 & 1,20 & 2,40 \\
\hline$C_{R}:$ & 17,44 & 13,52 & 18,32 & $19,20(\mathrm{CteCE})$ \\
POTmEC & 9,16 & 29,58 & 4,5 & - \\
\hline
\end{tabular}

Tabla 10.14: Coste de Configuraciones

En la Figura 10.20 se observa la gráfica comparativa de la evaluación de las configuraciones acorde al coste de procesamiento. 


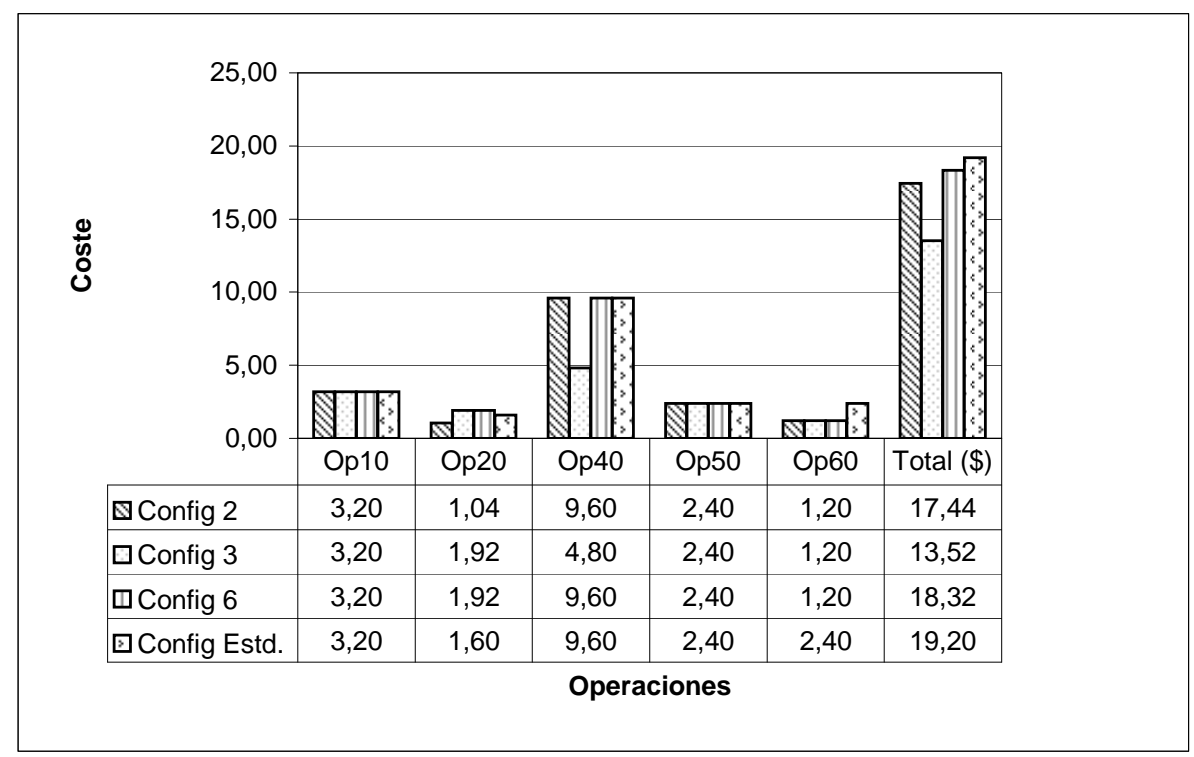

Figura 10.20: Comparativa de Configuraciones acorde al Coste

Como se indicó en la Sección 9.3.3, estos análisis previos permiten obtener una visión más clara respecto a la importancia de la definición de la configuración de la planta. Sin embargo, resulta necesario evaluar en conjunto tales atributos para identificar aquella que ofrece una mejor valoración global. Para ello, aplicaremos una técnica de evaluación multicriterio para normalizar los resultados obtenidos. Por tanto, se asignan pesos a cada uno de los atributos los cuales en conjunto de tal forma que conformaran el 100 por ciento del Total de la evaluación.

De esta forma, los pesos se asignaron como sigue: para el tiempo de procesamiento es del 0,4=>40\%, para el coste es el 0,4=>40\% y para el tipo de instancias el $0,2=>20 \%$. En la Tabla 10.15 se observa la evaluación global de las configuraciones.

\begin{tabular}{|l|c|c|c|c|}
\cline { 2 - 5 } \multicolumn{1}{c|}{} & \multicolumn{4}{c|}{ Configuraciones } \\
\hline \multicolumn{1}{c|}{ Criterios } & ID 2 & ID 3 & ID 6 & Estd. \\
\hline Tiempo de Procesamiento & 6,40 & 7,15 & 6,15 & 6,5 \\
Tipo de Instancias & 2,20 & 2,20 & 1,80 & 2,0 \\
Coste & 5,23 & 4,06 & 5,50 & 5,76 \\
\hline Totales: & 13,83 & 13,41 & 13,45 & 14,26 \\
\hline
\end{tabular}

Tabla 10.15: Evaluación Global de Configuraciones 


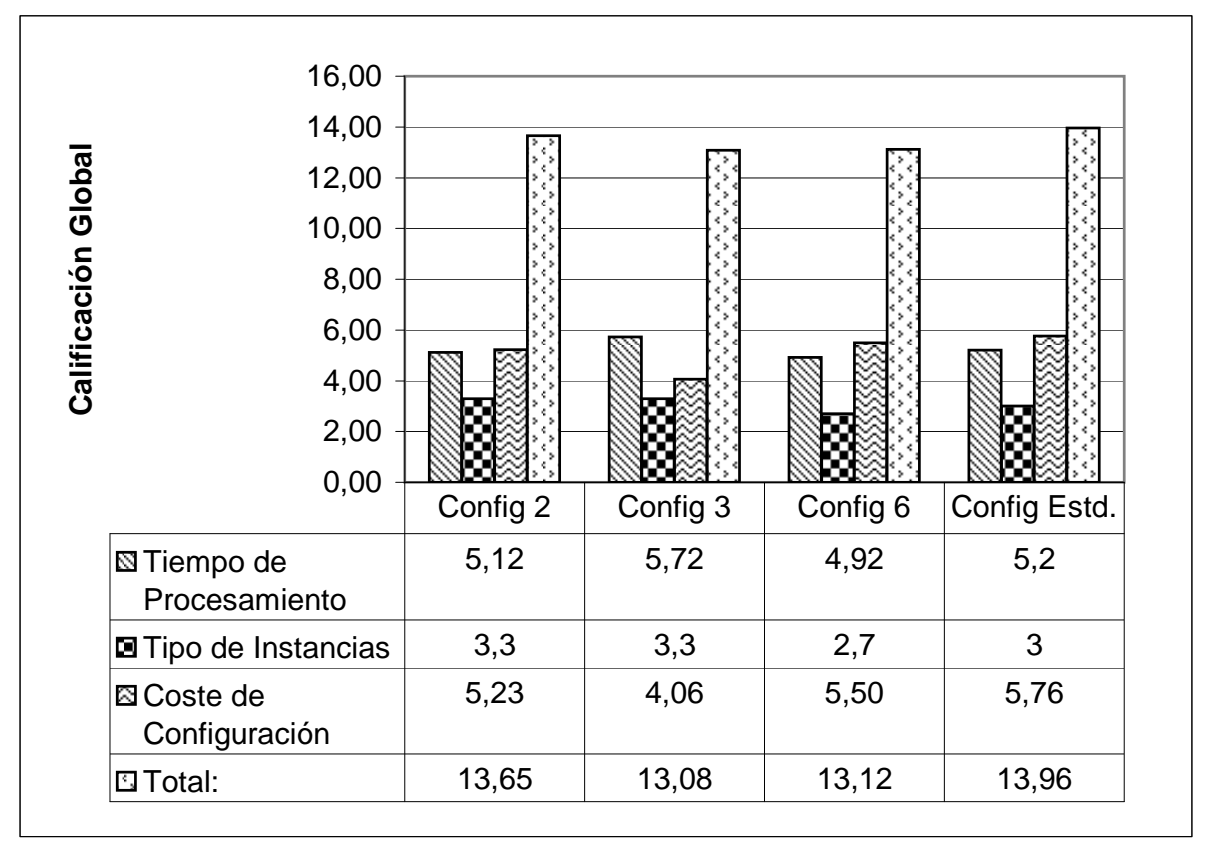

Figura 10.21: Comparativa de Calificación Global de Configuraciones

En la Figura 10.21 se observa la gráfica comparativa de la evaluación de las configuraciones acorde a la evaluación global.

De esta forma, es posible observar un panorama global de los beneficios e inconvenientes que ofrece cada una de las configuraciones. La evaluación global considera que quien obtenga una calificación menor será la mejor configuración. Sin embargo, es posible que se presente el caso en el cual no exista una diferencia significativa entre 2 o más configuraciones, como es el que se presenta en este análisis entre la Configuración 3 y 6 .

En este ejemplo, la configuración 3 obtiene la mejor calificación, pero es posible observar con respecto a la configuración 6 que su diferencia es mínima. La diferencia radica en 2 aspectos, mientras que en la Configuración 3 el tiempo de procesamiento es mayor con respecto a la Configuración 6, su coste es menor. Por tanto es posible realizar una segunda fase de análisis. En esta segunda fase se puede redefinir el objetivo basado en dos criterios: $a$ ) seleccionar la configuración con menor coste, o $b$ ) seleccionar la configuración con menor tiempo de procesamiento. De este modo, si se opta por el criterio $a$ ), la mejor configuración sería la Configuración 3 y si se opta por el $b$ ), la mejor configuración seria la Configuración 6. 


\subsubsection{Análisis de Tiempos Muertos por Configuraciones de Planta}

Para este análisis se consideró la fabricación del mismo producto gestionada mediante cinco órdenes de producción.

En la Figura 10.22 se observa la gráfica comparativa del Uso de Recursos utilizando diferentes configuraciones para el mismo número de órdenes.

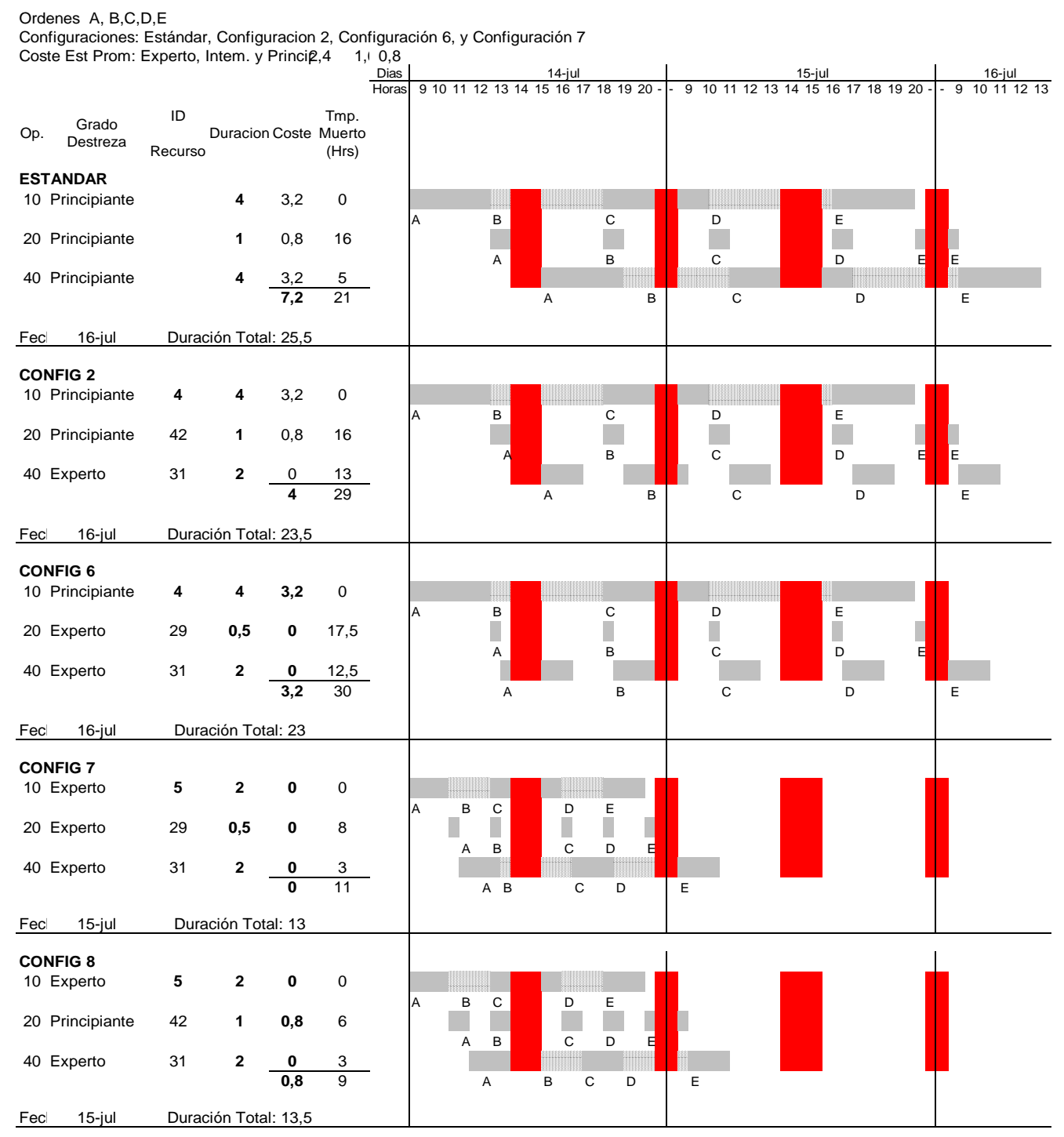

Figura 10.22: Comparativa de Uso de Recursos con Diferentes Configuraciones para 5 Ordenes 


\section{Asignación Directa de Tareas a Recursos.}

En la Figura 10.23 se observa la gráfica de la distribución de carga de trabajo de los recursos de la Planta de Fabricación cuando se aplica asignación directa de tareas a recursos en las cinco órdenes.

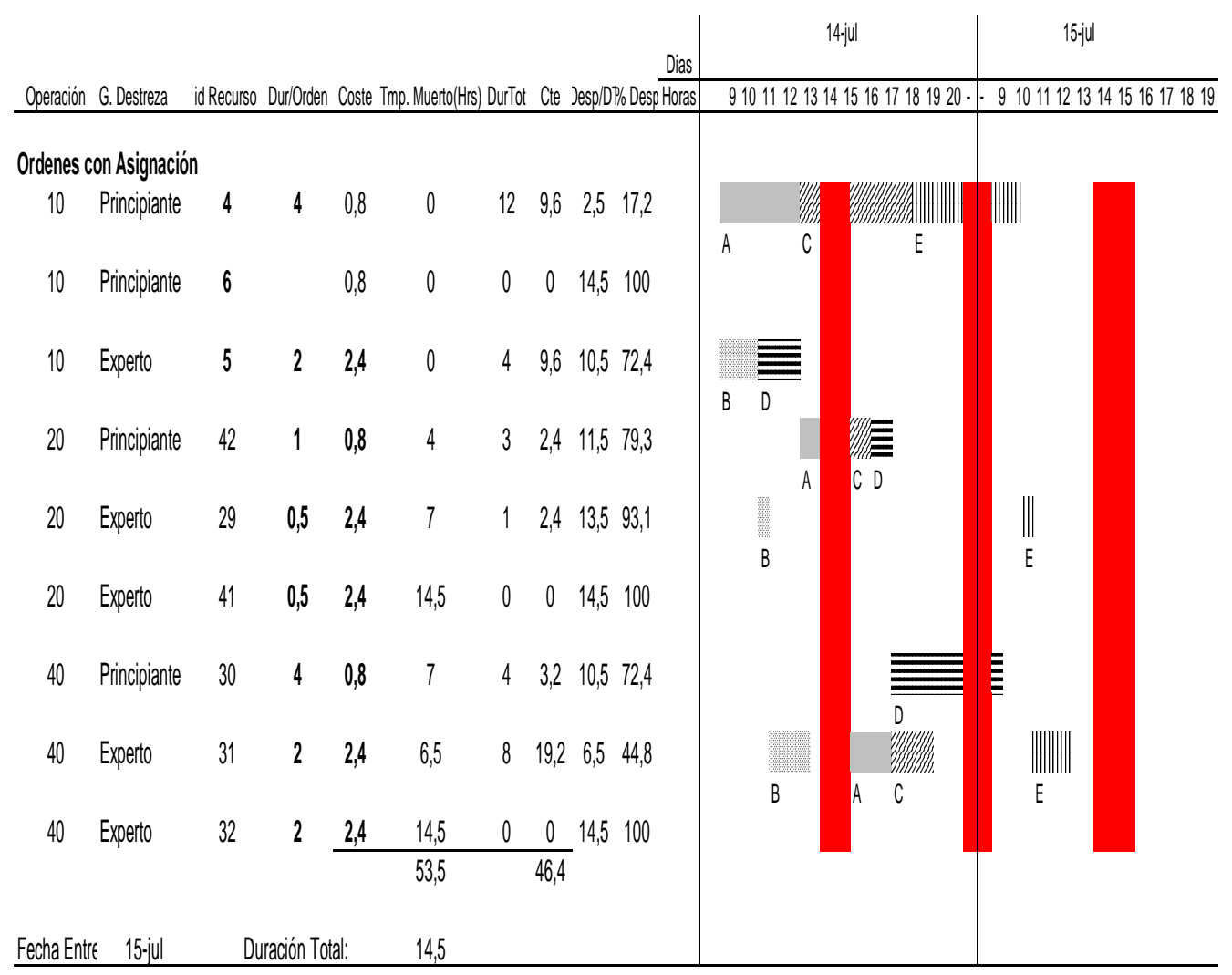

Figura 10.23: Distribución de Carga de Trabajo mediante Asignación directa para cinco órdenes

La asignación de tareas basada en una misma configuración trae consigo resultados tales como:

Situación: Cuándo el Usuario selecciona los recursos se requiere un menor tiempo para la asignación de tareas. Sin embargo, esto puede propiciar la aparición de cuellos de botella, subutilización de recursos y el aumento de tiempo muerto de la planta de fabricación. 
Solución: Una posible solución es la monitorización constante por parte del Usuario para identificar cuándo se está sobrecargando un recurso y cambie la asignación de tareas a otro recurso.

Observación: Esta situación promueve la subutilización de la capacidad de la planta de fabricación. Asimismo se desaprovecha el potencial del uso del paradigma de agentes y las técnicas de negociación, requiriendo que el Usuario este todo el tiempo monitorizando el comportamiento de la Planta para la toma de decisiones.

\section{Asignación por Negociación de Tareas a Recursos.}

En la Figura 10.24 se observa la gráfica de la distribución de carga de trabajo de los recursos de la Planta de Fabricación cuando se aplica asignación de tareas por negociación de recursos en las cinco órdenes.

\begin{tabular}{|c|c|c|c|c|c|c|c|c|c|c|c|c|}
\hline & & & & & & & & Dias & & & 14-jul & 15-jul \\
\hline Operación & G. Destreza & id Recul & Uur/Ord & Coste & Muertc & DurTot & Cte $)$ & esp $/ 1 \% \%$ Desp Horas & & 0111 & 14151617181920 & 910111213141516 \\
\hline Ordenes co & ion Negociac & & & & & & & & & & & \\
\hline 10 & Principiante & 4 & 4 & 0,8 & 0 & 4 & 3,2 & $4,5 \quad 52,9$ & B & & & \\
\hline 10 & Principiante & 6 & & 0,8 & 0 & 4 & 3,2 & $4,5 \quad 52,9$ & 7 & & & \\
\hline 10 & Experto & 5 & 2 & 2,4 & 0 & 6 & & $2,5 \quad 29,4$ & C & & & \\
\hline 20 & Principiante & 42 & 1 & 0,8 & 4 & & & $7,5 \quad 88,2$ & & D & & \\
\hline 20 & Experto & 29 & 0,5 & 2,4 & 5 & 1,5 & 3,6 & 782,4 & & & || & \\
\hline 20 & Experto & 41 & 0,5 & 2,4 & 4 & & & 894,1 & & & $E$ & \\
\hline 40 & Principiante & 30 & 4 & 0,8 & 5 & 2 & 1,6 & $6,5 \quad 76,5$ & & & 无 & \\
\hline 40 & Experto & 31 & 2 & 2,4 & 2,5 & 6 & 14,4 & $2,5 \quad 29,4$ & & & $|\|\||$ & \\
\hline 40 & Experto & 32 & 2 & 2,4 & $\frac{4,5}{25}$ & 2 & $\begin{array}{c}4,8 \\
47,2\end{array}$ & $6,5 \quad 76,5$ & & & & \\
\hline Fecha Entre & if $14-j u l$ & & ión To & $1: 8,5$ & 8,5 & & & & & & & \\
\hline
\end{tabular}

Figura 10.24: Distribución de Carga de Trabajo mediante Negociación para cinco órdenes

La asignación de tareas basada en técnicas de negociación trae consigo resultados tales como: 
Situación: Reducción de aparición de cuellos de botella, mejora en la utilización de recursos y distribución de carga de trabajo y la reducción de tiempo muerto de la planta de fabricación. La negociación sin embargo puede traer consigo un tiempo mayor para asignación de tareas.

Solución: Para la reducción del tiempo de negociación, una posible solución es la definición de algoritmos con la consideración de criterios que facilite la toma de decisiones al momento de asignar tareas.

Observación: Esta técnica reduce la aparición de cuellos de botella, ya que promueve la distribución uniforme de tareas. Así también se reduce el tiempo que el Usuario dedica a la monitorización constante.

\section{Combinación de Asignación de Tareas Directa y por Negociación a Recur-} sos.

En la Figura 10.25 se observa la gráfica de la distribución de carga de trabajo de los recursos de la Planta de Fabricación cuando se aplica asignación directa de tareas a recursos en 2 órdenes de producción (A,D) y la asignación por negociación en tres órdenes (B,C,E).

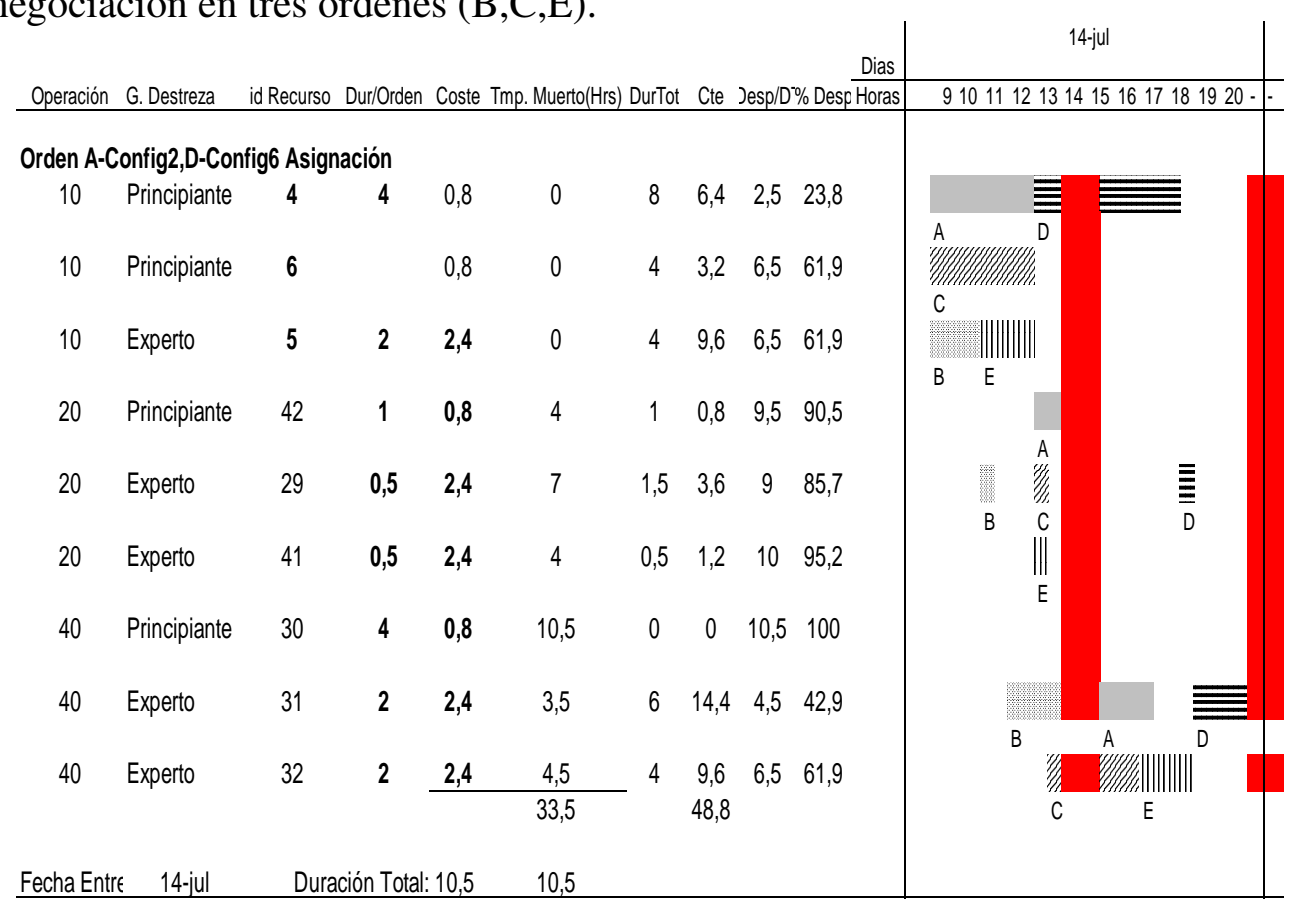

Figura 10.25: Distribución de Carga de Trabajo mediante Asignación y Negociación para cinco órdenes 
En la asignación combinada de tareas se pueden observar resultados tales como:

Situación: Posible aparición de cuellos de botella, posible subutilización de recursos, y posible reducción de tiempo muerto de la planta de fabricación. La asignación directa en este escenario, sigue propiciando el retraso de tareas (Orden D), con la aparición de cuellos de botellas. En cambio, la asignación por negociación promueve la distribución de trabajo.

Solución: Una posible solución es la monitorización constante por parte del Usuario para identificar cuando se está sobrecargando un recurso y reasignar tareas a otro recurso.

Observación: La mezcla de ambas técnicas promueve la distribución de trabajo. Sin embargo, con el uso prolongado de la asignación directa se corre el riesgo de subutilizar la capacidad de los recursos, requiriendo que el Usuario este todo el tiempo monitorizando el comportamiento de la Planta y tomar decisiones respecto a modificar las asignaciones.

\subsubsection{Aprendizaje de Agentes por refuerzo}

La información estadística obtenida es utilizada para efectos de aprendizaje de los agentes. Los agentes identifican aquellos estados en los que basados en simulaciones pasadas tomaron decisiones. Tales estados y las decisiones tomadas forman parte de las tuplas que se agregan a su base de conocimiento y que se utilizarán en procesos de negociación durante simulaciones futuras.

Los agentes de orden de producción pueden identificar aquellos recursos (máquinas, operarios, herramientas) que han sido utilizados en simulaciones pasadas en las operaciones asociadas al producto que tiene como objetivo fabricar. De esta forma, y basándonos en el planteamiento de la aplicación del aprendizaje por refuerzo presentado en la Sección 6.6. Los agentes de Recurso y de Orden se basan en las siguientes premisas.

En este caso de estudio, el agente de Orden de Producción con ID 225 tiene como objetivo: la fabricación de un Semiremolque38M-C3,EABS,SUSP.N y su entrega el 7 de abril de 2008. Por tanto, aplicando la estructura de la tupla que define el proceso de decisión de Markov para los Sistemas Multiagente, dicha estructura queda instanciada como sigue: 
$\mathbf{n}=$ Número total de agentes de orden lanzados en la Fase II - Simulación de Modelo: 2 Agentes de Orden de Fabricación.

$\mathbf{X}=$ Considerando que la Orden de Producción es quien inicia los procesos de comunicación y de negociación con los Recursos, los estados del Sistema comprenden:

- Identificar tareas pendientes de asignación de recursos. Para ello verifica el estado de las operaciones 10, 20, 40, 50 y 60 de la ruta del Semiremolque elegida (ruta: Semiremolque38M). En este caso las operaciones son secuenciales y no hay tareas paralelas.

- Negociación para la asignación de tareas. El objetivo es considerar el tiempo de procesamiento de cada una de las operaciones en donde se respete la fecha de entrega, por lo que la programación se inicia verificando la operación inicial y a partir de la fecha de entrega de dicha operación evitar los tiempos muertos durante el tiempo de fabricación.

- Monitorización del progreso de la Orden de Producción.

$\mathbf{U}=$ Las acciones que puede tomar el agente de Orden.

1. Obtener la siguiente tarea para asignar recursos. Mediante la monitorización recibe de los agentes la confirmación de la tarea aceptada por el(los) recursos y la(s) fecha(s) de entrega. De esta manera, basada en la fecha de entrega prometida y la lista de transiciones, para obtener la siguiente tarea se verifican dos cosas: 1) A partir de la tarea actual, se identifica(n) la(s) siguiente(s) tarea(s) destino. 2) A partir de dicha tarea destino, se verifica que todas sus tareas origen hayan sido asignadas y se toma como fecha inicial la fecha más lejana como la fecha mínima inicial de la tarea destino.

2. Identificación de agentes con servicios que incluyan un tipo de proceso específico. De acuerdo con la ruta de fabricación Semiremolque $38 M$, en la Tabla 10.16 se observan las operaciones con su tipo de proceso respectivo.

En el caso de la operación 10, para el proceso de soldadura, debido a que las máquinas requieren ser manipuladas los agentes que se ofrecen son los agentes de operarios WorkerWelding4 y WorkerWelding5. 


\begin{tabular}{|c|c|c|}
\hline Operación & Tipo de Proceso & Agentes Disponibles \\
\hline 10 & Soldadura & WorkerWelding4,Workerwelding5 \\
20 & Lavado & WorkerWashing3 \\
40 & Pintura & WorkerPainting8 \\
50 & Acabados & WorkerFinishing9 \\
60 & Acabados & WorkerFinishing11 \\
\hline
\end{tabular}

Tabla 10.16: Tipo de Proceso por Operación y Agentes que pueden atender las solicitudes.

3. Envío de solicitud de tarea, la cual incluye parámetros tales como: capacidad requerida en horas, fecha mínima y máxima de inicio y fin de tarea.

4. Recepción de propuestas de los agentes participantes. Ésta puede ser a favor o rechazar seguir participando. Para la operación 10 el WorkerWelding4 acepta y el WorkerWelding5 rechaza participar.

5. Selección del agente y asignación de tarea. En la operación 10, el agente de orden 225 le asigna la tarea de la operación 10 al agente WorkerWelding4. A su vez el agente WorkerWelding4 le confirma su aceptación y coloca la tarea en su cola de trabajos pendientes.

6. Recepción de avance en tareas asignadas. Para la operación 10, el agente WorkerWelding4 informará a la Orden del progreso en la fabricación (piezas terminadas y fecha/hora).

$\mathbf{f}=$ La función de probabilidad para la transición de estado estará dada por $f$ : $X x U x X \rightarrow[0,1]$. Cada vez que se asigna una tarea a un recurso, se guardan los datos de los elementos participantes relacionando, el producto, la operación y el recurso al que se le asignó la operación.

$\rho=$ Sera la función de recompensa dada por $\rho_{i}: X x U x X \rightarrow \mathcal{R}$.

De esta forma en las siguiente simulaciones, la orden aprende cuales son los recursos a los que puede asignarles la tarea de la operación asociada a un producto, ahorrando tiempo de negociación. Y por otra parte los recursos pueden identificar si anteriormente ya han aceptado trabajos relacionados con las operaciones de un producto específico.

\subsection{Conclusiones}

A lo largo de este caso de estudio hemos demostrado la aplicabilidad de nuestra propuesta de simulación soportada por agentes en un caso real del sector 
industrial. Asimismo, mediante el prototipo, hemos planteado cómo la Arquitectura del Entorno de Simulación, así como el Metamodelado para un Sistema de Fabricación soportados por el paradigma de agentes, ofrecen la funcionalidad necesaria para la definición de comportamientos complejos de un Sistema de Fabricación y su simulación. El entorno de simulación y los modelos están integrados por elementos inteligentes que interactúan respetando reglas de comportamiento predefinidas, pero que al mismo tiempo aportan flexibilidad y adaptabilidad mediante el aprendizaje continuo. Por tanto, en el prototipo se observa cómo dicha flexibilidad permite el diseño de modelos complejos, el diseño de experimentos, la simulación del comportamiento de los elementos que componen al modelo y la obtención de resultados que pueden ser utilizados para la toma de decisiones. La implementación del Entorno de Simulación basado en tales paradigmas traducida en una herramienta de fácil acceso a los Ingenieros de Diseño, hacen de ellos una herramienta con alto potencial de aplicación en el complejo entorno de la fabricación.

Esta propuesta permite a los Ingenieros de Diseño centrarse en la definición de los atributos de los elementos y la delimitación de agrupaciones (centros de trabajo) que se verán reflejados en comportamientos complejos. Por otra parte, el entorno de simulación ofrece al Ingeniero de Software la flexibilidad para integrar propuestas alternativas de control de la planta de fabricación mediante la integración de comportamientos adicionales que se activen bajo condiciones específicas. 



\section{Capitulo 11}

\section{Resultados de la Evaluación}

En este capítulo se presentan los resultados de la evaluación global de la implementación de la propuesta así como la evaluación de la funcionalidad del prototipo implementado y presentado en el Capítulo 8. Para la evaluación se aplican las bases presentadas en el Capítulo 9.

\subsection{Resultados de la Evaluación Global de la Im- plementación del Prototipo}

Tomando en consideración las métricas presentadas en la Sección 9.1, en esta sección se presentan los resultados de la evaluación global de la implementación del prototipo presentado en el Capítulo 8. La evaluación presenta el comportamiento del prototipo ante dichas métricas.

Facilidad de auditoria. La confirmación de cada uno de las etapas de comunicación entre los agentes es almacenada en la Base de Datos de tal manera que es posible identificar los agentes que han establecido comunicación, con quien se ha negociado, y a quien se le han asignado tareas durante la simulación. Las tablas de información que integran la Base de Datos, contienen claves de identificación que permiten vincular el detalle de las operaciones que se han llevado a cabo para la simulación de una orden de producción, el modelo, su configuración, y los resultados obtenidos.

Exactitud. Las tareas generan los resultados respetando los tipos de datos, y formato que serán almacenados en la base datos y que se intercambiarán entre los agentes como parte de mensajes con estructuras estándar. 
Consistencia. Todos los procesos se controlan mediante comportamientos predefinidos asociados a un tipo de agente. Los agentes utilizan protocolos de comunicación con mensajes que tienen estructuras estándar. Tales mensajes se procesan por los agentes acorde a comportamientos específicos, generando siempre acciones controladas con resultados consistentes.

Estandarización de datos. Los datos de entrada se han normalizado a fin de realizar operaciones sobre el mismo tipo de información, por otra parte la información generada tiene un formato predeterminado de tal manera que su análisis se puede realizar dentro de un marco común de interpretación.

Eficiencia de la ejecución. Durante el funcionamiento de la herramienta se puede comprobar la ejecución de las tareas solicitadas en los tiempos especificados. Algunas tareas son asíncronas y otras se activan acorde a los mensajes recibidos en los comportamientos.

Facilidad de expansión. Debido a que el prototipo de simulación se basa en la utilización de agentes, permite que se escale en dos sentidos: el comportamiento interno de los agentes y la facilidad para escalar los modelos creados. Los agentes actúan en base a la activación de comportamientos que se encapsulan como módulos independientes, lo que permite la integración de nuevos comportamientos que no afecten directamente a otros y en su caso sea posible la reutilización de los resultados de otros comportamientos o escalar los comportamientos existentes a fin de proporcionar una mayor flexibilidad. Por otra parte, los modelos creados pueden ser escalados integrando nuevos elementos al modelo. A dichos elementos se les asocian características específicas de una instancia, generándose un nuevo agente el cual puede comunicarse inmediatamente con el resto de los agentes.

Generalidad. La facilidad en la manipulación de metamodelos que se agrupan en tipos de holones sin especificar desde el inicio las características de las posibles instancias, permite al simulador la definición de una amplia variedad de modelos con características propias.

Modularidad. Debido a que los agentes actúan en base a la activación de comportamientos que se encapsulan como módulos independientes, se pueden integrar nuevos comportamientos que no afecten directamente a otros y en su caso sea posible la reutilización de los resultados de otros comportamientos o la expansión de los comportamientos existentes a fin de proporcionar una mayor flexibilidad. 
Facilidad de operación. Se han desarrollado interfaces de comunicación con el Usuario de tal manera que la ejecución de la simulación es transparente con respecto a las acciones que solicita el Usuario y los resultados que obtiene. De esta forma, los agentes que perciben las interacciones con el Usuario, integran dichos mensajes como parte de sus comportamientos procesándolos de forma transparente.

Autodocumentación. La herramienta ha sido desarrollada en el lenguaje Java y se ha empleado ECLIPSE como interfaz de desarrollo la cual proporciona una opción para la autogeneración de la documentación del programa. Cada vez que se crea o modifica un agente, es posible actualizar la documentación general de forma automática sin que el Usuario tenga que modificarla manualmente.

Facilidad de traza. Al igual que en control de auditoría, las tablas de información que integran la Base de Datos, contienen claves de identificación que permiten vincular el detalle de las operaciones que se han llevado a cabo para la simulación de una orden de producción, el modelo, su configuración, y los resultados obtenidos. Durante la simulación de una orden de producción, se crea además un archivo de texto que almacena las acciones en orden de la secuencia que van generando los agentes desde que inician sus actividades hasta que son eliminados.

\subsection{Resultados de la Evaluación de la Funciona- lidad del Prototipo}

Para llevar a cabo la evaluación de la Funcionalidad del prototipo de acuerdo con los criterios presentados en el Capítulo 9, se considera la participación de un grupo de ingenieros que han llevado a cabo el diseño de un Sistema de Fabricación. En dicha prueba se aplicó una evaluación basada en la lista de características ideales de una herramienta de simulación (Nikoukaran et al. 1999), se seleccionó un caso de estudio y se procedió a realizar su modelado con el prototipo implementado. Durante dicha prueba se utilizó un cuestionario que integra criterios acorde a cada una de las etapas:

- Durante el modelado: características tales como la medición de la facilidad y las opciones para integrar elementos en el modelo, posibilidades para configurar las atributos de cada uno, gama de opciones de recursos a 
utilizar (personal, maquinas, herramientas), y alcance en la definición de producto, orden de trabajo y de mantenimiento, son evaluados a criterio del ingeniero. La calificación asignada varía entre 1 y 5 , donde 5 se refiere a Fuerte (detallada) y 1 a Débil (poco detalle).

- Durante la simulación, en cambio, el ingeniero valora: características referentes a la animación de elementos, capacidad para controlar la ejecución de la simulación, capacidad de análisis de resultados, presentación de resultados en gráficas y reportes, capacidad para identificar estado actual de las entidades (en proceso, en espera, terminado). La calificación asignada varía entre 1 y 5 , donde 5 se refiere a Fuerte y 1 a Débil.

- Al finalizar la simulación el ingeniero valora: la capacidad de reutilización de los resultados para ejecutar replicaciones del modelo, opciones de visualización de resultados mediante reportes y gráficas, opciones para comparar las variaciones en los resultados de replicaciones y el grado de reutilización de los modelos generados previamente. La calificación asignada varía entre 1 y 5 , donde 5 se refiere a Fuerte y 1 a Débil.

Durante la evaluación de la Funcionalidad se han considerado los criterios presentados en las Secciones 9.2 y 9.3. El detalle de la evaluación de las características asociadas a dichos criterios se basa en las propuestas para cada etapa de la Simulación de Nikoukaran (Nikoukaran \& Paul 1999) y Banks et al. (Banks et al. 2001). Asimismo, en los resultados se presentan las aportaciones de la aplicación del paradigma de agentes.

Algunas características que no se han considerado para la evaluación se encuentran fuera del proceso de simulación (pero que se consideran como criterios importantes en la evaluación de una herramienta de simulación comercial), se refieren a funcionalidades adicionales. Entre ellas se encuentran: capacitación, documentación, tutoriales, soporte técnico y actualizaciones. Por otra parte, se han considerado también la facilidad para interoperar con otros entornos, la capacidad para integrar nuevos módulos o adaptar los existentes.

La evaluación de la interoperabilidad consiste en indicar las opciones que ofrece la herramienta, el tipo de entornos con los que se puede comunicar y mediante qué funcionalidad (uso de DLLS, archivos planos, etc.). En cuanto a la capacidad para escalar la herramienta, se indica qué utilidades ofrece para ello, alcance de las posibilidades de modificación de la herramienta en cuanto a personalización de interfaces y de los atributos asociados a los elementos del Sistema 
de Fabricación. La calificación asignada varía entre 1 y 5 , donde 5 se refiere a Fuerte y 1 a Débil.

En la Tabla 11.1 se muestra el detalle de las características contempladas en la etapa de Creación del Modelo y los resultados de la evaluación de las mismas. Para la segunda etapa correspondiente a la Simulación del Modelo: a) en la Tabla 11.1 se presenta la evaluación con respecto a la Creación de Modelos, b) en la Tabla 11.2 se presentan las características y resultados relacionados con el Entorno de Ejecución, c) en la Tabla 11.3 se presentan las características y resultados con respecto a la Animación, y d) en la Tabla 11.4 se presentan las características y resultados con respecto a la Experimentación y Análisis de los Resultados.

Basándonos en la información detallada presentada en las Tablas 11.1, 11.2, 11.3 y 11.4 , es posible presentar la evaluación global del entorno mediante la identificación de sus fortalezas o debilidades como se muestra en la Tabla 11.5.

Asimismo, la evaluación contempla, los requisitos de la nueva era de la fabricación y las aportaciones asociadas al paradigma de agentes que incluye la propuesta. En la Tabla 11.6 se presentan los requisitos que se ven afectados por las características del paradigma de agentes incluidos en la propuesta.

Las características asociadas a los Sistemas Multiagente que se contemplaron dentro de la propuesta permiten dar respuesta a los requisitos de la nueva era de la fabricación. Las características de movilidad y comunicación, favorecen la distribución de control puesto que permiten la definición de modelos con arquitecturas jerárquicas integradas por elementos proactivos, reactivos y autónomos con control propio sobre sus acciones. De tal manera que dichas características facilitan la definición de una Organización Distribuida.

En cuanto a la Reconfigurabilidad del Sistema de Fabricación, la definición de elementos flexibles, inteligentes, proactivos y tolerantes a fallos, permiten modelar un sistema de fabricación con un alto grado de adaptabilidad de los modelos conforme evolucionan las necesidades del diseñador. Esto facilita, por tanto, la Integración de la Empresa. Mediante la definición de los elementos del sistema de fabricación es posible integrar los atributos detalladamente, de tal forma que la reconfiguración de los mismos puede hacerse fácilmente (por ejemplo, la capacidad por hora, el tipo de proceso, el turno, el centro de trabajo y los algoritmos de control para los recursos de la planta y la ruta de fabricación a nivel 


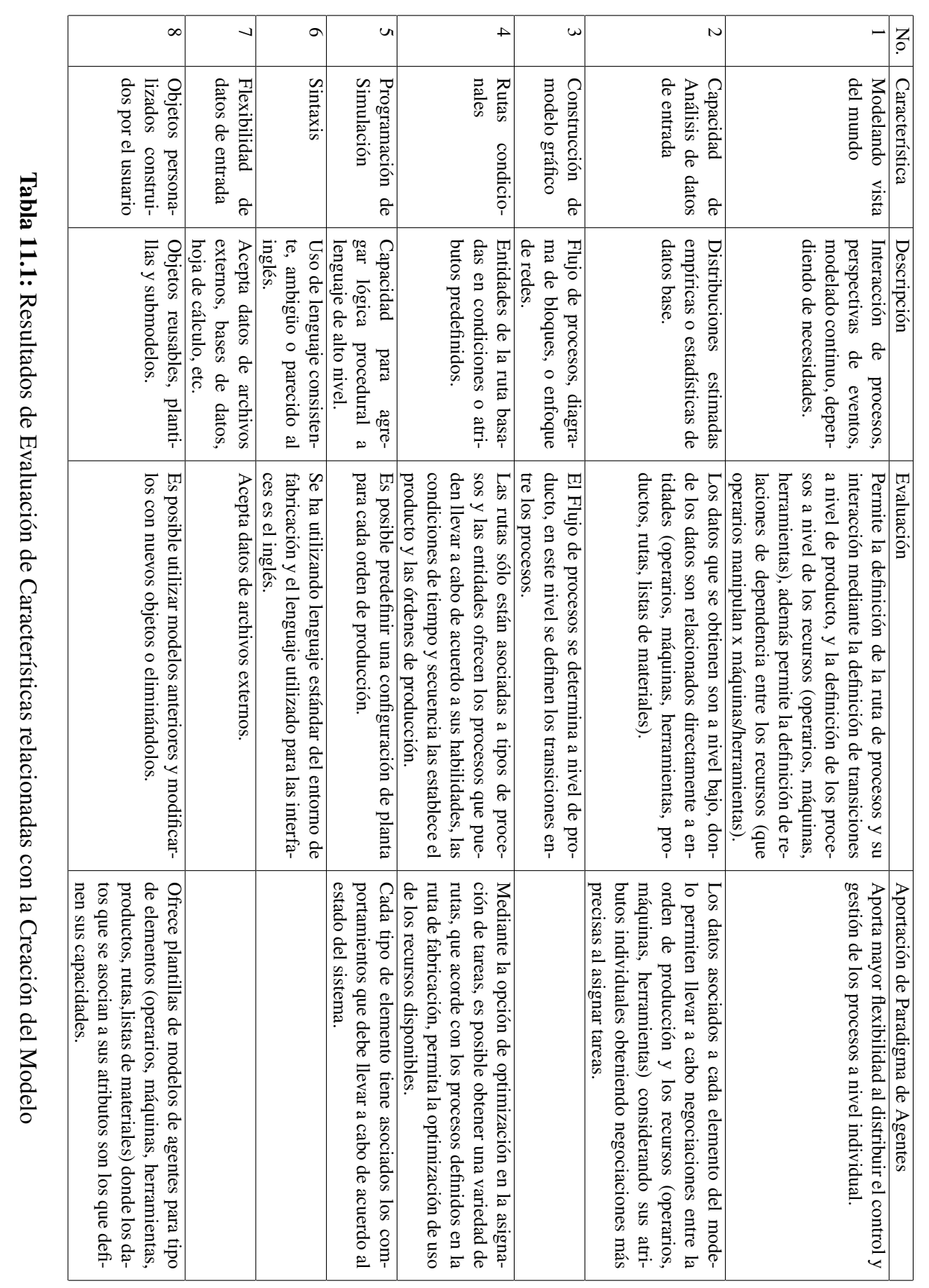




\begin{tabular}{|c|c|c|c|c|}
\hline 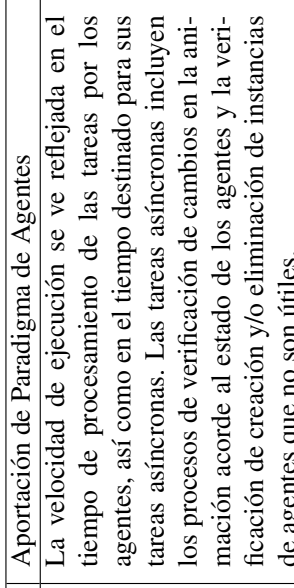 & 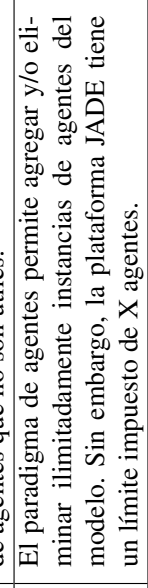 & 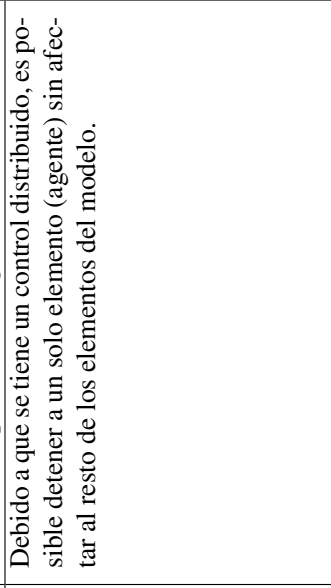 & 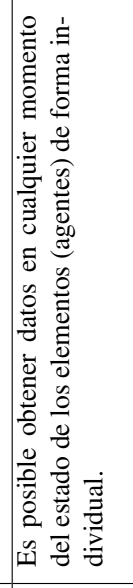 & 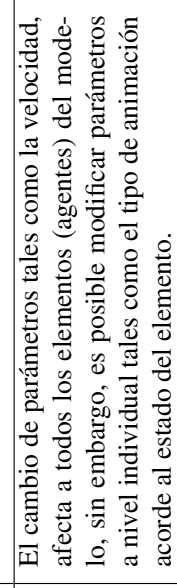 \\
\hline 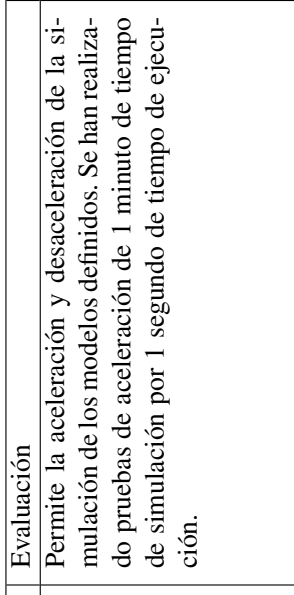 & 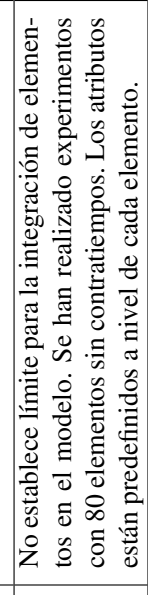 & 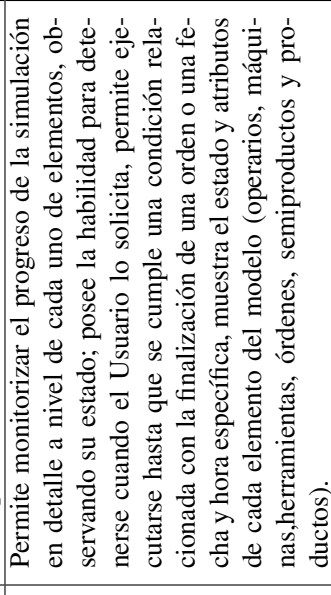 & 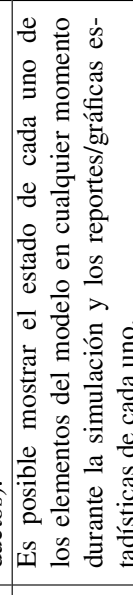 & 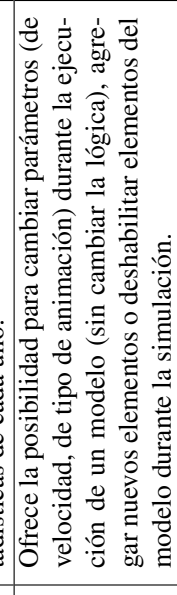 \\
\hline 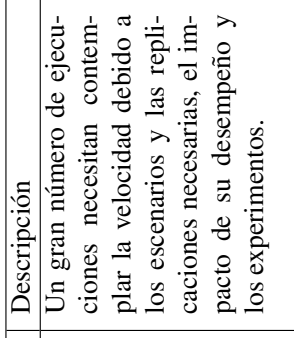 & 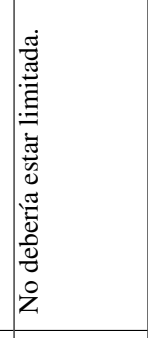 & 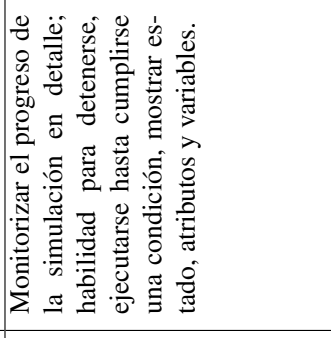 & 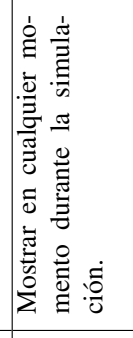 & 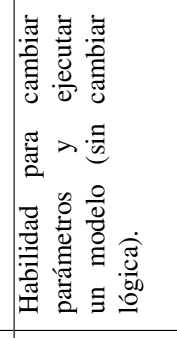 \\
\hline 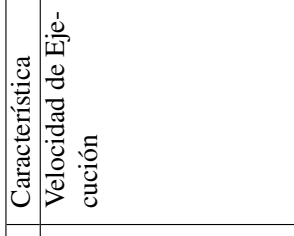 & 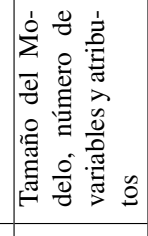 & 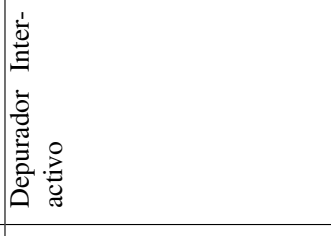 & 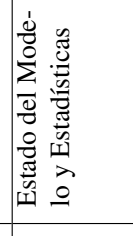 & 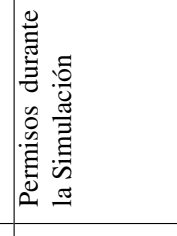 \\
\hline$\left.\dot{\dot{z}}\right|_{-}$ & $\mathrm{N}$ & $m$ & $\rightarrow$ & in \\
\hline
\end{tabular}




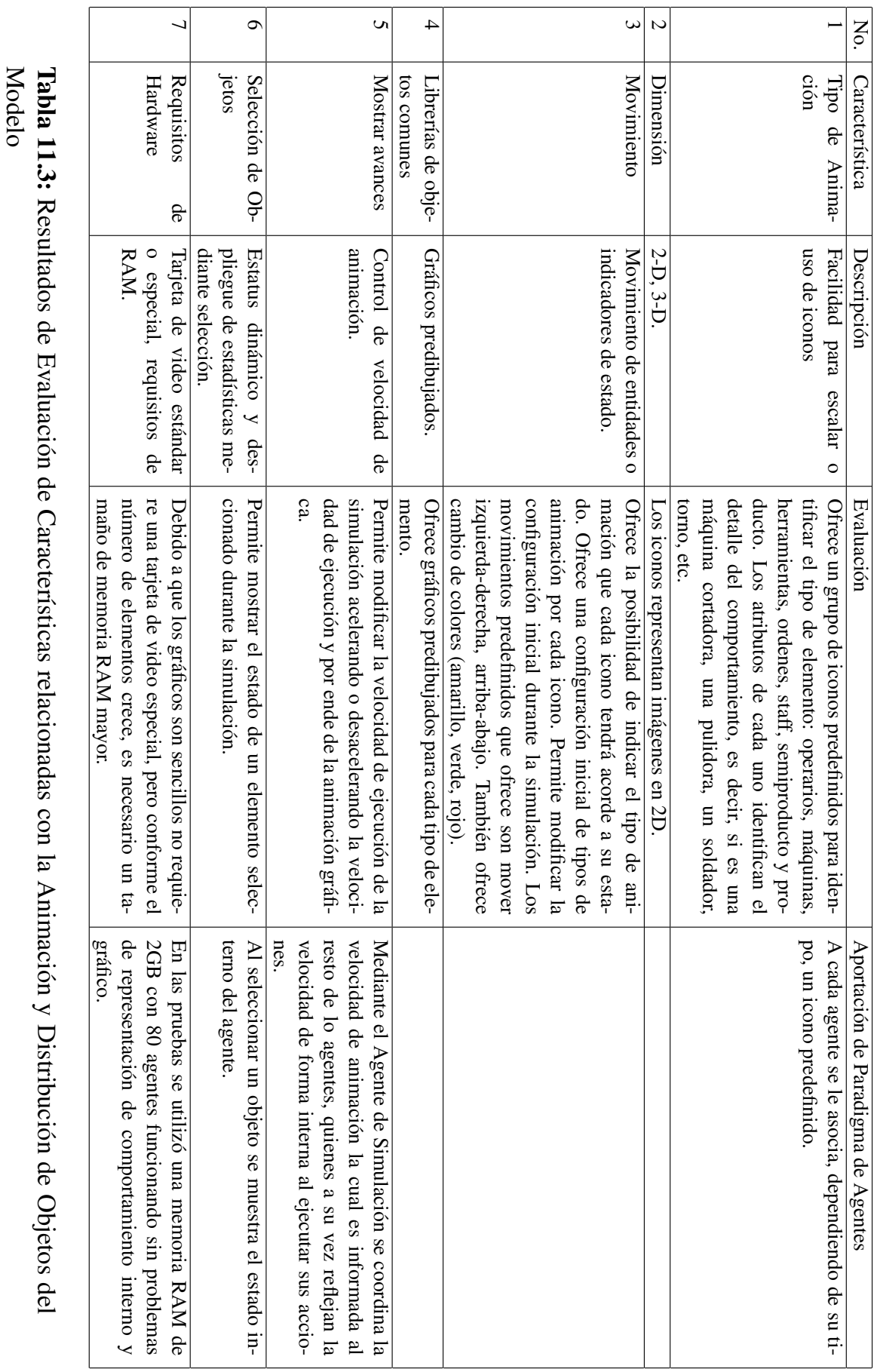




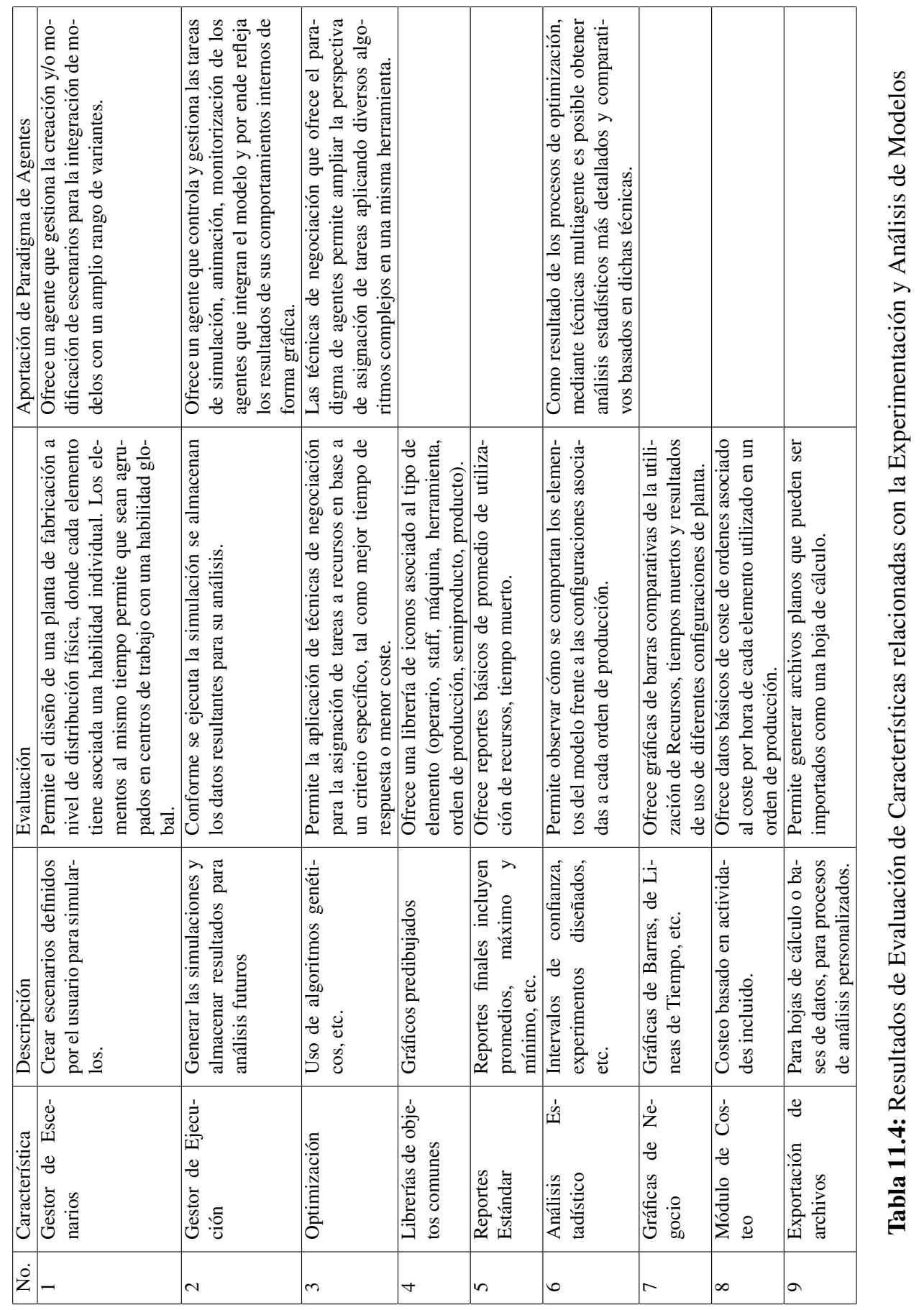




\begin{tabular}{|c|c|c|c|c|}
\hline Area & Característica & Débil & Medio & Fuerte \\
\hline \multirow{8}{*}{$\begin{array}{l}\text { Creación de } \\
\text { Modelo }\end{array}$} & Modelado de vista del mundo & & & $\mathrm{X}$ \\
\hline & Capacidad de análisis de Datos de Entrada & & $\mathrm{x}$ & \\
\hline & Construcción de Modelo Gráfico & & & $\mathrm{x}$ \\
\hline & Rutas Condicionales & & $\mathrm{x}$ & \\
\hline & Programación de Simulación & & $\mathrm{x}$ & \\
\hline & Sintaxis & & & $\mathrm{X}$ \\
\hline & Flexibilidad de Datos de Entrada & & $\mathrm{x}$ & \\
\hline & Objetos personalizados, construidos por el Usuario & $\mathrm{x}$ & & \\
\hline \multirow{5}{*}{$\begin{array}{l}\text { Simulación y } \\
\text { Análisis }\end{array}$} & Velocidad de Ejecución & & & $\mathrm{x}$ \\
\hline & Tamaño del modelo, número de variables y atributos & & & $\mathrm{X}$ \\
\hline & Depurador interactivo & & $\mathrm{x}$ & \\
\hline & Estado del Modelo y Estadísticas & & & $\mathrm{x}$ \\
\hline & Permisos durante la Simulación & & $\mathrm{x}$ & \\
\hline \multirow{7}{*}{ Animación } & Tipo de Animación & $\mathrm{x}$ & & \\
\hline & Dimensión & $\mathrm{x}$ & & \\
\hline & Movimiento & & $\mathrm{X}$ & \\
\hline & Librerías de objetos comunes & & $\mathrm{x}$ & \\
\hline & Mostrar avances & & & $\mathrm{x}$ \\
\hline & Selección de objetos & & & $\mathrm{X}$ \\
\hline & Requisitos de Hardware & & & $\mathrm{x}$ \\
\hline
\end{tabular}

Tabla 11.5: Grado de Fortaleza en las Fases I y II.

de producto). Asimismo, dicho detalle facilita la interacción e integración de los Humanos con el software y el hardware que componen el sistema de fabricación mediante interfaces de fácil manejo.

Tanto durante el modelado como durante la simulación, la arquitectura permite agregar elementos (recursos) al sistema de fabricación para satisfacer las necesidades emergentes en cualquier área de la planta de fabricación. Con ello, se favorece la escalabilidad del sistema.

Las características de inteligencia, comunicación y distribución de control de los elementos del sistema de fabricación, permiten prepararlos para la aparición de fallos. Mediante algoritmos complejos, los agentes pueden tomar decisiones y cooperar para realizar las acciones pertinentes para recuperar y mantener el sistema en operación ágilmente.

\subsection{Conclusiones}

En este capítulo se han presentado los resultados obtenidos al evaluar el prototipo desarrollado con respecto a la funcionalidad que debe ofrecer una herramienta de simulación. Para ello, se han evaluado cuatro áreas: a) la creación de 


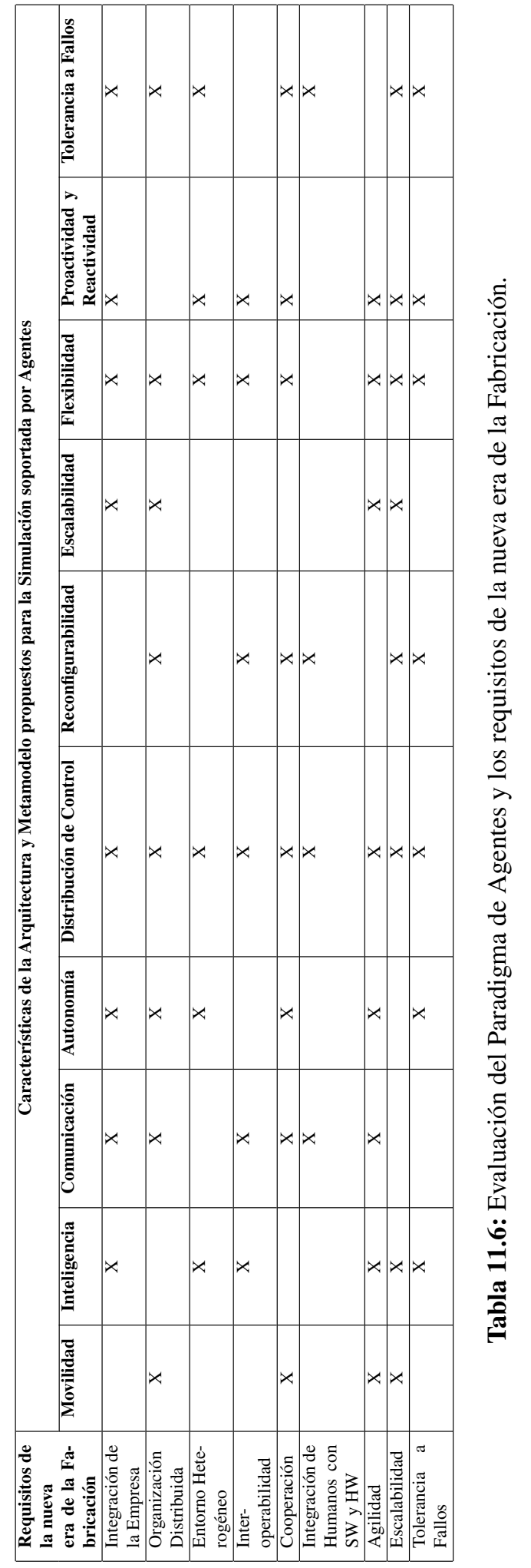


modelos, b) la simulación de modelos, c) la animación y distribución de los elementos del modelo y d) el diseño de experimentos y análisis de los resultados de la simulación de modelos. En cada área se han evaluado características de la funcionalidad de una herramienta de simulación y las mejoras obtenidas usando el paradigma de agentes.

En cuanto a la creación de modelos, el prototipo ofrece un alto grado de flexibilidad que permite el diseño a un nivel de detalle suficiente para definir comportamientos complejos. La interfaz proporcionada guía a un Usuario experto en el dominio de la fabricación para que, aún desconociendo el paradigma de Sistemas Multiagente le sea posible aprovechar las características de la tecnología SMA en la solución de problemas de planificación y control. En general, las herramientas de simulación actuales preparan modelos donde se definen rutas de procesos, las cuales se basan en el uso de funciones de probabilidad (por ejemplo, la distribución de Poisson) para la colocación de trabajos en las colas de los recursos disponibles. Por su parte, la arquitectura que se propone en este trabajo permite que durante la creación del modelo, mediante la definición de las rutas de fabricación a nivel de producto, se prepare al sistema para la ejecución de múltiples combinaciones de rutas de fabricación en la misma simulación. Con ello, se pueden reflejar comportamientos complejos más apegados a las situaciones reales que se presentan en el entorno de los Sistemas de Fabricación.

En la simulación del modelo, el número máximo de elementos del modelo está limitado por la capacidad del hardware y por la plataforma de JADE donde fue ejecutado. A diferencia de algunas de las herramientas de simulación actuales, el paradigma de agentes permite agregar o eliminar elementos durante la simulación del modelo, incorporando una mayor capacidad de forma automática, sin afectar el comportamiento general del Sistema de Fabricación. Esta opción ofrece una mayor flexibilidad cuando se realizan experimentos más complejos (por ejemplo, agregar nuevos equipos cuando se detecta un cuello de botella o inhabilitar un recurso para observar cómo se comporta el sistema ante un fallo inesperado). Asimismo, en el prototipo se puede definir el tipo de animación a nivel individual y cambiarlo antes y durante la simulación. Por un lado, es posible dar seguimiento al estado tanto a nivel general del avance de las ordenes cómo a nivel de cada uno de los recursos que componen el Sistema de Fabricación durante todo el proceso de simulación. Por otro lado, las herramientas de simulación en general, ofrecen reportes pero estos no son visibles mientras que la simulación no finaliza. 
Con respecto a la animación, proporciona iconos básicos que no pueden ser modificados, los gráficos actuales no pueden ser comparados con las herramientas de simulación comerciales que cuentan con diseñadores gráficos especializados para el manejo de imágenes en 3D. Sin embargo, la animación gráfica permite observar el estado de cada uno de los elementos del Sistema así como su detalle. Además, los resultados obtenidos permiten obtener información estadística suficiente para el análisis del comportamiento del sistema de fabricación que se simula. Como parte del trabajo futuro, se contempla la mejora de la interfaz gráfica durante la simulación.

En general, se observa que el prototipo ofrece en gran medida los aspectos globales que debe contener una herramienta de simulación. Asimismo, ofrece mejoras fundamentales para la simulación de comportamientos complejos de un Sistema Inteligente de Fábrica mediante el paradigma de agentes y todo esto de un modo transparente para el Usuario final. Asimismo, se observa cómo al integrar las características de los Sistemas Multiagente en el modelado de los Sistemas de Fabricación es posible cubrir los requisitos de la nueva Fabricación. Las características asociadas a la flexibilidad, la inteligencia, la autonomía, la proactividad y reactividad, y la distribución de control permiten adaptar los modelos a los requisitos de la nueva fabricación. 



\section{Capítulo 12}

\section{Conclusiones y Trabajo Futuro}

En este último capítulo se presentan las principales conclusiones de este trabajo, las líneas futuras de investigación, así como las publicaciones elaboradas como resultado del trabajo de investigación realizado.

\subsection{Aportaciones}

De acuerdo con los objetivos planteados, las principales aportaciones de esta tesis son:

- Definición de una Arquitectura Multiagente para la Simulación de Sistemas de Fabricación Inteligentes.

- Definición de un Metamodelo para el diseño de Sistemas de Fabricación Inteligentes soportado por agentes.

- Implementación de un prototipo para validar tanto el metamodelo como la arquitectura.

El campo de la simulación y su aplicación en los sistemas de fabricación ofrece una gran oportunidad de éxito de la Tecnología de los Sistemas Multiagente a fin de ampliar la visión del comportamiento de un sistema de fabricación. Por tanto en este trabajo hemos partido de la hipótesis de que "La simulación soportada por agentes puede proporcionar opciones de flexibilidad para incluir requisitos de fabricación asociados a la evolución de su entorno y por tanto permite simular escenarios de fabricación más cercanos a los que se presentan en el mundo real". 
Por una parte, se ha realizado un análisis de la teoría de la simulación dirigida por agentes, sus bases, beneficios, inconvenientes, y aplicaciones actuales. Asimismo, se realizó un análisis del proceso de simulación, metodologías, elementos, normas a considerar para la simulación de modelos. Por otra parte, se ha realizado un análisis de la simulación de Sistemas de Fabricación y los Sistemas Multiagente a fin de identificar las aportaciones del paradigma de los Sistemas Multiagente al aplicarlos en un entorno de simulación de una Planta de Fabricación. Tales aportaciones incluyen, la representación de comportamientos complejos, procesos de negociación, aprendizaje, asignación de tareas, control de tiempo en recursos tanto humanos como de equipamiento,etc.

Como resultado hemos definido una arquitectura de simulación que contempla la vinculación de dos Sistemas Multiagente, un Sistema Multiagente que actúa como una Planta de Fabricación Inteligente (basado en un metamodelo) y un Sistema Multiagente que actúa como una Herramienta de Simulación la cual sirve de interfaz entre la Planta de Fabricación y el Diseñador.

Para el modelado del Sistema Multiagente que actúa como un Sistema de Fabricación Inteligente, se utilizó la Arquitectura de Referencia PROSA (Brussel et al. 1998). En el primer Sistema Multiagente (Planta de Fabricación) definimos un Metamodelo que integra los aspectos relacionados con la estructura de la Planta de Fabricación, sus elementos básicos (Operarios, Máquinas, Herramientas y Staff, Productos, Materia Prima, Ordenes de Producción, etc.), características y habilidades de sus elementos, procesos de negociación y aprendizaje, control de las interacciones de sus elementos y su entorno. De este modo, en el modelado del Sistema Multiagente que actúa como Entorno de Simulación se aplicó la Metodología ANEMONA (Botti \& Giret. 2008). En el Segundo Sistema Multiagente, se integraron las funcionalidades básicas de una Herramienta de Simulación más las que aportan los Sistemas Multiagente, para ello, se contemplaron dos fases de operación: el Modelado y la Simulación. Durante la primera Fase se integraron las posibilidades de asistencia durante la creación del modelo así como la verificación de las reglas de modelado a fin de que dicho modelo pudiera actuar como un sistema multiagente en la segunda Fase.

El modelado permite diseñar la distribución física de la Planta de Fabricación y definir la estructura de los productos que pueden ser fabricados en dicha planta (dicha estructura incluye: la lista de materiales, la ruta de fabricación y sus transiciones). La definición del detalle de cada uno de los elementos, así como sus relaciones, permiten definir comportamientos complejos de los elementos de 
un Sistema de Fabricación así como los conceptos básicos que se utilizan en los ERP y MRP para su gestión y control.

La simulación además de representar gráficamente el comportamiento (lanzamiento de ordenes de producción, procesos de negociación, procesamiento de trabajos, etc.) de la Planta de Fabricación (primer Sistema Multiagente). La representación es resultado de la monitorización continua de los agentes de la Planta de Fabricación simulada. Los resultados de la simulación por tanto se utilizan para retroalimentar a los agentes, mediante procesos de aprendizaje (que serán aplicados en simulaciones futuras). Los resultados de la simulación permiten al mismo tiempo generar y mostrar reportes del comportamiento de los agentes de la planta. El nivel de detalle de la definición de los componentes del modelo permite al simulador representar comportamientos complejos propios de un Sistema de Fabricación real.

Con respecto a las herramientas de simulación actuales, al integrar características de flexibilidad, inteligencia, escalabilidad (asociadas a los Sistemas Multiagente) en la definición de la Arquitectura para el Entorno de Simulación, así como en el Metamodelo para el Sistema de Fabricación, la propuesta se vuelve más robusta ante las necesidades cambiantes para la Simulación de los Sistemas de Fabricación.

Con el objetivo de validar los modelos tanto de la Arquitectura del Entorno de simulación como del Metamodelo para un Sistema de Fabricación, se implementó un prototipo. Asimismo, se llevo a cabo la validación de la propuesta utilizando el prototipo con un caso de estudio: un sistema de fabricación de silos. Asimismo, el prototipo permitirá realizar pruebas de los trabajos realizados dentro del Grupo de Investigación relacionados con metodologías y desarrollo de Sistemas de Fabricación (Botti \& Giret. 2008).

El prototipo permite acercar el uso de la tecnología de agentes a Usuarios expertos en el área de Sistemas de Fabricación que no tienen relación con esta tecnología. El entorno proporcionado facilita el uso de herramientas de gestión inteligente, para optimizar el uso de la información proporcionada desde el sistema origen. Aporta mejoras dirigidas a la solución de los problemas con respecto a los requisitos de la nueva era de la fabricación. En consecuencia, se propone dicho entorno como una herramienta de ayuda en la toma de decisiones. El prototipo por si mismo, facilita la definición de comportamientos complejos al experto del dominio de Sistemas de Fabricación mediante el uso de interfaces 
sencillas. Esto facilita al Usuario el modelado y la simulación de las situaciones que se presentan continuamente y de forma espontánea en el Sistema Original.

Por tanto, resumimos las aportaciones de esta tesis como sigue:

- Análisis del proceso de simulación genérico e identificación de elementos clave del proceso de Simulación y de las funcionalidades de un Entorno de Simulación.

- Identificación de los elementos básicos de la Simulación de Sistemas de Fabricación y los comportamientos asociados a estos elementos.

- Evaluación de las Herramientas de Simulación para Sistemas de Fabricación actuales con respecto a los Requisitos de la Nueva Era de la Fabricación. Análisis e identificación de las aportaciones de la Teoría de los Sistemas Multiagente al campo de la Simulación.

- Identificación de las técnicas de Inteligencia Artificial aplicadas en los Sistemas Multiagente para mejorar las características de flexibilidad de los procesos de simulación de modelos de fabricación (creación y simulación).

- Definición de un Metamodelo soportado por Agentes que permite el diseño de modelos de Sistemas de Fabricación Inteligentes que aportan la flexibilidad necesaria para adaptarse a los requisitos de la nueva era de la fabricación.

- Definición de una Arquitectura de Simulación soportada por Agentes que permite la simulación de los comportamientos complejos de los elementos de un modelo de Sistemas de Fabricación Inteligente soportado por agentes.

- Definición de métricas para la evaluación de modelos y para las configuraciones de planta asociados a las ordenes de producción, así como una serie de métricas para la evaluación de los datos de producción generados en las simulaciones.

- Implementación de un prototipo para validar la arquitectura y el metamodelo propuestos.

- Validación de la arquitectura y el metamodelo mediante el uso del prototipo en un caso de estudio: un sistema de fabricación de silos. 


\subsection{Líneas Futuras de Investigación}

Debido al amplio campo de aplicación que ofrece la Simulación y los Sistemas Multiagente ante la representación de Sistemas de Fabricación, se destacan cinco líneas de investigación futuras: a) Integrar una base más amplia para la recomendación de configuraciones de planta, b) Simulación del Comportamiento del Almacén de Materia Prima y Subproductos, c) Integrar como módulo complementario a una Herramienta ERP para la simulación de la planta compartiendo en línea la Base de Datos del ERP. d) Conectar la Herramienta de Simulación a la Planta Física para así transformar la simulación a la representación real del comportamiento de la Planta de Fabricación.

En la primera línea futura, el proceso de recomendación de rutas y configuraciones para la fabricación de un producto es el siguiente paso en el proceso de aprendizaje del entorno de simulación. Como parte del trabajo futuro se pretende incluir las posibilidades de recomendación de configuraciones que puedan basarse en técnicas complejas para la toma de decisiones. Para este apartado se considera la utilización de técnicas para el filtrado de datos, razonamiento basado en casos, la integridad y seguridad de la recomendación y evaluación de recomendaciones. La clasificación basada en la valoración de las recomendaciones permitirá facilitar la retroalimentación al sistema.

Esta línea de trabajo futuro contempla la posibilidad de definir un Sistema de Recomendación. Un Sistema de Recomendación representa las preferencias del Usuario con el propósito de sugerir artículos para su compra o revisión. Una variedad de técnicas han sido propuestas para su aplicación en estos sistemas. Las técnicas incluyen las basadas en conocimiento, basadas en contenido, entre otras (Burke 2002). Los sistemas de recomendación utilizan las opiniones de los miembros de la comunidad para brindar soporte de forma individual en la identificación de información o productos que pudieran ser de su interés o relevante para sus necesidades (Konstan 2004). Las bases para nuestra propuesta se basan en considerar por una parte las calificaciones obtenidas para cada una de las configuraciones de planta a nivel de producto como el conjunto de las opiniones de los miembros de la comunidad. Y por otra parte se consideran a los objetivos que busca el Usuario cuando crea una orden de producción (tales como: la reducción del coste de producción de un producto o la reducción del tiempo de procesamiento) como las necesidades a cubrir. Sin embargo, en esta fase se integra solo la opción de ofrecer las configuraciones basándonos en las calificaciones obtenidas previamente. De esta forma, es posible recomendar la 
configuración con mejor calificación en cuanto al tiempo de procesamiento, al tipo de instancias, a la configuración con menor coste o mejor calificada por el Usuario.

En la segunda línea futura, se pretende añadir un módulo que permita observar al mismo tiempo el comportamiento del Almacén al interactuar con la Planta de Fabricación. En el Almacén se integrarán las principales estrategias de control de almacén tales como FIFO, control por lote/serie, y la distribución y manejo de materiales.

En la tercera línea, se dirige hacia el primer paso para la integración de la herramienta de simulación como módulo complementario de un ERP de tal manera que la base de datos actual del ERP sirva como soporte para inicializar el estado de la simulación y lanzar órdenes de producción y/o mantenimiento simuladas y evaluar así la capacidad de la planta sin afectar la planificación de órdenes real.

Finalmente, en la cuarta línea se pretende utilizar la Herramienta de Simulación como una Herramienta de Control de la Planta de Fabricación real, donde el Sistema Multiagente tiene comunicación en línea con los elementos físicos de la planta física. De esta forma sería posible al mismo tiempo que se tiene visualmente el control de la planta de fabricación, ejecutar simultáneamente una simulación de estado actual de la planta donde se le someta a situaciones excepcionales tales como: averías de equipos, adición de personal, lanzamiento de nuevas órdenes. Tales situaciones permitirán evaluar por ejemplo, posibles planes estratégicos de ventas futuras, integración de equipo con un alto coste de adquisición, etc.

\subsection{Publicaciones}

En esta sección presentamos las publicaciones que se han elaborado durante la realización de la presente tesis. Las publicaciones están clasificadas en: artículos en revistas, artículos en congresos internacionales, artículos en congresos nacionales y reporte técnico. 


\section{Artículos en revistas:}

- Identifying Roles, Goals and Scenarios at the Early Requirement Phase of Multiagent System Modeling,

Nancy Ruiz, Adriana Giret, Vicente Botti

Revista International Transactions on Systems Science and Applications (ITSSA), Volumen 3 Número 3,

ISSN 1751-1461(Impresa), ISSN 1751-147X(CD-ROM), pags. 249-256

Octubre 2007, Glasgow, Reino Unido.

- Agent-Based Simulator for Industry,

Nancy Ruiz, Adriana Giret, Vicente Botti.

Special edition of the International Journal of Computer Science (INFOCOMP), Ed. UFLA,

ISSN 1807-4545, pags. 35-42,

Febrero, 2007, Lavras, MG, Brasil.

- The Specification of an Agent-Supported Modeling for a Manufacturing System Simulation,

Nancy Ruiz, Adriana Giret, Vicente Botti.

Pendiente de Publicación en Revista International Transactions on Systems Science and Applications (ITSSA), Volumen 4 Número 3, ISSN 1751-1461(Impresa), ISSN 1751-147X(CD-ROM),

Enero 2008, Glasgow, Reino Unido.

- An Intelligent Simulation Environment for Manufacturing Systems, Nancy Ruiz, Adriana Giret, Vicente Botti.

Enviado a IEEE Transactions on Systems,Man and Cybernetics (SMC) Part C: Applications \& Reviews.

\section{Artículos en Congresos Internacionales:}

- Early Requirements Guidelines for Multiagent System Modeling, Nancy Ruiz, Adriana Giret, Vicente Botti Proceedings de IEEE Workshop on Distributed Intelligent Systems-Collective Intelligence and Its Applications (DIS 2006), Ed. IEEE Computer Society, ISBN 13-978-0-7695-2589-1(Impresa), ISBN 0-7695-2589-X (CD-ROM), pags. 183-188, 15-16 Junio 2006, Praga, República Checa 
- Towards an Agent-based Simulation Tool for Manufacturing Systems, Nancy Ruiz, Adriana Giret, Vicente Botti.

Proceedings of the 11th IEEE International Conference on Emerging Technologies and Factory Automation(ETFA 2006), Ed. IEEE Computer Society, ISBN 1-4244-0681-1, pags 703-709, 20-22 Septiembre 2006, Praga, República Checa

- An Agent-Supported Simulation Architecture for Manufacturing Systems, Nancy Ruiz, Adriana Giret, Vicente Botti.

Proceedings of the Agent-Directed Simulation (ADS'07) Part of the 2007 Spring Simulation Multiconference (SpringSim'07), ACM Press, Vol. 1, ISBN 1-56555-313-6, pags. 63-70, Marzo 25-29, 2007, Northfolk, Virginia, USA

- Holonic Architecture for a Multiagent-Based Simulation Tool, Nancy Ruiz, Adriana Giret, Vicente Botti.

Proceedings of the 9th International Conference on Enterprise Information Systems (ICEIS07), ISBN 978-972-8865-89-4, pags. 395-398, Junio 12-16, 2007, Funchal, Madeira, Portugal

- SimIShopF: An Agent-supported Simulation Tool for Intelligent Manufacturing Systems, Nancy Ruiz, Adriana Giret, Vicente Botti.

Pendiente de Publicación en the Agent-Directed Simulation (ADS'09) Part of the 2009 Spring Simulation Multiconference (SpringSim'09), ACM Press, Marzo 22-27, 2009, San Diego, California

\section{Artículos en Congresos Nacionales:}

- Agent-Supported Modeling and Simulation for Manufacturing Systems, Nancy Ruiz, Adriana Giret, Vicente Botti.

CAEPIA, II Workshop on Industrial Application of Distributed Intelligent Systems (INADIS07),

ISBN 978-84-611-8859-8, pags. 63-76,

Noviembre 12-16, 2007, Salamanca, España 
Reporte Técnico:

- An Extended Manufacturing Simulation Survey, Nancy Ruiz, Adriana Giret, Vicente Botti

Reporte Técnico Número DSIC-II/10/06

Departamento de Sistemas Informáticos y Computación, Universidad Politécnica de Valencia,

Abril 2006, Valencia, España 



\section{Bibliografía}

Abbot, C. (1994), Performance measurement in library and information services, ASLIB, London.

ADS, . (2008), 'Agent-directed simulation site'.

*http://www.eng.auburn.edu/ yilmaz/ADS.html

Albayak, S. (1999), Intelligent agents for telecomunication applications, in 'Third International Workshop, IATA '99', Vol. 1699 of Lecture Notes in Computer Science, Springer Verlang.

Albayak, S. \& Garijo, F. (1998), Intelligent agents for telecomunication applications, in 'Second International Workshop, IATA '98', Vol. 1437, Springer Verlang, pp. 413-416.

Albrecht, A. \& Gaffney, J. (1983), 'Software function, source lines of code, and development effort prediction: A software science validation', IEEE Transactions on Software Engineering 9(6).

Aldabi, T. \& Paul, R. (2001), 'Evaluation of tools for modeling manufacturing system design with multiple levels of detail', International Journal of Flexible Manufacturing Systems 13, 163-176.

Alder, M., Davis, A., Weihmayer \& Worrest, R. (1989), 'Conflict resolution strategies for nonhierarchical distributed agents', Distributed Artiificial Intelligence II, 139-162.

Allen, J. F. (1983), 'Maintaining knowledge about temporal intervals', Communications of the ACM 26(11), 832-843.

Alonso, E. \& Kudenko, D. (2001), 'Sistemas lógicos de múltiples agentes: arquitectura e implementación en simuladores de conflictos', Revista Iberoamericana de Inteligencia Artificial (13), 85-93. 
Amigoni, F. \& Somalvico, M. (2003), A multiagent systems for environmental perception, in 'Proceedings of the 3rd Conference on Artificial Intelligence Applications to the Environmental Science', American Meteorological Society.

A.M.Law \& McGomas, M. (1992), 'How to select simulation software for manufacturing applications', Industrial Engineering 24(7), 29-35.

Andreasen, M. \& Mortensen, N. (1998), A design language for synthesis and systematization, in 'Proceedings of the 9th Symposium fur Fertigungsgerechtes Konstruieren', Schnaittach.

Anussornnitisarn, P., Peralta, J. \& Nof, S. (2001), 'Time-out protocol for task allocation in multiagent systems', Journal of Intelligent Manufacturing $13,511-522$.

Arauz, J., Banerjee, S. \& Krishnamurthy, P. (2001), Maite: a scheme for improving the performance of tcp over wireless channels, in 'Vehicular Technology Conference', Vol. 1, pp. 252-256.

Araúzo, J. (2007), Control distribuido de sistemas de fabricaci'on flexibles: un enfoque basado en agentes, PhD thesis, Universidad de Valladolid, Valladolid, España.

AUML (2007), 'Agent auml', http://www.auml.org.

Axtell, R. (2000a), 'Effects of interaction topology and activation regime in several multi-agent systems', Multi-Agent Based Simulation pp. 33-48.

Axtell, R. (2000b), 'Why agents? on the varied motivations for agent computing in the social sciences', Center on Social and Economic Dynamics, Working Paper.

Bagrodia, R., Chandy, K. \& Misra, J. (1987), 'A message-based apporach to discrete-event simulation', IEEE Transactions on Software Engineering 13(6), 664-665.

Bakker, B., Steingrover, M. \& Schouten, R. (2005), Cooperative multi-agent reinforcement learning for traffic lights, in 'Workshop Copoerative Multi-agent Learning, 16th European Conference Machine Learning (ECML05)', Porto,Portugal. 
Balci, O. (1988), The implementation of four conceptual frameworks for simulation modeling in high-level languajes, in 'IEEE Proceedings of the 20th Winter Simulation Conference', ACM / SIGSIM, pp. 287-295.

Balci, O. (1998), Handbook of Simulation:Principles, Methodology, Advances, Applications, and Practice, John Wiley \& Sons Inc., chapter Verification, Validation and Testing, pp. 335-393.

Baldwin, L., Eldabi, T., Hlupic, V. \& Irani, Z. (2000), 'Enhancing simulation software for use in manufacturing', Logistics Information Management 13,.

Bandini, S., Manzoni, S. \& Vizzari, G. (2005), 'A spatially dependent communication model for ubiquitous systems', Environments for multi-agent systems LNCS 3374/2005, 74-90. First international workshop on environment for multi-agent systems.

Banks, J. (1991), Selecting simulation software, in 'The 1991 Winter Simulation Conference', pp. 15-20.

Banks, J. (1996), 'Interpreting simulation software checklist', ORMS Today 22(3), 74-78.

Banks, J. (1998), Handbook of Simulation:Principles, Methodology, Advances, Applications, and Practice, New York: John Wiley and Sons, chapter Principles of Simulation, pp. 3-30.

Banks, J., Aviles, E., Mc-Laughlin, J. \& Yuan, R. (1991), 'The simulator: new member of the simulation family', Interfaces 21(2), 76-86.

Banks, J., Carson, J., Nelson, B. \& Nicol, D. (2001), Discrete-Event System Simulation, Prentice-Hall,Englewood Cliffs,N.J.

Barata, J. \& Camarinha-Matos, L. (2003), 'Coalitions of manufacturing components for shop floor agility - the cobasa architecture', International Journal of Networking and Virtual Organisations 2(1), 50-77.

Barreteau, O., Bousquet, F. \& Attonaty, J. (2001), 'Role-playing games for openning the black box of multi-agent systems: method and lesson of its application to senegal river valley irrigated systems', Journal of Artificial Societies ans Social Simulation 4(2).

*http://jasss.soc.surrey.ac.uk/4/2/5.html 
Batty, M., Desyllas, J. \& Duxbury, E. (2003), 'The discrete dynamics of smallscale spatial events: agent-based models of mobility in carnivals and street parades', International Journal of Geographical Information Science 17(7), 673-697.

Baujard, O., Baujard, V., Aurel, S., Boyer, C. \& Appel, R. (1998), 'Marvin: A multi-agent softbot to retrieve multilingual medical information on the web', Medical Informatics 23(3), 187-191.

Bazzan, A. \& Klügl, F. (2005), 'Case studies on the braess paradox: Simulating route recommendation and learning in abstract and microscopic models', Trasportation Research Part C 13(4), 299-319.

Bazzan, A., Klugl, F. \& Ossowski, S. (2005), 'Agents in traffic and transportation: Exploring autonomy in logistics, management, simulation, and cooperative driving', Guest Editorial, Transportation Research Part C.

Belmonte, M., Pérez, J. \& Triguero, F. (2007), 'Interacción entre agentes: Utilidad, coaliciones y negociación', Revista Iberoamericana de Inteligencia Artificial 2(6), 57-67.

Benenson, I. (1998), 'Multi-agent simulations of residential dynamics in the city', Computers Environment and Urban Systems 22, 25-42.

Bentley, R., Lake, M. \& Shennan, S. (2005), 'Specialisation and wealth inequality in a model of a clustered economic network', Journal of Archeological Science (32), 1346-1356.

Bertalanffy, L. V. (1986), Teoria General de los Sistemas, Fondo de Cultura Economica.

Biemans, F. (1989), A Reference Model for Manufacturing Planning and Control, $\mathrm{PhD}$ thesis, University of Twente, Enschede.

Binmore, K. G. (1987), 'Modelling rational players i', Economics and Philosophy (3), 179-214.

Binmore, K. G. (1988), 'Modelling rational players ii', Economics and Philosophy (4), 9-55.

Biswas, G., Bagchi, S. \& Saad, A. (1995), Holonic planning and scheduling for 
assembly tasks, Technical Report TR CIS-95-01, Center for Intelligent Systems, Vanderbilt University.

Bitici, U., Carrie, A. \& McDevitt, L. (1997), 'Integrated performance measurement systems: an audit and development guide', The TQM Magazine 9(1), 46-53.

Bollacker, K., Lawrence, S. \& Giles, C. (1998), Citeseer: An autonomous web agent for automatic retrieval and identification of interesting publication, in 'Proceedings of the Second International ACM Conference on Autonomous Agents', ACM Press, New York, NY, St. Paul, MN, p. 116.

Boman, M., Davidsson, P., Skarmeas, N. \& Clark, K. (1998), Energy saving and added customer value in intelligent buildings, in 'Proc. Third Int. Conf. Practical Application of Intelligent Agents and Multi-Agent Technology', pp. 505-517.

Bond, A. H. \& Gasser, L. (1988), Readings in Distributed Artificial Intelligence, Morgan Kauffmann.

Bongaerts, L., Monostori, L., McFarlane, D. \& Kádár, B. (1998), Hierarchy in distributed shop floor control, in 'Proc. of the First Int. Workshop on intelligent Manufacturing Systems IMS Europe', Lausanne, pp. 97-113.

Bongaerts, L., Van Brussel, H. \& Valckenaers, P. (1998), Schedule execution using pertubation analysis, in 'Proc. of the IEEE International Confernece on Robotics and Automation (ICRA98)', Leuven, Belgium, pp. 97-113.

Botti, V. \& Giret, A. (2003), 'Aplicaciones industriales de los sistemas multiagente', www.fi.uba.ar/materias/7565/U5-industria.pdf.

Botti, V. \& Giret., A. (2008), ANEMONA. A Multi-Agent Methodology for Holonic Manufacturing Systems, Vol. XVI of Springer Series in Advanced Manufacturing, Springer. 214 p.

Bowling, M. \& Veloso, M. (2002), 'Multiagent learning using a variable learning rate', Artificial Intelligence 2, 215-250.

Breedam, A., Raes, J. \& Velde, K. (1990), 'Segmenting the simulation software market', OR Insight 3(2), 9-12. 
Brooks, R. A. (1991), Intelligence without reason, in J. Myopoulosm \& R. Reiter, eds, 'Proceedings of the 12th international joint conference on artificial intelligence (IJCAI91)', Morgan Kaufmann publishers Inc, Sidney,Australia, pp. 569-595.

Brown, S. (1996), Strategic Manufacturing for Competitiveness Advantage, Transforming Operations From Shop Floor to Strategy, Prentice Hall, London.

Brussel, H., Wyns, J., Valckenaers, P., Bongaerts, L. \& Peeters, P. (1998), 'Reference architecture for holonic manufacturing systems: Prosa', Сотриters in Industry 37(3), 255-274.

Burke, R. (2002), 'Hybrid recommender systems: Survey and experiments', User modeling and user-adapted interaction 12(4), 331-359.

Busetta, P., Rönnquist, R., Hodgson, A. \& Lucas, A. (1999), Jack intelligent agents - components for intelligent agents in java, Technical Report tr9901, AOS.

Busoniu, L., Babuska, R. \& DeSchutter, B. (2008), 'A comprehensive survey of multiagent reinforcement learning', IEEE Transactions on Systems, Man and Cybernetics-PartC 38(2), 156-172.

Bussmann, S. \& Schild, K. (2000), Self-organising manufacturing control: An industrial application of agent technology, in 'Proceedings of 4th International Conference Multi-Agent Systems’, Boston, MA, pp. 87-94.

Bussmann, S. \& Schild, K. (2001), An agent-based approach to the control of flexible production systems, in 'Proceedings of 8th IEEE International Conference on Emerging Technologies and Factory Automation', Vol. 2, Antibes, France, pp. 481-488.

Caglayan, A. \& Harrison, C. (1997), Agent sourcebook, Wiley Computer Publishing, New York.

Calderón, A. \& García, A. (2005), Aplicación de agentes en sistemas de automatización de la fabricación, in 'IX Congreso de Ingeniería de Organización’.

Caputo, F., DiGironimo, G. \& Marzano, A. (2006), A structured approach to 
simulate manufacturing systems in virtual environment, in 'Proceedings of XVIII Congreso International de Ingenería Gráfica'.

Carmorlinga, S. \& BArker, K. (2003), Multiagent Systems Storage Resource Allocation in a Peer-to-Peer Distributed File System.

Carrascosa, C. (2004), Meta-Razonamiento en Agents con Restricciones Temporales críticas, $\mathrm{PhD}$ thesis, Universidad Politécnica de Valencia, Valencia, España.

Carver, N. \& Lesser, V. (1991), A new framework for sensor interpretation: Planning to resolve sources of uncertainty, in 'Proceedings National Conference Aritifical Intelligence', pp. 724-731.

Castelfranchi, C., Falcone, R. \& Marzo, F. (2006), 'Being trusted in a social network: Trust as relational capital', Trust Management 3986/2006, 1932.

Chalupsky, H., Gil, Y., Knoblock, C., Lerman, K., Oh, J., Pynadath, D., Russ, T. \& Tambe, M. (2001), Electric elves: Applying agent technology to support human organizations, in 'Thirteenth Conference on Innovative Applications of Artificial Intelligence (IAAI01)', AAAI Press, Seattle, Washington.

Chan, F. \& Zhang, J. (2001), 'Modelling for agile manufacturing systems', International Journal of Production Research 39(11), 2313-2332.

Chaturvedi, A., Mehta, S., Dolk, D. \& Ayer, R. (2005), 'Agent-based simulation for computational experimentation: Developing an artificial labor market', European Journal of Operational Research 166(3), 694-716.

Chen, L. \& Sycara, K. (1998), Webmate: Personal agent for browsing and searching, in 'Proceedings of the Second International Conference on $\mathrm{Au}$ tonomous Agents', ACM Press, New York, NY, St. Paul, MN, pp. 132139.

Cherkassky, V. \& Mulier, F. (1998), Learning form Data, New York:Wiley.

Chirn, J.-L. \& McFarlane, D. (2000), A holonic component-base approach to reconfigurable manufacturing control architecture, in 'Proceedings of HolonMas00', London. 
Choy, K. \& Lee, W. (2000), 'Task allocation using case-based reasoning for distributed manufacturing systems', Logistics Informtion Management 13(3), 167-176.

Christensen, J. (1994), Holonic manufacturing systems - initial architecture and standards directions, in '1st Euro Conference on HMS', Hannover, Germany.

Christensen, J. (2002), Basic principles of hms architecture, in 'Proceedings HMS International Symposium'.

Cicirello, V. \& Smith, S. (2001), Insect societies and manufacturing, in 'Workshop on Artifical Intelligence and Manufacturing (IJCAI01). Working Notes.', AAAI SIGMAN, Seattle, W.A., pp. 26-32.

Cincotti, S., Focardi, S., Marchesi, M. \& Raberto, M. (2003), 'Who wins? study of long-run trader survival in an artificial stock market', Physica $A$ 324, 227-233.

Cockburn, D. \& Jennings, N. (1996), Foundations of Distributed Artificial Intelligence, Chapter 12, ARCHON: A Distributed Artificial Intelligence System for Industrial Applications, John Wiley and Sons.

Colombo, A., Schoop, R. \& Neubert, R. (2006), 'An agent-based intelligent control platform for industrial holonic manufacturing systems', IEEE Transactions on Industrial Electronics 53(1), 322-337.

Crites, R. \& Barto, A. (1998), 'Elevator group control using multiple reinforcement learning agents', Machine Learning 33(2-3), 235-262.

Cullen, R. (1999), 'Does performance measurement improve organizational effectiveness? a post modern analysis.', Performance Measurement and Metrics 1(1), 9-30.

Dautenhahn, K. (1996), 'Getting to know each other - artificial social intelligence for autonomous robots', Robotics and Autonomous Systems 16, 3333584 .

Davidsson, P. (2000), ‘Agent based social simulation', Journal of Artificial Societies and Social Simulation 5(1). 
Davidsson, P., Henesey, L., Ramstedt, L., Tornquist, J. \& Wernstedt, F. (2005), 'An analysis of agent-based approaches to transport logistcs', Trasportation Research Part C 13(4), 255-271.

Davis, L. \& Williams, G. (1994), 'Evaluation and selecting simulation software using the analityc hierarchy process', Integrating Manufacturing Systems 5(1), 23-32.

Davis, W. J. (1999), Simulation: technolgies in the new millenium, in 'Proceedings of the 1999 Winter Simulation Conference', pp. 141-147.

Deadman, P. (1999), 'Modeling individual behaviour and group performance in an intelligent agent-based simulator of the tragedy of the commons', Journal of Environment Management (56), 159-172.

Deadman, P. \& Gimblett, R. (1999), 'A role for goal-oriented autonomous agents in modeling people-environment interactions in forest creation', Mathematical and Computer Modeling 8(20), 121-133.

Decker, K., Pannu, A., Sycara, K. \& Williamson, M. (1997), Designing behaviors for information agents, in 'Proceedings First Int'l Conference Autonomous Agents', ACM Press, pp. 404-412.

Deen, S. (1994), A cooperation framework for holonic interactions in manufacturing, in 'Proc. of the Second International Working Conference on Cooperating Knowledge Based Systems (CKBS94)', UK, pp. 103-124.

Deen, S. (2003), Agent-Based MAnufacturing - Advances in the Holonic Approach, Springer-Verlang, Heildelberg,Germany.

Diala, D., Sidi-Ali, A., Abderrahman, E. \& Habib, C. (2007), Performance evaluation of manufacturing enterprises processes, in 'International Conference on Service Systems and Service Management 2007', pp. 1-4.

Dilts, D., Boyd, N. \& Whorms, H. (1991), 'The evolution of control architectures for automated manufacturing systems', Journal of Manufacturing Systems 10(1), 79-93.

Drogoul, A., Vanbergue, D. \& Meurisse, T. (2003), 'Multi-agent based simulation: Where are agents', Multi-Agent-Based Simulation III LNAI 2581, 1-15. 3rd International Workshop, MABS 2002, Bologna, Italy,July 2002. Revised Papers. 
ECLIPSE (2005), 'Plataforma de desarrollo'.

*http://www.eclipse.org/

EFNMS (2001), Benchmark definitions, Technical report, European Federation of National Maintenance Societies, Benchmarking Working Group, Copenhagen.

*http://www.efnms.org/efnms/publications/13defined101.doc

Eurescom (2001), 'A specification of a framework for agent oriented workflow management systems', http://www.eurescom.de/p̃ubdeliverables/P800-series/P815/D1/Vol2/P815D1Vol2.doc. Project P185: Communications Management Process Integration Using Software Agents.

Evans, J. (1991), Creative Thinking in the Decision and Management Sciences, South-Western Publishing, Cincinatti, Ohio.

Ferber, J., Michel, F. \& Baez, J. (2005), 'Integrating environments with organizations', Environments for multi-agent systems LNCS 3374/2005, 48-56. First international workshop on environment for multi-agent systems.

Férnandez, A. (2002), Manual y procedimientos de un sistema de calidad iso 9001-2000, Technical Report AS-37/02, Centro para la Calidad en Asturias, Instituto de Fomento Regional, Gijón, España.

Fernandez, F. \& Parker, L. (2001), 'Learning in large cooperative multirobot systems', International Journal Robot Autonomous 16(4), 217-226.

Finin, T., Fritzson, R., Mckay, D. \& McEntire, R. (1994), Kqml as an agent communication language, in 'Proc 3rd Int. Conf. On Information and Knowledge Management'.

FIPA (2001), Fipa interaction protocol library specification, Technical Report XC00025E.

Fishman, G. (1978), Conceptos y Métodos de la Simulación digital de Eventos Discretos, México: Limusa.

Fishwick, P. (1997), 'Computer simulation: growth through extensions', Transactions of the Society for Computer Simulation International 14(1), 13-23. 
Fletcher, M., Garcia-Herreros, E., Chritensen, J., Deen, S. \& Mittmann, R. (2000), An open architecture for holonic cooperation and autonomy, in 'Proc. of HoloMAS 2000,IEEE Computer Society', pp. 224-230.

Franco, M. \& Bourne, M. (2003), 'Factors that play a role in "managing through measures", Management Decision 41(8), 698-710.

Gabar, H., Shinohara, S., Shimada, Y. \& Suzuki, K. (2003), 'Experiment on distributed dynamic simulation safety design of chemical plants', Simulation Modelling Practice and Theory (11), 109-123.

Gal, T., Stewart, T. \& Hanne, T. (1999), Multicriteria Decision Making Advances in MCDM Models, Algorithms, Theory and Applications, Vol. 21 of International Series in Operations Research \& Management Science, Springer.

Gans, G., Jarke, M., Alkemeyer, G. \& Schmitz, D. (2005), 'Deliberation in a metadata-based modeling and simulation environment for interorganizational networks', Information Systems (30), 587-607.

Garijo, F. (2005), Telecomunicaciones. capítulo 6, in D. F. Aragón, ed., 'Agentes Software y Sistema Multiagente', Pearson, Prentice Hall, pp. 241-253.

Garijo, F., Tous, J., Corley, S. \& Tesselaar, M. (1998), 'Development of multiagent system for cooperative work with network negotiation capabilities', Lecture notes in Artificial Intelligence 1437, 222-232.

Gaud, N. (2007), Systèmes multi-agents holoniques : de l'analyse à l'implantation, PhD thesis, Université de Technologie de BelfortMontbéliard, Belfort-Montbéliard, Francia.

Gaussier, P., Moga, S., Banquet, J. \& Quoy, M. (1998), 'From perception-action loops to imitation processes: A bottom-up approach of learning by imitation', Applied Artificial Intelligence 12(7-8), 701-727.

Geuder, D. (1995), Object oriented modeling with simple ++, in 'Proc. of Winter Simulation Conference', pp. 534-540.

Gilbert, N. \& Troitzch, K. (2005), Simulation for the Social Scientist, 2nd edn, Open University Press. 
Giret, A. \& Botti, V. (2004a), 'Holons and agents', Journal of Intelligent Manufacturing 11(5), 645-659.

Giret, A. \& Botti, V. (2004b), 'Towards an abstract recursive agent', Integrated Computer-Aided Engineering 11(2), 165-177.

Gordillo, J., Giret, A. \& Botti, V. (2005), Distribución de órdenes de trabajo mediante algoritmos de asignación de recursos utilizando sistemas multiagentes, in 'Reunión de Otoño comunicaciones, computación, electrónica y exposición industrial ROC\&C' 2005', IEEE Sección México, pp. 116-119.

Gordillo, J., Giret, A. \& Botti, V. (2006), Implementación de algoritmo de equilibrio de temperatura en sistemas multiagentes para manufactura, in 'Reunión de Otoño comunicaciones, computación, electrónica y exposición industrial ROC\&C' 2006', IEEE Sección México, Acapulco,México, pp. 116-119.

Gouaïch, A., Michel, F. \& Guiraud, Y. (2005), 'Mic: A deployment environment for autonomous agents', Environments for multi-agent systems LNCS 3374/2005, 109-126. First international workshop on environment for multi-agent systems.

Grimes, G., Chalmes, S., Edwards, P. \& Preece, A. (2003), Granitenights - a multi-agent visit scheduler utilising semantic web technology, in 'Proceedings of the Conference on IA 2003'.

Hallé, S. \& Chaib-draa, B. (2005), 'A collaborative driving system based on multiagente modelling and simulations', Trasportation Research Part C 13(4), 320-345.

Hamilton, C. \& Tumay, K. (1997), Simulation Made Easy: A Manager's Guide, CRC Press, Boca Raton,Fl.USA.

Hammann, J. \& Markovitch, N. (1995), Introduction to arena [simulation software], in 'Simulation Conference Proceedings', Arlington, VA, USA, pp. 519-523. 
Hannebauer, M. \& Müller, S. (2001), Distributed constraint optimization for medical appointment scheduling, in 'Proceedings of 5th International Conference on Autonomous Agents', ACM Communications, Montreal, Quebec, Canada.

Hanneman, R. \& Patrick, S. (1997), 'On the uses of computer-assisted simulation modeling in the social sciences', Sociological Research Online 2(2). *http://www.socresonline.org.uk/socresonline/2/2/5.html

Harell, C. \& Tumay, K. (1996), Simulation Made Easy: A Manager's Guide, Industrial Engineering and Management Press, Norcross,GA.

Hasegawa, T., Gou, L., Tamura, S., Luh, P. \& Oblak, J. (1994), Holonic planning and scheduling architecture for manufacturing, in 'Proc. of the Second International Working Conference on Cooperating Knowledge Based Systems (CKBS94)', UK, pp. 125-139.

Hayashi, H. (1993), The ims international collaborative program, in 'Proc. 24th Int. Symp. Industrial Robots (ISIR)', Tokyo, Japan, pp. 3-6.

Hayes-Roth, B. (1985), 'A blackboard architectures for control', Artificial Intelligence 26(3), 251-321.

Heim, J. A. (1997), Integrating distributed simulation objects, in 'Proceedings of the 1997 Winter Simulation Conference', pp. 532-538.

Helleborg, A., Vizzari, G., Uhrmacher, A. \& Michel, F. (2007), 'Modelign dynamic environments in multiagent simulation', Autonomous Agnet and Multi-Agent Systems 14, 87-116.

Hlupic, V. (1997), 'Simulation software selection using simselect', Simulation 69(4), 231-239.

Hlupic, V. \& Paul, R. (1995a), 'A critical evaluation of four manufatcuring simulators', International Journal of Production Research 33(10), 27572766.

Hlupic, V. \& Paul, R. (1995b), 'Simulation software in manufacturing environments: a users' survey', Journal of Computing and Information Technology - CIT 1(3), 205-212. 
HMS (2004), 'Holonic manufacturing systems consortium web site', http://hms.ifw.uni-hannover.de.

Hodge, B. J., Anthony, W. \& Gales, L. (2003), Teoría de la Organización: un enfoque estratégico, Pearson Educación.

Hoeger, H. \& Jhones, J. (1996), 'Integrating concurrent and conservative distributed discrete event simulators', Simulation for Understanding 67(5), 303-314.

Holder, K. (1990), 'Selecting simulation software', OR Insight 3(4), 19-24.

Holland, J. H. (1992), Adaptation in natural and artificial systems, MIT Press, Cambridge, MA, USA.

Huberman, B. \& Clearwater, S. (1995), A multi-agent system for controlling building environments, in 'Proc. First Int. Conf. Multi-Agent Systems', AAAI Press: Menlo Park, Calif., pp. 171-176.

Hughes, L. (2000), Grounded representations for a robot team, in 'IEEE/RSJ International Conference on Intelligent Robots and Systems (IROS 2000)', Vol. 3, IEEE/IRSJ, Takamatsu, Japan, pp. 2248-2253.

IDS-scheer (2003), 'Geschäftsprozesse simulieren, analysieren und optimieren, aris simulation', IDS Scheer, white paper edition.

IMTR (1998), 'Integrated manufacturing technology roadmap for modeling and simulation', Modeling and Simulation Workshop Group and IMTR Roadmapping Project Team, Oak .

Ishiwaka, Y., Sato, T. \& Kakazu, Y. (2003), 'An approach to the pursuit problem on a heterogeneous multiagent system using reinforcement learning', International Journal Robot Autonomous 43(4), 245-256.

Itami, R. \& Deadman, P. (2000), 'Intelligent recreation agents in a virtual gis world', Complexity international $\mathbf{8}$.

Iwata, K. \& Onosato, M. (1995), 'A modeling and simulation architecture for virtual manufaturing systems', CIRP Annals 44(1), 399-402.

JADE (2005), 'Java agent development framework' .

*http://jade.tilab.com 
Jagannathan, V., Dodhiawala, R. \& Baum, L. (1989), Blackboard Architectures and Applications, Academic Press.

Jaso, C. (2004), El impacto de compranet en el gobierno electrónico en méxico, in 'EU-LAT Workshop on e-Government and e-Democracy'.

JAVA (2005), 'Lenguaje de desarrollo'.

*http://www.java.com/

Jennings, N. (1994), 'Cooperation in industrial multi-agent systems', World Scientific Series in Computer Science $\mathbf{4 3 .}$

Jennings, N., Corera, J. \& Laresgoti, I. (1995), Developing industrial multi-agent systems, in 'Proceeding of ICMAS 95', San Franciso,CA, pp. 423-430.

Jennings, N. R. (2000), 'On agent-based software engineering', Artificial Intelligence Journal .

Jennings, N. R. \& Wooldridge, M., eds (1998a), Agent Technology. Foundations, Applications and Markets, Springer-Verlang.

Jennings, N. R. \& Wooldridge, M., eds (1998b), Aplications of intelligent agents, Springer-Verlang.

Julian, V. \& Botti, V. (2003), 'Estudio de métodos de desarrollo de sistemas multiagente', Inteligencia Artificial, Revista Iberoamericana de Inteligencia Artificial (18), 65-80.

Julián, V., Rebollo, M. \& Carrascosa, C. (2001), 'Agentes de información', Revista Base, ISSN 1135-0695 (37), 56-62.

Kádár, B., Monostori, L. \& Szelke, E. (1998), 'An object oriented framework for developing distributed manufacturing architectures', Journal Intelligent Manufacturing 9(2), 173-179.

Klingstam, P. \& Gullander, P. (1999), 'Overview simulation tools for computeraided product engineering', Computers in Industry 38, 173-186.

Klugl, F., Fehler, M. \& Herrler, R. (2005), 'About the role of the environment in multi-agent simulations', Environments for multi-agent systems LNCS 3374/2005, 127-149. 
Klush, M. (2001), 'Information agent technology for teh internet: a survey', Data and Knowledge Engineering 36(3), 337-372.

Kochhar, A. (1989), Computer simulation of manufacturing systems - 3 decades of progress, in 'Proc. of the third European Simulation Congress', Edinburgh,UK, pp. 3-9.

Koestler, A. (1989), The ghost in the machine, Arkana Books.

Konstan, J. (2004), 'Introduction to recommender systems: Algorithms and evaluation', ACM Trans. Inf. Syst. 22(1), 1-4.

Kraus, S. (1997), 'Negotiation and cooperation in multi-agent environments', Artificial Intelligence (94), 79-97.

Krothapalli, N. \& Deshmukh, A. (1999), 'Design of negotiation protocols for multi-agent manufatcuring systems', International Journal of Production Research 37(7), 1601-1624.

Kuljis, J. (1996), Hci and simulation packages, in 'Proc. of the 1996 Winter Simulation Conference', California,USA, pp. 687-694.

Lander, S. \& Lesser, V. (1997), 'Sharing meta-information to guide cooperative search among heterogeneous reusable agents', IEEE Transactions Knowledge and Data Engineering 9(2), 193-208.

Larsen, M., Bilberg, A. \& Kirkby, P. (1997), Cals in denmark- danish sme's exhange of information throughout the product lifecyle, in 'Proceedings of the 8th International Conference and Exhibition on CALS and Electronic Commerce in Europe, CALS Europe 97', Frankfurt/M., Germany, pp. 196-208.

Larsen, M., Kirkby, L. \& Verterager, J. (1999), Alignment of product models and product state models - integration of the product lifecycle phases, in 'The 11th CALS Expo International \& 21st Century Commerce 1998 Conference Long Beach, USA,October, Proceedings of the 7th Mediterranean Conference on Control and Automation (MED99', Haifa, Israel, pp. 1990-2006.

Larsen, M., Sorensen, C. \& Langer, G. (2001), 'Development of a production meta product state model', Computers in Industry 46(3), 275-287. 
Larsson, J. \& Hayes-Roth, B. (1998), 'Guardian: An intelligente autonomous agent for medical monitoring and diagnosis', IEEE Intelligent Systems pp. 58-64.

Law, A. \& Haider, S. (1989), 'Selecting simulation software for manufacturing applications:practical guidelines and software survey', Industrial Engineering 34, 33-46.

Law, A. \& Kelton, W. (1991), Simulation Modelling and Analysis, McGraw-Hill, New York, N.Y.

Law, A. M. \& McComas, M. G. (1997), Simulations of manufacturing systems, in 'Proc. of the 1997 Winter Simulation Conference', pp. 86-89.

Law, A. M. \& McComas, M. G. (1999), Simulations of manufacturing systems, in 'Proc. of the 1999 Winter Simulation Conference', pp. 56-59.

Lee, G. (1997), 'Reconfigurability consideration design of components and manufacturing systems', International Journal of Advanced Manufacturing Technology 13, 376-386.

Lee, J. \& Oo, J. (2002), A multi-agent q-learning framework for optimizing stock trading systems, in '13th International Conference Database Expert Systems', Aix-Provence, France.

Lesser, V. (1999), 'Cooperative Multiagent systems: A Personal View of the State of Art', IEEE Transactions on Knowledge and Data Engineering 11(1), 133-142.

Lieberman, H. (1995), Letizia: An agent that assist web browsing, in 'Proceedings of the International Joint Conference on Artificial Intelligence', Vol. 1, Montreal, pp. 924-929.

Ligtenberg, A., Wachowicz, M., Bregt, A., Beulens, A. \& Kettenis, D. (2004), 'A design and application of multi-agent system for simulation of multiactor spatial planning', Journal of Environmental Management 72, 4355 .

Lin, E., Minis, L., Nau, D. \& Regli, W. (1994), Virtual manufacturing user workshop, Technical report, Lawrence Associates Inc. 
Lin, G. \& Solberg, J. (1992), 'Integrated shop floor control using autonomous agents', IEEE Transactions 24(3), 57-71.

Lin, G. \& Solberg, J. (1994), 'Autonomous control for open manufacturign systems', Computer control of flexible Manufacturing Systems pp. 169205.

Lind, J. (1999), 'A process model for the design of multi-agent systems', Lecture Notes in Computer Science.

Luke, S., Cioffi-Revilla, C., Panait, L. \& Sullivan, K. (2004), Mason: A new multi-agent simulation toolkit, in 'Proceedings of the 2004 SwarmFest Workshop'.

Mackulak, G., Savory, P. \& Cochran, J. (1994), 'Asertaining important features for industrial simulation environments', Simulation 63, 211-221.

Magnusson, J. \& Holm, T. (1996), 'The product model - cals technical goal', Forsvarets Materielverk.

Marík, V., Vrba, P., Maturana, F. \& Hall, K. (2005), Rockwell automation agents for manufacturing, in 'Proceedings of the fourth International join conference on Autonomous Agents and Multiagent Systems', pp. 107-113.

Mas Colell, A. (1997), 'Bargaining games', Cooperation: Game-Theoretic Approaches. Springer,NATO ASI Series .

Mason, C. \& Jonhson, R. (1989), 'Datms: A framework for distributed assumption based reasoning', Distributed Artiificial Intelligence 2, 239-317.

Mataric, M. (1996), Adaptation and Learning in Multi-Agent Systems, SpringerVerlang, New York, chapter 10, Learning in multi-robot systems, pp. 153-163.

Maturana, F. \& et. al. (2002), Using dynamically created decision-making organisation (holarchies) to plan, commit and execute control tasks in a chilledwater system, in 'Proceedings of the 3rd International Workshop on Industrial Applications of Holonic and Multi-Agent Systems', IEEE Computer Society, Aix-en-Provence,France, pp. 613-619.

Maturana, F. \& Norrie, D. (1996), 'Multi-agent mediator architecture for distributed manufacturing', Journal of Intelligent Manufacturing 7, 257-270. 
MCLean, C. \& et. al. (2003), Simulation standards: Current status, needs, and future directions, in 'Proceedings of the 2003 Winter Simulation Conference', pp. 2019-2026.

MCLean, C. \& Leong, S. (2002a), The expanding role of simulation in future manufacturing, in 'Proceedings of the 2001 Winter Simulation Conference', pp. 1478-1486.

MCLean, C. \& Leong, S. (2002b), A framework for standard modular simulation, in 'Proceedings of the 2002 Winter Simulation Conference', pp. 1613-1620.

Menczer, F. \& Belew, R. (2000), 'Adaptive retrieval agents: Internalizing local context and scaling up to teh web', Machine Learning Journal 39(2/3), 203-242.

Michel, F., Bommel, P. \& Ferber, J. (2002), Simulation interactive distribuée de sma par des sma, in 'Xémes journées Francophones pour $l^{\prime}$ 'Intelligence Artificielle Distribuée et les Systèmes Multi-Agents JFIADSMA 2002 (French workshop on Multi-Agent Systems)', Lille France.

Miller, S. \& Pegden, D. (2000), Introduction to manufacturing simulation, in 'Proc. of the 2000 Winter Simulation Conference', pp. 63-66.

Mills, J. \& Bourne, M. (2005), Performance improvement and resource coordination assessment, operations and global competitiveness, in '12th International Annual EurOMA Conference', Budapest, Hungary.

Moreno, A. (2005), Aplicaciones en medicina. capítulo 6, in D. F. Aragón, ed., 'Agentes Software y Sistema Multiagente', Pearson, Prentice Hall, pp. 203-220.

Moreno, A., V. A. \& Bocio, J. (2001), 'Management of hospital tems for organ transplants using multi-agent systems', Artficial Intelligence in Medicine, Lecture Notes in Computer Science 2101 pp. 413-416.

Morgan, B. (1984), Elements of Simulation, CRC Press.

Moriello, S. (2003), 'Sistemas complejos, caos y vida artificial', Red Científica .

Morris, W. (1967), 'On the art of modelling', Management Science 13(12), 707717. 
Moukas, A. \& Maes, P. (1998), 'Amalthea: An evolving multiagent information filtering and discovery system for the www', invited paper for first issue for the Journal of Autonomous Agents and Multi-Agent Systems .

Mouratidis, H., Giorgini, P. \& Manson, G. (2003), Modelling secure multiagent systems, in 'Proceedings of the Second International joint Conference on Autonomous Agents and Multiagent Systems AAMAS-03', Vol. ISBN:1-58113-683-8, ACM Press, Melbourne, Australia, pp. 859-866.

Mouratidis, H., Manson, G., Giorgini, P. \& Phil, I. (2002), Modelling and agentbased integrated health and social care information system for older people, in 'Proceedings of the Workshop on Agent Applications in Healt Care, 15th European Conference on Artificial Intelligence ECAI 2002', Lyon, France, pp. 1-9.

Muller, J. (1997), ‘The design of intelligence agents', LNAI 1037.

Musselman, K. (1994), Guidelines for simulation project success, in D. S. J.D. Tew, S. Manivannan \& A. Seila, eds, 'Proceedings of the 1994 Winter Simulation Conference', pp. 88-95.

MySQL (2005), 'Gestor de base de datos'. *http://dev.mysql.com/

Nash, J. (1950), 'The bargaining', Econometrica (28), 155-162.

Naylor, T. (1971), Computer Simulation Experiments with Models of Economic Systems, Wiley, Nueva York.

NCR (1995), 'Information technology and manufacturing: A research agenda', Committe to Study Information Technology and Manufacturing .

Neiman, D., Hildum, D., Lesser, V. \& Sandholm, T. (1994), Exploiting metalevel information in a distributed scheduling system, in 'Proceedings of 12th Nat'1 Conference Artificial Intelligence'.

Neyman, A. (1985), 'Bounded complexity justifies cooperation in the finitely repeated prisioner's dilemma', Economic Letters (19), 227-229.

Nii, H. (1986a), 'Blackboard systems: Blackboard application systems, blackboard systems from a knowledge engineering perspective', The AI Magazine 7(3), 82-106. 
Nii, H. (1986b), 'Blackboard systems: The blackboard model of problem solving and the evolution of blackboard architectures', The AI Magazine 7(2), 38-64.

Nikoukaran, J., Hulpic, V. \& Paul, R. (1999), 'A hierachical framework for evaluating simulation software', Simulation Practice and Theory 7(3), 219-231.

Nikoukaran, J. \& Paul, R. (1999), 'Software selection for simulation in manufacturing: a review', Simulation Practice and Theory 7(1), 1-14.

NIST, . (2007), 'Simulation-based manufacturing interoperability standards and testing', http://www.mel.nist.gov/programs/smist.htm. Manufacturing Systems Integration Program. Manufacturing Engineering Laboratory of National Institute of Standards and Technology, USA.

Noriega, P., Rodríguez-Aguilar, J. \& Sierra, C. (2005), Aplicaciones en comercio electrónico. capítulo 6, in D. F. Aragón, ed., 'Agentes Software y Sistema Multiagente', Pearson, Prentice Hall, pp. 231-241.

Oates, T., Nagendra, M. \& Lesser, V. (1997), Cooperative information gathering: A distributed problem solving approach, in 'IEE Proceedings Software Engineering, special issue on agent-based systems', Vol. 144.

Odell, J., Parunak, H. \& Bauer, B. (2001), 'Representing agent interaction protocols in uml', Agent-Oriented Software Engineering, Springer-Verlang pp. 121-140.

Okuyama, F., Bordini, R. \& daRochaCosta, A. (2005), 'Elms: An environment description language for multi-agent simulation', Environments for multi-agent systems LNCS 3374/2005, 91-108. First international workshop on environment for multi-agent systems.

Onosato, M. \& Iwata, K. (1993), 'Development of a virtual manufacturing system by integrating product models and factory models', CIRP Annals 42(1), 475-478.

Ossowski, S., Hernán-dez, J., Belmonte, M., Fernández, A., García-Serrano, A., 
Perez-de-la Cruz, J., Serrano, J. \& Trigue-ro, F. (2005), 'Decision support for traffic management based on organisational and communicative multiagent abstractions', Trasportation Research Part C 13(4), 272298.

P. Valckenaers, P., Bonneville, F., Van Brussel, H., Bongaerts, L. \& Wyns, J. (1994), Results of the holonic system benchmark at kuleuven, in 'Proc. of the Fourth Int. Conf. on Computer Integrated Manufacturing and $\mathrm{Au}-$ tomation Technology', Troy, New York, pp. 128-133.

Panurak, H. (1987), 'Manufacturing experience with the contract net', Distributed Artiificial Intelligence pp. 285-310.

Panurak, H. (1996), Implementing manufacturing agents, Workshop report, Sponsored by SFA project of NCMS in conjuntions with PAAM96.

Panurak, H. \& VanderBok, R. (1997), Managing emergent behavior in distributed control systems, in 'Proceedings IAS-TECH/97 Conference', Anaheim, CA.

Paris, D. \& Reeves-Hardcastle, P. (1996), Rapid prototyping using the labview environment, in 'AUTOTESTCON '96, 'Test Technology and Commercialization'. Conference Record', Dayton, OH, pp. 235-238.

Parunak, D. V. H. (1999), Multiagent Systems: A Modern Approach to Distributed Artifical Intelligence, MIT Press, chapter Industrial and Practical Applications of DAI.

Parunak, H. (1998), 'What can agents do in industry, and why?. an overview of industrially-oriented $\mathrm{r} \& \mathrm{~d}$ at cec', Cooperative Information Agents II - Learning, Mobility and Electronic Commerce for Information Discovery on the Internet, Lecture Notes in Computers Science,Springer 1435, 1-18.

Pavard, B. \& Dugdale, J. (2001), 'An introduction to complexity in social science', http://www.irit.fr/cos/training/complexity-tutorial/complexitytutorial.htm.

Pavón, J., Sansores, C. \& Gómez, J. (2008), 'Modelling and simulation of social systems with ingenias', International Journal Agent-Oriented Software Engineering 2(2), 196-221. 
Pegden, C., Shannon, R. \& Sadowski, R. (1995), Introduction to simulation using SIMAN, McGrawHill.

Peiro, J. (1991), Psicologia de la Organizacion. Universidad Nacional de Educacion a Distancia, Ed. Toran, S.A., Boston, Mass.

Peng, Y., Finin, T., Labrou, Y., Chu, B., Long, J., Tolone, W. \& Boughannam (1998), A multi-agent system for enterprise integration, in 'Proceedings of Third International Conference on the Practical Application of Intelligent Agents and Multi-Agents', London.

Pooch, U. \& Wall, J. (1993), Discrete Event Simulation, CRC Press, Boca Raton,Fl.USA.

Pritsker, A. (1998), Handbook of Simulation:Principles, Methodology, Advances, Applications, and Practice, John Wiley \& Sons Inc., chapter Principles of Simulation Modeling, pp. 31-51.

Pritsker, A., Sigal, C. \& Hammesfahr, R. (1989), SLAM II Network Models for Decision Support, Prentice-Hall, Englewood Cliffs, New Jersey.

PROTEGE (2005), 'Editor de ontologias'.

*http://protege.stanford.edu/

Proyecto-Europeo (2000), 'Masfit', http://www.masfit.net. Take-up action IST-2000-28221 EUTIST-AMI, entre IIIA-CSIC(Barcelona), AUTEC S.L.(empresa proveedora de tecnología de salas de subasta tradicionales) y las longas de Tarragona y Villanova i la Geltrú.

Rabelo, R. \& Camarinha-Matos, L. (1994), 'Multi-agent based dynamic scheduling', International Journal on Robotics and Computer Integrated Manufacturing II(4), 303-310.

Rabelo, R., Camarinha-Matos, L. \& Afsar-manesh, H. (1999), 'Multi-agentbased agile scheduling', Journal of Robotics and Autonomous Systems 27(1-2), 15-28.

Raberto, M., Cincotti, S., Focardi, S. \& Marchesi, M. (2001), 'Agent-based simulation of financial market', Physica A 299, 319-327. 
Raju, C., Nrahari, Y. \& Ravikumar, K. (2003), Reinforcement learning applications in dynamic pricing of retail markets, in 'IEEE International Conference E-Commerce (CEC03)', Newport Beach, CA.

Rannanjarvi, L. \& Heikkila, T. (1998), 'Software development for holonic manufacturing systems', Computation Industrial 37(3), 233-253.

Rasmussen, S. \& Barret, C. (1995), Elements of a theory of simulation, in 'European Conference of Artificial Intelligence', Paris, pp. 515-529.

Repast, . (2004), 'Repast,organization for architecture and development'. *http://repast.sourceforge.net/index.html

Righter, R. \& Walrand, J. (1989), Distributed simulation of discrete event systems, in 'Proceedings of the IEEE Simulation Conference', Vol. 77, pp. 99-113.

Rizo, R., Llorens, F. \& Pujol, M. (2002), Agentes Inteligentes: Sistemas Multiagentes y Aplicaciones, Editorial Club Universitario, D. L., chapter Arquitecturas y comunicación entre Agentes, pp. 181-214.

Rohrer, M. (1998), Handbook of Simulation:Principles, Methodology, Advances, Applications, and Practice, John Wiley \& Sons Inc., chapter Simulation of Manufacturing Systems and Material Handling, pp. 519-545.

Rosenschein, J. \& Zlotkin, G. (1994), Rules of Encounter: Designing Conventions for Automated Negotiation among Computers, MIT Press.

Rubinstein, A. (1982), 'Perfect equilibrium in a bargaining model', Econometrica (50), 97-109.

Rubinstein, A. (1986), 'Finite automata play the repeated prisioner's dilemma', Journal of Economic Theory (39), 83-96.

Ruiz, N., Giret, A. \& Botti, V. (2006a), An extended manufacturing simulation survey, Technical Report DSIC-II/10/06, Departmento de Sistemas Informáticos y Computación, Universidad Polit’écnica de Valencia.

Ruiz, N., Giret, A. \& Botti, V. (2006b), Towards an agent-based simulation tool for manufacturing systems, in 'Proceedings de 11th IEEE International Conference on Emerging Technologies and Factory Automation (ETFA06)', IEEE Computer Society, Praga, Czech Republic, pp. 797-804. 
Russell, S. \& Norvig, P. (1995), Artificial Intelligence: A Modern Approach, Prentice Hall International Editions.

Ryoke, M. \& Nakamori, Y. (2005), 'Agent-based approch to complex system modeling', International Journal of Operational Research 166(3), 717725.

Sadeh, N. \& Fox, M. (1989), Cortes:an exploration into micro-opportunistic jobshop scheduling, in 'Proceedings of Workshop on Manufacturing Production Scheduling', Detroit.

Sadowski, R. (1989), The simulation process: Avoiding the problems and pitfalls, in K. M. E.A. MacÑair \& P. Heidelberg, eds, 'Proceedings of the 1989 Winter Simulation Conference’, Washington,D.C., pp. 72-79.

Sansores, C. \& Pavón, J. (2006), 'Agent-based modeling of social complex systems', Current Topics in Artificial Intelligence 4177/2006, 99-102.

Sauter, T. \& Massotte, P. (2001), Enhancement of distributed product systems through software agents, in 'Proceedings of 8th IEEE International Conference on Emerging Technologies and Factory Automation', Vol. 1, Antibes, France, pp. 267-272.

Schaef, A., Shoham, Y. \& Tennenholtz, M. (1995), 'Adaptive load balancing: A study in multi-agent learning', Journal Artificial Intelligence Resources 2, 475-500.

Sen, S. \& Weiss, G. (1999), Multiagent Systems: A Modern Approach to Distributed Artifical Intelligence, Cambridge, MA., MIT: Press, chapter 6. Learning in Multiagent Systems, pp. 259-298.

Shapley, L. (1953), 'A value for n-person games', Contributions to the Theory of Games II, Annals of Mathematics Studies (28), 307-317.

Shen, W. \& Norrie, D. (1999), 'Agent-based systems for intelligent manufacturing: A state-of-art survey’, 1(2), 139-156.

Shen, W., Norrie, D. \& Barthes, J. (2001), Multi-Agent Systems for Concurrent Intelligent Design and Manufacturing, Taylor and Francis, London,UK.

Shen, W., Xue, D. \& Norrie, D. (1998), An agent-based manufacturing enterprise infrastructure for distributed integrated intelligent manufacturing 
systems, in 'Proceedings of Third International Conference on the Practical Application of Intelligent Agents and Multi-Agents', London,UK, pp. 23-25.

Simon, H. (1955), 'A behavioral model of rational choice', Quarterly Journal of Economics (64), 99-118.

Simon, H. (1990), 'Prediction and prescription in systems modeling', Operations Research 38(1), 7-14.

Sislak, D., Rehak, M. \& Pechoucek, M. (2005), A-globe: multi-agent platform with advanced simulation and visualization support, in 'Proceedings of the IEEE/WIC/ACM International Conference on Web Intelligence 2005', pp. 805-808.

Smith, V. (1976), Bidding and Auctioning for Procurement and Allocation, New York University Press, New York, chapter Bidding and Auctioning Institutions: Experimental Results, pp. 43-64.

Stadtler, H. \& Kilger, C. (2002), Supply Chain Management and Advanced Planning. Concepts, Models, Software and Case Studies, 3rd edn, SpringerVerlang Berling Heilderlberg New York, Germany.

Stam, A. \& Kuula, M. (1991), 'Selecting a flexible manufacturing system using multiple criteria analysis', International Journal of Production Research 29(4), 803-820.

Stephan, V., Debes, H. \& Gross, F. (2000), A reinforcement learning bases neural multiagent-systems for control of a combustion process, in 'IEEE-INNS-ENNS, International Joint Conference Neural Newtwork (IJCNN00)', Como, Italy, pp. 6217-6222.

Stone, P. \& Veloso, M. (2000), 'Multiagent systems: A survey from the machine learning perspective', Machine Learning 8(3), 345-383.

Suda, H. (1990), 'Future factory system forumulated in japan', Japanese Journal of Advanced Automation Technology 1-2(1), 67-76,58-66.

Sugawara, T. \& Murakami, K. (1992), . ' multi-agent diagnostic system for internetwork problems", in 'Proceedings INET'. 
Sycara, K., Roth, S., Sadeh, N. \& Fox, M. (1991), Distributed constrained heuristic search, in 'IEEE Trans. Systems, Man, and Cybernetics', number 6, pp. 1,446-1,461.

Tesauro, G. \& Kephart, J. (2002), 'Pricing in agent economies using multiagent q-learning', Autonomous Agents Multi-Agent Systems 5(3), 289-304.

The Mathworks, I. (2006), 'Matlab'.

*http://www.mathworks.es/access/helpdesk/help/techdoc/matlab.html

Thoman, D., Bain, J. \& Antle, C. (1969), 'Inference on the parameters of the weibull distribution', Technometrics 11(3), 445-460.

Tisue, S. \& Wilensky, U. (2004), Netlogo: A simple environment for modeling complexity, in 'International Conference on Complex Systems', Boston, MA.

Tonshoff, H., Seilonen, I., Teunis, G. \& Leitao, P. (2000), A mediator-based approach for decentralised production planning, scheduling and monitoring, in 'Proceedings of 2nd CIRP International Seminar on Intelligent Computation in Manufacturing Engineering', Capri, Italy, pp. 113-118.

Troitzsch, K. (2000), 'Methods of empirical social research', SICSS Summer School.

Tsu, W. \& Soo, V. (2001), Market performance of adaptive trading agents in synchronous double auctions, in "4th Pacific Rim International Workshop Multi-Agent Intelligent Systems: Specification Modelling Applications', Taipei, Taiwan.

Valckenaers, P., Heikkilá, T., Baumgaertel, H., McFarlane, D. \& Courtois, J. (1999), Towards a novel manufacturing control principle, in 'Proceedings of the Second International Workshop on Intelligent Manufacturing Systems', Leuven, Belgium, pp. 871-875.

Van Brussel, H., Valckenaers, P., Wyns, J., Bongaerts, L. \& Detand, J. (1996), Holonic manufacturing systems and iim, in 'IT and Manufacturing Partnerships, Conference on Integration in Manufacturing,', Galway,Ireland, pp. 185-196.

Veeramani, D. \& Wang, K.-J. (1997), 'Performance analysis of auction-based 
distributed shop-floor schemes form the perspective of the communication system', The International Journal of Flexible Manufacturing Systems 9, 121-143.

Veeramani, D., Wang, K.-J. \& Rojas, J. (1998), 'Modeling and simulation of auction-based shop-floor control using parallel computing', IIE Transactions 30(9), 773-783.

Vincent, S. (1998), Handbook of Simulation:Principles, Methodology, Advances, Applications, and Practice, John Wiley \& Sons Inc., chapter Input Data Analysis, pp. 55-92.

Vivacqua, A. \& Lieberman, H. (2000), Agents to assist in finding help, in 'Proceedings of the ACM Conference on Humans Factors in Computing Systems CHI2000', ACM Press, pp. 65-72.

von Neumann, J. \& Morgenstern, O. (1947), The Theory of Games and Economic Behavior, 2nd edn, Princeton University Press, Princeton, USA.

Vrba, P. \& Marík, V. (2005), 'Simulation in agent-based manufacturing control systems', Systems, Man and Cybernetics, IEEE Conference on 2, 17181723.

Wagner, J. \& Hollenbeck, J. (2004), Comportamiento Organizativo, Thomson.

Wartha, C., Peev, M., Borshchev, A. \& Filippov, A. (2002), Decision support tool-supply chain, in 'Proceedings of the Simulation Conference Winter 2002', Vol. 2, pp. 1297-1301.

Weibull, J. (1995), Evolutionary Game Theory, MIT Press, Cambridge, MA, USA.

Weiss, G. (1999), Multiagent Systems: A modern Approach to distribuited Artificial Intelligence, MIT Press.

Wiering, M. (2000), Multi-agent reinforcement learning for traffic light control, in '17th International Conference Machine Learning (ICML00)', Stanford,CA, pp. 1151-1158.

Wooldridge, M. (2002a), An Introduction to Multiagent Systems, WileyInterscience Publication. 
Wooldridge, M. (2002b), An Introduction to Multiagent Systems, Jonh Wiley and Sons.

Wooldridge, M. \& Jennings, N. R. (1995), 'Intelligent agents: Theory and practice', The Knowledge Engineering Review 10(2), 115-152.

Yan, X.-T., Borg, J. \& Juster, N. (2001), 'Concurrent modelling of component and realization systems to support proactive design for manufacture/assembly', Proceedings of the Institution of Mechanical Engineers, Part B 21(5), 1135-1141.

Yilmaz, L. \& Tuncer, I. (2007), Agent-directed simulation systems engineering, in 'Proceedings of the 2007 Summer Computer Simulation Conference', Society for Computer Simulation International, San Diego, California, pp. 897-904.

Yu, B. \& Popplewell, K. (1994), 'Metamodels in manufacturing: a review', International Journal of Production Research 32(4), 787-796.

Zeigler, B., Praehofer, H. \& Kim, T. (2000), Theory of Modeling and Simulation: Integrating Discrete Event and Continuous Complex Dynamic Systems, Academic Press.

Zeuthen, F. (1930), Problems of Monopoly and Economic Warfare, Routledge \& Kegan, P.

Ziergovel, G., Bithell, M., Washington, R. \& Downing, T. (2005), 'Agent-based social simulation: a method for assesing the impact of seasonal climate forecast applications among smallholder farmers', Agricultural Systems 83, $1-26$.

Zlotkin, G. \& Rosenschein, J. (1991), 'Cooperation and conflict resolution via negotiation among autonomous agents in noncooperative domains', IEEE Transanctions on Systems, Man and Cybernetics 21(6), 13171324. 